\title{
Sulfinates from Amines: A Radical Approach to Alkyl Sulfonyl Derivatives via Donor-Acceptor Activation of Pyridinium Salts
}

Jonathan A. Andrews, ${ }^{\text {a }}$ Dr. Loïc R. E. Pantaine, ${ }^{\text {a }}$ Dr. Christopher F. Palmer, ${ }^{b}$ Dr. Darren L. Poole, ${ }^{c}$ and Prof. Michael C. Willis ${ }^{a}{ }^{*}$

${ }^{*}$ michael.willis@chem.ox.ac.uk

a Department of Chemistry, University of Oxford, Chemistry Research Laboratory, Mansfield Road, Oxford, OX1 3TA, UK

${ }^{b}$ Evotec (UK) Limited, 114 Innovation Drive, Milton Park, Abingdon OX14 4RZ, UK

'GlaxoSmithKline Medicines Research Centre, Gunnells Wood Road, Stevenage, SG1 2NY, UK 


\section{Table of Contents}

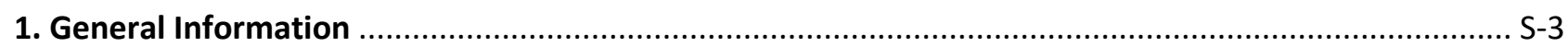

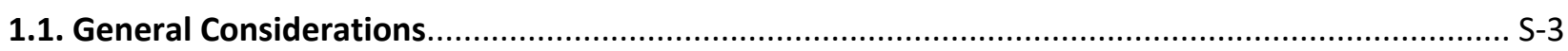

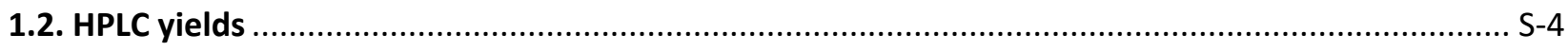

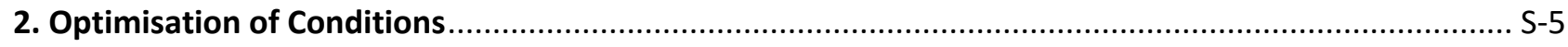

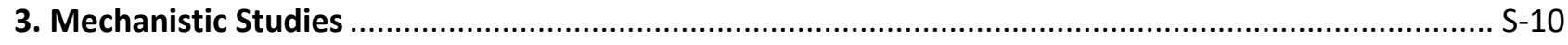

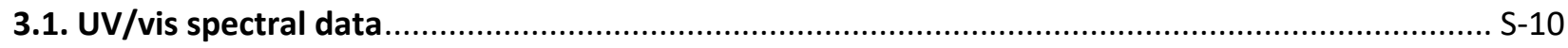

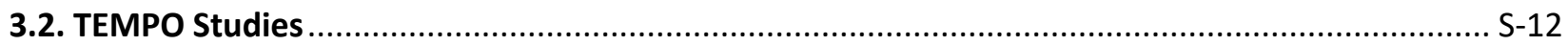

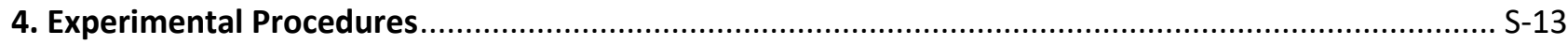

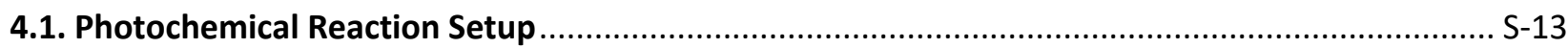

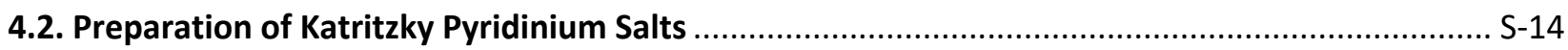

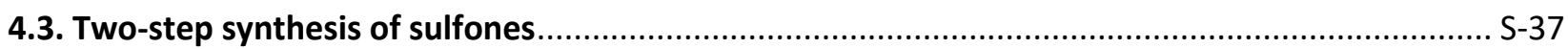

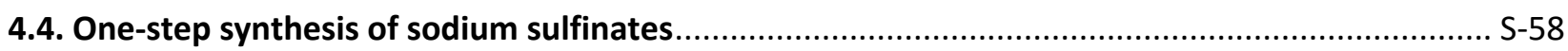

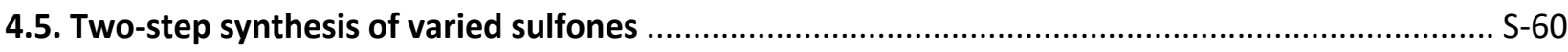

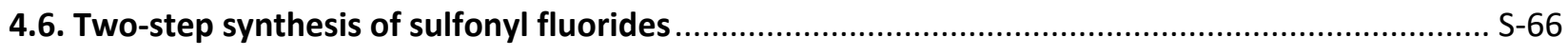

4.7. Two-step synthesis of primary sulfonamides ………................................................................. S-68

4.8. Two-step synthesis of substituted tertiary sulfonamides ….................................................... S-70

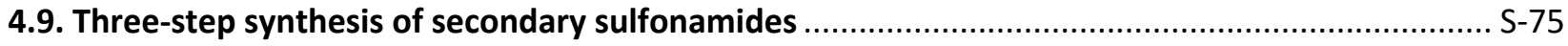

4.10. Characterisation of side-products and TEMPO products ...................................................... S-78

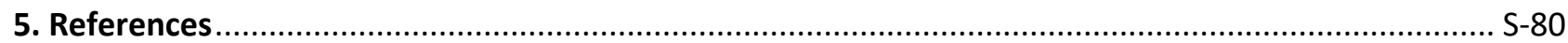

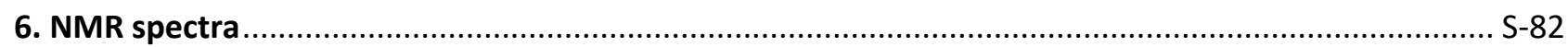

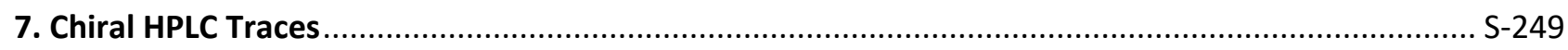




\section{General Information}

\subsection{General Considerations}

All reactions were performed under an inert $\mathrm{N}_{2}$ atmosphere with constant magnetic stirring, unless otherwise stated, using clean, oven dried glassware. All inert gases were sourced from the University of Oxford's internal supplies and dried through a $\mathrm{CaCl}_{2}$ drying columns. Reactions were monitored by thin-layer chromatography (TLC) which was performed on Merck Kieselgel 60 PF254 pre-coated aluminium backed TLC sheets and visualized by UV lamp ( $\lambda=254 \mathrm{~nm}$ and $365 \mathrm{~nm}$ ) and/ or staining using $\mathrm{KMnO}_{4}$, Ninhydrin solution or Hanessian's stain. All chemicals used were obtained from commercial sources, unless otherwise stated, including Sigma Aldrich, Fluorochem, Alfa Aesar and Strem. Dry solvents were obtained from the University of Oxford internal solvent drying system (Innovative Technology Inc. PS-400-7), wherein they are purified through dried alumina columns. Solvents used in the deaminative sulfination were degassed thoroughly by vigorously bubbling a stream of $\mathrm{N}_{2}$ through the solvent for a minimum of 30 mins prior to use. Solvents used for purification, or not needed dry, were reaction grade purchased from either Honeywells or Sigma Aldrich. When referring to the solvent 'Petrol', what is meant is the fractions of petroleum ether which boil within the range $40-60{ }^{\circ} \mathrm{C}$. Flash column chromatography was performed by loading the compound as an oil, a concentrated solution in dichloromethane or DMA onto a slurried column, using Geduran ${ }^{\circledR}$ Si $60,40-63$ micron silica gel, and eluted using the indicated solvent system.

${ }^{1} \mathrm{H}$ NMR spectra were recorded on a Brüker AVIII 400 Ultrashield spectrometer $(400 \mathrm{MHz}) ;{ }^{13} \mathrm{C}$ NMR spectra were recorded on a Brüker AVIII 400 Ultrashield spectrometer (101 MHz) using the ${ }^{1} \mathrm{H}$ decoupling method. ${ }^{19} \mathrm{~F}$ NMR spectra were recorded on a Brüker AVIII 400 Ultrashield spectrometer $(377 \mathrm{MHz})$ using the ${ }^{1} \mathrm{H}$ decoupling method. Acquisitions were carried out at room temperature unless otherwise stated. Chemical shifts $(\delta / \mathrm{ppm})$ are reported in parts per million (ppm) to two decimal places for ${ }^{1} \mathrm{H}$ spectra and one decimal place for ${ }^{13} \mathrm{C}$ and ${ }^{19} \mathrm{~F}$ spectra, and referenced relative to the residual solvent peak; ${ }^{1} \mathrm{H} \mathrm{NMR:} \mathrm{CHCl}_{3}(7.26 \mathrm{ppm})$, ${ }^{13} \mathrm{C} \mathrm{NMR} \mathrm{CHCl}_{3}(77 \mathrm{ppm}) .{ }^{19} \mathrm{~F} \mathrm{NMR}$ spectra were referenced externally to $\mathrm{CFCl}_{3}$. Coupling constants $(J)$ are given in Hertz $(\mathrm{Hz})$ and rounded to the nearest $0.5 \mathrm{~Hz}$. Proton multiplicity is assigned using the following abbreviations: singlet $(\mathrm{s})$, doublet $(\mathrm{d})$, triplet $(\mathrm{t})$, quartet $(\mathrm{q})$, quintet $(\mathrm{p})$, multiplet $(\mathrm{m})$, broad $(\mathrm{br})$, apparent (app.).

Low-resolution mass spectra were recorded on a Waters LCT Premier spectrometer (ESI) using EtOH: water: FA (90:10: 0.1). High resolution mass spectra were obtained via the University of Oxford in-house service, utilising using either a Bruker Daltronics microTOF spectrometer under electrospray ionisation conditions (ESI) or on a Waters LCT Premier spectrometer under the conditions of chemical ionisation (CI). Samples for mass spectra were prepared as $1 \mathrm{mg} / \mathrm{mL}$ solution in MeCN or MeOH (LRMS, HRMS-ESI) or submitted neat (HRMS-Cl). Values quoted are a ratio of mass to charge in Daltons to one decimal place for the low resolution spectrum and to four decimal places on the high resolution. For high resolution mass spectra, the mass found was compared to the mass calculated from the monoisotopic molecular formula, and all results were found to be within a 5 ppm error of the calculated values. 
Melting point values were measured using a Reichert melting point apparatus or a STUART Scientific Melting Point Apparatus SMP1 scientific hot-stage microscope, and are reported uncorrected. Infrared spectra were determined neat using a Brüker Tensor 27 FT-IR spectrometer with an internal range of $600-4000 \mathrm{~cm}^{-1}$, and all absorptions are given in wavenumbers $\left(\mathrm{cm}^{-1}\right)$.

Chiral SFC (supercritical fluid chromatography) separations were conducted on a Waters Acquity UPC2 system using Waters Empower software. Chiralpak ${ }^{\circledR}$ columns $(150 \times 3 \mathrm{~mm}$, particle size $3 \mu \mathrm{m})$ were used as specified in the text. Solvents used were of HPLC grade (purchased from Fisher Scientific, Sigma Aldrich or Rathburn).

\subsection{HPLC yields}

The HPLC data was obtained from an Agilent Technologies 1200 series HPLC Hypersol ODS, $5 \mu \mathrm{m}$ column (100 x $4.0 \mathrm{~mm}$ ) using an extended 12 min method $\mathrm{H}_{2} \mathrm{O}\left(0.1 \% \mathrm{v} / \mathrm{v} \mathrm{H}_{3} \mathrm{PO}_{4}\right)$ : MeCN (10 - 95\% (9 min) to 100\% (10.5 $\mathrm{min}$ ) to $10 \%(12 \mathrm{~min}))$. HPLC yields for the ligand screening were obtained via a standard calibration curve using authentic desired product and 1,3,5-triisopropylbenzene as an internal standard. Yields were calculated from absorbances at $210 \mathrm{~nm}$. The method was verified by product isolation which gave a result $\pm 2 \%$. 


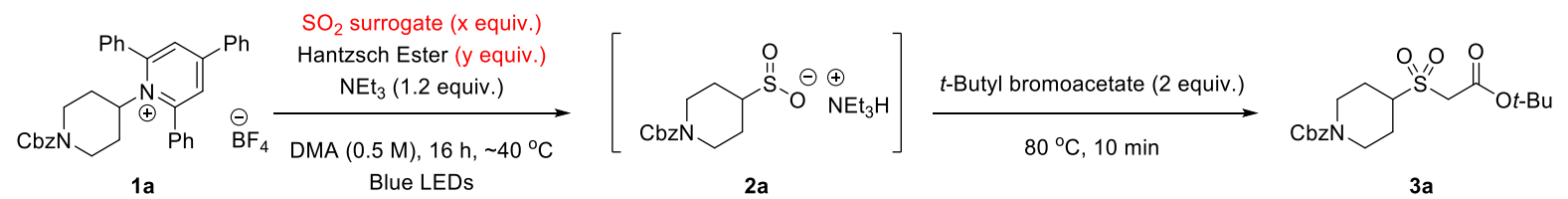

\begin{tabular}{|c|c|c|c|c|c|c|c|c|}
\hline & & \multicolumn{7}{|c|}{$\mathrm{SO}_{2}$ surrogate } \\
\hline & & $\begin{array}{c}\text { DABCO. }\left(\mathrm{SO}_{2}\right)_{2} \\
(0.6 \text { equiv. })\end{array}$ & $\begin{array}{c}\mathrm{Na}_{2} \mathrm{~S}_{2} \mathrm{O}_{5} \\
(1.2 \text { equiv.) }\end{array}$ & $\begin{array}{c}\mathrm{K}_{2} \mathrm{~S}_{2} \mathrm{O}_{5} \\
\text { (1.2 equiv.) }\end{array}$ & $\begin{array}{c}\text { TIMSO } \\
\text { (1.2 equiv.) }\end{array}$ & $\begin{array}{l}\text { NMMSO } \\
\text { (1.2 equiv.) }\end{array}$ & $\begin{array}{l}\text { DMAP.SO }{ }_{2} \\
\text { (1.2 equiv.) }\end{array}$ & $\begin{array}{l}\text { TMEDA. }\left(\mathrm{SO}_{2}\right)_{2} \\
(0.6 \text { equiv. })\end{array}$ \\
\hline \multirow{4}{*}{$\begin{array}{l}\text { Equivalents } \\
\text { Hantzsch } \\
\text { Ester }\end{array}$} & 0 & $0 \%$ & $18 \%$ & - & $24 \%$ & $15 \%$ & $15 \%$ & $15 \%$ \\
\hline & 0.5 & - & $40 \%$ & - & $65 \%$ & - & $47 \%$ & $44 \%$ \\
\hline & 1.1 & $46 \%$ & $62 \%$ & $49 \%$ & $78 \%$ & $81 \%$ & $79 \%$ & $74 \%$ \\
\hline & 3 & $39 \%$ & $31 \%$ & $41 \%$ & $71 \%$ & $60 \%$ & $64 \%$ & $72 \%$ \\
\hline
\end{tabular}

Table 1. Exploring $\mathrm{SO}_{2}$ surrogates and Hantzsch ester loading for secondary alkyl substrate $1 \mathrm{a} .0 .20 \mathrm{mmol}$ scale, yields of 3a calculated from HPLC analysis using 1,3,5-triisopropylbenzene as an internal standard.

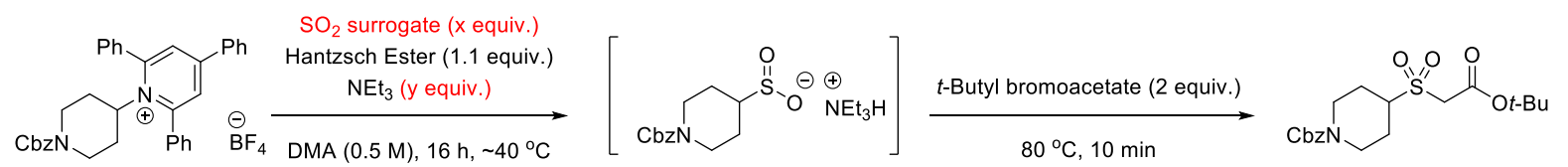

$1 \mathrm{a}$ Blue LEDs

$2 a$

$3 a$

\begin{tabular}{|c|c|c|c|c|c|c|c|c|}
\hline & & \multicolumn{7}{|c|}{$\mathrm{SO}_{2}$ surrogate } \\
\hline & & $\begin{array}{c}\text { DABCO. }\left(\mathrm{SO}_{2}\right)_{2} \\
(0.6 \text { equiv. })\end{array}$ & $\begin{array}{c}\mathrm{Na}_{2} \mathrm{~S}_{2} \mathrm{O}_{5} \\
\text { (1.2 equiv.) }\end{array}$ & $\begin{array}{c}\mathrm{K}_{2} \mathrm{~S}_{2} \mathrm{O}_{5} \\
\text { (1.2 equiv.) }\end{array}$ & $\begin{array}{c}\text { TIMSO } \\
\text { (1.2 equiv.) }\end{array}$ & $\begin{array}{l}\text { NMMSO } \\
\text { (1.2 equiv.) }\end{array}$ & $\begin{array}{l}\text { DMAP.SO }{ }_{2} \\
\text { (1.2 equiv.) }\end{array}$ & $\begin{array}{l}\text { TMEDA. }\left(\mathrm{SO}_{2}\right)_{2} \\
(0.6 \text { equiv. })\end{array}$ \\
\hline \multirow{5}{*}{$\begin{array}{l}\text { Equivalents } \\
\text { of } \mathrm{Et}_{3} \mathrm{~N}\end{array}$} & 0 & $0 \%$ & $0 \%$ & - & $39 \%$ & $65 \%$ & $24 \%$ & $7 \%$ \\
\hline & 0.5 & - & $63 \%$ & - & $79 \%$ & - & $66 \%$ & $56 \%$ \\
\hline & 1.2 & $46 \%$ & $76 \%$ & $49 \%$ & $78 \%$ & $81 \%$ & $79 \%$ & $74 \%$ \\
\hline & 2.4 & $73 \%$ & $72 \%$ & - & $73 \%$ & $75 \%$ & $79 \%$ & $70 \%$ \\
\hline & 3.6 & $82 \%$ & $75 \%$ & - & $76 \%$ & $78 \%$ & $75 \%$ & $75 \%$ \\
\hline
\end{tabular}

Table 2. Exploring $\mathrm{SO}_{2}$ surrogates and triethylamine loading for secondary alkyl substrate 1a. $0.20 \mathrm{mmol}$ scale, yields of 3a calculated from HPLC analysis using 1,3,5-triisopropylbenzene as an internal standard.

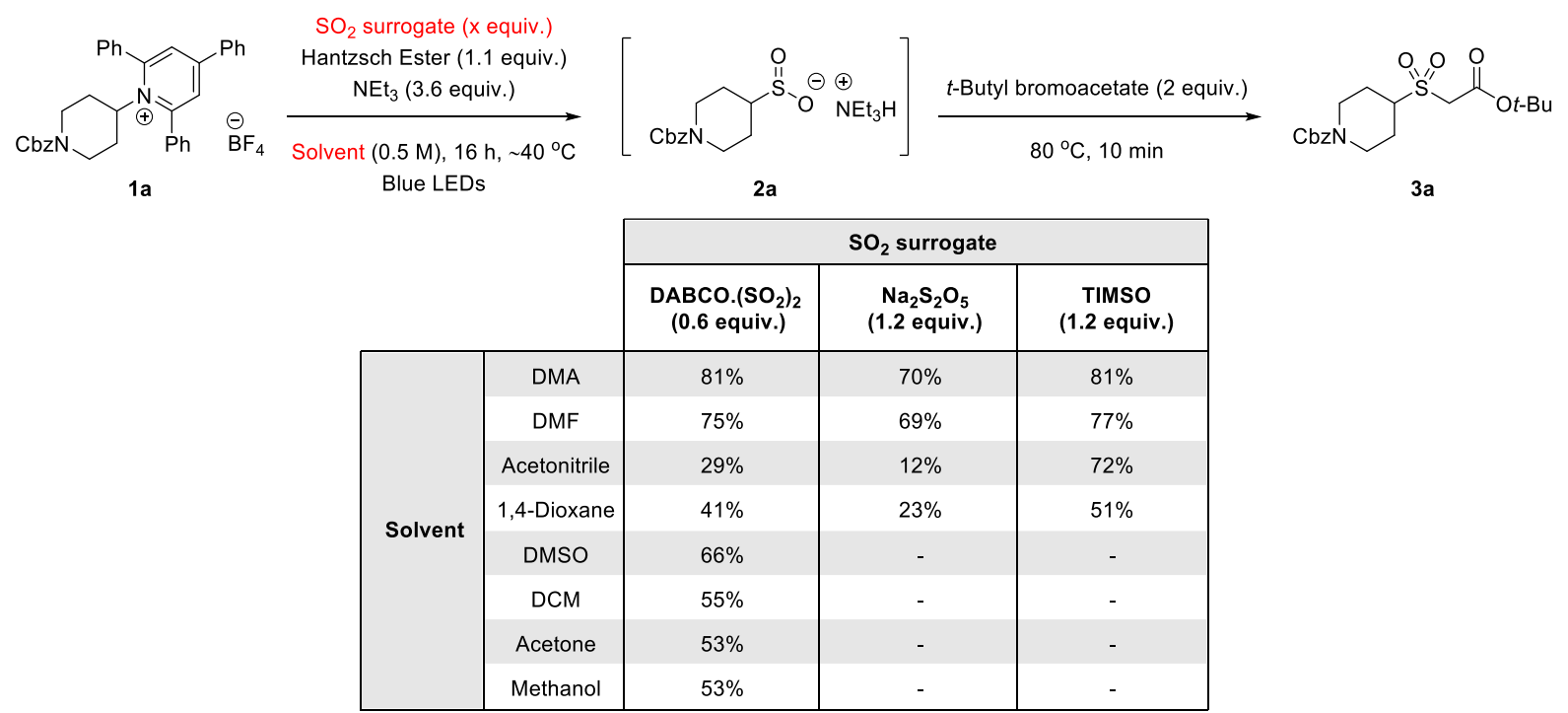

Table 3. Exploring $\mathrm{SO}_{2}$ surrogates and solvent for secondary alkyl substrate $1 \mathrm{a} .0 .20 \mathrm{mmol}$ scale, yields of $3 a$ calculated from HPLC analysis using 1,3,5-triisopropylbenzene as an internal standard. 

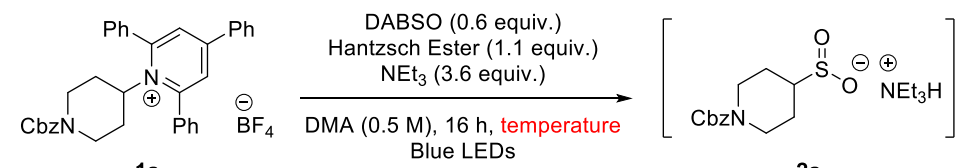

$\underset{t \text {-Butyl bromoacetate (2 equiv.) }}{ }$

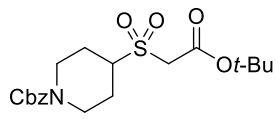

$2 a$

$3 a$

\begin{tabular}{|c|c|c|}
\cline { 2 - 3 } \multicolumn{1}{c|}{} & \multicolumn{2}{|c|}{$\mathbf{S O}_{2}$ surrogate } \\
\cline { 2 - 3 } & $\begin{array}{c}\text { DABCO.(SO } \\
\text { (0.6 })_{2}\end{array}$ & $\begin{array}{c}\mathrm{Na}_{2} \mathbf{S}_{2} \mathbf{O}_{5} \\
\mathbf{( 1 . 2 ~ e q u i v . ) ~}\end{array}$ \\
\hline$\sim 40{ }^{\circ} \mathrm{C}$ (vents sealed) & $81 \%$ & $75 \%$ \\
\hline$\sim 30^{\circ} \mathrm{C}$ (vents open with fan cooling) & $81 \%$ & $62 \%$ \\
\hline
\end{tabular}

Table 4. Exploring reaction temperature for secondary alkyl substrate $\mathbf{1 a} .0 .20 \mathrm{mmol}$ scale, yields of $\mathbf{3 a}$ calculated from HPLC analysis using 1,3,5-triisopropylbenzene as an internal standard.

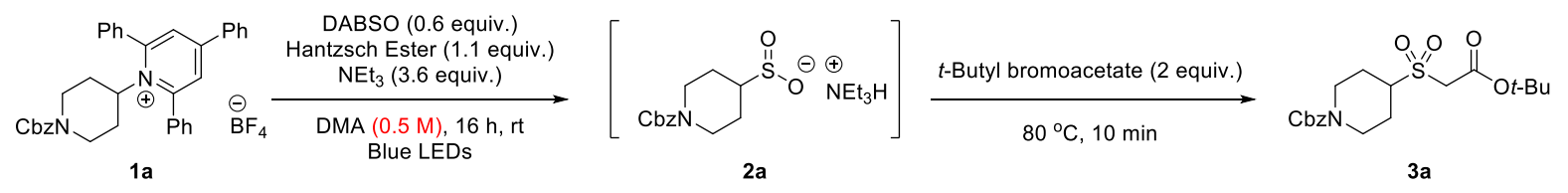

\begin{tabular}{|c|c|}
\hline Volume DMA & Yield \\
\hline $0.2 \mathrm{~mL}(1 \mathrm{M})$ & $75 \%$ \\
\hline $0.3 \mathrm{~mL}(0.67 \mathrm{M})$ & $73 \%$ \\
$0.4 \mathrm{~mL}(0.5 \mathrm{M})$ & $76 \%$ \\
$0.5 \mathrm{~mL}(0.4 \mathrm{M})$ & $77 \%$ \\
$0.6 \mathrm{~mL}(0.33 \mathrm{M})$ & $76 \%$ \\
$0.8 \mathrm{~mL}(0.25 \mathrm{M})$ & $66 \%$ \\
\hline
\end{tabular}

Table 5. Concentration screen for secondary alkyl substrate 1a. $0.20 \mathrm{mmol}$ scale, yields of 3 a calculated from HPLC analysis using 1,3,5-triisopropylbenzene as an internal standard.

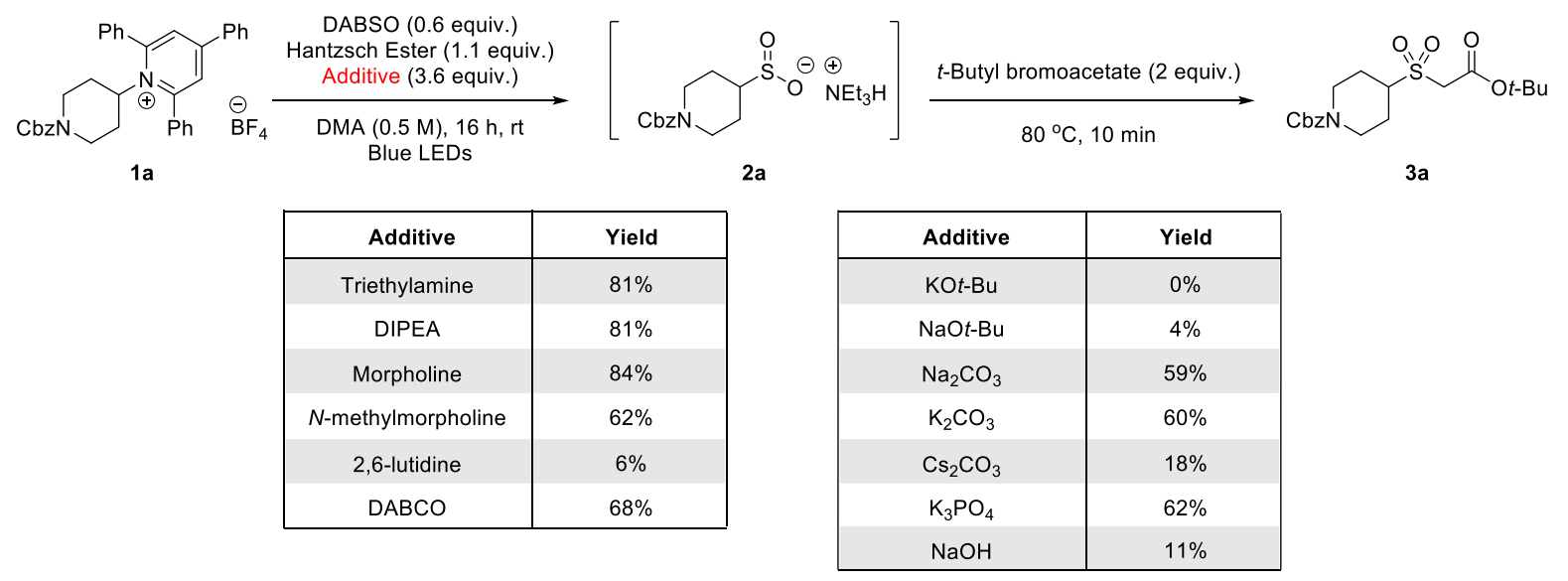

Table 6. Exploring additives for secondary alkyl substrate 1a. $0.20 \mathrm{mmol}$ scale, yields of 3a calculated from HPLC analysis using 1,3,5-triisopropylbenzene as an internal standard. 


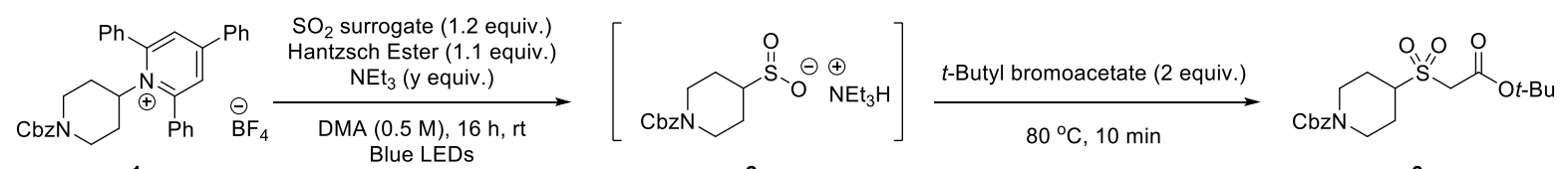

$1 a$ Blue LEDs

2a

$3 a$

\begin{tabular}{|c|c|}
\hline Modifications & Yield \\
\hline None & $82 \%$ \\
\hline Dark, $30^{\circ} \mathrm{C}$ & Trace \\
Dark, $60^{\circ} \mathrm{C}$ & $80 \%$ \\
\hline Rigorously dry and degassed solvents & $83 \%$ \\
Degassed, DMA from bottle & $79 \%$ \\
\hline Degassed, DMA $+5 \% \mathrm{H}_{2} \mathrm{O}$ & $73 \%$ \\
Under air, degassed dry DMA & $81 \%$ \\
Dry DMA sparged with air & $80 \%$ \\
\hline
\end{tabular}

Table 7. Variations from the optimised conditions for secondary alkyl substrate 1 a. $0.20 \mathrm{mmol}$ scale, yields of 3a calculated from HPLC analysis using 1,3,5-triisopropylbenzene as an internal standard.

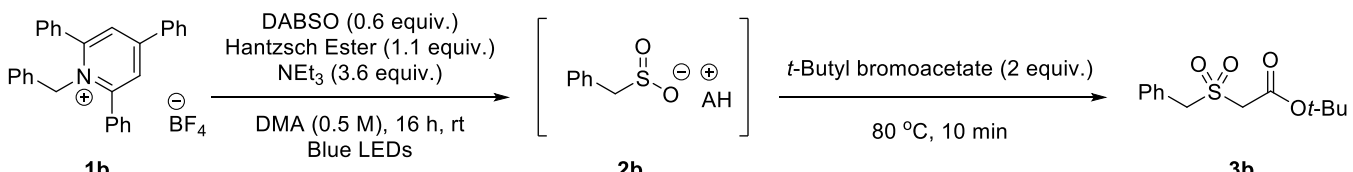

1b

$2 b$

3b

\begin{tabular}{|c|c|}
\hline Modifications & Yield \\
\hline None & $28 \%$ \\
${\text { No } \mathrm{NEt}_{3}}$ & $0 \%$ \\
1.2 equiv. $\mathrm{NEt}_{3}$ & $27 \%$ \\
2.4 equiv. DABSO & $27 \%$ \\
$2 \times$ Dilution (0.8 mL DMA) & $47 \%$ \\
Lower light intensity & $31 \%$ \\
$\mathrm{Na}_{2} \mathrm{~S}_{2} \mathrm{O}_{5}$ instead of DABSO & $7 \%$ \\
No Hantzsch Ester & $0 \%$ \\
\hline
\end{tabular}

Table 8. Initial optimisation studies for benzylic substrate $\mathbf{1 b}$. $0.20 \mathrm{mmol}$ scale, yields of $\mathbf{3 b}$ calculated from HPLC analysis using 1,3,5-triisopropylbenzene as an internal standard.

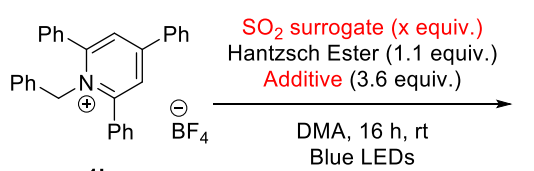

$1 b$

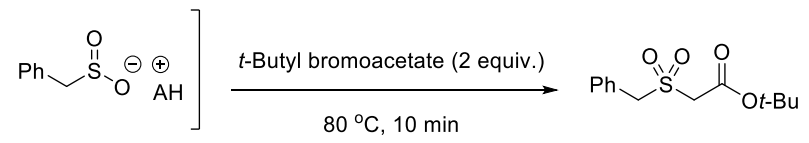

2b 3b

\begin{tabular}{|c|c|c|c|}
\hline & & \multicolumn{2}{|c|}{$\mathrm{SO}_{2}$ surrogate } \\
\hline & & $\begin{array}{c}\mathrm{DABCO}\left(\mathrm{SO}_{2}\right)_{2} \\
(0.6 \text { equiv. })\end{array}$ & $\begin{array}{c}\mathrm{Na}_{2} \mathrm{~S}_{2} \mathrm{O}_{5} \\
(1.2 \text { equiv.) }\end{array}$ \\
\hline \multirow{8}{*}{ Additive } & None & $9 \%$ & $70 \%$ \\
\hline & Triethylamine & $28 \%$ & $7 \%$ \\
\hline & DIPEA & $46 \%$ & - \\
\hline & Pyridine & $17 \%$ & - \\
\hline & 2,6-lutidine & $83 \%$ & $67 \%$ \\
\hline & 2,2,6,6-tetramethylpiperidine & $20 \%$ & - \\
\hline & 2,4,6-collidine & $82 \%$ & - \\
\hline & 2,6-di-tert-butylpyridine & $0 \%$ & - \\
\hline
\end{tabular}

Table 9. Base screen for benzylic substrate $\mathbf{1 b}$. $0.20 \mathrm{mmol}$ scale, yields of $\mathbf{3} \mathbf{b}$ calculated from HPLC analysis using 1,3,5-triisopropylbenzene as an internal standard. 


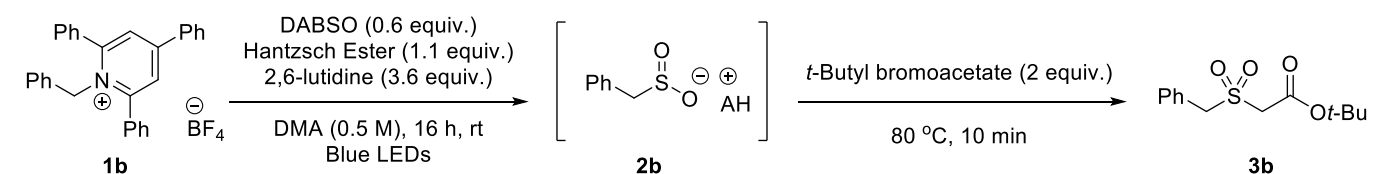

$1 \mathrm{~b}$ Blue LEDs

$2 \mathrm{~b}$

3b

\begin{tabular}{|c|c|}
\hline Modifications & Yield \\
\hline None & $83 \%$ \\
\hline 1.2 equiv. 2.6-lutidine & $68 \%$ \\
\hline 2.4 equiv. 2,6 -lutidine & $79 \%$ \\
Dark, $30^{\circ} \mathrm{C}$ & $54 \%$ \\
Dark, $40^{\circ} \mathrm{C}$ & $84 \%$ \\
No Hantzsch ester & $0 \%$ \\
\hline
\end{tabular}

Table 10. Variations from the optimised conditions for benzylic substrate $\mathbf{1 b}$. $0.20 \mathrm{mmol}$ scale, yields of $\mathbf{3 b}$ calculated from HPLC analysis using 1,3,5-triisopropylbenzene as an internal standard.
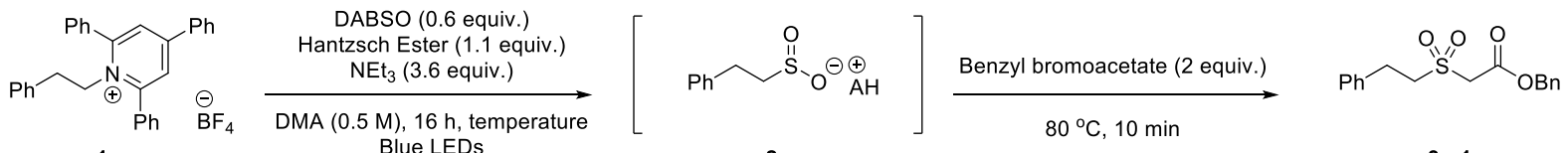
Blue LEDs

2c

Temperature

\begin{tabular}{|c|c|c|c|c|c|}
\hline & \multicolumn{4}{|c|}{ Temperature } \\
\hline & & $\begin{array}{c}\text { Ambient } \\
\text { Temp }\left(30^{\circ} \mathrm{C}\right)\end{array}$ & $60^{\circ} \mathrm{C}$ & $80^{\circ} \mathrm{C}$ & $90^{\circ} \mathrm{C}$ \\
\hline \multirow{17}{*}{ Additive } & Triethylamine & $0 \%$ & $0 \%$ & Trace & - \\
\hline & Morpholine & $0 \%$ & $33 \%$ & $28 \%$ & - \\
\hline & Pyrroline & $0 \%$ & $51 \%$ & $58 \%$ & - \\
\hline & Piperidine & - & $57 \%$ & $74 \%$ & $72 \%$ \\
\hline & Diethylamine & - & $47 \%$ & - & - \\
\hline & Diisopropylamine & - & $43 \%$ & $23 \%$ & - \\
\hline & Dicyclohexylamine & - & $37 \%$ & - & - \\
\hline & $N$-methylpiperazine & - & $49 \%$ & - & - \\
\hline & 2,6-lutidine & - & $0 \%$ & - & - \\
\hline & tert-butylamine & - & $0 \%$ & - & - \\
\hline & $n$-butylamine & - & $0 \%$ & - & - \\
\hline & DBU & $0 \%$ & $0 \%$ & - & - \\
\hline & 2,2,6,6-tetramethylguanidine & - & $0 \%$ & - & - \\
\hline & Dibenzylamine & - & $0 \%$ & - & - \\
\hline & DMAP & - & $0 \%$ & - & - \\
\hline & $\mathrm{N}$-methylanaline & - & $0 \%$ & - & - \\
\hline & Diphenylamine & - & $0 \%$ & - & - \\
\hline
\end{tabular}

Table 11. Base and temperature screen for primary alkyl substrate $1 \mathrm{c} .0 .20 \mathrm{mmol}$ scale, yields of $\mathbf{3 c - 1}$ calculated from HPLC analysis using 1,3,5-triisopropylbenzene as an internal standard. 


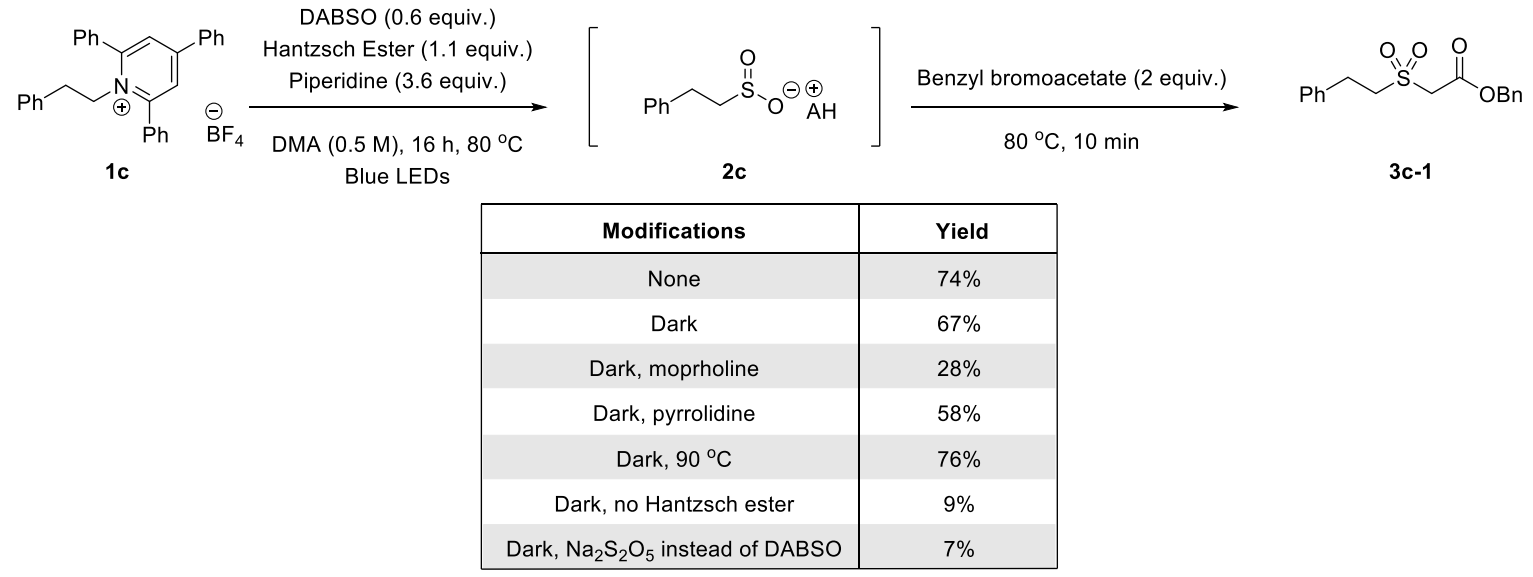

Table 12. Variations from the optimised conditions for primary alkyl substrate 1 c. $0.20 \mathrm{mmol}$ scale, yields of 3c-1 calculated from HPLC analysis using 1,3,5-triisopropylbenzene as an internal standard. 


\section{Mechanistic Studies}

\subsection{UV/vis spectral data}

In order to investigate EDA complex formation in our reaction mixtures, UV/vis absorption studies were performed on individual reaction components and mixtures to observe bathochromic shifts in absorption associated with complex formation. Spectra were acquired using a Perkin Elmer Lambda 20 spectrometer using quartz cuvettes (10 mm path length). Unless otherwise stated, all absorption spectra were recorded at $298 \mathrm{~K}$, with temperature control by a PTP-1 Peltier unit from Perkin Elmer, with baseline correction.

Concentrations of solutions:

- Katritzky salts 1a-c (0.1 M)

- Hantzsch Ester (0.11 M)

- $\operatorname{NEt}_{3}(0.36 \mathrm{M})$

- 2,6-lutidine $(0.36 \mathrm{M})$

- Piperidine $(0.36 \mathrm{M})$

- $\operatorname{DABCO}(0.06 \mathrm{M})$

In agreement with observations from Aggarwal and coworkers, ${ }^{1}$ binary mixtures of 1 a and Hantzsch ester or $\mathrm{Et}_{3} \mathrm{~N}$ in $\mathrm{DMA}$, showed a significant bathochromic shift when compared to the separate components, indicating the formation of EDA complexes. The ternary mixture showed a further bathochromic shift, indicating the formation of a ternary EDA complex (Figure S1). For benzylic substrate $\mathbf{1} \mathbf{b}$, the spectrum of the ternary mixture of $\mathbf{1} \mathbf{b}$, Hantzsch ester and 2,6-lutidine appeared to be identical to that of the binary mixture of $\mathbf{1} \mathbf{b}$ and Hantzsch ester, indicating that a ternary EDA complex does not form with 2,6-lutidine (Figure S2). This may result in reduced reactivity, and thus slower formation of the benzylic radical, which results in a reduced yield of side-products $\mathbf{4} \mathbf{b}$ and $\mathbf{5 b}$ and an improved yield of $\mathbf{3 b}$. For primary substrate $\mathbf{1 c}$, the spectrum of the ternary mixture of $\mathbf{1 c}$, Hantzsch ester and piperidine showed a further bathochromic shift and increase in absorbance compared to that using $\mathrm{Et}_{3} \mathrm{~N}$ (Figure S3). Previous studies have reported an increased stability for some EDA complexes when using secondary amine donors compared to that using tertiary amines. ${ }^{2-}$ ${ }^{4}$ This may contribute to the increased reactivity observed using piperidine with the primary substrates. 
UV/vis absorbance spectrum for 1a and components of optimised reaction mixture

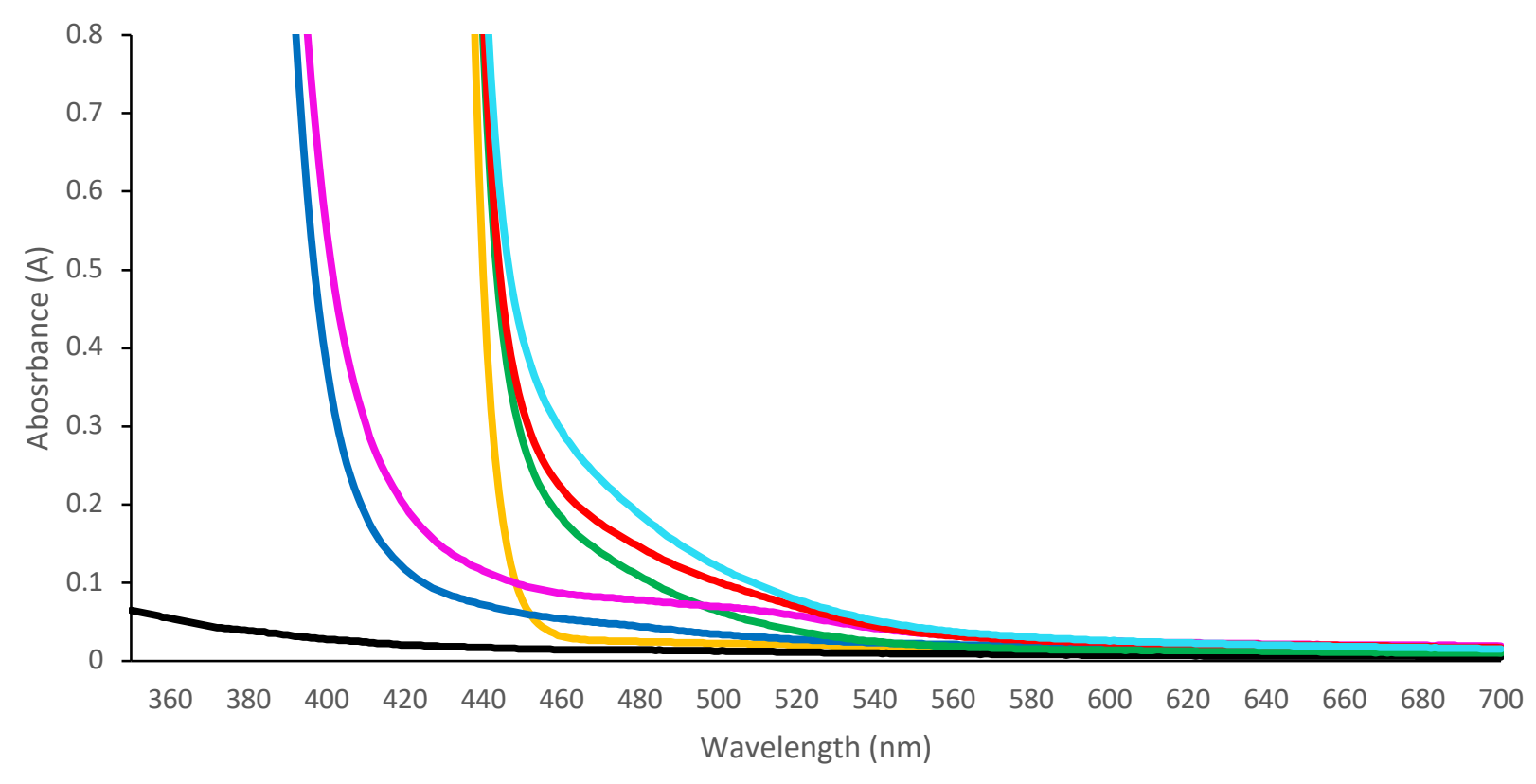

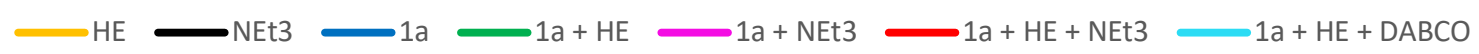

Figure S1. UV/vis absorbance spectra for components of secondary substrate 1a reaction mixture. Concentrations based on 1a (0.1 M in DMA).

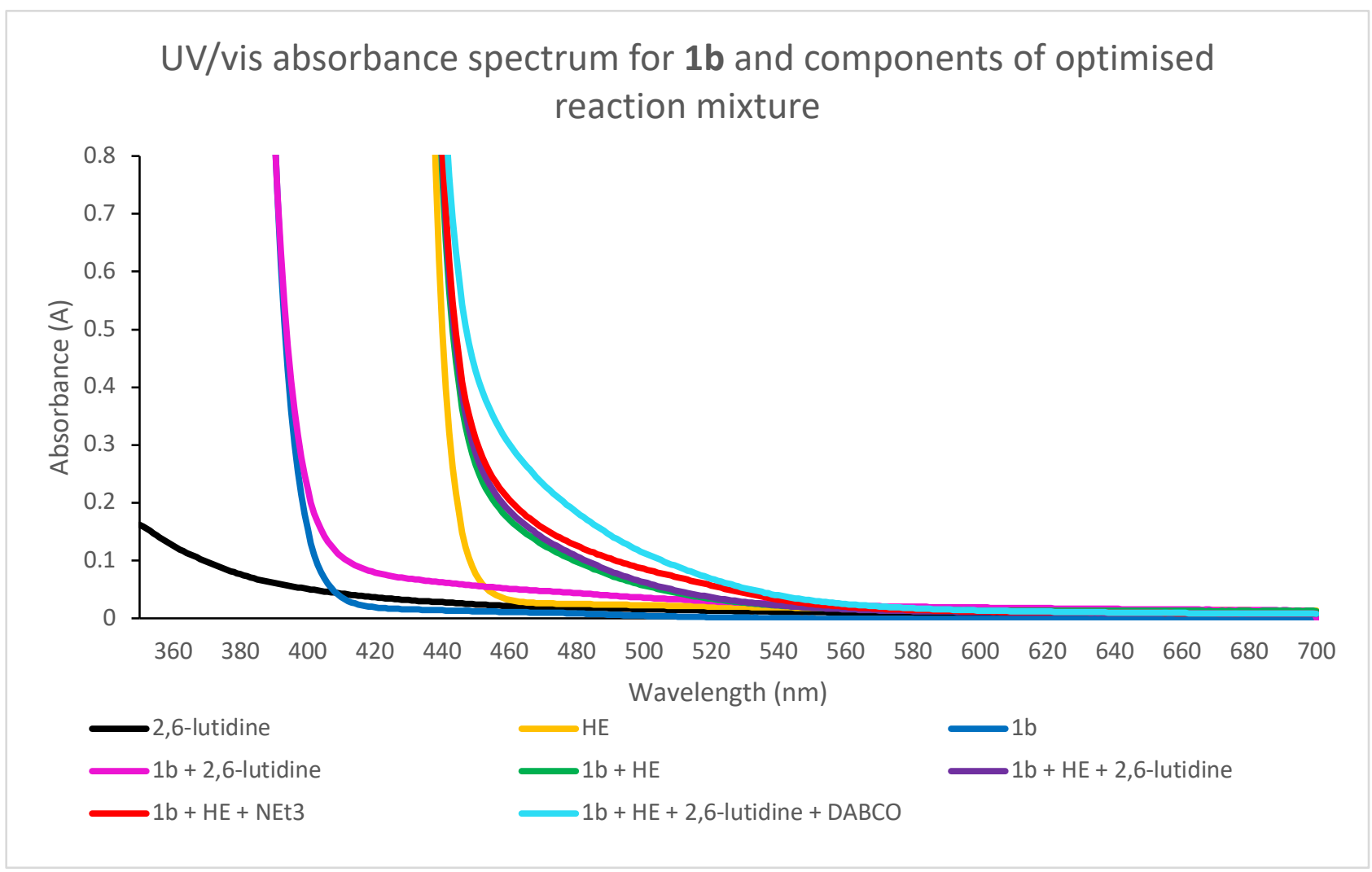

Figure S2. UV/vis absorbance spectra for components of benzylic substrate $\mathbf{1 b}$ reaction mixture. Concentrations based on $\mathbf{1 b}(0.1 \mathrm{M}$ in DMA). 


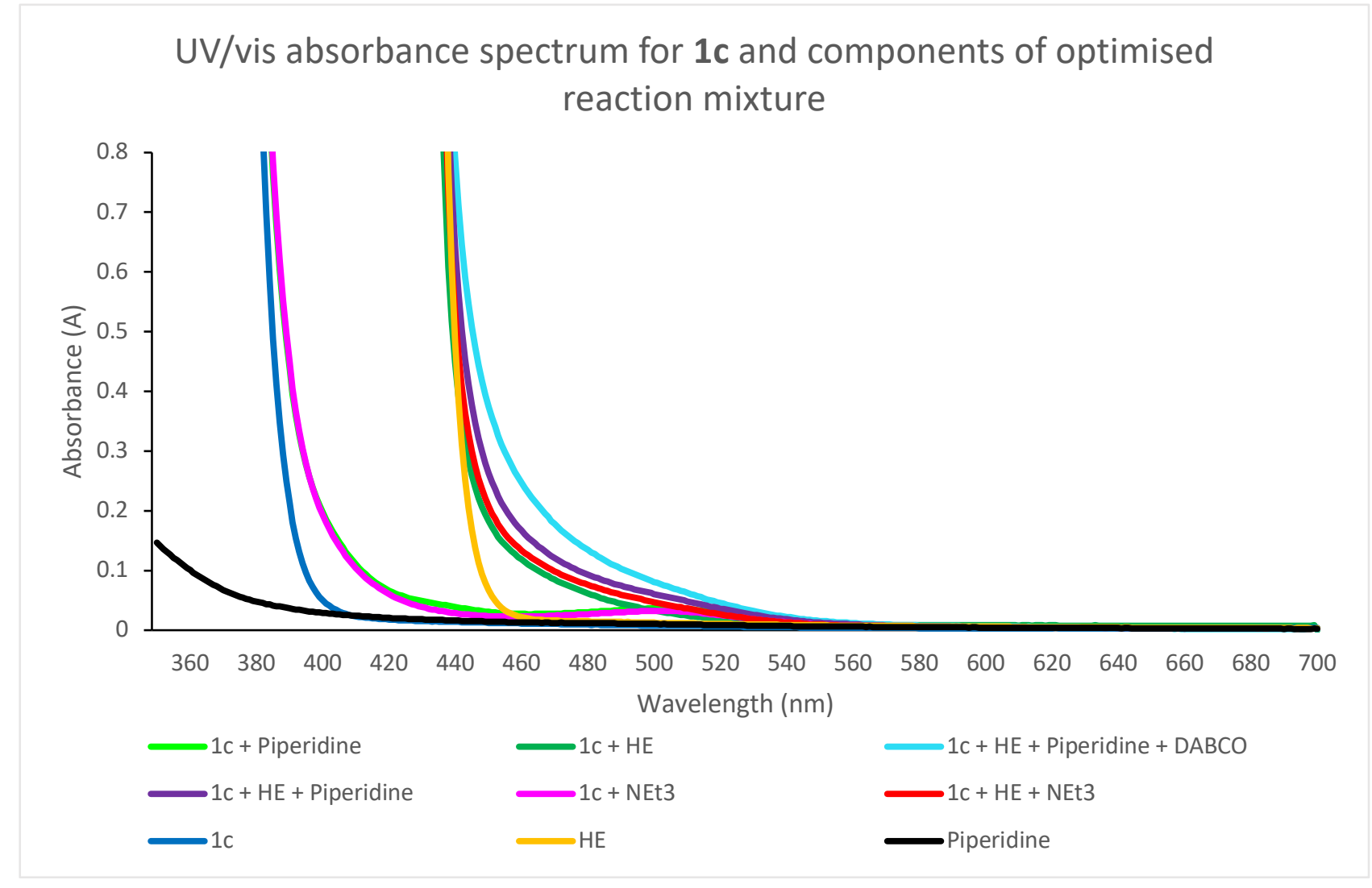

Figure S3. UV/vis absorbance spectra for components of primary substrate 1c reaction mixture. Concentrations based on 1c (0.1 M in DMA).

\subsection{TEMPO Studies}

For reactions of secondary and primary substrates $\mathbf{1 a}$ and $\mathbf{1 c}$, addition of TEMPO inhibited the reaction, and only starting materials were observed. For benzylic substrate $\mathbf{1 b}$, TEMPO-adduct $\mathbf{1 1}$ was isolated in good yield, demonstrating the increased reactivity of the benzylic system, and supporting the radical nature of the reaction.

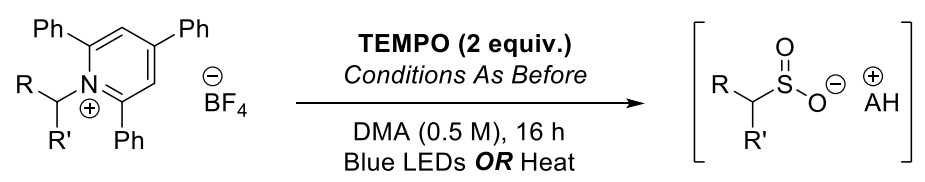<smiles></smiles>

No reaction (thermal or photoinduced)
$1 c$<smiles></smiles>

No reaction
$1 b$

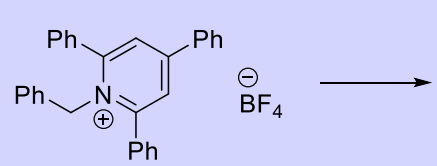

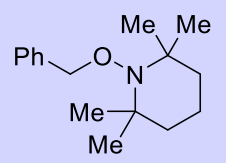

$11,73 \%$

Figure S4. TEMPO experiments. 


\section{Experimental Procedures}

\subsection{Photochemical Reaction Setup}

Blue LEDs, where specified, comprised a 40 W Kessil A160WE "Tuna Blue" aquarium LAMP or HepatoChem EvoluChem $^{\text {TM }} 450 \mathrm{PF} 18 \mathrm{~W} 450-455 \mathrm{~nm}$ LED spotlight. $0.20 \mathrm{mmol}$ scale reactions were carried out in $1.8 \mathrm{~mL}$ sample vials, fitted with a B14 Suba Seal ${ }^{\circledR}$ septum mounted in a EvoluChem ${ }^{\text {TM }}$ PhotoRedOx Box device. 10 $\mathrm{mmol}$ scale reactions were carried out in a round bottom flask. Fan cooling was used during reactions to give an internal temperature of $\sim 30^{\circ} \mathrm{C}$.

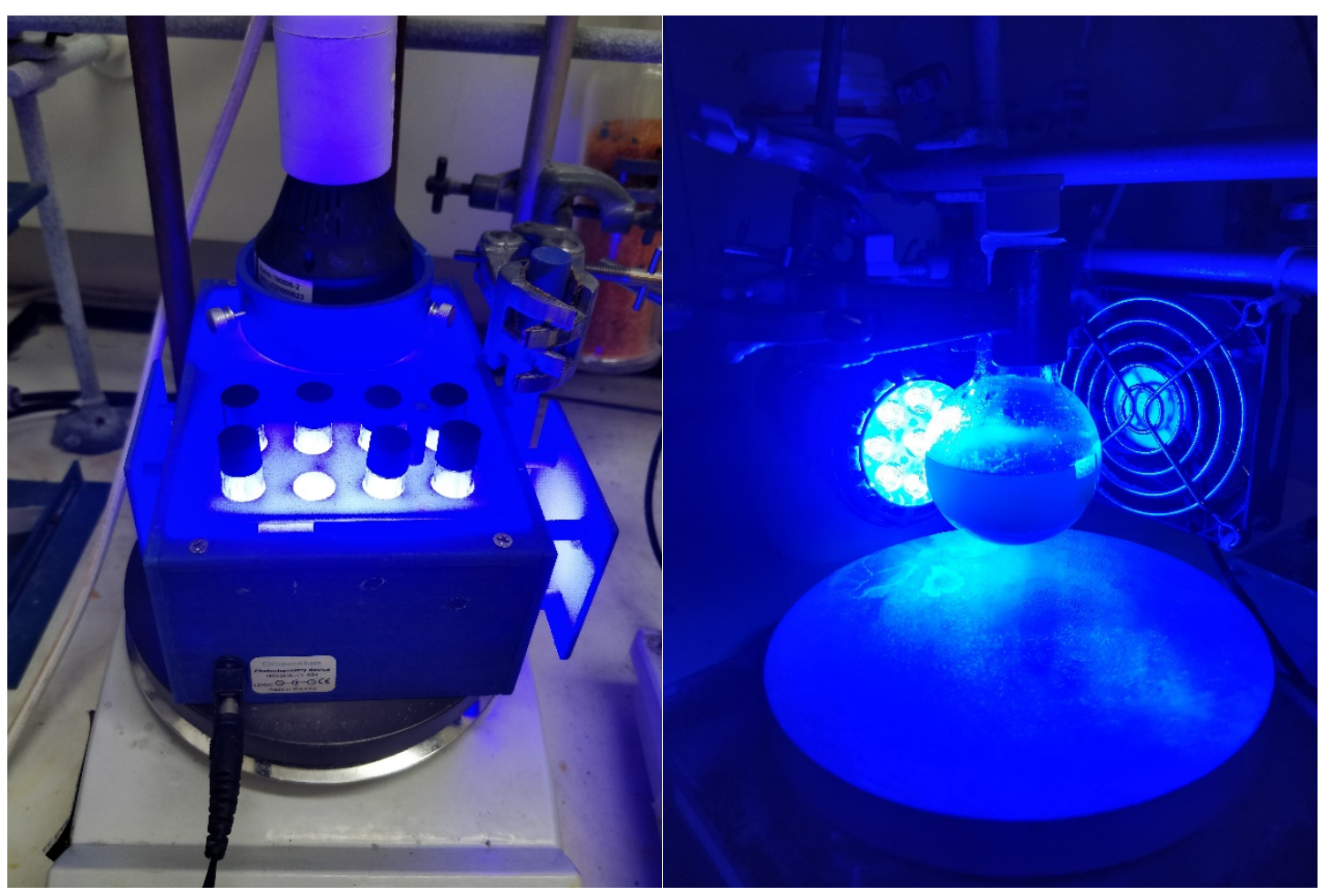

Figure S4. Left: Photobox used for $0.20 \mathrm{mmol}$ scale reactions. Right: Reaction setup for $10 \mathrm{mmol}$ scale reactions. 


\subsection{Preparation of Katritzky Pyridinium Salts}

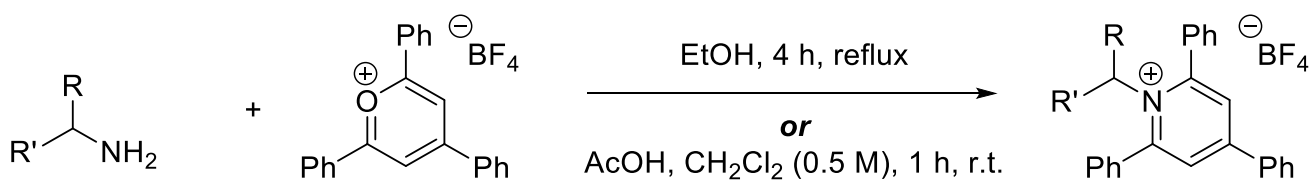

\section{General Procedure A:}

According to the procedure described by Watson and co-workers, ${ }^{5}$ the specified primary amine (1.2 equiv.) was added to a suspension of 2,4,6-triphenylpyrylium tetrafluoroborate (1.0 equiv.) and EtOH (1.0 M) in a round bottom flask. The flask was fitted with a reflux condenser and the mixture was stirred and heated to reflux in an aluminium heating block for $4 \mathrm{~h}$. The mixture was then allowed to cool to room temperature. If product precipitation occurred, the solid was filtered, washed with $\mathrm{EtOH}(3 \times 25 \mathrm{~mL})$, then $\mathrm{Et}_{2} \mathrm{O}(3 \times 25 \mathrm{~mL})$, and dried under high vacuum. If this did not occur, the solution was diluted with $\mathrm{Et}_{2} \mathrm{O}(2-3 \times$ volume of EtOH used) and vigorously stirred for $1 \mathrm{~h}$ to induce precipitation. The resulting solid pyridinium salt was filtered and washed with $\mathrm{Et}_{2} \mathrm{O}(3 \times 25 \mathrm{~mL})$. If product precipitation failed to occur at this point, it was subjected to flash column chromatography, eluting with $0-10 \%$ acetone $/ \mathrm{CH}_{2} \mathrm{Cl}_{2}$.

The corresponding amine hydrochloride salts can also be used by following a modified procedure: $\mathrm{Et}_{3} \mathrm{~N}$ (1.2 equiv.) was added to a mixture of the amine hydrochloride salt (1.2 equiv.) in $\mathrm{EtOH}(1.0 \mathrm{M})$. The mixture was then stirred for 30 mins at room temperature. 2,4,6-triphenylpyrylium tetrafluoroborate (1.0 equiv.) was then added and the reaction heated to reflux for $4 \mathrm{~h}$. The workup procedure was analogous to that described above, but precipitated product was washed with water $(3 \times 25 \mathrm{~mL})$ prior to washing with $\mathrm{EtOH}$ and $\mathrm{Et}_{2} \mathrm{O}$ as before.

\section{General Procedure B:}

According to the procedure described by Gabrielson and co-workers, ${ }^{6}$ 2,4,6-triphenylpyrylium tetrafluoroborate (1.0 equiv.) was added over $10 \mathrm{~min}$ to a stirred solution of the specified primary amine (1.3 equiv.) and $\mathrm{AcOH}$ ( 0.5 equiv.) in $\mathrm{CH}_{2} \mathrm{Cl}_{2}(0.5 \mathrm{M})$ in a round bottom flask. The reaction mixture was stirred at room temperature for $3 \mathrm{~h}$. If product precipitation occurred, the solid was filtered, washed with $\mathrm{EtOH}(3 \times 25$ $\mathrm{mL})$, then $\mathrm{Et}_{2} \mathrm{O}(3 \times 25 \mathrm{~mL})$, and dried under high vacuum. If product precipitation did not occur, solvent was removed in vacuo, the residue dissolved in boiling $\mathrm{EtOH}$ and the resulting solution vigorously stirred before being cooled to room temperature. If product precipitation still did not occur, the solution was diluted with $\mathrm{Et}_{2} \mathrm{O}(2-3 \times$ volume of EtOH used) and vigorously stirred for $1 \mathrm{~h}$ to induce precipitation. The resulting solid pyridinium salt was filtered and washed with $\mathrm{Et}_{2} \mathrm{O}(3 \times 25 \mathrm{~mL})$. If product precipitation failed to occur at this point, it was subjected to flash column chromatography, eluting with $0-10 \%$ acetone $/ \mathrm{CH}_{2} \mathrm{Cl}_{2}$.

The corresponding amine hydrochloride salts can also be used by following a modified procedure: $\mathrm{Et}_{3} \mathrm{~N}$ (1.3 equiv.) was added to a mixture of the amine hydrochloride salt (1.3 equiv.) in $\mathrm{CH}_{2} \mathrm{Cl}_{2}(0.5 \mathrm{M})$. The mixture was then stirred for $30 \mathrm{~min}$ at room temperature. $\mathrm{AcOH}$ ( 0.5 equiv.) was added in one portion followed by addition of 2,4,6-triphenylpyrylium tetrafluoroborate (1.0 equiv.) over 15 mins. The reaction was then stirred 
at room temperature overnight. The workup procedure was analogous to that described above, but precipitated product was washed with water $(3 \times 25 \mathrm{~mL})$ prior to washing with $\mathrm{EtOH}$ and $\mathrm{Et}_{2} \mathrm{O}$ as before.

1-(1-((Benzyloxy)carbonyl)piperidin-4-yl)-2,4,6-triphenylpyridin-1-ium tetrafluoroborate (1a)<smiles>O=C(c1ccccc1)N1CCC(c2cc(-c3ccccc3)cc(-c3ccccc3)[n+]2-c2ccccc2)CC1</smiles>

Prepared according to General Procedure A using benzyl 4-aminopiperidine-1-carboxylate $(2.81 \mathrm{~g}, 12 \mathrm{mmol}$, 1.2 equiv.) and 2,4,6-triphenylpyrylium tetrafluoroborate $(2.97 \mathrm{~g}, 7.50 \mathrm{mmol}, 1.0$ equiv.) in $\mathrm{EtOH}(7.5 \mathrm{~mL})$. Pyridinium salt 1a was isolated as a white solid (3.54 g, 77\%).

${ }^{1} \mathrm{H}$ NMR $\left(400 \mathrm{MHz}, \mathrm{CDCl}_{3}\right) \delta 7.82(\mathrm{~s}, 2 \mathrm{H}), 7.78-7.69(\mathrm{~m}, 6 \mathrm{H}), 7.64-7.43(\mathrm{~m}, 9 \mathrm{H}), 7.38-7.28(\mathrm{~m}, 3 \mathrm{H}), 7.24-$ $7.17(\mathrm{~m}, 2 \mathrm{H}), 4.95(\mathrm{~s}, 2 \mathrm{H}), 4.79(\mathrm{tt}, J=12.5,3.0 \mathrm{~Hz}, 1 \mathrm{H}), 4.18-3.85$ (br. $\mathrm{m}, 2 \mathrm{H}), 2.35-1.94(\mathrm{~m}, 4 \mathrm{H}), 1.82-$ 1.57 (br. m, 2H); ${ }^{13} \mathrm{C}$ NMR (101 MHz, $\left.\mathrm{CDCl}_{3}\right) \delta 157.3,155.7,154.9,136.4,134.1,133.8,132.2,131.3,129.8$, $129.4,129.2,128.7,128.5,128.3,127.9,69.7,67.4,44.4,32.6$ (br.) (1 aromatic carbon signal is not observed due to signal broadening); $\left.{ }^{19} \mathrm{~F} \mathrm{NMR} \mathrm{(377} \mathrm{MHz,} \mathrm{CDCl}_{3}\right) \delta-153.0$ (minor, ${ }^{10} \mathrm{BF}_{4}$ ), -153.0 (major, ${ }^{11} \mathrm{BF}_{4}$ ); LRMS (ESI) $\mathrm{m} / z 525.2\left(\left[\mathrm{M}-\mathrm{BF}_{4}\right]^{+}\right) ; \mathrm{HRMS}(\mathrm{ESI}) \mathrm{m} / z \mathrm{C}_{36} \mathrm{H}_{33} \mathrm{~N}_{2} \mathrm{O}_{2}{ }^{+}$requires $525.2537\left(\left[\mathrm{M}-\mathrm{BF}_{4}\right]^{+}\right)$, found 525.2529 .

Data is consistent with the literature. ${ }^{7}$

1-(1-(tert-Butoxycarbonyl)piperidin-4-yl)-2,4,6-triphenylpyridin-1-ium tetrafluoroborate (1d)<smiles></smiles>

Prepared according to General Procedure A using tert-butyl 4-aminopiperidine-1-carboxylate (1.20 g, 6.00 mmol, 1.2 equiv.) and 2,4,6-triphenylpyrylium tetrafluoroborate ( $1.98 \mathrm{~g}, 5.00 \mathrm{mmol}, 1.0$ equiv.) in EtOH (5.0 $\mathrm{mL})$. Pyridinium salt $1 \mathbf{d}$ was isolated as a white solid ( $2.18 \mathrm{~g}, 76 \%)$.

${ }^{1} \mathrm{H}$ NMR $\left(400 \mathrm{MHz}, \mathrm{CDCl}_{3}\right) \delta 7.82(\mathrm{~s}, 2 \mathrm{H}), 7.77-7.70(\mathrm{~m}, 6 \mathrm{H}), 7.63-7.44(\mathrm{~m}, 9 \mathrm{H}), 4.78(\mathrm{br} . \mathrm{tt}, J=12.5,3.0 \mathrm{~Hz}$, 1H), 4.12 - 3.72 (br. m, 2H), $2.34-1.92$ (br. m, 4H), $1.81-1.55$ (br. m, 2H), 1.31 (s, 9H); ${ }^{13} \mathrm{C} \mathrm{NMR} \mathrm{(101} \mathrm{MHz,}$ $\left.\mathrm{CDCl}_{3}\right) \delta 157.3,155.5,154.3,134.0,133.9,132.1,131.2,129.7,129.4,129.1,128.4,128.3,80.2,70.0,45.0$ (br.), 32.8 (br.), 28.4; ${ }^{19} \mathrm{~F}$ NMR (377 MHz, CDCl 3 ) $\delta$-153.0 (minor, ${ }^{10} \mathrm{BF}_{4}$ ), -153.1 (major, ${ }^{11} \mathrm{BF}_{4}$ ); LRMS (ESI) $\mathrm{m} / \mathrm{z}$ $491.2\left(\left[\mathrm{M}-\mathrm{BF}_{4}\right]^{+}\right)$; HRMS (ESI) $m / z \mathrm{C}_{33} \mathrm{H}_{35} \mathrm{~N}_{2} \mathrm{O}_{2}{ }^{+}$requires $491.2693\left(\left[\mathrm{M}-\mathrm{BF}_{4}\right]^{+}\right)$, found 491.2682.

Data is consistent with the literature. ${ }^{5}$

1-Tosylpiperidine-4-amine (1e-1) 


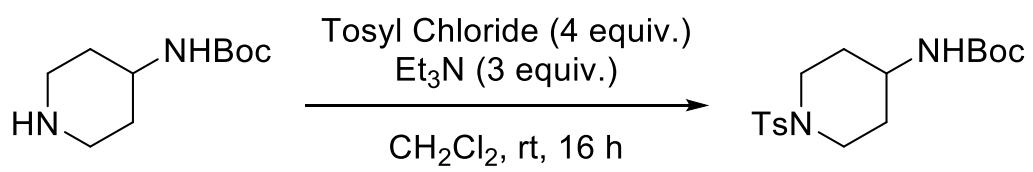

Prepared according to a modified procedure by Quan and co-workers. ${ }^{8}$ To a solution of $4(-N$-Bocamino)piperidine (553 mg, $3.00 \mathrm{mmol}, 1.0$ equiv.) anhydrous $\mathrm{CH}_{2} \mathrm{Cl}_{2}(15 \mathrm{~mL})$ cooled to $0{ }^{\circ} \mathrm{C}$ was added triethylamine ( $1.25 \mathrm{~mL}, 9.00 \mathrm{mmol}, 3.0$ equiv.) and tosyl chloride $(2.29 \mathrm{~g}, 12.0 \mathrm{mmol}, 4.0$ equiv.). The reaction mixture was stirred at rt overnight $(16 \mathrm{~h})$. The reaction was quenched with a saturated aqueous solution of $\mathrm{NaHCO}_{3}(20 \mathrm{~mL})$. The aqueous layer was extracted with ethyl acetate $(3 \times 40 \mathrm{~mL})$. The combined organic layers were dried using $\mathrm{MgSO}_{4}$, filtered and concentrated in vacuo. Purification by flash column chromatography (1:2 EtOAc:Petrol) afforded 1-tosylpiperidine-4-amine 1e-1 as a white solid (939 mg, 88\%).

$\mathbf{R}_{f}\left(30 \%\right.$ EtOAc in Petrol) $=0.23 ;{ }^{1} \mathbf{H}$ NMR $\left(400 \mathrm{MHz}, \mathrm{CDCl}_{3}\right) \delta 7.63(\mathrm{~d}, J=8.5 \mathrm{~Hz}, 2 \mathrm{H}), 7.32(\mathrm{~d}, J=8.0 \mathrm{~Hz}, 2 \mathrm{H})$, $4.48-4.34(m, 1 H), 3.77-3.59(m, 2 H), 3.47-3.27(m, 1 H), 2.49-2.33(m, 5 H), 2.02-1.90(m, 2 H), 1.47$ (ddd, $J=16.0,11.5,6.0 \mathrm{~Hz}, 2 \mathrm{H}), 1.41(\mathrm{~s}, 9 \mathrm{H}) ;{ }^{13} \mathrm{C} \mathrm{NMR}\left(101 \mathrm{MHz}, \mathrm{CDCl}_{3}\right) \delta 155.1,143.8,133.2,129.8,127.8$, 79.9 (br.), 47.2, 45.4, 31.9, 28.5, 21.7; HRMS (ESI) $m / z \mathrm{C}_{17} \mathrm{H}_{26} \mathrm{~N}_{2} \mathrm{O}_{4} \mathrm{SNa}^{+}$requires $377.1505\left([\mathrm{M}+\mathrm{Na}]^{+}\right)$, found 377.1507.

Data is consistent with the literature. ${ }^{8}$

1-Tosylpiperidin-4-amine hydrochloride (1e-2)<smiles>CC(C)(C)NC1CCN([13CH3])CC1</smiles>

To a solution of 1-tosylpiperidine-4-amine 1 e-1 $(922 \mathrm{mg}, 2.60 \mathrm{mmol}, 1.0$ equiv.) in EtOH (5.0 mL) was added $4 \mathrm{M} \mathrm{HCl}$ in dioxane $(10 \mathrm{~mL}, 40.0 \mathrm{mmol}, 15.4$ equiv.). The reaction mixture was stirred at $\mathrm{rt}$ overnight. The solvent was removed in vacuo to give 1-tosylpiperidin-4-amine hydrochloride $\mathbf{1 e - 2}$ as a white powder (620 $\mathrm{mg}, 83 \%)$.

${ }^{1} \mathrm{H}$ NMR $\left(400 \mathrm{MHz}, \mathrm{CD}_{3} \mathrm{OD}\right)$ 8 $7.73-7.63(\mathrm{~m}, 2 \mathrm{H}), 7.43(\mathrm{~d}, J=8.0 \mathrm{~Hz}, 2 \mathrm{H}), 3.92-3.78(\mathrm{~m}, 2 \mathrm{H}), 3.09(\mathrm{tt}, J=11.5$, $4.0 \mathrm{~Hz}, 1 \mathrm{H}), 2.47-2.34(\mathrm{~m}, 5 \mathrm{H}), 2.11-2.01(\mathrm{~m}, 2 \mathrm{H}), 1.66(\mathrm{qd}, J=12.5,4.5 \mathrm{~Hz}, 2 \mathrm{H}) ;{ }^{13} \mathrm{C} \mathrm{NMR}\left(101 \mathrm{MHz}, \mathrm{CD}_{3} \mathrm{OD}\right)$ $\delta 145.6,134.5,131.0,128.8,48.9,45.7,30.6,21.5 ;$ LRMS (ESI) $\mathrm{m} / \mathrm{z} 255.2\left([\mathrm{M}+\mathrm{H}]^{+}\right), 277.1\left([\mathrm{M}+\mathrm{Na}]^{+}\right)$; HRMS (ESI) $m / z \mathrm{C}_{12} \mathrm{H}_{19} \mathrm{~N}_{2} \mathrm{O}_{2} \mathrm{~S}^{+}$requires $255.1162\left([\mathrm{M}+\mathrm{H}]^{+}\right)$, found 255.1161 .

Data is consistent with the literature. ${ }^{9}$

2,4,6-Triphenyl-1-(1-tosylpiperidin-4-yl)pyridin-1-ium tetrafluoroborate (1e-3)

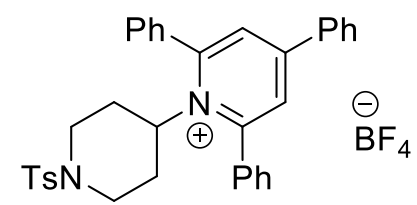


Prepared according to General Procedure A using 1-tosylpiperidin-4-amine hydrochloride 1e-2 (350 mg, $1.20 \mathrm{mmol}, 1.2$ equiv.), triethylamine (168 $\mu \mathrm{L}, 1.20 \mathrm{mmol}, 1.2$ equiv.), and 2,4,6-triphenylpyrylium tetrafluoroborate (400 mg, $1.00 \mathrm{mmol}, 1.0$ equiv.) in $\mathrm{EtOH}(1.0 \mathrm{~mL})$. Pyridinium salt 1e-3 was isolated as a white solid (340 mg, 54\%).

${ }^{1} \mathrm{H}$ NMR $\left(400 \mathrm{MHz}, \mathrm{CDCl}_{3}\right) \delta 7.79(\mathrm{~s}, 2 \mathrm{H}), 7.72-7.70(\mathrm{~m}, 6 \mathrm{H}), 7.62-7.51(\mathrm{~m}, 7 \mathrm{H}), 7.46(\mathrm{t}, \mathrm{J}=7.0 \mathrm{~Hz}, 2 \mathrm{H}), 7.40$ $(\mathrm{d}, J=8.0 \mathrm{~Hz}, 2 \mathrm{H}), 7.23(\mathrm{~d}, J=8.0 \mathrm{~Hz}, 2 \mathrm{H}), 4.55(\mathrm{t}, J=10.5 \mathrm{~Hz}, 1 \mathrm{H}), 3.60(\mathrm{~d}, J=10.0 \mathrm{~Hz}, 2 \mathrm{H}), 2.42(\mathrm{~s}, 3 \mathrm{H}), 2.28$ - $2.12(\mathrm{~m}, 2 \mathrm{H}), 1.94-1.67(\mathrm{~m}, 4 \mathrm{H}) ;{ }^{13} \mathrm{C}$ NMR $\left(101 \mathrm{MHz}, \mathrm{CDCl}_{3}\right) \delta 157.4,155.8,144.0,133.9,133.7,133.2$, $132.3,131.3,129.9,129.8,129.4,129.2,128.5,128.4,127.5,69.1,46.4,32.4,21.7 ;{ }^{19} \mathrm{~F} \mathrm{NMR}\left(377 \mathrm{MHz}, \mathrm{CDCl}_{3}\right)$

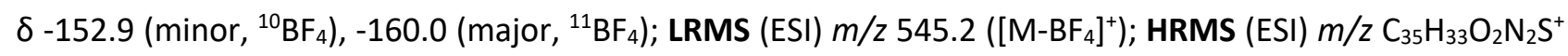
requires $545.2233\left(\left[\mathrm{M}-\mathrm{BF}_{4}\right]^{+}\right)$, found 545.2249 .

Data is consistent with the literature. ${ }^{9}$

1-Cyclobutyl-2,4,6-triphenylpyridin-1-ium tetrafluoroborate (1f)<smiles>c1ccc(-c2cc(-c3ccccc3)[n+](C3CCC3)c(-c3ccccc3)c2)cc1</smiles>

Prepared according to General Procedure A using cyclobutylamine (103 $\mu \mathrm{l}, 1.20 \mathrm{mmol}, 1.2$ equiv.) and 2,4,6triphenylpyrylium tetrafluoroborate (396 mg, $1.00 \mathrm{mmol}, 1.0$ equiv.) in $\mathrm{EtOH}(1.0 \mathrm{~mL}$ ). Pyridinium salt $\mathbf{1} f$ was isolated as a pale-yellow powder (333 mg, 74\%).

${ }^{1} \mathrm{H}$ NMR $\left(400 \mathrm{MHz}, \mathrm{CDCl}_{3}\right) \delta 7.88(\mathrm{dd}, J=8.0,1.5 \mathrm{~Hz}, 4 \mathrm{H}), 7.84(\mathrm{~s}, 2 \mathrm{H}), 7.82-7.77(\mathrm{~m}, 2 \mathrm{H}), 7.64-7.48(\mathrm{~m}, 9 \mathrm{H})$, $5.67-5.52(\mathrm{~m}, 1 \mathrm{H}), 1.91-1.73(\mathrm{~m}, 2 \mathrm{H}), 1.36-1.11(\mathrm{~m}, 5 \mathrm{H}) ;{ }^{13} \mathrm{C} \mathrm{NMR}\left(101 \mathrm{MHz}, \mathrm{CDCl}_{3}\right) \delta 157.5,155.6,134.2$, $134.2,132.2,131.6,129.9,129.7,129.5,128.2,126.3,64.4,33.2,14.3 ;{ }^{19} \mathrm{~F}$ NMR $\left(377 \mathrm{MHz}, \mathrm{CDCl}_{3}\right) \delta-153.1$ (minor, ${ }^{10} \mathrm{BF}_{4}$ ), -153.1 (major, ${ }^{11} \mathrm{BF}_{4}$ ); LRMS (ESI) $\mathrm{m} / z 362.2$ ([M-BF $]^{+}$); HRMS (ESI) $\mathrm{m} / z \mathrm{C}_{27} \mathrm{H}_{24} \mathrm{~N}^{+}$requires $362.1903\left(\left[\mathrm{M}-\mathrm{BF}_{4}\right]^{+}\right)$, found 362.1922 .

Data is consistent with the literature. ${ }^{10}$

1-Cyclopentyl-2,4,6-triphenylpyridin-1-ium tetrafluoroborate (1g)<smiles></smiles>

Prepared according to General Procedure A using cyclopentylamine (118 $\mu \mathrm{l}, 1.20 \mathrm{mmol}, 1.2$ equiv.) and 2,4,6triphenylpyrylium tetrafluoroborate $(396 \mathrm{mg}, 1.00 \mathrm{mmol}, 1.0$ equiv.) in $\mathrm{EtOH}(1.0 \mathrm{~mL})$. Pyridinium salt $1 \mathrm{~g}$ was isolated as a white solid (187 mg, 40\%). 
${ }^{1} \mathrm{H}$ NMR $\left(400 \mathrm{MHz}, \mathrm{CDCl}_{3}\right) \delta 7.83-7.70(\mathrm{~m}, 8 \mathrm{H}), 7.64-7.54(\mathrm{~m}, 6 \mathrm{H}), 7.54-7.43(\mathrm{~m}, 3 \mathrm{H}), 5.05(\mathrm{p}, \mathrm{J}=9.0 \mathrm{~Hz}$, $1 \mathrm{H}), 2.29-2.15(\mathrm{~m}, 2 \mathrm{H}), 2.09-1.95(\mathrm{~m}, 2 \mathrm{H}), 1.24-1.09(\mathrm{~m}, 2 \mathrm{H}), 1.01-0.87(\mathrm{~m}, 2 \mathrm{H}) ;{ }^{13} \mathrm{C} \mathrm{NMR}(101 \mathrm{MHz}$, $\mathrm{CDCl}_{3}$ ) $\delta 157.9,155.1,134.3,134.1,132.1,130.9,129.7,129.1,128.4,128.4,70.8,33.9,24.7$ (1 aromatic carbon signal is not observed due to signal broadening); ${ }^{19} \mathrm{~F}$ NMR $\left(377 \mathrm{MHz}, \mathrm{CDCl}_{3}\right) \delta-153.4$ (minor, $\left.{ }^{10} \mathrm{BF}_{4}\right)$, 153.4 (major, $\left.{ }^{11} \mathrm{BF}_{4}\right)$; LRMS (ESI) $\mathrm{m} / \mathrm{z} 376.2\left(\left[\mathrm{M}-\mathrm{BF}_{4}\right]^{+}\right)$; HRMS (ESI) $m / z \mathrm{C}_{28} \mathrm{H}_{26} \mathrm{~N}^{+}$requires $376.2062\left(\left[\mathrm{M}-\mathrm{BF}_{4}\right]^{+}\right)$, found 376.2062 .

Data is consistent with the literature. ${ }^{11}$

1-Cyclohexyl-2,4,6-triphenylpyridin-1-ium tetrafluoroborate (1h)<smiles></smiles>

Prepared according to General Procedure A using cyclohexylamine ( $275 \mu \mathrm{l}, 2.40 \mathrm{mmol}, 1.2$ equiv.) and 2,4,6triphenylpyrylium tetrafluoroborate (792 mg, $2.00 \mathrm{mmol}, 1.0$ equiv.) in EtOH ( $2.0 \mathrm{~mL}$ ). Pyridinium salt $1 \mathrm{~h}$ was isolated as a white solid (441 $\mathrm{mg}, 46 \%$ ).

${ }^{1} \mathrm{H}$ NMR $\left(400 \mathrm{MHz}, \mathrm{CDCl}_{3}\right) \delta 7.78(\mathrm{~s}, 2 \mathrm{H}), 7.77-7.69(\mathrm{~m}, 6 \mathrm{H}), 7.65-7.52(\mathrm{~m}, 6 \mathrm{H}), 7.55-7.40(\mathrm{~m}, 3 \mathrm{H}), 4.61$ $(\mathrm{tt}, J=12.0,3.0 \mathrm{~Hz}, 1 \mathrm{H}), 2.12(\mathrm{~d}, J=12.0 \mathrm{~Hz}, 2 \mathrm{H}), 1.57(\mathrm{dt}, J=13.5,3.5 \mathrm{~Hz}, 2 \mathrm{H}), 1.47(\mathrm{qd}, J=12.0,3.5 \mathrm{~Hz}, 2 \mathrm{H})$, 1.34 (br. d, $J=14.0 \mathrm{~Hz}, 1 \mathrm{H}$ ), 0.74 (qt, $J=13.0,3.0 \mathrm{~Hz}, 2 \mathrm{H}$ ), 0.60 (qt, $J=13.0,3.5 \mathrm{~Hz}, 1 \mathrm{H}) ;{ }^{13} \mathrm{C} \mathrm{NMR}(101 \mathrm{MHz}$, $\left.\mathrm{CDCl}_{3}\right) \delta 157.1,155.0,134.1,134.0,131.9,130.8,129.6,129.4,128.8,128.3,128.1,72.0,33.6,26.5,24.6 ;{ }^{19} \mathrm{~F}$ NMR (377 MHz, CDCl 3 ) $\delta$-153.3 (minor, ${ }^{10} \mathrm{BF}_{4}$ ), -153.3 (major, ${ }^{11} \mathrm{BF}_{4}$ ); LRMS (ESI) $\mathrm{m} / \mathrm{z} 390.2\left(\left[\mathrm{M}-\mathrm{BF}_{4}\right]^{+}\right.$); HRMS (ESI) $m / z \mathrm{C}_{29} \mathrm{H}_{28} \mathrm{~N}^{+}$requires $390.2216\left(\left[\mathrm{M}-\mathrm{BF}_{4}\right]^{+}\right)$, found 390.2217 .

Data is consistent with the literature. ${ }^{5}$

1-Isopropyl-2,4,6-triphenylpyridin-1-ium tetrafluoroborate (1i)<smiles></smiles>

Prepared according to General Procedure A using isopropylamine (102 $\mu \mathrm{l}, 1.20 \mathrm{mmol}, 1.2$ equiv.) and 2,4,6triphenylpyrylium tetrafluoroborate (396 mg, $1.00 \mathrm{mmol}, 1.0$ equiv.) in $\mathrm{EtOH}(1.0 \mathrm{~mL})$. Pyridinium salt $1 \mathrm{i}$ was isolated as a white solid (231 $\mathrm{mg}, 53 \%)$.

${ }^{1} \mathrm{H}$ NMR $\left(400 \mathrm{MHz}, \mathrm{CDCl}_{3}\right) \delta 7.81-7.68(\mathrm{~m}, 8 \mathrm{H}), 7.64-7.40(\mathrm{~m}, 9 \mathrm{H}), 5.11$ (hept, $\left.J=7.0 \mathrm{~Hz}, 1 \mathrm{H}\right), 1.35(\mathrm{~d}, J=$ $7.0 \mathrm{~Hz}, 6 \mathrm{H}) ;{ }^{13} \mathrm{C} \mathrm{NMR}\left(101 \mathrm{MHz}, \mathrm{CDCl}_{3}\right) \delta 157.2,155.3,134.2,134.0,132.0,131.0,129.7,129.6,129.0,128.5$, 128.4, 62.8, 23.5; ${ }^{19} \mathrm{~F}$ NMR (377 MHz, $\mathrm{CDCl}_{3}$ ) $\delta$-153.2 (minor, ${ }^{10} \mathrm{BF}_{4}$ ), -153.3 (major, ${ }^{11} \mathrm{BF}_{4}$ ); LRMS (ESI) $\mathrm{m} / \mathrm{z}$ $350.2\left(\left[\mathrm{M}-\mathrm{BF}_{4}\right]^{+}\right)$; HRMS (ESI) $m / z \mathrm{C}_{26} \mathrm{H}_{24} \mathrm{~N}^{+}$requires $350.1903\left(\left[\mathrm{M}-\mathrm{BF}_{4}\right]^{+}\right)$, found 350.1900 . 
Data is consistent with the literature. ${ }^{11}$

1-Cyclooctyl-2,4,6-triphenylpyridin-1-ium tetrafluoroborate (1j)<smiles></smiles>

Prepared according to General Procedure A using cyclooctanamine ( $335 \mu \mathrm{L}, 2.40 \mathrm{mmol}, 1.2$ equiv.), 2,4,6triphenylpyrylium tetrafluoroborate $(792 \mathrm{mg}, 2.00 \mathrm{mmol}, 1.0$ equiv.) in EtOH (2.0 mL). Pyridinium salt $\mathbf{1 j}$ was isolated as a white solid (565 mg, 56\%).

${ }^{1} \mathrm{H}$ NMR $\left(400 \mathrm{MHz}, \mathrm{CDCl}_{3}\right) \delta 7.95-7.33(\mathrm{~m}, 17 \mathrm{H}), 4.98(\mathrm{t}, J=8.5 \mathrm{~Hz}, 1 \mathrm{H}), 2.20$ (br. t, $\left.J=10.0 \mathrm{~Hz}, 2 \mathrm{H}\right), 1.61$ (app. q, $J=10.0 \mathrm{~Hz}, 2 \mathrm{H}), 1.45-1.44(\mathrm{~m}, 2 \mathrm{H}), 1.30-0.96(\mathrm{~m}, 7 \mathrm{H}), 0.61$ (br. $\mathrm{d}, J=7.0 \mathrm{~Hz}, 1 \mathrm{H}) ;{ }^{13} \mathrm{C} \mathrm{NMR}(101 \mathrm{MHz}$, $\left.\mathrm{CDCl}_{3}\right) \delta 156.4,154.6,134.0,134.0,131.5,130.6,129.3,128.6,128.1,127.9,126.3,71.1,35.8,25.7,25.5$, 25.3; ${ }^{19} \mathrm{~F}$ NMR (377 MHz, CDCl 3 ) $\delta$-153.4 (minor, ${ }^{10} \mathrm{BF}_{4}$ ), -153.5 (major, ${ }^{11} \mathrm{BF}_{4}$ ); LRMS (ESI) $\mathrm{m} / \mathrm{z} 418.2$ ([M-BF $]^{+}$); HRMS (ESI) $m / z \mathrm{C}_{31} \mathrm{H}_{32} \mathrm{~N}^{+}$requires $418.2529\left(\left[\mathrm{M}-\mathrm{BF}_{4}\right]^{+}\right)$, found 418.2529 .

Data is consistent with the literature. ${ }^{12}$

1-(2-Methylcyclohexyl)-2,4,6-triphenylpyridin-1-ium tetrafluoroborate (1k)<smiles></smiles>

Prepared according to General Procedure A using 2-methylcyclohexanamine (317 $\mu \mathrm{L}, 2.40 \mathrm{mmol}, 1.2$ equiv.), 2,4,6-triphenylpyrylium tetrafluoroborate (792 mg, $2.00 \mathrm{mmol}, 1.0$ equiv.) in EtOH (2.0 mL). Pyridinium salt $1 \mathbf{k}$ was isolated as a white solid (374 $\mathrm{mg}, 38 \%$ ).

${ }^{1} \mathrm{H}$ NMR $\left(400 \mathrm{MHz}, \mathrm{CDCl}_{3}\right) \delta 8.27-7.32(\mathrm{~m}, 17 \mathrm{H}), 4.31(\mathrm{t}, J=11.5 \mathrm{~Hz}, 1 \mathrm{H}), 2.48(\mathrm{~d}, J=12.5 \mathrm{~Hz}, 1 \mathrm{H}), 1.78(\mathrm{q}, J$ $=12.5 \mathrm{~Hz}, 1 \mathrm{H}), 1.62(\mathrm{~d}, J=11.5 \mathrm{~Hz}, 1 \mathrm{H}), 1.54-1.48(\mathrm{~m}, 2 \mathrm{H}), 1.35(\mathrm{~d}, J=11.5 \mathrm{~Hz}, 1 \mathrm{H}), 0.87-0.60(\mathrm{~m}, 5 \mathrm{H}), 0.53$ $(q, J=11.0 \mathrm{~Hz}, 1 \mathrm{H}) ;{ }^{13} \mathrm{C} \mathrm{NMR}\left(101 \mathrm{MHz}, \mathrm{CDCl}_{3}\right) \delta 158.4,156.8,155.2,134.2,133.8,133.6,132.2,131.4,131.1$, $129.7,129.6,129.2,128.9,128.6,128.4,128.2,126.9,77.6,36.2,35.6,34.2,26.2,25.1,18.4 ;{ }^{19} \mathrm{~F}$ NMR $(377$ $\mathrm{MHz}, \mathrm{CDCl}_{3}$ ) $\delta$-153.3 (minor, ${ }^{10} \mathrm{BF}_{4}$ ), -153.3 (major, ${ }^{11} \mathrm{BF}_{4}$ ); LRMS (ESI) $\mathrm{m} / \mathrm{z} 404.2$ ([M-BF $]^{+}$); HRMS (ESI) $\mathrm{m} / \mathrm{z}$ $\mathrm{C}_{30} \mathrm{H}_{30} \mathrm{~N}^{+}$requires $404.2373\left(\left[\mathrm{M}-\mathrm{BF}_{4}\right]^{+}\right.$), found 404.2373; IR (thin film, $v_{\max } / \mathrm{cm}^{-1}$ ) 2980, 1618, 1562, 1384, 1052, 704; m.p. $\left(\mathrm{CH}_{2} \mathrm{Cl}_{2}\right): 180-184^{\circ} \mathrm{C}$.

Note: mix of cis and trans diastereomers used, single diastereomer product undefined.

1-(Heptan-2-yl)-2,4,6-triphenylpyridin-1-ium tetrafluoroborate (1I) 
<smiles></smiles>

Prepared according to General Procedure A using heptan-2-amine ( $360 \mu \mathrm{L}, 2.40 \mathrm{mmol}, 1.2$ equiv.), 2,4,6triphenylpyrylium tetrafluoroborate (792 mg, $2.00 \mathrm{mmol}, 1.0$ equiv.) in EtOH (2.0 mL). Pyridinium salt $1 \mathrm{l}$ was isolated as a white solid (602 $\mathrm{mg}, 61 \%)$.

${ }^{1} \mathrm{H}$ NMR $\left(400 \mathrm{MHz}, \mathrm{CDCl}_{3}\right) \delta 7.97-7.39(\mathrm{~m}, 17 \mathrm{H}), 5.02-4.71(\mathrm{~m}, 1 \mathrm{H}), 1.84-1.71(\mathrm{~m}, 1 \mathrm{H}), 1.48-1.36(\mathrm{~m}$, 4H), $1.18-1.07(\mathrm{~m}, 2 \mathrm{H}), 1.07-0.91(\mathrm{~m}, 3 \mathrm{H}), 0.86-0.71(\mathrm{~m}, 4 \mathrm{H}) ;{ }^{13} \mathrm{C}$ NMR $\left(101 \mathrm{MHz}, \mathrm{CDCl}_{3}\right) \delta$ 157.3, 155.3, 134.1, 134.0, 132.1, 131.0, 129.7, 129.4, 129.0, 128.4, 67.2, 36.9, 31.0, 26.4, 22.4, 21.8, 14.0 (1 aromatic carbon signal is not observed due to signal broadening); ${ }^{19} \mathrm{~F}$ NMR $\left(377 \mathrm{MHz}, \mathrm{CDCl}_{3}\right) \delta-153.3\left(\right.$ minor, $\left.{ }^{10} \mathrm{BF}_{4}\right),-$ 153.4 (major, ${ }^{11} \mathrm{BF}_{4}$ ); LRMS (ESI) $\mathrm{m} / z 406.2\left(\left[\mathrm{M}-\mathrm{BF}_{4}\right]^{+}\right)$; HRMS (ESI) $m / z \mathrm{C}_{30} \mathrm{H}_{32} \mathrm{~N}^{+}$requires 406.2529 ([M-BF $]^{+}$), found 406.2523; IR (thin film, $\left.v_{\max } / \mathrm{cm}^{-1}\right)$ 2981, 1620, 1563, 1050, 763, 702; m.p. $\left(\mathrm{CH}_{2} \mathrm{Cl}_{2}\right): 150-155^{\circ} \mathrm{C}$.

Data is consistent with the literature. ${ }^{5}$

2,4,6-Triphenyl-1-(tetrahydro-2H-pyran-4-yl)pyridin-1-ium tetrafluoroborate (1m)<smiles>c1ccc(-c2cc(-c3ccccc3)c(-c3ccccc3)c(-c3ccccc3)c2)cc1</smiles>

Prepared according to General Procedure A using tetrahydro-2H-pyran-4-amine ( $247 \mu \mathrm{L}, 2.40 \mathrm{mmol}, 1.2$ equiv.), 2,4,6-triphenylpyrylium tetrafluoroborate (792 mg, $2.00 \mathrm{mmol}, 1.0$ equiv.) in EtOH (2.0 mL). Pyridinium salt $1 \mathrm{~m}$ was isolated as a white solid (640 mg, 67\%).

${ }^{1} \mathrm{H}$ NMR $\left(400 \mathrm{MHz}, \mathrm{CDCl}_{3}\right) \delta 7.75$ (app. d, $J=6.0 \mathrm{~Hz}, 6 \mathrm{H}$ ), 7.67 (app. d, $J=7.5 \mathrm{~Hz}, 2 \mathrm{H}$ ), $7.63-7.55(\mathrm{~m}, 6 \mathrm{H}$ ), 7.50 (app. t, $J=7.5 \mathrm{~Hz}, 1 \mathrm{H}$ ), 7.42 (app. t, $J=7.5 \mathrm{~Hz}, 2 \mathrm{H}$ ), 4.87 (t, $J=11.5 \mathrm{~Hz}, 1 \mathrm{H}$ ), 3.71 (dd, $J=11.5,3.0 \mathrm{~Hz}, 2 \mathrm{H}$ ), 2.79 $(\mathrm{t}, J=11.5 \mathrm{~Hz}, 2 \mathrm{H}), 2.06(\mathrm{~d}, J=11.5 \mathrm{~Hz}, 2 \mathrm{H}), 1.90-183(\mathrm{~m}, 2 \mathrm{H}) .{ }^{13} \mathrm{C} \mathrm{NMR}\left(101 \mathrm{MHz}, \mathrm{CDCl}_{3}\right) \delta$ 157.3, 155.4, $134.1,133.9,132.1,131.2,129.7,129.5,129.1,128.4,128.4,69.2,67.9,33.9 ;{ }^{19} \mathrm{~F} \mathrm{NMR}\left(377 \mathrm{MHz}, \mathrm{CDCl}_{3}\right) \delta$ 152.8 (minor, ${ }^{10} \mathrm{BF}_{4}$ ), -152.9 (major, ${ }^{11} \mathrm{BF}_{4}$ ); LRMS (ESI) $\mathrm{m} / \mathrm{z} 392.2$ ([M-BF $]^{+}$); HRMS (ESI) $\mathrm{m} / \mathrm{z} \mathrm{C}_{28} \mathrm{H}_{26} \mathrm{NO}^{+}$ requires 392.2009 $\left(\left[\mathrm{M}-\mathrm{BF}_{4}\right]^{+}\right)$, found 392.2006; IR (thin film, $\left.v_{\max } / \mathrm{cm}^{-1}\right)$ 2980, 1621, 1565, 1150, 1053, 705; m.p. $\left(\mathrm{CH}_{2} \mathrm{Cl}_{2}\right): 135^{\circ} \mathrm{C}$ (decomposition).

Data is consistent with the literature. ${ }^{10}$

2,4,6-Triphenyl-1-(tetrahydro-2H-thiopyran-4-yl)pyridin-1-ium tetrafluoroborate (1n) 
<smiles></smiles>

Prepared according to General Procedure A using tetrahydro-2H-thiopyran-4-amine ( $281 \mathrm{mg}, 2.40 \mathrm{mmol}, 1.2$ equiv.) and 2,4,6-triphenylpyrylium tetrafluoroborate (792 mg, $2.00 \mathrm{mmol}, 1.0$ equiv.) in EtOH (2.0 mL). Pyridinium salt $1 \mathrm{n}$ was isolated as an off-white solid (678 mg, 68\%).

${ }^{1} \mathrm{H}$ NMR $\left(400 \mathrm{MHz}, \mathrm{CDCl}_{3}\right) \delta 7.86$ (br. s, 2H), $7.77-7.73(\mathrm{~m}, 6 \mathrm{H}), 7.67-7.61(\mathrm{~m}, 6 \mathrm{H}), 7.56-7.47(\mathrm{~m}, 3 \mathrm{H}), 4.61$ $(\mathrm{t}, J=12.0 \mathrm{~Hz}, 1 \mathrm{H}), 2.51-2.42(\mathrm{~m}, 4 \mathrm{H}), 2.18(\mathrm{t}, J=12.5 \mathrm{~Hz}, 2 \mathrm{H}), 1.87(\mathrm{q}, J=12.0 \mathrm{~Hz}, 2 \mathrm{H}) ;{ }^{13} \mathrm{C} \mathrm{NMR}(101 \mathrm{MHz}$, $\mathrm{CDCl}_{3}$ ) $\delta 157.0,155.5,134.1,133.8,132.0,131.2,129.6,129.1$ (br.), 128.4, 71.0, 35.3, 29.70 (2 aromatic carbon signals not observed due to signal broadening);; ${ }^{19} \mathrm{~F}$ NMR $\left(377 \mathrm{MHz}, \mathrm{CDCl}_{3}\right) \delta-152.8$ (minor, $\left.{ }^{10} \mathrm{BF}_{4}\right)$, -

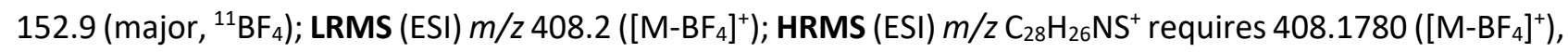
found 408.1778 .

Data is consistent with the literature. ${ }^{13}$

\section{1-(1,1-Dioxidotetrahydro-2H-thiopyran-4-yl)-2,4,6-triphenylpyridin-1-ium tetrafluoroborate (10)}<smiles></smiles>

$m$ CPBA (436 mg, $2.5 \mathrm{mmol}, 2.8$ equiv.) was added in one portion to a stirred, cold $\left(0^{\circ} \mathrm{C}\right.$ ) solution of $2,4,6$ triphenyl-1-(tetrahydro-2H-thiopyran-4-yl)pyridin-1-ium tetrafluoroborate (445 mg, $0.90 \mathrm{mmol}, 1.0$ equiv.) in chloroform $(4.5 \mathrm{~mL})$ and the reaction was allowed to warm to room temperature overnight. Pyridinium salt 10 precipitated out of the solution and was filtered off, washed with $\mathrm{Et}_{2} \mathrm{O}$, and isolated as a white solid (470 mg, $0.892 \mathrm{mmol}, 99 \%$ ).

${ }^{1} \mathrm{H}$ NMR (400 MHz, Acetone- $\left.d_{6}\right) \delta 8.36$ (br. s, $\left.2 \mathrm{H}\right), 8.18$ (br. d, $\left.J=8.3 \mathrm{~Hz}, 2 \mathrm{H}\right), 7.90-7.88(\mathrm{~m}, 4 \mathrm{H}), 7.69(\mathrm{~m}, 9 \mathrm{H})$, $5.20(\mathrm{t}, J=12.0 \mathrm{~Hz}, 1 \mathrm{H}), 2.91-2.77(\mathrm{~m}, 6 \mathrm{H}), 2.48-2.39(\mathrm{~m}, 2 \mathrm{H}) ;{ }^{13} \mathrm{C}$ NMR $\left(101 \mathrm{MHz}\right.$, Acetone- $\left.d_{6}\right) \delta 158.7$, 155.9, 134.8, 134.4, 133.3, 132.1, 130.6, 130.4 (br.), 129.9 (br.), 129.5, 128.7 (br.), 79.2, 68.5, 51.5; ${ }^{19}$ F NMR (377 MHz, Acetone- $d_{6}$ ) $\delta$-151.6 (minor, ${ }^{10} \mathrm{BF}_{4}$ ), -151.7 (major, ${ }^{11} \mathrm{BF}_{4}$ ); LRMS (ESI) $\mathrm{m} / \mathrm{z} 440.0\left(\left[\mathrm{M}-\mathrm{BF}_{4}\right]^{+}\right.$); HRMS (ESI) $\mathrm{m} / \mathrm{z} \mathrm{C}_{28} \mathrm{H}_{26} \mathrm{NO}_{2} \mathrm{~S}^{+}$requires $440.1679\left(\left[\mathrm{M}-\mathrm{BF}_{4}\right]^{+}\right.$), found 440.1682; IR (thin film, $v_{\max } / \mathrm{cm}^{-1}$ ) 3627, 3061, $2986,1620,1562,1296,1117,1059,769,706$; m.p. $\left(\mathrm{CH}_{2} \mathrm{Cl}_{2}\right)$ : $164^{\circ} \mathrm{C}$ (degradation). 
<smiles></smiles>

Prepared according to General Procedure A using 4-aminocyclohexanol (276 mg, $2.40 \mathrm{mmol}, 1.2$ equiv.), 2,4,6-triphenylpyrylium tetrafluoroborate (792 mg, $2.00 \mathrm{mmol}, 1.0$ equiv.) in EtOH ( $2.0 \mathrm{~mL}$ ). Pyridinium salt $1 p$ was isolated as a white solid (600 $\mathrm{mg}, 61 \%)$.

${ }^{1} \mathrm{H}$ NMR $\left(400 \mathrm{MHz}\right.$, acetone- $\left.d_{6}\right) \delta 8.28(\mathrm{~s}, 2 \mathrm{H}), 8.19-8.10(\mathrm{~m}, 2 \mathrm{H}), 7.91-7.81(\mathrm{~m}, 4 \mathrm{H}), 7.77-7.56(\mathrm{~m}, 9 \mathrm{H})$, $4.73(\mathrm{tt}, J=12.0,3.0 \mathrm{~Hz}, 1 \mathrm{H}), 3.67(\mathrm{~d}, J=4.5 \mathrm{~Hz}, 1 \mathrm{H}), 2.97(\mathrm{td}, J=11.0,5.0 \mathrm{~Hz}, 1 \mathrm{H}), 2.24(\mathrm{~d}, J=12.5 \mathrm{~Hz}, 2 \mathrm{H})$, $1.88-1.57(\mathrm{~m}, 4 \mathrm{H}), 0.77(\mathrm{qd}, J=12.5,3.0 \mathrm{~Hz}, 2 \mathrm{H}) ;{ }^{13} \mathrm{C}$ NMR $\left(101 \mathrm{MHz}\right.$, acetone- $\left.d_{6}\right) \delta 158.4,155.2,135.1$, $134.4,133.1,131.7,130.5,130.2,129.6,129.3,128.4,71.8,68.7,35.8,31.7 ;{ }^{19} \mathrm{~F}$ NMR $\left(377 \mathrm{MHz}\right.$, acetone- $\left.d_{6}\right)$ $\delta$-151.6 (minor, ${ }^{10} \mathrm{BF}_{4}$ ), -151.7 (major, ${ }^{11} \mathrm{BF}_{4}$ ); LRMS (ESI) $\mathrm{m} / \mathrm{z} 406.2\left(\left[\mathrm{M}-\mathrm{BF}_{4}\right]^{+}\right)$; HRMS (ESI) $\mathrm{m} / \mathrm{z} \mathrm{C}_{29} \mathrm{H}_{28} \mathrm{NO}^{+}$ requires $406.2165\left(\left[\mathrm{M}-\mathrm{BF}_{4}\right]^{+}\right)$, found 406.2161 .

Data is consistent with the literature. ${ }^{14}$

\section{1-(4-Carboxycyclohexyl)-2,4,6-triphenylpyridin-1-ium tetrafluoroborate (1q)}<smiles></smiles>

Prepared according to General Procedure A using 4-aminocyclohexanecarboxylic acid hydrochloride (413 $\mathrm{mg}, 2.40 \mathrm{mmol}, 1.2$ equiv.), triethylamine (336 $\mu \mathrm{L}, 2.40 \mathrm{mmol}, 1.2$ equiv.), and 2,4,6-triphenylpyrylium tetrafluoro-borate $(792 \mathrm{mg}, 2.00 \mathrm{mmol}, 1.0$ equiv.) in $\mathrm{EtOH}(2.0 \mathrm{~mL})$. Pyridinium salt $1 \mathrm{q}$ was isolated as a white solid (564 mg, 54\%).

${ }^{1} \mathrm{H}$ NMR $\left(400 \mathrm{MHz}, \mathrm{DMSO}-d_{6}\right) \delta 12.08$ (br. s, $\left.1 \mathrm{H}\right), 8.41$ (s, 2H), 8.24 (d, $\left.J=7.5 \mathrm{~Hz}, 2 \mathrm{H}\right), 7.78-7.58(\mathrm{~m}, 13 \mathrm{H})$, $4.42(\mathrm{t}, J=11.0 \mathrm{~Hz}, 1 \mathrm{H}), 2.16(\mathrm{~d}, J=11.0 \mathrm{~Hz}, 2 \mathrm{H}), 1.74(\mathrm{~d}, J=12.5 \mathrm{~Hz}, 2 \mathrm{H}), 1.55(\mathrm{t}, J=11.0 \mathrm{~Hz}, 1 \mathrm{H}), 1.40(\mathrm{q}, J=$ $11.5 \mathrm{~Hz}, 2 \mathrm{H}), 0.72(\mathrm{q}, J=12.5 \mathrm{~Hz}, 2 \mathrm{H}) ;{ }^{13} \mathrm{C}$ NMR (101 MHz, DMSO-d $)$ ) $175.1,157.1,153.3,133.9,132.8$, $132.5,130.9,129.6,129.4,128.7,128.7,126.9,70.3,41.2,31.7,28.7 ;{ }^{19} \mathrm{~F}$ NMR $\left(377 \mathrm{MHz}, \mathrm{DMSO}-d_{6}\right) \delta-148.3$ (minor, ${ }^{10} \mathrm{BF}_{4}$ ), 148.3 (major, ${ }^{11} \mathrm{BF}_{4}$ ); LRMS (ESI) $\mathrm{m} / \mathrm{z} 434.2$ ([M-BF $]^{+}$); HRMS (ESI) $\mathrm{m} / z \mathrm{C}_{30} \mathrm{H}_{28} \mathrm{NO}_{2}{ }^{+}$requires $434.2115\left(\left[\mathrm{M}-\mathrm{BF}_{4}\right]^{+}\right)$, found 434.2109; IR (thin film, $\left.v_{\max } / \mathrm{cm}^{-1}\right)$ 2978, 1710, 1620, 1141, 1058, 768, 705; m.p. $\left(\mathrm{CH}_{2} \mathrm{Cl}_{2}\right): 210-213^{\circ} \mathrm{C}$.

\section{1-(4-(Methoxycarbonyl)cyclohexyl)-2,4,6-triphenylpyridin-1-ium tetrafluoroborate (1r)}


<smiles></smiles>

Prepared according to General Procedure A using methyl 4-aminocyclohexane-1-carboxylate hydrochloride (465 mg, $2.40 \mathrm{mmol}, 1.2$ equiv.) and 2,4,6-triphenylpyrylium tetrafluoroborate (792 mg, $2.00 \mathrm{mmol}, 1.0$ equiv.) in $\mathrm{EtOH}(2.0 \mathrm{~mL})$ and triethylamine $(335 \mu \mathrm{l}, 2.40 \mathrm{mmol}, 1.20 \mathrm{mmol})$. Pyridinium salt $1 \mathrm{r}$ was isolated as an off-white solid (755 mg, 71\%).

${ }^{1} \mathrm{H}$ NMR $\left(400 \mathrm{MHz}, \mathrm{CDCl}_{3}\right) \delta 7.77-7.66(\mathrm{~m}, 8 \mathrm{H}), 7.62-7.53(\mathrm{~m}, 6 \mathrm{H}), 7.50-7.47(\mathrm{~m}, 1 \mathrm{H}), 7.42-7.39(\mathrm{~m}, 2 \mathrm{H})$, $4.61(\mathrm{t}, J=12.0 \mathrm{~Hz}, 1 \mathrm{H}), 3.52(\mathrm{~s}, 3 \mathrm{H}), 2.21(\mathrm{~d}, J=12.0 \mathrm{~Hz}, 2 \mathrm{H}), 1.82(\mathrm{~d}, J=13.0 \mathrm{~Hz}, 2 \mathrm{H}), 1.76-1.64(\mathrm{~m}, 1 \mathrm{H})$, $1.55(\mathrm{q}, J=12.5 \mathrm{~Hz}, 2 \mathrm{H}), 0.93(\mathrm{q}, J=12.5 \mathrm{~Hz}, 2 \mathrm{H}) ;{ }^{13} \mathrm{C} \mathrm{NMR}\left(101 \mathrm{MHz}, \mathrm{CDCl}_{3}\right) \delta 174.6,157.2,155.3,134.1$, 134.0, 132,0 131.1, 129.6, 129.4, 129.0, 128.4, 70.6, 51.8, 41.6, 32.2, 28.9; ${ }^{19} \mathrm{~F}$ NMR (377 MHz, $\left.\mathrm{CDCl}_{3}\right) \delta-153.1$ (minor, ${ }^{10} \mathrm{BF}_{4}$ ), -153.2 (major, ${ }^{11} \mathrm{BF}_{4}$ ); LRMS (ESI) $\mathrm{m} / \mathrm{z} 448.2\left(\left[\mathrm{M}-\mathrm{BF}_{4}\right]^{+}\right.$); HRMS (ESI) $\mathrm{m} / z \mathrm{C}_{31} \mathrm{H}_{30} \mathrm{NO}_{2}{ }^{+}$requires 448.2271 ([M-BF $\left.]^{+}\right)$, found 448.2262; IR (thin film, $v_{\max } / \mathrm{cm}^{-1}$ ) 2981, 1728, 1620, 1563, 1147, 1052, 766, 705; m.p. $\left(\mathrm{CH}_{2} \mathrm{Cl}_{2}\right): 206-209^{\circ} \mathrm{C}$.

\section{1-(1-(2,6-Dimethylphenoxy)propan-2-yl)-2,4,6-triphenylpyridin-1-ium tetrafluoroborate (1s)}<smiles></smiles>

Prepared according to General Procedure A using Mexiletine hydrochloride (518 mg, $2.40 \mathrm{mmol}, 1.2$ equiv.) and 2,4,6-triphenylpyrylium tetrafluoroborate $(792 \mathrm{mg}, 2.00 \mathrm{mmol}, 1.0$ equiv.) in EtOH $(2.0 \mathrm{~mL})$ and triethylamine $(335 \mu \mathrm{l}, 2.40 \mathrm{mmol}, 1.20 \mathrm{mmol})$. Pyridinium salt $1 \mathrm{~s}$ was purified by column chromatography (0$10 \%$ Acetone in $\mathrm{CH}_{2} \mathrm{Cl}_{2}$ ) and isolated as a yellow foam (555 mg, 50\%).

$\mathbf{R}_{f}\left(10 \%\right.$ Acetone in $\left.\mathrm{CH}_{2} \mathrm{Cl}_{2}\right)=0.43 ;{ }^{1} \mathbf{H}$ NMR $\left(400 \mathrm{MHz}, \mathrm{CDCl}_{3}\right) \delta 7.80$ (br. s, 4H), $7.73-7.69(\mathrm{~m}, 3 \mathrm{H}), 7.61-7.49$ $(\mathrm{m}, 8 \mathrm{H}), 7.46-7.41(\mathrm{~m}, 2 \mathrm{H}), 6.92-6.86(\mathrm{~m}, 3 \mathrm{H}), 5.52-5.38(\mathrm{~m}, 1 \mathrm{H}), 4.12(\mathrm{dd}, J=10.0,6.5 \mathrm{~Hz}, 1 \mathrm{H}), 3.51(\mathrm{dd}$, $J=10.0,7.0 \mathrm{~Hz}, 1 \mathrm{H}), 1.91(\mathrm{~s}, 6 \mathrm{H}), 1.53(\mathrm{~d}, J=7.0 \mathrm{~Hz}, 3 \mathrm{H}) ;{ }^{13} \mathrm{C}$ NMR $\left(101 \mathrm{MHz}, \mathrm{CDCl}_{3}\right) \delta 155.8,154.4,134.1$, 133.7, 132.1, 131.1, 130.1, 129.7, 129.4, 129.3, 129.0, 128.4, 124.6, 73.5, 65.4, 19.4, 16.3 (2 aromatic carbon signals are not observed due to signal broadening); ${ }^{19} \mathrm{~F} \mathrm{NMR}\left(377 \mathrm{MHz}, \mathrm{CDCl}_{3}\right) \delta-152.9$ (minor, $\left.{ }^{10} \mathrm{BF}_{4}\right),-153.0$ (major, $\left.{ }^{11} \mathrm{BF}_{4}\right)$; LRMS (ESI) $\mathrm{m} / z 470.2\left(\left[\mathrm{M}-\mathrm{BF}_{4}\right]^{+}\right)$; HRMS (ESI) $\mathrm{m} / z \mathrm{C}_{34} \mathrm{H}_{32} \mathrm{NO}^{+}$requires $470.2478\left(\left[\mathrm{M}-\mathrm{BF}_{4}\right]^{+}\right)$, found 470.2471 .

Data is consistent with the literature. ${ }^{15}$

(S)-1-(1-Hydroxy-3-phenylpropan-2-yl)-2,4,6-triphenylpyridin-1-ium tetrafluoroborate (1t) 
<smiles>OC[C@H](Cc1ccccc1)c1c(-c2ccccc2)cc(-c2ccccc2)cc1-c1ccccc1</smiles>

Prepared according to General Procedure A using L-phenylalaninol (182 mg, $1.20 \mathrm{mmol}, 1.2$ equiv.) and 2,4,6triphenylpyrylium tetrafluoroborate (396 mg, $1.00 \mathrm{mmol}, 1.0$ equiv.) in $\mathrm{EtOH}(1.0 \mathrm{~mL}$ ). Pyridinium salt 1t was isolated as a white powder (402 $\mathrm{mg}, 76 \%$ ).

${ }^{1} \mathrm{H}$ NMR $\left(400 \mathrm{MHz}, \mathrm{CDCl}_{3}\right) \delta 8.07-7.70(\mathrm{~m}, 7 \mathrm{H}), 7.72-7.37(\mathrm{~m}, 9 \mathrm{H}), 7.20-7.01(\mathrm{~m}, 4 \mathrm{H}), 6.59(\mathrm{~d}, \mathrm{~J}=7.0 \mathrm{~Hz}$, $2 \mathrm{H}), 5.43-5.31(\mathrm{~m}, 1 \mathrm{H}), 3.70(\mathrm{dd}, J=20.5,8.0 \mathrm{~Hz}, 2 \mathrm{H}), 3.53(\mathrm{dd}, J=10.0,6.0 \mathrm{~Hz}, 1 \mathrm{H}), 3.23(\mathrm{dd}, J=14.0,6.5$ $\mathrm{Hz}, 1 \mathrm{H}), 2.50$ (dd, $J=14.0,8.0 \mathrm{~Hz}, 1 \mathrm{H}) ;{ }^{13} \mathrm{C} \mathrm{NMR}\left(101 \mathrm{MHz}, \mathrm{CDCl}_{3}\right) \delta 155.2,135.7,133.7,133.6$ (br.), 132.5, 131.2 (br.), 130.2, 129.9, 129.2, 129.1, 128.7, 128.4, 127.6, 73.3, 62.6, 38.5 (two signals missing due to signal broadening); ${ }^{19} \mathrm{~F}$ NMR (377 MHz, $\mathrm{CDCl}_{3}$ ) $\delta$-152.9 (minor, ${ }^{10} \mathrm{BF}_{4}$ ), -152.9 (major, ${ }^{11} \mathrm{BF}_{4}$ ); LRMS (ESI) $\mathrm{m} / \mathrm{z} 442.2$ $\left(\left[\mathrm{M}-\mathrm{BF}_{4}\right]^{+}\right)$; HRMS (ESI) $m / z \mathrm{C}_{32} \mathrm{H}_{28} \mathrm{NO}^{+}$requires $442.2165\left(\left[\mathrm{M}-\mathrm{BF}_{4}\right]^{+}\right)$, found 442.2162 .

Data is consistent with the literature..$^{15}$

(S)-1-(1-methoxy-1-oxo-3-phenylpropan-2-yl)-2,4,6-triphenylpyridin-1-ium tetrafluoroborate (1u)<smiles></smiles>

Prepared according to General Procedure A using L-phenylalanine methyl ester hydrochloride (259 mg, $1.20 \mathrm{mmol}, 1.2$ equiv.) and 2,4,6-triphenylpyrylium tetrafluoroborate (396 mg, $1.00 \mathrm{mmol}, 1.0$ equiv.) in $\mathrm{EtOH}(1.0 \mathrm{~mL})$ and triethylamine $(167 \mu \mathrm{l}, 1.20 \mathrm{mmol}, 1.2$ equiv.). Pyridinium salt $1 \mathrm{u}$ was isolated as a paleyellow powder (138 mg, 25\%).

${ }^{1} \mathrm{H}$ NMR $\left(400 \mathrm{MHz}, \mathrm{CDCl}_{3}\right) \delta 7.97(\mathrm{~s}, 2 \mathrm{H}), 7.90-7.72(\mathrm{~m}, 4 \mathrm{H}), 7.67-7.45(\mathrm{~m}, 11 \mathrm{H}), 7.16-7.03(\mathrm{~m}, 3 \mathrm{H}), 6.78$ (dd, $J=7.5,1.5 \mathrm{~Hz}, 2 \mathrm{H}$ ), 5.66 (dd, $J=8.0,3.5 \mathrm{~Hz}, 1 \mathrm{H}$ ), 3.69 (s, 3H), 3.52 (dd, $J=14.5,3.5 \mathrm{~Hz}, 1 \mathrm{H}$ ), 2.90 (dd, $J=$ 14.5, 8.5 Hz, 1H); ${ }^{13} \mathrm{C}$ NMR (101 MHz, $\left.\mathrm{CDCl}_{3}\right) \delta 168.3,157.3,157.0,136.6,134.0,132.5,132.5,131.6,129.9$, $129.7,129.3,129.2,128.7,128.1,127.3,70.4,53.8,37.9 ;{ }^{19} \mathrm{~F}$ NMR $\left(377 \mathrm{MHz}, \mathrm{CDCl}_{3}\right) \delta$-153.1 (minor, $\left.{ }^{10} \mathrm{BF}_{4}\right)$, -153.1 (major, ${ }^{11} \mathrm{BF}_{4}$ ); LRMS (ESI) $\mathrm{m} / \mathrm{z} 470.2\left(\left[\mathrm{M}-\mathrm{BF}_{4}\right]^{+}\right.$); HRMS (ESI) $\mathrm{m} / \mathrm{z} \mathrm{C}_{33} \mathrm{H}_{28} \mathrm{NO}_{2}{ }^{+}$requires 470.2115 ([M$\left.\mathrm{BF}_{4}\right]^{+}$), found 470.2112 .

Data is consistent with the literature. ${ }^{11}$ 
<smiles></smiles>

Prepared according to General Procedure A using benzylamine (655 $\mu \mathrm{l}, 6 \mathrm{mmol}, 1.2$ equiv.) and 2,4,6triphenylpyrylium tetrafluoroborate $(1.98 \mathrm{~g}, 5 \mathrm{mmol}, 1.0$ equiv.) in EtOH $(5 \mathrm{~mL})$. Pyridinium salt $\mathbf{1 b}$ was isolated as a white solid ( $2.01 \mathrm{~g}, 83 \%)$.

${ }^{1} \mathrm{H}$ NMR $\left(400 \mathrm{MHz}, \mathrm{CDCl}_{3}\right) \delta 7.94(\mathrm{~s}, 2 \mathrm{H}), 7.85-7.78(\mathrm{~m}, 2 \mathrm{H}), 7.64(\mathrm{~d}, J=6.5 \mathrm{~Hz}, 4 \mathrm{H}), 7.61-7.42(\mathrm{~m}, 9 \mathrm{H}), 7.21$ $-7.12(\mathrm{~m}, 1 \mathrm{H}), 7.13-7.06(\mathrm{~m}, 2 \mathrm{H}), 6.46(\mathrm{~d}, J=7.5 \mathrm{~Hz}, 2 \mathrm{H}), 5.77(\mathrm{~s}, 2 \mathrm{H}) ;{ }^{13} \mathrm{C}$ NMR $\left(101 \mathrm{MHz}, \mathrm{CDCl}_{3}\right) \delta 157.8$, 156.5, 134.4, 134.0, 132.9, 132.5, 131.1, 130.0, 129.3, 129.2, 129.0, 128.4, 128.3, 126.7, 126.4, 58.4; ${ }^{19} \mathrm{~F} \mathrm{NMR}$

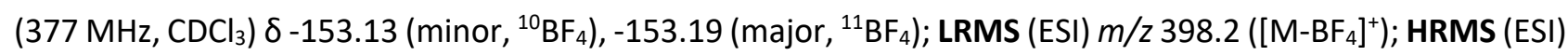
$m / z \mathrm{C}_{30} \mathrm{H}_{24} \mathrm{~N}^{+}$requires $398.1903\left(\left[\mathrm{M}-\mathrm{BF}_{4}\right]^{+}\right)$, found 398.1902.

Data is consistent with the literature. ${ }^{16}$

1-(4-Methoxybenzyl)-2,4,6-triphenylpyridin-1-ium tetrafluoroborate (1v)<smiles></smiles>

Prepared according to General Procedure B using 4-methozybenzylamine ( $340 \mu \mathrm{l}, 2.6 \mathrm{mmol}, 1.3$ equiv.) and 2,4,6-triphenylpyrylium tetrafluoroborate (792 mg, $2 \mathrm{mmol}, 1.0$ equiv.) in $\mathrm{CH}_{2} \mathrm{Cl}_{2}(4 \mathrm{~mL})$ and $\mathrm{AcOH}(57 \mu \mathrm{l}, 1$ mmol, 0.5 equiv.). The crude product was recrystallised from EtOH to give pyridinium salt $1 \mathbf{v}$ as a white powder (748 mg, 73\%).

${ }^{1} \mathrm{H}$ NMR $\left(400 \mathrm{MHz}, \mathrm{CDCl}_{3}\right) \delta 7.90(\mathrm{~s}, 2 \mathrm{H}), 7.78(\mathrm{dt}, J=6.8,1.3 \mathrm{~Hz}, 2 \mathrm{H}), 7.65(\mathrm{br} . \mathrm{d}, J=6.4 \mathrm{~Hz}, 4 \mathrm{H}), 7.57-7.46$ $(\mathrm{m}, 9 \mathrm{H}), 6.64-6.56(\mathrm{~m}, 2 \mathrm{H}), 6.34(\mathrm{~d}, J=8.5 \mathrm{~Hz}, 2 \mathrm{H}), 5.70(\mathrm{~s}, 2 \mathrm{H}), 3.70(\mathrm{~s}, 3 \mathrm{H}) ;{ }^{13} \mathrm{C} \mathrm{NMR}\left(101 \mathrm{MHz}, \mathrm{CDCl}_{3}\right) \delta$ 159.6, 157.6, 156.3, 133.9, 133.0, 132.5, 131.1, 129.9, 129.3, 129.3, 128.3, 127.9, 126.7, 126.1, 114.3, 58.0, 55.4; ${ }^{19} \mathrm{~F}$ NMR (377 MHz, CDCl 3 ) $\delta-153.00$ (minor, ${ }^{10} \mathrm{BF}_{4}$ ), -153.06 (major, ${ }^{11} \mathrm{BF}_{4}$ ); HRMS (ESI) $\mathrm{m} / \mathrm{z} \mathrm{C}_{31} \mathrm{H}_{26} \mathrm{NO}^{+}$ requires $428.2009\left(\left[\mathrm{M}-\mathrm{BF}_{4}\right]^{+}\right)$, found 428.2007 .

Data is consistent with the literature. ${ }^{15}$

1-(3-Methoxybenzyl)-2,4,6-triphenylpyridin-1-ium tetrafluoroborate (1w)<smiles></smiles>

Prepared according to General Procedure B using 3-methozybenzylamine (333 $\mu \mathrm{l}, 2.6 \mathrm{mmol}, 1.3$ equiv.) and 2,4,6-triphenylpyrylium tetrafluoroborate (792 mg, 2 mmol, 1.0 equiv.) in $\mathrm{CH}_{2} \mathrm{Cl}_{2}(4 \mathrm{~mL})$ and $\mathrm{AcOH}(57 \mu \mathrm{l}, 1$ 
mmol, 0.5 equiv.). The crude product was recrystallised from EtOH to give pyridinium salt $\mathbf{1} \mathbf{w}$ as a white powder (785 mg, 76\%).

${ }^{1} \mathrm{H}$ NMR $\left(400 \mathrm{MHz}, \mathrm{CDCl}_{3}\right) \delta 7.93(\mathrm{~s}, 2 \mathrm{H}), 7.80(\mathrm{dt}, J=6.5,1.5 \mathrm{~Hz}, 2 \mathrm{H}), 7.65(\mathrm{~d}, J=6.5 \mathrm{~Hz}, 4 \mathrm{H}), 7.61-7.42(\mathrm{~m}$, $9 \mathrm{H}), 7.03(\mathrm{t}, J=8.0 \mathrm{~Hz}, 1 \mathrm{H}), 6.69(\mathrm{dd}, J=8.0,2.0 \mathrm{~Hz}, 1 \mathrm{H}), 6.09-6.03(\mathrm{~m}, 1 \mathrm{H}), 5.96(\mathrm{t}, J=2.0 \mathrm{~Hz}, 1 \mathrm{H}), 5.73(\mathrm{~s}$, 2H), $3.61(\mathrm{~s}, 3 \mathrm{H}) ;{ }^{13} \mathrm{C}$ NMR (101 MHz, $\left.\mathrm{CDCl}_{3}\right) \delta$ 159.9, 157.7, 156.4, 135.8, 133.9, 132.9, 132.5, 131.1, 130.0, $129.9,129.3,129.3,128.3,126.6,118.4,113.8,112.3,58.3,55.3 ;{ }^{19} \mathrm{~F} \mathrm{NMR}\left(377 \mathrm{MHz}, \mathrm{CDCl}_{3}\right) \delta$-153.06 (minor, ${ }^{10} \mathrm{BF}_{4}$ ), -153.11 (major, ${ }^{11} \mathrm{BF}_{4}$ ); LRMS (ESI) $\mathrm{m} / \mathrm{z} 428.2\left(\left[\mathrm{M}-\mathrm{BF}_{4}\right]^{+}\right)$; HRMS (ESI) $\mathrm{m} / \mathrm{z} \mathrm{C}_{31} \mathrm{H}_{26} \mathrm{NO}^{+}$requires 428.2009 $\left(\left[\mathrm{M}-\mathrm{BF}_{4}\right]^{+}\right)$, found 428.2011 .

Data is consistent with the literature. ${ }^{17}$

1-(2-Methoxybenzyl)-2,4,6-triphenylpyridin-1-ium tetrafluoroborate (1x)<smiles></smiles>

Prepared according to General Procedure B using 2-methozybenzylamine ( $339 \mu \mathrm{l}, 2.6 \mathrm{mmol}, 1.3$ equiv.) and 2,4,6-triphenylpyrylium tetrafluoroborate ( $792 \mathrm{mg}, 2 \mathrm{mmol}, 1.0$ equiv.) in $\mathrm{CH}_{2} \mathrm{Cl}_{2}(4 \mathrm{~mL}$ ) and $\mathrm{AcOH}(57 \mu \mathrm{l}, 1$ mmol, 0.5 equiv.). The crude product was recrystallised from EtOH to give pyridinium salt $\mathbf{1} \mathbf{x}$ as a white powder (792 mg, 87\%).

${ }^{1} \mathrm{H}$ NMR $\left(400 \mathrm{MHz}, \mathrm{CDCl}_{3}\right) \delta 7.87(\mathrm{~s}, 2 \mathrm{H}), 7.80(\mathrm{dq}, J=6.5,2.0,1.5 \mathrm{~Hz}, 2 \mathrm{H}), 7.63(\mathrm{dd}, J=8.0,1.5 \mathrm{~Hz}, 4 \mathrm{H}), 7.58$ $-7.40(\mathrm{~m}, 9 \mathrm{H}), 7.10(\mathrm{td}, J=8.5,1.5 \mathrm{~Hz}, 1 \mathrm{H}), 6.65-6.54(\mathrm{~m}, 2 \mathrm{H}), 6.27(\mathrm{dd}, J=7.5,1.5 \mathrm{~Hz}, 1 \mathrm{H}), 5.72(\mathrm{~s}, 2 \mathrm{H})$, $3.46(\mathrm{~s}, 3 \mathrm{H}) ;{ }^{13} \mathrm{C}$ NMR $\left(101 \mathrm{MHz}, \mathrm{CDCl}_{3}\right) \delta 158.1,156.5,156.0,134.0,133.3,132.4,130.9,129.9,129.9,129.2$, 129.2, 128.6, 128.2, 126.3, 122.4, 120.6, 110.4, 55.8, 55.2; $\left.{ }^{19} \mathrm{~F} \mathrm{NMR} \mathrm{(377} \mathrm{MHz,} \mathrm{CDCl}\right)$ ס -153.15 (minor, ${ }^{10} \mathrm{BF}_{4}$ ),

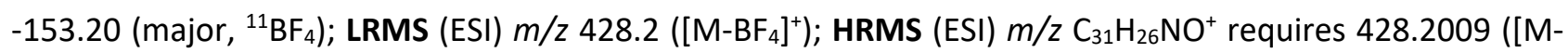
$\left.\mathrm{BF}_{4}\right]^{+}$), found 428.2009 .

Data is consistent with the literature. ${ }^{1}$

1-(4-Chlorobenzyl)-2,4,6-triphenylpyridin-1-ium tetrafluoroborate (1y)<smiles></smiles>

Prepared according to General Procedure A using 4-(chloromethyl)methanamine ( $292 \mu \mathrm{l}, 2.40 \mathrm{mmol}, 1.2$ equiv.) and 2,4,6-triphenylpyrylium tetrafluoroborate ( $792 \mathrm{mg}, 2 \mathrm{mmol}, 1.0$ equiv.) in EtOH ( $2 \mathrm{~mL}$ ). Pyridinium salt $1 \mathbf{y}$ was isolated as a white solid (869 $\mathrm{mg}, 84 \%)$. 
${ }^{1} \mathrm{H}$ NMR $\left(400 \mathrm{MHz}, \mathrm{CDCl}_{3}\right) \delta 7.92(\mathrm{~s}, 2 \mathrm{H}), 7.82-7.75(\mathrm{~m}, 2 \mathrm{H}), 7.69-7.62(\mathrm{~m}, 4 \mathrm{H}), 7.62-7.43(\mathrm{~m}, 9 \mathrm{H}), 7.11-$ $7.03(\mathrm{~m}, 2 \mathrm{H}), 6.46-6.38(\mathrm{~m}, 2 \mathrm{H}), 5.74(\mathrm{~s}, 2 \mathrm{H}) ;{ }^{13} \mathrm{C} \mathrm{NMR}\left(101 \mathrm{MHz}, \mathrm{CDCl}_{3}\right) \delta 157.6,156.7,134.5,133.9,132.8$, 132.7, 132.6, 131.3, 130.0, 129.4, 129.2, 129.1, 128.3, 127.8, 126.8, 57.7; $\left.{ }^{19} \mathrm{~F} \mathrm{NMR} \mathrm{(377} \mathrm{MHz,} \mathrm{CDCl}_{3}\right) \delta-152.84$ (minor, ${ }^{10} \mathrm{BF}_{4}$ ), - 152.90 (major, ${ }^{11} \mathrm{BF}_{4}$ ); LRMS (ESI) $\mathrm{m} / \mathrm{z}$ 432.2, $434.2\left(\left[\mathrm{M}-\mathrm{BF}_{4}\right]^{+}\right)$; HRMS (ESI) $\mathrm{m} / z \mathrm{C}_{30} \mathrm{H}_{23}{ }^{35} \mathrm{CIN}^{+}$ requires $432.1514\left(\left[\mathrm{M}-\mathrm{BF}_{4}\right]^{+}\right)$, found $432.1517, \mathrm{C}_{30} \mathrm{H}_{23}{ }^{37} \mathrm{ClN}^{+}$requires $434.1486\left(\left[\mathrm{M}-\mathrm{BF}_{4}\right]^{+}\right)$, found 434.1488 .

Data is consistent with the literature. ${ }^{16}$

2,4,6-Triphenyl-1-(4-(trifluoromethyl)benzyl)pyridin-1-ium tetrafluoroborate (1z)<smiles></smiles>

Prepared according to General Procedure A using 4-(trifluoromethyl)benzylamine (342 $\mu \mathrm{l}, 2.40 \mathrm{mmol}, 1.2$ equiv.) and 2,4,6-triphenylpyrylium tetrafluoroborate ( $792 \mathrm{mg}, 2 \mathrm{mmol}, 1.0$ equiv.) in EtOH ( $2 \mathrm{~mL}$ ). Pyridinium salt $1 \mathbf{z}$ was isolated as a white solid ( $854 \mathrm{mg}, 77 \%$ ).

${ }^{1} \mathrm{H}$ NMR $\left(400 \mathrm{MHz}, \mathrm{CDCl}_{3}\right) \delta 7.92(\mathrm{~s}, 2 \mathrm{H}), 7.77(\mathrm{~d}, J=7.7 \mathrm{~Hz}, 2 \mathrm{H}), 7.64(\mathrm{~d}, J=7.0 \mathrm{~Hz}, 4 \mathrm{H}), 7.56(\mathrm{t}, J=7.5 \mathrm{~Hz}$, $1 \mathrm{H}), 7.47(\mathrm{dt}, J=23.5,7.0 \mathrm{~Hz}, 8 \mathrm{H}), 7.35(\mathrm{~d}, J=8.0 \mathrm{~Hz}, 2 \mathrm{H}), 6.64(\mathrm{~d}, J=8.0 \mathrm{~Hz}, 2 \mathrm{H}), 5.83(\mathrm{~s}, 2 \mathrm{H}) ;{ }^{13} \mathrm{C} \mathrm{NMR}(101$ $\left.\mathrm{MHz}, \mathrm{CDCl}_{3}\right) \delta 157.6,156.9,138.1,133.8,132.7,132.6,131.3,130.6$ (q, $J=33.0 \mathrm{~Hz}$ ), 129.9, 129.4, 129.2, $128.4,126.9,126.8,125.9$ (q, $J=3.5 \mathrm{~Hz}$ ), 123.7 (q, $J=272.5 \mathrm{~Hz}), 57.8 ;{ }^{19} \mathrm{~F}$ NMR $\left(377 \mathrm{MHz}, \mathrm{CDCl}_{3}\right) \delta 62.78$, 152.61 (minor, ${ }^{10} \mathrm{BF}_{4}$ ), -152.66 (major, ${ }^{11} \mathrm{BF}_{4}$ ); LRMS (ESI) $\mathrm{m} / \mathrm{z} 466.2\left(\left[\mathrm{M}-\mathrm{BF}_{4}\right]^{+}\right.$); HRMS (ESI) $\mathrm{m} / \mathrm{z} \mathrm{C}_{31} \mathrm{H}_{23} \mathrm{~F}_{3} \mathrm{~N}^{+}$ requires $466.1777\left(\left[\mathrm{M}-\mathrm{BF}_{4}\right]^{+}\right)$, found 466.1768 .

Data is consistent with the literature. ${ }^{15}$

2,4,6-Triphenyl-1-(thiophen-2-ylmethyl)pyridin-1-ium tetrafluoroborate (1aa)<smiles></smiles>

Prepared according to General Procedure A using 2-thiophenemethylamine (267 $\mu \mathrm{l}, 2.40 \mathrm{mmol}, 1.2$ equiv.) and 2,4,6-triphenylpyrylium tetrafluoroborate (792 mg, $2 \mathrm{mmol}, 1.0$ equiv.) in EtOH ( $2 \mathrm{~mL}$ ), stirred at room temperature overnight. Pyridinium salt 1aa was isolated as a white solid (708 mg, 72\%).

${ }^{1} \mathrm{H}$ NMR $\left(400 \mathrm{MHz}, \mathrm{CDCl}_{3}\right) \delta 7.90(\mathrm{~s}, 2 \mathrm{H}), 7.81-7.71(\mathrm{~m}, 6 \mathrm{H}), 7.62-7.48(\mathrm{~m}, 9 \mathrm{H}), 7.11(\mathrm{dd}, J=5.0,1.0 \mathrm{~Hz}, 1 \mathrm{H})$, $6.68(\mathrm{dd}, J=5.0,3.5 \mathrm{~Hz}, 1 \mathrm{H}), 6.03(\mathrm{~d}, J=2.5 \mathrm{~Hz}, 1 \mathrm{H}), 5.95(\mathrm{~s}, 2 \mathrm{H}) ;{ }^{13} \mathrm{C}$ NMR $\left(101 \mathrm{MHz}, \mathrm{CDCl}_{3}\right) \delta 157.4,156.5$, $135.3,133.8,132.7,132.6,131.3,130.0,129.4,129.4,128.3,128.1,127.0,126.8,53.8$ (1 aromatic carbon signal is not observed due to signal broadening); ${ }^{19} \mathrm{~F} \mathrm{NMR}\left(377 \mathrm{MHz}, \mathrm{CDCl}_{3}\right.$ ) $\delta-152.98$ (minor, $\left.{ }^{10} \mathrm{BF}_{4}\right),-153.03$ (major, $\left.{ }^{11} \mathrm{BF}_{4}\right)$; LRMS (ESI) $\mathrm{m} / z$ 404.2 $\left(\left[\mathrm{M}-\mathrm{BF}_{4}\right]^{+}\right)$; HRMS (ESI) $\mathrm{m} / z \mathrm{C}_{28} \mathrm{H}_{22} \mathrm{NS}^{+}$requires $404.1467\left(\left[\mathrm{M}-\mathrm{BF}_{4}\right]^{+}\right)$, found 404.1470; IR (thin film, $v_{\max } / \mathrm{cm}^{-1}$ ) 3063, 3037, 1619, 1562, 1053, 759, 701; m.p. $\left(\mathrm{CH}_{2} \mathrm{Cl}_{2}\right): 131-134{ }^{\circ} \mathrm{C}$. 
2,4,6-Triphenyl-1-(pyridin-3-ylmethyl)pyridin-1-ium tetrafluoroborate (1ab)<smiles></smiles>

Prepared according to General Procedure A using 3-picolylamine ( $244 \mu \mathrm{l}, 2.40 \mathrm{mmol}, 1.2$ equiv.) and 2,4,6triphenylpyrylium tetrafluoroborate (792 mg, $2 \mathrm{mmol}, 1.0$ equiv.) in EtOH (2 mL). Pyridinium salt 1ab was isolated as a white solid (762 $\mathrm{mg}, 78 \%$ ).

${ }^{1} \mathrm{H}$ NMR $\left(400 \mathrm{MHz}, \mathrm{CDCl}_{3}\right) \delta 8.36(\mathrm{dd}, J=5.0,1.5 \mathrm{~Hz}, 1 \mathrm{H}), 7.91(\mathrm{~s}, 2 \mathrm{H}), 7.79-7.73(\mathrm{~m}, 2 \mathrm{H}), 7.70-7.62(\mathrm{~m}, 5 \mathrm{H})$, $7.60-7.43(\mathrm{~m}, 9 \mathrm{H}), 7.06(\mathrm{dd}, J=8.0,5.0 \mathrm{~Hz}, 1 \mathrm{H}), 6.93-6.86(\mathrm{~m}, 1 \mathrm{H}), 5.81(\mathrm{~s}, 2 \mathrm{H}) ;{ }^{13} \mathrm{C} \mathrm{NMR}\left(101 \mathrm{MHz}, \mathrm{CDCl}_{3}\right)$ $\delta 157.5,156.9,149.6,147.6,134.6,133.8,132.7,132.6$, 131.4, 123.0, 129.9, 129.5, 129.2 , 128.3, 126.9, 123.9, 56.1; ${ }^{19} \mathrm{~F}$ NMR (377 MHz, CDCl $)$ ) $\delta$-152.63 (minor, ${ }^{10} \mathrm{BF}_{4}$ ), -152.68 (major, ${ }^{11} \mathrm{BF}_{4}$ ); LRMS (ESI) m/z 399.2 ([M$\left.\mathrm{BF}_{4}\right]^{+}$); HRMS (ESI) $m / z \mathrm{C}_{29} \mathrm{H}_{23} \mathrm{~N}_{2}{ }^{+}$requires $399.1856\left(\left[\mathrm{M}-\mathrm{BF}_{4}\right]^{+}\right)$, found 399.1855.

Data is consistent with the literature. ${ }^{18}$

\section{2,4,6-Triphenyl-1-(pyridin-2-ylmethyl)pyridin-1-ium tetrafluoroborate (1ac)}<smiles></smiles>

Prepared according to General Procedure A using 2-picolylamine ( $374 \mu \mathrm{l}, 3.6 \mathrm{mmol}, 1.2$ equiv.) and 2,4,6triphenylpyrylium tetrafluoroborate (1.19 g, $3 \mathrm{mmol}, 1.0$ equiv.) in $\mathrm{EtOH}(2 \mathrm{~mL})$. Pyridinium salt 1ac was isolated as a white powder ( $842 \mathrm{mg}, 58 \%$ ).

${ }^{1} \mathrm{H}$ NMR $\left(400 \mathrm{MHz}, \mathrm{CDCl}_{3}\right) \delta 8.44(\mathrm{ddd}, J=5.0,1.5,1.0 \mathrm{~Hz}, 1 \mathrm{H}), 7.93(\mathrm{~s}, 2 \mathrm{H}), 7.82(\mathrm{dq}, J=6.5,2.5,2.0 \mathrm{~Hz}, 2 \mathrm{H})$, $7.64-7.51(\mathrm{~m}, 7 \mathrm{H}), 7.49-7.33(\mathrm{~m}, 7 \mathrm{H}),, 7.14(\mathrm{dd}, J=6.5,5.0 \mathrm{~Hz}, 1 \mathrm{H}), 6.51(\mathrm{~d}, J=8.0 \mathrm{~Hz}, 1 \mathrm{H}), 5.77(\mathrm{~s}, 2 \mathrm{H})$; ${ }^{13} \mathrm{C}$ NMR $\left(101 \mathrm{MHz}, \mathrm{CDCl}_{3}\right) \delta 157.7,156.0,153.4,149.3,136.9,134.2,133.1,132.3,130.9,129.9,129.0$, 128.2, 126.2, 123.2, 121.9, 59.1; ${ }^{19} \mathrm{~F}$ NMR (377 MHz, $\left.\mathrm{CDCl}_{3}\right)$ $\delta$-153.28 (minor, ${ }^{10} \mathrm{BF}_{4}$ ), -153.34 (major, ${ }^{11} \mathrm{BF}_{4}$ ); LRMS (ESI) $m / z 399.2\left(\left[M-\mathrm{BF}_{4}\right]^{+}\right)$; HRMS (ESI) $m / z \mathrm{C}_{29} \mathrm{H}_{23} \mathrm{~N}_{2}{ }^{+}$requires 399.1856 ([M-BF $]^{+}$), found 399.1858. Data is consistent with the literature. ${ }^{[5]}$

2-[(E)-3-Phenyl-allyl]-isoindole-1,3-dione (1ad-1)

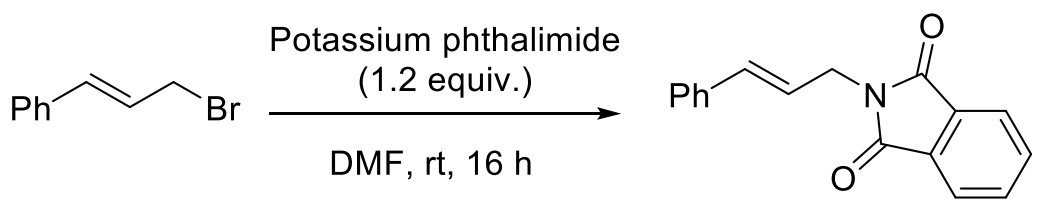

Prepared according to a modified procedure by Jørgensen and co-workers, ${ }^{19}$ to a solution of cinnamyl bromide (1.97 g, $10.0 \mathrm{mmol}, 1.0$ equiv.) anhydrous DMF $(25 \mathrm{~mL})$ was added potassium phthalimide $(2.22 \mathrm{~g}$, 
$12 \mathrm{mmol}, 1.2$ equiv.) in one portion. The reaction mixture was stirred at rt overnight. The reaction was diluted with $\mathrm{Et}_{2} \mathrm{O}(75 \mathrm{~mL})$, washed with brine $(4 \times 25 \mathrm{~mL})$, dried using $\mathrm{MgSO}_{4}$, filtered and concentrated in vacuo. The resulting pale orange crystals were recrystallised from toluene to afford 2-[(E)-3-phenyl-allyl]-isoindole-1,3dione 1 ad-1 as colourless crystals (2.32 g, 88\%).

$\mathbf{R}_{f}\left(20 \%\right.$ EtOAc in Petrol) $=0.28 ;{ }^{1} \mathbf{H}$ NMR $\left(400 \mathrm{MHz}, \mathrm{CDCl}_{3}\right) \delta 7.93-7.81(\mathrm{~m}, 2 \mathrm{H}), 7.77-7.67(\mathrm{~m}, 2 \mathrm{H}), 7.39-$ $7.16(\mathrm{~m}, 5 \mathrm{H}), 6.66(\mathrm{dt}, J=16.0,1.5 \mathrm{~Hz}, 1 \mathrm{H}), 6.26(\mathrm{dt}, J=16.0,6.5 \mathrm{~Hz}, 1 \mathrm{H}), 4.45(\mathrm{dd}, J=6.5,1.5 \mathrm{~Hz}, 2 \mathrm{H}) ;{ }^{13} \mathrm{C}$ NMR $\left(101 \mathrm{MHz}, \mathrm{CDCl}_{3}\right) \delta 168.1,136.4,134.1,134.0,132.3,128.7,128.0,126.7,123.5,122.9,39.8$; HRMS (ESI) $m / z \mathrm{C}_{17} \mathrm{H}_{14} \mathrm{NO}_{2}^{+}$requires $264.1019\left([\mathrm{M}+\mathrm{H}]^{+}\right)$, found 264.1021 .

Data is consistent with the literature. ${ }^{19}$

(E)-3-Phenylprop-2-en-1-ammonium chloride (1ad-2)<smiles>O=C1c2ccccc2C(=O)N1C/C=C/c1ccccc1</smiles>
i) Hydrazine monohydrate (3 equiv.) Methanol, reflux, $1 \mathrm{~h}$<smiles>NC/C=C/c1ccccc1</smiles>

ii) $2 \mathrm{M} \mathrm{HCl}$ in $\mathrm{Et}_{2} \mathrm{O}$

Prepared according to a modified procedure by Jørgensen and co-workers, ${ }^{19}$ To a solution of 2-[(E)-3-phenylallyl]-isoindole-1,3-dione 1ad-2 (2.24 g, $8.5 \mathrm{mmol}, 1.0$ equiv.) in EtOH (35 mL) was added hydrazine monohydrate ( $1.24 \mathrm{~mL}, 25.5 \mathrm{mmol}, 3.0$ equiv.). The reaction mixture was heated in an aluminium heating block to reflux with vigorous stirring under $\mathrm{N}_{2}$ for $1 \mathrm{~h}$. The reaction mixture was cooled to $0{ }^{\circ} \mathrm{C}$ and the precipitate filtered off. The filtrate was concentrated in vacuo and the resulting mixture resuspended in $\mathrm{Et}_{2} \mathrm{O}$ $(50 \mathrm{~mL})$, filtered and the filtrate concentrated in vacuo. The resultant yellow oil was dissolved in $\mathrm{EtOH}(10$ $\mathrm{mL}$ ) and converted into the hydrochloride using $2 \mathrm{M} \mathrm{HCl}$ in Et $2 \mathrm{O}(25 \mathrm{~mL}, 0.5 \mathrm{~mol}, 5.9$ equiv.). The mixture was concentrated in vacuo and the crude product recrystallised from toluene to give (E)-3-phenylprop-2-en-1ammonium chloride $\mathbf{1}$ ad-2 as colourless needles (526 mg, 29\%).

${ }^{1} \mathrm{H}$ NMR $\left(400 \mathrm{MHz}, \mathrm{CD}_{3} \mathrm{OD}\right) \delta 7.46(\mathrm{dt}, J=8.2,2.3 \mathrm{~Hz}, 2 \mathrm{H}), 7.39-7.25(\mathrm{~m}, 3 \mathrm{H}), 6.82(\mathrm{~d}, J=16.0 \mathrm{~Hz}, 1 \mathrm{H}), 6.31$ $(\mathrm{dt}, J=16.0,7.0 \mathrm{~Hz}, 1 \mathrm{H}), 3.72(\mathrm{~d}, J=7.0 \mathrm{~Hz}, 2 \mathrm{H}) ;{ }^{13} \mathrm{C}$ NMR $\left(101 \mathrm{MHz}, \mathrm{CD}_{3} \mathrm{OD}\right) \delta 137.8,137.1,129.8,129.6$, $127.8,121.2,42.5$.

Data is consistent with the literature. ${ }^{19}$

1-Cinnamyl-2,4,6-triphenylpyridin-1-ium tetrafluoroborate (1ad-3)<smiles></smiles> 
Prepared according to General Procedure B using (E)-3-phenylprop-2-en-1-ammonium chloride 1ad-2 (407 $\mathrm{mg}, 2.40 \mathrm{mmol}, 1.2$ equiv.) and 2,4,6-triphenylpyrylium tetrafluoroborate (792 mg, $2 \mathrm{mmol}, 1.0$ equiv.) in $\mathrm{CH}_{2} \mathrm{Cl}_{2}(4 \mathrm{~mL})$, triethylamine ( $335 \mu \mathrm{l}, 2.40 \mathrm{mmol}, 1.2$ equiv.) and $\mathrm{AcOH}(57 \mu \mathrm{l}, 1 \mathrm{mmol}, 0.5$ equiv.). Pyridinium salt 1 ad-3 was isolated as a white solid ( $278 \mathrm{mg}, 27 \%)$.

${ }^{1} \mathrm{H}$ NMR $\left(400 \mathrm{MHz}, \mathrm{CDCl}_{3}\right) \delta 7.92(\mathrm{~s}, 2 \mathrm{H}), 7.83-7.75(\mathrm{~m}, 6 \mathrm{H}), 7.63-7.49(\mathrm{~m}, 9 \mathrm{H}), 7.29-7.23(\mathrm{~m}, 3 \mathrm{H}), 7.12-$ $7.05(\mathrm{~m}, 2 \mathrm{H}), 5.76(\mathrm{dt}, J=16.0,6.5 \mathrm{~Hz}, 1 \mathrm{H}), 5.62(\mathrm{~d}, J=16.0 \mathrm{~Hz}, 1 \mathrm{H}), 5.23(\mathrm{dd}, J=6.5,1.5 \mathrm{~Hz}, 2 \mathrm{H}) ;{ }^{13} \mathrm{C} \mathrm{NMR}$ $\left(101 \mathrm{MHz}, \mathrm{CDCl}_{3}\right) \delta 157.1,156.3,136.4,135.0,134.1,132.9,132.3,131.2,129.9,129.4,128.9,128.8,128.4$, 128.3, 126.8, 126.8, 120.4, 57.4; ${ }^{19} \mathrm{~F}$ NMR (377 MHz, $\left.\mathrm{CDCl}_{3}\right) \delta$-153.21 (minor, ${ }^{10} \mathrm{BF}_{4}$ ), -153.27 (major, ${ }^{11} \mathrm{BF}_{4}$ ); LRMS (ESI) $m / z$ 424.2, ([M-BF $\left.]^{+}\right)$; HRMS (ESI) $m / z \mathrm{C}_{32} \mathrm{H}_{26} \mathrm{~N}^{+}$requires $424.2060\left(\left[\mathrm{M}-\mathrm{BF}_{4}\right]^{+}\right)$, found 424.2053 .

Data is consistent with the literature. ${ }^{20}$

\section{2-Isopropyl-4,6-diphenylpyrylium tetrafluoroborate (1ae-1)}

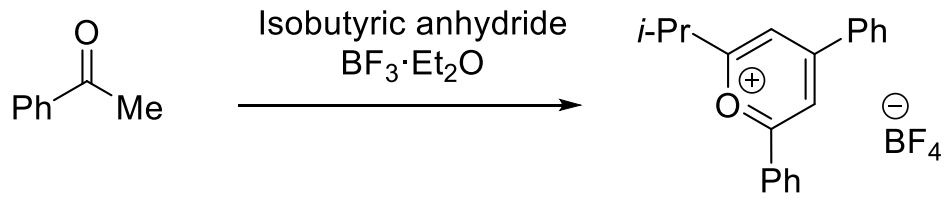

According to the procedure described by Boiko and co-workers, ${ }^{21}$ a mixture of acetophenone $(4.66 \mathrm{~mL}, 40$ mmol, 2.0 equiv.), isobutyric anhydride ( $13.3 \mathrm{~mL}, 80 \mathrm{mmol}, 4.0$ equiv.) and $\mathrm{BF}_{3} \cdot \mathrm{Et}_{2} \mathrm{O}$ ( $3.45 \mathrm{~mL}, 28 \mathrm{mmol}, 1.4$ equiv.) was heated in an aluminium heating block to reflux for $2 \mathrm{~h}$. The hot mixture was then poured into EtOAc and boiled for $5 \mathrm{~min}$. The solid pyrylium was then filtered and washed with boiling EtOAc. The solid product was then recrystalised from $\mathrm{AcOH}$ to give 2-isopropyl-4,6-diphenylpyrylium tetrafluoroborate 1ae-1 as bright yellow crystals ( $5.20 \mathrm{~g}, 78 \%)$.

${ }^{1} \mathrm{H}$ NMR $\left(400 \mathrm{MHz}\right.$, Acetone $\left.d_{6}\right) \delta 9.15(\mathrm{~d}, J=2.0 \mathrm{~Hz}, 1 \mathrm{H}), 8.61(\mathrm{~d}, J=1.5 \mathrm{~Hz}, 1 \mathrm{H}), 8.58-8.51(\mathrm{~m}, 2 \mathrm{H}), 8.50-$ $8.42(\mathrm{~m}, 2 \mathrm{H}), 7.92-7.83(\mathrm{~m}, 2 \mathrm{H}), 7.83-7.72(\mathrm{~m}, 4 \mathrm{H}), 3.79$ (hept, $J=7.0 \mathrm{~Hz}, 1 \mathrm{H}), 1.67(\mathrm{~d}, J=7.0 \mathrm{~Hz}, 6 \mathrm{H}) ;{ }^{13} \mathrm{C}$ NMR $\left(101 \mathrm{MHz}\right.$, Acetone $\left.d_{6}\right) \delta$ 206.3, 185.1, 173.1, 167.9, 136.2, 133.8, 131.0, 130.9, 130.7, 130.2, 129.5, 117.7, 116.3, 35.7, 20.6; ${ }^{19} \mathrm{~F}$ NMR (377 MHz, Acetone $\left.d_{6}\right) \delta$-151.58 (minor, ${ }^{10} \mathrm{BF}_{4}$ ), -151.63 (major, ${ }^{11} \mathrm{BF}_{4}$ ); LRMS

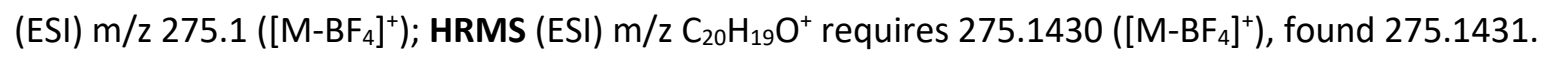

Data is consistent with the literature. ${ }^{22}$

\section{2-Isopropyl-4,6-diphenyl-1-(1-phenylethyl)pyridin-1-ium tetrafluoroborate (1ae-2)}<smiles></smiles><smiles>C[C@H](N)c1ccccc1</smiles><smiles></smiles>

According to the procedure described by Katritzky and co-workers, ${ }^{22}$ 1-phenylethanamine (356 $\mu \mathrm{L}, 2.76$ mmol, 2.0 equiv.) and 2-isopropyl-4,6-diphenylpyrylium tetrafluoroborate 1ae-1 (500 mg, $1.38 \mathrm{mmol}, 1.0$ 
equiv.) were stirred in $\mathrm{CH}_{2} \mathrm{Cl}_{2}(20 \mathrm{~mL})$ over $3 \AA$ molecular sieves for $48 \mathrm{~h}$ at $\mathrm{rt}$. $\mathrm{Et}_{2} \mathrm{O}(40 \mathrm{~mL})$ was then added to give the crude product. The crude product was then recrystallised from EtOH to give pyridinium salt 1ae-2 as a white crystalline solid (302 mg, 47\%).

${ }^{1} \mathrm{H}$ NMR $\left(400 \mathrm{MHz}, \mathrm{CDCl}_{3}\right) \delta 7.95(\mathrm{~d}, J=2.5 \mathrm{~Hz}, 1 \mathrm{H}), 7.90-7.75(\mathrm{~m}, 4 \mathrm{H}), 7.69-7.48(\mathrm{~m}, 7 \mathrm{H}), 7.43(\mathrm{t}, J=7.5$ $\mathrm{Hz}, 2 \mathrm{H}), 7.34(\mathrm{t}, J=7.0 \mathrm{~Hz}, 1 \mathrm{H}), 7.11(\mathrm{~d}, J=8.0 \mathrm{~Hz}, 2 \mathrm{H}), 6.24(\mathrm{q}, J=7.0 \mathrm{~Hz}, 1 \mathrm{H}), 3.32(\mathrm{p}, J=6.5 \mathrm{~Hz}, 1 \mathrm{H}), 2.14(\mathrm{~d}$, $J=7.0 \mathrm{~Hz}, 3 \mathrm{H}), 1.46(\mathrm{~s}, 3 \mathrm{H}), 0.74(\mathrm{~d}, J=6.0 \mathrm{~Hz}, 3 \mathrm{H}) ;{ }^{13} \mathrm{C} \mathrm{NMR}\left(101 \mathrm{MHz}, \mathrm{CDCl}_{3}\right) \delta$ 166.7, 156.7, 156.6, 138.0, 134.3, 133.9, 132.1, 131.2, 130.0, 129.8, 129.7, 129.0, 128.9, 128.4, 127.8, 126.0, 125.4, 64.7, 32.4, 24.0, 22.1, 18.6 (note isopropyl carbons are non-equivalent), ${ }^{22}{ }^{19} \mathrm{~F} \mathrm{NMR}\left(377 \mathrm{MHz}, \mathrm{CDCl}_{3}\right) \delta-152.98$ (minor, ${ }^{10} \mathrm{BF}_{4}$ ), 153.03 (major, $\left.{ }^{11} \mathrm{BF}_{4}\right) ; \mathrm{LRMS}(\mathrm{ESI}) \mathrm{m} / z$ 378.2 $\left(\left[\mathrm{M}-\mathrm{BF}_{4}\right]^{+}\right) ; \mathrm{HRMS}(\mathrm{ESI}) \mathrm{m} / z \mathrm{C}_{28} \mathrm{H}_{28} \mathrm{~N}^{+}$requires $378.2216\left(\left[\mathrm{M}-\mathrm{BF}_{4}\right]^{+}\right)$, found 378.2211 .

Data is consistent with the literature. ${ }^{22}$

1-Phenethyl-2,4,6-triphenylpyridin-1-ium tetrafluoroborate (1c)<smiles></smiles>

Prepared according to General Procedure A using phenethylamine ( $756 \mu \mathrm{l}, 6 \mathrm{mmol}, 1.2$ equiv.) and 2,4,6triphenylpyrylium tetrafluoroborate (1.98 g, $5 \mathrm{mmol}, 1.0$ equiv.) in EtOH ( $2 \mathrm{~mL})$. Pyridinium salt $1 \mathrm{c}$ was isolated as a white solid (2.39 $\mathrm{g}, 96 \%)$.

${ }^{1} \mathrm{H}$ NMR $\left(400 \mathrm{MHz}, \mathrm{CDCl}_{3}\right) \delta 7.93(\mathrm{~s}, 2 \mathrm{H}), 7.81(\mathrm{dt}, J=6.5,1.5 \mathrm{~Hz}, 6 \mathrm{H}), 7.70-7.50(\mathrm{~m}, 9 \mathrm{H}), 7.18-7.11(\mathrm{~m}, 1 \mathrm{H})$, $7.11-7.03(\mathrm{~m}, 2 \mathrm{H}), 6.34-6.28(\mathrm{~m}, 2 \mathrm{H}), 4.72-4.63(\mathrm{~m}, 2 \mathrm{H}), 2.76-2.68(\mathrm{~m}, 2 \mathrm{H}) ;{ }^{13} \mathrm{C} \mathrm{NMR}\left(101 \mathrm{MHz}, \mathrm{CDCl}_{3}\right)$ $\delta 156.8,156.2$, 135.3, 134.2, 132.9, 132.3, 131.3, 129.9, 129.5, 129.3, 129.0, 128.3, 128.3, 127.5, 126.9, 56.0, 35.8; ${ }^{19} \mathrm{~F}$ NMR (377 MHz, CDCl $) \delta$-153.27 (minor, ${ }^{10} \mathrm{BF}_{4}$ ), -153.33 (major, ${ }^{11} \mathrm{BF}_{4}$ ); LRMS (ESI) $\mathrm{m} / \mathrm{z} 412.2$ ([M$\left.\mathrm{BF}_{4}\right]^{+}$); HRMS (ESI) $m / z \mathrm{C}_{31} \mathrm{H}_{26} \mathrm{~N}^{+}$requires $412.2060\left(\left[\mathrm{M}-\mathrm{BF}_{4}\right]^{+}\right)$, found 412.2062 .

Data is consistent with the literature. ${ }^{5}$

1-Neopentyl-2,4,6-triphenylpyridin-1-ium tetrafluoroborate (1af)<smiles></smiles>

Prepared according to General Procedure A using neopentylamine ( $283 \mu \mathrm{l}, 2.40 \mathrm{mmol}, 1.2$ equiv.) and 2,4,6triphenylpyrylium tetrafluoroborate $(792 \mathrm{mg}, 5 \mathrm{mmol}, 1.0$ equiv.) in EtOH (2 mL). Pyridinium salt 1af was isolated as a white solid (671 $\mathrm{mg}, 72 \%)$.

${ }^{1} \mathrm{H}$ NMR $\left(400 \mathrm{MHz}, \mathrm{CDCl}_{3}\right) \delta 8.31-8.11(\mathrm{~m}, 2 \mathrm{H}), 7.91(\mathrm{~s}, 2 \mathrm{H}), 7.82(\mathrm{dd}, J=8.0,1.5 \mathrm{~Hz}, 2 \mathrm{H}), 7.69-7.50(\mathrm{~m}$, 11H), 5.02 (s, 2H), $0.46(\mathrm{~s}, 9 \mathrm{H}) ;{ }^{13} \mathrm{C}$ NMR $\left(101 \mathrm{MHz}, \mathrm{CDCl}_{3}\right) \delta 159.1,155.7,134.5,133.6,132.7,131.5,130.5$, 
130.0, 129.7, 128.3, 126.7, 64.0, 35.6, 29.0; ${ }^{19} \mathrm{~F}$ NMR (377 MHz, $\mathrm{CDCl}_{3}$ ) $\delta-152.92$ (minor, ${ }^{10} \mathrm{BF}_{4}$ ), -152.97

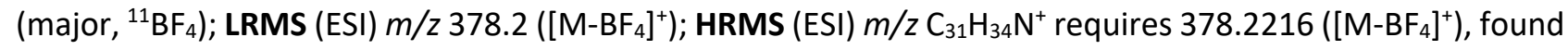
378.2228.

Data is consistent with the literature. ${ }^{23}$

1-Octyl-2,4,6-triphenylpyridin-1-ium tetrafluoroborate (1ag)<smiles>CCCCCCCC[n+]1c(-c2ccccc2)cc(-c2ccccc2)cc1-c1ccccc1</smiles>

Prepared according to General Procedure A using octylamine (397 $\mu \mathrm{l}, 2.40 \mathrm{mmol}, 1.2$ equiv.) and 2,4,6triphenylpyrylium tetrafluoroborate $(792 \mathrm{mg}, 5 \mathrm{mmol}, 1.0$ equiv.) in $\mathrm{EtOH}(2 \mathrm{~mL})$. Pyridinium salt 1ag was isolated as a white solid (737 $\mathrm{g}, 73 \%)$.

${ }^{1} \mathrm{H}$ NMR $\left(400 \mathrm{MHz}, \mathrm{CDCl}_{3}\right) \delta 7.83(\mathrm{~s}, 2 \mathrm{H}), 7.82-7.76(\mathrm{~m}, 4 \mathrm{H}), 7.76-7.71(\mathrm{~m}, 2 \mathrm{H}), 7.63-7.56(\mathrm{~m}, 6 \mathrm{H}), 7.57-$ $7.45(\mathrm{~m}, 3 \mathrm{H}), 4.44-4.35(\mathrm{~m}, 2 \mathrm{H}), 1.43(\mathrm{dq}, J=8.0,5.0 \mathrm{~Hz}, 2 \mathrm{H}), 1.13(\mathrm{p}, J=7.0 \mathrm{~Hz}, 2 \mathrm{H}), 1.00(\mathrm{dq}, J=9.0,6.5$ $\mathrm{Hz}, 2 \mathrm{H}), 0.95-0.85(\mathrm{~m}, 2 \mathrm{H}), 0.80(\mathrm{t}, J=7.5 \mathrm{~Hz}, 3 \mathrm{H}), 0.78-0.69(\mathrm{~m}, 4 \mathrm{H}) ;{ }^{13} \mathrm{C} \mathrm{NMR}\left(101 \mathrm{MHz}, \mathrm{CDCl}_{3}\right) \delta 156.5$, 155.7, 134.1, 132.8, 132.0, 131.0, 129.7, 129.3, 129.1, 128.1, 126.7, 54.7, 31.4, 29.6, 28.5, 27.9, 26.0, 22.4, 14.0; ${ }^{19} \mathrm{~F}$ NMR (377 MHz, CDCl $)$ ) $\delta$-153.38 (minor, ${ }^{10} \mathrm{BF}_{4}$ ), -153.44 (major, ${ }^{11} \mathrm{BF}_{4}$ ); LRMS (ESI) $\mathrm{m} / \mathrm{z} 420.2$ ([M$\left.\left.\mathrm{BF}_{4}\right]^{+}\right) ; \operatorname{HRMS}(\mathrm{ESI}) \mathrm{m} / z \mathrm{C}_{31} \mathrm{H}_{34} \mathrm{~N}^{+}$requires $420.2662\left(\left[\mathrm{M}-\mathrm{BF}_{4}\right]^{+}\right)$, found 420.2668 .

Data is consistent with the literature. ${ }^{24}$

1-(2-(Cyclohex-1-en-1-yl)ethyl)-2,4,6-triphenylpyridin-1-ium tetrafluoroborate (1ah)<smiles></smiles>

Prepared according to General Procedure A using 2-(1-cyclohexenyl)-ethylamine (335 $\mu \mathrm{l}, 2.40 \mathrm{mmol}, 1.2$ equiv.) and 2,4,6-triphenylpyrylium tetrafluoroborate ( $792 \mathrm{mg}, 2 \mathrm{mmol}, 1.0$ equiv.) in EtOH ( $2 \mathrm{~mL}$ ). Pyridinium salt 1ah was isolated as a white solid ( $883 \mathrm{mg}, 88 \%)$.

${ }^{1} \mathrm{H}$ NMR $\left(400 \mathrm{MHz}, \mathrm{CDCl}_{3}\right) \delta 7.86-7.78(\mathrm{~m}, 6 \mathrm{H}), 7.76-7.70(\mathrm{~m}, 2 \mathrm{H}), 7.63-7.57(\mathrm{~m}, 6 \mathrm{H}), 7.56-7.45(\mathrm{~m}, 3 \mathrm{H})$, $4.84-4.78(\mathrm{~m}, 1 \mathrm{H}), 4.47-4.38(\mathrm{~m}, 2 \mathrm{H}), 2.05-1.97(\mathrm{~m}, 2 \mathrm{H}), 1.76-1.66(\mathrm{~m}, 2 \mathrm{H}), 1.31(\mathrm{p}, J=3.0 \mathrm{~Hz}, 4 \mathrm{H}), 1.15$ - $1.05(\mathrm{~m}, 2 \mathrm{H}) ;{ }^{13} \mathrm{C}$ NMR $\left(101 \mathrm{MHz}, \mathrm{CDCl}_{3}\right) \delta$ 156.6, 155.8, 134.2, 132.9, 132.2, 131.8, 131.1, 129.8, 129.4, $129.3,128.2,126.8,125.6,54.3,38.3,27.2,25.1,22.4,21.7 ;{ }^{19} \mathrm{~F}$ NMR $\left(377 \mathrm{MHz}, \mathrm{CDCl}_{3}\right.$ ) $\delta$-153.36 (minor, ${ }^{10} \mathrm{BF}_{4}$ ), -153.41 (major, ${ }^{11} \mathrm{BF}_{4}$ ); LRMS (ESI) $\mathrm{m} / z 416.2\left(\left[\mathrm{M}-\mathrm{BF}_{4}\right]^{+}\right)$; HRMS (ESI) $\mathrm{m} / z \mathrm{C}_{31} \mathrm{H}_{30} \mathrm{~N}^{+}$requires 416.2349 $\left(\left[\mathrm{M}-\mathrm{BF}_{4}\right]^{+}\right)$, found 416.2349 .

Data is consistent with the literature. ${ }^{16}$ 
1-(2,2-Dimethoxyethyl)-2,4,6-triphenylpyridin-1-ium tetrafluoroborate (1ai)<smiles></smiles>

Prepared according to General Procedure A using aminoacetaldehyde dimethyl acetal $(261 \mu \mathrm{l}, 2.40 \mathrm{mmol}$, 1.2 equiv.) and 2,4,6-triphenylpyrylium tetrafluoroborate (792 mg, $2 \mathrm{mmol}, 1.0$ equiv.) in EtOH ( $2 \mathrm{~mL}$ ). Pyridinium salt 1ai was isolated as a white solid ( $820 \mathrm{mg}, 85 \%)$.

${ }^{1} \mathrm{H}$ NMR $\left(400 \mathrm{MHz}, \mathrm{CDCl}_{3}\right) \delta 7.87(\mathrm{~s}, 2 \mathrm{H}), 7.85-7.75(\mathrm{~m}, 6 \mathrm{H}), 7.64-7.49(\mathrm{~m}, 9 \mathrm{H}), 4.77(\mathrm{~d}, \mathrm{~J}=5.5 \mathrm{~Hz}, 2 \mathrm{H}), 3.95$ $(\mathrm{t}, J=5.5 \mathrm{~Hz}, 1 \mathrm{H}), 2.92(\mathrm{~s}, 6 \mathrm{H}) ;{ }^{13} \mathrm{C}$ NMR $\left(101 \mathrm{MHz}, \mathrm{CDCl}_{3}\right) \delta 157.8,156.1,133.9,133.3,132.5,131.2,129.9$, 129.8, 129.4, 128.3, 126.4, 101.2, 55.2, 54.9; ${ }^{19} \mathrm{~F}$ NMR (377 MHz, $\mathrm{CDCl}_{3}$ ) $\delta$-153.15 (minor, ${ }^{10} \mathrm{BF}_{4}$ ), -153.20 (major, $\left.{ }^{11} \mathrm{BF}_{4}\right)$; LRMS (ESI) $\mathrm{m} / \mathrm{z} 396.2\left(\left[\mathrm{M}-\mathrm{BF}_{4}\right]^{+}\right)$; HRMS (ESI) $\mathrm{m} / z \mathrm{C}_{27} \mathrm{H}_{26} \mathrm{NO}_{2}{ }^{+}$requires $396.1958\left(\left[\mathrm{M}-\mathrm{BF}_{4}\right]^{+}\right.$), found 396.1958.

Data is consistent with the literature..$^{25}$

1-(4-Cyanophenethyl)-2,4,6-triphenylpyridin-1-ium tetrafluoroborate (1aj)<smiles>N#Cc1ccc(CC[n+]2c(-c3ccccc3)cc(-c3ccccc3)cc2-c2ccccc2)cc1</smiles>

Prepared according to General Procedure A using 4-Cyanophenylethylamine hydrochloride (438 mg, 2.40 mmol, 1.2 equiv.) and 2,4,6-triphenylpyrylium tetrafluoroborate ( $792 \mathrm{mg}, 2 \mathrm{mmol}, 1.0$ equiv.) in EtOH ( $2 \mathrm{~mL}$ ) and triethylamine ( $335 \mu \mathrm{l}, 2.40 \mathrm{mmol}, 1.20 \mathrm{mmol})$. Pyridinium salt 1aj was isolated as a white solid (472 mg, $45 \%)$.

${ }^{1} \mathrm{H}$ NMR $\left(400 \mathrm{MHz}, \mathrm{CDCl}_{3}\right) \delta 7.85(\mathrm{~s}, 2 \mathrm{H}), 7.85-7.78(\mathrm{~m}, 4 \mathrm{H}), 7.76-7.70(\mathrm{~m}, 2 \mathrm{H}), 7.69-7.58(\mathrm{~m}, 6 \mathrm{H}), 7.58-$ $7.52(\mathrm{~m}, 1 \mathrm{H}), 7.51-7.44(\mathrm{~m}, 2 \mathrm{H}), 7.31(\mathrm{~d}, J=8.5 \mathrm{~Hz}, 2 \mathrm{H}), 6.37(\mathrm{~d}, J=8.5 \mathrm{~Hz}, 2 \mathrm{H}), 4.64-4.50(\mathrm{~m}, 2 \mathrm{H}), 2.89-$ $2.74(\mathrm{~m}, 2 \mathrm{H}) ;{ }^{13} \mathrm{C}$ NMR $\left(101 \mathrm{MHz}, \mathrm{CDCl}_{3}\right) \delta 156.5,141.0,134.1,132.7,132.6,132.4,131.4,129.8,129.6,129.2$, $129.1,128.3,127.0,118.4,111.3,55.1,35.6$ (one signal missing due to signal broadening); ${ }^{19} \mathrm{~F} \mathrm{NMR} \mathrm{(377} \mathrm{MHz,}$ $\mathrm{CDCl}_{3}$ ) $\delta$-152.82 (minor, ${ }^{10} \mathrm{BF}_{4}$ ), -152.87 (major, ${ }^{11} \mathrm{BF}_{4}$ ); LRMS (ESI) $\mathrm{m} / z 437.2$ ([M-BF $]^{+}$); HRMS (ESI) $\mathrm{m} / \mathrm{z}$ $\mathrm{C}_{32} \mathrm{H}_{25} \mathrm{~N}_{2}{ }^{+}$requires $437.2012\left(\left[\mathrm{M}-\mathrm{BF}_{4}\right]^{+}\right)$, found 437.2027.

Data is consistent with the literature. ${ }^{16}$

1-(4-(tert-Butoxy)-4-oxobutyl)-2,4,6-triphenylpyridin-1-ium tetrafluoroborate (1ak)<smiles></smiles> 
Prepared according to General Procedure A using tert-butyl 4-aminobutanoate hydrochloride (470 mg, 2.40 mmol, 1.2 equiv.) and 2,4,6-triphenylpyrylium tetrafluoroborate ( $792 \mathrm{mg}, 2 \mathrm{mmol}, 1.0$ equiv.) in EtOH ( $2 \mathrm{~mL}$ ) and triethylamine ( $335 \mu \mathrm{l}, 2.40 \mathrm{mmol}, 1.20 \mathrm{mmol}$ ). Pyridinium salt 1ak was isolated as a white solid (610 $\mathrm{mg}$, $57 \%)$.

${ }^{1} \mathrm{H}$ NMR $\left(400 \mathrm{MHz}, \mathrm{CDCl}_{3}\right) \delta 7.87(\mathrm{~s}, 2 \mathrm{H}), 7.85-7.79(\mathrm{~m}, 4 \mathrm{H}), 7.79-7.73(\mathrm{~m}, 2 \mathrm{H}), 7.65-7.59(\mathrm{~m}, 6 \mathrm{H}), 7.57-$ $7.48(\mathrm{~m}, 3 \mathrm{H}), 4.51(\mathrm{t}, J=7.5 \mathrm{~Hz}, 2 \mathrm{H}), 1.76-1.67(\mathrm{~m}, 4 \mathrm{H}), 1.26(\mathrm{~s}, 9 \mathrm{H}) ;{ }^{13} \mathrm{C} \mathrm{NMR}\left(101 \mathrm{MHz}, \mathrm{CDCl}_{3}\right) \delta 170.7$, 156.8, 156.1, 134.2, 132.9, 132.2, 131.2, 129.8, 129.5, 129.2, 128.3, 127.0, 81.1, 54.4, 32.2, 28.0, 25.2; ${ }^{19} \mathrm{~F}$ NMR (377 MHz, CDCl 3 ) $\delta$-153.34 (minor, ${ }^{10} \mathrm{BF}_{4}$ ), -153.39 (major, ${ }^{11} \mathrm{BF}_{4}$ ); LRMS (ESI) $\mathrm{m} / z 450.2\left(\left[\mathrm{M}-\mathrm{BF}_{4}\right]^{+}\right.$);

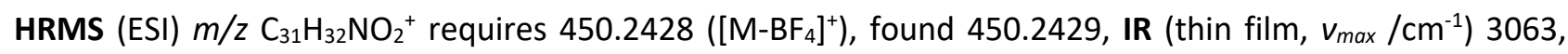
$2980,1723,1623,1154,1054,704$; m.p. $\left(\mathrm{CH}_{2} \mathrm{Cl}_{2}\right): 163-165^{\circ} \mathrm{C}$.

1-(2-(1H-Indol-3-yl)ethyl)-2,4,6-triphenylpyridin-1-ium tetrafluoroborate (1al)<smiles></smiles>

Prepared according to General Procedure A using tryptamine (385 mg, $2.40 \mathrm{mmol}, 1.2$ equiv.) and 2,4,6triphenylpyrylium tetrafluoroborate $(792 \mathrm{mg}, 2 \mathrm{mmol}, 1.0$ equiv.) in $\mathrm{EtOH}(2 \mathrm{~mL})$. Pyridinium salt 1al was isolated as a white solid (528 $\mathrm{mg}, 49 \%)$.

${ }^{1} \mathrm{H}$ NMR $\left(400 \mathrm{MHz}\right.$, DMSO- $\left.d_{6}\right) \delta 10.81(\mathrm{~s}, 1 \mathrm{H}), 8.47(\mathrm{~s}, 2 \mathrm{H}), 8.26(\mathrm{~d}, J=7.0 \mathrm{~Hz}, 2 \mathrm{H}), 7.88-7.58(\mathrm{~m}, 13 \mathrm{H}), 7.27$ $(\mathrm{d}, J=8.0 \mathrm{~Hz}, 1 \mathrm{H}), 6.99(\mathrm{t}, J=7.5 \mathrm{~Hz}, 1 \mathrm{H}), 6.71(\mathrm{t}, J=7.5 \mathrm{~Hz}, 1 \mathrm{H}), 6.53(\mathrm{~s}, 1 \mathrm{H}), 6.30(\mathrm{~d}, J=8.0 \mathrm{~Hz}, 1 \mathrm{H}), 4.63-$ $4.51(\mathrm{~m}, 2 \mathrm{H}), 2.82-2.71(\mathrm{~m}, 2 \mathrm{H}) ;{ }^{13} \mathrm{C}$ NMR $\left(101 \mathrm{MHz}, \mathrm{DMSO}-d_{6}\right) \delta 156.1,154.2,136.0,133.2,133.1,132.4$, $130.9,129.6,129.3,129.1,128.7,126.3,126.0,123.0,121.3,118.5,117.0,111.5,108.0,55.3,25.1 ;{ }^{19} \mathrm{~F} \mathrm{NMR}$ (377 MHz, DMSO- $d_{6}$ ) $\delta$-148.22 (minor, ${ }^{10} \mathrm{BF}_{4}$ ), -148.28 (major, ${ }^{11} \mathrm{BF}_{4}$ ); LRMS (ESI) $\mathrm{m} / \mathrm{z} 451.2\left(\left[\mathrm{M}-\mathrm{BF}_{4}\right]^{+}\right.$); HRMS (ESI) $m / z \mathrm{C}_{33} \mathrm{H}_{27} \mathrm{~N}_{2}{ }^{+}$requires $451.2169\left(\left[\mathrm{M}-\mathrm{BF}_{4}\right]^{+}\right)$, found 451.2164 .

Data is consistent with the literature. ${ }^{16}$

1-(2-(Dimethylamino)ethyl)-2,4,6-triphenylpyridin-1-ium tetrafluoroborate (1am)<smiles></smiles>

Prepared according to General Procedure A using $N, N$-dimethylethylenediamine ( $258 \mu \mathrm{l}, 2.40 \mathrm{mmol}, 1.2$ equiv.) and 2,4,6-triphenylpyrylium tetrafluoroborate ( $792 \mathrm{mg}, 2 \mathrm{mmol}, 1.0$ equiv.) in EtOH ( $2 \mathrm{~mL}$ ). Pyridinium salt 1am was isolated as a pale-yellow solid (836 mg, 90\%).

${ }^{1}{ }^{H}$ NMR $\left(400 \mathrm{MHz}\right.$, DMSO- $\left.d_{6}\right) \delta 8.47(\mathrm{~s}, 2 \mathrm{H}), 8.31-8.23(\mathrm{~m}, 2 \mathrm{H}), 7.94-7.86(\mathrm{~m}, 4 \mathrm{H}), 7.76-7.58(\mathrm{~m}, 9 \mathrm{H}), 4.48$ $(\mathrm{t}, J=7.0 \mathrm{~Hz}, 2 \mathrm{H}), 2.20(\mathrm{t}, J=7.0 \mathrm{~Hz}, 2 \mathrm{H}), 1.62(\mathrm{~s}, 6 \mathrm{H}) ;{ }^{13} \mathrm{C}$ NMR $\left(101 \mathrm{MHz}, \mathrm{DMSO}-d_{6}\right) \delta 156.4,154.2,133.1$, 
$133.1,132.4,131.0,129.6,129.5,129.1,128.7,125.9,56.8,51.9,44.3 ;{ }^{19} \mathbf{F}$ NMR $\left(377 \mathrm{MHz}, \mathrm{DMSO}-d_{6}\right) \delta-$ 153.11 (minor, ${ }^{10} \mathrm{BF}_{4}$ ), - 153.16 (major, ${ }^{11} \mathrm{BF}_{4}$ ); LRMS (ESI) $\mathrm{m} / \mathrm{z} 379.2\left(\left[\mathrm{M}-\mathrm{BF}_{4}\right]^{+}\right.$); HRMS (ESI) $\mathrm{m} / \mathrm{z} \mathrm{C}_{27} \mathrm{H}_{27} \mathrm{~N}_{2}{ }^{+}$

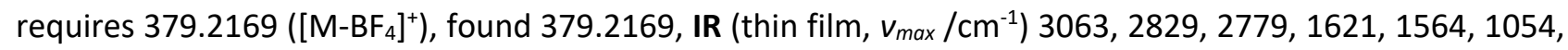
703; m.p. $\left(\mathrm{CH}_{2} \mathrm{Cl}_{2}\right): 260-264^{\circ} \mathrm{C}$.

(S)-1-(5-((tert-Butoxycarbonyl)amino)-5-carboxypentyl)-2,4,6-triphenylpyridin-1-ium

tetrafluoroborate (1an)<smiles></smiles>

Prepared according to General Procedure A using $N_{\alpha}$-(tert-butoxycarbonyl)-L-lysine (591 mg, $2.40 \mathrm{mmol}, 1.2$ equiv.) and 2,4,6-triphenylpyrylium tetrafluoroborate ( $792 \mathrm{mg}, 2 \mathrm{mmol}, 1.0$ equiv.) in EtOH ( $2 \mathrm{~mL}$ ). Pyridinium salt 1 an was isolated as a white solid ( $835 \mathrm{mg}, 67 \%)$.

${ }^{1} \mathrm{H}$ NMR $\left(400 \mathrm{MHz}, \mathrm{CDCl}_{3}\right) \delta 7.80(\mathrm{~s}, 2 \mathrm{H}), 7.79-7.67(\mathrm{~m}, 6 \mathrm{H}), 7.61-7.40(\mathrm{~m}, 9 \mathrm{H}), 6.84(\mathrm{~s}, 1 \mathrm{H}), 5.03(\mathrm{~d}, J=7.5$ $H z, 1 H), 4.42-4.28(m, 2 H), 3.95-3.83(m, 1 H), 1.50-1.40(m, 2 H), 1.37(s, 9 H), 1.25-1.16(m, 1 H), 1.08$ $(\mathrm{dq}, J=14.0,7.5 \mathrm{~Hz}, 1 \mathrm{H}), 0.85-0.69(\mathrm{~m}, 2 \mathrm{H}) ;{ }^{13} \mathrm{C} \mathrm{NMR}\left(101 \mathrm{MHz}, \mathrm{CDCl}_{3}\right) \delta 174.8,156.6,155.8,155.6,134.1$, $132.8,132.2,131.3,129.8,129.5,129.1,128.2,126.8,80.1,54.5,52.9,31.0,29.2,28.4,22.0 ;{ }^{19} \mathrm{~F}$ NMR $(377$ $\mathrm{MHz}, \mathrm{CDCl}_{3}$ ) $\delta$-152.19 (minor, ${ }^{10} \mathrm{BF}_{4}$ ), -152.24 (major, ${ }^{11} \mathrm{BF}_{4}$ ); LRMS (ESI) $\mathrm{m} / \mathrm{z} 537.2\left(\left[\mathrm{M}-\mathrm{BF}_{4}\right]^{+}\right)$; HRMS (ESI) $\mathrm{m} / \mathrm{z}$ $\mathrm{C}_{34} \mathrm{H}_{37} \mathrm{~N}_{2} \mathrm{O}_{4}{ }^{+}$requires $537.2748\left(\left[\mathrm{M}-\mathrm{BF}_{4}\right]^{+}\right)$, found $537.2745 ;[\boldsymbol{\alpha}]_{\mathbf{D}}^{25}+21.5^{\circ}\left(\mathrm{c}=1.0, \mathrm{CHCl}_{3}\right)$.

Data is consistent with the literature. ${ }^{16}$

2,4,6-Triphenyl-1-(2-sulfoethyl)pyridin-1-ium tetrafluoroborate (1ao)<smiles></smiles>

Prepared according to General Procedure A using taurine (300 mg, $2.40 \mathrm{mmol}, 1.2$ equiv.) and 2,4,6triphenylpyrylium tetrafluoroborate ( $792 \mathrm{mg}, 2 \mathrm{mmol}, 1.0$ equiv.) in EtOH ( $2 \mathrm{~mL}$ ). A few drops of triethylamine were added to solubilise the taurine. Purification by flash column chromatography $(0-100 \%$ acetone in $\mathrm{CH}_{2} \mathrm{Cl}_{2}$ ) afforded pyridinium salt 1ao as an off-white solid (727 mg, 72\%).

${ }^{1} \mathrm{H}$ NMR $\left(400 \mathrm{MHz}\right.$, DMSO- $\left.d_{6}\right) \delta 8.38(\mathrm{~s}, 2 \mathrm{H}), 8.31(\mathrm{~s}, 1 \mathrm{H}), 8.24(\mathrm{~d}, J=7.0 \mathrm{~Hz}, 2 \mathrm{H}), 7.90-7.81(\mathrm{~m}, 4 \mathrm{H}), 7.73-$ $7.56(\mathrm{~m}, 9 \mathrm{H}), 4.75-4.65(\mathrm{~m}, 2 \mathrm{H}), 2.47-2.39(\mathrm{~m}, 2 \mathrm{H}) ;{ }^{13} \mathrm{C}$ NMR $\left(101 \mathrm{MHz}\right.$, DMSO- $\left.d_{6}\right) \delta 156.6,153.9,133.2$, $133.1,132.3,130.8,129.5,129.3,129.0,128.6,125.9,52.3,48.9 ;{ }^{19} \mathrm{~F}$ NMR $\left(377 \mathrm{MHz}\right.$, DMSO-d $\left.{ }_{6}\right) \delta-148.23$ (minor, ${ }^{10} \mathrm{BF}_{4}$ ), -148.29 (major, ${ }^{11} \mathrm{BF}_{4}$ ); LRMS (ESI) $\mathrm{m} / z 416.0\left(\left[\mathrm{M}-\mathrm{BF}_{4}\right]^{+}\right)$; HRMS (ESI) $\mathrm{m} / z \mathrm{C}_{25} \mathrm{H}_{22} \mathrm{NO}_{3} \mathrm{~S}^{+}$requires $416.1315\left(\left[\mathrm{M}-\mathrm{BF}_{4}\right]^{+}\right)$, found 416.1313 , IR (thin film, $v_{\max } / \mathrm{cm}^{-1}$ ) 3406, 3058, 1620, 1563, 1179, 1029, 701; m.p. $\left(\mathrm{CH}_{2} \mathrm{Cl}_{2}\right): 252-254^{\circ} \mathrm{C}$ (decomposition). 
2,4,6-Triphenyl-1-(4-sulfamoylphenethyl)pyridin-1-ium tetrafluoroborate (1ap)<smiles>NS(=O)(=O)c1ccc(CC[n+]2c(-c3ccccc3)cc(-c3ccccc3)cc2-c2ccccc2)cc1</smiles>

Prepared according to General Procedure A using 4-(2-aminoethyl)benzenesulfonamide (481 mg, $2.40 \mathrm{mmol}$, 1.2 equiv.) and 2,4,6-triphenylpyrylium tetrafluoroborate (792 mg, $2 \mathrm{mmol}, 1.0$ equiv.) in EtOH ( $2 \mathrm{~mL}$ ). Pyridinium salt 1ap was isolated as a white solid (1.06 g, 92\%).

${ }^{1} \mathrm{H}$ NMR $\left(400 \mathrm{MHz}, \mathrm{DMSO}-d_{6}\right) \delta 8.48(\mathrm{~s}, 2 \mathrm{H}), 8.30-8.23(\mathrm{~m}, 2 \mathrm{H}), 7.80-7.60(\mathrm{~m}, 13 \mathrm{H}), 7.54(\mathrm{~d}, J=8.5 \mathrm{~Hz}, 2 \mathrm{H})$, $7.33(\mathrm{~s}, 2 \mathrm{H}), 6.60(\mathrm{~d}, J=8.5 \mathrm{~Hz}, 2 \mathrm{H}), 4.61(\mathrm{t}, J=7.5 \mathrm{~Hz}, 2 \mathrm{H}), 2.70(\mathrm{t}, J=7.5 \mathrm{~Hz}, 2 \mathrm{H}) ;{ }^{13} \mathrm{C} \mathrm{NMR}(101 \mathrm{MHz}$, DMSO$\left.d_{6}\right) \delta 156.2,154.5,143.1,139.7,133.1,132.9,132.5,131.0,129.6,129.3,129.2,128.8,128.7,126.1,126.0$, 55.3, 34.3; ${ }^{19} \mathrm{~F}$ NMR (377 MHz, DMSO- $d_{6}$ ) $\delta-148.25$ (minor, ${ }^{10} \mathrm{BF}_{4}$ ), -148.31 (major, ${ }^{11} \mathrm{BF}_{4}$ ); LRMS (ESI) $\mathrm{m} / \mathrm{z}$ $491.2\left(\left[\mathrm{M}-\mathrm{BF}_{4}\right]^{+}\right) ; \mathrm{HRMS}(\mathrm{ESI}) \mathrm{m} / z \mathrm{C}_{31} \mathrm{H}_{27} \mathrm{~N}_{2} \mathrm{O}_{2} \mathrm{~S}^{+}$requires $491.1788\left(\left[\mathrm{M}-\mathrm{BF}_{4}\right]^{+}\right)$, found 491.1782.

Data is consistent with the literature. ${ }^{16}$ 


\subsection{Two-step synthesis of sulfones}

General Procedure C: Secondary alkyl Katritzky salts

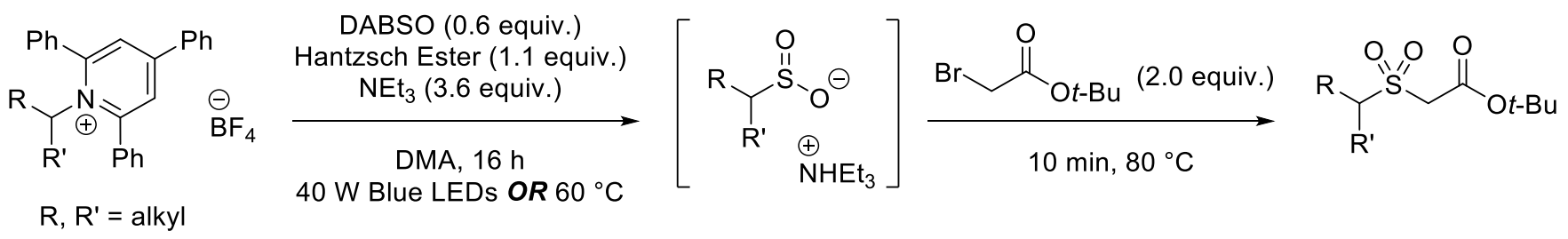

Hantzsch ester (1.1 equiv.), DABSO (0.6 equiv.) and Katritzky pyridinium salt (1.0 equiv.) were added to an oven-dried $1.9 \mathrm{~mL}$ reaction vial. The vial was then sealed with a rubber septum, evacuated and backfilled with $\mathrm{N}_{2}(\times 3)$. Degassed, anhydrous DMA $(0.5 \mathrm{M})$ was then added, followed by degassed, anhydrous triethylamine (3.6 equiv.). The reaction was stirred under blue LED irradiation at ambient temperature (General Procedure C-1, see Figure S4 for experimental setups for $0.20 \mathrm{mmol}$ and $10 \mathrm{mmol}$ reactions) or heated to $60^{\circ} \mathrm{C}$ in an aluminium heating block (General Procedure C-2) for $16 \mathrm{~h}$. tert-Butylbromoacetate (59 $\mu \mathrm{L}$ ) was then added and the reaction heated to $80^{\circ} \mathrm{C}$ in an aluminium heating block for $10 \mathrm{~min}$. The reaction mixture was cooled to room temperature. Without workup, the crude reaction mixture was loaded directly to silica gel and purified by flash column chromatography to afford the sulfone product.

Benzyl 4-((2-(tert-butoxy)-2-oxoethyl)sulfonyl)piperidine-1-carboxylate (3a)<smiles>CCCCOC(=O)CS(=O)(=O)C1CCN(C(C)(C)C)CC1</smiles>

Prepared according to General Procedure C-1 using Katritzky salt 1a (123 mg, $0.20 \mathrm{mmol}, 1.0$ equiv.), Hantzsch ester ( $55.7 \mathrm{mg}, 0.22 \mathrm{mmol}, 1.1$ equiv.), triethylamine (102 $\mu \mathrm{L}, 0.72 \mathrm{mmol}, 3.6$ equiv.), DABSO (28.8 $\mathrm{mg}, 0.12 \mathrm{mmol}, 0.6$ equiv.) and tert-butyl 2-bromoacetate ( $59 \mu \mathrm{L}, 0.40 \mathrm{mmol}, 2.0$ equiv.) in DMA ( $0.4 \mathrm{~mL}, 0.5$ M). Purification by flash column chromatography (20 - 30\% EtOAc in Petrol) afforded sulfone $\mathbf{3 a}$ as a colourless oil (66.1 mg, 83\%).

Also prepared according to General Procedure C-2, affording sulfone 3a as a colourless oil (63.9 mg, 80\%).

$\mathbf{R}_{\boldsymbol{f}}\left(30 \%\right.$ EtOAc in Petrol) $=0.14 ;{ }^{1} \mathrm{H}$ NMR $\left(400 \mathrm{MHz}, \mathrm{CDCl}_{3}\right) \delta 7.41-7.28(\mathrm{~m}, 5 \mathrm{H}), 5.13(\mathrm{~s}, 2 \mathrm{H}), 4.37$ (app. br. s, $2 \mathrm{H}$ ), $3.86(\mathrm{~s}, 2 \mathrm{H}$ ), 3.52 (tt, $J=12.0,4.0 \mathrm{~Hz}, 1 \mathrm{H}$ ), 2.86 (app. br. s, $2 \mathrm{H}$ ), 2.11 (app. br. d, $J=12.8 \mathrm{~Hz}, 2 \mathrm{H}$ ), 1.81 (qd, $J=12.0,4.5 \mathrm{~Hz}, 2 \mathrm{H}), 1.50(\mathrm{~s}, 9 \mathrm{H}) ;{ }^{13} \mathrm{C}$ NMR $\left(101 \mathrm{MHz}, \mathrm{CDCl}_{3}\right) \delta 162.2,155.1,136.6,128.7,128.3,128.2,84.5$, 67.6, 59.0, 56.1, 42.8, 28.0, 24.3; HRMS (ESI) $m / z \mathrm{C}_{19} \mathrm{H}_{26} \mathrm{NO}_{6} \mathrm{~S}^{-}$requires 396.1484 ([M-H] $\left.]^{-}\right)$, found 396.1484; IR (thin film, $v_{\max } / \mathrm{cm}^{-1}$ ) 2980, 2939, 1698, 1432, 1320, 1215, 1119.

tert-Butyl 4-((2-(tert-butoxy)-2-oxoethyl)sulfonyl)piperidine-1-carboxylate (3d) 
<smiles>CCC(C)OC(=O)CS(=O)(=O)C1CCN(C(C)(C)C)CC1</smiles>

Prepared according to General Procedure C-1 using Katritzky salt 1d (115.7 mg, 0.20 mmol, 1.0 equiv.), Hantzsch ester ( $55.7 \mathrm{mg}, 0.22 \mathrm{mmol}, 1.1$ equiv.), triethylamine (102 $\mu \mathrm{L}, 0.72 \mathrm{mmol}, 3.6$ equiv.), DABSO (28.8 $\mathrm{mg}, 0.12 \mathrm{mmol}, 0.6$ equiv.) and tert-butyl 2-bromoacetate ( $59 \mu \mathrm{L}, 0.40 \mathrm{mmol}, 2.0$ equiv.) in DMA (0.4 mL, 0.5 M). Purification by flash column chromatography (20-30\% EtOAc in Petrol) afforded sulfone $\mathbf{3} \mathbf{d}$ as an offwhite solid (65.0 mg, 89\%).

Also prepared according to General Procedure C-1 and C-2 on a $10 \mathrm{mmol}$ scale, affording sulfone $\mathbf{3 d}$ as an off-white solid ( $3.14 \mathrm{~g}, 86 \%$ and $3.05 \mathrm{~g}, 84 \%$, respectively).

$\mathbf{R}_{\boldsymbol{f}}\left(30 \%\right.$ EtOAc in Petrol) $=0.30 ;{ }^{1} \mathrm{H}$ NMR $\left(400 \mathrm{MHz}, \mathrm{CDCl}_{3}\right) \delta 4.29$ (app. br. s, 2H), $3.86(\mathrm{~s}, 2 \mathrm{H}), 3.48$ (tt, $J=$ 12.0, 3.5 Hz, 1H), 2.76 (app. br. s, 2H), 2.09 (app. br. d, $J=13.5 \mathrm{~Hz}, 2 \mathrm{H}$ ), $1.86-1.71(\mathrm{~m}, 2 \mathrm{H}$ ), 1.50 (s, 9H), 1.45 (s, 9H); ${ }^{13} \mathrm{C}$ NMR $\left(101 \mathrm{MHz}, \mathrm{CDCl}_{3}\right) \delta$ 162.2, 154.4, 84.4, 80.3, 59.3, 56.0, 42.6 (br.), 28.5, 28.0, 24.4; LRMS (ESI) $m / z 386.2\left([\mathrm{M}+\mathrm{Na}]^{+}\right)$; HRMS (ESI) $m / z \mathrm{C}_{16} \mathrm{H}_{29} \mathrm{NO}_{6} \mathrm{SNa}^{+}$requires $386.1608\left([\mathrm{M}+\mathrm{Na}]^{+}\right)$, found 386.1610 .

Data is consistent with the literature. ${ }^{26}$

tert-Butyl 2-((1-tosylpiperidin-4-yl)sulfonyl)acetate (3e)<smiles>CCCCOC(=O)CS(=O)(=O)C1CCN([As])CC1</smiles>

Prepared according to General Procedure C-1 using Katritzky salt 1e-3 (126.5 mg, 0.20 mmol, 1.0 equiv.), Hantzsch ester ( $55.7 \mathrm{mg}, 0.22 \mathrm{mmol}, 1.1$ equiv.), triethylamine (102 $\mu \mathrm{L}, 0.72 \mathrm{mmol}, 3.6$ equiv.), DABSO (28.8 $\mathrm{mg}, 0.12 \mathrm{mmol}, 0.6$ equiv.) and tert-butyl 2-bromoacetate ( $59 \mu \mathrm{L}, 0.40 \mathrm{mmol}, 2.0$ equiv.) in DMA (0.4 mL, 0.5 M). Purification by flash column chromatography (10 - 30\% EtOAc in Petrol) afforded sulfone $3 e$ as a white solid (74 mg, 89\%).

$\mathbf{R}_{f}\left(30 \%\right.$ EtOAc in Petrol) = 0.30; ${ }^{1} \mathrm{H}$ NMR $\left(400 \mathrm{MHz}, \mathrm{CDCl}_{3}\right) \delta 7.62(\mathrm{~d}, J=8.0 \mathrm{~Hz}, 2 \mathrm{H}), 7.33(\mathrm{~d}, J=8.0 \mathrm{~Hz}, 2 \mathrm{H})$, 3.91 (br. dt, $J=12.0,3.0 \mathrm{~Hz}, 2 \mathrm{H}$ ), $3.83(\mathrm{~s}, 2 \mathrm{H}), 3.28(\mathrm{tt}, J=12.0,4.0 \mathrm{~Hz}, 1 \mathrm{H}), 2.43(\mathrm{~s}, 3 \mathrm{H}), 2.37$ (td, $J=12.0,2.0$ $\mathrm{Hz}, 2 \mathrm{H}), 2.15$ (br. dd, $J=12.0,2.0 \mathrm{~Hz}, 2 \mathrm{H}), 1.97$ (qd, $J=12.0,4.0 \mathrm{~Hz}, 2 \mathrm{H}), 1.45(\mathrm{~s}, 9 \mathrm{H}) ;{ }^{13} \mathrm{C} \mathrm{NMR}(101 \mathrm{MHz}$, $\left.\mathrm{CDCl}_{3}\right) \delta 162.2,144.1,133.0,130.0,127.7,84.5,58.0,56.1,44.9,27.9,24.1,21.6$; LRMS (ESI) $\mathrm{m} / \mathrm{z} 440.2$ $\left([\mathrm{M}+\mathrm{Na}]^{+}\right)$; HRMS (ESI) $\mathrm{m} / z \mathrm{C}_{18} \mathrm{H}_{27} \mathrm{NO}_{6} \mathrm{~S}_{2} \mathrm{Na}^{+}$requires $440.1162\left([\mathrm{M}+\mathrm{Na}]^{+}\right)$, found 440.1170; IR (thin film, $v_{\max }$ $\left./ \mathrm{cm}^{-1}\right) 2980,1729,1320,1161,936,725 ;$ m.p. $\left(\mathrm{CH}_{2} \mathrm{Cl}_{2}\right): 145-148^{\circ} \mathrm{C}$.

tert-Butyl 2-(cyclobutylsulfonyl)acetate (3f) 


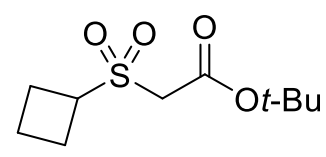

Prepared according to General Procedure E using 1-(1-cyclobutyl)-2,4,6-triphenylpyridin-1-ium tetrafluoroborate If (89.9 mg, $0.20 \mathrm{mmol}, 1.0$ equiv.), Hantzsch ester (55.7 mg, $0.22 \mathrm{mmol}, 1.1$ equiv.), piperidine (71 $\mu \mathrm{L}, 0.72 \mathrm{mmol}, 3.6$ equiv.), DABSO (28.8 mg, $0.12 \mathrm{mmol}, 0.6$ equiv.) and tert-butyl 2bromoacetate (59 $\mu \mathrm{L}, 0.40 \mathrm{mmol}, 2.0$ equiv.) in DMA (0.4 mL, $0.5 \mathrm{M})$. Purification by flash column chromatography (20\% EtOAc in Petrol) afforded sulfone $\mathbf{3 f}$ as a colourless oil ( $37.7 \mathrm{mg}, 80 \%)$.

$\mathbf{R}_{f}\left(20 \%\right.$ EtOAc in Petrol) $=0.26 ;{ }^{1} \mathbf{H}$ NMR $\left(400 \mathrm{MHz}, \mathrm{CDCl}_{3}\right) \delta 4.15(\mathrm{pd}, J=8.5,0.5 \mathrm{~Hz}, 1 \mathrm{H}), 3.73(\mathrm{~s}, 2 \mathrm{H}), 2.65-$ $2.50(\mathrm{~m}, 2 \mathrm{H}), 2.37-2.24(\mathrm{~m}, 2 \mathrm{H}), 2.16-1.97(\mathrm{~m}, 2 \mathrm{H}), 1.48(\mathrm{~s}, 9 \mathrm{H}) ;{ }^{13} \mathrm{C}$ NMR $\left(101 \mathrm{MHz}, \mathrm{CDCl}_{3}\right) \delta$ 162.1, 84.0, 56.9, 54.7, 27.9, 22.5, 17.1; LRMS (ESI) $m / z 257.0$ ([M+Na] $]^{+}$); HRMS (ESI) $m / z \mathrm{C}_{10} \mathrm{H}_{18} \mathrm{O}_{4} \mathrm{SNa}^{+}$requires 257.0818 $\left([\mathrm{M}+\mathrm{Na}]^{+}\right)$, found 257.0820; IR (thin film, $\left.v_{\max } / \mathrm{cm}^{-1}\right)$ 2981, 2950, 1728, 1370. 1322, 1133, 1100.

tert-Butyl 2-(cyclopentylsulfonyl)acetate (3g)

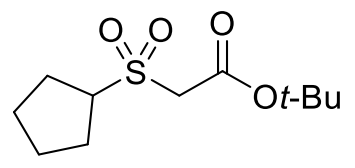

Prepared according to General Procedure C-1 using 1-(1-cyclopentyl)-2,4,6-triphenylpyridin-1-ium tetrafluoroborate $1 \mathrm{~g}$ (93.0 mg, $0.20 \mathrm{mmol}, 1.0$ equiv.), Hantzsch ester (55.7 mg, $0.22 \mathrm{mmol}, 1.1$ equiv.), triethylamine (102 $\mu \mathrm{L}, 0.72 \mathrm{mmol}, 3.6$ equiv.), DABSO (28.8 mg, $0.12 \mathrm{mmol}, 0.6$ equiv.) and tert-butyl 2bromoacetate (59 $\mu \mathrm{L}, 0.40 \mathrm{mmol}, 2.0$ equiv.) in DMA $(0.4 \mathrm{~mL}, 0.5 \mathrm{M})$. Purification by flash column chromatography ( $2-5 \%$ EtOAc in Petrol) afforded sulfone $3 \mathrm{~g}$ as a colourless oil ( $40 \mathrm{mg}, 81 \%$ ).

$\mathbf{R}_{f}(5 \%$ EtOAc in Petrol $)=0.23 ;{ }^{1} \mathbf{H}$ NMR $\left(400 \mathrm{MHz}, \mathrm{CDCl}_{3}\right) \delta 3.84(\mathrm{~s}, 2 \mathrm{H}), 3.85-3.76(\mathrm{~m}, 1 \mathrm{H}), 2.18-1.97(\mathrm{~m}$, 4H), $1.91-1.76(\mathrm{~m}, 2 \mathrm{H}), 1.75-1.60(\mathrm{~m}, 2 \mathrm{H}), 1.50(\mathrm{~s}, 9 \mathrm{H}) ;{ }^{13} \mathrm{C} \mathrm{NMR}\left(101 \mathrm{MHz}, \mathrm{CDCl}_{3}\right) \delta 162.3,84.1,61.6,57.9$, 28.0, 26.9, 26.2; LRMS (ESI) $\mathrm{m} / z 271.0\left([\mathrm{M}+\mathrm{Na}]^{+}\right)$; HRMS (ESI) $\mathrm{m} / z \mathrm{C}_{11} \mathrm{H}_{20} \mathrm{O}_{4} \mathrm{SNa}^{+}$requires $271.0975\left([\mathrm{M}+\mathrm{Na}]^{+}\right)$, found 271.0972; IR (thin film, $v_{\max } / \mathrm{cm}^{-1}$ ) 2979, 1728, 1370, 1309, 1130, 707.

Data is consistent with the literature. ${ }^{26}$

tert-Butyl 2-(cyclohexylsulfonyl)acetate (3h)<smiles>CCCCOC(=O)CS(=O)(=O)C1CCCCC1</smiles>

Prepared according to General Procedure C-1 using 1-(1-cyclohexyl)-2,4,6-triphenylpyridin-1-ium tetrafluoroborate $\mathbf{1 h}$ (95.5 mg, $0.20 \mathrm{mmol}, 1.0$ equiv.), Hantzsch ester (55.7 mg, $0.22 \mathrm{mmol}, 1.1$ equiv.), triethylamine (102 $\mu \mathrm{L}, 0.72 \mathrm{mmol}, 3.6$ equiv.), DABSO (28.8 mg, $0.12 \mathrm{mmol}, 0.6$ equiv.) and tert-butyl 2- 
bromoacetate ( $59 \mu \mathrm{L}, 0.40 \mathrm{mmol}, 2.0$ equiv.) in DMA (0.4 mL, $0.5 \mathrm{M})$. Purification by flash column chromatography ( $2-5 \%$ EtOAc in Petrol) afforded sulfone $3 \mathrm{~h}$ as a colourless oil ( $43 \mathrm{mg}, 82 \%)$.

$\mathbf{R}_{f}(10 \%$ EtOAc in Petrol $)=0.28 ;{ }^{1} \mathbf{H}$ NMR $\left(400 \mathrm{MHz}, \mathrm{CDCl}_{3}\right) \delta 3.84(\mathrm{~s}, 2 \mathrm{H}), 3.28(\mathrm{tt}, \mathrm{J}=12.0,3.5 \mathrm{~Hz}, 1 \mathrm{H}), 2.29-$ $2.10(\mathrm{~m}, 2 \mathrm{H}), 1.99-1.86(\mathrm{~m}, 2 \mathrm{H}), 1.77-1.67(\mathrm{~m}, 1 \mathrm{H}), 1.57$ (ddd, J =25.0, 12.0, 3.5 Hz, 2H), $1.49(\mathrm{~s}, 9 \mathrm{H}), 1.40-$ $1.12(\mathrm{~m}, 3 \mathrm{H}) ;{ }^{13} \mathrm{C}$ NMR $\left(101 \mathrm{MHz}, \mathrm{CDCl}_{3}\right) \delta 162.2,84.0,61.2,55.9,28.0,25.1,25.1,24.9$; LRMS (ESI) $\mathrm{m} / \mathrm{z} 285.0$ $\left([\mathrm{M}+\mathrm{Na}]^{+}\right)$; HRMS (ESI) $\mathrm{m} / z \mathrm{C}_{12} \mathrm{H}_{22} \mathrm{O}_{4} \mathrm{SNa}^{+}$requires $285.1131\left([\mathrm{M}+\mathrm{Na}]^{+}\right)$, found 285.1131; IR (thin film, $v_{\max }$ $\left./ \mathrm{cm}^{-1}\right)$ 2936, 1729, 1315, 1132, 1099, 700.

Data is consistent with the literature. ${ }^{26}$

tert-Butyl 2-(isopropylsulfonyl)acetate (3i)<smiles>CCCOC(=O)CS(=O)(=O)C(C)C</smiles>

Prepared according to General Procedure C-1 using 1-isopropyl-2,4,6-triphenylpyridin-1-ium tetrafluoroborate 1i ( $87.5 \mathrm{mg}, 0.20 \mathrm{mmol}, 1.0$ equiv.), Hantzsch ester (55.7 mg, $0.22 \mathrm{mmol}, 1.1$ equiv.), triethylamine (102 $\mu \mathrm{L}, 0.72 \mathrm{mmol}, 3.6$ equiv.), DABSO (28.8 mg, $0.12 \mathrm{mmol}, 0.6$ equiv.) and tert-butyl 2bromoacetate ( $59 \mu \mathrm{L}, 0.40 \mathrm{mmol}, 2.0$ equiv.) in DMA $(0.4 \mathrm{~mL}, 0.5 \mathrm{M})$. Purification by flash column chromatography (2 - 5\% EtOAc in Petrol) afforded sulfone $\mathbf{3 i}$ as a colourless oil (36.2 mg, 81\%).

Also prepared according to General Procedure C-2, affording sulfone $\mathbf{3 i}$ as a colourless oil (36.8 mg, $83 \%)$.

$\mathbf{R}_{f}\left(10 \%\right.$ EtOAc in Petrol) $=0.14 ;{ }^{1} \mathbf{H}$ NMR $\left(400 \mathrm{MHz}, \mathrm{CDCl}_{3}\right) \delta 3.87(\mathrm{~s}, 2 \mathrm{H}), 3.54$ (hept, $\left.J=7.0 \mathrm{~Hz}, 1 \mathrm{H}\right), 1.50(\mathrm{~s}$, 9H), 1.42 (d, $J=7.0 \mathrm{~Hz}, 6 \mathrm{H}) ;{ }^{13} \mathrm{C}$ NMR $\left(101 \mathrm{MHz}, \mathrm{CDCl}_{3}\right) \delta$ 162.2, 84.1, 56.0, 53.4, 28.0, 15.2; LRMS (ESI) $\mathrm{m} / \mathrm{z}$ $245.0\left([\mathrm{M}+\mathrm{Na}]^{+}\right)$; HRMS (ESI) $\mathrm{m} / z \mathrm{C}_{9} \mathrm{H}_{18} \mathrm{O}_{4} \mathrm{SNa}^{+}$requires $245.0818\left([\mathrm{M}+\mathrm{Na}]^{+}\right)$, found 245.0818; IR (thin film, $\left.v_{\max } / \mathrm{cm}^{-1}\right)$ 2981, 1730, 1371, 1319, 1259, 1132.

tert-Butyl 2-(cyclooctylsulfonyl)acetate (3j)

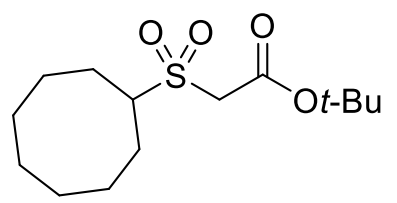

Prepared according to General Procedure C-1 using 1-(1-cyclooctyl)-2,4,6-triphenylpyridin-1-ium tetrafluoroborate $1 \mathbf{j}$ (101 mg, $0.20 \mathrm{mmol}, 1.0$ equiv.), Hantzsch ester (55.7 mg, $0.22 \mathrm{mmol}, 1.1$ equiv.), triethylamine (102 $\mu \mathrm{L}, 0.72 \mathrm{mmol}, 3.6$ equiv.), DABSO (28.8 mg, $0.12 \mathrm{mmol}, 0.6$ equiv.) and tert-butyl 2bromoacetate ( $59 \mu \mathrm{L}, 0.40 \mathrm{mmol}, 2.0$ equiv.) in DMA $(0.4 \mathrm{~mL}, 0.5 \mathrm{M})$. Purification by flash column chromatography (0-0.5\% EtOAc in Toluene) afforded sulfone $\mathbf{3} \mathbf{j}$ as a colourless oil (52 $\mathrm{mg}, 90 \%$ ). 
$\mathbf{R}_{f}(5 \%$ EtOAc in Toluene $)=0.32 ;{ }^{1} \mathrm{H}$ NMR $\left(400 \mathrm{MHz}, \mathrm{CDCl}_{3}\right) \delta 3.86(\mathrm{~s}, 2 \mathrm{H}), 3.47(\mathrm{tt}, \mathrm{J}=9.5,3.5 \mathrm{~Hz}, 1 \mathrm{H}), 2.22-$ $2.14(\mathrm{~m}, 2 \mathrm{H}), 1.89-1.74(\mathrm{~m}, 4 \mathrm{H}), 1.70-1.40(\mathrm{~m}, 8 \mathrm{H}), 1.50(\mathrm{~s}, 9 \mathrm{H}) ;{ }^{13} \mathrm{C}$ NMR $\left(101 \mathrm{MHz}, \mathrm{CDCl}_{3}\right) \delta$ 162.3, 84.0, 61.9, 56.0, 28.0, 26.4, 26.2, 25.5, 25.3; LRMS (ESI) $m / z$ 313.2 ([M+Na] $\left.]^{+}\right)$; HRMS (ESI) $m / z \mathrm{C}_{14} \mathrm{H}_{26} \mathrm{O}_{4} \mathrm{SNa}^{+}$requires 313.1444 $\left([\mathrm{M}+\mathrm{Na}]^{+}\right)$, found 313.1445; IR (thin film, $\left.v_{\max } / \mathrm{cm}^{-1}\right)$ 2980, 2928, 1730, 1370, 1317, 1134.

tert-Butyl 2-((2-methylcyclohexyl)sulfonyl)acetate (3k)<smiles>CCCCOC(=O)CS(=O)(=O)C1CCCCC1C</smiles>

Prepared according to General Procedure C-1 using 1-(2-methylcyclohexyl)-2,4,6-triphenylpyridin-1-ium tetra-fluoroborate $1 \mathbf{k}(98.0 \mathrm{mg}, 0.20 \mathrm{mmol}, 1.0$ equiv.), Hantzsch ester (55.7 mg, $0.22 \mathrm{mmol}, 1.1$ equiv.), triethylamine (102 $\mu \mathrm{L}, 0.72 \mathrm{mmol}, 3.6$ equiv.), DABSO (28.8 mg, $0.12 \mathrm{mmol}, 0.6$ equiv.) and tert-butyl 2bromoacetate ( $59 \mu \mathrm{L}, 0.40 \mathrm{mmol}, 2.0$ equiv.) in DMA $(0.4 \mathrm{~mL}, 0.5 \mathrm{M})$. Purification by flash column chromatography ( $0-1 \%$ EtOAc in Toluene) afforded sulfone $\mathbf{3 k}$ as a colourless oil (54 mg, 90\%, 2:7 diastereomeric ratio $\mathbf{A}: \mathbf{B})$.

$\mathbf{R}_{f}\left(5 \%\right.$ EtOAc in Petrol) $=0.17 ;{ }^{1} \mathbf{H}$ NMR $\left(400 \mathrm{MHz}, \mathrm{CDCl}_{3}\right) 3.97(\mathrm{~d}, J=14.5 \mathrm{~Hz}, 1 \mathrm{H}$, dia B), $3.88(\mathrm{~d}, J=14.0 \mathrm{~Hz}$, 1H, dia A), $3.79-3.70(\mathrm{~m}, 1 \mathrm{H}$ dia $\mathbf{A}$ and $1 \mathrm{H}$ dia B), $3.46(\mathrm{dt}, J=11.5,4.0 \mathrm{~Hz}, 1 \mathrm{H}$, dia A), 3.04 (ddd, $J=11.5$, 10.0, 4.0 Hz, 1H, dia B), 2.60 (app. dp, J = 10.5, 3.5 Hz, 1H, dia A), $2.24-2.12$ (m, 1H, dia B), 1.99 (app. dddq, $J=13.0,9.5,6.5,3.5 \mathrm{~Hz}, 1 \mathrm{H}, \mathrm{dia} \mathbf{B}), 1.93-1.78(\mathrm{~m}, 3 \mathrm{H}$ dia $\mathbf{A}$ and $2 \mathrm{H}$ dia $\mathbf{B}), 1.77-1.62(\mathrm{~m}, 2 \mathrm{H}$ dia $\mathbf{A}$ and $1 \mathrm{H}$ dia B), $1.62-1.51$ (m, 2H dia A and $1 \mathrm{H}$ dia B), 1.49 (s, 9H dia $\mathbf{A}$ and $10 \mathrm{H}$ dia B), $1.35-1.25$ (m, 1H dia $\mathbf{A}$ and $2 \mathrm{H}$ dia B), $1.22\left(\mathrm{~d}, J=6.5 \mathrm{~Hz}, 3 \mathrm{H}\right.$, dia B), $1.16\left(\mathrm{~d}, J=7.0 \mathrm{~Hz}, 3 \mathrm{H}\right.$, dia A); ${ }^{13} \mathrm{C} \mathrm{NMR}\left(101 \mathrm{MHz}, \mathrm{CDCl}_{3}\right) \delta$ for major dia : 162.4 (dias A and B), 84.0 (dias A and B), 67.7 (dia B), 63.9 (dia A), 58.2 (dia B), 57.3 (dia A), 35.4 (dia B), 33.5 (dia A), 32.3 (dia B), 28.0 (dias A and B), 27.8 (dia A), 26.7 (dia B), 25.6 (dia A), $25.2(\operatorname{dia}$ B), 24.9 (dia B), 21.4 (dia B), 20.7 (dia A), 19.5 (dia A), 13.6 (dia A); LRMS (ESI) $\mathrm{m} / z 299.0$ ([M+Na] $]^{+}$); HRMS (ESI) $\mathrm{m} / \mathrm{z}$ $\mathrm{C}_{13} \mathrm{H}_{24} \mathrm{O}_{4} \mathrm{SNa}^{+}$requires $299.1288\left([\mathrm{M}+\mathrm{Na}]^{+}\right)$, found 299.1287; IR (thin film, $v_{\max } / \mathrm{cm}^{-1}$ ) 2933, 1731, 1370, 1313, $1161,1135$.

tert-Butyl 2-(heptan-2-ylsulfonyl)acetate (3I)

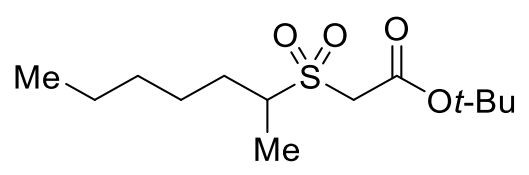

Prepared according to General Procedure C-1 using 1-(Heptan-2-yl)-2,4,6-triphenylpyridin-1-ium tetrafluoroborate 1 l (101 mg, 0.20 mmol, 1.0 equiv.), Hantzsch ester (55.7 mg, 0.22 mmol, 1.1 equiv.), triethylamine (102 $\mu \mathrm{L}, 0.72 \mathrm{mmol}, 3.6$ equiv.), DABSO (28.8 mg, $0.12 \mathrm{mmol}, 0.6$ equiv.) and tert-butyl 2bromoacetate ( $59 \mu \mathrm{L}, 0.40 \mathrm{mmol}, 2.0$ equiv.) in DMA $(0.4 \mathrm{~mL}, 0.5 \mathrm{M})$. Purification by flash column chromatography (0 - 1\% EtOAc in Toluene) afforded sulfone $3 \mathbf{l}$ as a colourless oil ( $50 \mathrm{mg}, 90 \%)$. 
$\mathbf{R}_{f}(1 \%$ EtOAc in Toluene $)=0.25 ;{ }^{1} \mathrm{H}$ NMR $\left(400 \mathrm{MHz}, \mathrm{CDCl}_{3}\right) \delta 3.89(\mathrm{~d}, \mathrm{~J}=14.5 \mathrm{~Hz}, 1 \mathrm{H}), 3.84(\mathrm{~d}, \mathrm{~J}=14.5 \mathrm{~Hz}, 1 \mathrm{H})$, $3.40-3.32(\mathrm{~m}, 1 \mathrm{H}), 2.02-1.95(\mathrm{~m}, 1 \mathrm{H}), 1.68-1.45(\mathrm{~m}, 2 \mathrm{H}), 1.49(\mathrm{~s}, 9 \mathrm{H}), 1.40(\mathrm{~d}, J=7.0 \mathrm{~Hz}, 3 \mathrm{H}), 1.36-1.27$ (m, 5H), 0.89 (app. t, $J=6.5 \mathrm{~Hz}, 3 \mathrm{H}) ;{ }^{13} \mathrm{C}$ NMR (101 MHz, CDCl $\left.)\right) \delta 162.2,84.1,58.0,56.2,31.6,28.5,28.0$, 26.3, 22.5, 14.1, 12.8; LRMS (ESI) $\mathrm{m} / z 301.2\left([\mathrm{M}+\mathrm{Na}]^{+}\right)$; HRMS (ESI) $\mathrm{m} / z \mathrm{C}_{13} \mathrm{H}_{26} \mathrm{O}_{4} \mathrm{SNa}^{+}$requires 301.1444 $\left([\mathrm{M}+\mathrm{Na}]^{+}\right)$, found 301.1444; IR (thin film, $\left.v_{\max } / \mathrm{cm}^{-1}\right)$ 2981, 1730, 1372, 1315, 1156, 954.

tert-Butyl 2-((tetrahydro-2H-pyran-4-yl)sulfonyl)acetate (3m)<smiles>CCC(C)OC(=O)CS(=O)(=O)C1CCOCC1</smiles>

Prepared according to General Procedure C-1 using 1-(tetrahydro-2H-pyran-4-yl)-2,4,6-triphenylpyridin-1ium tetra-fluoroborate $1 \mathrm{~m}$ (96.0 mg, $0.20 \mathrm{mmol}, 1.0$ equiv.), Hantzsch ester (55.7 mg, $0.22 \mathrm{mmol}, 1.1$ equiv.), triethylamine (102 $\mu \mathrm{L}, 0.72 \mathrm{mmol}, 3.6$ equiv.), DABSO (28.8 mg, $0.12 \mathrm{mmol}, 0.6$ equiv.) and tert-butyl 2bromoacetate (59 $\mu \mathrm{L}, 0.40 \mathrm{mmol}, 2.0$ equiv.) in DMA (0.4 mL, $0.5 \mathrm{M})$. Purification by flash column chromatography (10 - 20\% EtOAc in Petrol) afforded sulfone $3 \mathrm{~m}$ as a colourless oil (48 mg, 91\%).

$\mathbf{R}_{\boldsymbol{f}}\left(40 \%\right.$ EtOAc in Petrol) $=0.30 ;{ }^{1} \mathbf{H}$ NMR $\left(400 \mathrm{MHz}, \mathrm{CDCl}_{3}\right) \delta 4.12(\mathrm{ddd}, J=11.5,4.0,2.0 \mathrm{~Hz}, 2 \mathrm{H}), 3.85(\mathrm{~s}, 2 \mathrm{H})$, $3.62-3.54(\mathrm{~m}, 1 \mathrm{H}), 3.42(\mathrm{td}, J=11.5,3.0 \mathrm{~Hz}, 2 \mathrm{H}), 2.05-1.87(\mathrm{~m}, 4 \mathrm{H}), 1.49(\mathrm{~s}, 9 \mathrm{H}) ;{ }^{13} \mathrm{C} \mathrm{NMR}(101 \mathrm{MHz}, \mathrm{CDCl})$

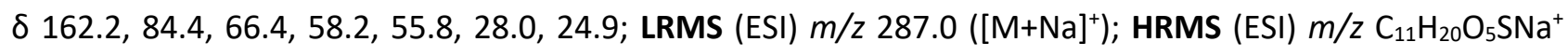
requires $287.0924\left([\mathrm{M}+\mathrm{Na}]^{+}\right)$, found 287.0924; IR (thin film, $\left.v_{\max } / \mathrm{cm}^{-1}\right)$ 2979, 2854, 1729, 1318, $1128,1084$.

tert-Butyl 2-((tetrahydro-2H-thiopyran-4-yl)sulfonyl)acetate (3n)<smiles>CCC(C)OC(=O)CS(=O)(=O)C1CCSCC1</smiles>

Prepared according to General Procedure C-1 using 2,4,6-triphenyl-1-(tetrahydro-2H-thiopyran-4-yl)pyridin1-ium tetrafluoroborate $1 \mathrm{n}$ (99 mg, 0.20 mmol, 1.0 equiv.), Hantzsch ester (55.7 mg, 0.22 mmol, 1.1 equiv.), triethylamine (102 $\mu \mathrm{L}, 0.72 \mathrm{mmol}, 3.6$ equiv.), DABSO (28.8 mg, $0.12 \mathrm{mmol}, 0.6$ equiv.) and tert-butyl 2bromoacetate (59 $\mu \mathrm{L}, 0.40 \mathrm{mmol}, 2.0$ equiv.) in DMA (0.4 mL, $0.5 \mathrm{M})$. Purification by flash column chromatography (0 - 20\% EtOAc in Petrol) afforded sulfone $3 \mathrm{n}$ as a colourless oil (48 mg, $86 \%$ ).

$\mathbf{R}_{\boldsymbol{f}}\left(30 \%\right.$ EtOAc in Petrol) $=0.43 ;{ }^{1} \mathbf{H}$ NMR $\left(400 \mathrm{MHz}, \mathrm{CDCl}_{3}\right) \delta 3.86(\mathrm{~s}, 2 \mathrm{H}), 3.34(\mathrm{tt}, \mathrm{J}=12.0,3.0 \mathrm{~Hz}, 1 \mathrm{H}), 2.82-$ $2.69(\mathrm{~m}, 4 \mathrm{H}), 2.49$ (br. dd, J = 13.0, 3.5 Hz, 2H), 1.98 (qd, J = 12.0, 4.0 Hz, 2H), $1.50(\mathrm{~s}, 9 \mathrm{H}) ;{ }^{13} \mathrm{C} \mathrm{NMR}(101 \mathrm{MHz}$, $\left.\mathrm{CDCl}_{3}\right) \delta 162.1,84.4,60.8,55.7,28.0,27.5,26.3$; LRMS (ESI) $\mathrm{m} / \mathrm{z} 303.0\left([\mathrm{M}+\mathrm{Na}]^{+}\right)$; HRMS (ESI) $\mathrm{m} / z$ $\mathrm{C}_{11} \mathrm{H}_{20} \mathrm{O}_{4} \mathrm{~S}_{2} \mathrm{Na}^{+}$requires $303.0695\left([\mathrm{M}+\mathrm{Na}]^{+}\right)$, found 303.0696; IR (thin film, $v_{\max } / \mathrm{cm}^{-1}$ ) 2980, 2890, 1730, 1371, 1319, 1156, 1135, 955. 


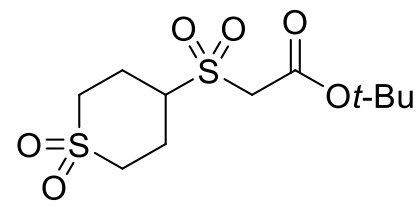

Prepared according to General Procedure C-1 using 1-(1,1-dioxidotetrahydro-2H-thiopyran-4-yl)-2,4,6triphenylpyridin-1-ium tetrafluoroborate 10 (105.5 mg, 0.20 mmol, 1.0 equiv.), Hantzsch ester (55.7 mg, 0.22 mmol, 1.1 equiv.), triethylamine (102 $\mu \mathrm{L}, 0.72 \mathrm{mmol}, 3.6$ equiv.), DABSO ( $28.8 \mathrm{mg}, 0.12 \mathrm{mmol}, 0.6$ equiv.) and tert-butyl 2-bromoacetate ( $59 \mu \mathrm{L}, 0.40 \mathrm{mmol}, 2.0$ equiv.) in DMA (0.4 mL, $0.5 \mathrm{M})$. Purification by flash column chromatography (30 - 50\% EtOAc in Petrol) afforded sulfone 30 as a white solid (47 mg, 75\%).

Also prepared according to General Procedure C-2, affording sulfone 30 as a white solid (43.1 mg, $69 \%)$.

$\mathbf{R}_{f}\left(50 \%\right.$ EtOAc in Petrol) $=0.27 ;{ }^{1} \mathbf{H}$ NMR $\left(400 \mathrm{MHz}, \mathrm{CDCl}_{3}\right) \delta 3.95(\mathrm{~s}, 2 \mathrm{H}), 3.65-3.59(\mathrm{~m}, 1 \mathrm{H}), 3.37-3.32(\mathrm{~m}$, 2H), $3.08-3.01(\mathrm{~m}, 2 \mathrm{H}), 2.64-2.54(\mathrm{~m}, 4 \mathrm{H}), 1.50(\mathrm{~s}, 9 \mathrm{H}) ;{ }^{13} \mathrm{C} \mathrm{NMR}\left(101 \mathrm{MHz}, \mathrm{CDCl}_{3}\right) \delta$ 162.0, 85.0, 56.8, 56.2, 48.9, 28.0, 23.6; LRMS (ESI) $m / z 335.0\left([\mathrm{M}+\mathrm{Na}]^{+}\right)$; HRMS (ESI) $m / z \mathrm{C}_{11} \mathrm{H}_{20} \mathrm{O}_{6} \mathrm{~S}_{2} \mathrm{Na}^{+}$requires $335.0594\left([\mathrm{M}+\mathrm{Na}]^{+}\right)$, found 335.0595; IR (thin film, $v_{\max } / \mathrm{cm}^{-1}$ ) 2982, 2890, 1728, 1319, 1248, 1119, 905, 707; m.p. $\left(\mathrm{CH}_{2} \mathrm{Cl}_{2}\right): 124-$ $130{ }^{\circ} \mathrm{C}$.

tert-Butyl 2-((4-hydroxycyclohexyl)sulfonyl)acetate (3p)<smiles>CCCCOC(=O)CS(=O)(=O)C1CCC(O)CC1</smiles>

Prepared according to General Procedure C-1 using 1-(4-hydroxycyclohexyl)-2,4,6-triphenylpyridin-1-ium tetrafluoroborate $1 p(99.0 \mathrm{mg}, 0.20 \mathrm{mmol}, 1.0$ equiv.), Hantzsch ester (55.7 mg, $0.22 \mathrm{mmol}, 1.1$ equiv.), triethylamine (102 $\mu \mathrm{L}, 0.72 \mathrm{mmol}, 3.6$ equiv.), DABSO (28.8 mg, $0.12 \mathrm{mmol}, 0.6$ equiv.) and tert-butyl 2bromoacetate ( $59 \mu \mathrm{L}, 0.40 \mathrm{mmol}, 2.0$ equiv.) in DMA $(0.4 \mathrm{~mL}, 0.5 \mathrm{M})$. Purification by flash column chromatography (50-70\% EtOAc in Petrol) afforded sulfone $3 p$ as a colourless oil (42 mg, 75\%, 3:4 diastereomeric ratio $\mathbf{A}: \mathbf{B})$.

$\mathbf{R}_{f}(70 \%$ EtOAc in Petrol $)=0.29 \& 0.34 ;{ }^{1} \mathbf{H}$ NMR $\left(400 \mathrm{MHz}, \mathrm{CDCl}_{3}\right) \delta 4.10-4.05(\mathrm{~m}, 1 \mathrm{H}, \mathrm{dia} \mathbf{A}), 3.86(\mathrm{~s}, 2 \mathrm{H}$, dia A), $3.86(\mathrm{~s}, 2 \mathrm{H}$, dia B), $3.70-3.59(\mathrm{~m}, 1 \mathrm{H}$, dia B), 3.29 (qt, $J=12.5,3.5 \mathrm{~Hz}, 1 \mathrm{H}$ dia A and $1 \mathrm{H}$ dia B), $2.28-1.91$ (m, 4H dia A and 6H dia B), $1.81-1.54$ (m, 3H dia A and 3H dia B), 1.49 (s, 9H, dia B), 1.49 (s, 9H, dia A), 1.41 - 1.27 (m, 2H dia A); ${ }^{13} \mathrm{C}$ NMR (101 MHz, $\mathrm{CDCl}_{3}$ ) $\delta 162.3$ (dia B), 162.1 (dia A), 84.3 (dia A), 84.1 (dia B), 69.2 (dia A), 64.4 (dia B), 60.6 (dia B), 59.8 (dia A), 56.4 (dia A), 55.9 (dia B), 33.7 (dia A), 31.4 (dia B), 28.0 (dias A and B), 23.3 (dia A), 18.9 (dia B); LRMS (ESI) $\mathrm{m} / z 301.0$ ([M+Na $\left.]^{+}\right)$; HRMS (ESI) $m / z \quad \mathrm{C}_{12} \mathrm{H}_{22} \mathrm{O}_{5} \mathrm{SNa}^{+}$requires $301.1080\left([\mathrm{M}+\mathrm{Na}]^{+}\right)$, found 301.1082; IR (thin film, $\left.v_{\max } / \mathrm{cm}^{-1}\right)$ 3524, 2940, 1728, 1312, 1131, 1069. 


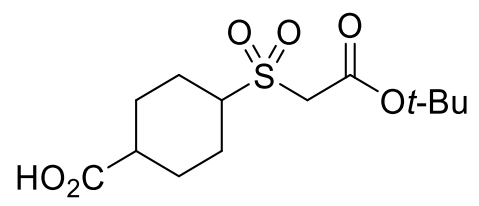

Prepared according to General Procedure C-1 using 1-(4-carboxycyclohexyl)-2,4,6-triphenyl-pyridin-1-ium tetrafluoroborate 1q (104 mg, 0.20 mmol, 1.0 equiv.), Hantzsch ester (55.7 mg, 0.22 mmol, 1.1 equiv.), triethylamine (102 $\mu \mathrm{L}, 0.72 \mathrm{mmol}, 3.6$ equiv.), DABSO (28.8 mg, $0.12 \mathrm{mmol}, 0.6$ equiv.) and tert-butyl 2bromoacetate ( $59 \mu \mathrm{L}, 0.40 \mathrm{mmol}, 2.0$ equiv.) in DMA $(0.4 \mathrm{~mL}, 0.5 \mathrm{M})$. Purification by flash column chromatography (10-30\% EtOAc in Petrol with $2 \% \mathrm{AcOH}$ ) afforded sulfone $\mathbf{3 q}$ as a colourless oil (51 mg, $83 \%$, 2:3 diastereomeric ratio $\mathbf{A}: \mathbf{B})$.

$\mathbf{R}_{f}(30 \%$ EtOAc in Petrol with $2 \% \mathrm{AcOH})=0.18 ;{ }^{1} \mathbf{H} \mathbf{N M R}\left(400 \mathrm{MHz}, \mathrm{CDCl}_{3}\right) \delta 3.87(\mathrm{~s}, 2 \mathrm{H}, \mathrm{dia} \mathbf{B}), 3.86(\mathrm{~s}, 2 \mathrm{H}$, dia A), $3.41-3.27$ ( $m, 1 \mathrm{H}$ dia $\mathbf{A}$ and $1 \mathrm{H}$ dia B), $2.79-2.69(\mathrm{~m}, 1 \mathrm{H}$, dia B), $2.44-2.20(\mathrm{~m}, 5 \mathrm{H}$ dia $\mathbf{A}$ and $2 \mathrm{H}$ dia B), 2.08 (br. dd, $J=13.5,3.0 \mathrm{~Hz}, 2 \mathrm{H}$, dia B), 1.86 (ddd, $J=15.0,13.0,3.5 \mathrm{~Hz}, 2 \mathrm{H}$, dia B), $1.74-1.53$ (m, 4H, dia A and 2H dia B), 1.50 (s, 9H, dia B), 1.50 (s, 9H, dia A); ${ }^{13}$ C NMR (101 MHz, CDCl $) \delta 180.4$ (dia A), 179.9 (dia B), 162.3 (dia B), 162.1 (dia A), 84.4 (dia A), 84.2 (dia B), 60.2 (dia B), 60.0 (dia A), 56.2 (dia A), 56.0 (dia B), 41.7 (dia A), 38.3 (dia B), 28.0 (dias A and B), 27.3 (dia A), 25.6 (dia B), 23.9 (dia A), 21.8 (dia B); LRMS (ESI) m/z 305.1 ([M-H]); HRMS (ESI) $\mathrm{m} / z \mathrm{C}_{13} \mathrm{H}_{21} \mathrm{O}_{6} \mathrm{~S}^{-}$requires 305.1064 ([M-H] ), found 305.1061; IR (thin film, $v_{\max } / \mathrm{cm}^{-}$ $\left.{ }^{1}\right) 2939,1726,1704,1314,1131,735$.

Methyl 4-((2-(tert-butoxy)-2-oxoethyl)sulfonyl)cyclohexane-1-carboxylate (3r)<smiles>CCCCOC(=O)CS(=O)(=O)C1CCC(C(C)C)CC1</smiles>

Prepared according to General Procedure C-1 using 1-(4-(methoxycarbonyl)cyclohexyl)-2,4,6triphenylpyridin-1-ium tetrafluoroborate $1 \mathrm{r}(107 \mathrm{mg}, 0.20 \mathrm{mmol}, 1.0$ equiv.), Hantzsch ester (55.7 mg, 0.22 mmol, 1.1 equiv.), triethylamine (102 $\mu \mathrm{L}, 0.72 \mathrm{mmol}, 3.6$ equiv.), DABSO ( $28.8 \mathrm{mg}, 0.12 \mathrm{mmol}, 0.6$ equiv.) and tert-butyl 2-bromoacetate ( $59 \mu \mathrm{L}, 0.40 \mathrm{mmol}, 2.0$ equiv.) in DMA ( $0.4 \mathrm{~mL}, 0.5 \mathrm{M})$. Purification by flash column chromatography (10 - 20\% EtOAc in Petrol) afforded sulfone $3 r$ as an orange oil (58 mg, 90\%, 2:3 diastereomeric ratio $\mathbf{A}: \mathbf{B})$.

$\mathbf{R}_{\boldsymbol{f}}\left(30 \%\right.$ EtOAc in Petrol) $=0.29 ;{ }^{1} \mathbf{H}$ NMR $\left(400 \mathrm{MHz}, \mathrm{CDCl}_{3}\right) \delta 3.85(\mathrm{~s}, 2 \mathrm{H}, \operatorname{dia} \mathbf{A}), 3.83(\mathrm{~s}, 2 \mathrm{H}, \operatorname{dia} \mathbf{B}), 3.69(\mathrm{~s}, 3 \mathrm{H}$, $\operatorname{dia} \mathbf{B}$ ), 3.67 (s, 3H, dia A), $3.37-3.26$ (m, 1H, dia A, 1H, dia B), 2.66 (app. p, J=4.5 Hz, 1H, dia B), $2.36-2.18$ (m, 5H dia A and $2 \mathrm{H}$ dia B), $2.07-2.02(\mathrm{~m}, 2 \mathrm{H}$, dia B), 1.81 (qd, $J=13.5,13.0,3.5 \mathrm{~Hz}, 2 \mathrm{H}$, dia B), $1.70-1.45$ (m, 4H dia A and 2H dia B), 1.49 (s, 9H, dia A), 1.49 (s, 9H, dia B); $\left.{ }^{13} \mathbf{C ~ N M R ~ ( 1 0 1 ~ M H z , ~ C D C l ~}{ }_{3}\right) \delta 175.1$ (dia A), 174.2 (dia B), 162.2 (dia B), 162.0 (dia A), 84.2 (dia A), 84.1 (dia B), 60.2 (dia B), 60.0 (dia A), 56.1 (dia A), 56.0 
(dia B), 52.0 (dia B), 51.9 (dia A), 41.9 (dia A), 38.4 (dia B), 27.9 (dias A and B), 27.4 (dia A), 25.8 (dia B), 24.0 (dia A), 21.8 (dia B); LRMS (ESI) $m / z 343.0\left([\mathrm{M}+\mathrm{Na}]^{+}\right.$); HRMS (ESI) $m / z \quad \mathrm{C}_{14} \mathrm{H}_{24} \mathrm{O}_{6} \mathrm{SNa}^{+}$requires 343.1186 $\left([\mathrm{M}+\mathrm{Na}]^{+}\right)$, found 343.1188; IR (thin film, $v_{\max } / \mathrm{cm}^{-1}$ ) 2980, 1727, 1371, 1132, 898, 702.

tert-Butyl 2-((1-(2,6-dimethylphenoxy)propan-2-yl)sulfonyl)acetate (3s)<smiles>CCCCOC(=O)CS(=O)(=O)C(C)COc1c(C)cccc1C</smiles>

Prepared according to General Procedure C-1 using 1-(1-(2,6-dimethylphenoxy)propan-2-yl)-2,4,6triphenylpyridin-1-ium tetrafluoroborate $1 \mathrm{~s}$ ( $111.5 \mathrm{mg}, 0.20 \mathrm{mmol}, 1.0$ equiv.), Hantzsch ester (55.7 mg, 0.22 mmol, 1.1 equiv.), triethylamine (102 $\mu \mathrm{L}, 0.72 \mathrm{mmol}, 3.6$ equiv.), DABSO ( $28.8 \mathrm{mg}, 0.12 \mathrm{mmol}, 0.6$ equiv.) and tert-butyl 2-bromoacetate ( $59 \mu \mathrm{L}, 0.40 \mathrm{mmol}, 2.0$ equiv.) in DMA (0.4 mL, $0.5 \mathrm{M})$. Purification by flash column chromatography (10 - 20\% EtOAc in Petrol) afforded sulfone 3 s as a yellow oil (58 $\mathrm{mg}, 85 \%)$.

$\mathbf{R}_{f}\left(5 \%\right.$ EtOAc in Toluene) $=0.40 ;{ }^{1} \mathbf{H}$ NMR $\left(400 \mathrm{MHz}, \mathrm{CDCl}_{3}\right) \delta 7.03$ (br. d, $\left.J=7.5 \mathrm{~Hz}, 2 \mathrm{H}\right), 6.96(\mathrm{dd}, J=8.5,6.0$ $\mathrm{Hz}, 1 \mathrm{H}), 4.50(\mathrm{~d}, J=14.5 \mathrm{~Hz}, 1 \mathrm{H}), 4.21(\mathrm{dd}, J=10.0,8.5 \mathrm{~Hz}, 1 \mathrm{H}), 4.14-4.07(\mathrm{~m}, 1 \mathrm{H}), 4.03(\mathrm{~d}, J=14.5 \mathrm{~Hz}, 1 \mathrm{H})$, $3.87(\mathrm{dd}, J=10.0,4.0 \mathrm{~Hz}, 1 \mathrm{H}), 2.30(\mathrm{~s}, 6 \mathrm{H}), 1.50(\mathrm{~s}, 9 \mathrm{H}), 1.48(\mathrm{~d}, J=7.0 \mathrm{~Hz}, 3 \mathrm{H}) ;{ }^{13} \mathrm{C} \mathrm{NMR}\left(101 \mathrm{MHz}, \mathrm{CDCl}_{3}\right) \delta$ 162.4 154.7, 130.9, 129.2, 124.8, 83.9, 71.0, 59.7, 57.7, 28.0, 16.4, 9.3; LRMS (ESI) m/z 365.0 ([M+Na] $]^{+}$; HRMS (ESI) $\mathrm{m} / \mathrm{z} \mathrm{C}_{17} \mathrm{H}_{26} \mathrm{O}_{5} \mathrm{SNa}^{+}$requires $365.1393\left([\mathrm{M}+\mathrm{Na}]^{+}\right.$), found 365.1394; IR (thin film, $v_{\max } / \mathrm{cm}^{-1}$ ) 2979, 1733, 1477, 1323, 1197, 1159, 1116.

tert-Butyl 2-((1-hydroxy-3-phenylpropan-2-yl)sulfonyl)acetate (3t)<smiles>CCC(C)OC(=O)CS(=O)(=O)C(CO)Cc1ccccc1</smiles>

Prepared according to General Procedure C-1 using 1-(1-hydroxy-3-phenylpropan-2-yl)-2,4,6-triphenylpyridin-1-ium tetrafluoroborate $1 \mathrm{t}(96.0 \mathrm{mg}, 0.20 \mathrm{mmol}, 1.0$ equiv.), Hantzsch ester ( $55.7 \mathrm{mg}, 0.22 \mathrm{mmol}, 1.1$ equiv.), triethylamine (102 $\mu \mathrm{L}, 0.72 \mathrm{mmol}, 3.6$ equiv.), DABSO ( $28.8 \mathrm{mg}, 0.12 \mathrm{mmol}, 0.6$ equiv.) and tert-butyl 2-bromoacetate ( $59 \mu \mathrm{L}, 0.40 \mathrm{mmol}, 2.0$ equiv.) in DMA (0.4 mL, $0.5 \mathrm{M})$. Purification by flash column chromatography (10 - 30\% EtOAc in Petrol) afforded sulfone $3 \mathrm{t}$ as a colourless oil ( $54 \mathrm{mg}, 86 \%)$.

Also prepared according to General Procedure C-2, affording sulfone $3 \mathrm{t}$ as a colourless oil (58.6 mg, 93\%).

$\mathbf{R}_{\boldsymbol{f}}\left(30 \%\right.$ EtOAc in Petrol) $=0.31 ;{ }^{1} \mathbf{H}$ NMR $\left(400 \mathrm{MHz}, \mathrm{CDCl}_{3}\right) \delta 7.37-7.31(\mathrm{~m}, 2 \mathrm{H}), 7.30-7.24(\mathrm{~m}, 3 \mathrm{H}), 4.09(\mathrm{~d}$, $J=14.5 \mathrm{~Hz}, 1 \mathrm{H}), 4.00(\mathrm{dd}, J=12.5,2.5 \mathrm{~Hz}, 1 \mathrm{H}), 3.93(\mathrm{dd}, J=12.5,7.0 \mathrm{~Hz}, 1 \mathrm{H}), 3.87-3.77(\mathrm{~m}, 2 \mathrm{H}), 3.83(\mathrm{~d}, J=$ $14.5 \mathrm{~Hz}, 1 \mathrm{H}$ ), 3.35 (dd, J = 14.0, $4.5 \mathrm{~Hz}, 1 \mathrm{H}$ ), 2.92 (dd, $J=14.0,10.5 \mathrm{~Hz}, 1 \mathrm{H}), 2.47$ (br. s, $1 \mathrm{H}), 1.51(\mathrm{~s}, 9 \mathrm{H}) ;{ }^{13} \mathrm{C}$ NMR $\left(101 \mathrm{MHz}_{\mathrm{CDCl}}\right) \delta 162.4,136.4,129.4,129.1,127.4,84.5,65.7,59.3,59.2,30.4,28.0$; LRMS (ESI) $\mathrm{m} / \mathrm{z}$ 
$337.0\left([\mathrm{M}+\mathrm{Na}]^{+}\right)$; HRMS (ESI) $\mathrm{m} / z \mathrm{C}_{15} \mathrm{H}_{22} \mathrm{O}_{5} \mathrm{SNa}^{+}$requires $337.1080\left([\mathrm{M}+\mathrm{Na}]^{+}\right)$, found 337.1080; IR (thin film, $\left.v_{\max } / \mathrm{cm}^{-1}\right) 3522,2935,1729,1312,1112,702$. 
General Procedure D: Benzylic Katritzky salts

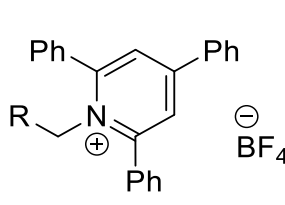

$\mathrm{R}=$ aryl, cinnamyl
DABSO (0.6 equiv.) Hantzsch Ester (1.1 equiv.) 2,6-lutidine (3.6 equiv.)

DMA, $16 \mathrm{~h}$ $40 \mathrm{~W}$ Blue LEDs OR $40^{\circ} \mathrm{C}$<smiles>[R]C[S@@](=O)[O-]</smiles>

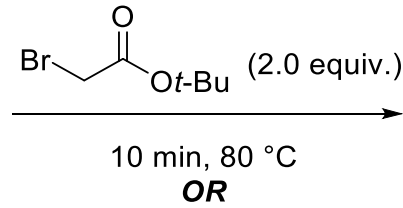

$6 \mathrm{~h}, \mathrm{rt}$<smiles>[R]CS(=O)(=O)CC(=O)OCCCC</smiles>

Hantzsch ester (1.1 equiv.), DABSO (0.6 equiv.) and Katritzky pyridinium salt (1.0 equiv.) were added to an oven-dried $1.9 \mathrm{~mL}$ reaction vial. The vial was then sealed with a rubber septum, evacuated and backfilled with $\mathrm{N}_{2}(\times 3)$. Degassed, anhydrous DMA (0.5 M) was then added, followed by degassed, anhydrous 2,6lutidine (3.6 equiv.). The reaction was stirred under blue LED irradiation at ambient temperature (General Procedure D-1, see figure S1 for experimental setup) or heated to $40{ }^{\circ} \mathrm{C}$ in an aluminium heating block (General Procedure D-2) for $16 \mathrm{~h}$. tert-Butylbromoacetate (2.0 equiv.) was then added and the reaction was heated to $80^{\circ} \mathrm{C}$ in an aluminium heating block for $10 \mathrm{~min}$ or at room temperature for 6 hours. The reaction mixture was cooled to room temperature. Without workup, the crude reaction mixture was loaded directly to silica gel and purified by flash column chromatography to afford the sulfone product.

tert-Butyl 2-(benzylsulfonyl)acetate (3b)<smiles>CCCOC(=O)CS(=O)(=O)Cc1ccccc1</smiles>

Prepared according to General Procedure D-1 using 1-benzyl-2,4,6-triphenylpyridin-1-ium tetrafluoroborate 1b (97.1 mg, 0.20 mmol, 1.0 equiv.), Hantzsch ester ( 55.7 mg, 0.22 mmol, 1.1 equiv.), 2,6-lutidine ( $83 \mu \mathrm{L}, 0.72$ mmol, 3.6 equiv.), DABSO ( $28.8 \mathrm{mg}, 0.12 \mathrm{mmol}, 0.6$ equiv.) and tert-butyl 2-bromoacetate $(59 \mu \mathrm{L}, 0.40 \mathrm{mmol}$, 2.0 equiv.) in DMA ( $0.4 \mathrm{~mL}, 0.5 \mathrm{M})$. Purification by flash column chromatography ( $5-10 \%$ EtOAc in Petrol) afforded sulfone $\mathbf{3 b}$ as a white crystalline solid (44.5 $\mathrm{mg}, 82 \%)$.

Also prepared according to General Procedure D-2, affording sulfone $\mathbf{3 b}$ as a white crystalline solid (45.3 mg, $84 \%)$.

$\mathbf{R}_{f}\left(10 \%\right.$ EtOAc in Petrol) $=0.14 ;{ }^{1} \mathrm{H}$ NMR $\left(400 \mathrm{MHz}, \mathrm{CDCl}_{3}\right) \delta 7.52-7.47(\mathrm{~m}, 2 \mathrm{H}), 7.44-7.39(\mathrm{~m}, 3 \mathrm{H}), 4.50(\mathrm{~s}$,

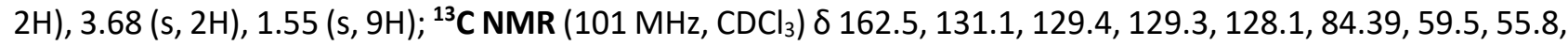
28.1; LRMS (ESI) $\mathrm{m} / \mathrm{z} 293.0\left([\mathrm{M}+\mathrm{Na}]^{+}\right)$; HRMS (ESI) $\mathrm{m} / \mathrm{z} \mathrm{C}_{13} \mathrm{H}_{18} \mathrm{O}_{4} \mathrm{SNa}^{+}$requires $293.0818\left([\mathrm{M}+\mathrm{Na}]^{+}\right)$, found 293.0818.

Data is consistent with the literature. ${ }^{26}$

tert-Butyl 2-((4-methoxybenzyl)sulfonyl)acetate (3v)<smiles>CCCCOC(=O)CS(=O)(=O)Cc1ccc(OC)cc1</smiles> 
Prepared according to General Procedure D-1 using 1-(4-methoxybenzyl)-2,4,6-triphenylpyridin-1-ium tetrafluoroborate $1 \mathbf{v}$ (103.1 mg, 0.20 mmol, 1.0 equiv.), Hantzsch ester ( $55.7 \mathrm{mg}, 0.22 \mathrm{mmol}, 1.1$ equiv.), 2,6lutidine ( $83 \mu \mathrm{L}, 0.72 \mathrm{mmol}, 3.6$ equiv.), DABSO (28.8 $\mathrm{mg}, 0.12 \mathrm{mmol}, 0.6$ equiv.) and tert-butyl 2bromoacetate ( $59 \mu \mathrm{L}, 0.40 \mathrm{mmol}, 2.0$ equiv.) in DMA $(0.4 \mathrm{~mL}, 0.5 \mathrm{M})$. Purification by flash column chromatography (10 - 20\% EtOAc in Petrol) afforded sulfone $3 v$ as an off-white solid (41.3 mg, 69\%).

Also prepared according to General Procedure D-2, affording sulfone $\mathbf{3 v}$ as an off-white solid (39.8 mg, 66\%). $\mathbf{R}_{f}\left(20 \%\right.$ EtOAc in Petrol) $=0.22 ;{ }^{1} \mathrm{H}$ NMR $\left(400 \mathrm{MHz}, \mathrm{CDCl}_{3}\right) \delta 7.44-7.37(\mathrm{~m}, 2 \mathrm{H}), 6.96-6.89(\mathrm{~m}, 2 \mathrm{H}), 4.43(\mathrm{~s}$, 2H), 3.81 (s, 3H), 3.66 (s, 2H), $1.54(\mathrm{~s}, 9 \mathrm{H}) ;{ }^{13} \mathrm{C}$ NMR (101 MHz, CDCl $) \delta 162.5,160.5,132.3,119.9,114.7$, 84.3, 58.8, 55.6, 55.5, 28.1; LRMS (ESI) $m / z 323.0$ ([M+Na] $]^{+}$); HRMS (ESI) $m / z \mathrm{C}_{14} \mathrm{H}_{20} \mathrm{O}_{5} \mathrm{SNa}^{+}$requires 323.0924 $\left([\mathrm{M}+\mathrm{Na}]^{+}\right)$, found 323.0923; IR (thin film, $\left.v_{\max } / \mathrm{cm}^{-1}\right)$ 2980, 2936, 1728, 1514, 1326, 1252, 1162; m.p. $\left(\mathrm{CH}_{2} \mathrm{Cl}_{2}\right)$ : $61-63^{\circ} \mathrm{C}$.

tert-Butyl 2-((3-methoxybenzyl)sulfonyl)acetate (3w)<smiles>CCCCOC(=O)CS(=O)(=O)Cc1cccc(OC)c1</smiles>

Prepared according to General Procedure D-1 using 1-(3-methoxybenzyl)-2,4,6-triphenylpyridin-1-ium tetrafluoroborate $1 \mathbf{w}$ (103.1 mg, 0.20 mmol, 1.0 equiv.), Hantzsch ester (55.7 mg, 0.22 mmol, 1.1 equiv.), 2,6lutidine (83 $\mu \mathrm{L}, 0.72 \mathrm{mmol}, 3.6$ equiv.), DABSO (28.8 mg, $0.12 \mathrm{mmol}, 0.6$ equiv.) and tert-butyl 2bromoacetate ( $59 \mu \mathrm{L}, 0.40 \mathrm{mmol}, 2.0$ equiv.) in DMA $(0.4 \mathrm{~mL}, 0.5 \mathrm{M})$. Purification by flash column chromatography (10 - 20\% EtOAc in Petrol) afforded sulfone $3 \mathbf{w}$ as an off-white solid (43.7mg, 73\%).

$\mathbf{R}_{f}\left(20 \%\right.$ EtOAc in Petrol) $=0.15 ;{ }^{1} \mathbf{H}$ NMR $\left(400 \mathrm{MHz}, \mathrm{CDCl}_{3}\right) \delta 7.34-7.28(\mathrm{~m}, 1 \mathrm{H}), 7.09-7.01(\mathrm{~m}, 2 \mathrm{H}), 6.93$ (ddd, $J=8.5,2.5,1.0 \mathrm{~Hz}, 1 \mathrm{H}), 4.46(\mathrm{~s}, 2 \mathrm{H}), 3.81(\mathrm{~s}, 3 \mathrm{H}), 3.69(\mathrm{t}, \mathrm{s}, 2 \mathrm{H}), 1.54(\mathrm{~s}, 9 \mathrm{H}) ;{ }^{13} \mathrm{C} \mathrm{NMR}\left(101 \mathrm{MHz}, \mathrm{CDCl}_{3}\right)$ $\delta 162.4,160.1,130.2,129.4,123.2,116.4,115.0,84.3,59.4,55.8,55.4,28.0 ;$ LRMS (ESI) $\mathrm{m} / \mathrm{z} 323.0$ ([M+Na] $\left.]^{+}\right)$ $\left.299.0([\mathrm{M}-\mathrm{H}]]^{-}\right)$; HRMS (ESI) $\mathrm{m} / z \mathrm{C}_{14} \mathrm{H}_{20} \mathrm{O}_{5} \mathrm{SNa}^{+}$requires $323.0924\left([\mathrm{M}+\mathrm{Na}]^{+}\right)$, found 323.0922; IR (thin film, $v_{\max }$ $\left./ \mathrm{cm}^{-1}\right)$ 2980, 2938, 1727, 1491, 1325, 1121, 695.

tert-Butyl 2-((2-methoxybenzyl)sulfonyl)acetate (3x)<smiles>CCCOC(=O)CS(=O)(=O)Cc1ccccc1OC</smiles>

Prepared according to General Procedure D-1 using 1-(2-methoxybenzyl)-2,4,6-triphenylpyridin-1-ium tetrafluoroborate $1 \mathbf{x}$ (103.1 mg, $0.20 \mathrm{mmol}, 1.0$ equiv.), Hantzsch ester ( $55.7 \mathrm{mg}, 0.22 \mathrm{mmol}, 1.1$ equiv.), 2,6lutidine ( $83 \mu \mathrm{L}, 0.72 \mathrm{mmol}, 3.6$ equiv.), DABSO (28.8 mg, $0.12 \mathrm{mmol}, 0.6$ equiv.) and tert-butyl 2bromoacetate ( $59 \mu \mathrm{L}, 0.40 \mathrm{mmol}, 2.0$ equiv.) in DMA $(0.4 \mathrm{~mL}, 0.5 \mathrm{M})$. Purification by flash column chromatography (10 - 20\% EtOAc in Petrol) afforded sulfone $3 x$ as an off-white solid $(40.0 \mathrm{mg}, 67 \%)$. 
$\mathbf{R}_{f}\left(20 \%\right.$ EtOAc in Petrol) $=0.19 ;{ }^{1} \mathbf{H}$ NMR $\left(400 \mathrm{MHz}, \mathrm{CDCl}_{3}\right) \delta 7.43(\mathrm{dd}, J=7.5,1.5 \mathrm{~Hz}, 1 \mathrm{H}), 7.36$ (ddd, $J=8.5$, 7.5, $2.0 \mathrm{~Hz}, 1 \mathrm{H}), 6.99(\mathrm{td}, J=7.5,1.0 \mathrm{~Hz}, 1 \mathrm{H}), 6.94(\mathrm{dd}, J=8.5,1.0 \mathrm{~Hz}, 1 \mathrm{H}), 4.59(\mathrm{~s}, 2 \mathrm{H}), 3.86(\mathrm{~s}, 3 \mathrm{H}), 3.81(\mathrm{~s}$, 2H), 1.53 (s, 9H); ${ }^{13} \mathrm{CNMR}\left(101 \mathrm{MHz}, \mathrm{CDCl}_{3}\right) \delta 162.2,158.0,132.8,130.9,121.2,116.5,111.4,84.0,57.3,55.8$, 54.3, 28.0; LRMS (ESI) $\mathrm{m} / \mathrm{z} 323.0\left([\mathrm{M}+\mathrm{Na}]^{+}\right), 299.0\left([\mathrm{M}-\mathrm{H}]^{-}\right)$; HRMS (ESI) $\mathrm{m} / z \mathrm{C}_{14} \mathrm{H}_{20} \mathrm{O}_{5} \mathrm{SNa}^{+}$requires 323.0924 $\left([\mathrm{M}+\mathrm{Na}]^{+}\right)$, found 323.0923; IR (thin film, $\left.v_{\max } / \mathrm{cm}^{-1}\right)$ 2980, 2934, 1730, 1496, 1325, 1252, 1131; m.p. $\left(\mathrm{CH}_{2} \mathrm{Cl}_{2}\right)$ : $71-74{ }^{\circ} \mathrm{C}$.

tert-Butyl 2-((4-chlorobenzyl)sulfonyl)acetate (3y)

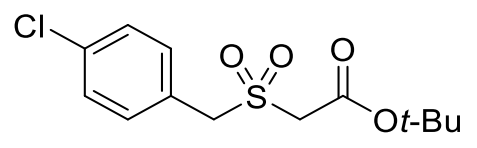

Prepared according to General Procedure D-1 using 1-(4-chlorobenzyl)-2,4,6-triphenylpyridin-1-ium tetrafluoroborate $1 \mathbf{y}$ (104.0 mg, 0.20 mmol, 1.0 equiv.), Hantzsch ester ( $55.7 \mathrm{mg}, 0.22 \mathrm{mmol}, 1.1$ equiv.), 2,6lutidine (83 $\mu \mathrm{L}, 0.72 \mathrm{mmol}, 3.6$ equiv.), DABSO (28.8 $\mathrm{mg}, 0.12 \mathrm{mmol}, 0.6$ equiv.) and tert-butyl 2bromoacetate ( $59 \mu \mathrm{L}, 0.40 \mathrm{mmol}, 2.0$ equiv.) in DMA $(0.4 \mathrm{~mL}, 0.5 \mathrm{M})$. Purification by flash column chromatography (10 - 20\% EtOAc in Petrol) afforded sulfone $3 y$ as an off-white solid (48.1 mg, 79\%).

Also prepared according to General Procedure D-2, affording sulfone $3 y$ as an off-white solid (46.7 mg, 77\%).

$\mathbf{R}_{f}\left(20 \%\right.$ EtOAc in Petrol) $=0.32 ;{ }^{1} \mathrm{H}$ NMR $\left(400 \mathrm{MHz}, \mathrm{CDCl}_{3}\right) \delta 7.47-7.41(\mathrm{~m}, 2 \mathrm{H}), 7.40-7.34(\mathrm{~m}, 2 \mathrm{H}), 4.47(\mathrm{~s}$,

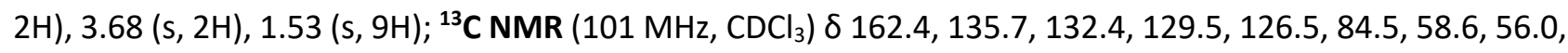
28.0; LRMS (ESI) $m / z$ 303.0, 305.0 ([M-H]); HRMS (ESI) $m / z \mathrm{C}_{13} \mathrm{H}_{17}{ }^{35} \mathrm{ClO}_{4} \mathrm{SNa}^{+}$requires $327.0428\left([\mathrm{M}+\mathrm{Na}]^{+}\right)$, found 327.0428, $\mathrm{C}_{13} \mathrm{H}_{17}{ }^{37} \mathrm{ClO}_{4} \mathrm{SNa}^{+}$requires $329.0398\left([\mathrm{M}+\mathrm{Na}]^{+}\right)$, found 329.0398 ; IR (thin film, $v_{\max } / \mathrm{cm}^{-1}$ ) $2981,2936,1727,1493,1327,1097,834$; m.p. $\left(\mathrm{CH}_{2} \mathrm{Cl}_{2}\right): 103-105^{\circ} \mathrm{C}$.

tert-Butyl 2-((4-(trifluoromethyl)benzyl)sulfonyl)acetate (3z)<smiles>CCCOC(=O)CS(=O)(=O)Cc1ccc(C(F)(F)F)cc1</smiles>

Prepared according to General Procedure D-1 using 2,4,6-triphenyl-1-(4-(trifluoromethyl)benzyl)pyridin-1ium tetrafluoroborate $1 \mathbf{z}$ (110.6 mg, 0.20 mmol, 1.0 equiv.), Hantzsch ester ( $55.7 \mathrm{mg}, 0.22 \mathrm{mmol}, 1.1$ equiv.), 2,6-lutidine ( $83 \mu \mathrm{L}, 0.72 \mathrm{mmol}, 3.6$ equiv.), DABSO (28.8 mg, $0.12 \mathrm{mmol}, 0.6$ equiv.) and tert-butyl 2bromoacetate ( $59 \mu \mathrm{L}, 0.40 \mathrm{mmol}, 2.0$ equiv.) in DMA (0.4 mL, $0.5 \mathrm{M})$. Step 1 was carried out for a shorter time of $8 \mathrm{~h}$, followed by the alkylation step at room temperature for $5 \mathrm{~h}$. Purification by flash column chromatography (10 - 20\% EtOAc in Petrol) afforded sulfone $3 z$ as an off-white solid (50.9 mg, 75\%).

Also prepared according to General Procedure D-2, using a shorter reaction time for step 1 of $8 \mathrm{~h}$, followed by alkylation at room temperature for $5 \mathrm{~h}$, affording sulfone $3 z$ as an off-white solid ( $43.0 \mathrm{mg}, 64 \%$ ). 
$\mathbf{R}_{f}\left(20 \%\right.$ EtOAc in Petrol) $=0.29 ;{ }^{1} \mathbf{H}$ NMR $\left(400 \mathrm{MHz}, \mathrm{CDCl}_{3}\right) \delta 7.68(\mathrm{~d}, J=8.0 \mathrm{~Hz}, 2 \mathrm{H}), 7.64(\mathrm{~d}, J=8.5 \mathrm{~Hz}, 2 \mathrm{H})$, $4.57(\mathrm{~s}, 2 \mathrm{H}), 3.70(\mathrm{~s}, 2 \mathrm{H}), 1.54(\mathrm{~s}, 9 \mathrm{H}) ;{ }^{13} \mathrm{C}$ NMR $\left(101 \mathrm{MHz}, \mathrm{CDCl}_{3}\right) \delta 162.4,131.9,131.6,131.5\left(\mathrm{q}, J_{\mathrm{CF}}=32.5\right.$ $\mathrm{Hz}), 126.1\left(\mathrm{q}, J_{\mathrm{CF}}=3.5 \mathrm{~Hz}\right), 123.9\left(\mathrm{q}, J_{\mathrm{CF}}=272.5 \mathrm{~Hz}\right), 84.7,58.8,56.4,28.0 ;{ }^{19} \mathrm{~F}$ NMR $\left(377 \mathrm{MHz}, \mathrm{CDCl}_{3}\right) \delta-62.9$; LRMS (ESI) $\mathrm{m} / \mathrm{z} 337.0$ ([M-H] $\left.]^{-}\right)$; HRMS (ESI) $\mathrm{m} / \mathrm{z} \mathrm{C}_{14} \mathrm{H}_{16} \mathrm{~F}_{3} \mathrm{O}_{4} \mathrm{~S}^{-}$requires 337.0716 ([M-H] $]^{-}$), found 337.0731; IR (thin film, $\left.v_{\max } / \mathrm{cm}^{-1}\right) 2992,2937,1728,1318,1165,1131,1069 ;$ m.p. $\left(\mathrm{CH}_{2} \mathrm{Cl}_{2}\right): 103-106^{\circ} \mathrm{C}$.

tert-Butyl 2-((thiophen-2-ylmethyl)sulfonyl)acetate (3aa)

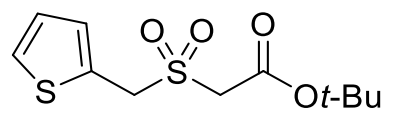

Prepared according to General Procedure D-1 using 2,4,6-triphenyl-1-(thiophen-2-ylmethyl)pyridin-1-ium tetrafluoroborate 1aa ( $98.3 \mathrm{mg}, 0.20 \mathrm{mmol}, 1.0$ equiv.), Hantzsch ester ( $55.7 \mathrm{mg}, 0.22 \mathrm{mmol}, 1.1$ equiv.), 2,6lutidine ( $83 \mu \mathrm{L}, 0.72 \mathrm{mmol}, 3.6$ equiv.), DABSO (28.8 mg, $0.12 \mathrm{mmol}, 0.6$ equiv.) and tert-butyl 2bromoacetate (59 $\mathrm{LL}, 0.40 \mathrm{mmol}, 2.0$ equiv.) in DMA $(0.4 \mathrm{~mL}, 0.5 \mathrm{M})$. Purification by flash column chromatography (10 - 20\% EtOAc in Petrol) afforded sulfone 3aa as a colourless oil (31.9 mg, 58\%).

$\mathbf{R}_{f}\left(20 \%\right.$ EtOAc in Petrol) $=0.25 ;{ }^{1} \mathbf{H}$ NMR $\left(400 \mathrm{MHz}, \mathrm{CDCl}_{3}\right) \delta 7.39(\mathrm{dd}, J=5.0,1.0 \mathrm{~Hz}, 1 \mathrm{H}), 7.25(\mathrm{dd}, J=2.0,1.0$ $\mathrm{Hz}, 1 \mathrm{H}), 7.07(\mathrm{dd}, J=5.0,3.5 \mathrm{~Hz}, 1 \mathrm{H}), 4.71(\mathrm{~s}, 2 \mathrm{H}), 3.76(\mathrm{~s}, 2 \mathrm{H}), 1.54(\mathrm{~s}, 9 \mathrm{H}) ;{ }^{13} \mathrm{C} \mathrm{NMR}\left(101 \mathrm{MHz}, \mathrm{CDCl}_{3}\right) \delta 162.3$, 130.9, 128.6, 128.3, 127.8, 84.6, 55.3, 54.1, 28.0; LRMS (ESI) $\mathrm{m} / \mathrm{z} 299.0$ ([M+Na] $]^{+}$; HRMS (ESI) $\mathrm{m} / \mathrm{z}$ $\mathrm{C}_{11} \mathrm{H}_{16} \mathrm{O}_{4} \mathrm{~S}_{2} \mathrm{Na}^{+}$requires $299.0382\left([\mathrm{M}+\mathrm{Na}]^{+}\right)$, found 299.0383; IR (thin film, $v_{\max } / \mathrm{cm}^{-1}$ ) 2980, 2934, 1728, 1328, $1256,1139,712$.

tert-Butyl 2-((pyridin-3-ylmethyl)sulfonyl)acetate (3ab)

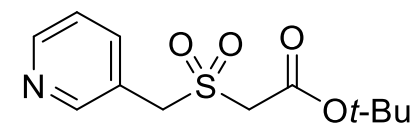

Prepared according to General Procedure D-1 using 2,4,6-triphenyl-1-(pyridin-3-ylmethyl)pyridin-1-ium tetrafluoroborate $1 \mathrm{ab}$ ( $97.3 \mathrm{mg}, 0.20 \mathrm{mmol}, 1.0$ equiv.), Hantzsch ester ( $55.7 \mathrm{mg}, 0.22 \mathrm{mmol}, 1.1$ equiv.), 2,6lutidine ( $83 \mu \mathrm{L}, 0.72 \mathrm{mmol}, 3.6$ equiv.), DABSO (28.8 mg, $0.12 \mathrm{mmol}, 0.6$ equiv.) and tert-butyl 2bromoacetate $(59 \mu \mathrm{L}, 0.40 \mathrm{mmol}, 2.0$ equiv.) in DMA $(0.4 \mathrm{~mL}, 0.5 \mathrm{M})$. The alkylation step was carried out at room temperature for $5 \mathrm{~h}$. Purification by flash column chromatography (60\% EtOAc in Petrol) afforded sulfone $3 a b$ as an off-white solid (27.1 $\mathrm{mg}, 50 \%)$.

$\mathbf{R}_{f}\left(60 \%\right.$ EtOAc in Petrol) $=0.17 ;{ }^{1} \mathbf{H}$ NMR $\left(400 \mathrm{MHz}, \mathrm{CDCl}_{3}\right) 8.75-8.62(\mathrm{~m}, 2 \mathrm{H}), 7.86(\mathrm{dt}, J=8.0,2.0 \mathrm{~Hz}, 1 \mathrm{H})$, $7.36(\mathrm{dd}, J=8.0,5.0 \mathrm{~Hz}, 1 \mathrm{H}), 4.52(\mathrm{~s}, 2 \mathrm{H}), 3.70(\mathrm{~s}, 2 \mathrm{H}), 1.54(\mathrm{~s}, 9 \mathrm{H}) ;{ }^{13} \mathrm{C} \mathrm{NMR}\left(101 \mathrm{MHz}, \mathrm{CDCl}_{3}\right) \delta 162.2,151.7$, 150.7, 138.6, 124.3, 124.0, 84.8, 56.6, 56.3, 28.0; LRMS (ESI) m/z $272.0\left([\mathrm{M}+\mathrm{H}]^{+}\right), 270.0\left([\mathrm{M}-\mathrm{H}]^{-}\right)$; HRMS (ESI) $\mathrm{m} / \mathrm{z} \mathrm{C}_{12} \mathrm{H}_{18} \mathrm{NO}_{4} \mathrm{~S}^{+}$requires $272.0951\left([\mathrm{M}+\mathrm{H}]^{+}\right)$, found 272.0949; IR (thin film, $\left.v_{\max } / \mathrm{cm}^{-1}\right)$ 2982, 2934, 1727, 1326, 1160, 1119, 713; m.p. $\left(\mathrm{CH}_{2} \mathrm{Cl}_{2}\right): 124-126^{\circ} \mathrm{C}$.

tert-Butyl 2-(cinnamylsulfonyl)acetate (3ad) 
<smiles>CCC(C)OC(=O)CS(=O)(=O)C/C=C/c1ccccc1</smiles>

Prepared according to General Procedure D-1 using 1-cinnamyl-2,4,6-triphenylpyridin-1-ium tetrafluoroborate 1ad-3 (102.3 mg, 0.20 mmol, 1.0 equiv.), Hantzsch ester (55.7 mg, 0.22 mmol, 1.1 equiv.), 2,6-lutidine ( $83 \mu \mathrm{L}, 0.72 \mathrm{mmol}, 3.6$ equiv.), DABSO (28.8 mg, $0.12 \mathrm{mmol}, 0.6$ equiv.) and tert-butyl 2bromoacetate ( $59 \mu \mathrm{L}, 0.40 \mathrm{mmol}, 2.0$ equiv.) in DMA $(0.4 \mathrm{~mL}, 0.5 \mathrm{M})$. Purification by flash column chromatography (10 - 20\% EtOAc in Petrol) afforded sulfone 3ad as an off-white solid (48.1 mg, 79\%).

$\mathbf{R}_{\boldsymbol{f}}\left(20 \%\right.$ EtOAc in Petrol) $=0.33 ;{ }^{1} \mathbf{H}$ NMR $\left(400 \mathrm{MHz}, \mathrm{CDCl}_{3}\right) \delta 7.45-7.40(\mathrm{~m}, 2 \mathrm{H}), 7.38-7.28(\mathrm{~m}, 3 \mathrm{H}), 6.82(\mathrm{~d}$, $J=16.0 \mathrm{~Hz}, 1 \mathrm{H}), 6.26(\mathrm{dt}, J=16.0,7.5 \mathrm{~Hz}, 1 \mathrm{H}), 4.13(\mathrm{~d}, J=8.0 \mathrm{~Hz}, 2 \mathrm{H}), 3.88(\mathrm{~s}, 2 \mathrm{H}), 1.53(\mathrm{~s}, 9 \mathrm{H}) ;{ }^{13} \mathrm{C}$ NMR $(101$ $\left.\mathrm{MHz}, \mathrm{CDCl}_{3}\right) \delta 162.3,140.0,135.6,129.0,128.9,127.0,115.3,84.3,57.5,56.2,28.0$; LRMS (ESI) $\mathrm{m} / \mathrm{z} 319.0$ $\left([\mathrm{M}+\mathrm{Na}]^{+}\right)$; HRMS (ESI) $m / z \mathrm{C}_{15} \mathrm{H}_{20} \mathrm{O}_{4} \mathrm{SNa}^{+}$requires $319.0975\left([\mathrm{M}+\mathrm{Na}]^{+}\right)$, found 319.0975; IR (thin film, $v_{\max }$ $\left./ \mathrm{cm}^{-1}\right) 2981,2935,1728,1370,1325,1131,1102,753 ;$ m.p. $\left(\mathrm{CH}_{2} \mathrm{Cl}_{2}\right): 83-85^{\circ} \mathrm{C}$.

tert-butyl 2-((1-phenylethyl)sulfonyl)acetate (3ae)<smiles>CCCOC(=O)CS(=O)(=O)C(C)c1ccccc1</smiles>

Prepared according to General Procedure D-1 using 2-Isopropyl-4,6-diphenyl-1-(1-phenylethyl)pyridin-1-ium tetrafluoroborate 1ae (93.1 mg, 0.20 mmol, 1.0 equiv.), Hantzsch ester ( $55.7 \mathrm{mg}, 0.22 \mathrm{mmol}, 1.1$ equiv.), 2,6lutidine ( $83 \mu \mathrm{L}, 0.72 \mathrm{mmol}, 3.6$ equiv.), DABSO (28.8 $\mathrm{mg}, 0.12 \mathrm{mmol}, 0.6$ equiv.) and tert-butyl 2bromoacetate (59 $\mathrm{\mu L}, 0.40 \mathrm{mmol}, 2.0$ equiv.) in DMA $(0.4 \mathrm{~mL}, 0.5 \mathrm{M})$. Purification by flash column chromatography (5 - 10\% EtOAc in Petrol) afforded sulfone 3ae as a colourless oil (41.9 mg, 74\%).

$\mathbf{R}_{f}\left(10 \%\right.$ EtOAc in Petrol) $=0.19 ;{ }^{1} \mathbf{H}$ NMR $\left(400 \mathrm{MHz}, \mathrm{CDCl}_{3}\right) \delta 7.55-7.50(\mathrm{~m}, 2 \mathrm{H}), 7.43-7.37(\mathrm{~m}, 3 \mathrm{H}), 4.74$ (q, $J=7.0 \mathrm{~Hz}, 1 \mathrm{H}), 3.65(\mathrm{~d}, J=15.0 \mathrm{~Hz}, 1 \mathrm{H}), 3.56(\mathrm{~d}, J=15.0 \mathrm{~Hz}, 1 \mathrm{H}), 1.80(\mathrm{~d}, J=7.0 \mathrm{~Hz}, 3 \mathrm{H}), 1.52(\mathrm{~s}, 9 \mathrm{H}) ;{ }^{13} \mathrm{C} \mathrm{NMR}$ $\left(101 \mathrm{MHz}, \mathrm{CDCl}_{3}\right) \delta 162.5,134.4,129.5,129.4,129.2,84.1,62.9,55.3,28.1,13.3$; LRMS (ESI) $\mathrm{m} / \mathrm{z} 307.1$ $\left([\mathrm{M}+\mathrm{Na}]^{+}\right)$; HRMS (ESI) $m / z \mathrm{C}_{14} \mathrm{H}_{20} \mathrm{O}_{4} \mathrm{SNa}^{+}$requires $307.0975\left([\mathrm{M}+\mathrm{Na}]^{+}\right)$, found 307.0974 ; IR (thin film, $v_{\max }$ $\left./ \mathrm{cm}^{-1}\right)$ 2981, 2939, 1729, 1456, 1370, 1323, 1154. 
General Procedure E: Primary Katritzky salts

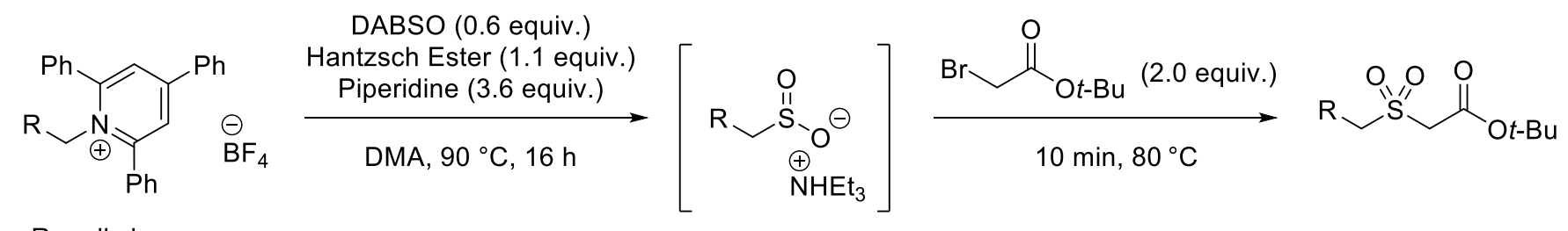

$\mathrm{R}=\mathrm{alkyl}$

Hantzsch ester (1.1 equiv.), DABSO (0.6 equiv.) and Katritzky pyridinium salt (1.0 equiv.) were added to an oven-dried $1.9 \mathrm{~mL}$ reaction vial. The vial was then sealed with a rubber septum, evacuated and backfilled with $\mathrm{N}_{2}(\times 3)$. Degassed, dry DMA (0.5 M) was then added, followed by degassed, dry piperidine (3.6 equiv.). The reaction was heated to $90^{\circ} \mathrm{C}$ in an aluminium heating block for $16 \mathrm{~h}$. tert-butylbromoacetate (2.0 equiv.) was then added and the reaction heated to $80^{\circ} \mathrm{C}$ in an aluminium heating block for $10 \mathrm{~min}$. The reaction mixture was cooled to room temperature. Without workup, the crude reaction mixture was loaded directly to silica gel and purified by flash column chromatography to afford the sulfone product.

\section{Benzyl 2-(phenethylsulfonyl)acetate (3c-1)}<smiles>O=C(CS(=O)(=O)CCc1ccccc1)OCc1ccccc1</smiles>

Prepared according to General Procedure E using 1-phenethyl-2,4,6-triphenylpyridin-1-ium tetrafluoroborate 1c (99.9 mg, 0.20 mmol, 1.0 equiv.), Hantzsch ester (55.7 mg, 0.22 mmol, 1.1 equiv.), piperidine ( $71 \mu \mathrm{L}, 0.72 \mathrm{mmol}, 3.6$ equiv.), DABSO ( $28.8 \mathrm{mg}, 0.12 \mathrm{mmol}, 0.6$ equiv.) and benzyl 2 -bromoacetate (63 $\mu \mathrm{L}, 0.40 \mathrm{mmol}, 2.0$ equiv.) in DMA $(0.4 \mathrm{~mL}, 0.5 \mathrm{M})$. Purification by flash column chromatography (10 $20 \%$ EtOAc in Petrol) afforded sulfone $3 \mathrm{c}-1$ as a colourless oil (53.1 mg, 83\%).

$\mathbf{R}_{f}(20 \%$ EtOAc in Petrol $)=0.17 ;{ }^{1} \mathbf{H}$ NMR $\left(400 \mathrm{MHz}, \mathrm{CDCl}_{3}\right) \delta 7.42-7.35(\mathrm{~m}, 5 \mathrm{H}), 7.35-7.22(\mathrm{~m}, 3 \mathrm{H}), 7.20-$ $7.15(\mathrm{~m}, 2 \mathrm{H}), 5.23(\mathrm{~s}, 2 \mathrm{H}), 3.90(\mathrm{~s}, 2 \mathrm{H}), 3.55-3.47(\mathrm{~m}, 2 \mathrm{H}), 3.19-3.11(\mathrm{~m}, 2 \mathrm{H}) ;{ }^{13} \mathrm{C} \mathrm{NMR}\left(101 \mathrm{MHz}, \mathrm{CDCl}_{3}\right) \delta$ 162.9, 137.1, 134.5, 129.1, 129.0, 128.9, 128.7, 128.7, 127.3, 68.5, 57.7, 54.9, 28.3; LRMS (ESI) $\mathrm{m} / \mathrm{z} 341.0$ $\left([\mathrm{M}+\mathrm{Na}]^{+}\right) 317.0\left([\mathrm{M}-\mathrm{H}]^{-}\right)$; HRMS (ESI) $\mathrm{m} / 2 \mathrm{C}_{17} \mathrm{H}_{18} \mathrm{O}_{4} \mathrm{SNa}^{+}$requires $341.0818\left([\mathrm{M}+\mathrm{Na}]^{+}\right)$, found 341.0819; IR (thin film, $v_{\max } / \mathrm{cm}^{-1}$ ) 2942, 1737, 1455, 1317, 1111, 749, 697.

tert-Butyl 2-(phenethylsulfonyl)acetate (3c-2)<smiles>CCC(C)OC(=O)CS(=O)(=O)CCc1ccccc1</smiles>

Prepared according to General Procedure E using 1-phenethyl-2,4,6-triphenylpyridin-1-ium tetrafluoroborate 1c (99.9 mg, $0.20 \mathrm{mmol}, 1.0$ equiv.), Hantzsch ester (55.7 mg, $0.22 \mathrm{mmol}, 1.1$ equiv.), piperidine (71 $\mu \mathrm{L}, 0.72 \mathrm{mmol}, 3.6$ equiv.), DABSO (28.8 mg, $0.12 \mathrm{mmol}, 0.6$ equiv.) and tert-butyl 2- 
bromoacetate ( $59 \mu \mathrm{L}, 0.40 \mathrm{mmol}, 2.0$ equiv.) in DMA (0.4 mL, $0.5 \mathrm{M})$. Purification by flash column chromatography (10 - 20\% EtOAc in Petrol) afforded sulfone $3 \mathrm{c}-2$ as an off-white solid (46.4 mg, 81\%).

$\mathbf{R}_{f}\left(20 \%\right.$ EtOAc in Petrol) $=0.41 ;{ }^{1} \mathbf{H}$ NMR $\left(400 \mathrm{MHz}, \mathrm{CDCl}_{3}\right) \delta 7.38-7.29(\mathrm{~m}, 2 \mathrm{H}), 7.29-7.22(\mathrm{~m}, 3 \mathrm{H}), 3.78(\mathrm{~s}$, $2 \mathrm{H}), 3.59-3.50(\mathrm{~m}, 2 \mathrm{H}), 3.22-3.13(\mathrm{~m}, 2 \mathrm{H}), 1.50(\mathrm{~s}, 9 \mathrm{H}) ;{ }^{13} \mathrm{C} \mathrm{NMR}\left(101 \mathrm{MHz}, \mathrm{CDCl}_{3}\right) \delta 162.2,137.4,129.1$, 128.7, 127.3, 84.3, 58.8, 54.8, 28.4, 28.0; LRMS (ESI) $\mathrm{m} / \mathrm{z} 307.0\left([\mathrm{M}+\mathrm{Na}]^{+}\right), 283.0\left([\mathrm{M}-\mathrm{H}]^{-}\right)$; HRMS (ESI) $\mathrm{m} / \mathrm{z}$ $\mathrm{C}_{14} \mathrm{H}_{20} \mathrm{O}_{4} \mathrm{SNa}^{+}$requires $307.0975\left([\mathrm{M}+\mathrm{Na}]^{+}\right)$, found 307.0973; IR (thin film, $v_{\max } / \mathrm{cm}^{-1}$ ) 2981, 2937, 1729, 1319, $1159,1102,700$; m.p. $\left(\mathrm{CH}_{2} \mathrm{Cl}_{2}\right): 61-64{ }^{\circ} \mathrm{C}$.

tert-Butyl 2-(neopentylsulfonyl)acetate (3af)

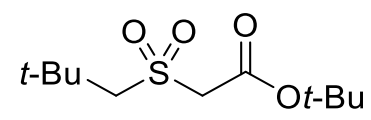

Prepared according to General Procedure E using 1-neopentyl-2,4,6-triphenylpyridin-1-ium tetrafluoroborate 1 af $(93.1 \mathrm{mg}, 0.20 \mathrm{mmol}, 1.0$ equiv.), Hantzsch ester (55.7 mg, $0.22 \mathrm{mmol}, 1.1$ equiv.), piperidine ( $71 \mu \mathrm{L}, 0.72 \mathrm{mmol}, 3.6$ equiv.), DABSO (28.8 mg, $0.12 \mathrm{mmol}, 0.6$ equiv.) and tert-butyl 2bromoacetate ( $59 \mu \mathrm{L}, 0.40 \mathrm{mmol}, 2.0$ equiv.) in DMA $(0.4 \mathrm{~mL}, 0.5 \mathrm{M})$. Purification by flash column chromatography ( $70 \% \mathrm{CH}_{2} \mathrm{Cl}_{2}$ in Petrol) afforded sulfone 3 af as a pale-yellow oil ( $\left.35.7 \mathrm{mg}, 71 \%\right)$.

$\mathbf{R}_{\boldsymbol{f}}\left(70 \% \mathrm{CH}_{2} \mathrm{Cl}_{2}\right.$ in Petrol) = 0.4; ${ }^{1} \mathrm{H}$ NMR $\left(400 \mathrm{MHz}, \mathrm{CDCl}_{3}\right) \delta 3.82(\mathrm{~s}, 2 \mathrm{H}), 3.23(\mathrm{~s}, 2 \mathrm{H}), 1.51(\mathrm{~s}, 9 \mathrm{H}), 1.22(\mathrm{~s}, 9 \mathrm{H})$; ${ }^{13} \mathrm{C}$ NMR $\left(101 \mathrm{MHz} \mathrm{CDCl}_{3}\right) \delta 162.5,84.1,64.5,61.9,32.3,29.9,28.0 ;$ LRMS (ESI) $\mathrm{m} / \mathrm{z} 273.0\left([\mathrm{M}+\mathrm{Na}]^{+}\right)$; HRMS (ESI) $\mathrm{m} / z \mathrm{C}_{11} \mathrm{H}_{22} \mathrm{O}_{4} \mathrm{SNa}^{+}$requires $273.1131\left([\mathrm{M}+\mathrm{Na}]^{+}\right)$, found 273.1133; IR (thin film, $v_{\max } / \mathrm{cm}^{-1}$ ) 2959, 1480, 1730, 1370, 1324, 1135, 1098.

tert-Butyl 2-(octylsulfonyl)acetate (3ag)<smiles>CCCCCCCCCS(=O)(=O)CC(=O)OCCCC</smiles>

Prepared according to General Procedure E using 1-octyl-2,4,6-triphenylpyridin-1-ium tetrafluoroborate 1ag (101.5 mg, 0.20 mmol, 1.0 equiv.), Hantzsch ester ( $55.7 \mathrm{mg}, 0.22 \mathrm{mmol}, 1.1$ equiv.), piperidine (71 $\mu \mathrm{L}, 0.72$ mmol, 3.6 equiv.), DABSO ( $28.8 \mathrm{mg}, 0.12 \mathrm{mmol}, 0.6$ equiv.) and tert-butyl 2-bromoacetate ( $59 \mu \mathrm{L}, 0.40 \mathrm{mmol}$, 2.0 equiv.) in DMA (0.4 mL, $0.5 \mathrm{M})$. Purification by flash column chromatography $\left(70-80 \% \mathrm{CH}_{2} \mathrm{Cl}_{2}\right.$ in Petrol) afforded sulfone 3 ag as an off-white solid (54.6 mg, 93\%).

$\mathbf{R}_{f}\left(80 \% \mathrm{CH}_{2} \mathrm{Cl}_{2}\right.$ in Petrol $)=0.47 ;{ }^{1} \mathbf{H}$ NMR $\left(400 \mathrm{MHz}, \mathrm{CDCl}_{3}\right) \delta 3.84(\mathrm{~s}, 2 \mathrm{H}), 3.27-3.17(\mathrm{~m}, 2 \mathrm{H}), 1.93-1.78(\mathrm{~m}$, $2 \mathrm{H}), 1.50(\mathrm{~s}, 9 \mathrm{H}), 1.44(\mathrm{q}, J=7.5 \mathrm{~Hz}, 2 \mathrm{H}), 1.36-1.22(\mathrm{~m}, 8 \mathrm{H}), 0.92-0.84(\mathrm{~m}, 3 \mathrm{H}) ;{ }^{13} \mathrm{C} \mathrm{NMR}\left(101 \mathrm{MHz}, \mathrm{CDCl}_{3}\right)$ $\delta$ 162.3, 84.2, 58.6, 53.7, 31.8, 29.1, 29.0, 28.5, 28.0, 22.7, 22.1, 14.2; LRMS (ESI) $m / z 315.2$ ([M+Na] $]^{+}$); HRMS (ESI) $\mathrm{m} / z \mathrm{C}_{14} \mathrm{H}_{28} \mathrm{O}_{4} \mathrm{SNa}^{+}$requires $315.1601\left([\mathrm{M}+\mathrm{Na}]^{+}\right)$, found 315.1600; IR (thin film, $v_{\max } / \mathrm{cm}^{-1}$ ) 2927, 2857, $1730,1370,1323,1135,1101$. 


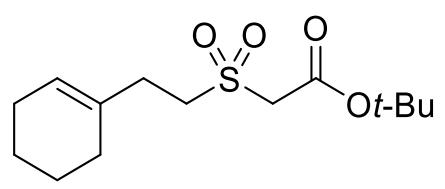

Prepared according to General Procedure E using 1-(2-(cyclohex-1-en-1-yl)ethyl)-2,4,6-triphenylpyridin-1ium tetrafluoroborate $1 \mathrm{ah}$ ( $100.7 \mathrm{mg}, 0.20 \mathrm{mmol}, 1.0$ equiv.), Hantzsch ester (55.7 mg, $0.22 \mathrm{mmol}, 1.1$ equiv.), piperidine (71 $\mu \mathrm{L}, 0.72 \mathrm{mmol}, 3.6$ equiv.), DABSO (28.8 mg, $0.12 \mathrm{mmol}, 0.6$ equiv.) and tert-butyl 2bromoacetate (59 $\mu \mathrm{L}, 0.40 \mathrm{mmol}, 2.0$ equiv.) in DMA (0.4 mL, $0.5 \mathrm{M})$. Purification by flash column chromatography $\left(70-80 \% \mathrm{CH}_{2} \mathrm{Cl}_{2}\right.$ in Petrol) afforded sulfone 3 ah as a colourless oil ( $\left.49.5 \mathrm{mg}, 86 \%\right)$.

$\mathbf{R}_{\boldsymbol{f}}\left(70 \% \mathrm{CH}_{2} \mathrm{Cl}_{2}\right.$ in Petrol) $=0.34 ;{ }^{1} \mathbf{H}$ NMR $\left(400 \mathrm{MHz}, \mathrm{CDCl}_{3}\right) \delta 5.53(\mathrm{~s}, 1 \mathrm{H}), 3.85(\mathrm{~s}, 2 \mathrm{H}), 3.37-3.27(\mathrm{~m}, 2 \mathrm{H}), 2.52$ $-2.41(\mathrm{~m}, 2 \mathrm{H}), 2.05-1.88(\mathrm{~m}, 4 \mathrm{H}), 1.67-1.59(\mathrm{~m}, 2 \mathrm{H}), 1.59-1.52(\mathrm{~m}, 2 \mathrm{H}), 1.50(\mathrm{~s}, 9 \mathrm{H}) ;{ }^{13} \mathrm{C} \mathrm{NMR}(101 \mathrm{MHz}$, $\left.\mathrm{CDCl}_{3}\right) \delta 162.2,133.5,124.1,84.2,58.8,52.2,30.2,28.2,28.0,25.3,22.8,22.3$; LRMS (ESI) $\mathrm{m} / \mathrm{z} 311.0$ $\left([\mathrm{M}+\mathrm{Na}]^{+}\right)$; HRMS (ESI) $\mathrm{m} / z \mathrm{C}_{14} \mathrm{H}_{24} \mathrm{O}_{4} \mathrm{SNa}^{+}$requires $311.1288\left([\mathrm{M}+\mathrm{Na}]^{+}\right)$, found 311.1288; IR (thin film, $v_{\max }$ $\left./ \mathrm{cm}^{-1}\right)$ 2978, 2934, 1729, 1370, 1323, 1163, 1136.

\section{tert-Butyl 2-((2,2-dimethoxyethyl)sulfonyl)acetate (3ai)}<smiles>CCCCOC(=O)CS(=O)(=O)CC(OC)OC</smiles>

Prepared according to General Procedure E using 1-(2,2-dimethoxyethyl)-2,4,6-triphenylpyridin-1-ium tetrafluoroborate 1ai ( $96.7 \mathrm{mg}, 0.20 \mathrm{mmol}, 1.0$ equiv.), Hantzsch ester (55.7 mg, $0.22 \mathrm{mmol}, 1.1$ equiv.), piperidine (71 $\mu \mathrm{L}, 0.72 \mathrm{mmol}, 3.6$ equiv.), DABSO (28.8 mg, $0.12 \mathrm{mmol}, 0.6$ equiv.) and tert-butyl 2bromoacetate ( $59 \mu \mathrm{L}, 0.40 \mathrm{mmol}, 2.0$ equiv.) in DMA (0.4 mL, $0.5 \mathrm{M})$. Purification by flash column chromatography (20\% EtOAc in Petrol) afforded sulfone 3ai as a colourless oil (23.5 mg, 44\%).

$\mathbf{R}_{f}\left(20 \%\right.$ EtOAc in Petrol) $=0.24 ;{ }^{1} \mathbf{H}$ NMR $\left(400 \mathrm{MHz}, \mathrm{CDCl}_{3}\right) \delta 4.88(\mathrm{t}, J=5.5 \mathrm{~Hz}, 1 \mathrm{H}), 4.01(\mathrm{~s}, 2 \mathrm{H}), 3.57(\mathrm{~d}, J=$ $5.5 \mathrm{~Hz}, 2 \mathrm{H}), 3.41$ (s, 6H), 1.50 (s, 9H); ${ }^{13} \mathrm{C}$ NMR (101 MHz, CDCl $) \delta 162.3,99.7,84.1,59.3,55.3,54.1,28.0$; LRMS (ESI) $\mathrm{m} / \mathrm{z} 291.0\left([\mathrm{M}+\mathrm{Na}]^{+}\right)$; HRMS (ESI) $\mathrm{m} / \mathrm{z} \mathrm{C}_{10} \mathrm{H}_{20} \mathrm{O}_{6} \mathrm{SNa}^{+}$requires $291.0873\left([\mathrm{M}+\mathrm{Na}]^{+}\right)$, found 291.0873; IR (thin film, $v_{\max } / \mathrm{cm}^{-1}$ ) 2981, 2938, 1730, 1371, 1325, 1110, 1070.

tert-Butyl 2-((4-cyanophenethyl)sulfonyl)acetate (3aj)<smiles>CCCCOC(=O)CS(=O)(=O)CCc1ccc(C#N)cc1</smiles>

Prepared according to General Procedure E using 1-(4-cyanophenethyl)-2,4,6-triphenylpyridin-1-ium tetrafluoroborate 1aj (104.9 mg, 0.20 mmol, 1.0 equiv.), Hantzsch ester (55.7 mg, 0.22 mmol, 1.1 equiv.), piperidine (71 $\mu \mathrm{L}, 0.72 \mathrm{mmol}, 3.6$ equiv.), DABSO (28.8 mg, $0.12 \mathrm{mmol}, 0.6$ equiv.) and tert-butyl 2- 
bromoacetate ( $59 \mu \mathrm{L}, 0.40 \mathrm{mmol}, 2.0$ equiv.) in DMA (0.4 mL, $0.5 \mathrm{M})$. Purification by flash column chromatography ( $40-50 \% \mathrm{Et}_{2} \mathrm{O}$ in Petrol) afforded sulfone 3 aj as a white crystalline solid (30.8 $\left.\mathrm{mg}, 50 \%\right)$.

$\mathbf{R}_{f}\left(50 \% \mathrm{Et}_{2} \mathrm{O}\right.$ in Petrol $)=0.14 ;{ }^{1} \mathbf{H}$ NMR $\left(400 \mathrm{MHz}, \mathrm{CDCl}_{3}\right) \delta 7.66-7.60(\mathrm{~m}, 2 \mathrm{H}), 7.38(\mathrm{~d}, \mathrm{~J}=8.5 \mathrm{~Hz}, 2 \mathrm{H}), 3.85(\mathrm{~s}$, 2H), $3.58-3.51(\mathrm{~m}, 2 \mathrm{H}), 3.27-3.20(\mathrm{~m}, 2 \mathrm{H}), 1.50(\mathrm{~s}, 9 \mathrm{H}) ;{ }^{13} \mathrm{C} \mathrm{NMR}\left(101 \mathrm{MHz}, \mathrm{CDCl}_{3}\right) \delta 162.2,143.0,132.8$, 129.5, 118.7, 111.4, 84.7, 59.0, 53.9, 28.2, 28.0; LRMS (ESI) $\mathrm{m} / \mathrm{z} 332.0\left([\mathrm{M}+\mathrm{Na}]^{+}\right), 308.1$ ([M-H] $\left.]^{-}\right)$; HRMS (ESI) $\mathrm{m} / \mathrm{z} \mathrm{C}_{15} \mathrm{H}_{19} \mathrm{O}_{4} \mathrm{NSNa}^{+}$requires $332.0927\left([\mathrm{M}+\mathrm{Na}]^{+}\right.$), found 332.0927; IR (thin film, $v_{\max } / \mathrm{cm}^{-1}$ ) 2980, 2937, 2229, 1729, 1371, 1329, 1161; m.p. (MeOH): $117-119^{\circ} \mathrm{C}$.

tert-Butyl 4-((2-(tert-butoxy)-2-oxoethyl)sulfonyl)butanoate (3ak)<smiles>CCC(C)OC(=O)CCCS(=O)(O)(O)CC(=O)OCC(C)(C)C</smiles>

Prepared according to General Procedure E using 1-(4-(tert-butoxy)-4-oxobutyl)-2,4,6-triphenylpyridin-1ium tetrafluoroborate 1ak (107.5 mg, 0.20 mmol, 1.0 equiv.), Hantzsch ester (55.7 mg, 0.22 mmol, 1.1 equiv.), piperidine (71 $\mu \mathrm{L}, 0.72 \mathrm{mmol}, 3.6$ equiv.), DABSO (28.8 mg, $0.12 \mathrm{mmol}, 0.6$ equiv.) and tert-butyl 2bromoacetate ( $59 \mu \mathrm{L}, 0.40 \mathrm{mmol}, 2.0$ equiv.) in DMA $(0.4 \mathrm{~mL}, 0.5 \mathrm{M})$. Purification by flash column chromatography (50\% $\mathrm{Et}_{2} \mathrm{O}$ in Petrol) afforded sulfone 3ak as a colourless oil (48.2 mg, 75\%).

$\mathbf{R}_{f}\left(50 \% \mathrm{Et}_{2} \mathrm{O}\right.$ in Petrol $)=0.4 ;{ }^{1} \mathbf{H}$ NMR $\left(400 \mathrm{MHz}, \mathrm{CDCl}_{3}\right) \delta 3.87(\mathrm{~s}, 2 \mathrm{H}), 3.34-3.28(\mathrm{~m}, 2 \mathrm{H}), 2.42(\mathrm{t}, J=7.0 \mathrm{~Hz}$,

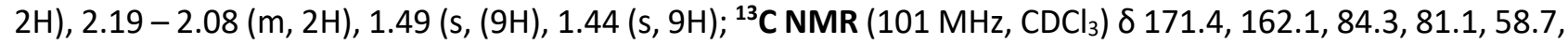
52.7, 33.6, 28.2, 28.0, 17.8; LRMS (ESI) $m / z 345.0$ ([M+Na] $]^{+}$); HRMS (ESI) $m / z \mathrm{C}_{14} \mathrm{H}_{26} \mathrm{O}_{6} \mathrm{SNa}^{+}$requires 345.1342 $\left([\mathrm{M}+\mathrm{Na}]^{+}\right)$, found 345.1342; IR (thin film, $v_{\max } / \mathrm{cm}^{-1}$ ) 2979, 2936, 1722, 1369, 1323, 1155, 1132.

tert-Butyl 2-((2-(1H-indol-3-yl)ethyl)sulfonyl)acetate (3al)

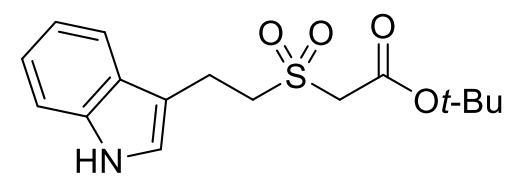

Prepared according to General Procedure E using 1-(2-(1H-indol-3-yl)ethyl)-2,4,6-triphenylpyridin-1-ium tetrafluoroborate 1al (107.7 mg, 0.20 mmol, 1.0 equiv.), Hantzsch ester (55.7 mg, 0.22 mmol, 1.1 equiv.), piperidine (71 $\mu \mathrm{L}, 0.72 \mathrm{mmol}, 3.6$ equiv.), DABSO (28.8 mg, $0.12 \mathrm{mmol}, 0.6$ equiv.) and tert-butyl 2bromoacetate ( $59 \mu \mathrm{L}, 0.40 \mathrm{mmol}, 2.0$ equiv.) in DMA $(0.4 \mathrm{~mL}, 0.5 \mathrm{M})$. Purification by flash column chromatography (20 - 30\% EtOAc in Petrol) afforded sulfone 3al as a colourless oil (35.6 mg, 55\%).

$\mathbf{R}_{f}\left(30 \%\right.$ EtOAc in Petrol) $=0.19 ;{ }^{1} \mathbf{H}$ NMR $(400 \mathrm{MHz}, \mathrm{CDCl})$ ) $8.10(\mathrm{~s}, 1 \mathrm{H}), 7.66-7.59(\mathrm{~m}, 1 \mathrm{H}), 7.39(\mathrm{dt}, J=8.0$, $1.0 \mathrm{~Hz}, 1 \mathrm{H}$ ), 7.23 (ddd, J = 8.0, 7.0,1.0 Hz, 1H), 7.16 (ddd, J=8.0, 7.0, 1.0 Hz, 1H), 7.09 (d, J=2.5 Hz, $1 \mathrm{H}), 3.81$ (s, 2H), $3.69-3.58(\mathrm{~m}, 2 \mathrm{H}), 3.42-3.31(\mathrm{~m}, 2 \mathrm{H}), 1.48(\mathrm{~s}, 9 \mathrm{H}) ;{ }^{13} \mathrm{C} \mathrm{NMR}\left(101 \mathrm{MHz}, \mathrm{CDCl}_{3}\right) \delta 162.2,136.5,126.8$, $122.7,122.3,120.0,118.6,111.8,111.5,84.3,58.9,53.9,28.0,18.6$; LRMS (ESI) $m / z 346.0\left([\mathrm{M}+\mathrm{Na}]^{+}\right), 322.0$ 
([M-H] $\left.]^{-}\right)$HRMS (ESI) $m / z \mathrm{C}_{16} \mathrm{H}_{21} \mathrm{NO}_{4} \mathrm{SNa}^{+}$requires $346.1084\left(\left[\mathrm{M}+\mathrm{Na}^{+}\right)\right.$, found 346.1084; IR (thin film, $v_{\max } / \mathrm{cm}^{-}$ 1) $3413,2979,2934,1724,1317,1095,741$.

tert-Butyl 2-((2-(dimethylamino)ethyl)sulfonyl)acetate (3am)

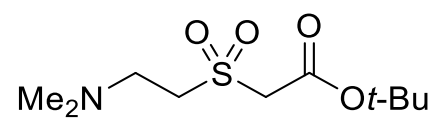

Prepared according to General Procedure E using 1-(2-(dimethylamino)ethyl)-2,4,6-triphenylpyridin-1-ium tetrafluoroborate $1 \mathrm{am}$ ( $93.3 \mathrm{mg}, 0.20 \mathrm{mmol}, 1.0$ equiv.), Hantzsch ester ( $55.7 \mathrm{mg}, 0.22 \mathrm{mmol}, 1.1$ equiv.), piperidine (71 $\mu \mathrm{L}, 0.72 \mathrm{mmol}, 3.6$ equiv.), DABSO (28.8 mg, $0.12 \mathrm{mmol}, 0.6$ equiv.) and tert-butyl 2bromoacetate ( $59 \mu \mathrm{L}, 0.40 \mathrm{mmol}, 2.0$ equiv.) in DMA $(0.4 \mathrm{~mL}, 0.5 \mathrm{M})$. Purification by flash column chromatography (0 - 2\% MeOH in EtOAc) afforded sulfone 3am as a white solid (37.4 mg, 74\%).

$\mathbf{R}_{f}\left(2 \% \mathrm{MeOH}\right.$ in EtOAc) $=0.28 ;{ }^{1} \mathrm{H}$ NMR $\left(400 \mathrm{MHz}, \mathrm{DMSO}-d_{6}\right) \delta 4.30(\mathrm{~s}, 2 \mathrm{H}), 3.39(\mathrm{t}, J=6.5 \mathrm{~Hz}, 2 \mathrm{H}), 2.68(\mathrm{t}, J=$ $6.5 \mathrm{~Hz}, 2 \mathrm{H}), 2.17(\mathrm{~s}, 6 \mathrm{H}), 1.44(\mathrm{~s}, 9 \mathrm{H}) ;{ }^{13} \mathrm{C}$ NMR (101 MHz, DMSO-d $\left.d_{6}\right) \delta$ 162.3, 82.6, 59.2, 52.0, 50.5, 44.7, 27.5; LRMS (ESI) $\mathrm{m} / \mathrm{z} 252.0\left([\mathrm{M}+\mathrm{H}]^{+}\right)$; HRMS (ESI) $\mathrm{m} / \mathrm{z} \mathrm{C}_{10} \mathrm{H}_{22} \mathrm{NO}_{4} \mathrm{~S}^{+}$requires $252.1264\left([\mathrm{M}+\mathrm{H}]^{+}\right)$, found 252.1265; IR (thin film, $\left.v_{\max } / \mathrm{cm}^{-1}\right) 2927,1728,1571,1371,1306,1060,1020 ;$ m.p. $\left(\mathrm{CH}_{2} \mathrm{Cl}_{2}\right)$ : $205-208^{\circ} \mathrm{C}$ (decomposition).

(S)-6-((2-(tert-Butoxy)-2-oxoethyl)sulfonyl)-2-((tert-butoxycarbonyl)amino)hexanoic acid (3an)<smiles>CCC(C)OC(=O)CS(=O)(=O)CCCC[C@H](NC(=O)OCc1ccccc1)C(=O)O</smiles>

Prepared according to General Procedure E using (S)-1-(5-((tert-butoxycarbonyl)amino)-5-carboxypentyl)2,4,6-triphenylpyridin-1-ium tetrafluoroborate 1an (124.9 mg, 0.20 mmol, 1.0 equiv.), Hantzsch ester (55.7 $\mathrm{mg}, 0.22 \mathrm{mmol}, 1.1$ equiv.), piperidine ( $71 \mu \mathrm{L}, 0.72 \mathrm{mmol}, 3.6$ equiv.), DABSO ( $28.8 \mathrm{mg}, 0.12 \mathrm{mmol}, 0.6$ equiv.) and tert-butyl 2-bromoacetate ( $59 \mu \mathrm{L}, 0.40 \mathrm{mmol}, 2.0$ equiv.) in DMA ( $0.4 \mathrm{~mL}, 0.5 \mathrm{M})$. Purification by flash column chromatography (50\% EtOAc in Petrol with $2 \% \mathrm{AcOH}$ ) afforded sulfone 3 an as a colourless oil (56.4 $\mathrm{mg}, 78 \%)$.

$\mathbf{R}_{\boldsymbol{f}}(50 \%$ EtOAc in Petrol with $2 \% \mathrm{AcOH})=0.21 ;{ }^{1} \mathrm{H} \mathbf{N M R}\left(400 \mathrm{MHz}, \mathrm{CDCl}_{3}\right) \delta 8.78(\mathrm{~s}, 1 \mathrm{H}), 5.16(\mathrm{~d}, J=8.0 \mathrm{~Hz}$, $1 \mathrm{H}), 4.42-4.08(\mathrm{~m}, 1 \mathrm{H}), 3.87(\mathrm{~s}, 2 \mathrm{H}) 3.34-3.16(\mathrm{~m}, 2 \mathrm{H}), 2.00-1.82(\mathrm{~m}, 3 \mathrm{H}), 1.82-1.67(\mathrm{~m}, 1 \mathrm{H}), 1.66-1.53$

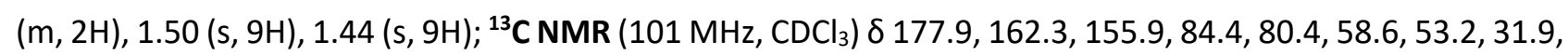
29.7, 28.5, 28.0, 24.3, 21.6; LRMS (ESI) $\mathrm{m} / \mathrm{z} 408.1$ ([M-H]'); HRMS (ESI) $\mathrm{m} / 2 \mathrm{C}_{17} \mathrm{H}_{30} \mathrm{NO}_{8} \mathrm{~S}^{-}$requires 408.1698 $\left([\mathrm{M}-\mathrm{H}]^{-}\right)$), found 408.1692; IR (thin film, $\left.v_{\max } / \mathrm{cm}^{-1}\right)$ 3350, 2979, 2935, 1716, 1370, 1327, 1162; $[\boldsymbol{\alpha}]_{\mathrm{D}}^{25}+14.7^{\circ}$ (c $\left.=1.0, \mathrm{CHCl}_{3}\right)$.

2-((2-(tert-butoxy)-2-oxoethyl)sulfonyl)ethane-1-sulfonic acid (3ao) 


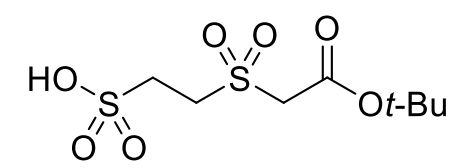

Prepared according to General Procedure E using 2,4,6-triphenyl-1-(2-sulfoethyl)pyridin-1-ium tetrafluoroborate 1 ao (100.7 mg, 0.20 mmol, 1.0 equiv.), Hantzsch ester (55.7 mg, 0.22 mmol, 1.1 equiv.), piperidine (71 $\mu \mathrm{L}, 0.72 \mathrm{mmol}, 3.6$ equiv.), DABSO (28.8 mg, $0.12 \mathrm{mmol}, 0.6$ equiv.) and tert-butyl 2bromoacetate (59 $\mu \mathrm{L}, 0.40 \mathrm{mmol}, 2.0$ equiv.) in DMA (0.4 mL, $0.5 \mathrm{M})$. Purification by flash column chromatography $(0-2 \% \mathrm{AcOH}$ in $5 \% \mathrm{MeOH}$ in EtOAc) afforded sulfone 3ao as a white crystalline solid (43.7 $\mathrm{mg}, 76 \%)$.

$\mathbf{R}_{f}\left(5 \% \mathrm{MeOH}\right.$ in EtOAc with 2\% AcOH) $=0.08 ;{ }^{1} \mathbf{H} \mathbf{N M R}\left(400 \mathrm{MHz}, \mathrm{DMSO}-d_{6}\right) \delta 4.33(\mathrm{~s}, 2 \mathrm{H}), 3.53-3.46(\mathrm{~m}$, 2H), $2.80-2.74(\mathrm{~m}, 2 \mathrm{H}), 1.44(\mathrm{~s}, 9 \mathrm{H}) ;{ }^{13} \mathrm{C}$ NMR (101 MHz, DMSO-d $) \delta 162.3,82.7,58.2,50.5,42.9,27.5$; LRMS (ESI) $\mathrm{m} / \mathrm{z} 287.0$ ([M-H] $\left.]^{-}\right) ;$HRMS (ESI) $\mathrm{m} / \mathrm{z} \mathrm{C}_{8} \mathrm{H}_{15} \mathrm{O}_{7} \mathrm{~S}_{2}^{-}$requires 287.0265 ([M-H] $\left.]^{-}\right)$, found 287.0260; IR (thin film, $v_{\max } / \mathrm{cm}^{-1}$ ) 3423, 2984, 1731, 1564, 1416, 1320, 1206; m.p. (MeOH): $49-51{ }^{\circ} \mathrm{C}$.

tert-Butyl 2-((4-sulfamoylphenethyl)sulfonyl)acetate (3ap)<smiles>CCCOC(=O)CS(=O)(=O)CCc1ccc(S(N)(=O)=O)cc1</smiles>

Prepared according to General Procedure E using 2,4,6-triphenyl-1-(4-sulfamoylphenethyl)pyridin-1-ium tetrafluoroborate 1 ap (115.7 mg, 0.20 mmol, 1.0 equiv.), Hantzsch ester (55.7 mg, 0.22 mmol, 1.1 equiv.), piperidine (71 $\mu \mathrm{L}, 0.72 \mathrm{mmol}, 3.6$ equiv.), DABSO (28.8 mg, $0.12 \mathrm{mmol}, 0.6$ equiv.) and tert-butyl 2bromoacetate (59 $\mu \mathrm{L}, 0.40 \mathrm{mmol}, 2.0$ equiv.) in DMA (0.4 mL, $0.5 \mathrm{M})$. Purification by flash column chromatography (40 - 50\% EtOAc in Petrol) afforded sulfone 3ap as a white crystalline solid (56.4 mg, $78 \%$ ). $\mathbf{R}_{f}(50 \%$ EtOAc in Petrol $)=0.26 ;{ }^{1} \mathbf{H}$ NMR $\left(400 \mathrm{MHz}, \mathrm{DMSO}-d_{6}\right) \delta 7.76(\mathrm{~d}, J=8.5 \mathrm{~Hz}, 2 \mathrm{H}), 7.48(\mathrm{~d}, J=8.5 \mathrm{~Hz}, 2 \mathrm{H})$, $7.32(\mathrm{~s}, 2 \mathrm{H}), 4.31(\mathrm{~s}, 2 \mathrm{H}), 3.63-3.57(\mathrm{~m}, 2 \mathrm{H}), 3.16-3.09(\mathrm{~m}, 2 \mathrm{H}), 1.43(\mathrm{~s}, 9 \mathrm{H}) ;{ }^{13} \mathrm{C}$ NMR $\left(101 \mathrm{MHz}, \mathrm{DMSO}-d_{6}\right)$ $\delta$ 162.3, 142.6, 142.4, 129.1, 126.0, 82.9, 58.2, 53.7, 27.6, 27.1; LRMS (ESI) m/z $386.0\left([\mathrm{M}+\mathrm{Na}]^{+}\right), 362.0([\mathrm{M}-$ $\mathrm{H}^{-}$); HRMS (ESI) $m / z \mathrm{C}_{14} \mathrm{H}_{21} \mathrm{NO}_{6} \mathrm{~S}_{2} \mathrm{Na}^{+}$requires $386.0702\left([\mathrm{M}+\mathrm{Na}]^{+}\right)$, found 386.0702; IR (thin film, $v_{\max } / \mathrm{cm}^{-1}$ ) 3357, 3267, 2937, 1726, 1311, 1160, 1096. 


\subsection{One-step synthesis of sodium sulfinates}

\section{General Procedure F:}

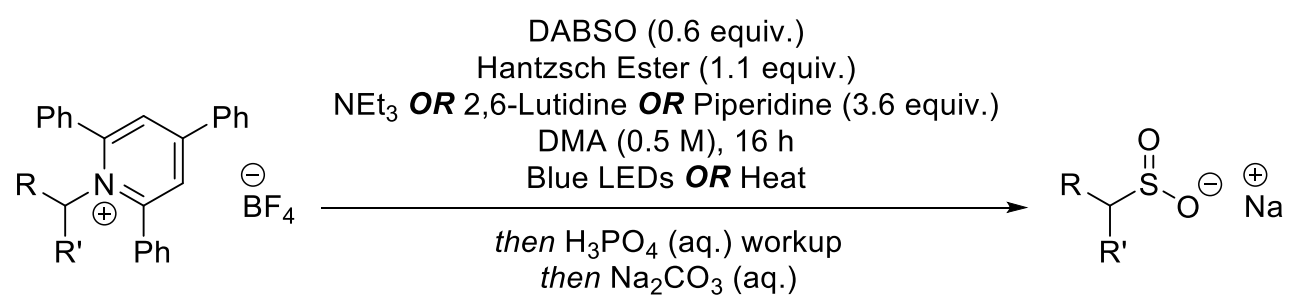

Hantzsch ester (1.1 equiv.), DABSO (0.6 equiv.) and Katritzky pyridinium salt (1.0 equiv.) were added to an oven-dried $1.9 \mathrm{~mL}$ reaction vial. The vial was then sealed with a rubber septum, evacuated and backfilled with $\mathrm{N}_{2}(\times 3)$. Degassed, dry DMA (0.5 M) was then added, followed by degassed, dry triethylamine, 2,6lutidine or piperidine (3.6 equiv.). The reaction was stirred under blue LED irradiation at ambient temperature (General Procedure F-1) or heated to $90^{\circ} \mathrm{C}$ in an aluminium heating block (General Procedure F-2) for $16 \mathrm{~h}$. Upon completion, aq. $\mathrm{H}_{3} \mathrm{PO}_{4}(5 \mathrm{~mL}, 2 \mathrm{M})$ and brine $(12 \mathrm{~mL})$ were added to the mixture. The mixture was then extracted with $\mathrm{Et}_{2} \mathrm{O}(2 \times 10 \mathrm{~mL})$. The combined organic phases were washed with further aq. $\mathrm{H}_{3} \mathrm{PO}_{4}(5 \mathrm{~mL}$, $2 \mathrm{M})$ and brine $(12 \mathrm{~mL})$, and then extracted with a $10 \% \mathrm{Na}_{2} \mathrm{CO}_{3}$ solution $(3 \times 10 \mathrm{~mL})$. The aqueous layer was concentrated in vacuo by forming an azeotrope with acetonitrile. EtOH $(20 \mathrm{~mL})$ was added, and the mixture refluxed for 10 mins. The mixture was then cooled to $0{ }^{\circ} \mathrm{C}$, filtered through a $\mathrm{P} 4$ frit and the residue washed with cold EtOH. The extraction process was repeated, and the combined extracts evaporated in vacuo to give the sodium sulfinate as a white solid.

\section{Sodium 1-((benzyloxy)carbonyl)piperidine-4-sulfinate (2a)}<smiles>O=C(OCc1ccccc1)N1CCC(S(=O)(=O)[O-])CC1</smiles>

Prepared according to General Procedure F-1 using 1-(1-((benzyloxy)carbonyl)piperidin-4-yl)-2,4,6triphenylpyridin-1-ium tetrafluoroborate $1 \mathrm{a}$ (122.5 mg, $0.20 \mathrm{mmol}, 1.0$ equiv.), Hantzsch ester (55.7 mg, 0.22 mmol, 1.1 equiv.), triethylamine (102 $\mu \mathrm{L}, 0.72 \mathrm{mmol}, 3.6$ equiv.) and DABSO ( $28.8 \mathrm{mg}, 0.12 \mathrm{mmol}, 0.6$ equiv.) in DMA (0.4 mL, $0.5 \mathrm{M})$ to afford sulfinate salt $2 \mathrm{a}$ as a white solid $(50.2 \mathrm{mg}, 82 \%)$.

${ }^{1} \mathrm{H}$ NMR $\left(400 \mathrm{MHz}, \mathrm{MeOD}-d_{4}\right) \delta 7.42-7.24(\mathrm{~m}, 5 \mathrm{H}), 5.11(\mathrm{~s}, 2 \mathrm{H}), 4.21(\mathrm{~d}, J=13.0 \mathrm{~Hz}, 2 \mathrm{H}), 2.97-2.77(\mathrm{~m}, 2 \mathrm{H})$, $2.02(\mathrm{tt}, J=12.0,4.0 \mathrm{~Hz}, 1 \mathrm{H}), 1.95-1.85(\mathrm{~m}, 2 \mathrm{H}), 1.43(\mathrm{qd}, J=12.5,4.5 \mathrm{~Hz}, 2 \mathrm{H}) ;{ }^{13} \mathrm{C}$ NMR $(101 \mathrm{MHz}, \mathrm{MeOD}-$ $\left.d_{4}\right) \delta 156.9,138.2,129.5,129.1,128.8,68.2,65.2,44.8,25.4$; LRMS (ESI) $m / z 282.0$ ([M-Na]); HRMS (ESI) $m / z$ $\mathrm{C}_{13} \mathrm{H}_{16} \mathrm{NO}_{4} \mathrm{~S}^{-}$requires 282.0806 ([M-Na]'), found 282.0800; IR (thin film, $v_{\max } / \mathrm{cm}^{-1}$ ) 3379, 2951, 1677, 1624, 1471, 1395; m.p. (MeOH): $268-271^{\circ} \mathrm{C}$ (decomposition).

\section{Sodium phenylmethanesulfinate (2b)}


<smiles>O=S([O-])Cc1ccccc1</smiles>

Prepared according to General Procedure F-1 using 1-benzyl-2,4,6-triphenylpyridin-1-ium tetrafluoroborate 1b (97.1 mg, $0.20 \mathrm{mmol}, 1.0$ equiv.), Hantzsch ester ( $55.7 \mathrm{mg}, 0.22 \mathrm{mmol}, 1.1$ equiv.), triethylamine (102 $\mu \mathrm{L}$, $0.72 \mathrm{mmol}, 3.6$ equiv.) and DABSO ( $28.8 \mathrm{mg}, 0.12 \mathrm{mmol}, 0.6$ equiv.) in DMA ( $0.4 \mathrm{~mL}, 0.5 \mathrm{M})$ to afford sulfinate salt $\mathbf{2} \mathbf{b}$ as a white solid ( $27.3 \mathrm{mg}, 77 \%)$.

${ }^{1} \mathrm{H}$ NMR $\left(400 \mathrm{MHz}, \mathrm{MeOD}-d_{4}\right) \delta 7.30-7.25(\mathrm{~m}, 4 \mathrm{H}), 7.23-7.17(\mathrm{~m}, 1 \mathrm{H}), 3.51(\mathrm{~s}, 2 \mathrm{H}) ;{ }^{13} \mathrm{C}$ NMR $(101 \mathrm{MHz}$, MeOD- $d_{4}$ ) $\delta$ 134.7, 130.9, 129.2, 127.7, 70.9; LRMS (ESI) $\mathrm{m} / \mathrm{z} 155.0$ ([M-Na]') 155.0; HRMS (ESI) $\mathrm{m} / \mathrm{z}_{7} \mathrm{C}_{7} \mathrm{H}_{7} \mathrm{O}_{2} \mathrm{~S}^{-}$ requires $155.0172\left([\mathrm{M}-\mathrm{Na}]^{-}\right)$, found 155.0161.

Data is consistent with the literature. ${ }^{27}$

Sodium 2-phenylethane-1-sulfinate (2c)<smiles>O=S([O-])CCc1ccccc1</smiles>

$\mathrm{Na}$

Prepared according to General Procedure F-2 using 1-phenethyl-2,4,6-triphenylpyridin-1-ium tetrafluoroborate 1c (99.9 mg, 0.20 mmol, 1.0 equiv.), Hantzsch ester (55.7 mg, 0.22 mmol, 1.1 equiv.), triethylamine (102 $\mu \mathrm{L}, 0.72 \mathrm{mmol}, 3.6$ equiv.) and DABSO (28.8 mg, $0.12 \mathrm{mmol}, 0.6$ equiv.) in DMA (0.4 mL, $0.5 \mathrm{M}$ ) to afford sulfinate salt $\mathbf{2 c}$ as a white solid (33.3 $\mathrm{mg}, 87 \%$ ).

${ }^{1} \mathrm{H}$ NMR $\left(400 \mathrm{MHz}, \mathrm{MeOD}-d_{4}\right) \delta 7.29-7.20(\mathrm{~m}, 4 \mathrm{H}), 7.18-7.11(\mathrm{~m}, 1 \mathrm{H}), 2.94-2.85(\mathrm{~m}, 2 \mathrm{H}), 2.57-2.48(\mathrm{~m}$, $2 \mathrm{H}) ;{ }^{13} \mathrm{C}$ NMR $\left(101 \mathrm{MHz}, \mathrm{MeOD}-d_{4}\right) \delta 142.5,129.4,126.9,64.6,29.7$ (1 aromatic carbon signal is not observed due to signal overlap); LRMS (ESI) $\mathrm{m} / \mathrm{z} 169.0$ ([M-Na] $]^{-}$); HRMS (ESI) $\mathrm{m} / \mathrm{z} \mathrm{C}_{8} \mathrm{H}_{9} \mathrm{O}_{2} \mathrm{~S}^{-}$requires 169.0329 ([M-Na] ), found 169.0317 .

Data is consistent with the literature. ${ }^{27}$ 


\subsection{Two-step synthesis of varied sulfones}

\section{Benzyl 4-(propylsulfonyl)piperidine-1-carboxylate (6a)}<smiles>CCCS(=O)(=O)C1CCN(C(=O)O)CC1</smiles>

Hantzsch ester (55.2 mg, $0.22 \mathrm{mmol}, 1.1$ equiv.), DABSO (28.8 mg, $0.12 \mathrm{mmol}, 0.6$ equiv.) and 1-(1((benzyloxy)carbonyl)piperidin-4-yl)-2,4,6-triphenylpyridin-1-ium tetrafluoroborate 1a (122.5 mg, 0.20 mmol, 1.0 equiv.) were added to an oven-dried $1.9 \mathrm{~mL}$ reaction vial. The vial was then sealed with a rubber septum, evacuated and backfilled with $\mathrm{N}_{2}(\times 3)$. Degassed, dry DMA $(0.4 \mathrm{~mL}, 0.5 \mathrm{M})$ was then added, followed by degassed, dry triethylamine (102 $\mu \mathrm{L}, 0.72 \mathrm{mmol}, 3.6$ equiv.). The reaction was stirred under blue LED irradiation at ambient temperature for $16 \mathrm{~h}$. 1-lodopropane ( $40 \mu \mathrm{L}, 0.40 \mathrm{mmol}, 2.0$ equiv.) was then added and the reaction heated to $100{ }^{\circ} \mathrm{C}$ in an aluminium heating block for $30 \mathrm{~min}$. The reaction was cooled to room temperature, and without workup, the crude reaction mixture was loaded directly to silica gel and purified by flash column chromatography (50\% EtOAc in Petrol) to afford sulfone 6 a as a colourless oil ( $55.3 \mathrm{mg}, 85 \%$ ). $\mathbf{R}_{f}\left(50 \%\right.$ EtOAc in Petrol) $=0.26 ;{ }^{1} \mathbf{H}$ NMR $\left(400 \mathrm{MHz}, \mathrm{CDCl}_{3}\right) \delta 7.41-7.28(\mathrm{~m}, 5 \mathrm{H}), 5.13(\mathrm{~s}, 2 \mathrm{H}), 4.37$ (app. br. s, $2 \mathrm{H}), 2.98(\mathrm{tt}, J=12.0,3.5 \mathrm{~Hz}, 1 \mathrm{H}), 2.92-2.74(\mathrm{~m}, 4 \mathrm{H}), 2.09$ (app. br. $\mathrm{d}, J=13.0 \mathrm{~Hz}, 2 \mathrm{H}), 1.96-1.84(\mathrm{~m}, 2 \mathrm{H})$, $1.84-1.71(\mathrm{~m}, 2 \mathrm{H}), 1.09(\mathrm{t}, J=7.5 \mathrm{~Hz}, 3 \mathrm{H}) ;{ }^{13} \mathrm{C}$ NMR $\left(101 \mathrm{MHz}, \mathrm{CDCl}_{3}\right) \delta 155.1,136.5,128.7,128.3,128.2$, 67.6, 59.0, 51.4, 42.9, 24.7, 15.2, 13.4; LRMS (ESI) $\mathrm{m} / \mathrm{z} 348.0$ ([M+Na] $]^{+}$); HRMS (ESI) $m / z \mathrm{C}_{16} \mathrm{H}_{23} \mathrm{NO}_{4} \mathrm{SNa}^{+}$ requires $348.1240\left([\mathrm{M}-\mathrm{Na}]^{+}\right)$, found 348.1241 ; IR (thin film, $v_{\max } / \mathrm{cm}^{-1}$ ) 2967, 2935, 1693, 1429, 1262, 1212, 1130.

\section{Benzyl 4-(benzylsulfonyl)piperidine-1-carboxylate (6b)}<smiles>O=C(O)N1CCC(S(=O)(=O)Cc2ccccc2)CC1</smiles>

Hantzsch ester (55.2 mg, $0.22 \mathrm{mmol}, 1.1$ equiv.), DABSO (28.8 mg, $0.12 \mathrm{mmol}, 0.6$ equiv.) and 1-(1((benzyloxy)carbonyl)piperidin-4-yl)-2,4,6-triphenylpyridin-1-ium tetrafluoroborate 1a (122.5 mg, 0.20 mmol, 1.0 equiv.) were added to an oven-dried $1.9 \mathrm{~mL}$ reaction vial. The vial was then sealed with a rubber septum, evacuated and backfilled with $\mathrm{N}_{2}(\times 3)$. Degassed, dry DMA $(0.4 \mathrm{~mL}, 0.5 \mathrm{M})$ was then added, followed by degassed, dry triethylamine (102 $\mu \mathrm{L}, 0.72 \mathrm{mmol}, 3.6$ equiv.). The reaction was stirred under blue LED irradiation at ambient temperature for $16 \mathrm{~h}$. Benzyl bromide $(48 \mu \mathrm{L}, 0.40 \mathrm{mmol}, 2.0$ equiv.) was then added and the reaction heated to $80^{\circ} \mathrm{C}$ in an aluminium heating block ${ }^{\circ} \mathrm{C}$ for $10 \mathrm{~min}$. The reaction was cooled to room temperature, and without workup, the crude reaction mixture was loaded directly to silica gel and purified by flash column chromatography (40\% EtOAc in Petrol) to afford sulfone $6 \mathbf{b}$ as a colourless oil (63.9 $\mathrm{mg}, 86 \%)$. 
$\mathbf{R}_{f}\left(40 \%\right.$ EtOAc in Petrol) $=0.16 ;{ }^{1} \mathbf{H}$ NMR $\left(400 \mathrm{MHz}, \mathrm{CDCl}_{3}\right) \delta 7.46-7.28(\mathrm{~m}, 10 \mathrm{H}), 5.12(\mathrm{~s}, 2 \mathrm{H}), 4.30$ (app. br. s, $2 \mathrm{H}$ ), 4.20 (s, 2H), 2.91 (tt, J=11.8, 3.7 Hz, 1H), 2.75 (app. br. s, 2H), 2.02 (app. br. d, J = 11.0 Hz, 2H), $1.88-$ 1.69 (br. m, 2H); ${ }^{13} \mathrm{C}$ NMR (101 MHz, $\left.\mathrm{CDCl}_{3}\right) \delta 154.9,136.4,130.6,129.1,128.6,128.5,128.2,128.0,127.4$, 67.4, 57.1, 56.6, 42.6, 24.5; LRMS (ESI) $m / z$ 396.0 ([M+Na] $]^{+}$); HRMS (ESI) $m / z \mathrm{C}_{20} \mathrm{H}_{24} \mathrm{NO}_{4} \mathrm{~S}^{+}$requires 374.1420 $\left([\mathrm{M}+\mathrm{H}]^{+}\right)$, found 374.1420; IR (thin film, $v_{\max } / \mathrm{cm}^{-1}$ ) 2931, 2868, 1690, 1430, 1309, 1213, 1116.

\section{Benzyl 4-(allylsulfonyl)piperidine-1-carboxylate (6c)}<smiles>C=CCS(=O)(=O)C1CCN(C(C)(C)C)CC1</smiles>

Hantzsch ester (55.2 mg, $0.22 \mathrm{mmol}, 1.1$ equiv.), DABSO (28.8 mg, $0.12 \mathrm{mmol}, 0.6$ equiv.) and 1-(1((benzyloxy)carbonyl)piperidin-4-yl)-2,4,6-triphenylpyridin-1-ium tetrafluoroborate 1a (122.5 mg, 0.20 $\mathrm{mmol}, 1.0$ equiv.) were added to an oven-dried $1.9 \mathrm{~mL}$ reaction vial. The vial was then sealed with a rubber septum, evacuated and backfilled with $\mathrm{N}_{2}(\times 3)$. Degassed, dry DMA $(0.4 \mathrm{~mL}, 0.5 \mathrm{M})$ was then added, followed by degassed, dry triethylamine (102 $\mu \mathrm{L}, 0.72 \mathrm{mmol}, 3.6$ equiv.). The reaction was stirred under blue LED irradiation at ambient temperature for $16 \mathrm{~h}$. Allyl bromide ( $35 \mu \mathrm{L}, 0.40 \mathrm{mmol}, 2.0$ equiv.) was then added and the reaction heated to $80{ }^{\circ} \mathrm{C}$ in an aluminium heating block for $10 \mathrm{~min}$. The reaction was cooled to room temperature, and without workup, the crude reaction mixture was loaded directly to silica gel and purified by flash column chromatography (40\% EtOAc in Petrol) to afford sulfone $6 \mathrm{c}$ as a colourless oil ( $53.6 \mathrm{mg}, 83 \%$ ). $\mathbf{R}_{f}\left(40 \%\right.$ EtOAc in Petrol) $=0.14 ;{ }^{1} \mathbf{H}$ NMR $\left(400 \mathrm{MHz}, \mathrm{CDCl}_{3}\right) \delta 7.40-7.28(\mathrm{~m}, 5 \mathrm{H}), 5.91(\mathrm{ddt}, J=17.5,10.0,7.5$ $\mathrm{Hz}, 1 \mathrm{H}$ ), $5.54-5.38$ (m, 2H), 5.12 (s, 2H), 4.34 (app. br. s, 2H), 3.70 (d, J = 7.5 Hz, 2H), 3.09 (tt, J = 12.0, $4.0 \mathrm{~Hz}$, 1H), 2.81 (app. br. s, 2H), 2.05 (app. br. d, $J=12.5 \mathrm{~Hz}, 2 \mathrm{H}$ ), $1.89-1.70$ (br. m, 2H); ${ }^{13} \mathrm{C} \mathrm{NMR}\left(101 \mathrm{MHz}, \mathrm{CDCl}_{3}\right)$ $\delta$ 155.0, 136.5, 128.6, 128.2, 128.0, 124.7, 124.7, 67.5, 57.4, 55.0, 42.7, 24.4; LRMS (ESI) $\mathrm{m} / \mathrm{z} 346.0$ ([M+Na] $]^{+}$); HRMS (ESI) $\mathrm{m} / \mathrm{z} \mathrm{C}_{16} \mathrm{H}_{21} \mathrm{NO}_{4} \mathrm{SNa}^{+}$requires $346.1084\left([\mathrm{M}+\mathrm{Na}]^{+}\right.$), found 346.1084; IR (thin film, $v_{\max } / \mathrm{cm}^{-1}$ ) 2926, $2864,1692,1425,1311,1212,1130$.

\section{Benzyl 4-(phenylsulfonyl)piperidine-1-carboxylate (6d)}<smiles>O=[N+]1CCC(S(=O)(=O)c2ccccc2)CC1</smiles>

Hantzsch ester (55.2 mg, $0.22 \mathrm{mmol}, 1.1$ equiv.), DABSO (28.8 mg, $0.12 \mathrm{mmol}, 0.6$ equiv.) and 1-(1((benzyloxy)carbonyl)piperidin-4-yl)-2,4,6-triphenylpyridin-1-ium tetrafluoroborate 1a (122.5 mg, 0.20 mmol, 1.0 equiv.) were added to an oven-dried $1.9 \mathrm{~mL}$ reaction vial. The vial was then sealed with a rubber septum, evacuated and backfilled with $\mathrm{N}_{2}(\times 3)$. Degassed, dry DMA $(0.4 \mathrm{~mL}, 0.5 \mathrm{M})$ was then added, followed by degassed, dry triethylamine (102 $\mu \mathrm{L}, 0.72 \mathrm{mmol}, 3.6$ equiv.). The reaction was stirred under blue LED irradiation at ambient temperature for $16 \mathrm{~h}$. Diphenyliodonium triflate (172 mg, $0.40 \mathrm{mmol}, 2.0$ equiv.) was 
then added and the reaction heated to $100^{\circ} \mathrm{C}$ in an aluminium heating block for $3 \mathrm{~h}$. The reaction was cooled to room temperature, and without workup, the crude reaction mixture was loaded directly to silica gel and purified by flash column chromatography (30\% EtOAc in Petrol) to afford sulfone $6 \mathbf{d}$ as a colourless oil (62.5 $\mathrm{mg}, 87 \%)$.

$\mathbf{R}_{f}\left(30 \%\right.$ EtOAc in Petrol) $=0.12 ;{ }^{1} \mathrm{H}$ NMR $\left(400 \mathrm{MHz}, \mathrm{CDCl}_{3}\right) \delta 7.87(\mathrm{dt}, J=8.5,1.5 \mathrm{~Hz}, 2 \mathrm{H}), 7.73-7.65(\mathrm{~m}, 1 \mathrm{H})$, $7.63-7.54(\mathrm{~m}, 2 \mathrm{H}), 7.41-7.28(\mathrm{~m}, 5 \mathrm{H}), 5.10(\mathrm{~s}, 2 \mathrm{H}), 4.31$ (app. br. s, 2H), $3.05(\mathrm{tt}, J=12.0,3.5 \mathrm{~Hz}, 1 \mathrm{H}), 2.74$ (app. br. s, 2H), 2.01 (app. br. d, $J=13.0 \mathrm{~Hz}, 2 \mathrm{H}), 1.63$ (qd, $J=12.5,4.5 \mathrm{~Hz}, 2 \mathrm{H}) ;{ }^{13} \mathrm{C} \mathrm{NMR}\left(101 \mathrm{MHz}, \mathrm{CDCl}_{3}\right) \delta$ 155.0, 136.7, 136.6, 134.2, 129.4, 129.3, 128.7, 128.3, 128.1, 67.6, 61.7, 42.9, 25.2; LRMS (ESI) $\mathrm{m} / \mathrm{z} 382.0$ $\left([\mathrm{M}+\mathrm{Na}]^{+}\right)$; HRMS (ESI) $m / z \mathrm{C}_{19} \mathrm{H}_{21} \mathrm{NO}_{4} \mathrm{SNa}^{+}$requires $382.1084\left([\mathrm{M}+\mathrm{Na}]^{+}\right)$, found 382.1083; IR (thin film, $v_{\max }$ $\left./ \mathrm{cm}^{-1}\right)$ 2932, 2861, 1698, 1447, 1306, 1214, 1146.

\section{Benzyl 4-((2-hydroxycyclohexyl)sulfonyl)piperidine-1-carboxylate (6e)}<smiles>O=[N+]1CCC(S(=O)(=O)C2CCCC[C@H]2O)CC1</smiles>

Hantzsch ester (55.2 mg, $0.22 \mathrm{mmol}, 1.1$ equiv.), DABSO (28.8 mg, $0.12 \mathrm{mmol}, 0.6$ equiv.) and 1-(1((benzyloxy)carbonyl)piperidin-4-yl)-2,4,6-triphenylpyridin-1-ium tetrafluoroborate 1a (122.5 mg, 0.20 mmol, 1.0 equiv.) were added to an oven-dried $1.9 \mathrm{~mL}$ reaction vial. The vial was then sealed with a rubber septum, evacuated and backfilled with $\mathrm{N}_{2}(\times 3)$. Degassed, dry DMA $(0.4 \mathrm{~mL}, 0.5 \mathrm{M})$ was then added, followed by degassed, dry triethylamine (102 $\mu \mathrm{L}, 0.72 \mathrm{mmol}, 3.6$ equiv.). The reaction was stirred under blue LED irradiation at ambient temperature for $16 \mathrm{~h}$. Cyclohexene oxide $(162 \mu \mathrm{L}, 1.6 \mathrm{mmol}, 8.0$ equiv.) and water $(0.5$ $\mathrm{mL}$ ) was then added and the reaction heated to $100{ }^{\circ} \mathrm{C}$ in an aluminium heating block for $48 \mathrm{~h}$. The reaction was cooled to room temperature, and the reaction partitioned between EtOAc and water. The organic layer was washed with water, brine, dried using $\mathrm{MgSO}_{4}$, filtered, and solvent removed in vacuo. The crude reaction mixture was loaded onto silica gel and purified by flash column chromatography (50-60\% EtOAc in Petrol) to afford sulfone $3 e$ as two diastereomers $6 \mathrm{e}-\mathbf{1}(50.5 \mathrm{mg}, 66 \%)$ and $6 \mathrm{e}-\mathbf{2}(12.0 \mathrm{mg}, 16 \%)$ as colourless oils (82\% total).

\section{6e-1}

$\mathbf{R}_{f}\left(60 \%\right.$ EtOAc in Petrol) = 0.25; ${ }^{1} \mathrm{H}$ NMR $\left(400 \mathrm{MHz}, \mathrm{CDCl}_{3}\right) \delta 7.40-7.28(\mathrm{~m}, 5 \mathrm{H}), 5.12(\mathrm{~s}, 2 \mathrm{H}), 4.34$ (br. s, 2H), $4.05-3.94$ (br. m, 1H), 3.43 (br. s, 1H), $3.21-2.90$ (br. m, 2H), $2.90-2.72$ (m, 2H), $2.21-2.04$ (br. m, 4H), $1.92-1.70$ (br. $\mathrm{m}, 4 \mathrm{H}), 1.55$ (qd, $J=13.0,4.0 \mathrm{~Hz}, 1 \mathrm{H}), 1.41-1.19(\mathrm{~m}, 3 \mathrm{H}) ;{ }^{13} \mathrm{C}$ NMR $\left(101 \mathrm{MHz}, \mathrm{CDCl}_{3}\right) \delta 155.1$, 136.6, 128.7, 128.3, 128.1, 69.2, 67.5, 63.3, 59.9, 43.0, 42.8, 35.2, 25.0, 24.7, 23.9, 23.7; LRMS (ESI) $\mathrm{m} / \mathrm{z} 404.0$ $\left([\mathrm{M}+\mathrm{Na}]^{+}\right)$; HRMS (ESI) $\mathrm{m} / z \mathrm{C}_{19} \mathrm{H}_{27} \mathrm{NO}_{5} \mathrm{SNa}^{+}$requires $404.1502\left([\mathrm{M}+\mathrm{Na}]^{+}\right)$, found 404.1497; IR (thin film, $v_{\max }$ $\left./ \mathrm{cm}^{-1}\right)$ 3464, 2936, 2860, 1702, 1452, 1215, 1131.

\section{$6 e-2$}


$\mathbf{R}_{f}(60 \%$ EtOAc in Petrol $)=0.12 ;{ }^{1} \mathrm{H}$ NMR $\left(400 \mathrm{MHz}, \mathrm{CDCl}_{3}\right) \delta 7.39-7.29(\mathrm{~m}, 5 \mathrm{H}), 5.13(\mathrm{~s}, 2 \mathrm{H}), 4.29(\mathrm{~s}, 2 \mathrm{H}), 3.95$ (ddd, $J=11.5,9.0,4.5 \mathrm{~Hz}, 1 \mathrm{H}), 3.67-3.54(\mathrm{~m}, 1 \mathrm{H}), 2.96-2.71(\mathrm{~m}, 3 \mathrm{H}), 2.20(\mathrm{~d}, J=3.5 \mathrm{~Hz}, 1 \mathrm{H}), 2.15-1.93$ (m, 4H), $1.79-1.63(\mathrm{~m}, 4 \mathrm{H}), 1.52(\mathrm{qd}, J=12.5,4.0 \mathrm{~Hz}, 1 \mathrm{H}), 1.36-1.22(\mathrm{~m}, 3 \mathrm{H}) ;{ }^{13} \mathrm{C} \mathrm{NMR}\left(101 \mathrm{MHz}, \mathrm{CDCl}_{3}\right) \delta$ $155.2,136.7,128.7,128.2,128.1,85.8,73.4,67.5,62.1,43.2,33.4,32.3,24.3,24.1,23.8 ;$ LRMS (ESI) $\mathrm{m} / \mathrm{z}$ $404.2\left([\mathrm{M}+\mathrm{Na}]^{+} \text {); HRMS (ESI) } \mathrm{m} / z \mathrm{C}_{19} \mathrm{H}_{27} \mathrm{NO}_{5} \mathrm{SNa}^{+} \text {requires } 404.1502 \text { ([M+Na }\right]^{+}$), found 404.1499; IR (thin film, $\left.v_{\max } / \mathrm{cm}^{-1}\right) 3362,2937,2861,1701,1452,1215,1119$.

Benzyl 4-(benzo[d]thiazol-2-ylsulfonyl)piperidine-1-carboxylate (6f)

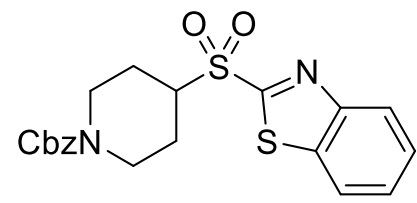

Hantzsch ester (55.2 mg, $0.22 \mathrm{mmol}, 1.1$ equiv.), DABSO (28.8 mg, $0.12 \mathrm{mmol}, 0.6$ equiv.) and 1-(1((benzyloxy)carbonyl)piperidin-4-yl)-2,4,6-triphenylpyridin-1-ium tetrafluoroborate 1a (122.5 mg, 0.20 mmol, 1.0 equiv.) were added to an oven-dried $1.9 \mathrm{~mL}$ reaction vial. The vial was then sealed with a rubber septum, evacuated and backfilled with $\mathrm{N}_{2}(\times 3)$. Degassed, dry DMA $(0.4 \mathrm{~mL}, 0.5 \mathrm{M})$ was then added, followed by degassed, dry triethylamine (102 $\mu \mathrm{L}, 0.72 \mathrm{mmol}, 3.6$ equiv.). The reaction was stirred under blue LED irradiation at ambient temperature for $16 \mathrm{~h}$. 2-Chlorobenzothiazole ( $50 \mu \mathrm{L}, 0.40 \mathrm{mmol}, 2.0$ equiv.) was then added and the reaction heated to $100^{\circ} \mathrm{C}$ in an aluminium heating block for $1.5 \mathrm{~h}$. The reaction was cooled to room temperature, and without workup, the crude reaction mixture was loaded directly to silica gel and purified by flash column chromatography (30\% EtOAc in Petrol) to afford sulfone $\mathbf{6 f}$ as a colourless oil (57.8 $\mathrm{mg}, 69 \%)$.

$\mathbf{R}_{\boldsymbol{f}}\left(30 \%\right.$ EtOAc in Petrol) $=0.16 ;{ }^{1} \mathbf{H}$ NMR $\left(400 \mathrm{MHz}, \mathrm{CDCl}_{3}\right) \delta 8.27-8.21(\mathrm{~m}, 1 \mathrm{H}), 8.06-8.00(\mathrm{~m}, 1 \mathrm{H}), 7.70-$ $7.58(\mathrm{~m}, 2 \mathrm{H}), 7.40-7.27(\mathrm{~m}, 5 \mathrm{H}), 5.11$ (s, 2H), 4.35 (app. br. s, 2H), 3.64 (tt, J=12.0, $3.5 \mathrm{~Hz}, 1 \mathrm{H}$ ), 2.85 (app. br. s, 2H), 2.01 (app. br. d, $J=13.0 \mathrm{~Hz}, 2 \mathrm{H}$ ), 1.88 (qd, $J=12.5,4.5 \mathrm{~Hz}, 2 \mathrm{H}) ;{ }^{13} \mathrm{C}$ NMR $\left(101 \mathrm{MHz}, \mathrm{CDCl}_{3}\right) \delta 164.3$, 155.0, 153.0, 137.1, 136.5, 128.6, 128.3, 128.3, 128.1, 127.9, 125.7, 122.4, 67.6, 61.1, 42.8, 24.8; LRMS (ESI) $\mathrm{m} / \mathrm{z} 439.0\left([\mathrm{M}+\mathrm{Na}]^{+}\right)$; HRMS (ESI) $\mathrm{m} / z \mathrm{C}_{20} \mathrm{H}_{20} \mathrm{~N}_{2} \mathrm{O}_{4} \mathrm{~S}_{2} \mathrm{Na}^{+}$requires $439.0757\left([\mathrm{M}+\mathrm{Na}]^{+}\right)$, found 439.0756; IR (thin film, $v_{\max } / \mathrm{cm}^{-1}$ ) 2930, 2864, 1690, 1423, 1324, 1213, 1144.

\section{Benzyl 4-(methylsulfonyl)piperidine-1-carboxylate (6g)}<smiles>CC(F)(F)N1CCC(S(C)(=O)=O)CC1</smiles>

Hantzsch ester (55.2 mg, $0.22 \mathrm{mmol}, 1.1$ equiv.), DABSO (28.8 mg, $0.12 \mathrm{mmol}, 0.6$ equiv.) and 1-(1((benzyloxy)carbonyl)piperidin-4-yl)-2,4,6-triphenylpyridin-1-ium tetrafluoroborate 1a (122.5 mg, 0.20 mmol, 1.0 equiv.) were added to an oven-dried $1.9 \mathrm{~mL}$ reaction vial. The vial was then sealed with a rubber septum, evacuated and backfilled with $\mathrm{N}_{2}(\times 3)$. Degassed, dry DMA $(0.4 \mathrm{~mL}, 0.5 \mathrm{M})$ was then added, followed 
by degassed, dry triethylamine (102 $\mu \mathrm{L}, 0.72 \mathrm{mmol}, 3.6$ equiv.). The reaction was stirred under blue LED irradiation at ambient temperature for $16 \mathrm{~h}$. Methyl iodide ( $40 \mu \mathrm{L}, 0.60 \mathrm{mmol}, 3.0$ equiv.) was then added and the reaction stirred at room temperature for $6 \mathrm{~h}$. Without workup, the crude reaction mixture was loaded directly to silica gel and purified by flash column chromatography (70\% EtOAc in Petrol) to afford sulfone $\mathbf{6 g}$ as a colourless oil (53.9 $\mathrm{mg}, 91 \%)$.

$\mathbf{R}_{f}\left(70 \%\right.$ EtOAc in Petrol) $=0.27 ;{ }^{1} \mathbf{H}$ NMR $\left(400 \mathrm{MHz}_{\mathrm{CDCl}}\right) \delta 7.40-7.29(\mathrm{~m}, 5 \mathrm{H}), 5.12(\mathrm{~s}, 2 \mathrm{H}), 4.38$ (app. br. s, $2 \mathrm{H}$ ), 2.98 (tt, $J=12.0,3.5 \mathrm{~Hz}, 1 \mathrm{H}$ ), 2.82 (app. s, 5H), 2.13 (app. br. d, $J=12.5 \mathrm{~Hz}, 2 \mathrm{H}$ ), $1.81-1.64(\mathrm{~m}, 2 \mathrm{H}) ;{ }^{13} \mathrm{C}$ NMR $\left(101 \mathrm{MHz}, \mathrm{CDCl}_{3}\right) \delta$ 155.0, 136.5, 128.6, 128.3, 128.1, 67.5, 60.5, 42.8, 37.4, 25.0; LRMS (ESI) $\mathrm{m} / \mathrm{z} 320.0$ $\left([\mathrm{M}+\mathrm{Na}]^{+}\right)$; HRMS (ESI) $\mathrm{m} / z \mathrm{C}_{14} \mathrm{H}_{20} \mathrm{NO}_{4} \mathrm{~S}^{+}$requires $298.1108\left([\mathrm{M}+\mathrm{H}]^{+}\right)$, found 298.1109; IR (thin film, $v_{\max } / \mathrm{cm}^{-}$ $\left.{ }^{1}\right)$ 2930, 2863, 1692, 1431, 1303, 1261, 1213.

((Methylsulfonyl)methyl)benzene (6h)<smiles>CS(=O)(=O)Cc1ccccc1</smiles>

Hantzsch ester ( $55.2 \mathrm{mg}, 0.22 \mathrm{mmol}, 1.1$ equiv.), DABSO ( $28.8 \mathrm{mg}, 0.12 \mathrm{mmol}, 0.6$ equiv.) and 1-benzyl-2,4,6triphenylpyridin-1-ium tetrafluoroborate $1 \mathrm{~b}(97.1 \mathrm{mg}, 0.20 \mathrm{mmol}, 1.0$ equiv.) were added to an oven-dried $1.9 \mathrm{~mL}$ reaction vial. The vial was then sealed with a rubber septum, evacuated and backfilled with $\mathrm{N}_{2}(\times 3)$. Degassed, dry DMA (0.4 mL, 0.5 M) was then added, followed by degassed, dry 2,6-lutidine ( $83 \mu \mathrm{L}, 0.72 \mathrm{mmol}$, 3.6 equiv.). The reaction was stirred under blue LED irradiation at ambient temperature for $16 \mathrm{~h}$. Methyl iodide ( $40 \mu \mathrm{L}, 0.60 \mathrm{mmol}, 3.0$ equiv.) was then added and the reaction stirred at room temperature for $6 \mathrm{~h}$. Without workup, the crude reaction mixture was loaded directly to silica gel and purified by flash column chromatography (40\% EtOAc in Petrol) to afford sulfone $6 \mathrm{~h}$ as a white solid (25.6 mg, 75\%).

$\mathbf{R}_{f}\left(40 \%\right.$ EtOAc in Petrol) $=0.25 ;{ }^{1} \mathrm{H}$ NMR $\left(400 \mathrm{MHz}, \mathrm{CDCl}_{3}\right) \delta 7.46-7.35(\mathrm{~m}, 5 \mathrm{H}), 4.25(\mathrm{~s}, 2 \mathrm{H}), 2.75(\mathrm{~s}, 3 \mathrm{H}) ;{ }^{13} \mathrm{C}$ NMR (101 MHz, $\left.\mathrm{CDCl}_{3}\right) \delta 130.6,129.2,128.4,61.4,39.1 ;$ LRMS (ESI) $\mathrm{m} / \mathrm{z} 193.0\left([\mathrm{M}+\mathrm{Na}]^{+}\right) ;$HRMS (ESI) $\mathrm{m} / \mathrm{z}$ $\mathrm{C}_{8} \mathrm{H}_{10} \mathrm{O}_{2} \mathrm{SNa}^{+}$requires $193.0294\left(\left[\mathrm{M}+\mathrm{Na}^{+}\right]\right)$, found 193.0297 .

Data is consistent with the literature. ${ }^{27}$

\section{(2-(Methylsulfonyl)ethyl)benzene (6i)}

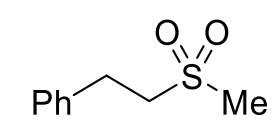

Hantzsch ester ( $55.2 \mathrm{mg}, 0.22 \mathrm{mmol}, 1.1$ equiv.), DABSO ( $28.8 \mathrm{mg}, 0.12 \mathrm{mmol}, 0.6$ equiv.) and 1-phenethyl2,4,6-triphenylpyridin-1-ium tetrafluoroborate $1 \mathrm{c}(99.9 \mathrm{mg}, 0.20 \mathrm{mmol}, 1.0$ equiv.) were added to an ovendried $1.9 \mathrm{~mL}$ reaction vial. The vial was then sealed with a rubber septum, evacuated and backfilled with $\mathrm{N}_{2}$ ( $\times 3)$. Degassed, dry DMA (0.4 mL, $0.5 \mathrm{M})$ was then added, followed by degassed, dry piperidine $(71 \mu \mathrm{L}, 0.72$ mmol, 3.6 equiv.). The reaction was heated to $90{ }^{\circ} \mathrm{C}$ in an aluminium heating block for $16 \mathrm{~h}$. Methyl iodide $(62 \mu \mathrm{L}, 1.00 \mathrm{mmol}, 5.0$ equiv.) was then added and the reaction stirred at room temperature for $6 \mathrm{~h}$. Without 
workup, the crude reaction mixture was loaded directly to silica gel and purified by flash column chromatography (30\% EtOAc in Petrol) to afford sulfone $6 \mathbf{i}$ as a white solid (26.8 $\mathrm{mg}, 73 \%$ ).

$\mathbf{R}_{f}\left(30 \%\right.$ EtOAc in Petrol) $=0.22 ;{ }^{1} \mathbf{H}$ NMR $\left(400 \mathrm{MHz}, \mathrm{CDCl}_{3}\right) \delta 7.38-7.29(\mathrm{~m}, 2 \mathrm{H}), 7.29-7.21(\mathrm{~m}, 3 \mathrm{H}), 3.34-$ $3.26(\mathrm{~m}, 2 \mathrm{H}), 3.21-3.12(\mathrm{~m}, 2 \mathrm{H}), 2.82(\mathrm{~s}, 3 \mathrm{H}) ;{ }^{13} \mathrm{C} \mathrm{NMR}\left(101 \mathrm{MHz}, \mathrm{CDCl}_{3}\right) \delta 137.5,129.1,128.5,127.3,56.3$, 41.1, 28.7; LRMS (ESI) $\mathrm{m} / \mathrm{z} 207.0\left([\mathrm{M}+\mathrm{Na}]^{+}\right)$; HRMS (ESI) $\mathrm{m} / \mathrm{z} \mathrm{C}_{9} \mathrm{H}_{12} \mathrm{O}_{2} \mathrm{SNa}^{+}$requires $207.0450\left([\mathrm{M}+\mathrm{Na}]^{+}\right)$, found 207.0453.

Data is consistent with the literature. ${ }^{27}$ 


\subsection{Two-step synthesis of sulfonyl fluorides}

\section{Benzyl 4-(fluorosulfonyl)piperidine-1-carboxylate (7a)}<smiles></smiles>

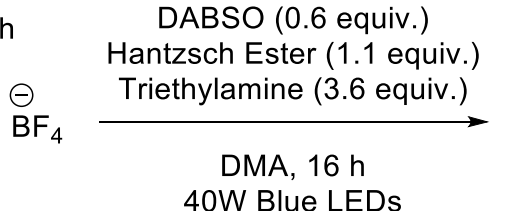<smiles>CCNCCCOS(=O)C1CCN(C(C)(C)C)CC1</smiles>
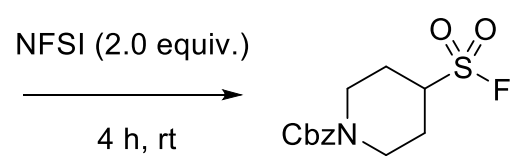

Hantzsch ester (55.2 mg, $0.22 \mathrm{mmol}, 1.1$ equiv.), DABSO (28.8 mg, $0.12 \mathrm{mmol}, 0.6$ equiv.) and 1-(1((benzyloxy)carbonyl)piperidin-4-yl)-2,4,6-triphenylpyridin-1-ium tetrafluoroborate 1a (122.5 mg, 0.20 mmol, 1.0 equiv.) were added to an oven-dried $1.9 \mathrm{~mL}$ reaction vial. The vial was then sealed with a rubber septum, evacuated and backfilled with $\mathrm{N}_{2}(\times 3)$. Degassed, dry DMA $(0.4 \mathrm{~mL}, 0.5 \mathrm{M})$ was then added, followed by degassed, dry triethylamine $(102 \mu \mathrm{L}, 0.72 \mathrm{mmol}, 3.6$ equiv.). The reaction was stirred under blue LED irradiation at ambient temperature for $16 \mathrm{~h}$. NFSI (126.1 $\mathrm{mg}, 0.40 \mathrm{mmol}, 2.0$ equiv.) was then added and the reaction stirred at room temperature for $4 \mathrm{~h}$. Without workup, the crude reaction mixture was loaded directly to silica gel and purified by flash column chromatography $\left(100 \% \mathrm{CH}_{2} \mathrm{Cl}_{2}\right)$ to afford sulfonyl fluoride $7 \mathrm{a}$ as a white crystalline solid (51.4 mg, 85\%).

$\mathbf{R}_{f}\left(100 \% \mathrm{CH}_{2} \mathrm{Cl}_{2}\right)=0.23 ;{ }^{1} \mathbf{H}$ NMR $\left(400 \mathrm{MHz}, \mathrm{CDCl}_{3}\right) \delta 7.44-7.29(\mathrm{~m}, 5 \mathrm{H}), 5.14(\mathrm{~s}, 2 \mathrm{H}), 4.46-4.23$ (app. br. s, $2 \mathrm{H}$ ), 3.47 (ttd, $J=12.0,4.0,2.0 \mathrm{~Hz}, 1 \mathrm{H}$ ), 2.90 (app. br. s, 2H), 2.24 (app. br. d, $J=12.0 \mathrm{~Hz}, 2 \mathrm{H}$ ), 1.90 (br. qd, $J=$ 12.0, 11.5, 4.0 Hz, 2H). ${ }^{13} \mathrm{C}$ NMR $\left(101 \mathrm{MHz}, \mathrm{CDCl}_{3}\right) \delta 154.9,136.4,128.7,128.4,128.2,67.8,58.9\left(\mathrm{~d}, J_{\mathrm{CF}}=15.1\right.$ $\mathrm{Hz}$ ), 42.5, 25.9; ${ }^{19} \mathrm{~F}$ NMR (377 MHz, $\left.\mathrm{CDCl}_{3}\right) \delta$ 42.3; HRMS (ESI) $\mathrm{m} / \mathrm{z} \mathrm{C}_{13} \mathrm{H}_{16} \mathrm{FNO}_{4} \mathrm{SNa}^{+}$requires 324.0676 $\left([\mathrm{M}+\mathrm{Na}]^{+}\right)$, found 324.0677; IR (thin film, $\left.v_{\max } / \mathrm{cm}^{-1}\right) 2938,2868,1698,1400,1183,1015,760 ;$ m.p. $\left(\mathrm{CH}_{2} \mathrm{Cl}_{2}\right)$ : $87-89^{\circ} \mathrm{C}$.

\section{Phenylmethanesulfonyl fluoride (7b)}<smiles></smiles>

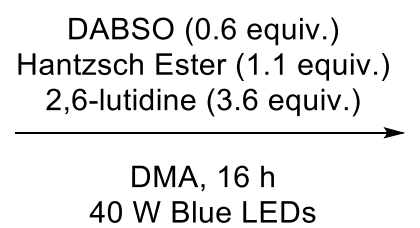

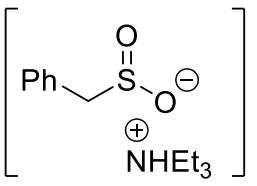

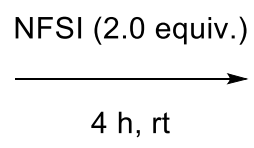<smiles>O=S(=O)(F)CSc1ccccc1</smiles>

Hantzsch ester ( $55.2 \mathrm{mg}, 0.22 \mathrm{mmol}, 1.1$ equiv.), DABSO ( $28.8 \mathrm{mg}, 0.12 \mathrm{mmol}, 0.6$ equiv.) and 1-benzyl-2,4,6triphenylpyridin-1-ium tetrafluoroborate $1 \mathrm{~b}(97.1 \mathrm{mg}, 0.20 \mathrm{mmol}, 1.0$ equiv.) were added to an oven-dried $1.9 \mathrm{~mL}$ reaction vial. The vial was then sealed with a rubber septum, evacuated and backfilled with $\mathrm{N}_{2}(\times 3)$. Degassed, dry DMA (0.4 mL, 0.5 M) was then added, followed by degassed, dry 2,6-lutidine ( $84 \mu \mathrm{L}, 0.72 \mathrm{mmol}$, 3.6 equiv.). The reaction was stirred under blue LED irradiation at ambient temperature for $16 \mathrm{~h}$. NFSI (126.1 $\mathrm{mg}, 0.40 \mathrm{mmol}, 2.0$ equiv.) was then added and the reaction stirred at room temperature for $4 \mathrm{~h}$. Without workup, the crude reaction mixture was loaded directly to silica gel and purified by flash column chromatography (5\% EtOAc in Petrol) to afford sulfonyl fluoride $7 \mathbf{b}$ as a white crystalline solid (26.1 mg, $75 \%$ ). 
$\mathbf{R}_{f}\left(100 \% \mathrm{CH}_{2} \mathrm{Cl}_{2}\right)=0.23 ;{ }^{1} \mathrm{H}$ NMR $\left(400 \mathrm{MHz}, \mathrm{CDCl}_{3}\right) \delta 7.51-7.40(\mathrm{~m}, 5 \mathrm{H}), 4.60(\mathrm{~d}, J=3.0 \mathrm{~Hz}, 2 \mathrm{H}) ;{ }^{13} \mathrm{C}$ NMR $(101$ $\left.\mathrm{MHz}_{\mathrm{CDCl}}\right) \delta 130.78,130.03,129.44,125.62,57.0\left(\mathrm{~d}, \mathrm{~J}_{\mathrm{CF}}=18.0 \mathrm{~Hz}\right) ;{ }^{19} \mathrm{~F}$ NMR $\left(377 \mathrm{MHz}, \mathrm{CDCl}_{3}\right) \delta 51.4(\mathrm{t}, J=$ $3.5 \mathrm{~Hz})$.

Data is consistent with the literature. ${ }^{28}$

\section{2-Phenylethane-1-sulfonyl fluoride (7c)}

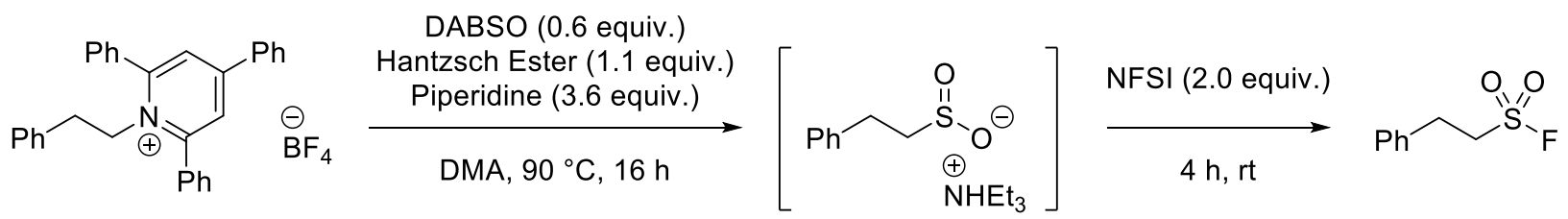

Hantzsch ester (55.2 mg, $0.22 \mathrm{mmol}, 1.1$ equiv.), DABSO (28.8 mg, $0.12 \mathrm{mmol}, 0.6$ equiv.) and 1-phenethyl2,4,6-triphenylpyridin-1-ium tetrafluoroborate $1 \mathrm{c}$ (100 mg, $0.20 \mathrm{mmol}, 1.0$ equiv.) were added to an ovendried $1.9 \mathrm{~mL}$ reaction vial. The vial was then sealed with a rubber septum, evacuated and backfilled with $\mathrm{N}_{2}$ ( $\times 3)$. Degassed, dry DMA (0.4 mL, $0.5 \mathrm{M})$ was then added, followed by degassed, dry piperidine $(71 \mu \mathrm{L}, 0.72$ mmol, 3.6 equiv.). The reaction was heated to $90^{\circ} \mathrm{C}$ in an aluminium heating block for $16 \mathrm{~h}$. NFSI (126.1 mg, $0.40 \mathrm{mmol}, 2.0$ equiv.) was then added and the reaction stirred at room temperature for $4 \mathrm{~h}$. Without workup, the crude reaction mixture was loaded directly to silica gel and purified by flash column chromatography ( $3-5 \%$ EtOAc in Petrol) to afford sulfonyl fluoride $7 \mathrm{c}$ as a white crystalline solid (30.5 mg, $80 \%)$.

$\mathbf{R}_{\boldsymbol{f}}\left(5 \%\right.$ EtOAc in Petrol) $=0.15 ;{ }^{1} \mathbf{H} \mathbf{N M R}\left(400 \mathrm{MHz}, \mathrm{CDCl}_{3}\right) \delta 7.40-7.27(\mathrm{~m}, 3 \mathrm{H}), 7.25-7.19(\mathrm{~m}, 2 \mathrm{H}), 3.68-$ $3.58(\mathrm{~m}, 2 \mathrm{H}), 3.29-3.19(\mathrm{~m}, 2 \mathrm{H}) ;{ }^{13} \mathrm{C}$ NMR $\left(101 \mathrm{MHz}, \mathrm{CDCl}_{3}\right) \delta 136.1,129.3,128.5,127.8,52.3(\mathrm{~d}, J=16.0$ $\mathrm{Hz}), 29.8 ;{ }^{19} \mathrm{~F}$ NMR $\left(377 \mathrm{MHz}, \mathrm{CDCl}_{3}\right) \delta 53.2(\mathrm{t}, J=4.5 \mathrm{~Hz})$.

Data is consistent with the literature. ${ }^{29}$ 


\subsection{Two-step synthesis of primary sulfonamides}

\section{Benzyl 4-sulfamoylpiperidine-1-carboxylate (8a)}

$\begin{gathered}\mathrm{DMA}, 16 \mathrm{~h} \\ \text { 40W Blue LEDs }\end{gathered}$
$\mathrm{NEt}_{3}(3.6$ equiv.)

Hantzsch ester $(55.7 \mathrm{mg}, 0.22 \mathrm{mmol}, 1.1$ equiv.), DABSO (28.8 mg, $0.12 \mathrm{mmol}, 0.6$ equiv.) and 1-(1((benzyloxy)carbonyl)piperidin-4-yl)-2,4,6-triphenylpyridin-1-ium tetrafluoroborate 1a (122.5 mg, 0.20 mmol, 1.0 equiv.) were added to an oven-dried $1.9 \mathrm{~mL}$ reaction vial. The vial was then sealed with a rubber septum, evacuated and backfilled with $\mathrm{N}_{2}(\times 3)$. Degassed, dry DMA $(0.4 \mathrm{~mL}, 0.5 \mathrm{M})$ was then added, followed by degassed, dry triethylamine (102 $\mu \mathrm{L}, 0.72 \mathrm{mmol}, 3.6$ equiv.). The reaction was stirred under blue LED irradiation at ambient temperature for $16 \mathrm{~h}$. Hydroxylamine- $O$-sulfonic acid ( $45.2 \mathrm{mg}, 0.40 \mathrm{mmol}, 2.0$ equiv.) was then added and the reaction stirred at room temperature for $24 \mathrm{~h}$. Without workup, the crude reaction mixture was loaded directly to silica gel and purified by flash column chromatography (60\% EtOAc in Petrol) to afford sulfonamide $8 \mathrm{a}$ as a colourless oil $(52.6 \mathrm{mg}, 88 \%)$.

$\mathbf{R}_{\boldsymbol{f}}\left(60 \%\right.$ EtOAc in Petrol) $=0.19 ;{ }^{1} \mathbf{H}$ NMR $\left(400 \mathrm{MHz}, \mathrm{CDCl}_{3}\right) \delta 7.40-7.30(\mathrm{~m}, 5 \mathrm{H}), 5.13(\mathrm{~s}, 2 \mathrm{H}), 4.48(\mathrm{~s}, 2 \mathrm{H}), 4.37$ (app. br. s, 2H), 3.08 (tt, $J=12.0,3.5 \mathrm{~Hz}, 1 \mathrm{H}$ ), 2.83 (app. br. s, 2H), 2.19 (app. br. d, $J=13 \mathrm{~Hz}, 2 \mathrm{H}$ ), $1.81-1.66$ (br. m, 2H); $\left.{ }^{13} \mathrm{C} \mathrm{NMR} \mathrm{(101} \mathrm{MHz,} \mathrm{CDCl}\right) \delta 155.2,136.6,128.7,128.3,128.1,67.6,60.7,43.0,26.2$; LRMS (ESI) $\mathrm{m} / \mathrm{z} 321.0\left([\mathrm{M}+\mathrm{Na}]^{+}\right), 297.0\left([\mathrm{M}-\mathrm{H}]^{-}\right)$; HRMS (ESI) $\mathrm{m} / \mathrm{z} \mathrm{C}_{13} \mathrm{H}_{18} \mathrm{~N}_{2} \mathrm{O}_{4} \mathrm{SNa}^{+}$requires $321.0879\left([\mathrm{M}+\mathrm{Na}]^{+}\right)$, found 321.0877; IR (thin film, $v_{\max } / \mathrm{cm}^{-1}$ ) 3249, 2960, 1679, 1436, 1323, 1279, 1218. 1142; m.p. $\left(\mathrm{CH}_{2} \mathrm{Cl}_{2}\right): 126-129$ ${ }^{\circ} \mathrm{C}$.

\section{Phenylmethanesulfonamide (8b)}

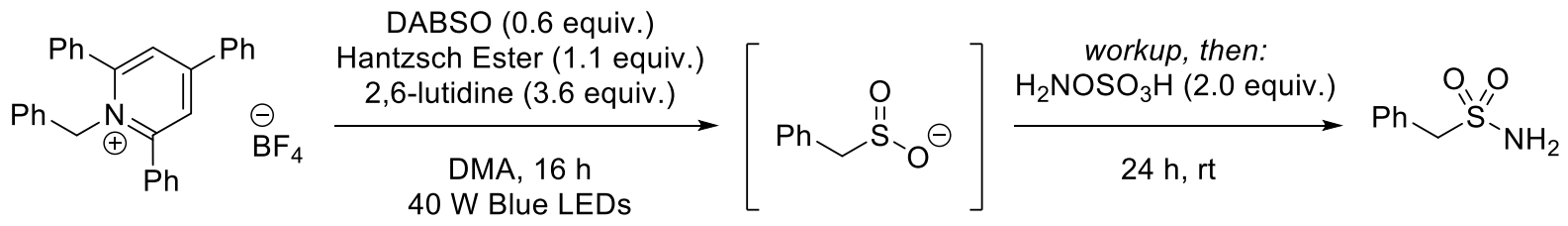

Hantzsch ester (55.7 mg, $0.22 \mathrm{mmol}, 1.1$ equiv.), DABSO (28.8 mg, $0.12 \mathrm{mmol}, 0.6$ equiv.) and 1-benzyl-2,4,6triphenylpyridin-1-ium tetrafluoroborate $1 \mathrm{~b}(97.1 \mathrm{mg}, 0.20 \mathrm{mmol}, 1.0$ equiv.) were added to an oven-dried $1.9 \mathrm{~mL}$ reaction vial. The vial was then sealed with a rubber septum, evacuated and backfilled with $\mathrm{N}_{2}(\times 3)$. Degassed, dry DMA (0.4 mL, $0.5 \mathrm{M}$ ) was then added, followed by degassed, dry 2,6-lutidine ( $84 \mu \mathrm{L}, 0.72 \mathrm{mmol}$, 3.6 equiv.). The reaction was stirred under blue LED irradiation at ambient temperature for $16 \mathrm{~h}$. Upon completion, aq. $\mathrm{H}_{3} \mathrm{PO}_{4}(3 \mathrm{~mL}, 2 \mathrm{M})$ and brine $(1 \mathrm{~mL})$ were added to the mixture. The mixture was then extracted with $\mathrm{CH}_{2} \mathrm{Cl}_{2}(3 \times 2 \mathrm{~mL})$. The combined organic phase was transferred to a round-bottom flask and DIPEA (3.0 equiv.) was added. Hydroxylamine- $O$-sulfonic acid $(45.2 \mathrm{mg}, 0.40 \mathrm{mmol}, 2.0$ equiv.) and sodium acetate $(32.8 \mathrm{mg}, 0.40 \mathrm{mmol}, 2.0$ equiv.) were then added and the reaction stirred at room temperature for 
$24 \mathrm{~h}$. The reaction mixture was concentrated in vacuo, and the crude reaction mixture was then loaded directly to silica gel and purified by flash column chromatography ( $40 \%$ EtOAcin Petrol) to afford sulfonamide $\mathbf{8 b}$ as a white crystalline solid ( $25.3 \mathrm{mg}, 74 \%)$.

Also prepared according to a modified procedure using $\mathrm{Na}_{2} \mathrm{~S}_{2} \mathrm{O}_{5}(45.6 \mathrm{mg}, 0.24 \mathrm{mmol}, 1.2$ equiv.) instead of DABSO, and no 2,6-lutidine was added. Hydroxylamine-O-sulfonic acid ( $45.2 \mathrm{mg}, 0.40 \mathrm{mmol}, 2.0$ equiv.) and sodium acetate ( $32.8 \mathrm{mg}, 0.40 \mathrm{mmol}, 2.0$ equiv.) were added to the crude reaction mixture without workup, affording sulfonamide $\mathbf{8 b}(23.5 \mathrm{mg}, 69 \%)$.

$\mathbf{R}_{f}\left(40 \%\right.$ EtOAc in Petrol) $=0.23 ;{ }^{1} \mathrm{H}$ NMR $\left(400 \mathrm{MHz}\right.$, DMSO-d $\left.d_{6}\right) \delta 7.43-7.31(\mathrm{~m}, 5 \mathrm{H}), 6.83(\mathrm{~s}, 2 \mathrm{H}), 4.26(\mathrm{~s}, 2 \mathrm{H})$; ${ }^{13}$ C NMR (101 MHz, DMSO- $d_{6}$ ) $\delta$ 130.8, 130.8, 128.3, 127.9, 60.2; LRMS (ESI) m/z 170.0 ([M-H]); HRMS (ESI) $m / z \mathrm{C}_{7} \mathrm{H}_{8} \mathrm{NO}_{2} \mathrm{~S}^{-}$requires $170.0281\left([\mathrm{M}-\mathrm{H}]^{-}\right)$, found 170.0276 .

Data is consistent with the literature. ${ }^{30}$

\section{2-Phenylethane-1-sulfonamide (8c)}

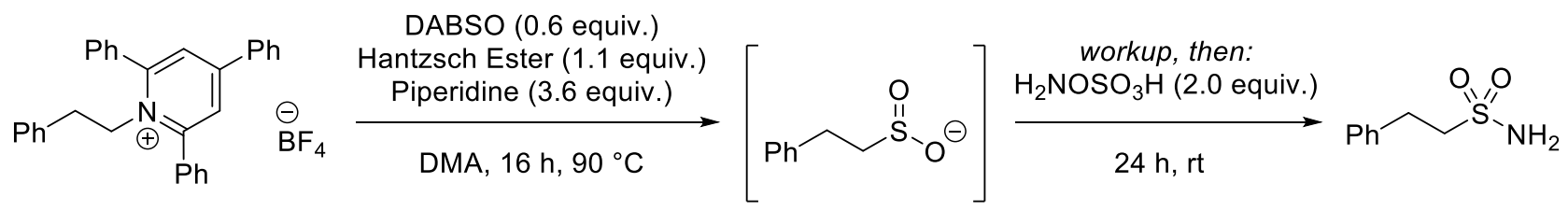

Hantzsch ester ( $55.2 \mathrm{mg}, 0.22 \mathrm{mmol}, 1.1$ equiv.), DABSO (28.8 mg, $0.12 \mathrm{mmol}, 0.6$ equiv.) and 1-phenethyl2,4,6-triphenylpyridin-1-ium tetrafluoroborate 1c (99.9 mg, $0.20 \mathrm{mmol}, 1.0$ equiv.) were added to an ovendried $1.9 \mathrm{~mL}$ reaction vial. The vial was then sealed with a rubber septum, evacuated and backfilled with $\mathrm{N}_{2}$ (×3). Degassed, dry DMA (0.4 mL, $0.5 \mathrm{M})$ was then added, followed by degassed, dry piperidine $(71 \mu \mathrm{L}, 0.72$ mmol, 3.6 equiv.). The reaction was heated to $90^{\circ} \mathrm{C}$ in an aluminium heating block for $16 \mathrm{~h}$. Upon completion, aq. $\mathrm{H}_{3} \mathrm{PO}_{4}(3 \mathrm{~mL}, 2 \mathrm{M})$ and brine $(1 \mathrm{~mL})$ were added to the mixture. The mixture was then extracted with $\mathrm{CH}_{2} \mathrm{Cl}_{2}(3 \times 2 \mathrm{~mL})$. The combined organic phase was transferred to a round-bottom flask and DIPEA (105 $\mu \mathrm{L}$, $0.60 \mathrm{mmol}, 3.0$ equiv.) was added. Hydroxylamine- $O$-sulfonic acid ( $45.2 \mathrm{mg}, 0.40 \mathrm{mmol}, 2.0$ equiv.) and sodium acetate $(32.8 \mathrm{mg}, 0.40 \mathrm{mmol}, 2.0$ equiv.) were then added and the reaction stirred at room temperature for $24 \mathrm{~h}$. The reaction mixture was concentrated in vacuo, and the crude reaction mixture was then loaded directly to silica gel and purified by flash column chromatography (40\% EtOAc in Petrol) to afford sulfonamide $8 \mathrm{c}$ as a white crystalline solid (26.3 $\mathrm{mg}, 71 \%)$.

$\mathbf{R}_{f}\left(40 \%\right.$ EtOAc in Petrol) $=0.25 ;{ }^{1} \mathbf{H}$ NMR $\left(400 \mathrm{MHz}\right.$, DMSO- $\left.d_{6}\right) \delta 7.36-7.17(\mathrm{~m}, 5 \mathrm{H}), 6.87(\mathrm{~s}, 2 \mathrm{H}), 3.28-3.20$ (m, 2H), $3.04-2.96(m, 2 H) ;{ }^{13}$ C NMR (101 MHz, DMSO- $\left.d_{6}\right) \delta 138.8,128.6,128.3,126.4,55.5,29.6$; LRMS (ESI) $\mathrm{m} / \mathrm{z} 184.1\left([\mathrm{M}-\mathrm{H}]^{-}\right)$; HRMS (ESI) $\mathrm{m} / 2 \mathrm{C} \mathrm{C}_{8} \mathrm{H}_{10} \mathrm{NO}_{2} \mathrm{~S}^{-}$requires 184.0438 ([M-H] $\left.]^{-}\right)$, found 184.0434.

Data is consistent with the literature. ${ }^{31}$ 


\subsection{Two-step synthesis of substituted tertiary sulfonamides}

\section{General Procedure G:}

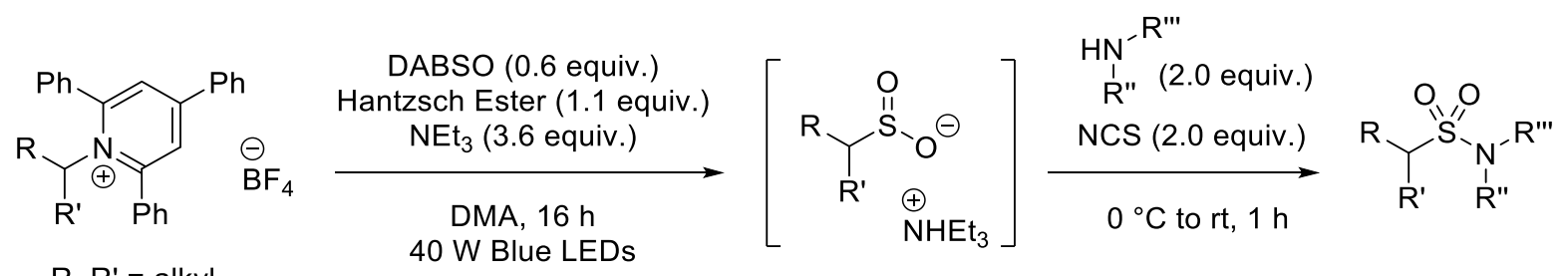

Hantzsch ester (1.1 equiv.), DABSO (0.6 equiv.) and Katritzky pyridinium salt (1.0 equiv.) were added to an oven-dried $1.9 \mathrm{~mL}$ reaction vial. The vial was then sealed with a rubber septum, evacuated and backfilled with $\mathrm{N}_{2}(\times 3)$. Degassed, dry DMA (0.5 M) was then added, followed by degassed, dry triethylamine (3.6 equiv.). The reaction was stirred under blue LED irradiation at ambient temperature for $16 \mathrm{~h}$. After cooling the solution to $0{ }^{\circ} \mathrm{C}$, amine (2.0 equiv.) and NCS (2.0 equiv.) were added and the reaction stirred at $\mathrm{rt}$ for $1 \mathrm{~h}$. Without workup, the crude reaction mixture was loaded directly to silica gel and purified by flash column chromatography to afford the sulfonamide product.

\section{Benzyl 4-(morpholinosulfonyl)piperidine-1-carboxylate (9a)}<smiles>O=[W]N1CCC(S(=O)(=O)N2CCOCC2)CC1</smiles>

Prepared according to General Procedure G using 1-(1-((benzyloxy)carbonyl)piperidin-4-yl)-2,4,6triphenylpyridin-1-ium tetrafluoroborate $1 \mathrm{a}$ (122.5 mg, $0.20 \mathrm{mmol}, 1.0$ equiv.), Hantzsch ester (55.7 mg, 0.22 mmol, 1.1 equiv.), triethylamine (102 $\mu \mathrm{L}, 0.72 \mathrm{mmol}, 3.6$ equiv.), DABSO ( $28.8 \mathrm{mg}, 0.12 \mathrm{mmol}, 0.6$ equiv.), NCS (53.4 mg, $0.40 \mathrm{mmol}, 2.0$ equiv.) and morpholine ( $34.5 \mu \mathrm{L}, 0.40 \mathrm{mmol}, 2.0$ equiv.) in DMA (0.4 mL, 0.5 M). Purification by flash column chromatography (60\% EtOAc in Petrol) afforded sulfonamide 9a as a colourless oil (56.8 mg, 77\%).

$\mathbf{R}_{f}\left(60 \%\right.$ EtOAc in Petrol) = 0.23; ${ }^{1} \mathrm{H}$ NMR $\left(400 \mathrm{MHz}, \mathrm{CDCl}_{3}\right) \delta 7.42-7.30(\mathrm{~m}, 5 \mathrm{H}), 5.13(\mathrm{~s}, 2 \mathrm{H}), 4.33$ (br. s, 2H), $3.76-3.68(\mathrm{~m}, 4 \mathrm{H}), 3.41-3.31(\mathrm{~m}, 4 \mathrm{H}), 3.06(\mathrm{tt}, J=12.0,3.5 \mathrm{~Hz}, 1 \mathrm{H}), 2.80$ (br. app. s, 2H), 2.06 (br. app. d, J $=12.5 \mathrm{~Hz}, 2 \mathrm{H}$ ), 1.82 - 1.65 (br. m, 2H); ${ }^{13} \mathrm{C}$ NMR $\left(101 \mathrm{MHz}, \mathrm{CDCl}_{3}\right) \delta$ 155.1, 136.6, 128.7, 128.3, 128.2, 67.6, 67.2, 59.5, 46.6, 43.0, 26.1; LRMS (ESI) $\mathrm{m} / \mathrm{z} 391.1$ ([M+Na] $]^{+}$; HRMS (ESI) $\mathrm{m} / \mathrm{z} \mathrm{C}_{17} \mathrm{H}_{24} \mathrm{~N}_{2} \mathrm{O}_{5} \mathrm{SNa}^{+}$requires $391.1298\left([\mathrm{M}+\mathrm{Na}]^{+}\right)$; found 391.1299; IR (thin film, $\left.v_{\max } / \mathrm{cm}^{-1}\right)$ 2918, 2861, 1775, 1705, 1431, 1179, 953; m.p. $\left(\mathrm{CH}_{2} \mathrm{Cl}_{2}\right) 101-104^{\circ} \mathrm{C}$.

Benzyl 4-((4-(2-chlorodibenzo[b,f][1,4]oxazepin-11-yl)piperazin-1-yl)sulfonyl)piperidine-1-carboxylate (9b) 


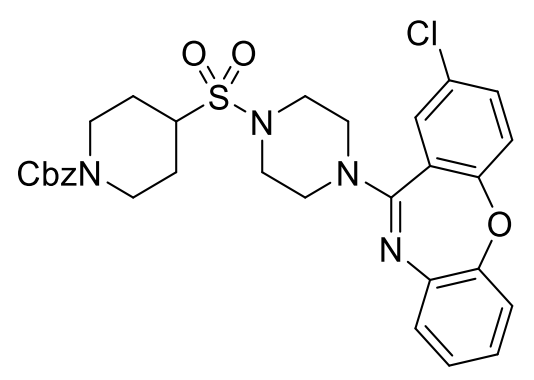

Prepared according to General Procedure G using 1-(1-((benzyloxy)carbonyl)piperidin-4-yl)-2,4,6triphenylpyridin-1-ium tetrafluoroborate 1a (122.5 mg, 0.20 mmol, 1.0 equiv.), Hantzsch ester ( $55.7 \mathrm{mg}, 0.22$ mmol, 1.1 equiv.), triethylamine (102 $\mu \mathrm{L}, 0.72 \mathrm{mmol}, 3.6$ equiv.), DABSO (28.8 mg, $0.12 \mathrm{mmol}, 0.6$ equiv.), NCS (53.4 mg, $0.40 \mathrm{mmol}, 2.0$ equiv.) and Amoxapine ( $125.5 \mathrm{mg}, 0.40 \mathrm{mmol}, 2.0$ equiv.) in DMA (0.4 mL, 0.5 M). Purification by flash column chromatography (20-30\% EtOAc in Petrol) afforded sulfonamide $9 \mathrm{~b}$ as a colourless oil (66.9 mg, 56\%).

$\mathbf{R}_{\boldsymbol{f}}\left(30 \%\right.$ EtOAc in Petrol) $=0.18 ;{ }^{1} \mathrm{H}$ NMR $\left(400 \mathrm{MHz}, \mathrm{CDCl}_{3}\right) \delta 7.41(\mathrm{dd}, J=8.5,2.5 \mathrm{~Hz}, 1 \mathrm{H}), 7.38-7.28(\mathrm{~m}, 6 \mathrm{H})$, $7.20(\mathrm{~d}, J=8.5 \mathrm{~Hz}, 1 \mathrm{H}), 7.17-7.07(\mathrm{~m}, 3 \mathrm{H}), 7.06-6.99(\mathrm{~m}, 1 \mathrm{H}), 5.13$ (s, 2H), 4.33 (app. br. s, 2H), 3.60 (app. br. s, 4H), 3.47 (app. br. s, 4H), 3.08 (tt, $J=12.0,3.5 \mathrm{~Hz}, 1 \mathrm{H}$ ), 2.79 (app. br. s, 2H), 2.07 (app. br. d, $J=12.0 \mathrm{~Hz}$, 2H), 1.84 - 1.64 (br. m, 2H); ${ }^{13} \mathrm{C}$ NMR (101 MHz, CDCl $) \delta$ 159.5, 158.5, 155.0, 151.8, 139.7, 136.6, 133.1, 130.6, 128.9, 128.7, 128.3, 128.1, 127.2, 126.0, 125.24, 124., 123.0, 120.3, 67.5, 59.6, 48.1, 46.0, 43.0, 26.1; LRMS (ESI) $\mathrm{m} / \mathrm{z} 595.2\left([\mathrm{M}+\mathrm{H}]^{+}\right)$; HRMS (ESI) $\mathrm{m} / \mathrm{z} \quad \mathrm{C}_{30} \mathrm{H}_{32} \mathrm{Cl}^{35} \mathrm{~N}_{4} \mathrm{O}_{5} \mathrm{~S}^{+}$requires $595.1776\left([\mathrm{M}+\mathrm{H}]^{+}\right)$; found 595.1770; IR (thin film, $v_{\max } / \mathrm{cm}^{-1}$ ) 2929, 2858, 1698, 1587, 1471, 1215, 1143.

\section{Benzyl 4-((6,7-dihydrothieno[3,2-c]pyridin-5(4H)-yl)sulfonyl)piperidine-1-carboxylate (9c)}

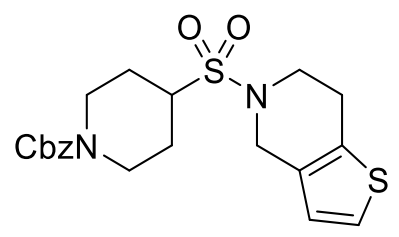

Prepared according to General Procedure G using 1-(1-((benzyloxy)carbonyl)piperidin-4-yl)-2,4,6triphenylpyridin-1-ium tetrafluoroborate 1a (122.5 mg, 0.20 mmol, 1.0 equiv.), Hantzsch ester (55.7 mg, 0.22 mmol, 1.1 equiv.), triethylamine (102 $\mu \mathrm{L}, 0.72 \mathrm{mmol}, 3.6$ equiv.), DABSO ( $28.8 \mathrm{mg}, 0.12 \mathrm{mmol}, 0.6$ equiv.), NCS (53.4 mg, $0.40 \mathrm{mmol}, 2.0$ equiv.) and 4,5,6,7-tetrahydrothieno[3,2-c]pyridine (55.7 mg, $0.40 \mathrm{mmol}, 2.0$ equiv.) in DMA (0.4 mL, $0.5 \mathrm{M})$. Purification by flash column chromatography ( $20-30 \%$ EtOAc in Petrol) afforded sulfonamide $9 \mathrm{c}$ as a colourless oil (68.2 $\mathrm{mg}, 81 \%)$.

$\mathbf{R}_{\boldsymbol{f}}\left(30 \%\right.$ EtOAc in Petrol) $=0.18 ;{ }^{1} \mathbf{H}$ NMR $\left(400 \mathrm{MHz}, \mathrm{CDCl}_{3}\right) \delta 7.42-7.29(\mathrm{~m}, 5 \mathrm{H}), 7.14(\mathrm{~d}, J=5.0 \mathrm{~Hz}, 1 \mathrm{H}), 6.75$ $(\mathrm{d}, J=5.0 \mathrm{~Hz}, 1 \mathrm{H}), 5.12(\mathrm{~s}, 2 \mathrm{H}), 4.45(\mathrm{~s}, 2 \mathrm{H}), 4.31$ (app. br. s, 2H), $3.66(\mathrm{t}, J=5.5 \mathrm{~Hz}, 2 \mathrm{H}), 3.10(\mathrm{tt}, J=12.0,3.5$ Hz, 1H), $2.92(t, J=5.5 \mathrm{~Hz}, 2 \mathrm{H}$ ), 2.78 (app. br. s, 2H), 2.06 (app. br. d, $J=12.5 \mathrm{~Hz}, 2 \mathrm{H}$ ), $1.83-1.66$ (br. m, 2H); ${ }^{13} \mathrm{C}$ NMR $\left(101 \mathrm{MHz}, \mathrm{CDCl}_{3}\right) \delta$ 155.0, 136.5, 133.0, 131.1, 128.6, 128.2, 128.1, 124.6, 123.8, 67.4, 59.9, 46.0, 
44.1, 43.0, 26.1, 25.9; LRMS (ESI) $\mathrm{m} / \mathrm{z} 443.2$ ([M+Na $]^{+}$); HRMS (ESI) $m / z \mathrm{C}_{20} \mathrm{H}_{24} \mathrm{~N}_{2} \mathrm{O}_{4} \mathrm{~S}_{2} \mathrm{Na}^{+}$requires 443.1070 $\left([\mathrm{M}+\mathrm{Na}]^{+}\right)$; found 443.1068; IR (thin film, $\left.v_{\max } / \mathrm{cm}^{-1}\right)$ 2960, 2861, 1695, 1430, 1318, 1215, 1139.

Benzyl 4-((1H-imidazol-1-yl)sulfonyl)piperidine-1-carboxylate (9d)

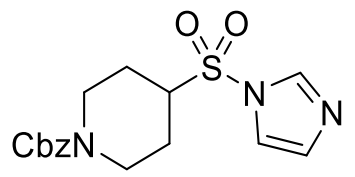

Prepared according to General Procedure G using 1-(1-((benzyloxy)carbonyl)piperidin-4-yl)-2,4,6triphenylpyridin-1-ium tetrafluoroborate 1a (122.5 mg, 0.20 mmol, 1.0 equiv.), Hantzsch ester (55.7 mg, 0.22 mmol, 1.1 equiv.), triethylamine (102 $\mu \mathrm{L}, 0.72 \mathrm{mmol}, 3.6$ equiv.), DABSO ( $28.8 \mathrm{mg}, 0.12 \mathrm{mmol}, 0.6$ equiv.), NCS (53.4 mg, $0.40 \mathrm{mmol}, 2.0$ equiv.) and imidazole ( $27.2 \mathrm{mg}, 0.40 \mathrm{mmol}, 2.0$ equiv.) in DMA (0.4 mL, $0.5 \mathrm{M}$ ). Purification by flash column chromatography (70\% EtOAc in Petrol) afforded sulfonamide $9 \mathrm{~d}$ as a colourless oil (46.1 mg, 66\%).

$\mathbf{R}_{\boldsymbol{f}}(70 \%$ EtOAc in Petrol $)=0.19 ;{ }^{1} \mathbf{H}$ NMR $\left(400 \mathrm{MHz}, \mathrm{CDCl}_{3}\right) \delta 7.83(\mathrm{~s}, 1 \mathrm{H}), 7.34-7.20(\mathrm{~m}, 5 \mathrm{H}), 7.19(\mathrm{t}, J=1.5$ Hz, 1H), $7.15-7.08(\mathrm{~m}, 1 \mathrm{H}$ ), 5.04 (s, 2H), 4.27 (app. br. s, 2H), 3.24 (tt, $J=12.0,3.5 \mathrm{~Hz}, 1 \mathrm{H}$ ), 2.70 (app. br. s, 2H), 1.92 (app. br. $d, J=12.5 \mathrm{~Hz}, 2 \mathrm{H}), 1.67-1.54(\mathrm{~m}, 2 \mathrm{H}) ;{ }^{13} \mathrm{C} \mathrm{NMR}\left(101 \mathrm{MHz}, \mathrm{CDCl}_{3}\right) \delta$ 154.9, 137.5, 136.3, 131.6, 128.7, 128.4, 128.2, 118.4, 67.8, 62.8, 42.6, 25.7; LRMS (ESI) $\mathrm{m} / \mathrm{z} 350.1$ ([M+H] $]^{+}$); HRMS (ESI) $\mathrm{m} / \mathrm{z}$ $\mathrm{C}_{16} \mathrm{H}_{20} \mathrm{~N}_{3} \mathrm{O}_{4} \mathrm{~S}^{+}$requires $350.1169\left([\mathrm{M}+\mathrm{H}]^{+}\right)$; found 350.1168; IR (thin film, $v_{\max } / \mathrm{cm}^{-1}$ ) 3123, 2960, 1696, 1430, $1376,1219,1168$.

\section{1-(Benzylsulfonyl)pyrrolidine (9e)}

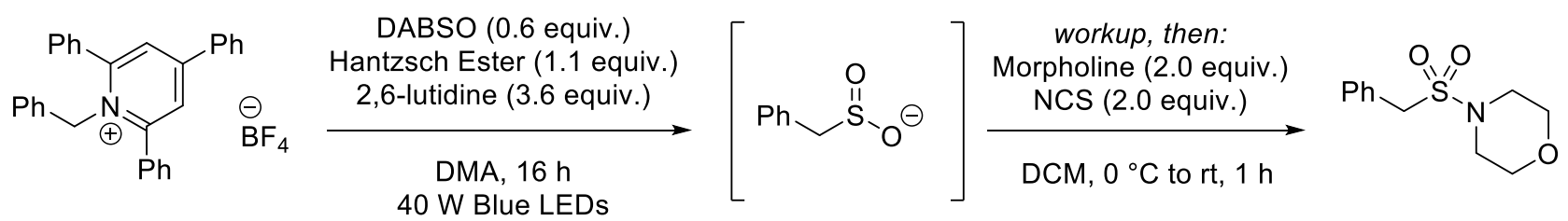

Hantzsch ester (55.2 mg, $0.22 \mathrm{mmol}, 1.1$ equiv.), DABSO (28.8 mg, $0.12 \mathrm{mmol}, 0.6$ equiv.) and 1-benzyl-2,4,6triphenylpyridin-1-ium tetrafluoroborate $1 \mathrm{~b}(97.1 \mathrm{mg}, 0.20 \mathrm{mmol}, 1.0$ equiv.) were added to an oven-dried $1.9 \mathrm{~mL}$ reaction vial. The vial was then sealed with a rubber septum, evacuated and backfilled with $\mathrm{N}_{2}(\times 3)$. Degassed, dry DMA (0.4 mL, $0.5 \mathrm{M})$ was then added, followed by degassed, dry 2,6-lutidine ( $84 \mu \mathrm{L}, 0.72 \mathrm{mmol}$, 3.6 equiv.). The reaction was stirred under blue LED irradiation at ambient temperature for $16 \mathrm{~h}$. Upon completion, aq. $\mathrm{H}_{3} \mathrm{PO}_{4}(3 \mathrm{~mL}, 2 \mathrm{M})$ and brine $(1 \mathrm{~mL})$ were added to the mixture. The mixture was then extracted with $\mathrm{CH}_{2} \mathrm{Cl}_{2}(3 \times 2 \mathrm{~mL})$. The combined organic phase was transferred to a round-bottom flask and DIPEA (105 $\mu \mathrm{L}, 0.60 \mathrm{mmol}, 3.0$ equiv.) was added. NCS ( $53.4 \mathrm{mg}, 0.40 \mathrm{mmol}, 2.0$ equiv.) and morpholine (34.5 $\mu \mathrm{L}, 0.40 \mathrm{mmol}, 2.0$ equiv.) were added and the reaction stirred at $\mathrm{rt}$ for $1 \mathrm{~h}$. The crude reaction mixture was concentrated in vacuo, and the crude reaction mixture was then loaded directly to silica gel and purified by 
flash column chromatography (30\% EtOAc in Petrol) to afford sulfonamide 9 e as a white crystalline solid (33.8 $\mathrm{mg}, 70 \%)$.

Also prepared according a modified procedure using $\mathrm{Na}_{2} \mathrm{~S}_{2} \mathrm{O}_{5}(45.6 \mathrm{mg}, 0.24 \mathrm{mmol}, 1.2$ equiv.) instead of DABSO, and no 2,6-lutidine was added. NCS $(53.4 \mathrm{mg}, 0.40 \mathrm{mmol}, 2.0$ equiv.) and morpholine (34.5 $\mu \mathrm{L}, 0.40$ mmol, 2.0 equiv.) were added to the crude reaction mixture without workup, affording the title product (33.9 $\mathrm{mg}, 70 \%)$.

$\mathbf{R}_{f}\left(30 \%\right.$ EtOAc in Petrol) $=0.18 ;{ }^{1} \mathbf{H}$ NMR $\left(400 \mathrm{MHz}, \mathrm{CDCl}_{3}\right) \delta 7.48-7.32(\mathrm{~m}, 5 \mathrm{H}), 4.23(\mathrm{~s}, 2 \mathrm{H}), 3.61(\mathrm{t}, J=4.5$ $\mathrm{Hz}, 4 \mathrm{H}), 3.10(\mathrm{t}, J=4.5 \mathrm{~Hz}, 4 \mathrm{H}) ;{ }^{13} \mathrm{C}$ NMR $\left(101 \mathrm{MHz}, \mathrm{CDCl}_{3}\right) \delta 130.8,129.0,129.0,128.7,66.8,56.9,46.2$; LRMS (ESI) $\mathrm{m} / \mathrm{z} 264.0\left([\mathrm{M}+\mathrm{Na}]^{+}\right)$; HRMS (ESI) $\mathrm{m} / z \mathrm{C}_{11} \mathrm{H}_{16} \mathrm{NO}_{3} \mathrm{~S}^{+}$requires $242.0845\left([\mathrm{M}+\mathrm{H}]^{+}\right)$, found 242.0847.

Data is consistent with the literature. ${ }^{32}$

\section{4-(Phenethylsulfonyl)morpholine (9f)}

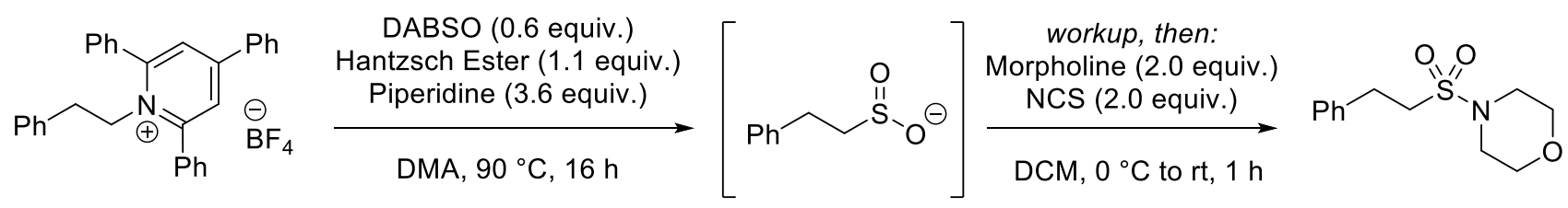

Hantzsch ester ( $55.2 \mathrm{mg}, 0.22 \mathrm{mmol}, 1.1$ equiv.), DABSO ( $28.8 \mathrm{mg}, 0.12 \mathrm{mmol}, 0.6$ equiv.) and 1-phenethyl2,4,6-triphenylpyridin-1-ium tetrafluoroborate $1 \mathrm{c}$ (100 mg, $0.20 \mathrm{mmol}, 1.0$ equiv.) were added to an ovendried $1.9 \mathrm{~mL}$ reaction vial. The vial was then sealed with a rubber septum, evacuated and backfilled with $\mathrm{N}_{2}$ (×3). Degassed, dry DMA (0.4 mL, 0.5 M) was then added, followed by degassed, dry piperidine $(71 \mu \mathrm{L}, 0.72$ mmol, 3.6 equiv.). The reaction was heated to $90^{\circ} \mathrm{C}$ in an aluminium heating block for $16 \mathrm{~h}$. Upon completion, aq. $\mathrm{H}_{3} \mathrm{PO}_{4}(3 \mathrm{~mL}, 2 \mathrm{M})$ and brine $(1 \mathrm{~mL})$ were added to the mixture. The mixture was then extracted with $\mathrm{CH}_{2} \mathrm{Cl}_{2}(3 \times 2 \mathrm{~mL})$. The combined organic phase was transferred to a round-bottom flask and DIPEA (105 $\mu \mathrm{L}$, $0.60 \mathrm{mmol}, 3.0$ equiv.) was added. NCS $(53.4 \mathrm{mg}, 0.40 \mathrm{mmol}, 2.0$ equiv.) and morpholine ( $34.5 \mu \mathrm{L}, 0.40 \mathrm{mmol}$, 2.0 equiv.) were added and the reaction stirred at $\mathrm{rt}$ for $1 \mathrm{~h}$. The crude reaction mixture was concentrated in vacuo, and the crude reaction mixture was then loaded directly to silica gel and purified by flash column chromatography (30\% EtOAc in Petrol) to afford sulfonamide 9 f as a white crystalline solid (34.3 mg, 67\%).

$\mathbf{R}_{f}\left(30 \% \mathrm{CH}_{2} \mathrm{Cl}_{2}\right.$ in Petrol) $=0.24 ;{ }^{1} \mathbf{H}$ NMR $\left(400 \mathrm{MHz}, \mathrm{CDCl}_{3}\right) \delta 7.37-7.29(\mathrm{~m}, 2 \mathrm{H}), 7.29-7.19(\mathrm{~m}, 3 \mathrm{H}), 3.83-$

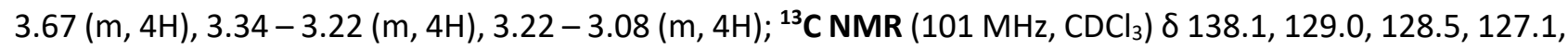
66.7, 50.5, 45.9, 29.4; LRMS (ESI) $\mathrm{m} / \mathrm{z} 278.0$ ([M+Na] $]^{+}$); HRMS (ESI) $\mathrm{m} / z \mathrm{C}_{12} \mathrm{H}_{18} \mathrm{NO}_{3} \mathrm{~S}^{+}$requires 256.1002 $\left([\mathrm{M}+\mathrm{H}]^{+}\right)$, found 256.1003; IR (thin film, $\left.v_{\max } / \mathrm{cm}^{-1}\right)$ 2920, 2857, 1453, 1323, 1156, 1111, 949; m.p. $\left(\mathrm{CH}_{2} \mathrm{Cl}_{2}\right) 78$ $-80^{\circ} \mathrm{C}$.

\section{1-(Phenethylsulfonyl)piperidine (9g)}




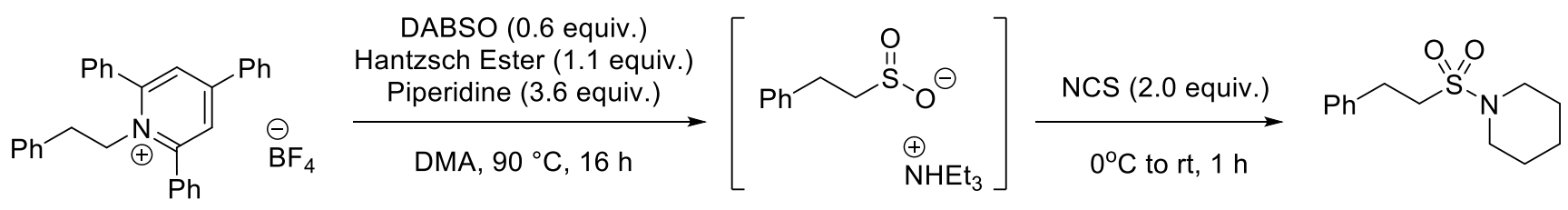

Hantzsch ester ( $55.7 \mathrm{mg}, 0.22 \mathrm{mmol}, 1.1$ equiv.), DABSO ( $28.8 \mathrm{mg}, 0.12 \mathrm{mmol}, 0.6$ equiv.) and 1-phenethyl2,4,6-triphenylpyridin-1-ium tetrafluoroborate $1 \mathrm{c}(99.9 \mathrm{mg}, 0.20 \mathrm{mmol}, 1.0$ equiv.) were added to an ovendried $1.9 \mathrm{~mL}$ reaction vial. The vial was then sealed with a rubber septum, evacuated and backfilled with $\mathrm{N}_{2}$ (× 3). Degassed, dry DMA (0.4 mL, $0.5 \mathrm{M})$ was then added, followed by degassed, dry piperidine $(71 \mu \mathrm{L}, 0.72$ mmol, 3.6 equiv.). The reaction was heated to $90^{\circ} \mathrm{C}$ in an aluminium heating block for $16 \mathrm{~h}$. After cooling the solution to $0{ }^{\circ} \mathrm{C}, \mathrm{NCS}(53.4 \mathrm{mg}, 0.40 \mathrm{mmol}, 2.0$ equiv.) was added and the reaction stirred at $\mathrm{rt}$ for $1 \mathrm{~h}$. Without workup, the crude reaction mixture was loaded directly to silica gel and purified by flash column chromatography ( $70-80 \% \mathrm{CH}_{2} \mathrm{Cl}_{2}$ in Petrol) to afford sulfonamide $9 \mathrm{~g}$ as a white crystalline solid (37.1 $\mathrm{mg}$, $73 \%)$.

$\mathbf{R}_{f}\left(80 \% \mathrm{CH}_{2} \mathrm{Cl}_{2}\right.$ in Petrol $)=0.28 ;{ }^{1} \mathbf{H}$ NMR $\left(400 \mathrm{MHz}, \mathrm{CDCl}_{3}\right) \delta 7.36-7.29(\mathrm{~m}, 2 \mathrm{H}), 7.27-7.18(\mathrm{~m}, 3 \mathrm{H}), 3.28-$ $3.21(\mathrm{~m}, 4 \mathrm{H}), 3.13$ (app. s, 4H), $1.70-1.60(\mathrm{~m}, 4 \mathrm{H}), 1.60-1.52(\mathrm{~m}, 2 \mathrm{H}) ;{ }^{13} \mathrm{C}$ NMR $\left(101 \mathrm{MHz}, \mathrm{CDCl}_{3}\right) \delta 138.5$, 129.0, 128.5, 127.0, 50.9, 46.8, 29.5, 25.8, 24.0; LRMS (ESI) $\mathrm{m} / \mathrm{z} 254.2\left([\mathrm{M}+\mathrm{H}]^{+}\right), 276.0\left([\mathrm{M}+\mathrm{Na}]^{+}\right)$; HRMS (ESI) $m / z \mathrm{C}_{13} \mathrm{H}_{20} \mathrm{NO}_{2} \mathrm{~S}^{+}$requires $254.1209\left([\mathrm{M}+\mathrm{H}]^{+}\right)$, found 254.1209 .

Data is consistent with the literature. ${ }^{33}$ 


\subsection{Three-step synthesis of secondary sulfonamides}

\section{General Procedure H:}

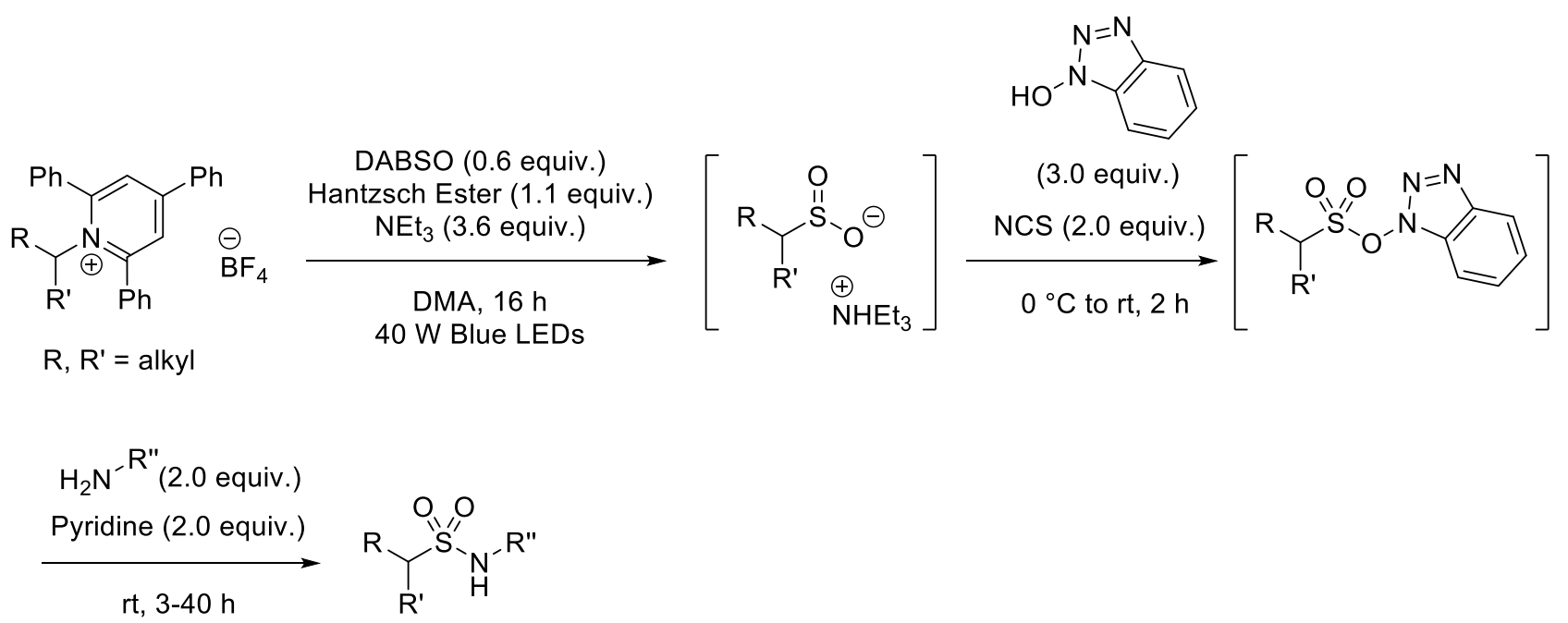

Hantzsch ester (1.1 equiv.), DABSO (0.6 equiv.) and Katritzky pyridinium salt (1.0 equiv.) were added to an oven-dried $1.9 \mathrm{~mL}$ reaction vial. The vial was then sealed with a rubber septum, evacuated and backfilled with $\mathrm{N}_{2}(\times 3)$. Degassed, dry DMA (0.5 M) was then added, followed by degassed, dry triethylamine (3.6 equiv.). The reaction was stirred under blue LED irradiation at ambient temperature for $16 \mathrm{~h}$. HOBt hydrate (80\%) (3.0 equiv.) was then added and the reaction stirred for 30 mins. After cooling the solution to $0{ }^{\circ} \mathrm{C}, \mathrm{NCS}$ (2.0 equiv.) was added and the reaction stirred at $\mathrm{rt}$ for $2 \mathrm{~h}$. Pyridine (2.0 equiv.) and primary amine (2.0 equiv.) were then added and the reaction stirred at rt until completion. Without workup, the crude reaction mixture was loaded directly to silica gel and purified by flash column chromatography to afford the sulfonamide product.

Amine hydrochloride salts could also be used to prepare sulfonamides. Amine hydrochloride (3.0 equiv.) and DIPEA (3.0 equiv.) were stirred for 30 mins in DMA $(0.2 \mathrm{~mL}, 0.3 \mathrm{M})$ before adding to the crude reaction mixture containing the HOBt sulfonate ester and the reaction stirred until completion. The sulfonamide product was then isolated as above.

Benzyl 4-( $N$-benzylsulfamoyl)piperidine-1-carboxylate (10a)<smiles>O=C(c1ccccc1)N1CCC(S(=O)(=O)NCc2ccccc2)CC1</smiles>

Prepared according to General Procedure H using 1-(1-((benzyloxy)carbonyl)piperidin-4-yl)-2,4,6triphenylpyridin-1-ium tetrafluoroborate 1a (122.5 mg, 0.20 mmol, 1.0 equiv.), Hantzsch ester ( $55.7 \mathrm{mg}, 0.22$ mmol, 1.1 equiv.), triethylamine (102 $\mu \mathrm{L}, 0.72 \mathrm{mmol}, 3.6$ equiv.), DABSO ( $28.8 \mathrm{mg}, 0.12 \mathrm{mmol}, 0.6$ equiv.), HOBt (80\%) (101.3 mg, $0.60 \mathrm{mmol}, 3.0$ equiv.), NCS (53.4 mg, $0.40 \mathrm{mmol}, 2.0$ equiv.) and benzylamine (44 $\mu \mathrm{L}$, $0.40 \mathrm{mmol}, 2.0$ equiv.) and pyridine ( $32 \mu \mathrm{L}, 0.40 \mathrm{mmol}, 2.0$ equiv.) in DMA (0.4 mL, $0.5 \mathrm{M})$. Purification by 
flash column chromatography (40\% EtOAc in Petrol) afforded sulfonamide $10 \mathrm{a}$ as a white solid (58.6 mg, $75 \%)$.

$\mathbf{R}_{\boldsymbol{f}}\left(40 \%\right.$ EtOAc in Petrol) $=0.18 ;{ }^{1} \mathrm{H}$ NMR $\left(400 \mathrm{MHz}, \mathrm{CDCl}_{3}\right) \delta 7.39-7.27(\mathrm{~m}, 10 \mathrm{H}), 5.11(\mathrm{~s}, 2 \mathrm{H}), 4.88$ (app. s, 1H), $4.36-4.17$ (br. m, 4H), 2.83 (tt, $J=12.0,3.5 \mathrm{~Hz}, 1 \mathrm{H}$ ), 2.67 (app. br. s, 2H), 2.03 (app. br. d, $J=12.5 \mathrm{~Hz}$, 2H), 1.75 - 1.59 (br. m, 2H); ${ }^{13} \mathrm{C}$ NMR (101 MHz, CDCl $) \delta 155.1,137.3,136.5,128.9,128.6,128.2,128.2$, 128.1, 128.0, 67.5, 59.9, 47.6, 42.9, 25.9; LRMS (ESI) $\mathrm{m} / \mathrm{z} 411.2\left([\mathrm{M}+\mathrm{Na}]^{+}\right), 387.2\left([\mathrm{M}-\mathrm{H}]^{-}\right)$; HRMS (ESI) $\mathrm{m} / \mathrm{z}$ $\mathrm{C}_{20} \mathrm{H}_{25} \mathrm{~N}_{2} \mathrm{O}_{4} \mathrm{~S}^{+}$requires $389.1530\left([\mathrm{M}+\mathrm{H}]^{+}\right)$; found 389.1523; IR (thin film, $v_{\max } / \mathrm{cm}^{-1}$ ) 3256, 2980, 1684, 1435, $1319,1217,1141 ;$ m.p. $\left(\mathrm{CH}_{2} \mathrm{Cl}_{2}\right) 121-124^{\circ} \mathrm{C}$.

\section{Benzyl 4-(N-(4-methoxyphenyl)sulfamoyl)piperidine-1-carboxylate (10b)}<smiles>COc1ccc(NS(=O)(=O)C2CCN(C(=O)OCc3ccccc3)CC2)cc1</smiles>

Prepared according to General Procedure H using 1-(1-((benzyloxy)carbonyl)piperidin-4-yl)-2,4,6triphenylpyridin-1-ium tetrafluoroborate $1 \mathrm{a}$ ( $122.5 \mathrm{mg}, 0.20 \mathrm{mmol}, 1.0$ equiv.), Hantzsch ester (55.7 mg, 0.22 mmol, 1.1 equiv.), triethylamine (102 $\mu \mathrm{L}, 0.72 \mathrm{mmol}, 3.6$ equiv.), DABSO ( $28.8 \mathrm{mg}, 0.12 \mathrm{mmol}, 0.6$ equiv.), HOBt (80\%) (101.3 mg, $0.60 \mathrm{mmol}, 3.0$ equiv.), NCS (53.4 mg, $0.40 \mathrm{mmol}, 2.0$ equiv.) and $p$-anisidine (49.3 $\mathrm{mg}, 0.40 \mathrm{mmol}, 2.0$ equiv.) and pyridine ( $32 \mu \mathrm{L}, 0.40 \mathrm{mmol}, 2.0$ equiv.) in DMA ( $0.4 \mathrm{~mL}, 0.5 \mathrm{M}$ ). Purification by flash column chromatography $\left(70-80 \% \mathrm{Et}_{2} \mathrm{O}\right.$ in Petrol) afforded sulfonamide $10 \mathrm{~b}$ as an off-white solid (64.3 mg, 79\%).

$\mathbf{R}_{f}\left(40 \%\right.$ EtOAc in Petrol) $=0.2 ;{ }^{1} \mathbf{H}$ NMR $\left(400 \mathrm{MHz}, \mathrm{CDCl}_{3}\right) \delta 7.39-7.28(\mathrm{~m}, 5 \mathrm{H}), 7.21-7.14(\mathrm{~m}, 2 \mathrm{H}), 6.88-$ $6.83(\mathrm{~m}, 2 \mathrm{H}), 5.11$ (s, 2H), 4.28 (app. br. s, 2H), 3.79 (s, 3H), 3.09 (tt, $J=12.0,3.5 \mathrm{~Hz}, 1 \mathrm{H}$ ), 2.75 (app. br. s, 2H), 2.07 (app. br. d, $J=11.5 \mathrm{~Hz}, 2 \mathrm{H}), 1.87-1.69$ (br. m, 2H); ${ }^{13} \mathrm{C}$ NMR $\left(101 \mathrm{MHz}, \mathrm{CDCl}_{3}\right) \delta 13 \mathrm{C} \mathrm{NMR} \mathrm{(101} \mathrm{MHz,}$ $\mathrm{CDCl}_{3}$ ) $\delta 157.9,155.1,136.5,129.3,128.7,128.3,128.1,124.2,114.9,67.6,58.0,55.6,42.9,25.9 ;$ LRMS (ESI) $\mathrm{m} / \mathrm{z} 427.0\left([\mathrm{M}+\mathrm{Na}]^{+}\right), 403.0\left([\mathrm{M}-\mathrm{H}]^{-}\right)$; HRMS (ESI) $\mathrm{m} / \mathrm{z} \mathrm{C}_{20} \mathrm{H}_{24} \mathrm{~N}_{2} \mathrm{O}_{5} \mathrm{SNa}^{+}$requires $427.1298\left([\mathrm{M}+\mathrm{Na}]^{+}\right)$; found 427.1300; IR (thin film, $v_{\max } / \mathrm{cm}^{-1}$ ) 3250, 2960, 1675, 1510, 1280, 1213, 1147; m.p. $\left(\mathrm{CH}_{2} \mathrm{Cl}_{2}\right) 104-107^{\circ} \mathrm{C}$.

Benzyl (S)-4-(N-(1-methoxy-1-oxopropan-2-yl)sulfamoyl)piperidine-1-carboxylate (10c)<smiles>COC(=O)C(Cc1ccccc1)NS(=O)(=O)C1CCN(C(=O)OCc2ccccc2)CC1</smiles>

Prepared according to General Procedure H using 1-(1-((benzyloxy)carbonyl)piperidin-4-yl)-2,4,6triphenylpyridin-1-ium tetrafluoroborate $1 \mathrm{a}(122.5 \mathrm{mg}, 0.20 \mathrm{mmol}, 1.0$ equiv.), Hantzsch ester (55.7 mg, 0.22 mmol, 1.1 equiv.), triethylamine (102 $\mu \mathrm{L}, 0.72 \mathrm{mmol}, 3.6$ equiv.), DABSO (28.8 mg, $0.12 \mathrm{mmol}, 0.6$ equiv.), HOBt (80\%) (101.3 mg, $0.60 \mathrm{mmol}, 3.0$ equiv.), NCS (53.4 mg, $0.40 \mathrm{mmol}, 2.0$ equiv.) and L-Alanine methyl 
ester hydrochloride ( $55.8 \mathrm{mg}, 0.40 \mathrm{mmol}, 2.0$ equiv.) and pyridine $(32 \mu \mathrm{L}, 0.40 \mathrm{mmol}, 2.0$ equiv.) in DMA (0.4 $\mathrm{mL}, 0.5 \mathrm{M}$ ). Purification by flash column chromatography (40\% EtOAc in Petrol) afforded sulfonamide 10c as a pale-yellow oil $(67.5 \mathrm{mg}, 73 \%)$.

$\mathbf{R}_{f}\left(40 \%\right.$ EtOAc in Petrol) $=0.18 ;{ }^{1} \mathrm{H}$ NMR $\left(400 \mathrm{MHz}, \mathrm{CDCl}_{3}\right) \delta 7.37-7.06(\mathrm{~m}, 10 \mathrm{H}), 5.03(\mathrm{~s}, 2 \mathrm{H}), 4.86(\mathrm{~d}, J=9.5$ Hz, 1H), 4.21 (td, J = 9.0, 4.5 Hz, 1H), 4.07 (app. br. s, 2H), 3.69 (s, 3H), 3.09 (dd, J = 14.0, 4.5 Hz, 1H), 2.82 (dd, $J=14.0,9.0 \mathrm{~Hz}, 1 \mathrm{H}$ ), 2.46 (app. br. s, 3H), 1.78 (app. br. d, $J=12.5 \mathrm{~Hz}, 1 \mathrm{H}$ ), 1.65 (app. br. d, $J=12.5 \mathrm{~Hz}, 1 \mathrm{H}$ ), 1.41 (qd, $J=12.5,4.5 \mathrm{~Hz}, 1 \mathrm{H}), 1.29$ (qd, $J=12.5,4.5 \mathrm{~Hz}, 1 \mathrm{H}) ;{ }^{13} \mathrm{C} \mathrm{NMR}\left(101 \mathrm{MHz}, \mathrm{CDCl}_{3}\right) \delta 172.4,155.0,136.6$, 136.0, 129.6, 128.9, 128.7, 128.3, 128.1, 127.6, 67.5, 60.2, 58.0, 52.9, 42.8, 42.8, 39.6, 25.5; LRMS (ESI) $\mathrm{m} / \mathrm{z}$ $483.2\left([\mathrm{M}+\mathrm{Na}]^{+}\right)$, $\left.459.1([\mathrm{M}-\mathrm{H}]]^{-}\right)$; HRMS (ESI) $\mathrm{m} / z \mathrm{C} \mathrm{C}_{23} \mathrm{H}_{29} \mathrm{~N}_{2} \mathrm{O}_{6} \mathrm{~S}^{+}$requires $461.1741\left([\mathrm{M}+\mathrm{H}]^{+}\right)$; found 461.1740; IR (thin film, $v_{\max } / \mathrm{cm}^{-1}$ ) 3251, 2957, 1745, 1704, 1434, 1279, 1217; ee determined by chiral SFC using a Chiralpak ${ }^{\circledR} \mathrm{IC}$ column, 1500 psi, $30^{\circ} \mathrm{C}$; flow: $1.5 \mathrm{~mL} / \mathrm{min}$; 1 - 30\% MeOH in $5 \mathrm{~min}, 98 \%$ ee (major enantiomer $t_{R}=4.305$ min, minor enantiomer $\left.t_{R}=4.497 \mathrm{~min}\right) ;[\alpha]_{D}^{25}-13.0^{\circ}\left(c=1.0, \mathrm{CHCl}_{3}\right)$. 


\subsection{Characterisation of side-products and TEMPO products}

\section{1,4-Dibenzyl-2,4,6-triphenyl-1,4-dihydropyridine (5b)}<smiles></smiles>

Hantzsch ester (55.2 mg, $0.22 \mathrm{mmol}, 1.1$ equiv.), DABSO (28.8 mg, $0.12 \mathrm{mmol}, 0.6$ equiv.) and 1-benzyl-2,4,6triphenylpyridin-1-ium tetrafluoroborate $1 \mathbf{b}(97.1 \mathrm{mg}, 0.20 \mathrm{mmol}, 1.0$ equiv.) were added to an oven-dried $1.9 \mathrm{~mL}$ reaction vial. The vial was then sealed with a rubber septum, evacuated and backfilled with $\mathrm{N}_{2}(\times 3)$. Degassed, dry DMA (0.4 mL, 0.5 M) was then added, followed by degassed, dry triethylamine (102 $\mu \mathrm{L}, 0.72$ mmol, 3.6 equiv.). The reaction was stirred under blue LED irradiation at ambient temperature for $16 \mathrm{~h}$. Without workup, the crude reaction mixture was loaded directly to silica gel and purified by flash column chromatography (20\% $\mathrm{CH}_{2} \mathrm{Cl}_{2}$ in Petrol) to afford dihydropyridine $\mathbf{5 b}$ as a colourless oil $(9.6 \mathrm{mg}, 20 \%)$.

$\mathbf{R}_{f}\left(20 \% \mathrm{CH}_{2} \mathrm{Cl}_{2}\right.$ in Petrol $)=0.27 ;{ }^{1} \mathrm{H}_{\mathrm{NMR}}\left(400 \mathrm{MHz}, \mathrm{CDCl}_{3}\right) \delta 7.52-7.46(\mathrm{~m}, 4 \mathrm{H}), 7.43-7.32(\mathrm{~m}, 6 \mathrm{H}), 7.24-$ $7.19(\mathrm{~m}, 4 \mathrm{H}), 7.15-6.99(\mathrm{~m}, 5 \mathrm{H}), 6.99-6.93(\mathrm{~m}, 2 \mathrm{H}), 6.70(\mathrm{dt}, J=8.0,2.5 \mathrm{~Hz}, 2 \mathrm{H}), 6.65-6.60(\mathrm{~m}, 2 \mathrm{H}), 5.12$ (s, 2H), $4.18(\mathrm{~s}, 2 \mathrm{H}), 2.93(\mathrm{~s}, 2 \mathrm{H}) ;{ }^{13} \mathrm{C}$ NMR $\left(101 \mathrm{MHz}, \mathrm{CDCl}_{3}\right) \delta$ 149.9, 142.5, 138.3, 138.3, 137.6, 131.0, 128.5, 128.4, 128.3, 128.1, 128.1, 127.9, 127.4, 127.1, 127.0, 125.9, 125.4, 114.3, 51.9, 51.6, 44.6; LRMS (ESI) $\mathrm{m} / \mathrm{z}$ $490.2\left([\mathrm{M}+\mathrm{H}]^{+}\right)$; HRMS (ESI) $m / z \mathrm{C}_{37} \mathrm{H}_{32} \mathrm{~N}^{+}$requires $490.2529\left([\mathrm{M}+\mathrm{H}]^{+}\right)$, found 490.2529; IR (thin film, $v_{\max } / \mathrm{cm}^{-}$ $\left.{ }^{1}\right) 3028,1655,1600,1494,1446,1387,698$.

\section{1,4-Diphenethyl-2,4,6-triphenyl-1,4-dihydropyridine (5c)}<smiles>C1=C(c2ccccc2)N(CCc2ccccc2)C(c2ccccc2)=CC1(CCc1ccccc1)c1ccccc1</smiles>

Hantzsch ester ( $55.2 \mathrm{mg}, 0.22 \mathrm{mmol}, 1.1$ equiv.), DABSO ( $28.8 \mathrm{mg}, 0.12 \mathrm{mmol}, 0.6$ equiv.) and 1-phenethyl2,4,6-triphenylpyridin-1-ium tetrafluoroborate $1 \mathrm{c}(99.9 \mathrm{mg}, 0.20 \mathrm{mmol}, 1.0$ equiv.) were added to an ovendried $1.9 \mathrm{~mL}$ reaction vial. The vial was then sealed with a rubber septum, evacuated and backfilled with $\mathrm{N}_{2}$ (× 3). Degassed, dry DMA (0.4 mL, $0.5 \mathrm{M})$ was then added, followed by degassed, dry triethylamine (102 $\mu \mathrm{L}$, $0.72 \mathrm{mmol}, 3.6$ equiv.). The reaction was stirred under blue LED irradiation at ambient temperature for $16 \mathrm{~h}$. Without workup, the crude reaction mixture was loaded directly to silica gel and purified by flash column chromatography ( $20 \% \mathrm{CH}_{2} \mathrm{Cl}_{2}$ in Petrol) to afford dihydropyridine $\mathbf{5 c}$ as a colourless oil ( $\left.83 \mathrm{mg}, 14 \%\right)$.

$\mathbf{R}_{f}\left(20 \% \mathrm{CH}_{2} \mathrm{Cl}_{2}\right.$ in Petrol $)=0.27 ;{ }^{1} \mathbf{H}$ NMR $\left(400 \mathrm{MHz}, \mathrm{CDCl}_{3}\right) \delta 7.61-7.51(\mathrm{~m}, 6 \mathrm{H}), 7.46-7.32(\mathrm{~m}, 7 \mathrm{H}), 7.25-$ $7.08(\mathrm{~m}, 5 \mathrm{H}), 7.02-6.94(\mathrm{~m}, 3 \mathrm{H}), 6.47(\mathrm{dt}, J=7.5,3.5 \mathrm{~Hz}, 2 \mathrm{H}), 5.17(\mathrm{~s}, 2 \mathrm{H}), 3.29-3.17(\mathrm{~m}, 2 \mathrm{H}), 2.72-2.62$ (m, 2H), $2.37-2.26(\mathrm{~m}, 2 \mathrm{H}), 2.19-2.10(\mathrm{~m}, 2 \mathrm{H}) ;{ }^{13} \mathrm{C}$ NMR $\left(101 \mathrm{MHz}, \mathrm{CDCl}_{3}\right) \delta 151.5,143.2,139.3,138.2$, 
128.6, $128.5,128.5,128.4,128.2,128.2,128.2,126.5,125.9,125.7,125.7,113.4,50.1,46.8,44.0,34.9,32.8$; LRMS (ESI) $m / z 518.2\left([\mathrm{M}+\mathrm{H}]^{+}\right)$; HRMS (ESI) $\mathrm{m} / z \mathrm{C}_{39} \mathrm{H}_{36} \mathrm{~N}^{+}$requires $518.2842\left([\mathrm{M}+\mathrm{H}]^{+}\right)$, found 518.2842 .

Data is consistent with the literature. ${ }^{1}$

1-(Benzyloxy)-2,2,6,6-tetramethylpiperidine (11)

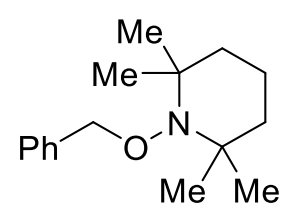

Hantzsch ester (55.2 mg, $0.22 \mathrm{mmol}, 1.1$ equiv.), DABSO (28.8 mg, $0.12 \mathrm{mmol}, 0.6$ equiv.), 1-benzyl-2,4,6triphenylpyridin-1-ium tetrafluoroborate $\mathbf{1 b}(97.1 \mathrm{mg}, 0.20 \mathrm{mmol}, 1.0$ equiv.) and TEMPO (62.5 $\mathrm{mg}, 0.40$ mmol, 2.0 equiv.) were added to an oven-dried $1.9 \mathrm{~mL}$ reaction vial. The vial was then sealed with a rubber septum, evacuated and backfilled with $\mathrm{N}_{2}(\times 3)$. Degassed, dry DMA $(0.4 \mathrm{~mL}, 0.5 \mathrm{M})$ was then added, followed by degassed, dry 2,6-lutidine ( $84 \mu \mathrm{L}, 0.72 \mathrm{mmol}, 3.6$ equiv.). The reaction was stirred under blue LED irradiation at ambient temperature for $16 \mathrm{~h}$. Without workup, the crude reaction mixture was loaded directly to silica gel and purified by flash column chromatography Purification by flash column chromatography ( $3 \%$ $\mathrm{Et}_{2} \mathrm{O}$ in Petrol) to afforded TEMPO adduct 11 as a colourless oil (36.1 mg, 73\%).

$\mathbf{R}_{f}\left(3 \% \mathrm{Et}_{2} \mathrm{O}\right.$ in Petrol $)=0.25 ;{ }^{1} \mathbf{H}$ NMR $\left(400 \mathrm{MHz}, \mathrm{CDCl}_{3}\right) \delta 7.42-7.33(\mathrm{~m}, 4 \mathrm{H}), 7.32-7.27(\mathrm{~m}, 1 \mathrm{H}), 4.85(\mathrm{~s}, 2 \mathrm{H})$, $1.68-1.33(\mathrm{~m}, 6 \mathrm{H}), 1.28(\mathrm{~s}, 6 \mathrm{H}), 1.18(\mathrm{~s}, 6 \mathrm{H}) ;{ }^{13} \mathrm{C}$ NMR $\left(101 \mathrm{MHz}, \mathrm{CDCl}_{3}\right) \delta 138.5,128.4,127.6,127.4,78.9$, 60.2, 39.9, 33.3, 20.5, 17.3; LRMS (ESI) $\mathrm{m} / \mathrm{z} 248.2\left([\mathrm{M}+\mathrm{H}]^{+}\right)$; HRMS (ESI) $\mathrm{m} / z \mathrm{C}_{16} \mathrm{H}_{26} \mathrm{NO}^{+}$requires 284.2009 $\left([\mathrm{M}+\mathrm{H}]^{+}\right)$, found 284.2010 .

Data is consistent with the literature. ${ }^{34}$ 


\section{References}

1. Wu, J.; Grant, P. S.; Li, X.; Noble, A.; Aggarwal, V. K., Catalyst-Free Deaminative Functionalizations of Primary Amines by Photoinduced Single-Electron Transfer. Angew. Chem. Int. Ed. 2019, 58, 5697-5701.

2. Anunziata, J.; Singh, J.; Silber, J. J., $n-\pi$ Electron donor-acceptor complexes. I. Aliphatic amines with benzonitrile. Can. J. Chem. 1981, 59, 1291-1296.

3. Singh, J. O.; Anunziata, J. D.; Silber, J. J., $n-\pi$ Electron donor-acceptor complexes. II. Aliphatic amines with dinitrobenzenes. Can. J. Chem. 1985, 63, 903-907.

4. Anunziata, J. D.; Galaverna, N. S.; Singh, J. O.; Silber, J. J., $n-\pi$ Electron donor-acceptor complexes. III. Aliphatic amines with dicyanobenzenes. Electric and steric effects of the $\mathrm{N}$-substituents on complex formation. Can. J. Chem. 1986, 64, 1491-1495.

5. Basch, C. H.; Liao, J.; Xu, J.; Piane, J. J.; Watson, M. P., Harnessing Alkyl Amines as Electrophiles for NickelCatalyzed Cross Couplings via C-N Bond Activation. J. Am. Chem. Soc. 2017, 139, 5313-5316.

6. Awartani, R.; Sakizadeh, K.; Gabrielsen, B., The preparation and react ions of phenyl-substituted pyrylium and pyridinium salts: Nucleophilic substitution of an amino group by pyridine. J. Chem. Educ. 1986, 63, 172-172.

7. Yue, H.; Zhu, C.; Shen, L.; Geng, Q.; Hock, K. J.; Yuan, T.; Cavallo, L.; Rueping, M., Nickel-catalyzed C-N bond activation: Activated primary amines as alkylating reagents in reductive cross-coupling. Chem. Sci. 2019, 10, 44304435.

8. Yue, H.; Lu, F.; Shen, C.; Quan, J. M., Structure-based design of benzo[e]isoindole-1,3-dione derivatives as selective GSK-3 $\beta$ inhibitors to activate Wnt/ $\beta$-catenin pathway. Bioorg. Chem. 2015, 61, 21-27.

9. Zhu, Z.-F.; Chen, G.-L.; Liu, F., Ruthenium-catalysed meta-selective CAr-H bond alkylation via a deaminative strategy. Chem. Commun. 2021, 57, 3411-3414.

10.Martin-Montero, R.; Yatham, V. R.; Yin, H.; Davies, J.; Martin, R., Ni-catalyzed Reductive Deaminative Arylation at sp 3 Carbon Centers. Org. Lett. 2019, 21, 2947-2951.

11.Klauck, F. J. R.; James, M. J.; Glorius, F., Deaminative Strategy for the Visible-Light-Mediated Generation of Alkyl Radicals. Angew. Chem. Int. Ed. 2017, 56, 12336-12339.

12.Hu, J.; Wang, G.; Li, S.; Shi, Z., Selective C-N Borylation of Alkyl Amines Promoted by Lewis Base. Angew. Chem. Int. Ed. 2018, 57, 15227-15231.

13.Wang, X.; Kuang, Y.; Ye, S.; Wu, J., Photoredox-catalyzed synthesis of sulfones through deaminative insertion of sulfur dioxide. Chem. Commun. 2019, 55, 14962-14964.

14.Yi, J.; Badir, S. O.; Kammer, L. M.; Ribagorda, M.; Molander, G. A., Deaminative Reductive Arylation Enabled by Nickel/Photoredox Dual Catalysis. Org. Lett. 2019, 21, 3346-3351.

15.Ociepa, M.; Turkowska, J.; Gryko, D., Redox-Activated Amines in C( sp 3$)-C($ sp ) and C( sp 3 )-C( sp 2 ) Bond Formation Enabled by Metal-Free Photoredox Catalysis. ACS Catal. 2018, 8, 11362-11367.

16.Wu, J.; He, L.; Noble, A.; Aggarwal, V. K., Photoinduced Deaminative Borylation of Alkylamines. J. Am. Chem. Soc. 2018, 140, 10700-10704.

17.Xia, Q.; Li, Y.; Wang, X.; Dai, P.; Deng, H.; Zhang, W.-H., Visible Light-Driven $\alpha$-Alkylation of N-Aryl tetrahydroisoquinolines Initiated by Electron Donor-Acceptor Complexes. Org. Lett. 2020, 22, 7290-7294.

18.Liao, J.; Guan, W.; Boscoe, B. P.; Tucker, J. W.; Tomlin, J. W.; Garnsey, M. R.; Watson, M. P., Transforming Benzylic Amines into Diarylmethanes: Cross-Couplings of Benzylic Pyridinium Salts via C-N Bond Activation. Org. Lett. 2018, 20, 3030-3033.

19.Jensen, T.; Pedersen, H.; Bang-Andersen, B.; Madsen, R.; Jørgensen, M., Palladium-Catalyzed Aryl AminationHeck Cyclization Cascade: A One-Flask Approach to 3-Substituted Indoles. Angew. Chem. Int. Ed. 2008, 47, 888-890. 20.Malet, R.; Moreno-Mañas, M.; Parella, T.; Pleixats, R., (I,2-Bis(diphenylphosphino)ethane)(f/3-l-arylallyl)palladium Tetrafluoroborates. Distribution of the Positive Charge Density by Correlation of NMR Chemical Shifts with Hammett Substituent Constants. Organometallics 1995, 14, 2463-2463.

21.Markina, T. A.; Boiko, N. N., Synthesis of 2-methyl-4,6-diarylpyrilium tetrafluoroborates. Chemistry of Heterocyclic Compounds 1985, 21, 138-141.

22.Katritzky, A. R.; Vassilatos, S. N.; Alajarin-Ceron, M., Rotational equilibria in 1,2,6-trisubstituted pyridinium cations and reactions of 2-Isopropylpyrylium cations. Org. Magn. Reson. 1983, 21, 587-595.

23.Katritzky, A. R.; Lloyd, J. M.; Patel, R. C., The Preparation of Pyridiniums from Pyryliums. J. Chem. Soc., Perkin Trans. 1 1982, 117-123. 
24.Katritzky, A. R.; Aurrecoechea, J. M., Alkylation of Monosubstituted Malonate Anions With Pyridinium and Quinolinium Salts. Synthesis 1987, 1987, 342-345.

25.Katritzky, A. R.; Burgess, K.; Patel, R. C., The Synthesis of Condensed Quinolizinium Systems. Heterocycles 1981, $15,1175-1178$.

26.Rocke, B. N.; Bahnck, K. B.; Herr, M.; Lavergne, S.; Mascitti, V.; Perreault, C.; Polivkova, J.; Shavnya, A., Synthesis of sulfones from organozinc reagents, DABSO, and alkyl halides. Org. Lett. 2014, 16, 154-157.

27.Day, J. J.; Neill, D. L.; Xu, S.; Xian, M., Benzothiazole Sulfinate: A Sulfinic Acid Transfer Reagent under OxidationFree Conditions. Org. Lett. 2017, 19, 3819-3822.

28.Davies, A. T.; Curto, J. M.; Bagley, S. W.; Willis, M. C., One-pot palladium-catalyzed synthesis of sulfonyl fluorides from aryl bromides. Chem. Sci. 2017, 8, 1233-1237.

29.Laudadio, G.; Bartolomeu, A. D. A.; Verwijlen, L. M. H. M.; Cao, Y.; De Oliveira, K. T.; Noël, T., Sulfonyl Fluoride Synthesis through Electrochemical Oxidative Coupling of Thiols and Potassium Fluoride. J. Am. Chem. Soc. 2019, 141, 11832-11836.

30.Tan, B. Y.-H.; Teo, Y.-C.; Seow, A.-H., Low Catalyst Loadings for Ligand-Free Copper(I)-Oxide-Catalyzed N -Arylation of Methanesulfonamide in Water. Eur. J. Org. Chem. 2014, 2014, 1541-1546.

31.Katohgi, M.; Togo, $\mathrm{H}$., Oxidatively sonochemical dealkylation of various $\mathrm{N}$-alkylsulfonamides to free sulfonamides and aldehydes. Tetrahedron 2001, 57, 7481-7486.

32.Zhou, G.; Ting, P.; Aslanian, R.; Piwinski, J. J., A useful Pd-catalyzed Negishi coupling approach to benzylic sulfonamide derivatives. Org. Lett. 2008, 10, 2517-2520.

33.Zilaout, H.; Van Den Hoogenband, A.; De Vries, J.; Lange, J. H. M.; Terpstra, J. W., The first examples of rhodiumcatalyzed 1,4-conjugate addition reactions of arylboronic acids with ethenesulfonamides. Tetrahedron Lett. 2011, 52, 5934-5939.

34.Uchikura, T.; Toda, M.; Mouri, T.; Fujii, T.; Moriyama, K.; Ibáñez, I.; Akiyama, T., Radical Hydroalkylation and Hydroacylation of Alkenes by the Use of Benzothiazoline under Thermal Conditions. J. Org. Chem. 2020, 85, 1271512723. 


\section{NMR spectra}

NMR spectra of 1-(1-((benzyloxy)carbonyl)piperidin-4-yl)-2,4,6-triphenylpyridin-1-ium tetrafluoroborate (1a)
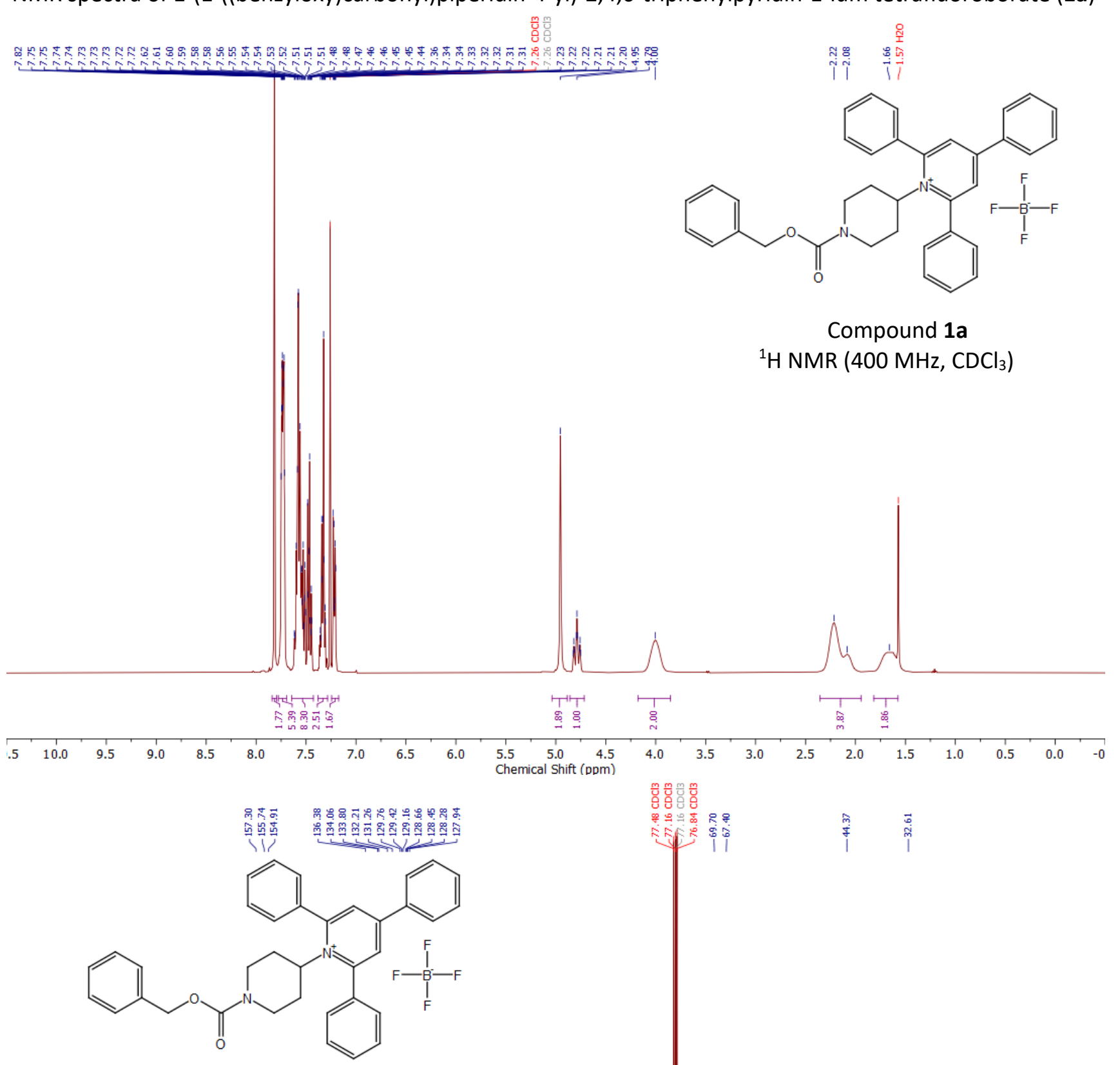

Compound 1a

$\left.{ }^{13} \mathrm{C} \mathrm{NMR} \mathrm{(101} \mathrm{MHz,} \mathrm{CDCl}_{3}\right)$

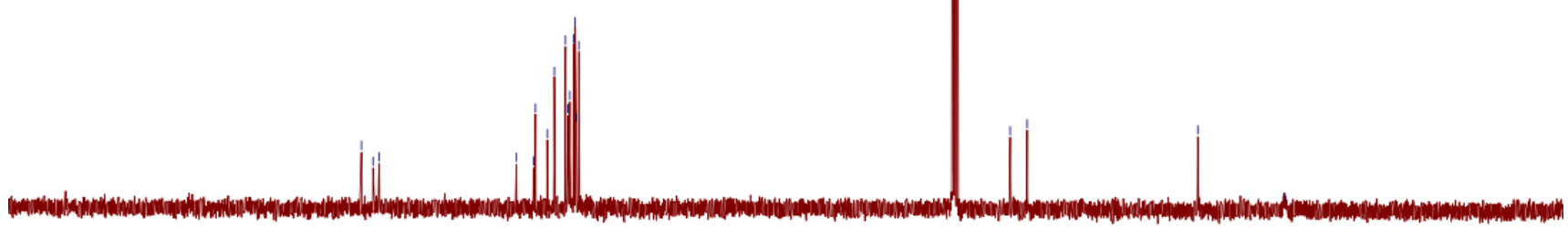

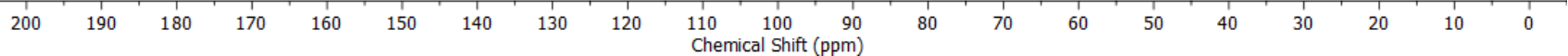




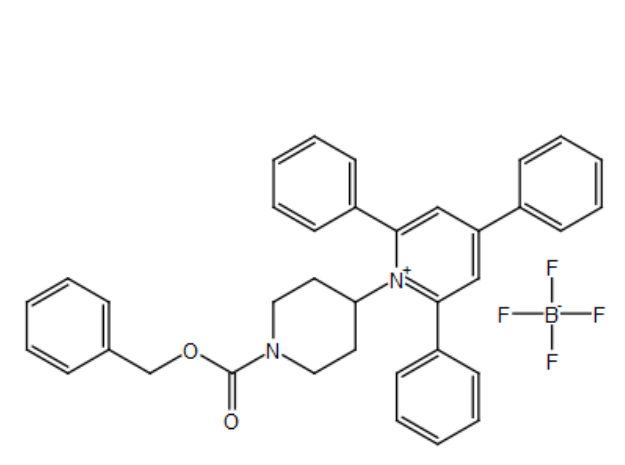

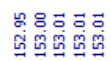

Compound 1a

${ }^{19} \mathrm{~F} \mathrm{NMR} \mathrm{(377} \mathrm{MHz,} \mathrm{CDCl}_{3}$ )

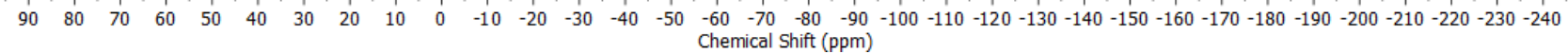


NMR spectra of 1-(1-(tert-butoxycarbonyl)piperidin-4-yl)-2,4,6-triphenylpyridin-1-ium tetrafluoroborate (1d)<smiles></smiles>

Compound 1d

${ }^{1} \mathrm{H}$ NMR $\left(400 \mathrm{MHz}, \mathrm{CDCl}_{3}\right)$

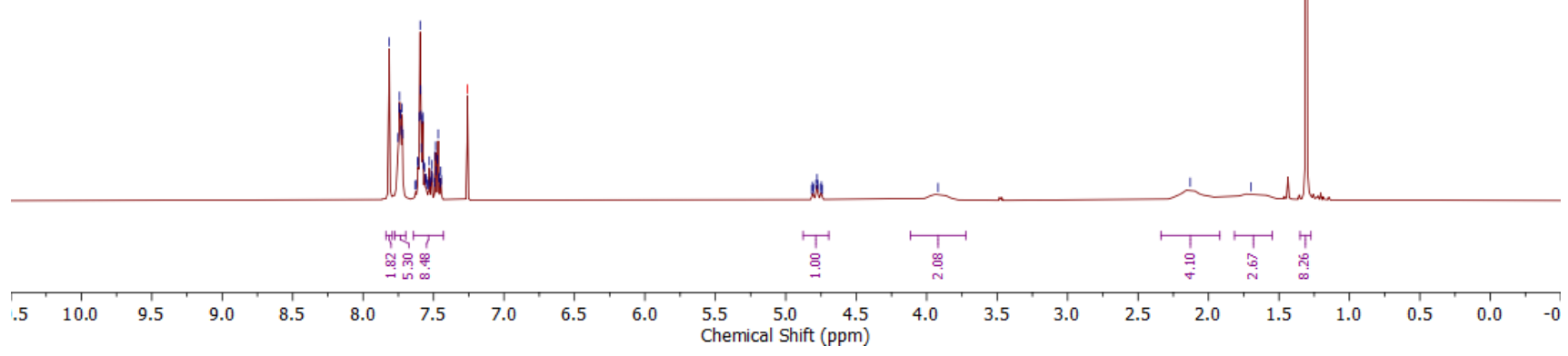<smiles></smiles>

Compound 1d

${ }^{13} \mathrm{C}$ NMR (101 MHz, $\mathrm{CDCl}_{3}$ )

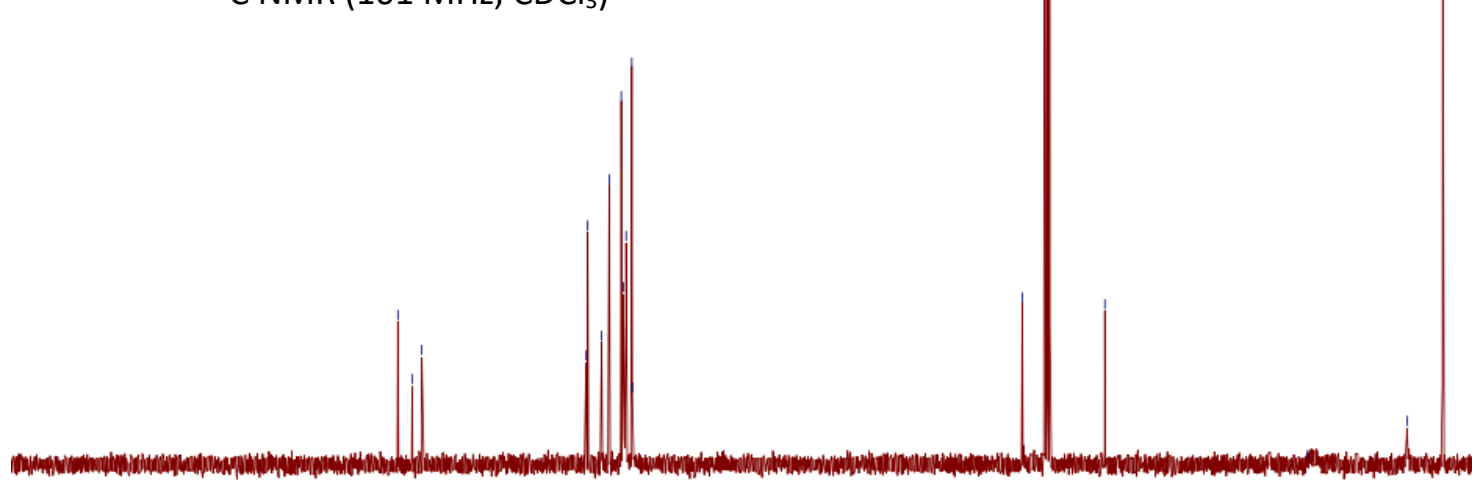


<smiles></smiles>

Compound 1d

$\left.{ }^{19} \mathrm{~F} \mathrm{NMR} \mathrm{(377} \mathrm{MHz,} \mathrm{CDCl} 3\right)$

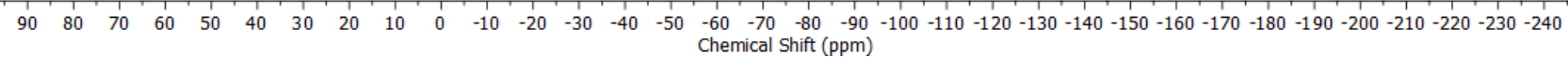


NMR spectra of 1-tosylpiperidine-4-amine (1e-1)
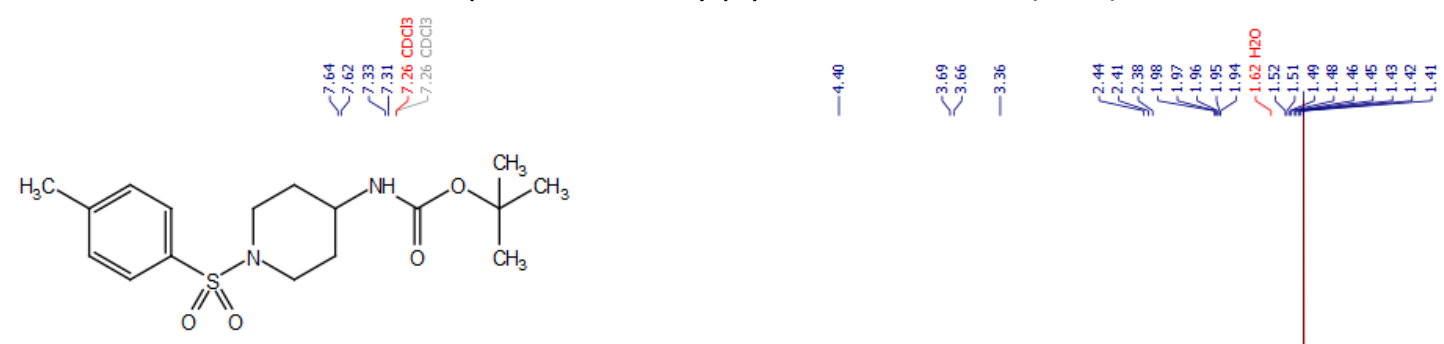

Compound 1e-1

${ }^{1} \mathrm{H}$ NMR $\left(400 \mathrm{MHz}, \mathrm{CDCl}_{3}\right)$
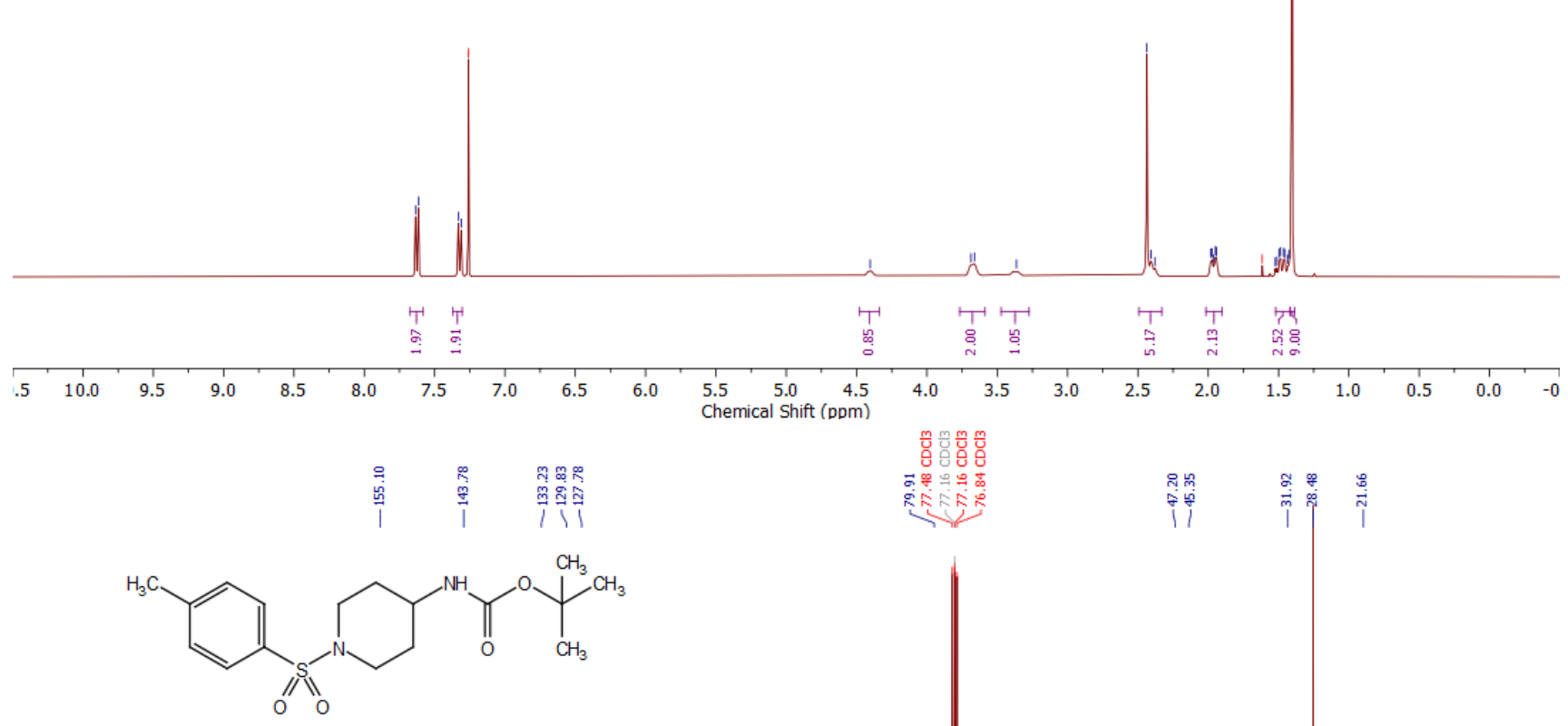

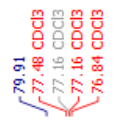

Compound 1e-1

${ }^{13} \mathrm{C}$ NMR $\left(101 \mathrm{MHz}, \mathrm{CDCl}_{3}\right)$

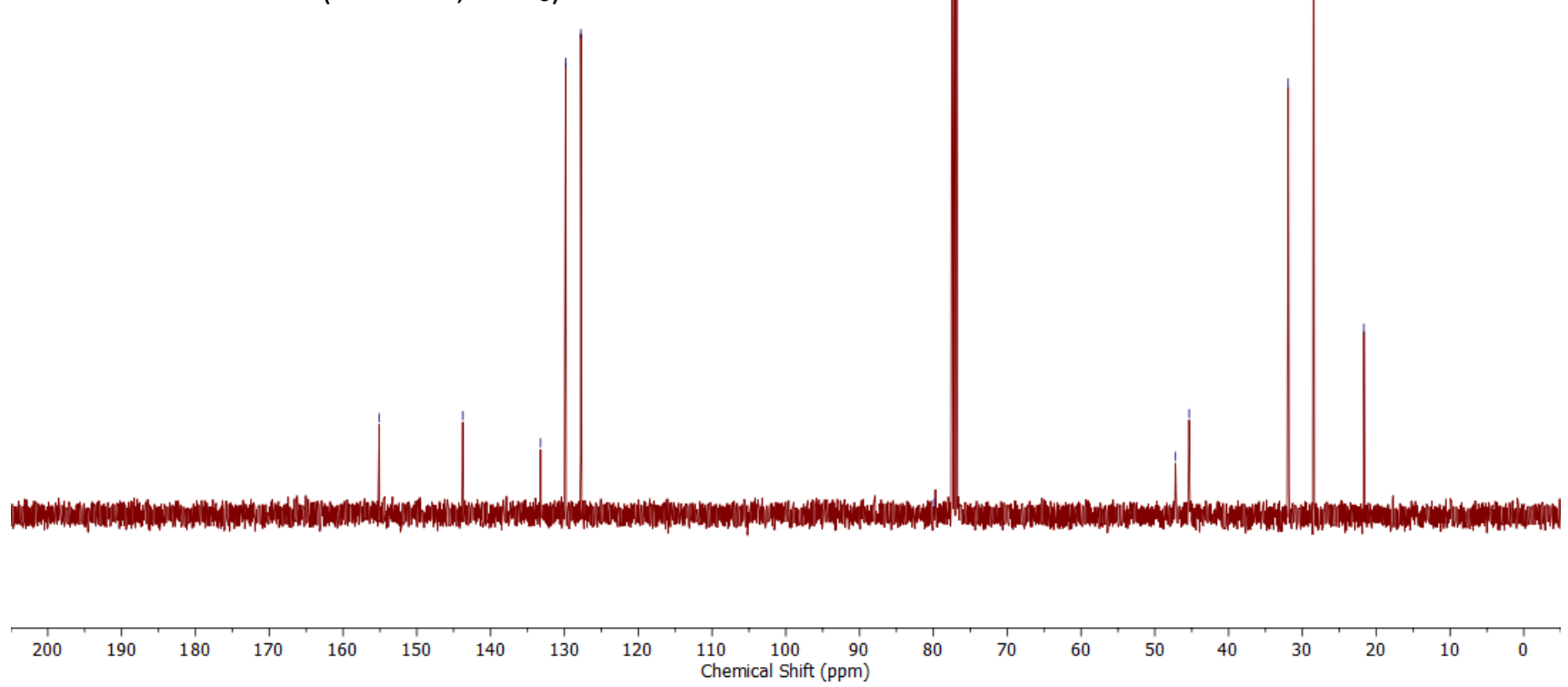


NMR spectra of 1-tosylpiperidin-4-amine hydrochloride (1e-2)

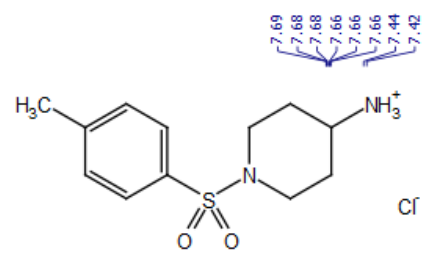

Compound 1e-2

${ }^{1} \mathrm{H}$ NMR $\left(400 \mathrm{MHz}, \mathrm{CDCl}_{3}\right)$
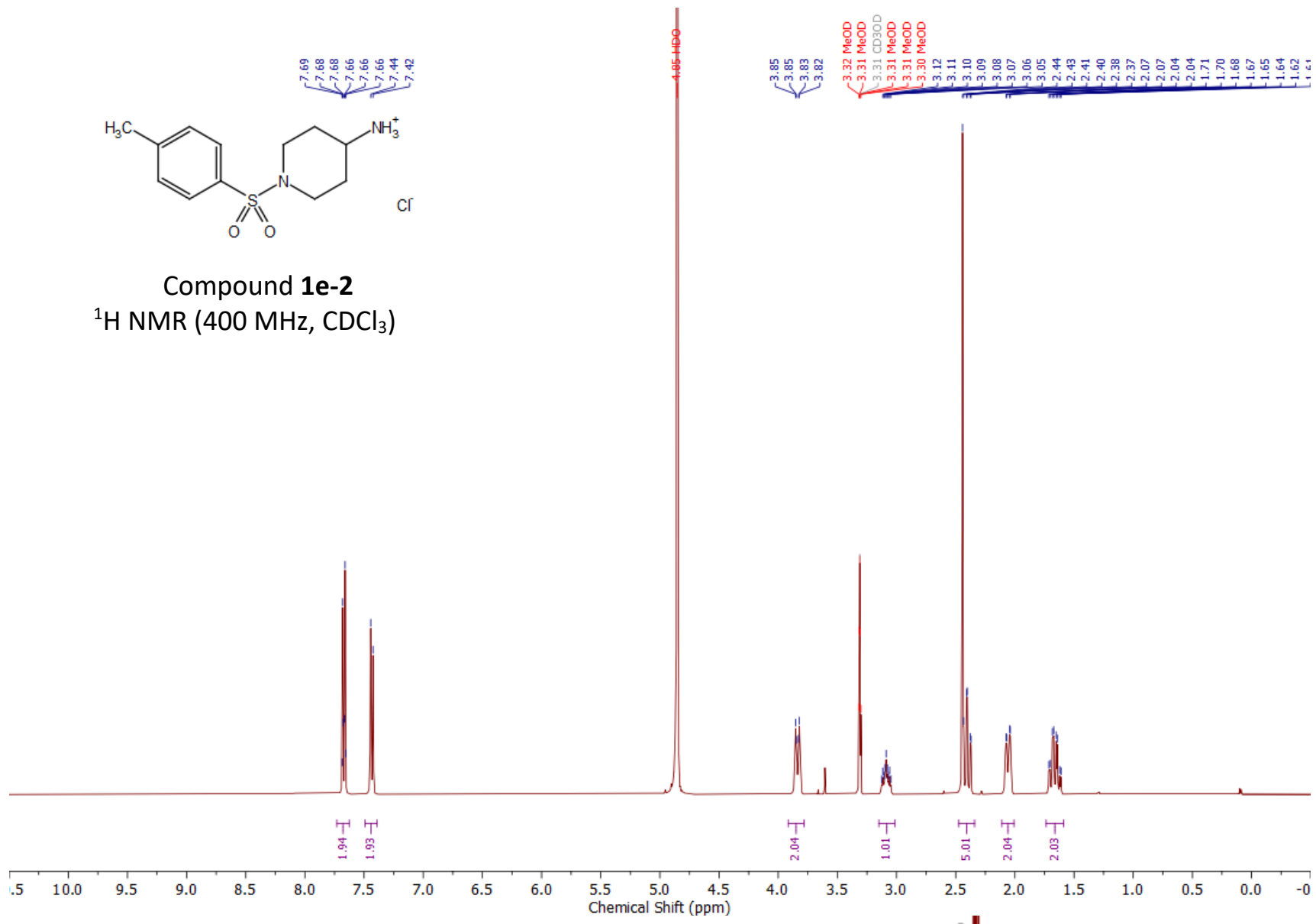<smiles>CNC1CCN(S(=O)(=O)c2ccc(C)cc2)CC1</smiles>

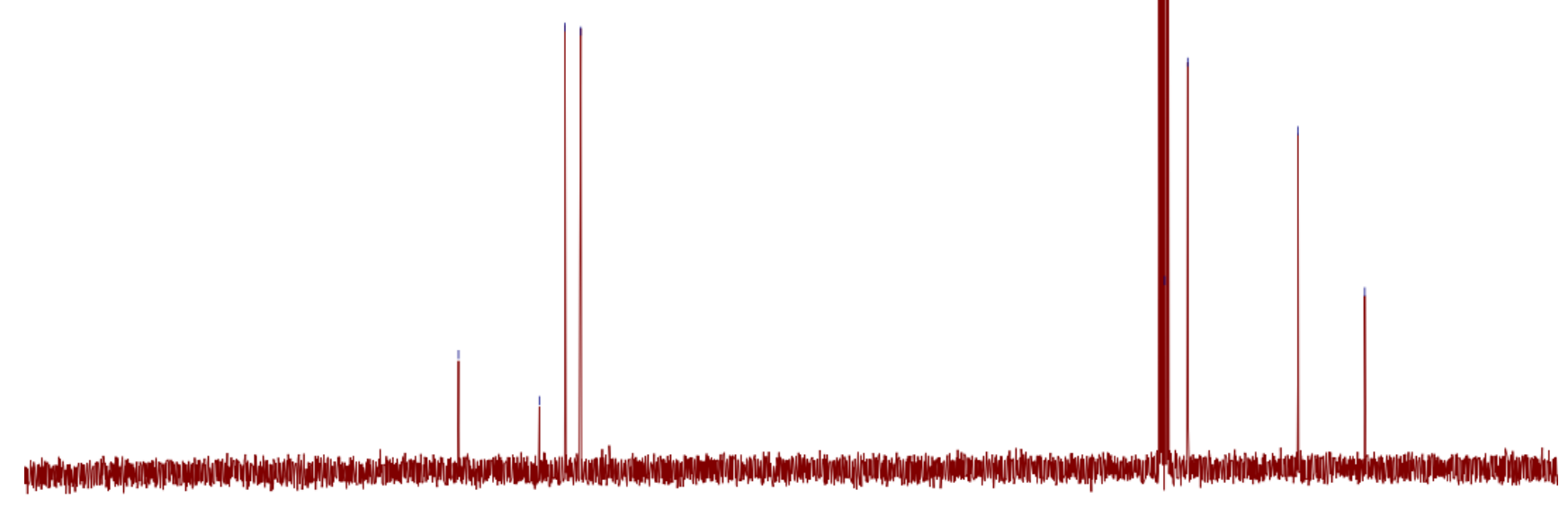

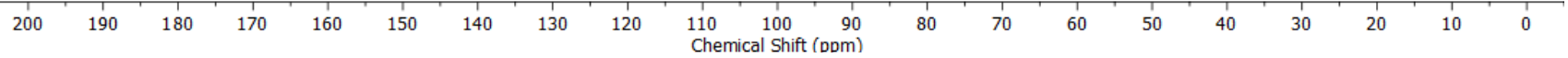


NMR spectra of 2,4,6-triphenyl-1-(1-tosylpiperidin-4-yl)pyridin-1-ium tetrafluoroborate (1e-3)

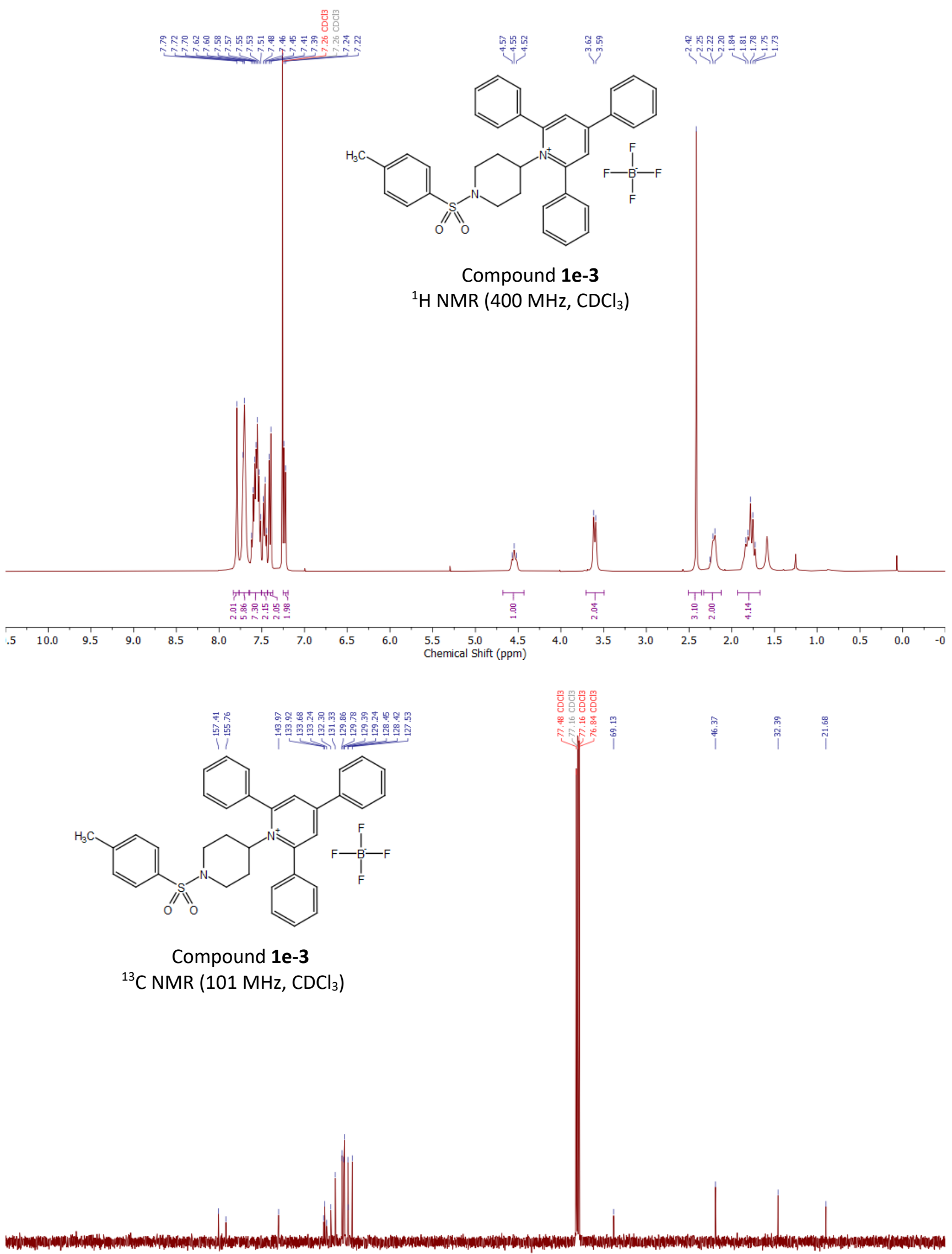

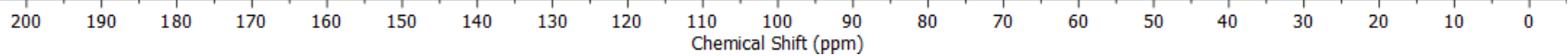




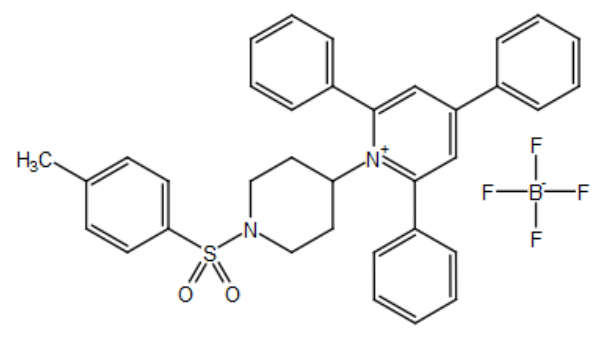

Compound 1e-3

${ }^{19} \mathrm{~F}$ NMR (377 MHz, $\mathrm{CDCl}_{3}$ )

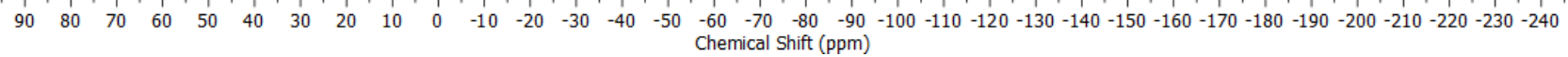


NMR spectra of 1-cyclobutyl-2,4,6-triphenylpyridin-1-ium tetrafluoroborate (1f)

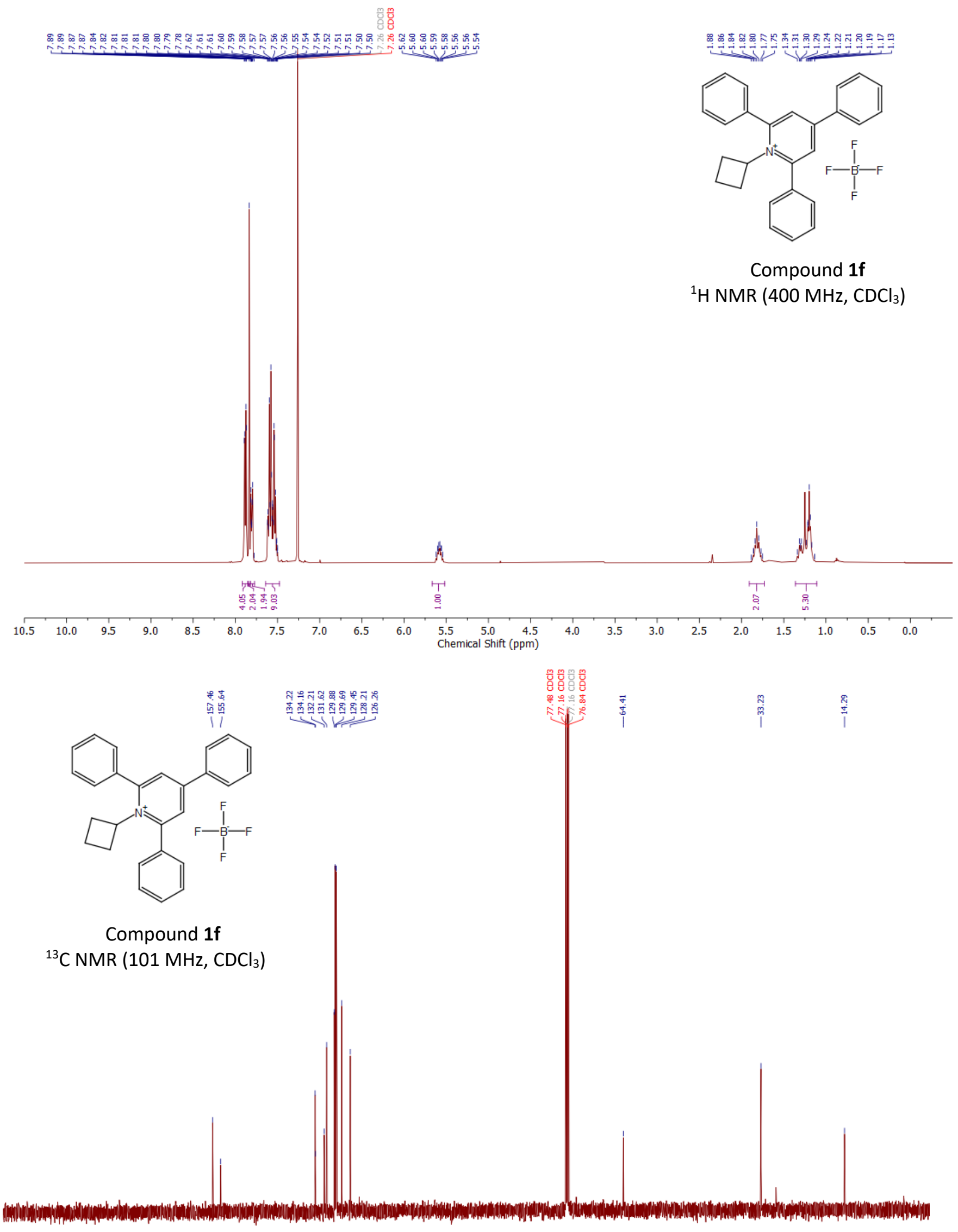

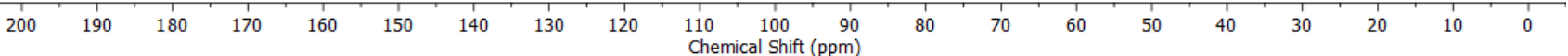




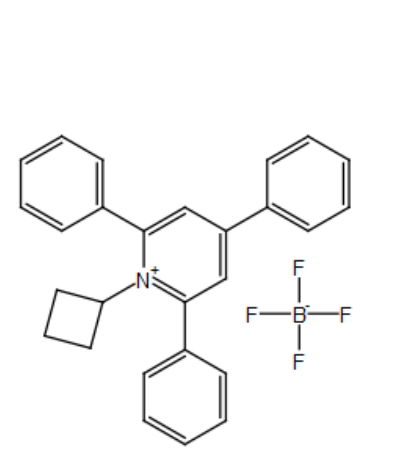

Compound 1f

${ }^{19} \mathrm{~F} \mathrm{NMR} \mathrm{(377} \mathrm{MHz,} \mathrm{CDCl}_{3}$ )

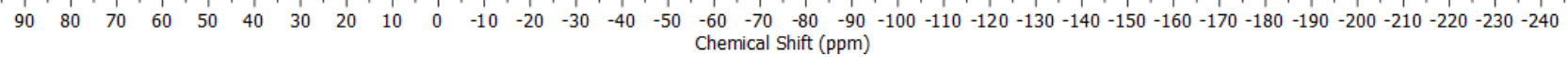


NMR spectra of 1-cyclopentyl-2,4,6-triphenylpyridin-1-ium tetrafluoroborate (1g)<smiles>FP(F)(F)(F)c1cc(-c2ccccc2)cc(-c2ccccc2)[n+]1C1CCCC1</smiles>

Compound $1 \mathrm{~g}$

${ }^{1} \mathrm{H} \mathrm{NMR}\left(400 \mathrm{MHz}, \mathrm{CDCl}_{3}\right)$

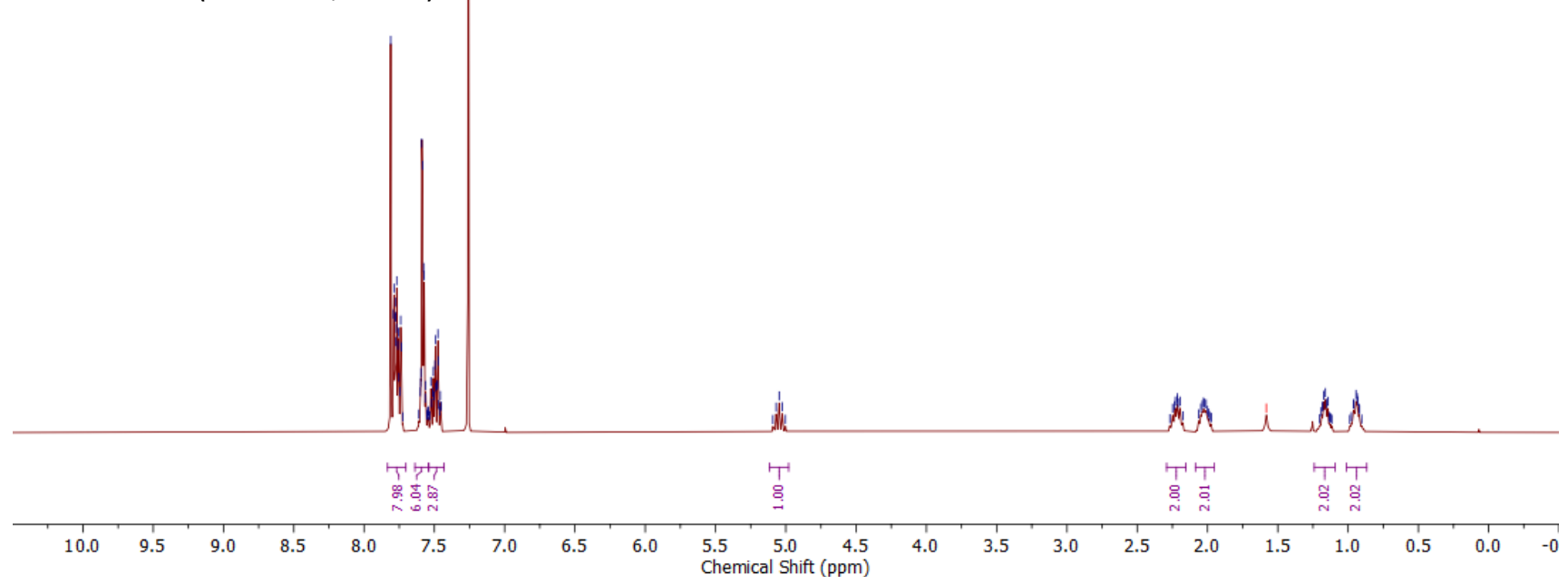<smiles>FP(F)(F)(F)c1cccc(-c2cc(-c3ccccc3)[n+](C3CCCC3)c(-c3ccccc3)c2)c1</smiles>

Compound $1 \mathrm{~g}$

${ }^{13} \mathrm{C}$ NMR $\left(101 \mathrm{MHz}, \mathrm{CDCl}_{3}\right)$

$\begin{array}{llllllllll}200 & 190 & 180 & 170 & 160 & 150 & 140 & 130 & 120 & \begin{array}{l}110 \\ \text { Chemical Shift (pom) }\end{array}\end{array}$


<smiles></smiles>

Compound $1 \mathrm{~g}$

${ }^{19} \mathrm{~F}$ NMR (377 MHz, $\mathrm{CDCl}_{3}$ ) 
NMR spectra of 1-cyclohexyl-2,4,6-triphenylpyridin-1-ium tetrafluoroborate (1h)

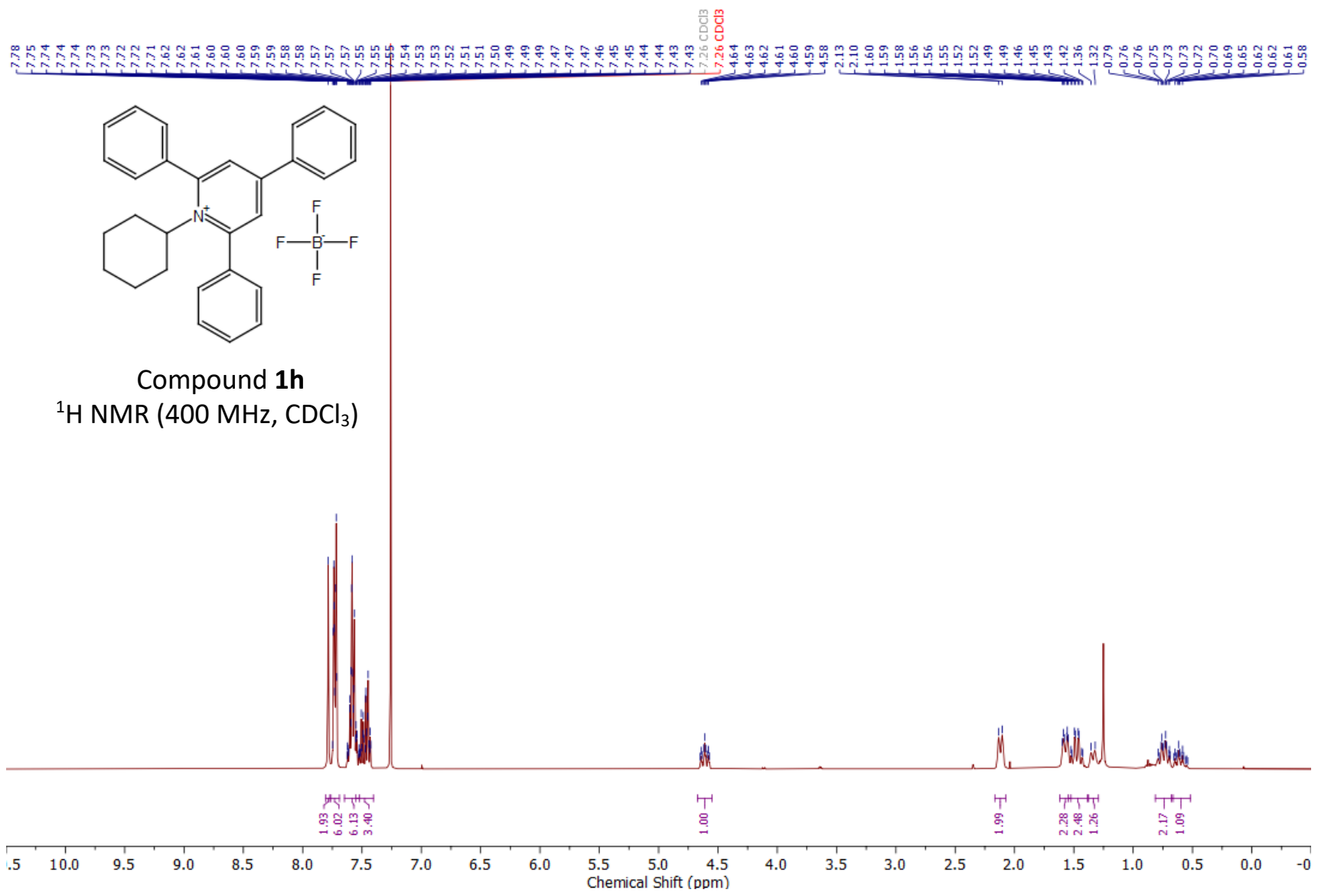<smiles>FC(F)(F)F</smiles>

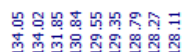

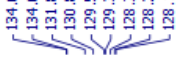

${ }^{13} \mathrm{C}$ NMR $\left(101 \mathrm{MHz}, \mathrm{CDCl}_{3}\right)$

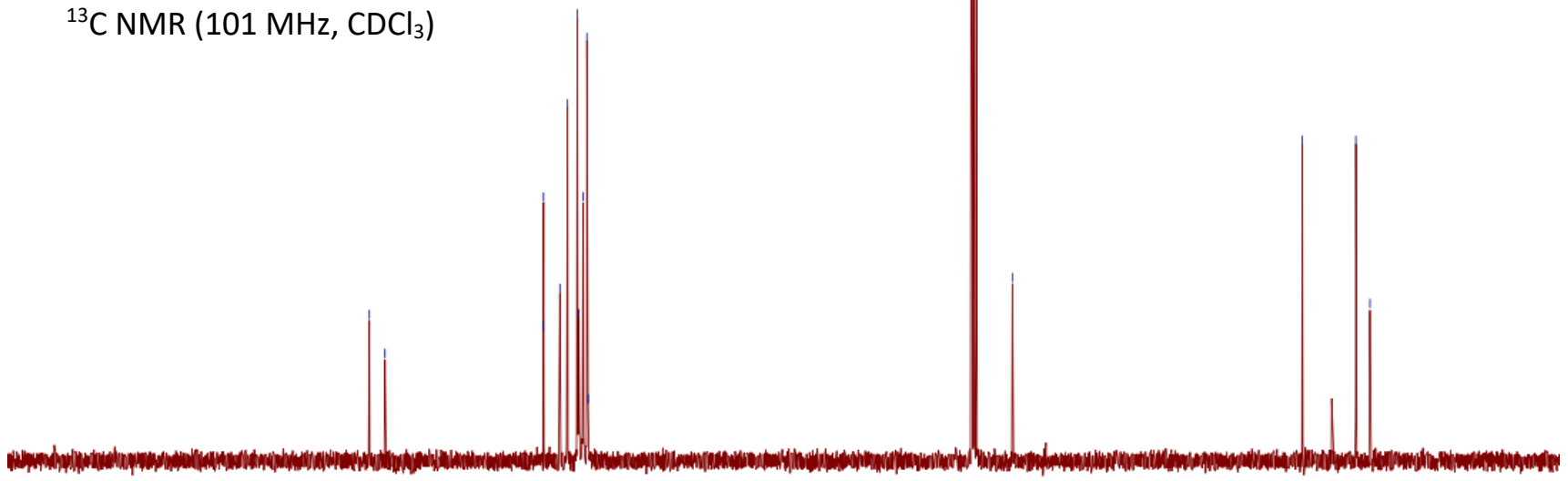

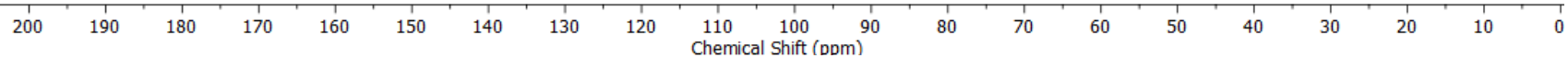




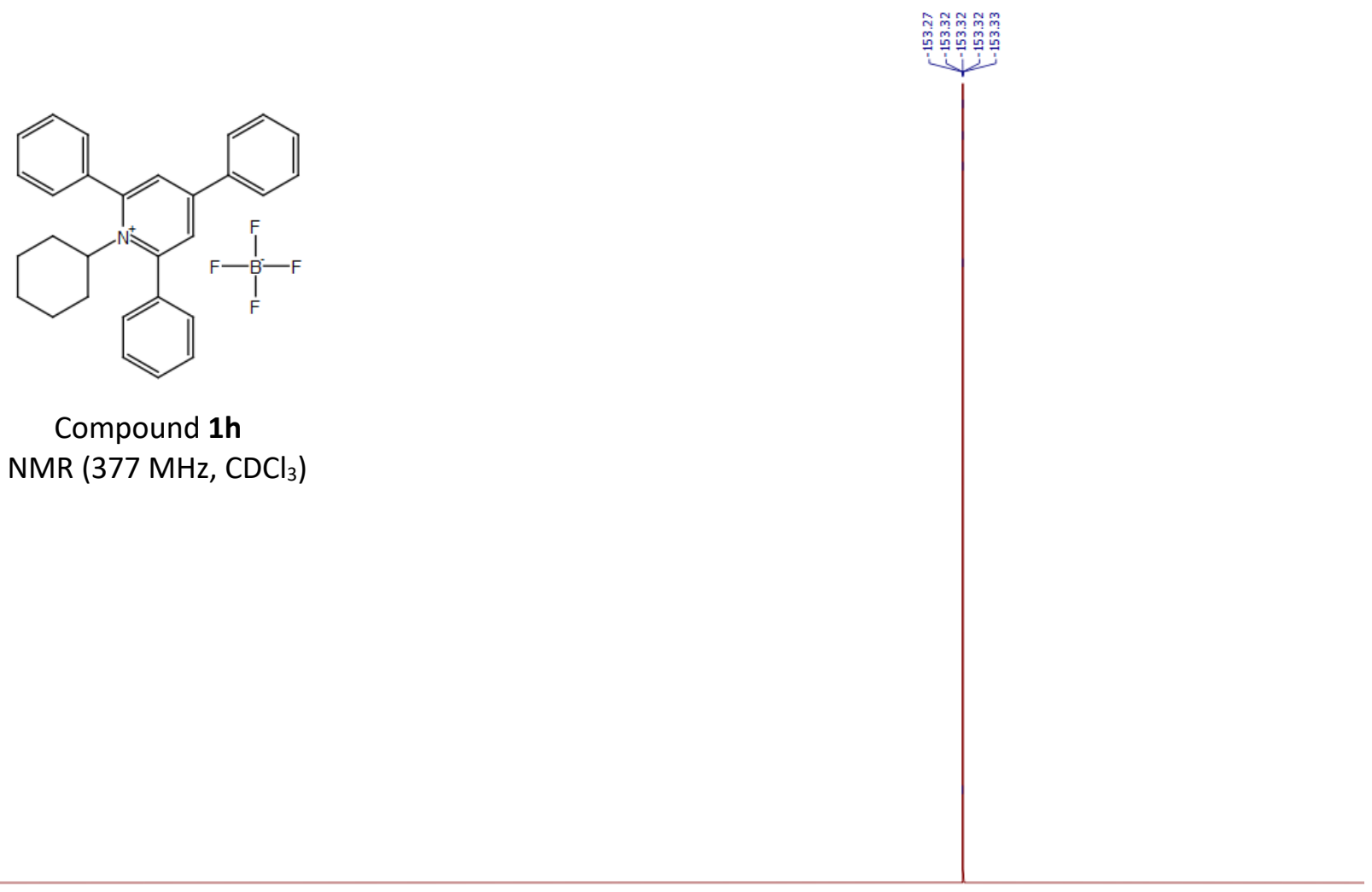

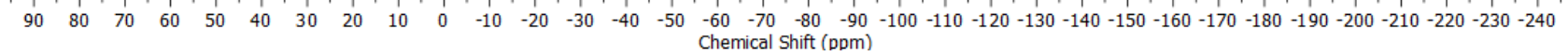


NMR spectra of 1-isopropyl-2,4,6-triphenylpyridin-1-ium tetrafluoroborate (1i)

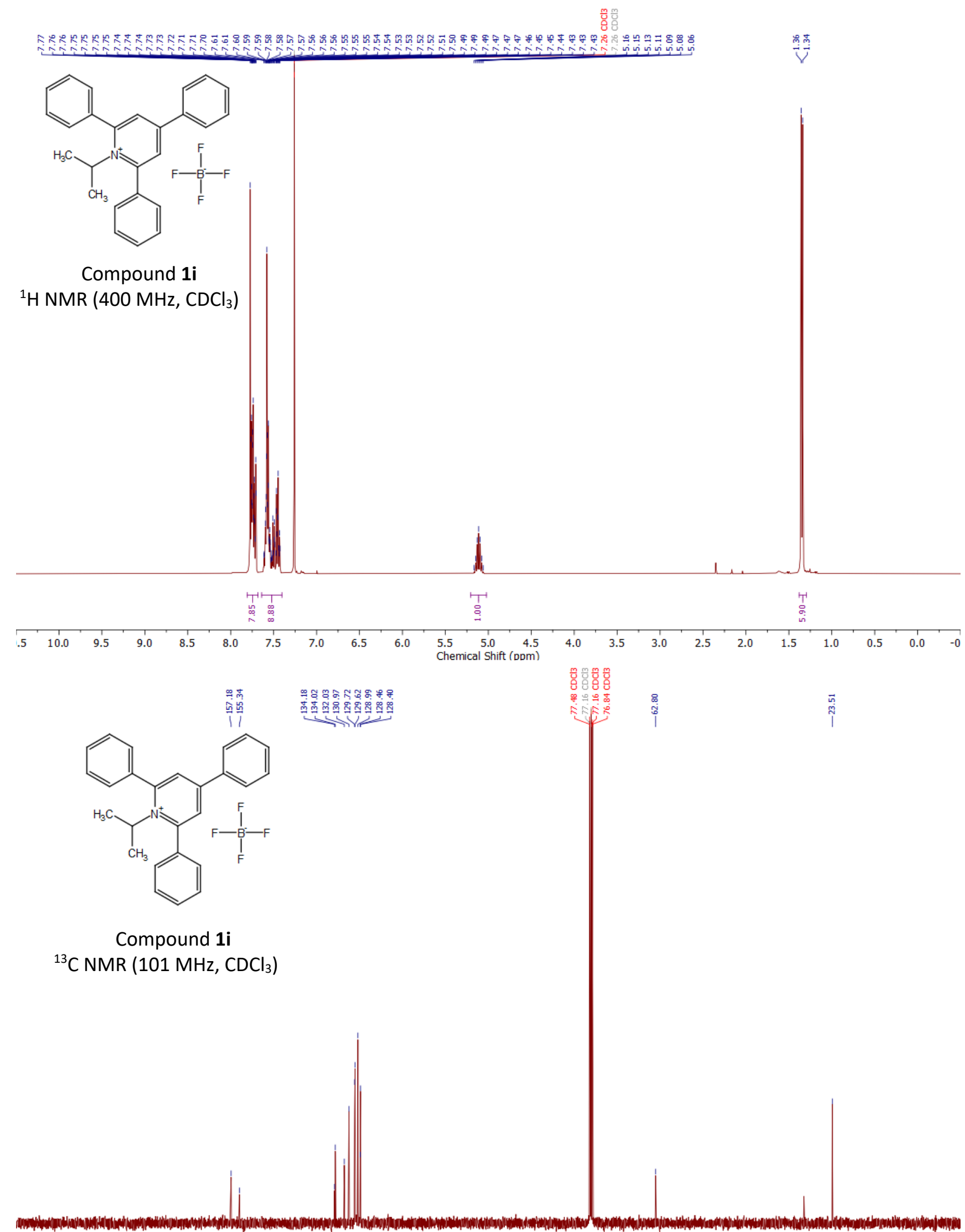

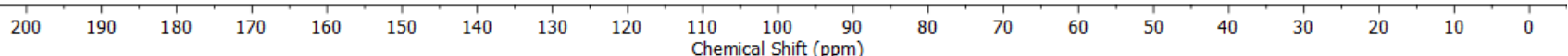




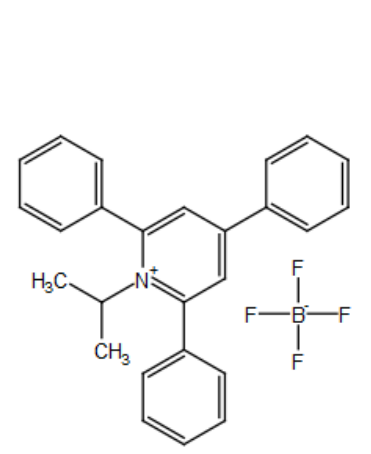

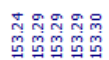

Compound $\mathbf{1 i}$

${ }^{19} \mathrm{~F}$ NMR (377 MHz, $\mathrm{CDCl}_{3}$ )

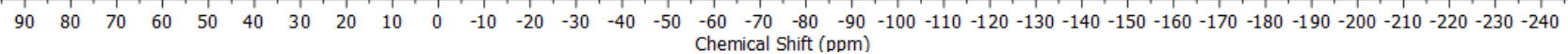


NMR spectra of 1-cyclooctyl-2,4,6-triphenylpyridin-1-ium tetrafluoroborate (1j)

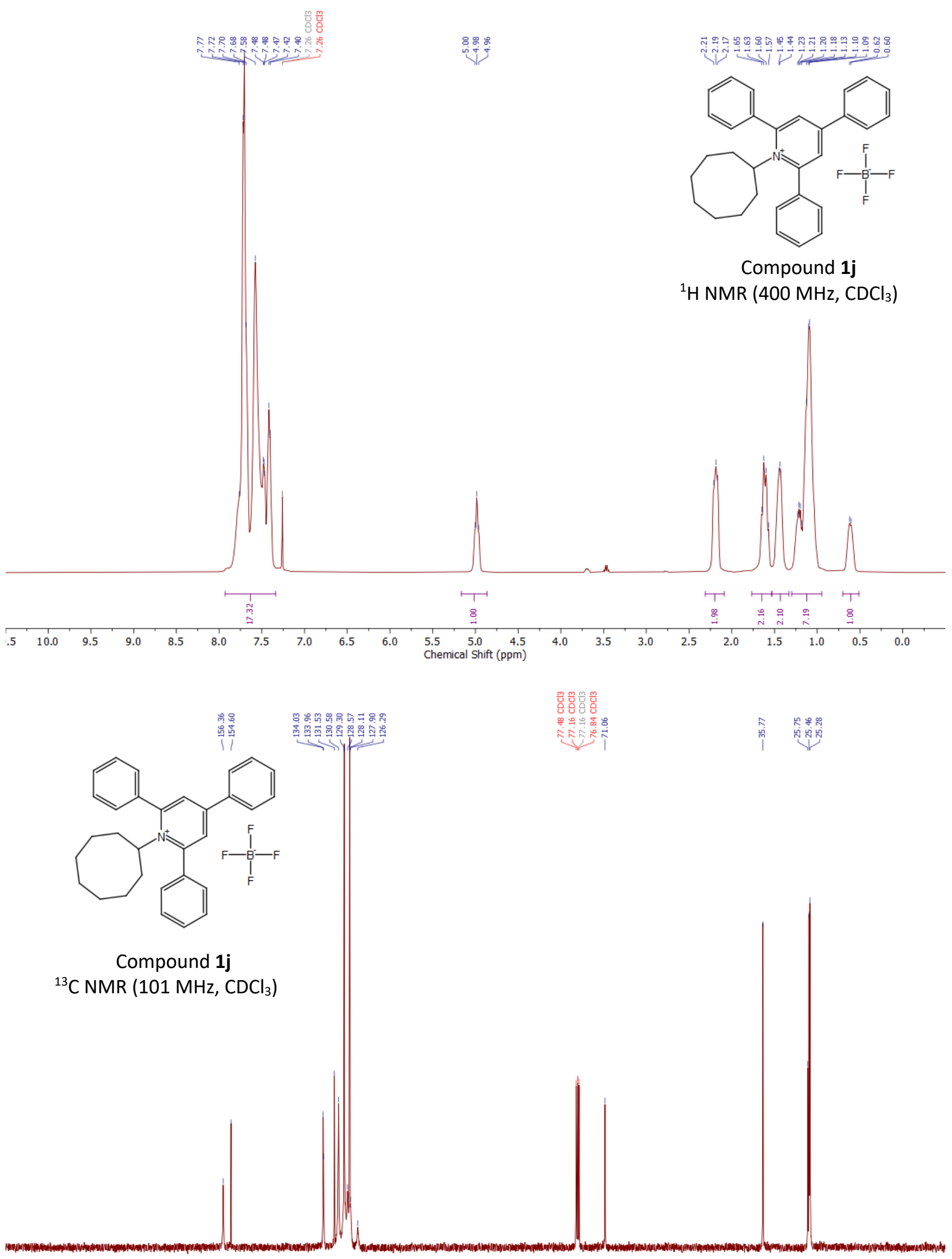

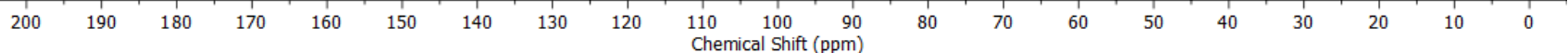




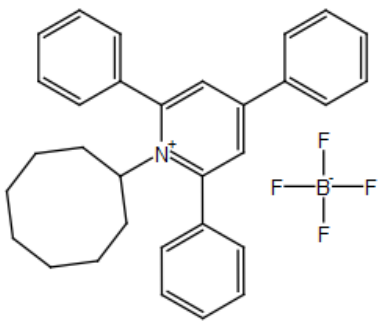

Compound $\mathbf{1 j}$

${ }^{19} \mathrm{~F} \mathrm{NMR} \mathrm{(377} \mathrm{MHz,} \mathrm{CDCl}_{3}$ )

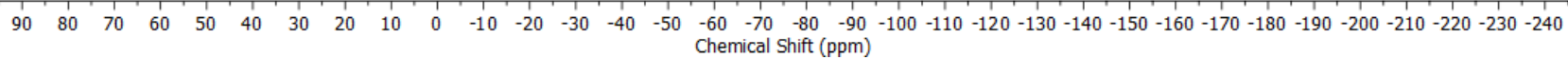


NMR spectra of 1-(2-methylcyclohexyl)-2,4,6-triphenylpyridin-1-ium tetrafluoroborate (1k)

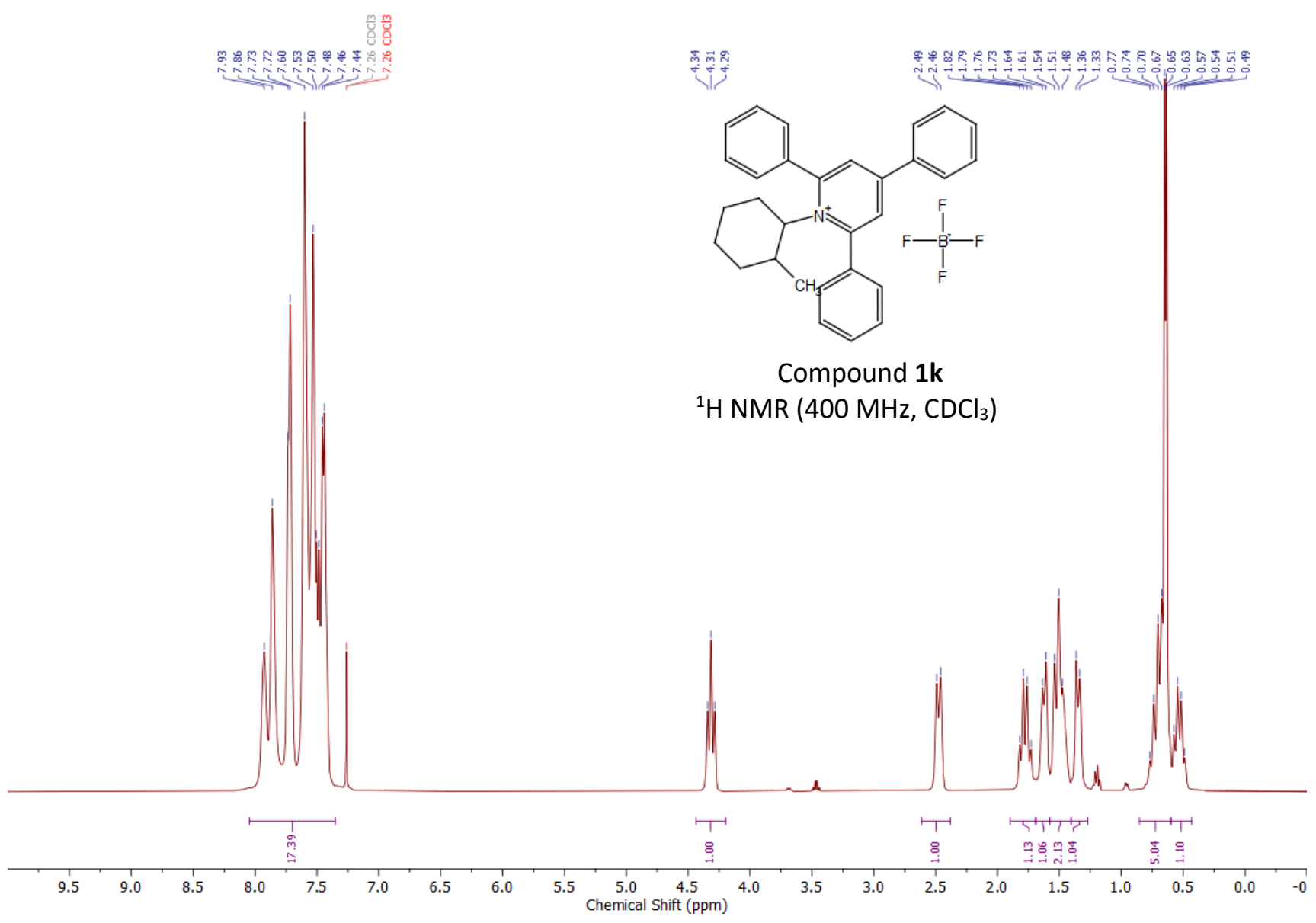<smiles></smiles>

Compound $\mathbf{1 k}$

${ }^{13} \mathrm{C}$ NMR $\left(101 \mathrm{MHz}, \mathrm{CDCl}_{3}\right)$

$\begin{array}{lllllllllll}200 & 190 & 180 & 170 & 160 & 150 & 140 & 130 & 120 & \begin{array}{l}110 \\ \text { Chemical Shift (ppm) }\end{array}\end{array}$




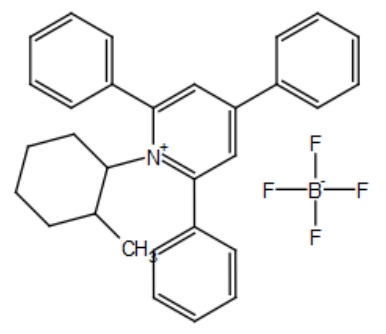

Compound $\mathbf{1 k}$

${ }^{19} \mathrm{~F} \mathrm{NMR}\left(377 \mathrm{MHz}, \mathrm{CDCl}_{3}\right)$

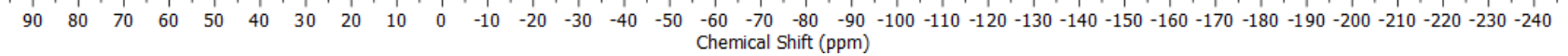


NMR spectra of 1-(heptan-2-yl)-2,4,6-triphenylpyridin-1-ium tetrafluoroborate (1I)

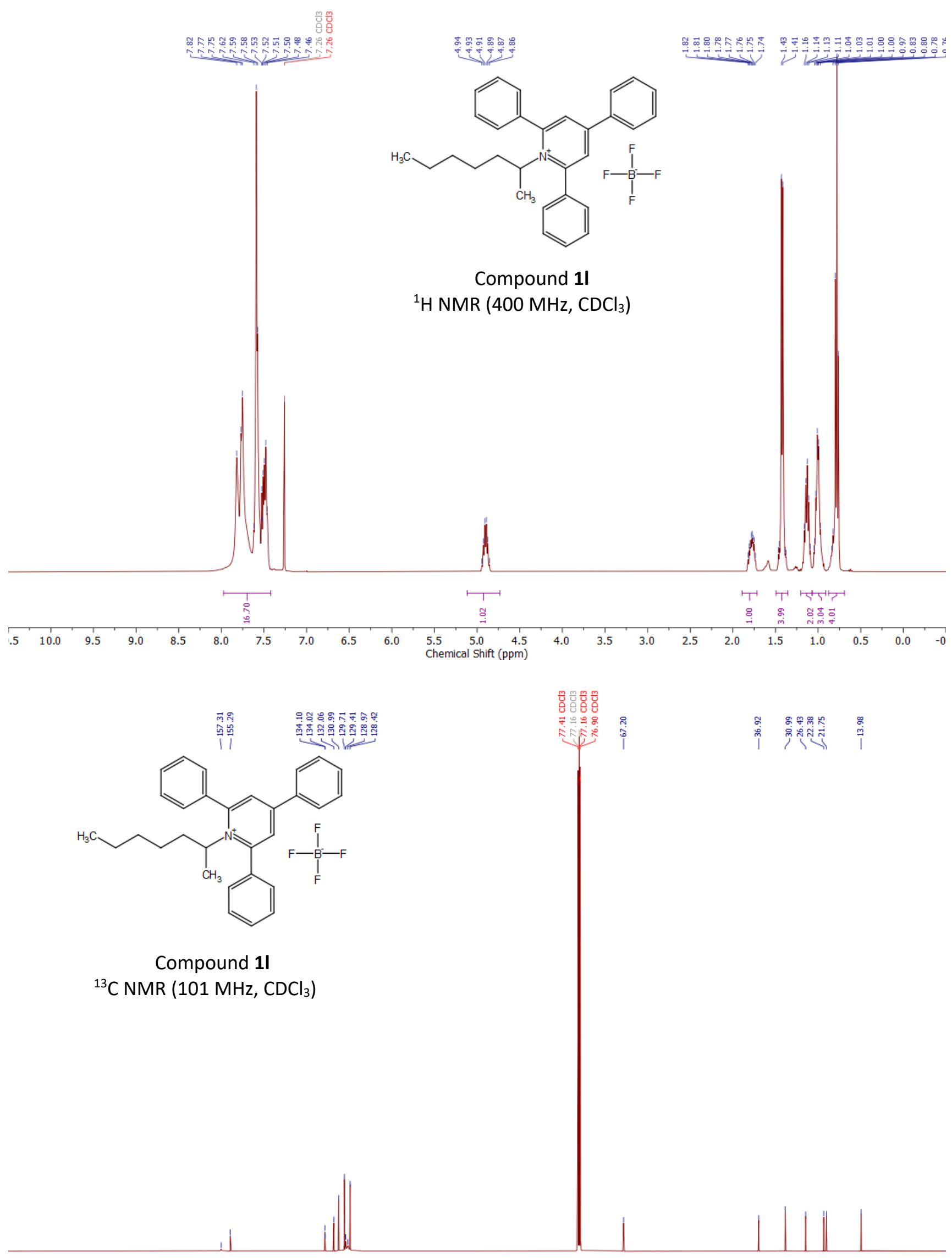

$\begin{array}{llllllllll}200 & 190 & 180 & 170 & 160 & 150 & 140 & 130 & 120 & \begin{array}{c}110 \\ \text { Chemical Shift (ppm) }\end{array}\end{array}$ 


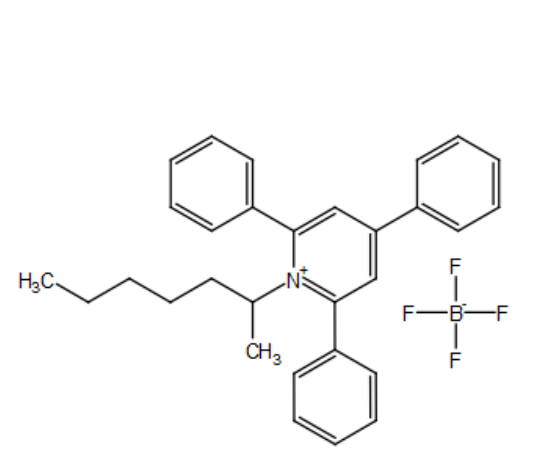

Compound 11

${ }^{19} \mathrm{~F} \mathrm{NMR}\left(377 \mathrm{MHz}, \mathrm{CDCl}_{3}\right)$

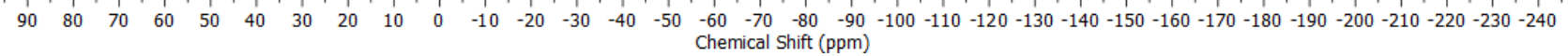


NMR spectra of 2,4,6-Triphenyl-1-(tetrahydro-2H-pyran-4-yl)pyridin-1-ium tetrafluoroborate (1m)
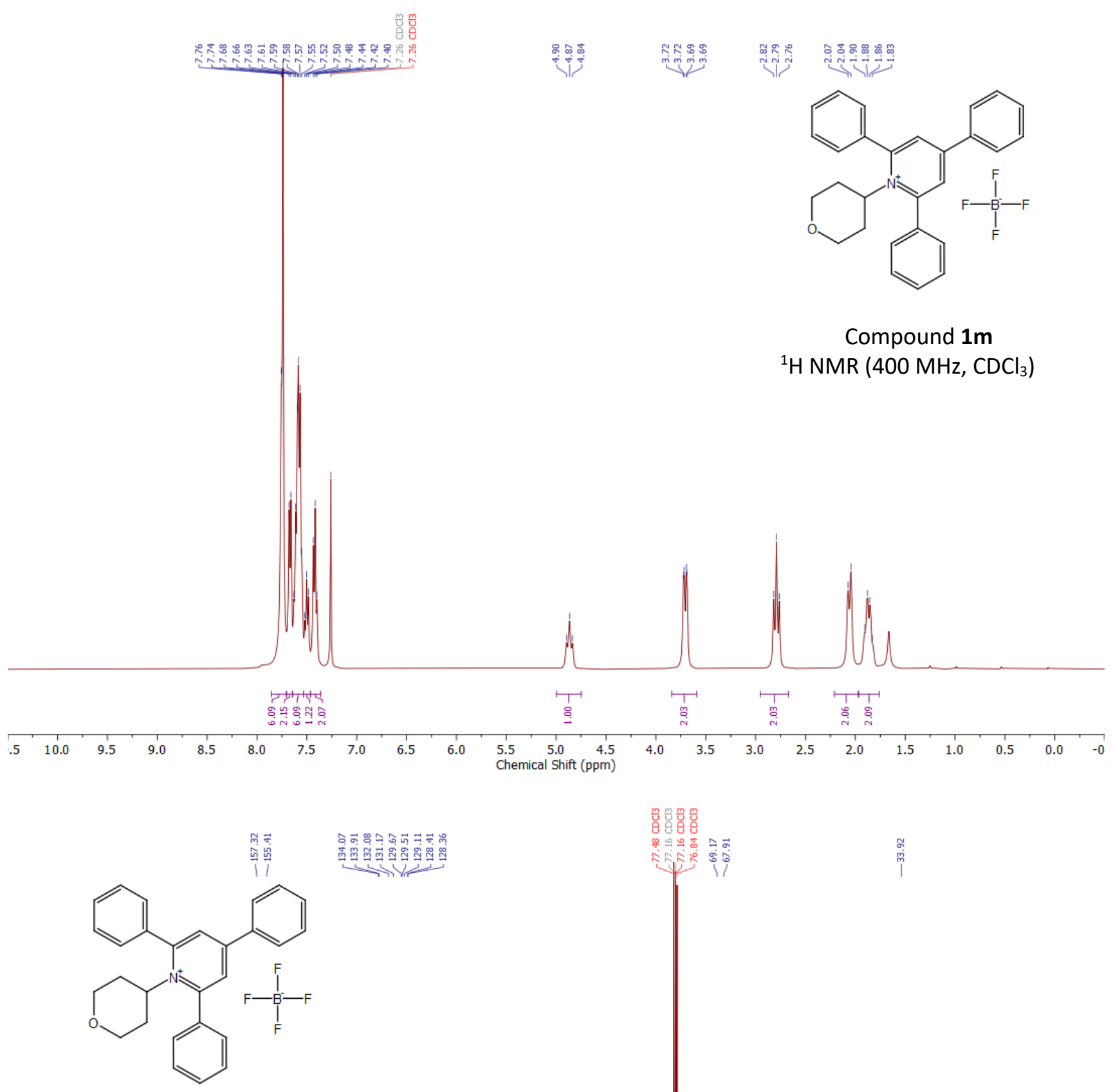

Compound $1 \mathrm{~m}$

${ }^{13} \mathrm{C}$ NMR $\left(101 \mathrm{MHz}, \mathrm{CDCl}_{3}\right)$ 


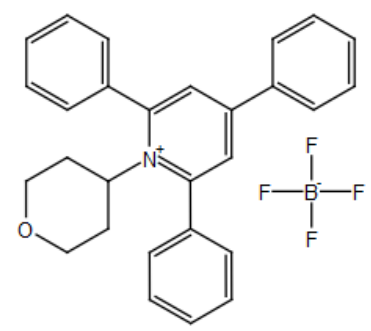

Compound $1 \mathrm{~m}$

$\left.{ }^{19} \mathrm{~F} \mathrm{NMR} \mathrm{(377} \mathrm{MHz}, \mathrm{CDCl}_{3}\right)$

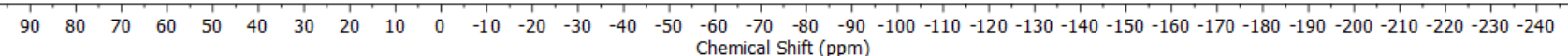


NMR spectra of 2,4,6-triphenyl-1-(tetrahydro-2H-thiopyran-4-yl)pyridin-1-ium tetrafluoroborate (1n)
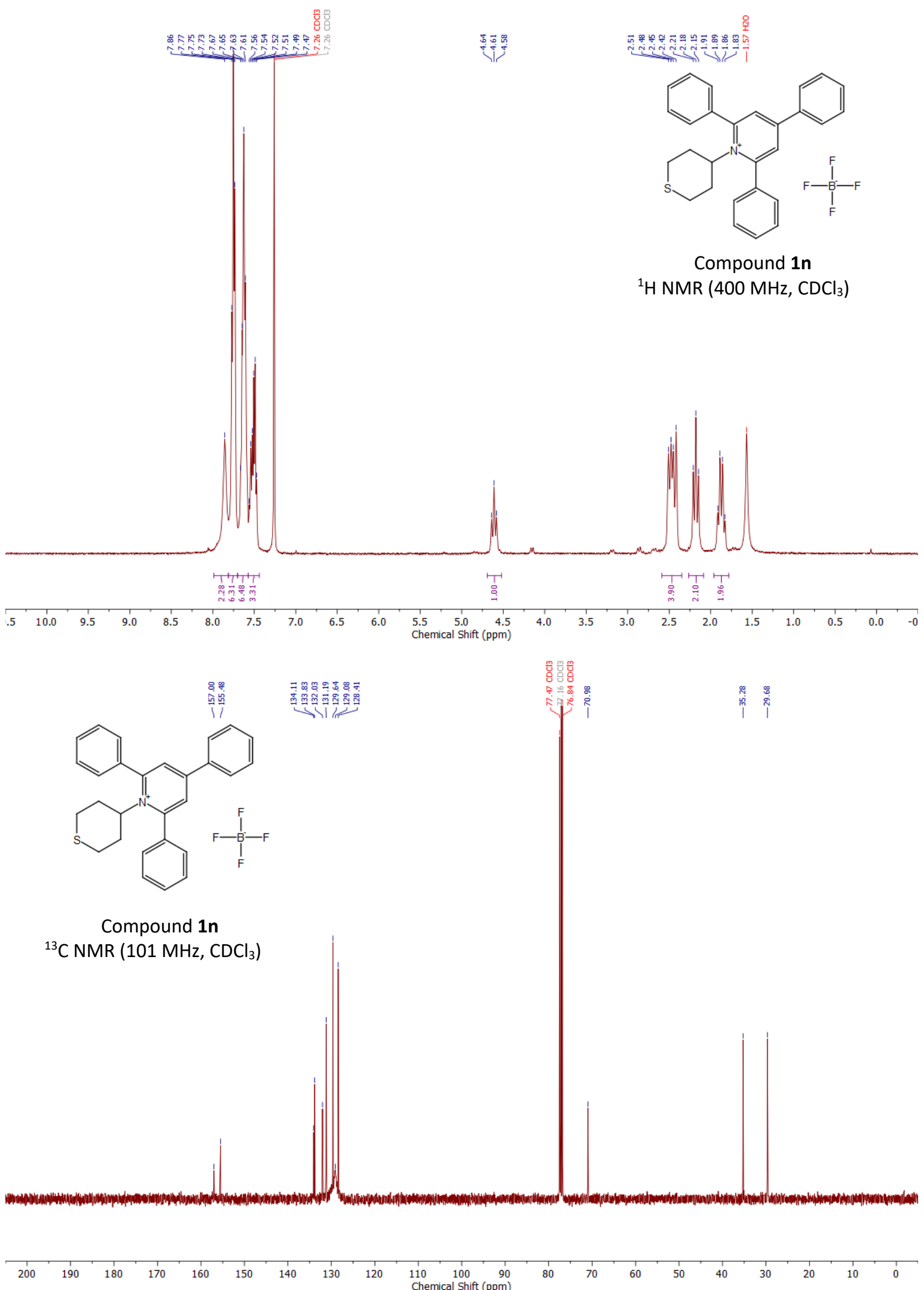


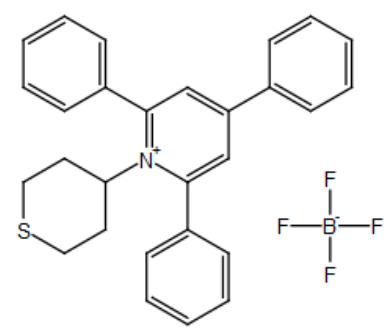

Compound 1n

${ }^{19} \mathrm{~F} \mathrm{NMR}\left(377 \mathrm{MHz}, \mathrm{CDCl}_{3}\right)$ 
NMR spectra of 1-(1,1-dioxidotetrahydro-2H-thiopyran-4-yl)-2,4,6-triphenylpyridin-1-ium tetrafluoroborate (10)
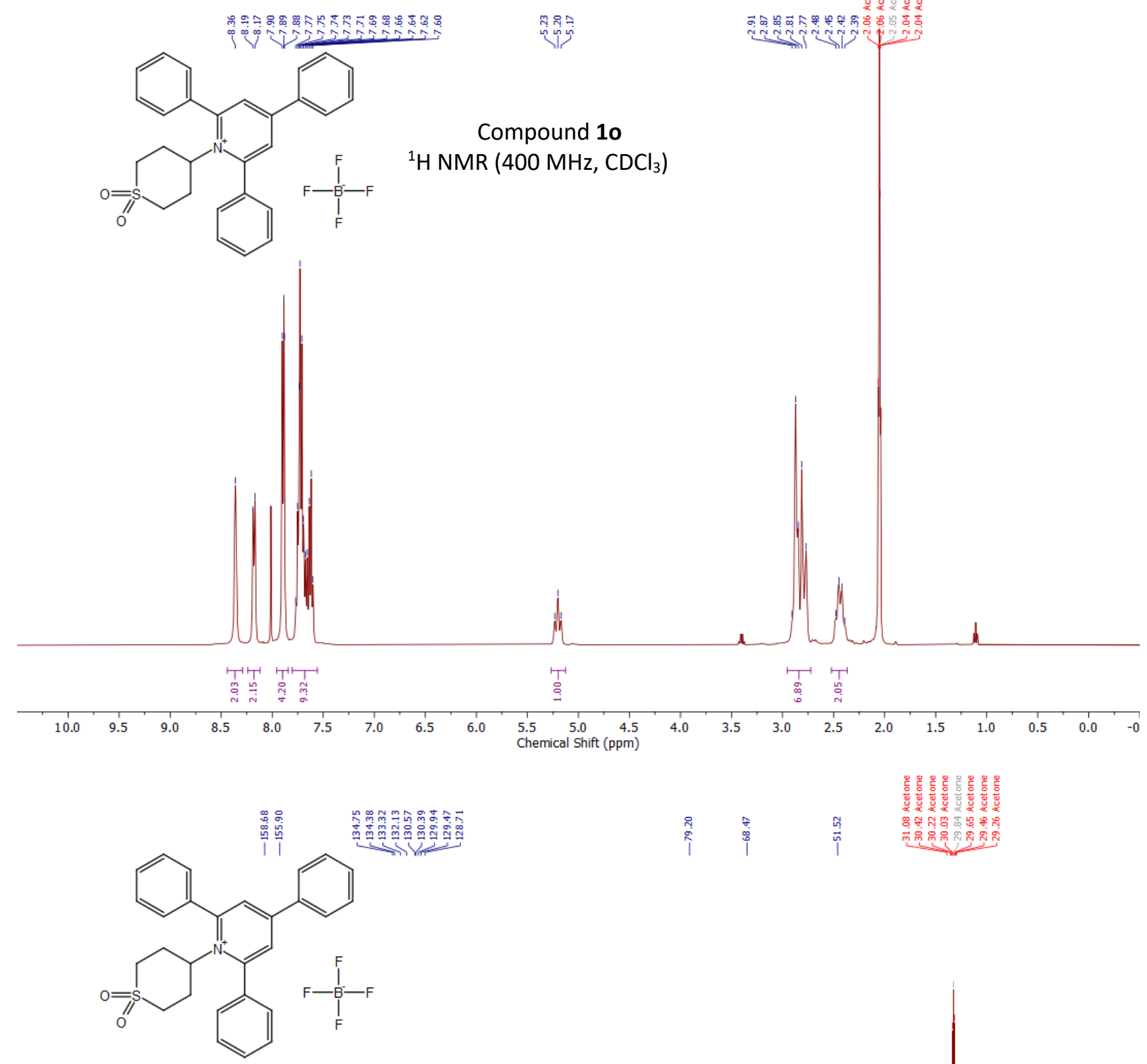

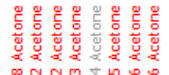

Compound 10

$\left.{ }^{13} \mathrm{C} \mathrm{NMR} \mathrm{(101} \mathrm{MHz,} \mathrm{CDCl}_{3}\right)$

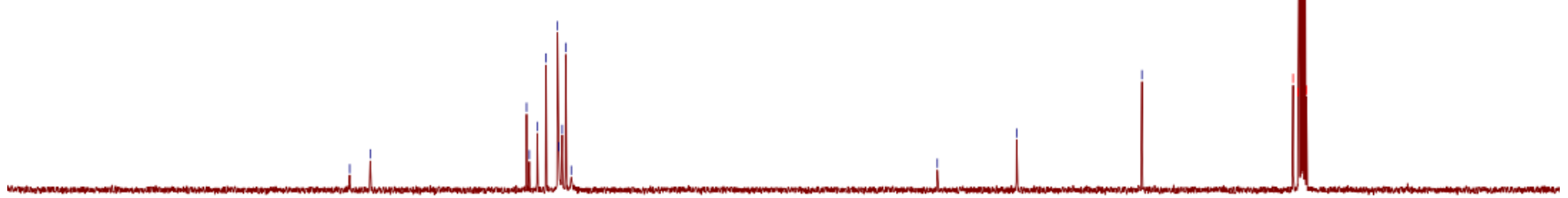




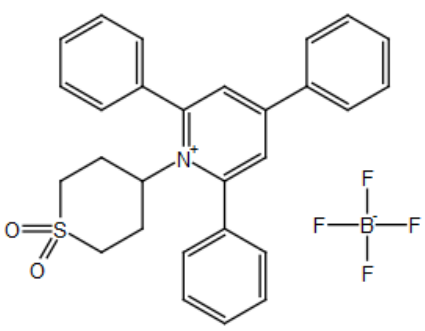

Compound 10

${ }^{19} \mathrm{~F}$ NMR (377 MHz, $\mathrm{CDCl}_{3}$ ) 
NMR spectra of 1-(4-hydroxycyclohexyl)-2,4,6-triphenylpyridin-1-ium tetrafluoroborate (1p)

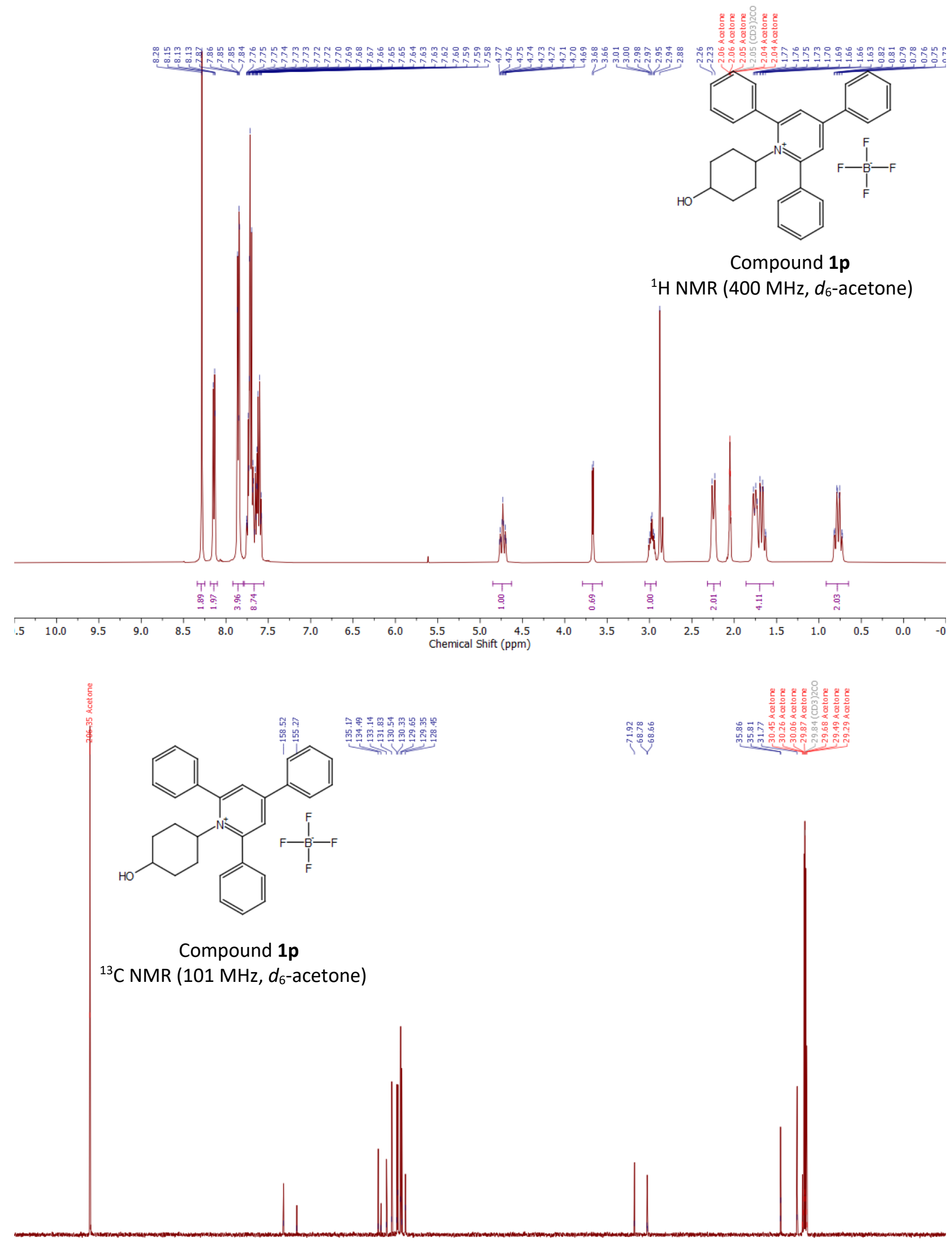

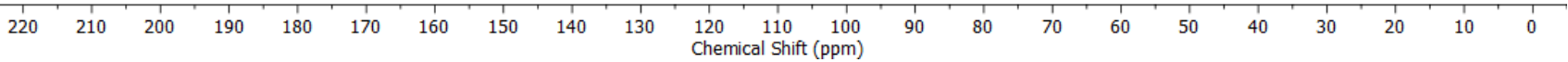




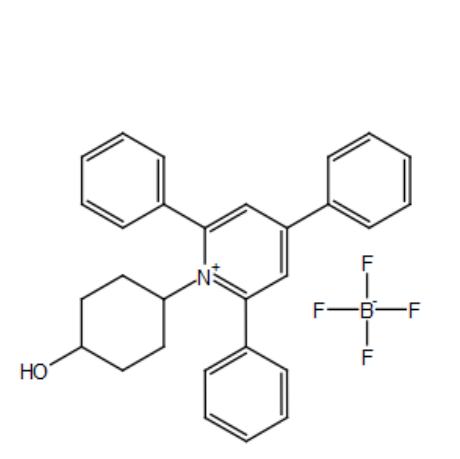

Compound 1p

${ }^{19} \mathrm{~F}$ NMR (377 MHz, $d_{6}$-acetone)

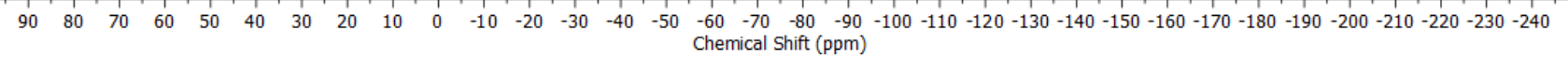


NMR spectra of 1-(4-carboxycyclohexyl)-2,4,6-triphenylpyridin-1-ium tetrafluoroborate (1q)<smiles></smiles>

Compound 1q

${ }^{1} \mathrm{H}$ NMR (400 MHz, $d_{6}$-DMSO)
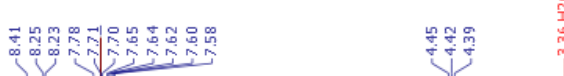

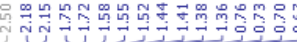
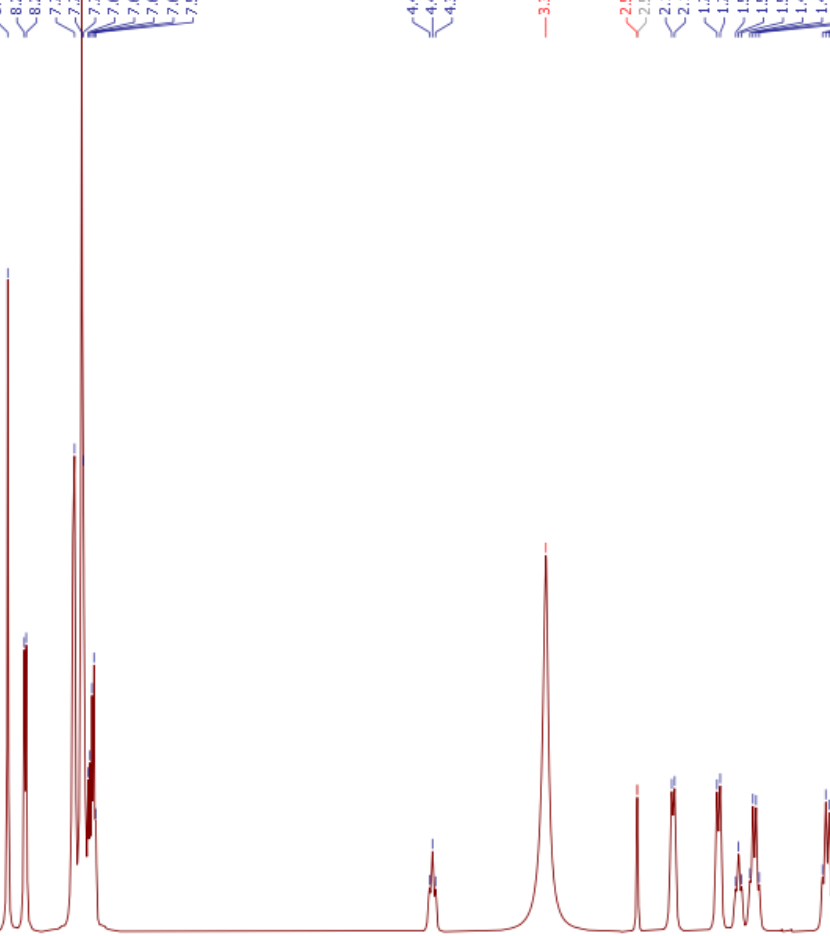

एग

욕

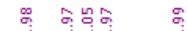

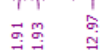

$\begin{array}{cc}8 & 7 \\ \text { Chemical Shift (ppm) }\end{array}$

$+$
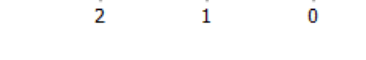<smiles></smiles>

Compound 1q

${ }^{13} \mathrm{C}$ NMR (101 MHz, $d_{6}$-DMSO)

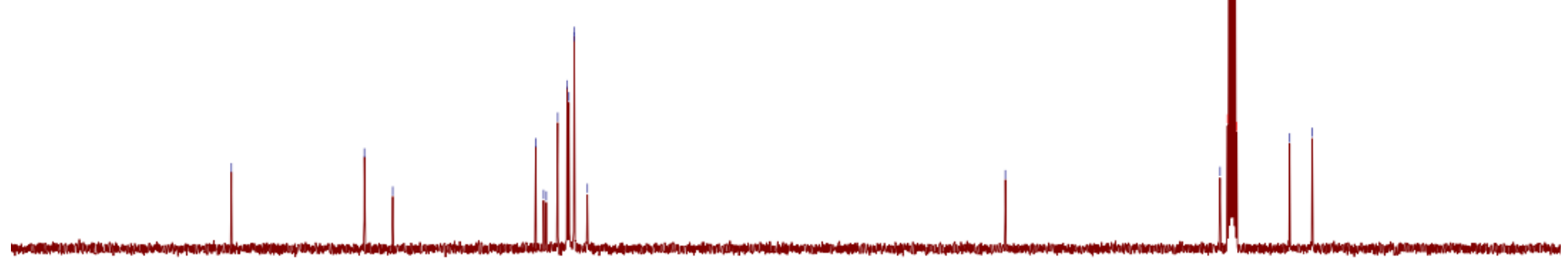




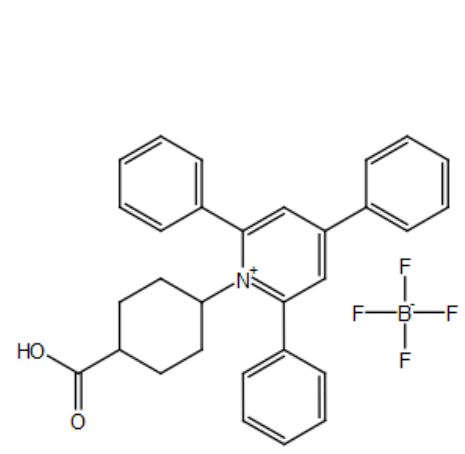

Compound 1q

${ }^{19} \mathrm{~F}$ NMR (377 MHz, $d_{6}$-DMSO)

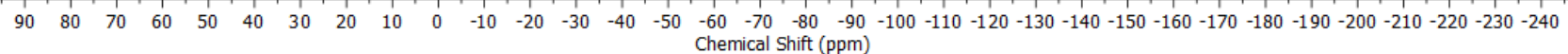


NMR spectra of 1-(4-(methoxycarbonyl)cyclohexyl)-2,4,6-triphenylpyridin-1-ium tetrafluoroborate (1r)
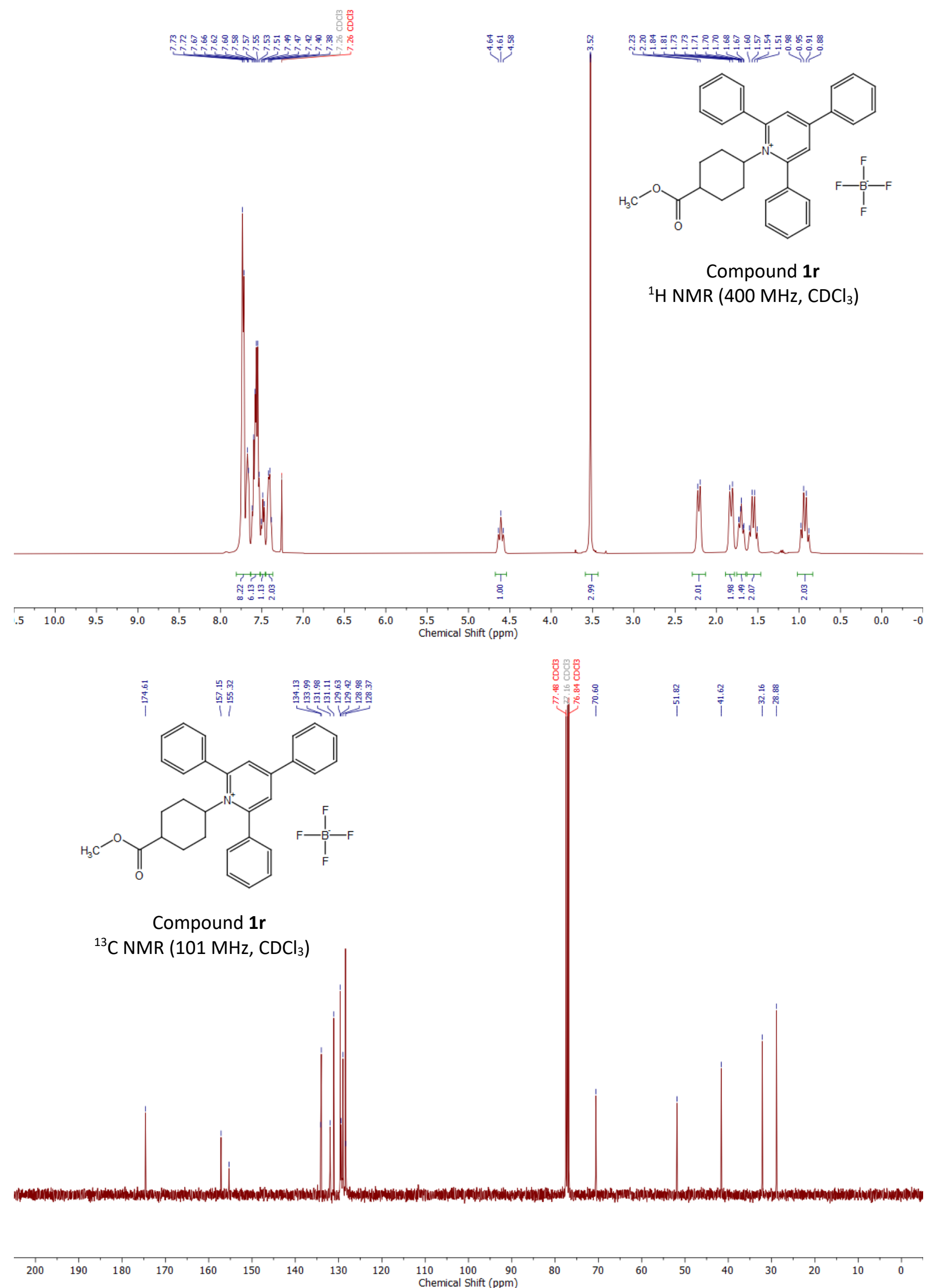


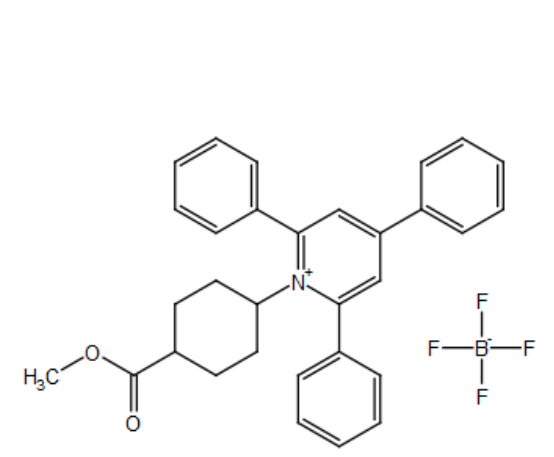

Compound $\mathbf{1 r}$

${ }^{19} \mathrm{~F} \mathrm{NMR} \mathrm{(377} \mathrm{MHz,} \mathrm{CDCl}_{3}$ )

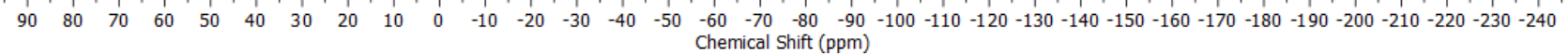


NMR spectra of 1-(1-(2,6-dimethylphenoxy)propan-2-yl)-2,4,6-triphenylpyridin-1-ium tetrafluoroborate (1s)

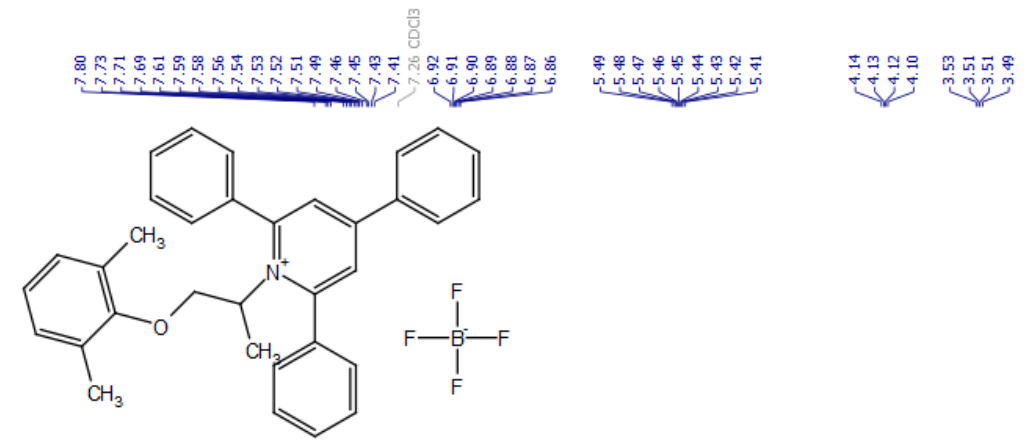

Compound 1s

${ }^{1} \mathrm{H} \mathrm{NMR}\left(400 \mathrm{MHz}, \mathrm{CDCl}_{3}\right)$

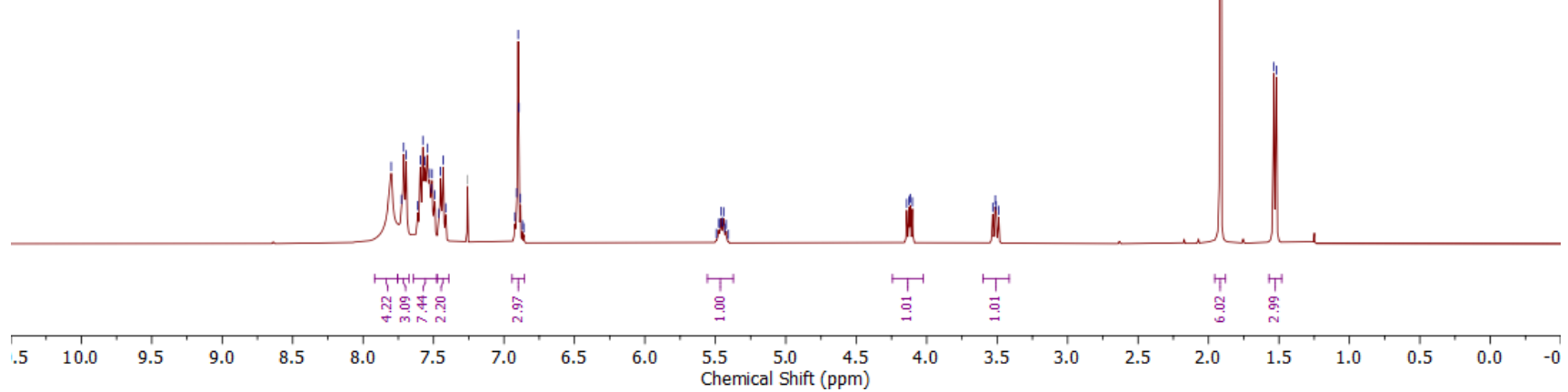<smiles></smiles>

Compound 1s

${ }^{13} \mathrm{C} \mathrm{NMR}\left(101 \mathrm{MHz}, \mathrm{CDCl}_{3}\right)$
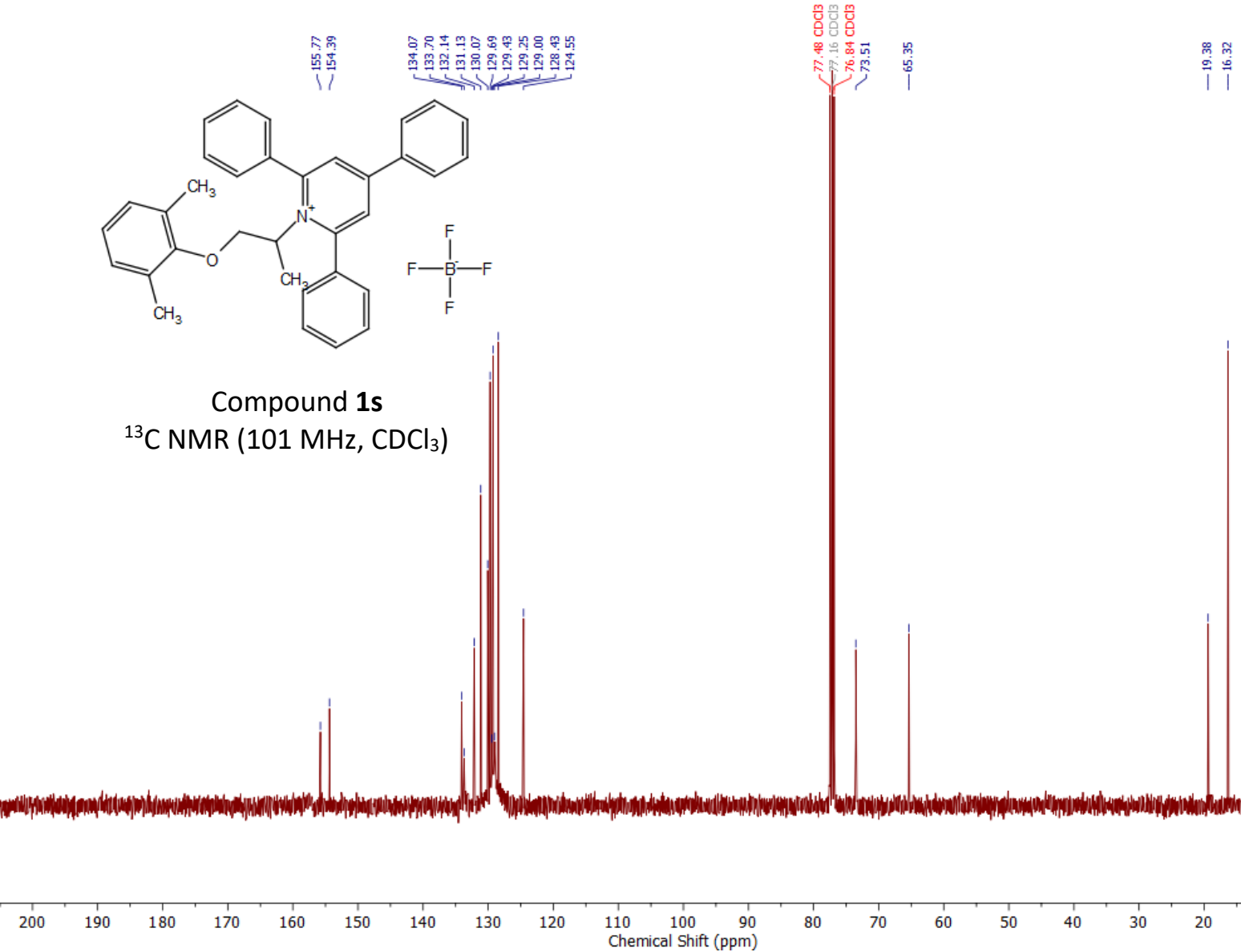

$\begin{array}{llllllllll}200 & 190 & 180 & 170 & 160 & 150 & 140 & 130 & 120 & \begin{array}{l}110 \\ \text { Chemical Shift }(\mathrm{ppm})\end{array}\end{array}$ 


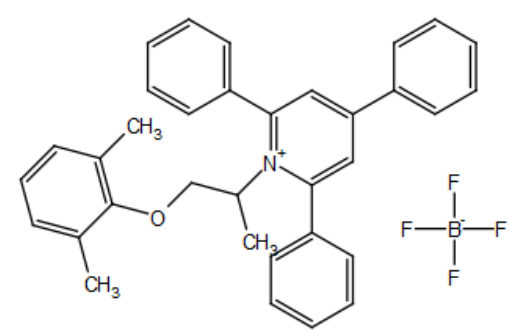

Compound 1s

${ }^{19} \mathrm{~F} \mathrm{NMR}\left(377 \mathrm{MHz}, \mathrm{CDCl}_{3}\right)$

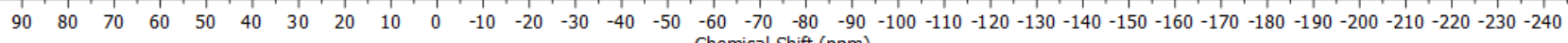
Chemical Shift (ppm) 
NMR spectra of (S)-1-(1-hydroxy-3-phenylpropan-2-yl)-2,4,6-triphenylpyridin-1-ium tetrafluoroborate (1t)

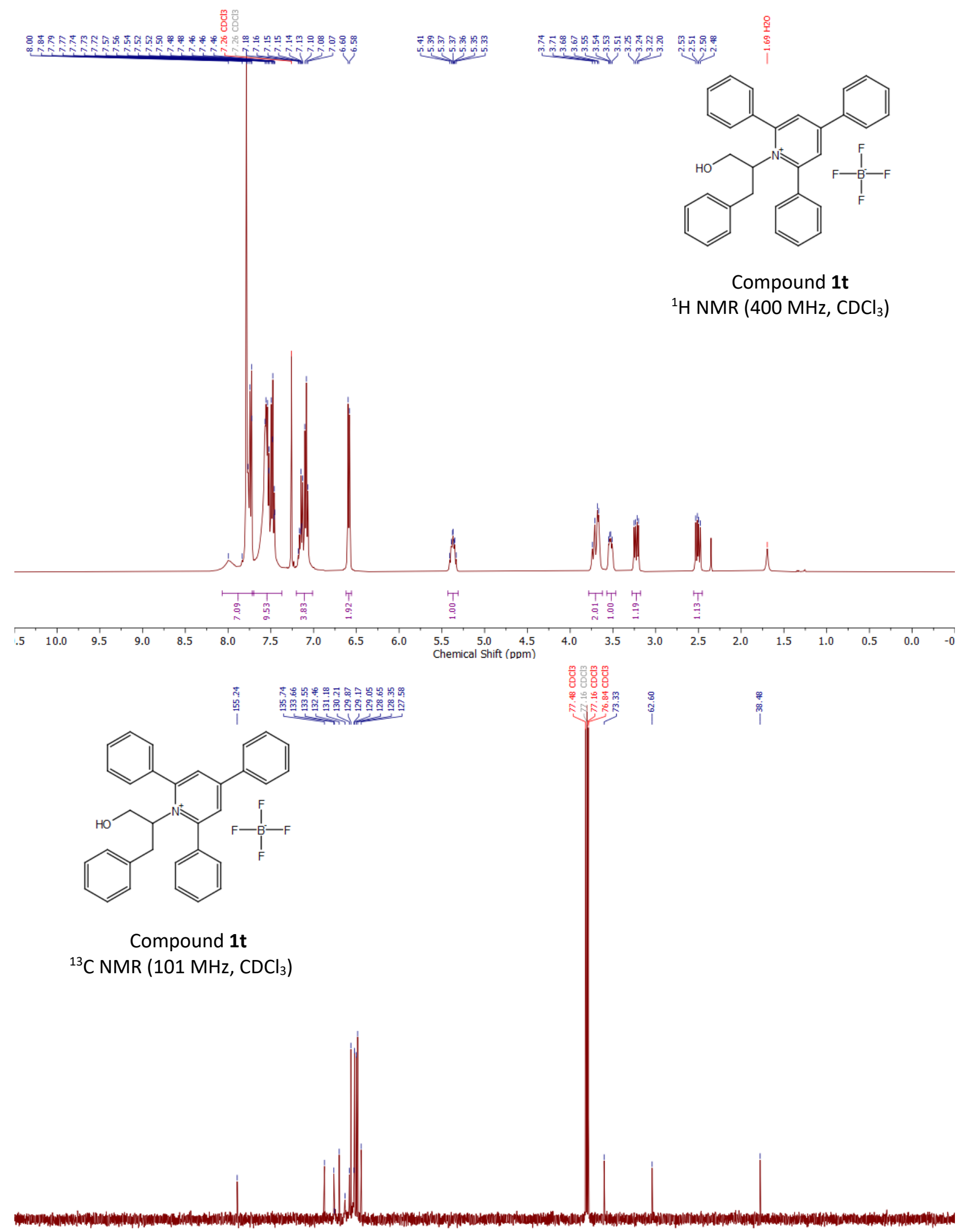

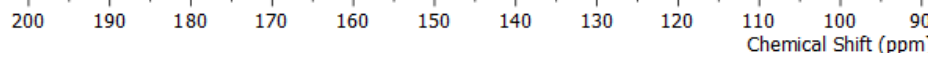


<smiles></smiles>

Compound 1t

${ }^{19}$ F NMR (377 MHz, $\mathrm{CDCl}_{3}$ )

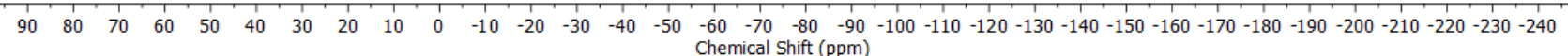


NMR spectra of (S)-1-(1-methoxy-1-oxo-3-phenylpropan-2-yl)-2,4,6-triphenylpyridin-1-ium tetrafluoroborate (1u)
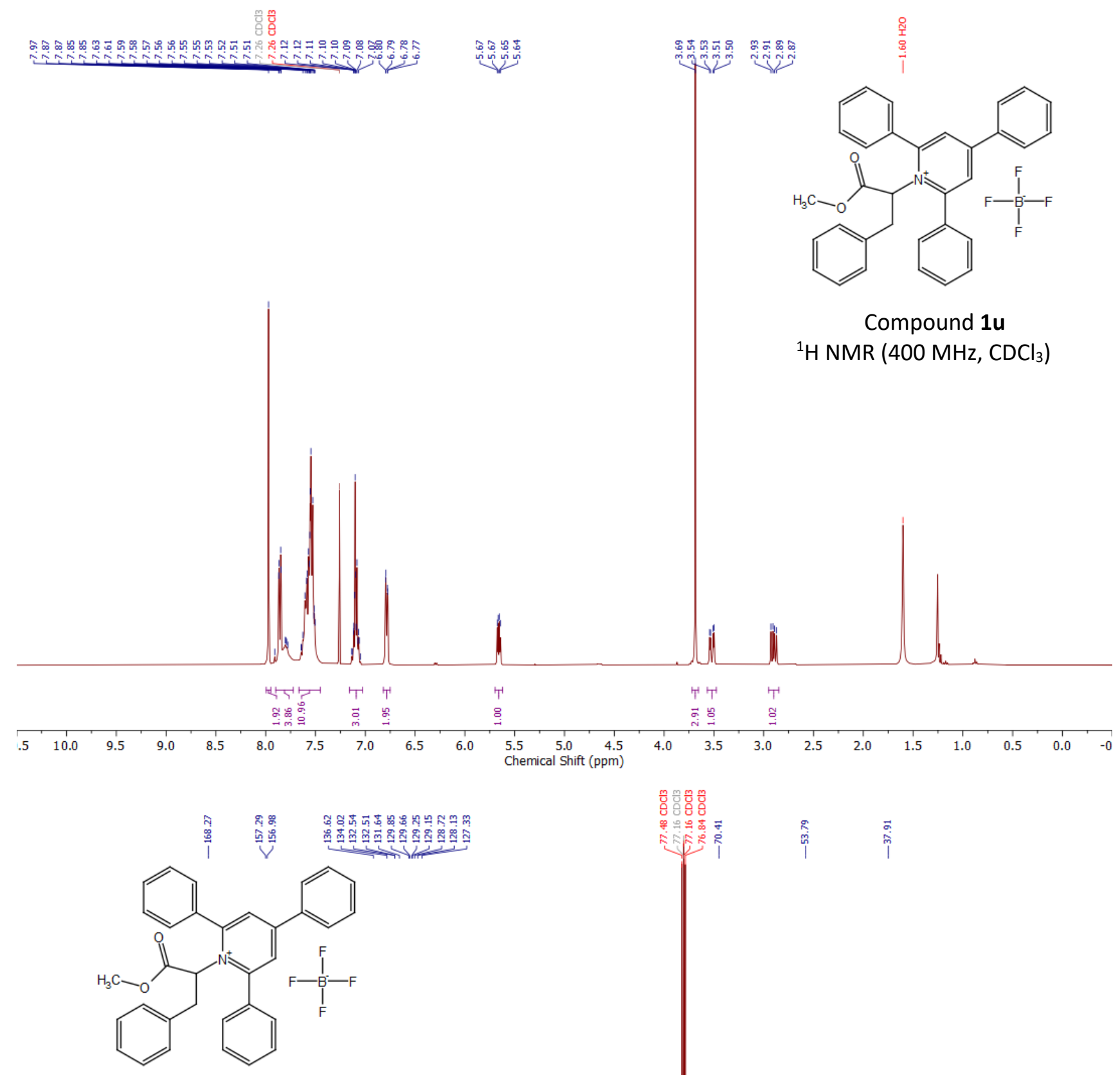

Compound 1u

${ }^{13} \mathrm{C}$ NMR $\left(101 \mathrm{MHz}, \mathrm{CDCl}_{3}\right)$

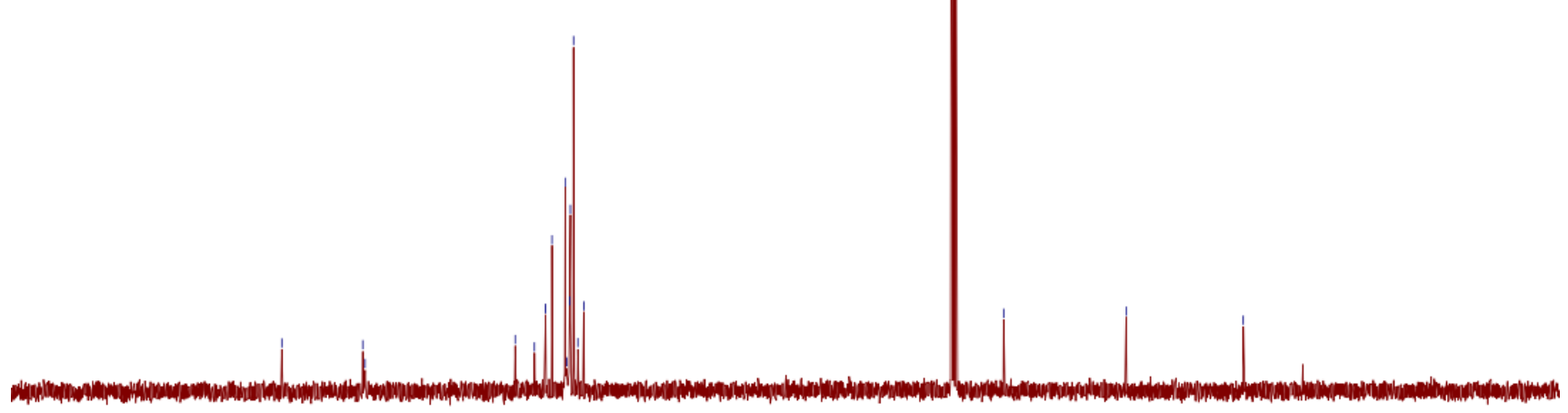

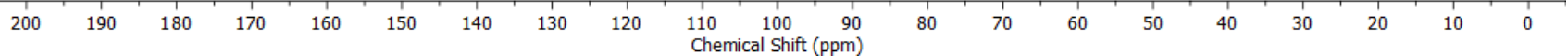


<smiles></smiles>

Compound $\mathbf{1 u}$

${ }^{19} \mathrm{~F}$ NMR (377 MHz, $\mathrm{CDCl}_{3}$ ) 
NMR spectra of 1-benzyl-2,4,6-triphenylpyridin-1-ium tetrafluoroborate (1b)

응 몽

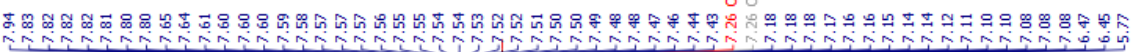

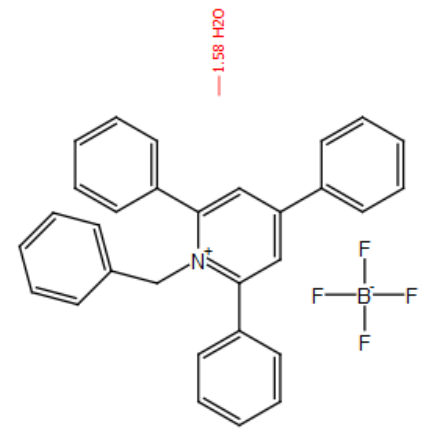

Compound 1b

${ }^{1} \mathrm{H} \mathrm{NMR}\left(400 \mathrm{MHz}, \mathrm{CDCl}_{3}\right)$

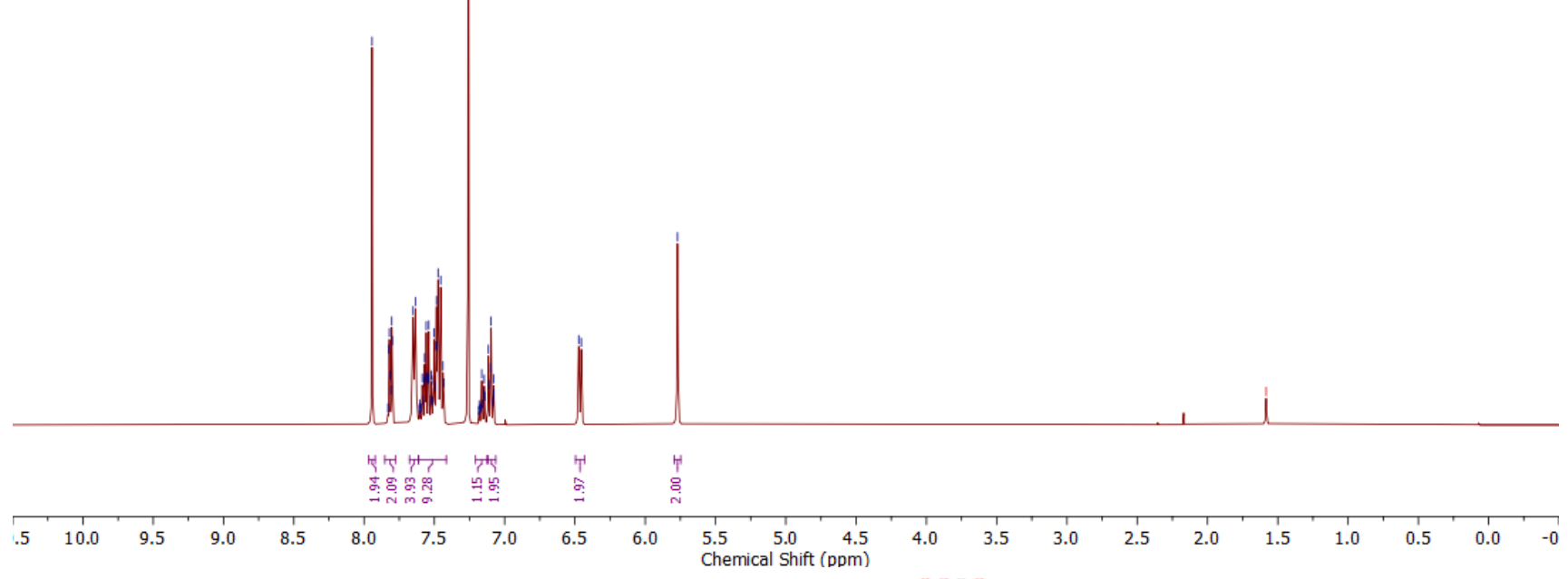<smiles>FC(F)(F)F</smiles>

Compound $\mathbf{1 b}$

${ }^{13} \mathrm{C}$ NMR (101 MHz, $\left.\mathrm{CDCl}_{3}\right)$

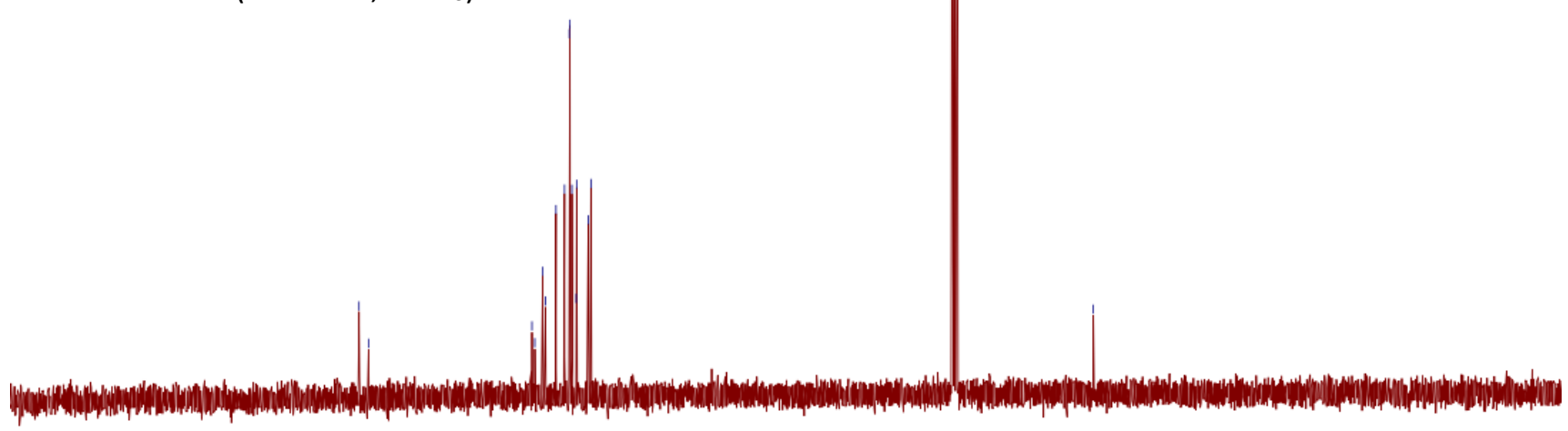

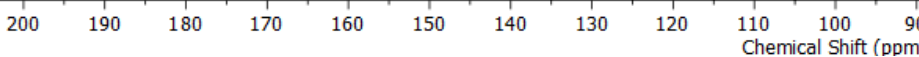




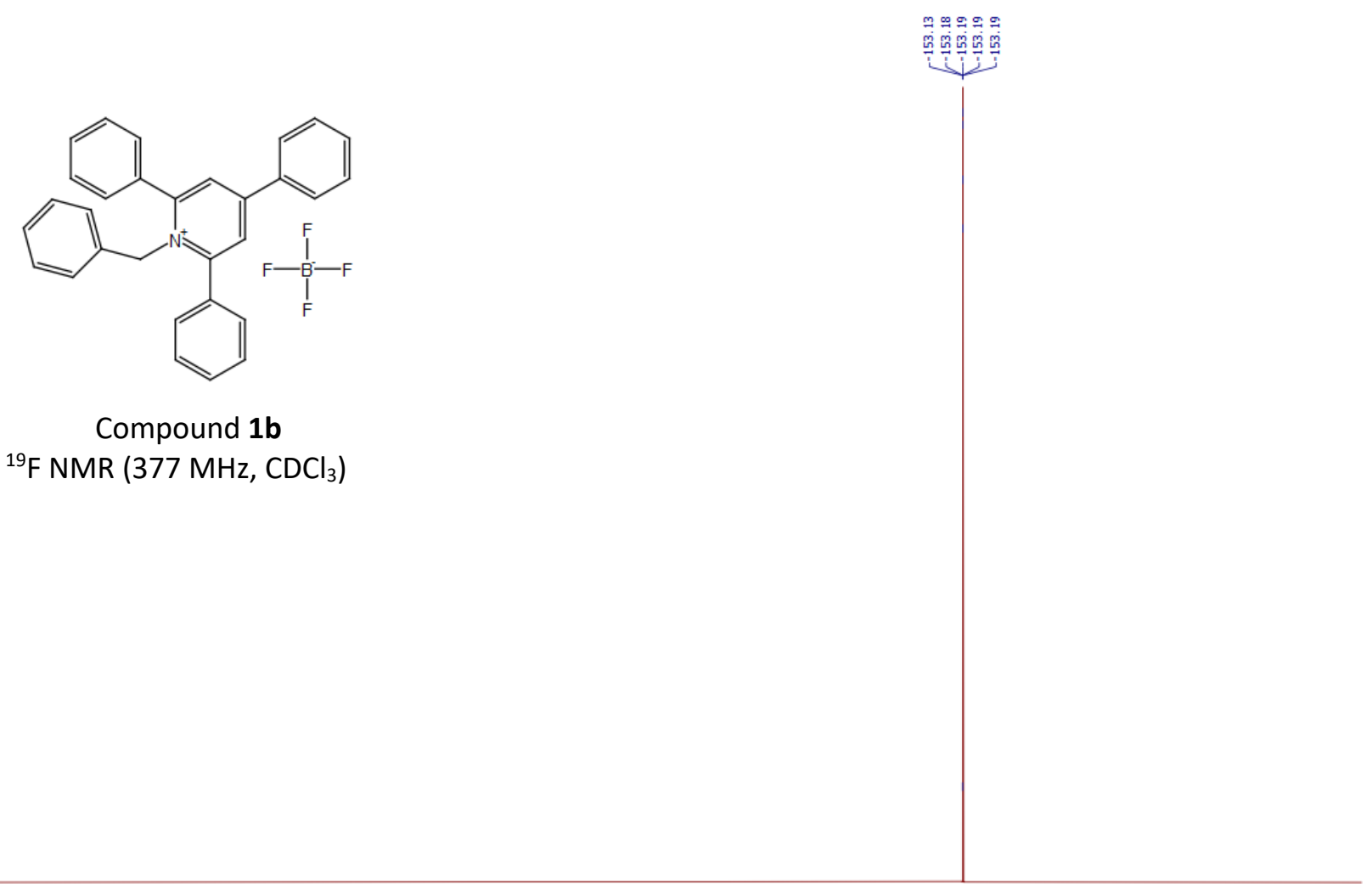

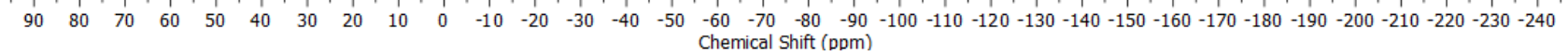


NMR spectra of 1-(4-methoxybenzyl)-2,4,6-triphenylpyridin-1-ium tetrafluoroborate (1v)

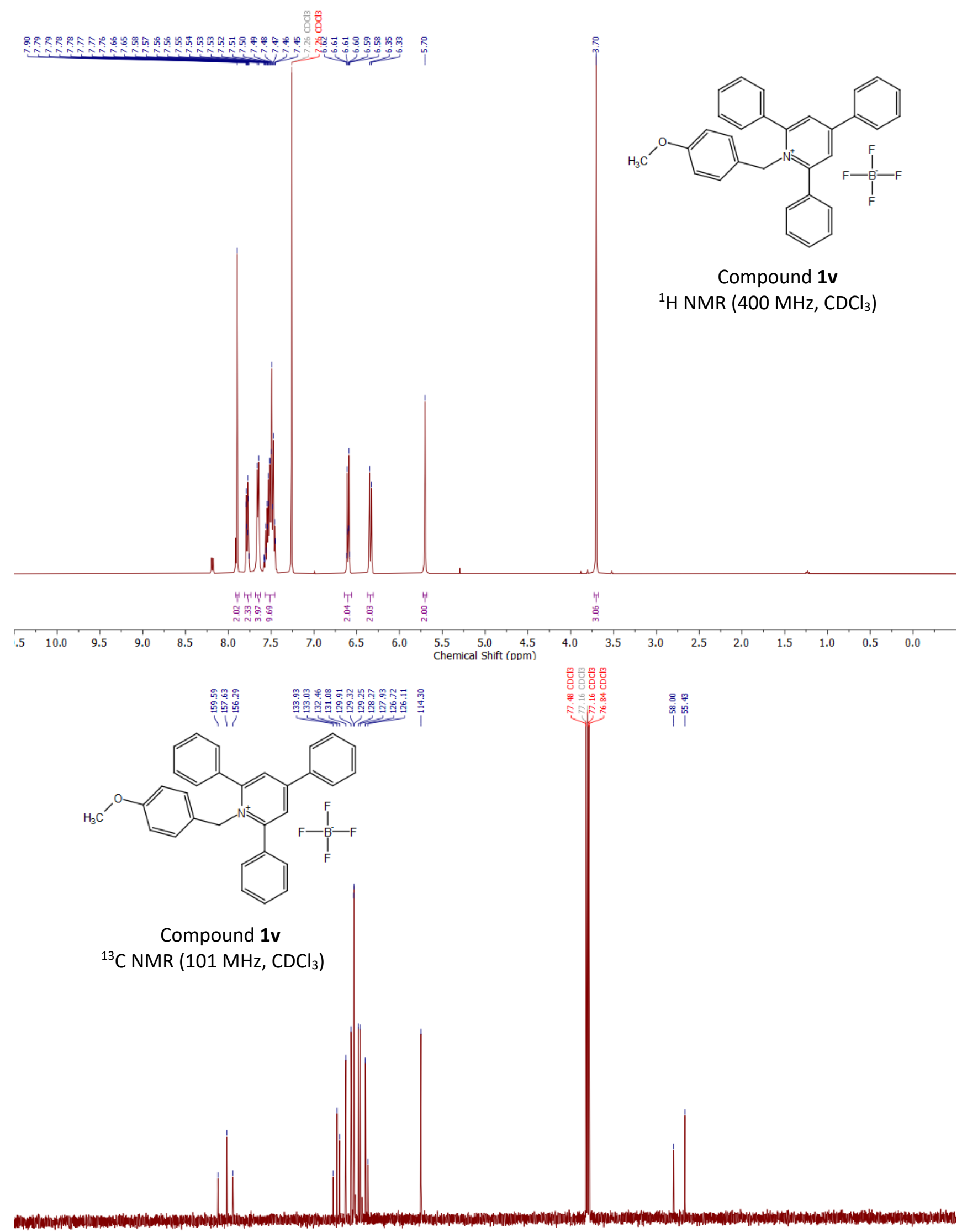

$\begin{array}{llllllllllllllllllllll}200 & 190 & 180 & 170 & 160 & 150 & 140 & 130 & 120 & \begin{array}{c}110 \\ \text { Chemical Shift }(\mathrm{Dbm})\end{array} & 80 & 70 & 60 & 50 & 40 & 30 & 20 & 10 & 0\end{array}$




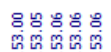

은

(c)

Compound 1v

${ }^{19} \mathrm{~F} \mathrm{NMR} \mathrm{(377} \mathrm{MHz,} \mathrm{CDCl}_{3}$ )

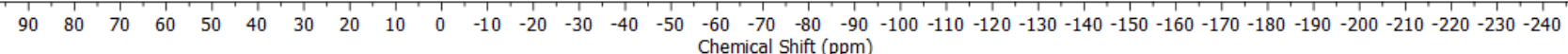


NMR spectra of 1-(3-methoxybenzyl)-2,4,6-triphenylpyridin-1-ium tetrafluoroborate (1w)

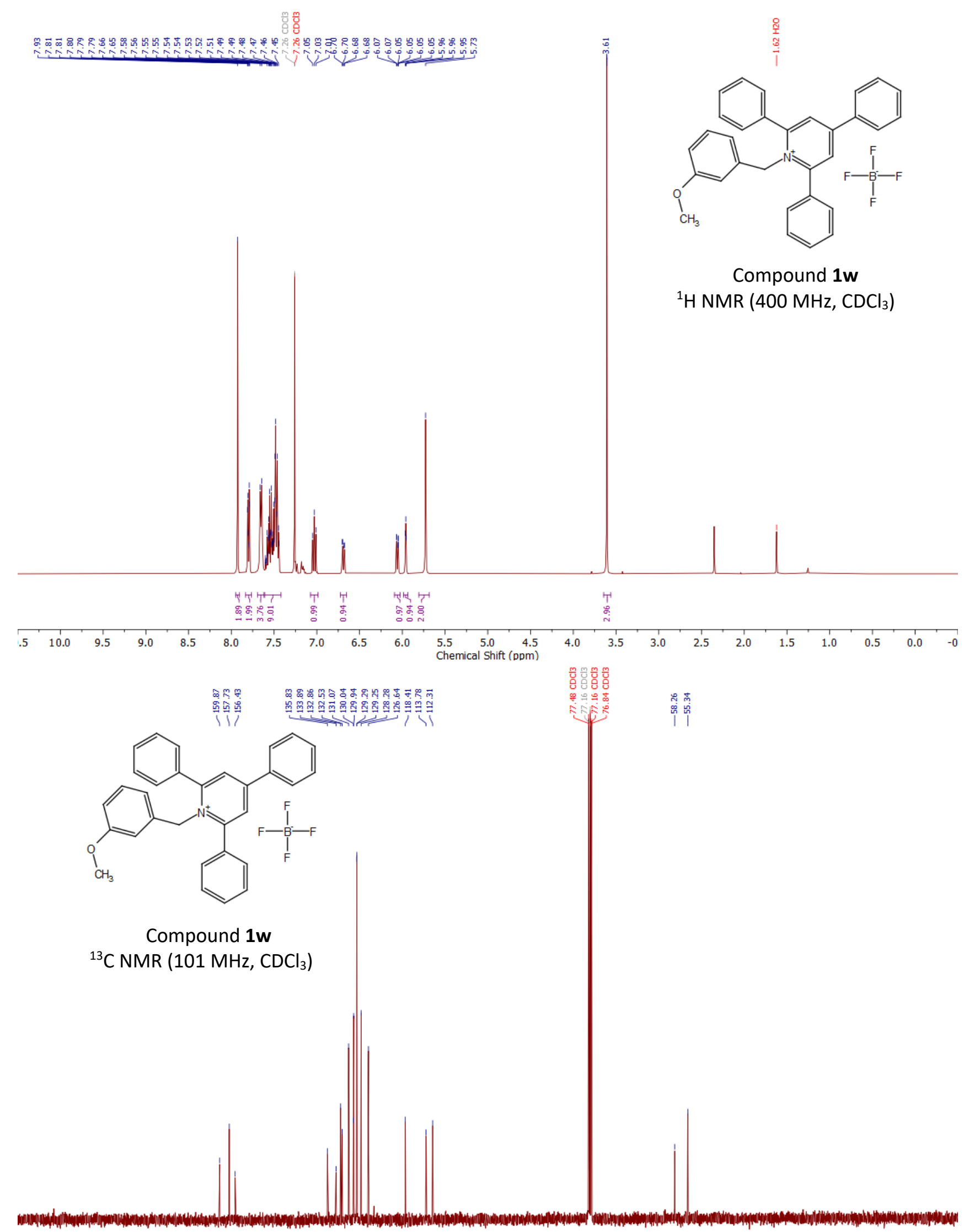

(101 $\mathrm{MHz}_{\mathrm{CDCl}}$

$\begin{array}{llllllllllll}200 & 190 & 180 & 170 & 160 & 150 & 140 & 130 & 120 & 110 & 100 & 90\end{array}$ Chemical Shift (pom) 


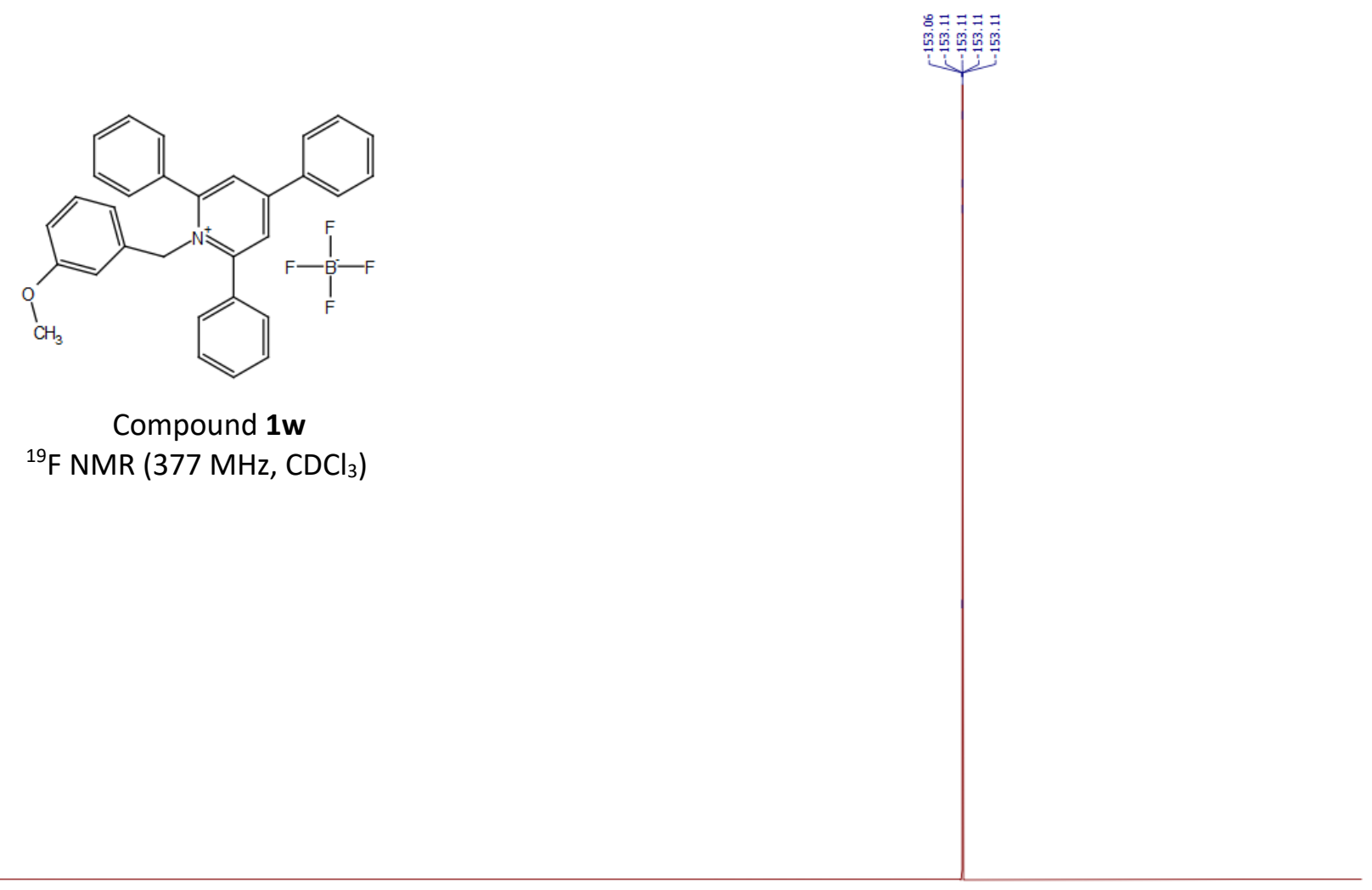

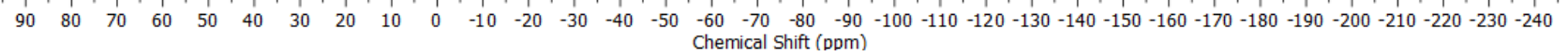


NMR spectra of 1-(2-methoxybenzyl)-2,4,6-triphenylpyridin-1-ium tetrafluoroborate (1x)

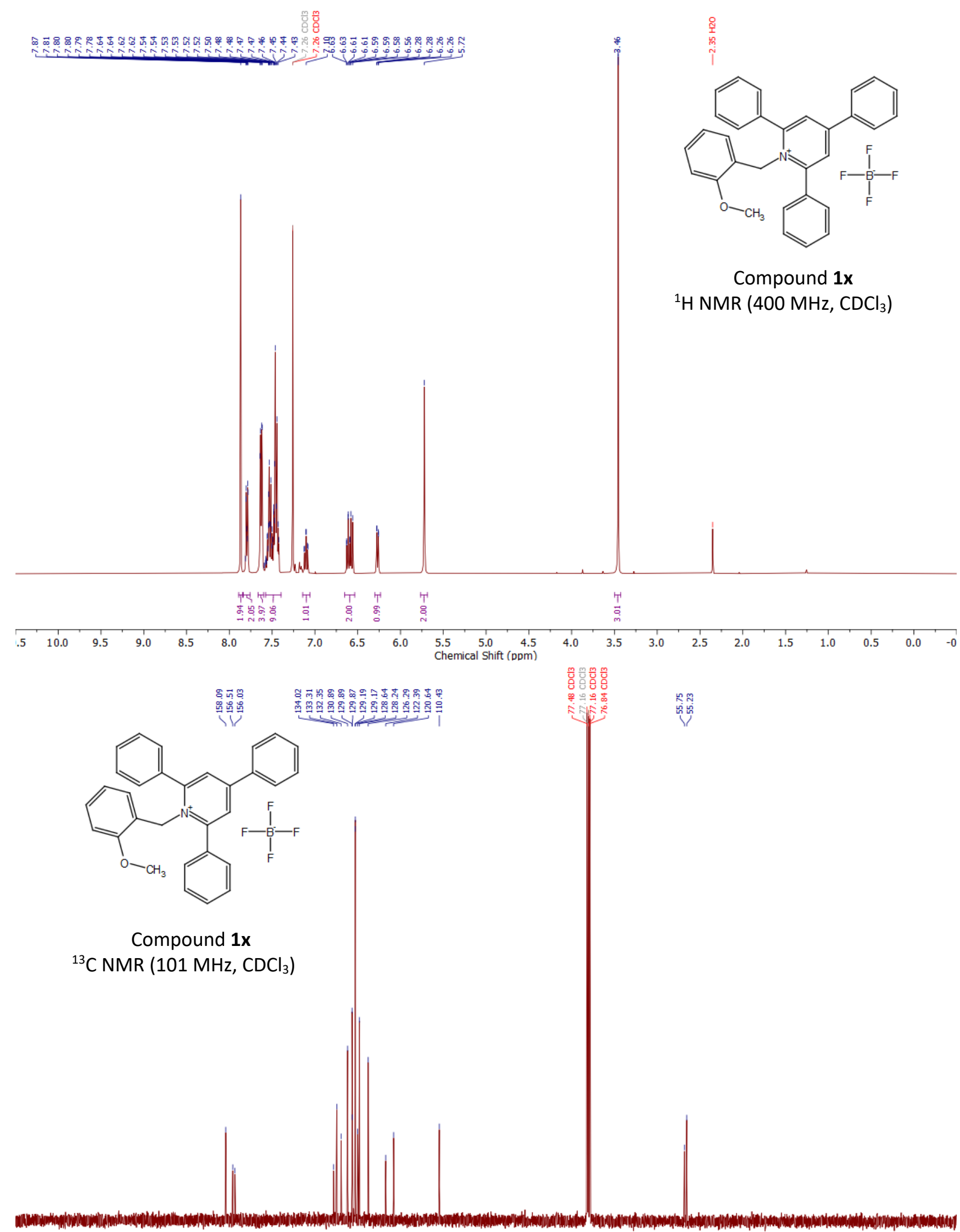

$\begin{array}{llllllllllllllllllllll}200 & 190 & 180 & 170 & 160 & 150 & 140 & 130 & 120 & \begin{array}{c}110 \\ \text { Chemical Shift (ovm) }\end{array} & 80 & 70 & 60 & 50 & 40 & 30 & 20 & 10 & 0\end{array}$ 


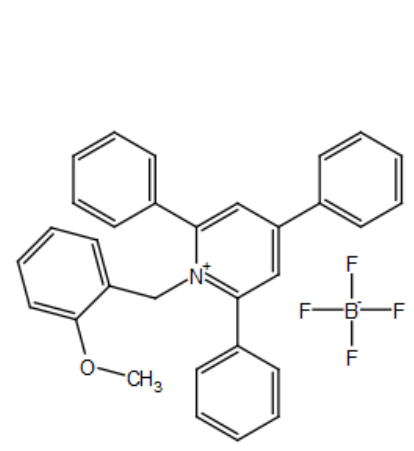

느 ำ ำ

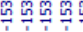

Compound $\mathbf{1 x}$

${ }^{19}$ F NMR (377 MHz, $\mathrm{CDCl}_{3}$ )

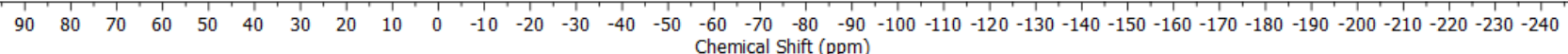


NMR spectra of 1-(4-chlorobenzyl)-2,4,6-triphenylpyridin-1-ium tetrafluoroborate (1y)

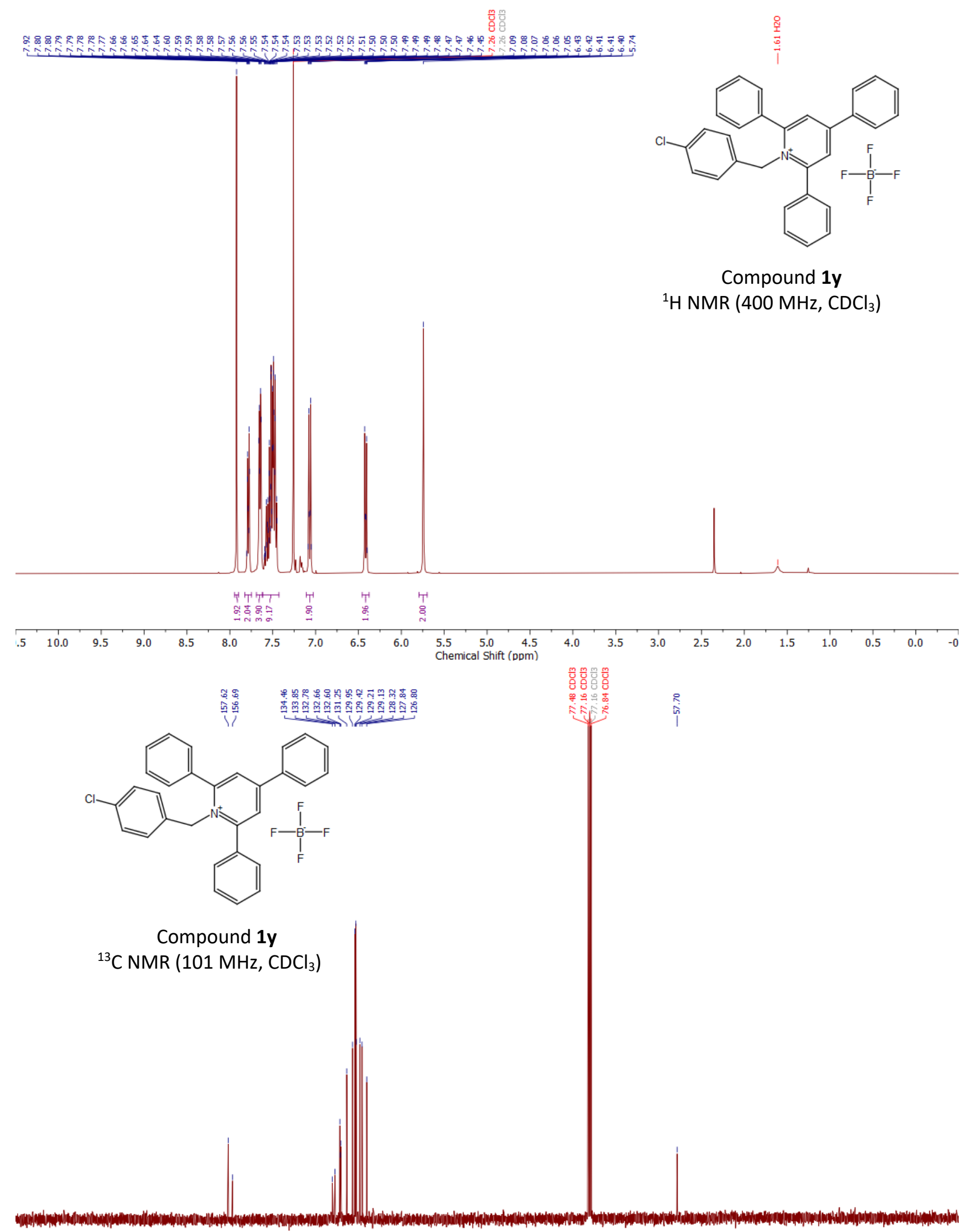

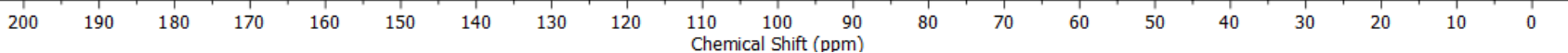




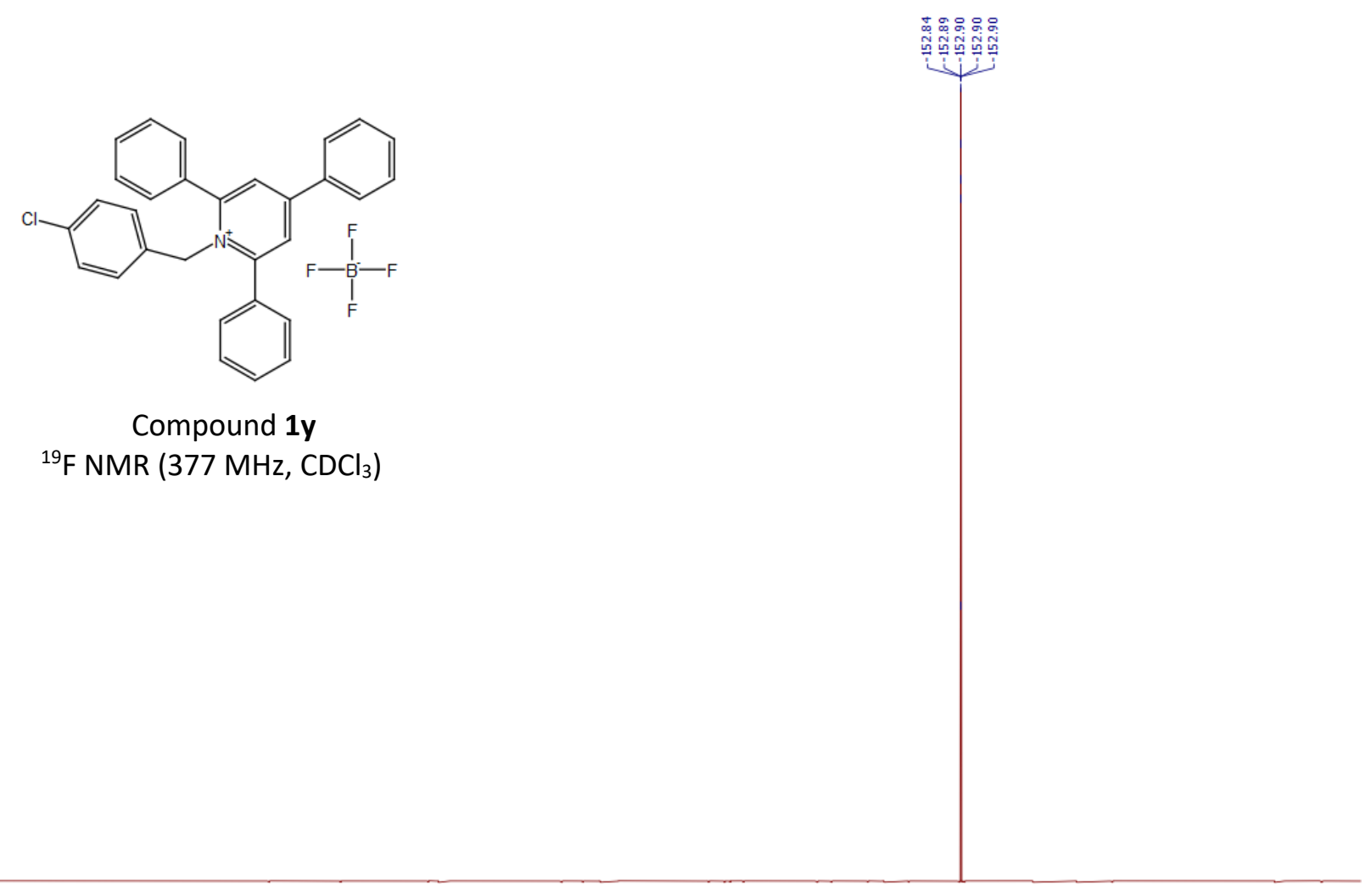

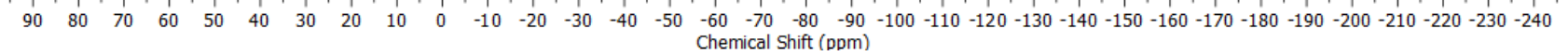


NMR spectra of 2,4,6-triphenyl-1-(4-(trifluoromethyl)benzyl)pyridin-1-ium tetrafluoroborate (1z)

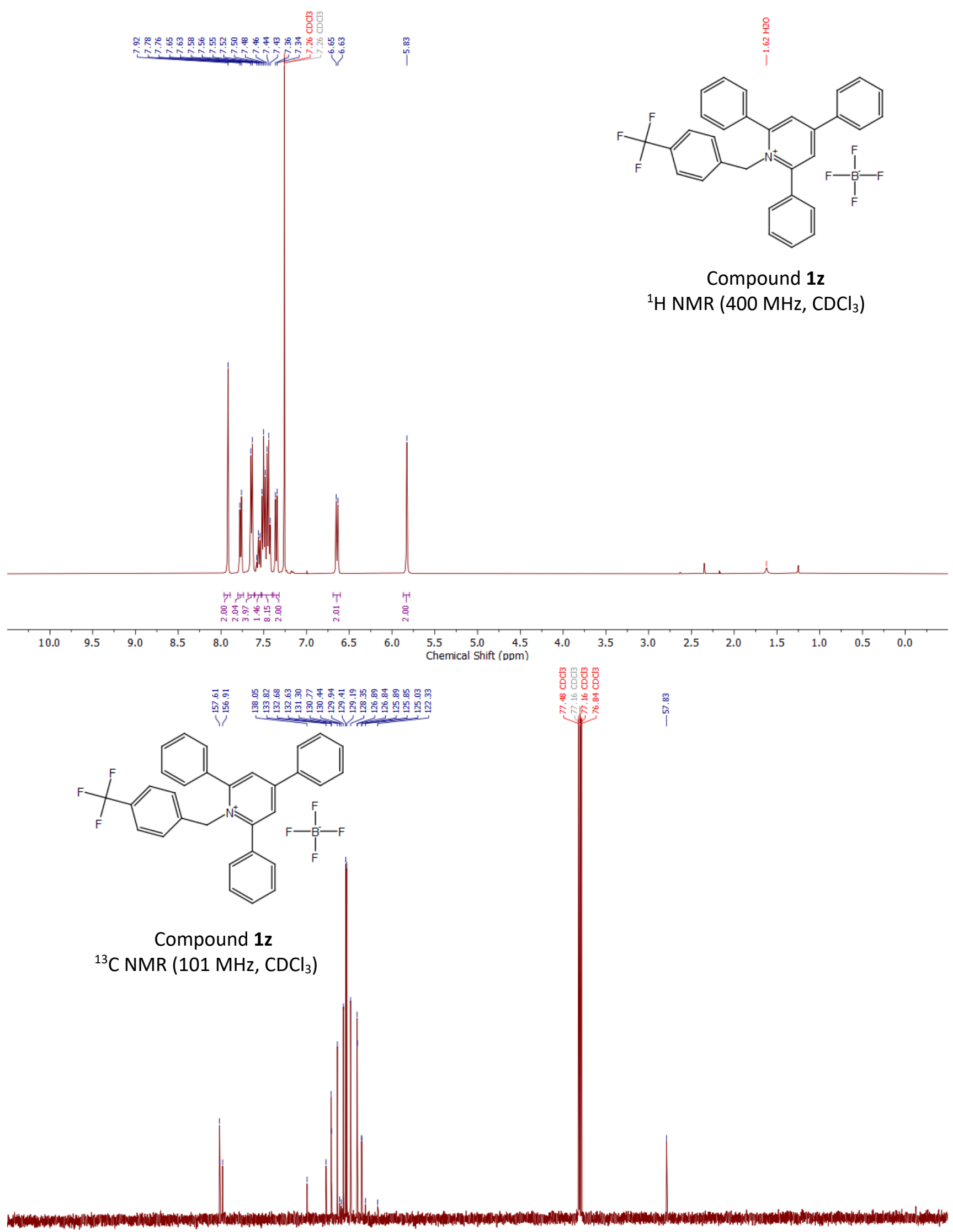

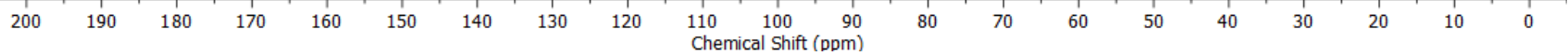




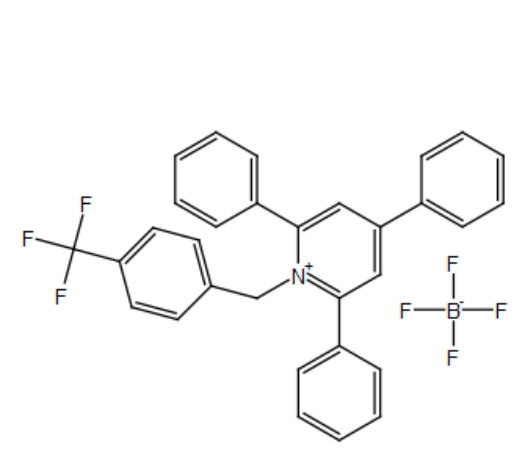

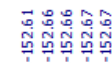

Compound $\mathbf{1 z}$

${ }^{19} \mathrm{~F}$ NMR (377 MHz, $\mathrm{CDCl}_{3}$ )

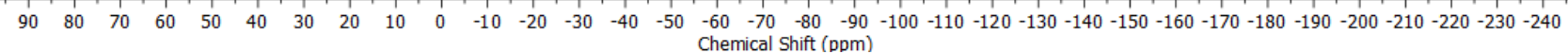


NMR spectra of 2,4,6-triphenyl-1-(thiophen-2-ylmethyl)pyridin-1-ium tetrafluoroborate (1aa)

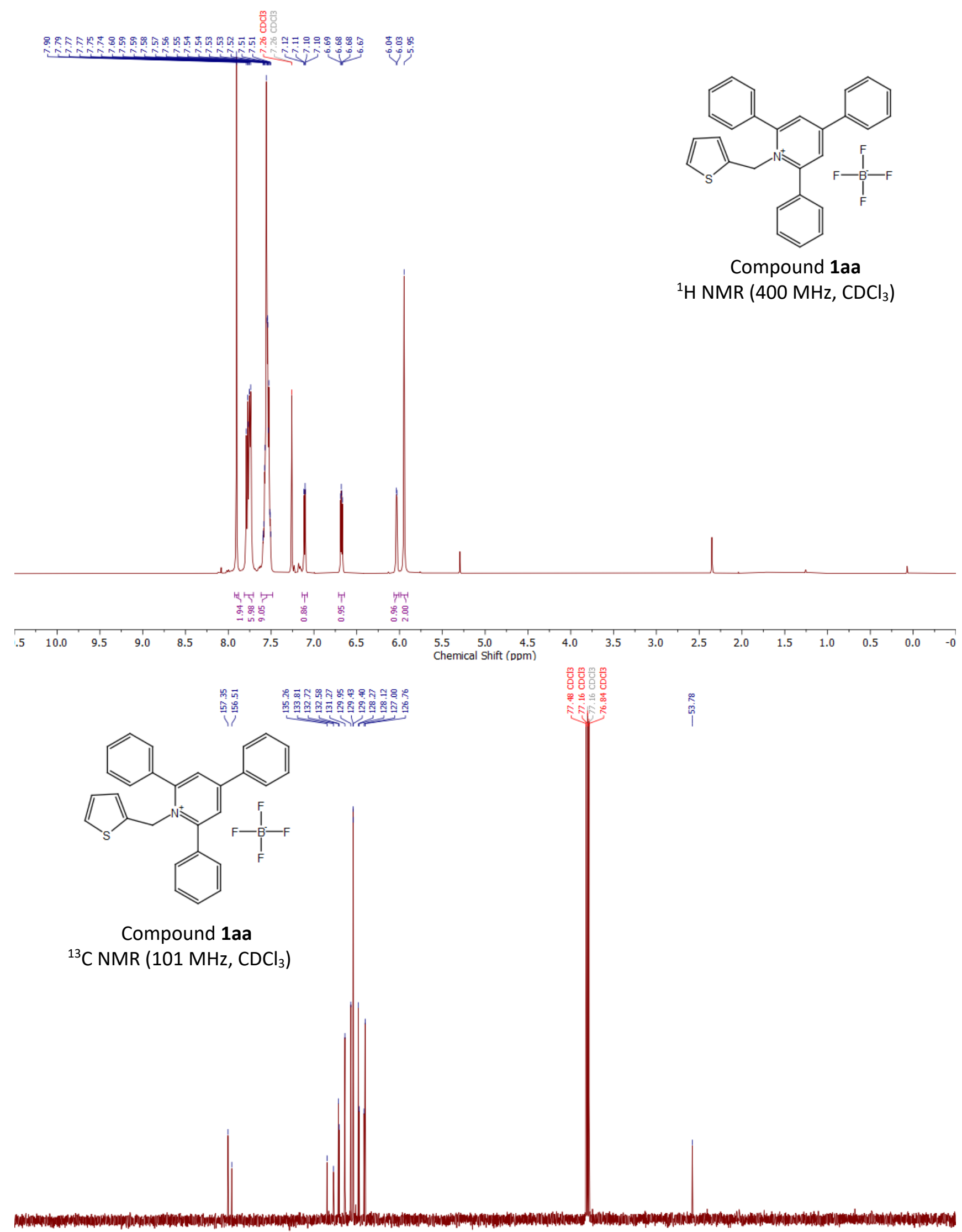

$\begin{array}{llllllllll}200 & 190 & 180 & 170 & 160 & 150 & 140 & 130 & 120 & \begin{array}{c}110 \\ \text { Chemical Shift (pom) }\end{array}\end{array}$ 


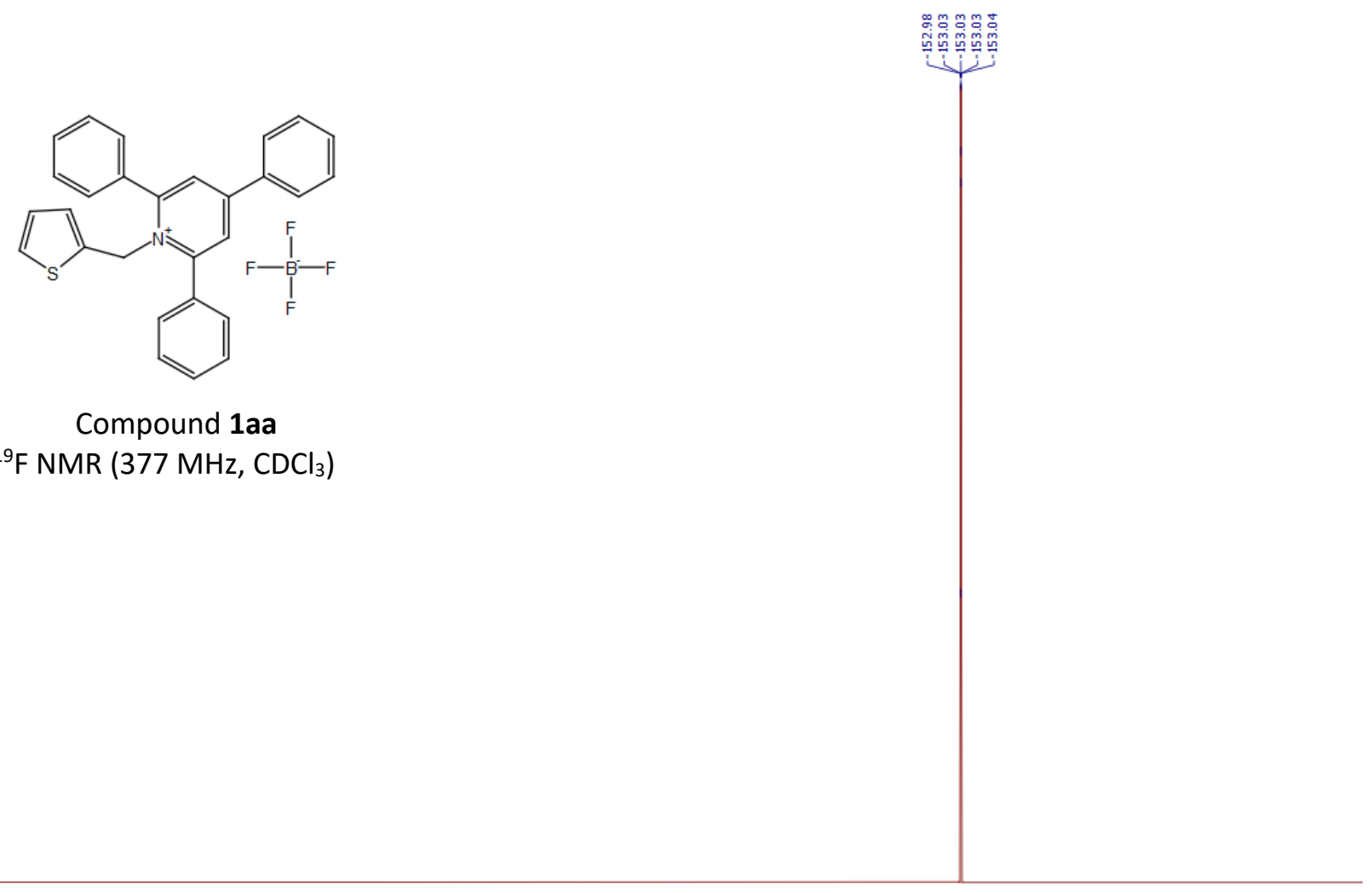

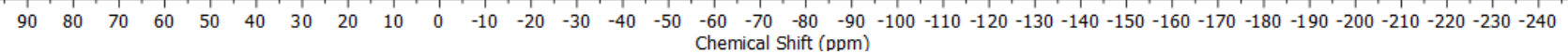


NMR spectra of 2,4,6-triphenyl-1-(pyridin-3-ylmethyl)pyridin-1-ium tetrafluoroborate (1ab)

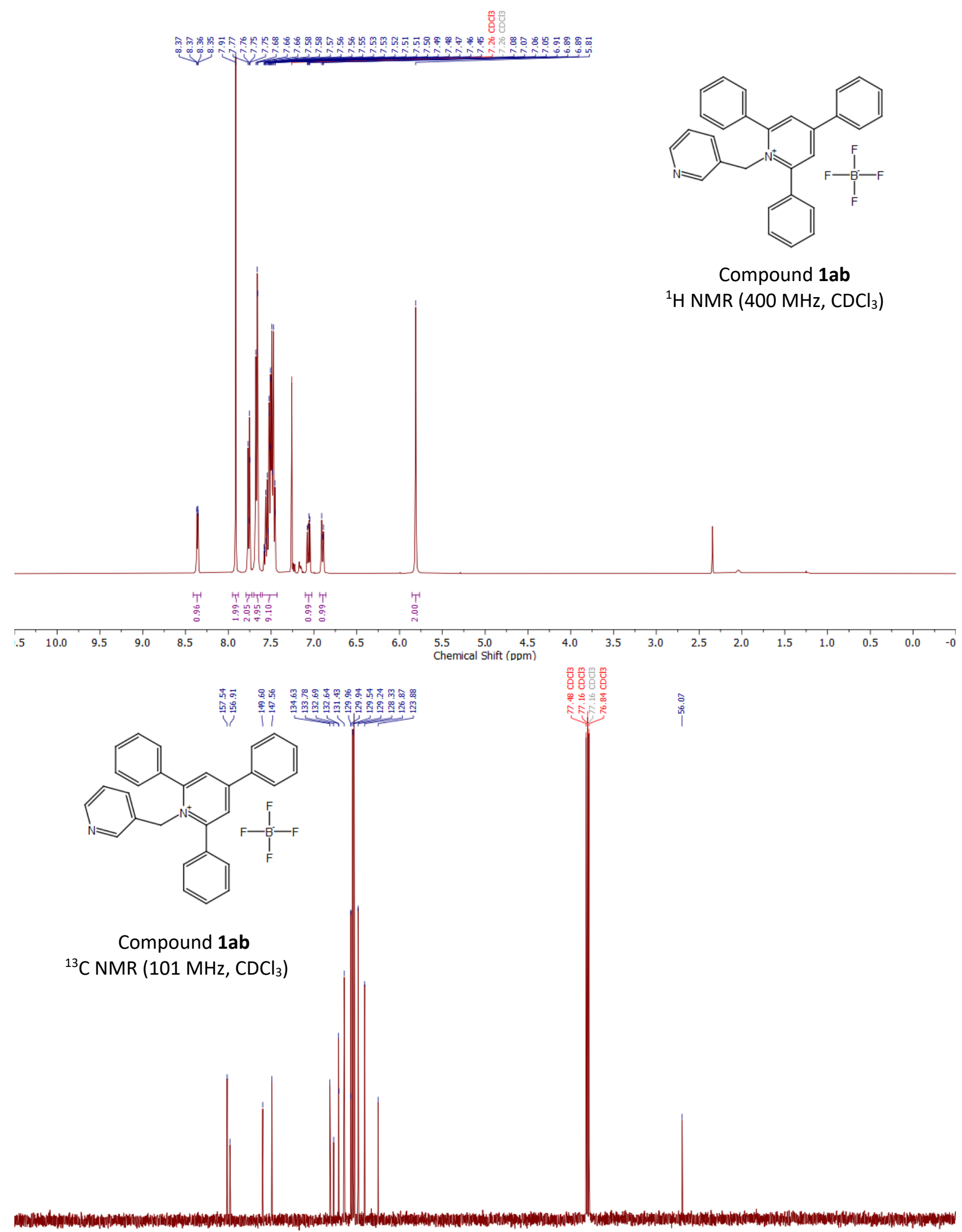

$\begin{array}{llllllllll}200 & 190 & 180 & 170 & 160 & 150 & 140 & 130 & 120 & \begin{array}{c}110 \\ \text { Chemical Shift (pom) }\end{array}\end{array}$ 


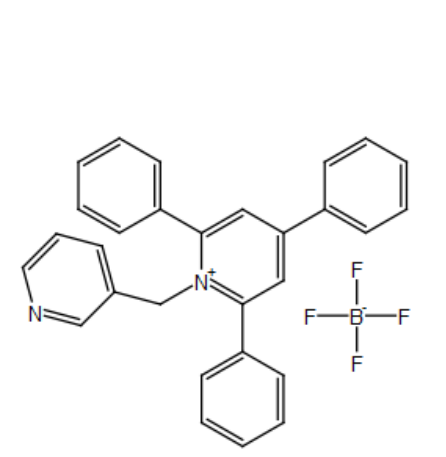

Compound 1ab

${ }^{19} \mathrm{~F}$ NMR (377 MHz, $\mathrm{CDCl}_{3}$ )

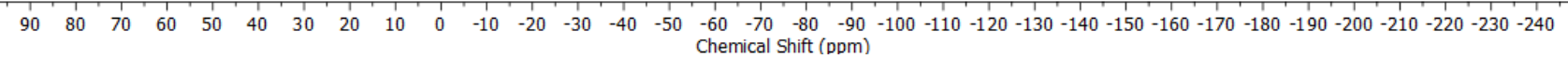


NMR spectra of 2,4,6-triphenyl-1-(pyridin-3-ylmethyl)pyridin-1-ium tetrafluoroborate (1ac)

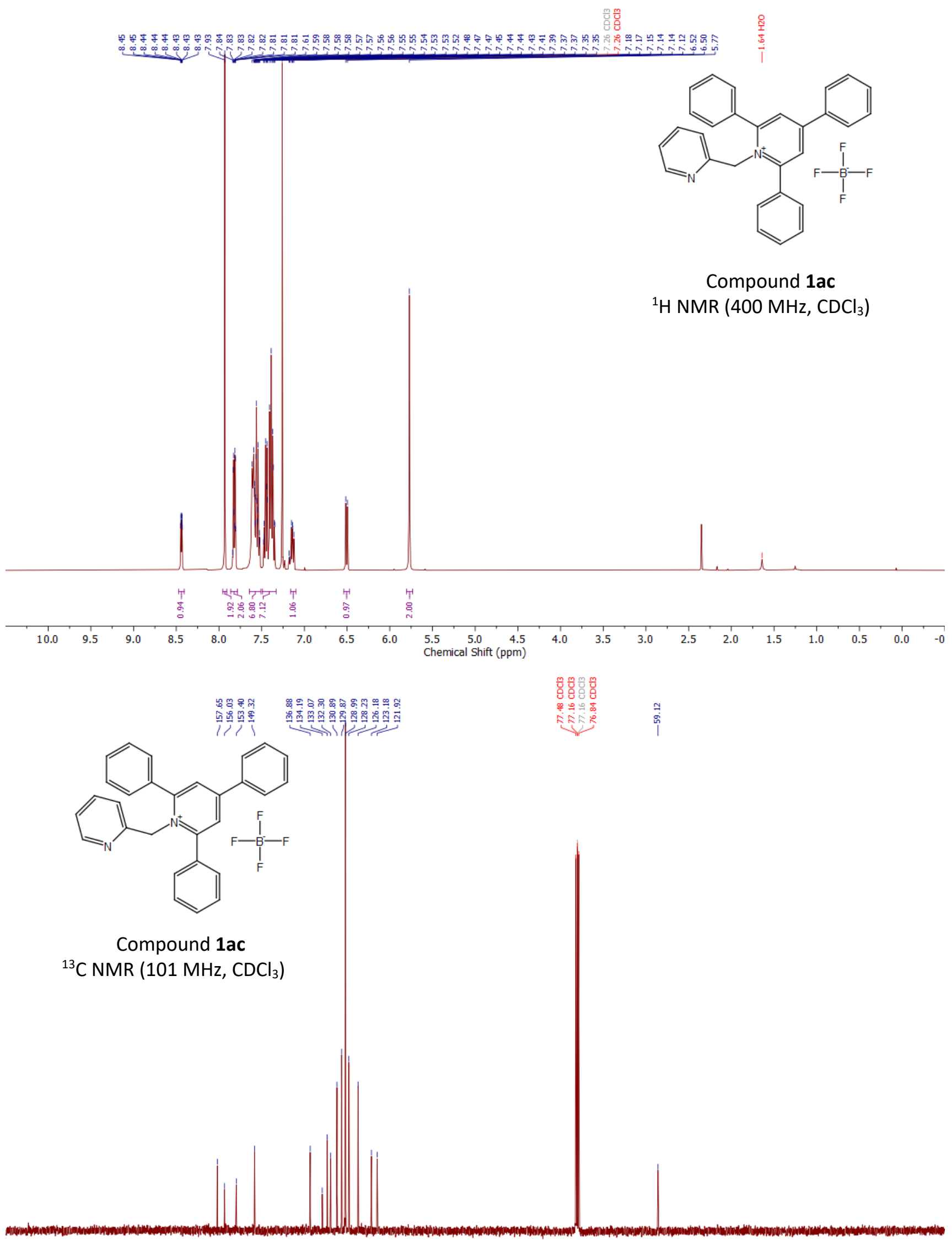

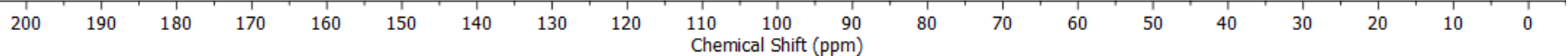




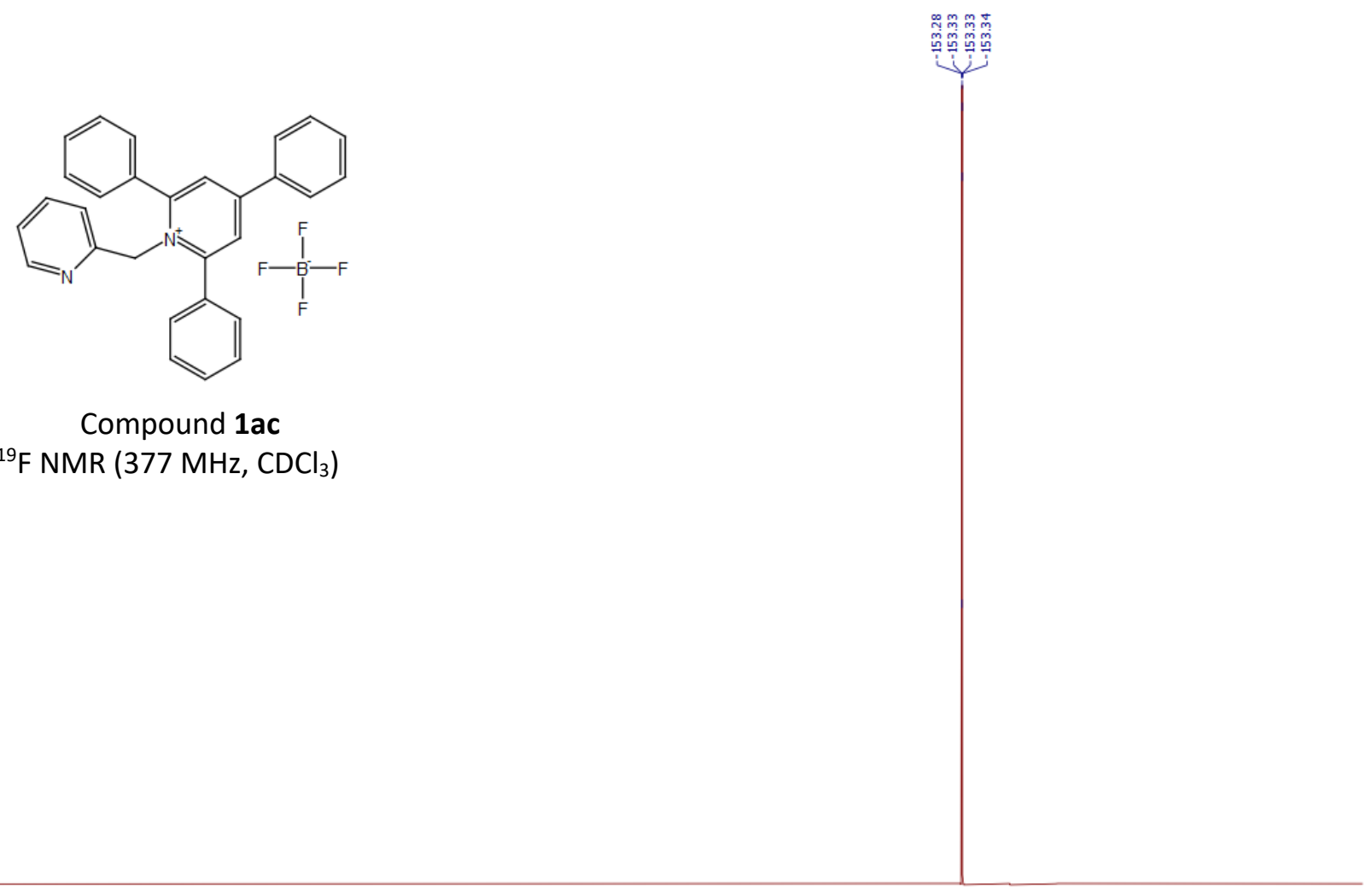

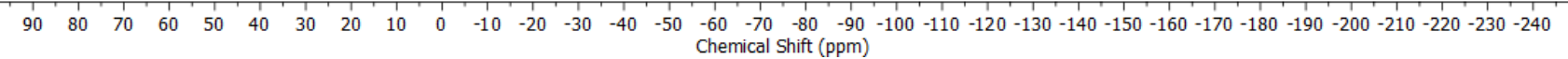


NMR spectra of 2-[(E)-3-phenyl-allyl]-isoindole-1,3-dione (1ad-1)

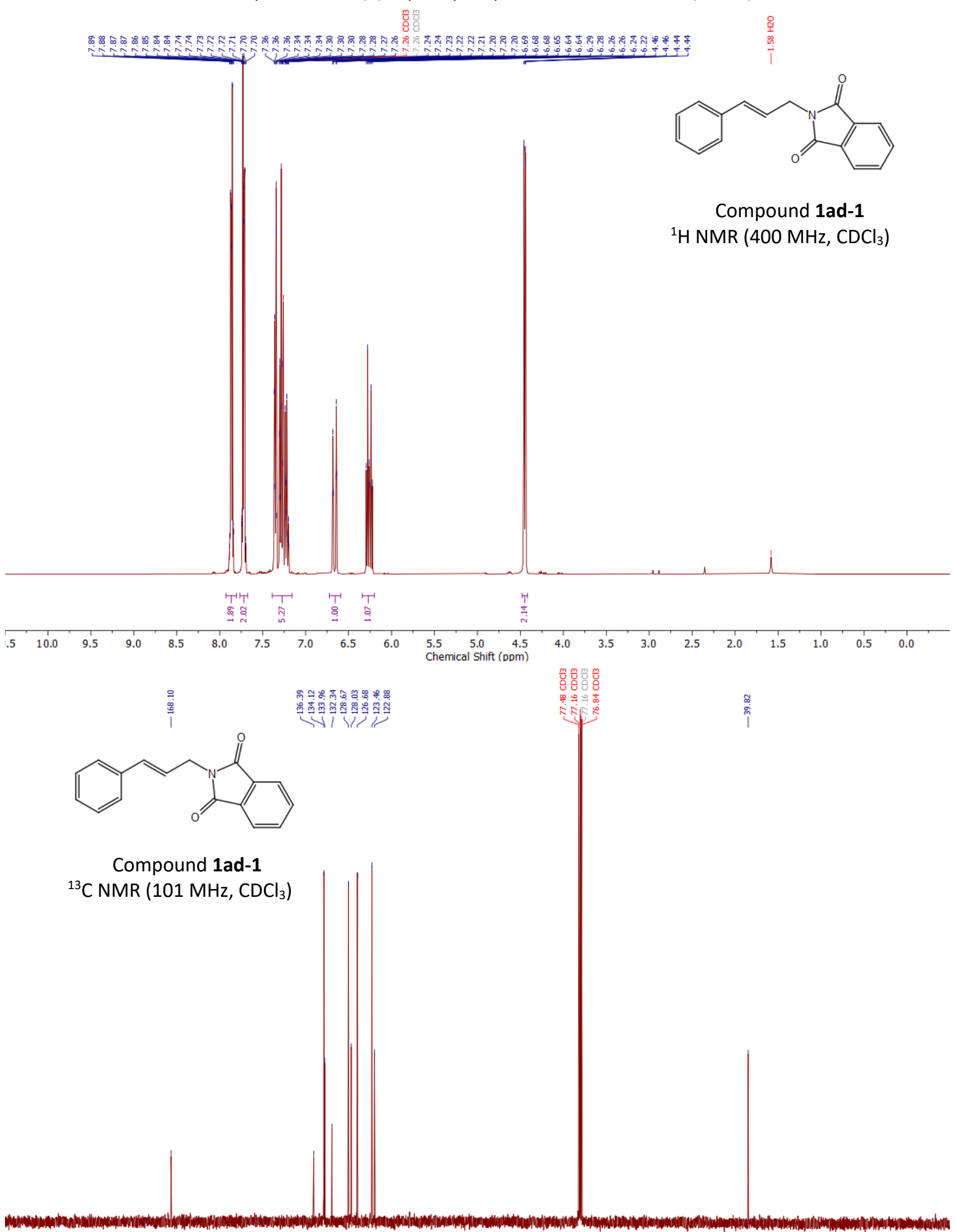

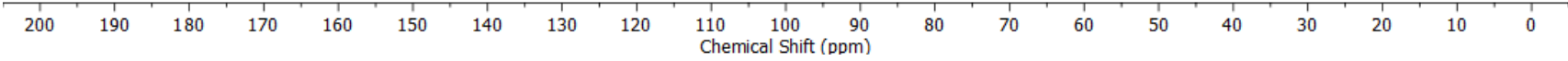


NMR spectra of (E)-3-phenyl-allyl-ammonium chloride (1ad-2)

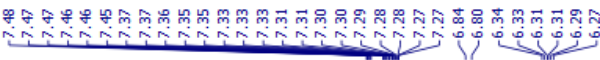<smiles>[NH-]C/C=C/c1ccccc1</smiles>

Compound 1ad-2

${ }^{1} \mathrm{H}$ NMR (400 MHz, $\left.d_{4}-\mathrm{MeOD}\right)$
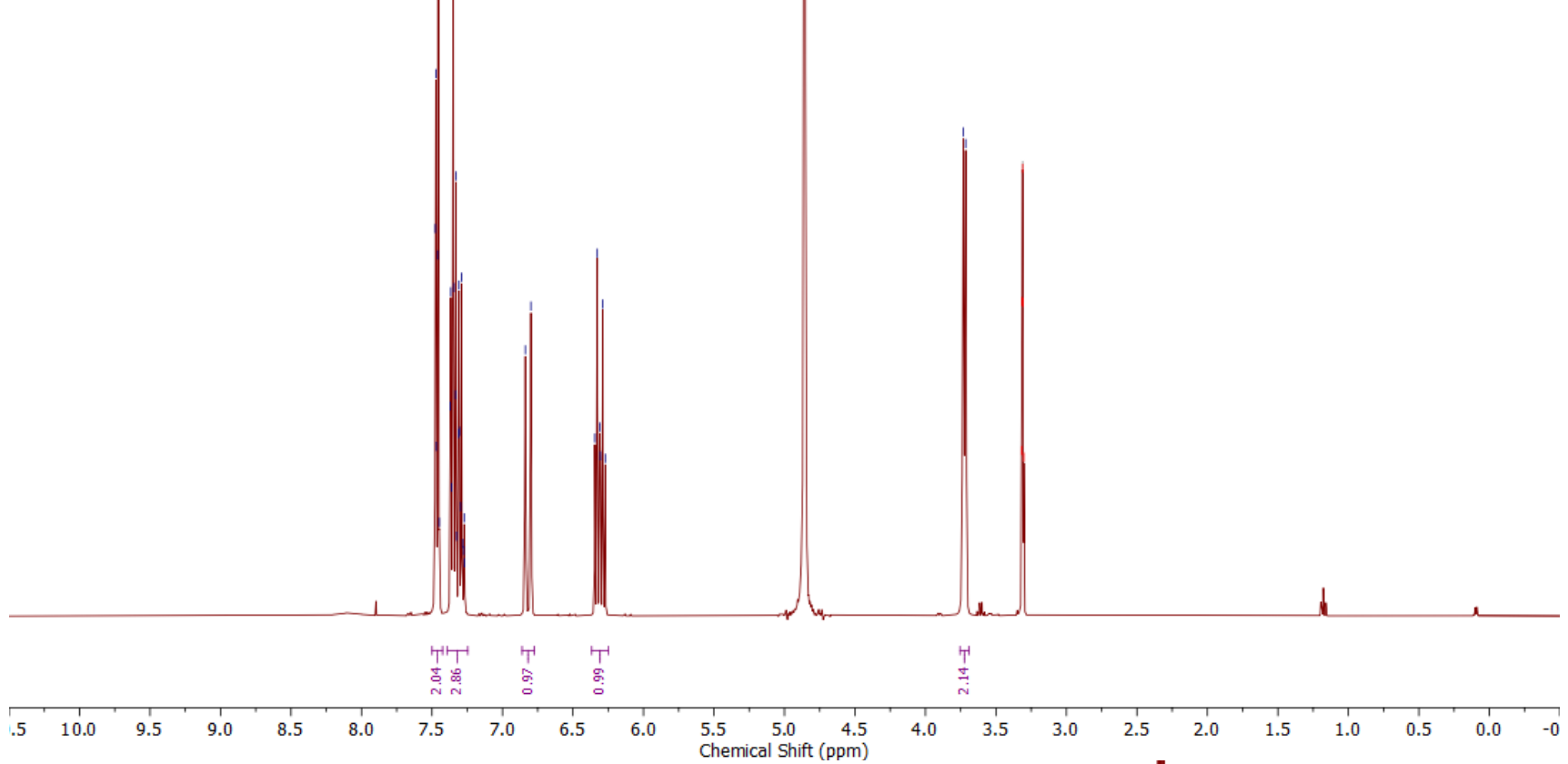

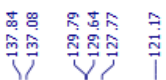<smiles>[NH3+]C/C=C/c1ccccc1</smiles>

Compound 1ad-2

${ }^{13} \mathrm{C}$ NMR (101 MHz, $\left.d_{4}-\mathrm{MeOD}\right)$

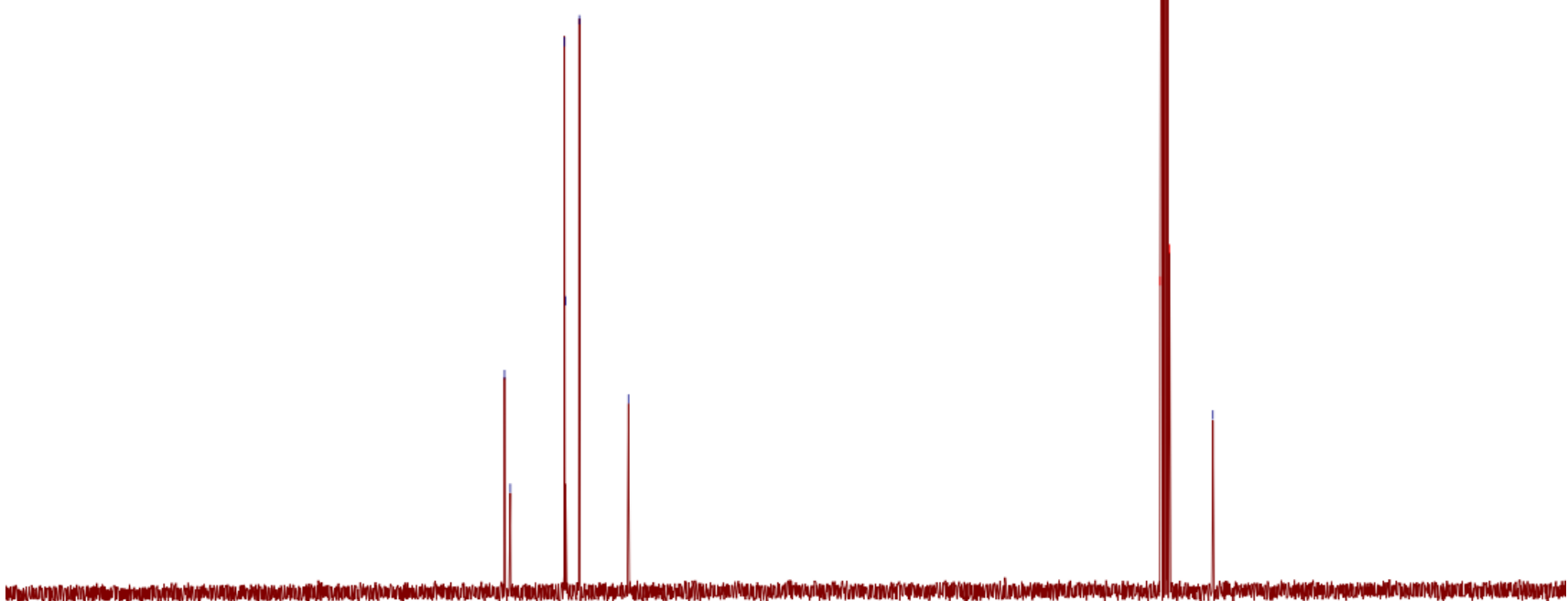


NMR spectra of 1-cinnamyl-2,4,6-triphenylpyridin-1-ium tetrafluoroborate (1ad-3)

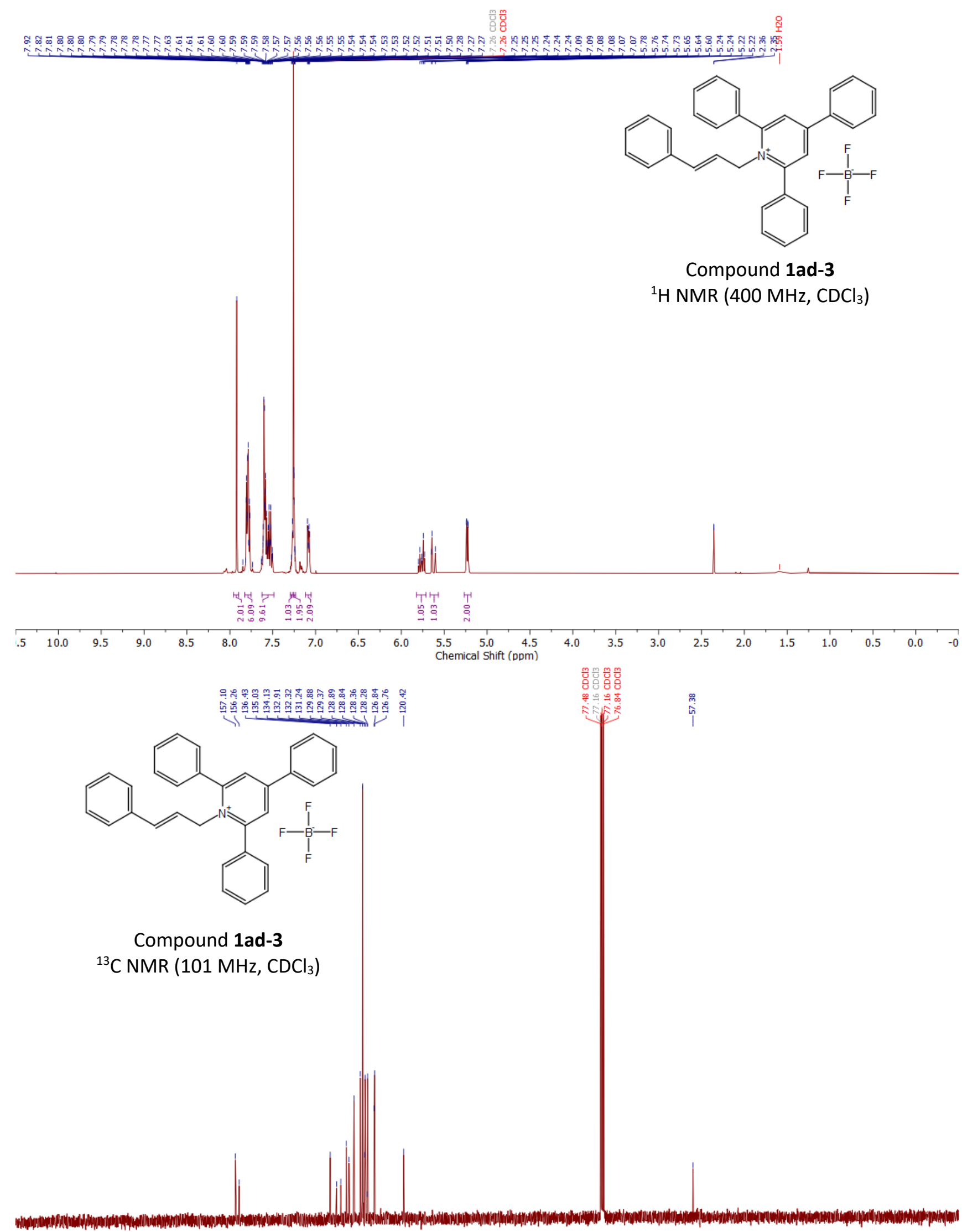

$\begin{array}{lllllllllllllllllllll}1 & 1 \\ 200 & 190 & 180 & 170 & 160 & 150 & 140 & 130 & 120 & \begin{array}{c}110 \\ \text { Chemical Shift }(\mathrm{Dbm})\end{array} & 90 & 70 & 60 & 50 & 40 & 30 & 20 & 10 & 0\end{array}$




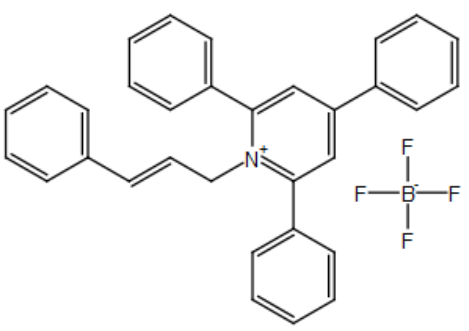

Compound 1ad-3

${ }^{19} \mathrm{~F} \mathrm{NMR} \mathrm{(377} \mathrm{MHz,} \mathrm{CDCl}_{3}$ )

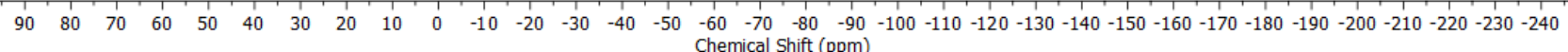


NMR spectra of 2-isopropyl-4,6-diphenylpyrylium tetrafluoroborate (1ae-1)

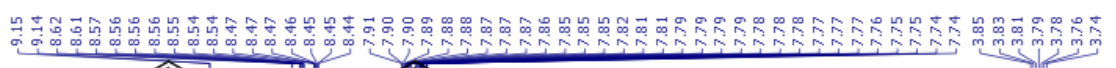<smiles>Cc1cccc(-c2cc(-c3ccccc3)cc(C(C)C)[o+]2)c1</smiles>

Compound 1ae-1

${ }^{1} \mathrm{H}$ NMR (400 MHz, $d_{6}$-acetone)
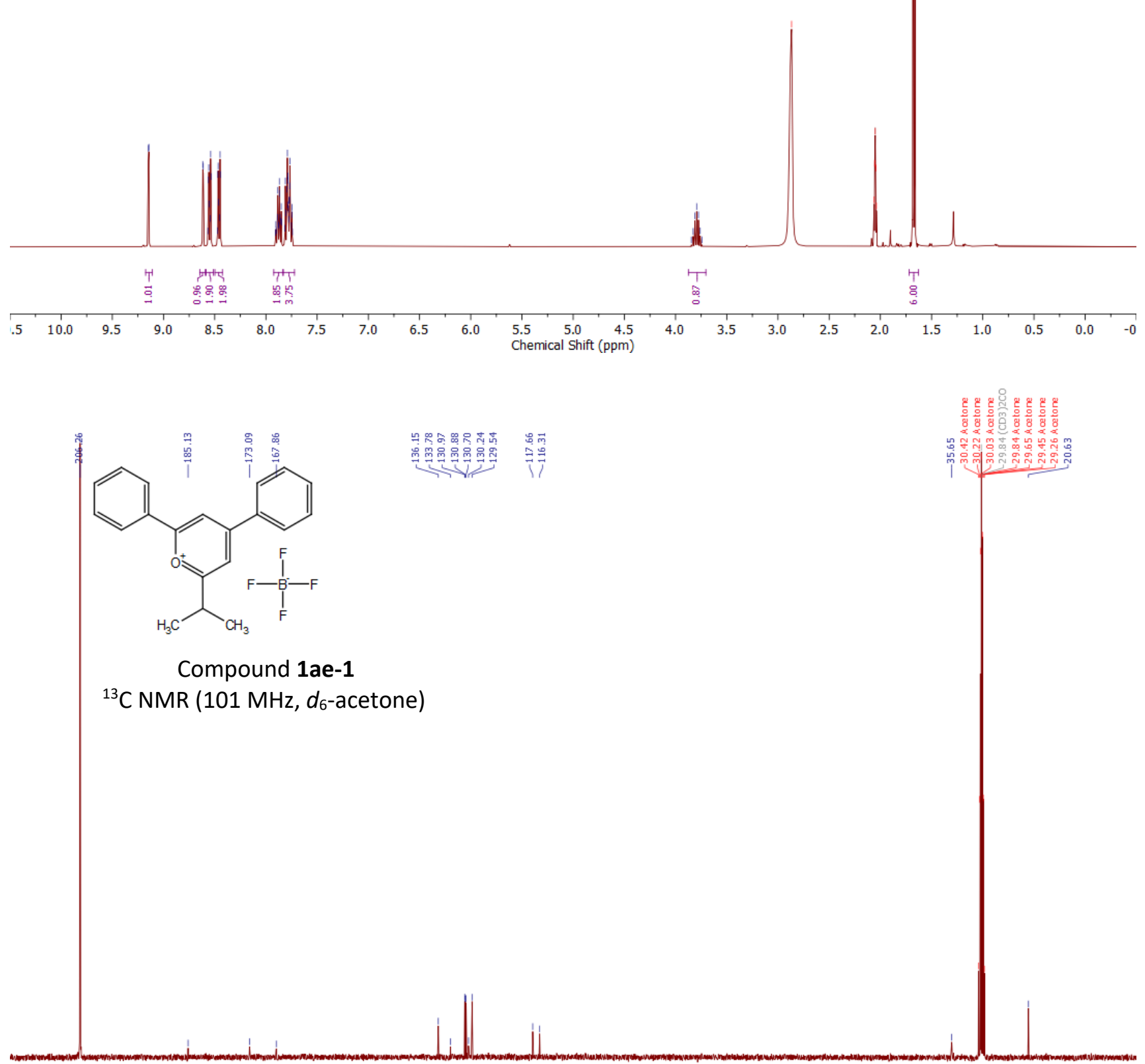

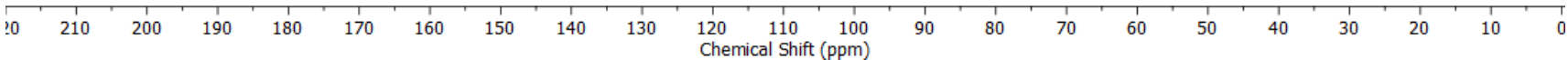


<smiles></smiles>

Compound 1ae-1

${ }^{19} \mathrm{~F}$ NMR (377 MHz, $d_{6}$-acetone)

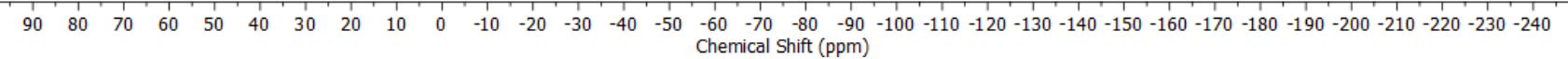


NMR spectra of 2-isopropyl-4,6-diphenyl-1-(1-phenylethyl)pyridin-1-ium tetrafluoroborate (1ae-2)
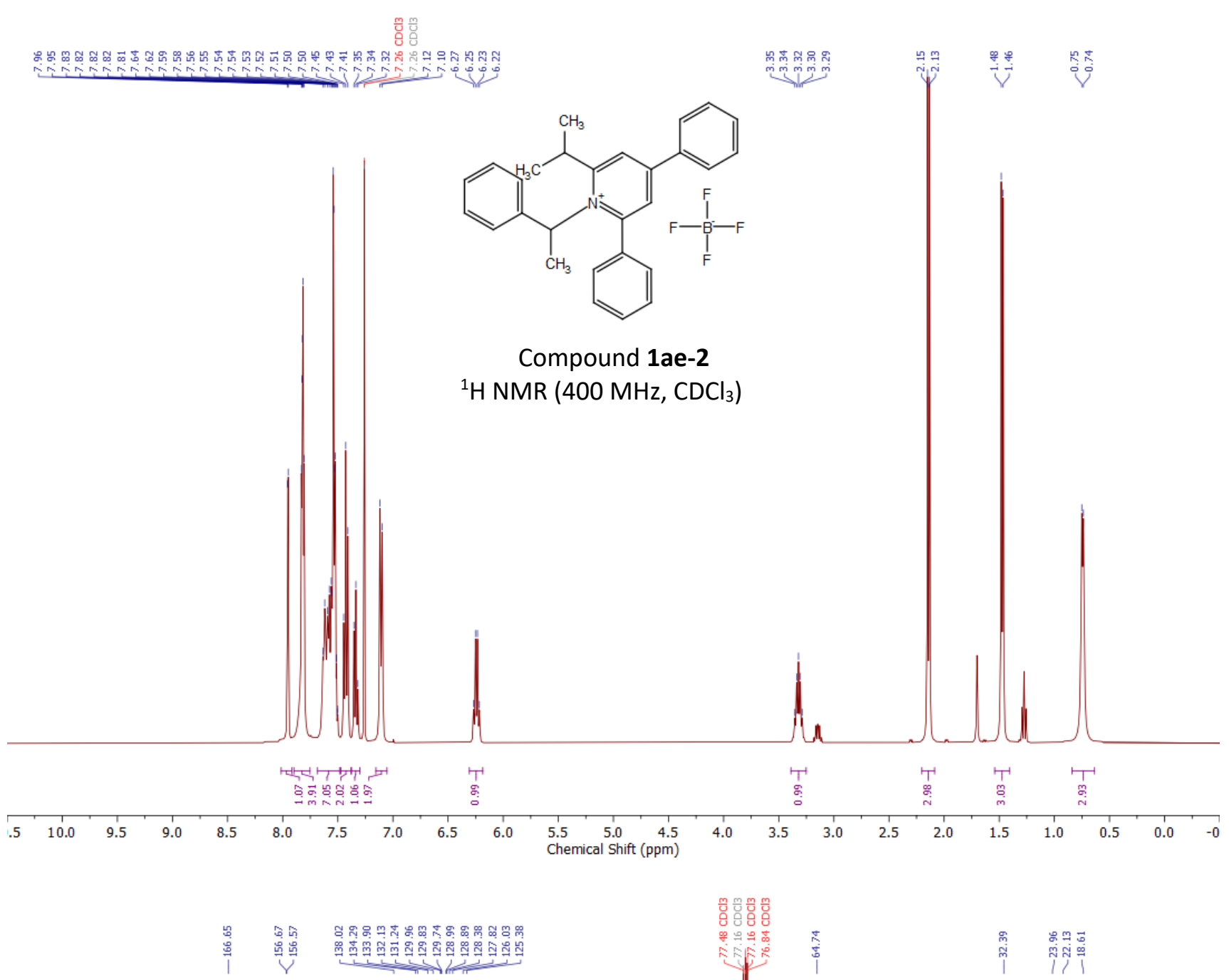<smiles></smiles>

Compound 1ae-2

$\left.{ }^{13} \mathrm{C} \mathrm{NMR} \mathrm{(101} \mathrm{MHz,} \mathrm{CDCl}_{3}\right)$ 
<smiles></smiles>

Compound 1ae-2

${ }^{19} \mathrm{~F} \mathrm{NMR} \mathrm{(377} \mathrm{MHz,} \mathrm{CDCl}_{3}$ )

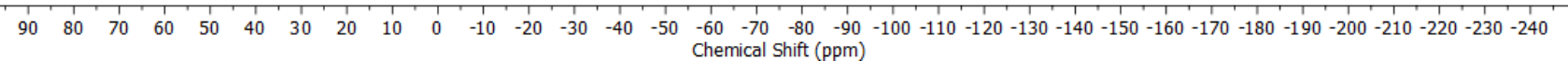


NMR spectra of 1-phenethyl-2,4,6-triphenylpyridin-1-ium tetrafluoroborate (1c)

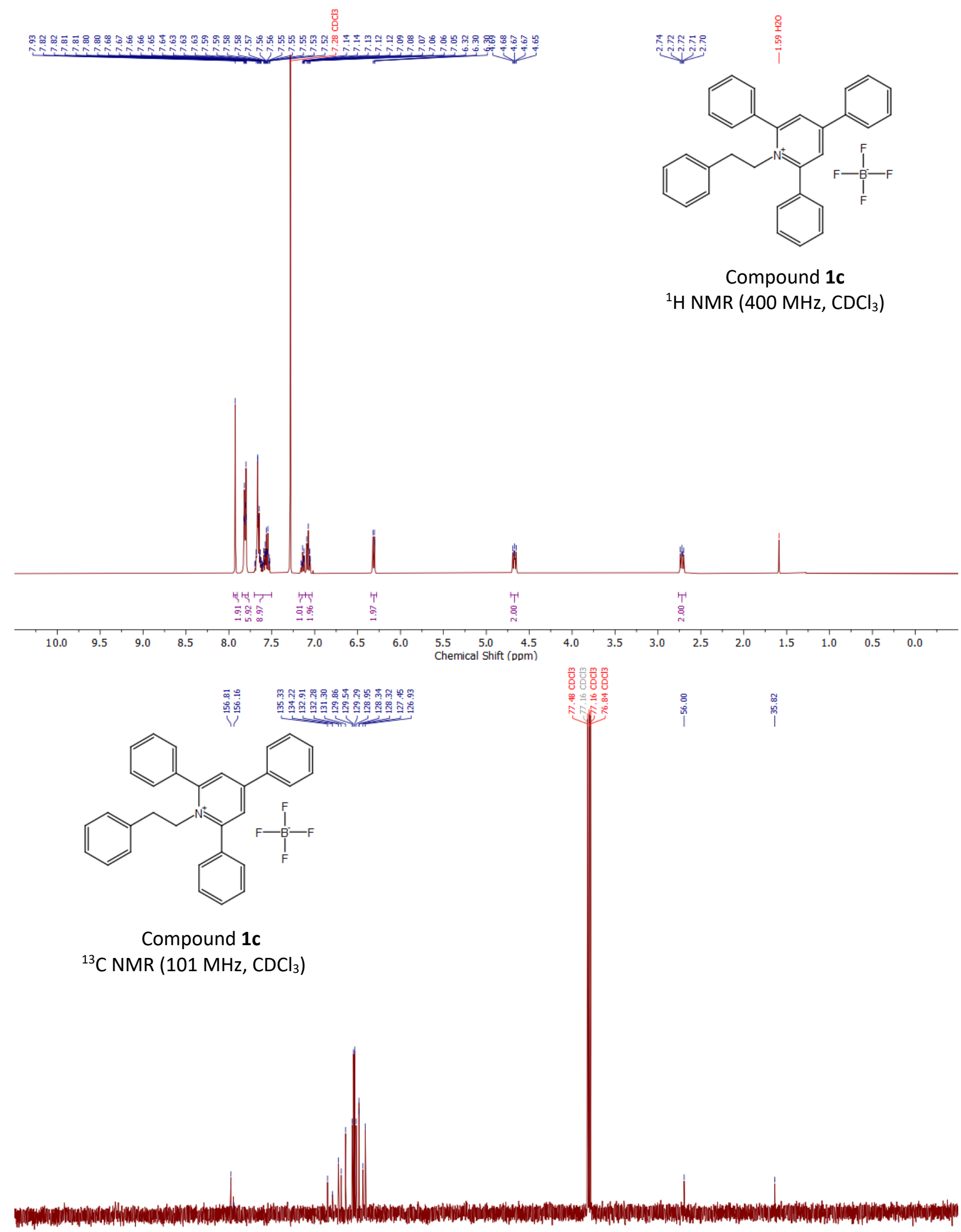

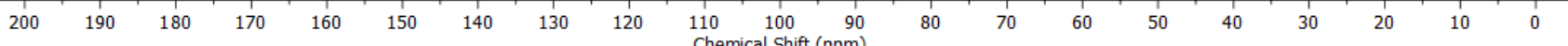




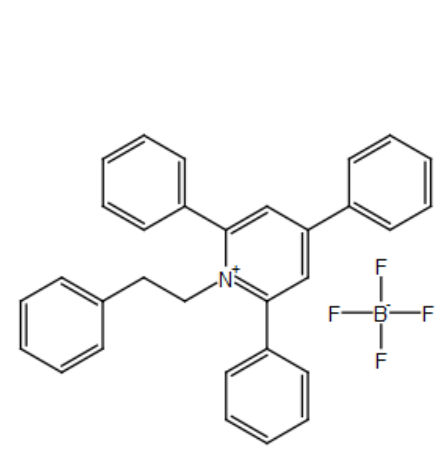

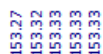

更

Compound 1c

${ }^{19}$ F NMR (377 MHz, $\mathrm{CDCl}_{3}$ )

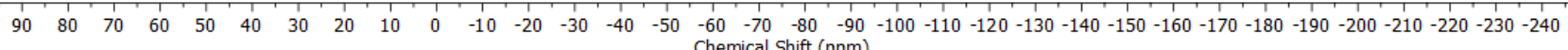
Chemical Shift (ppm) 
NMR spectra of 1-neopentyl-2,4,6-triphenylpyridin-1-ium tetrafluoroborate (1af)

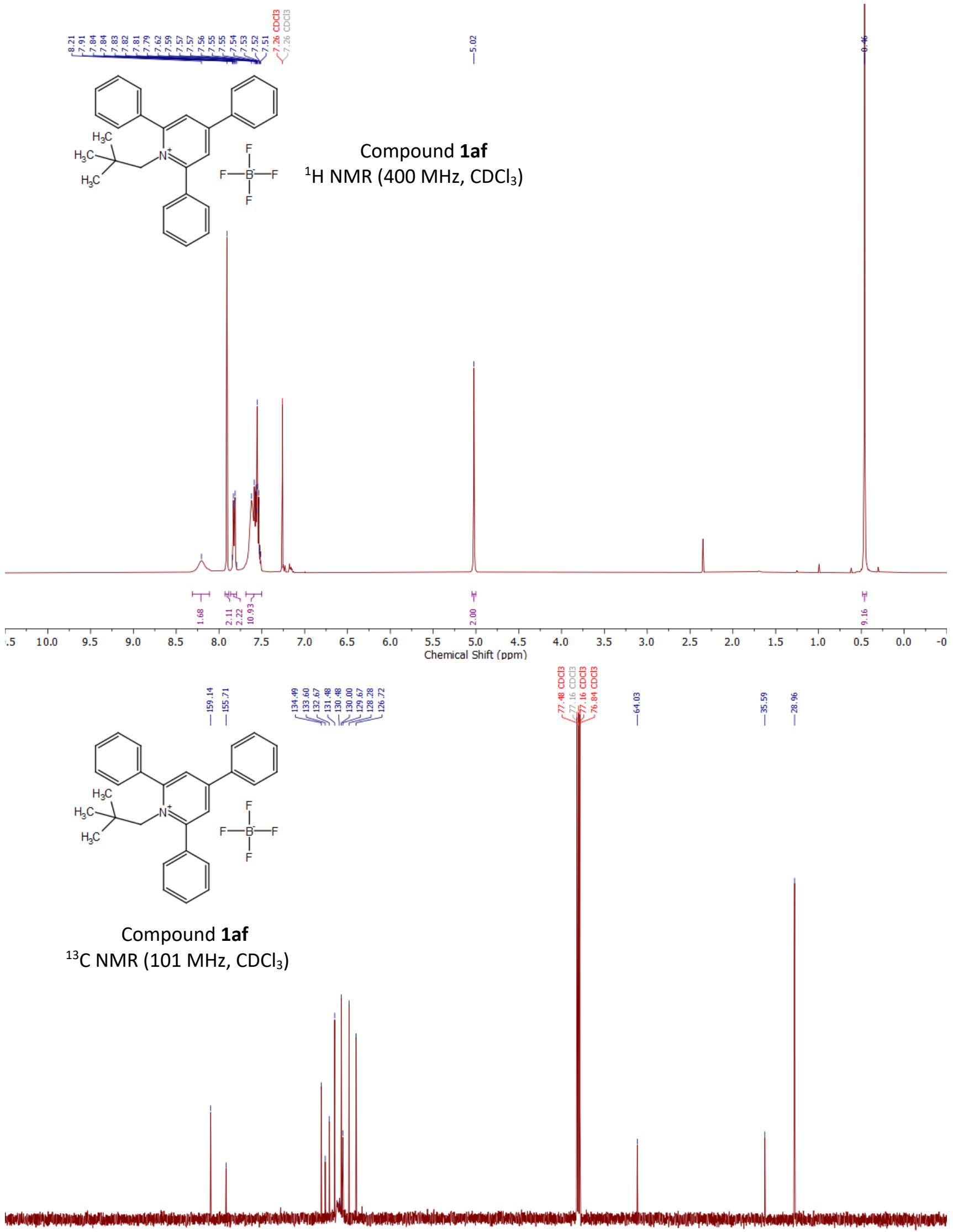

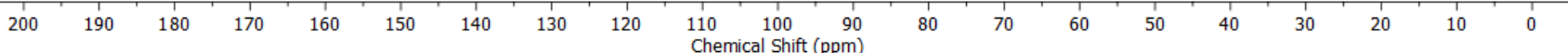




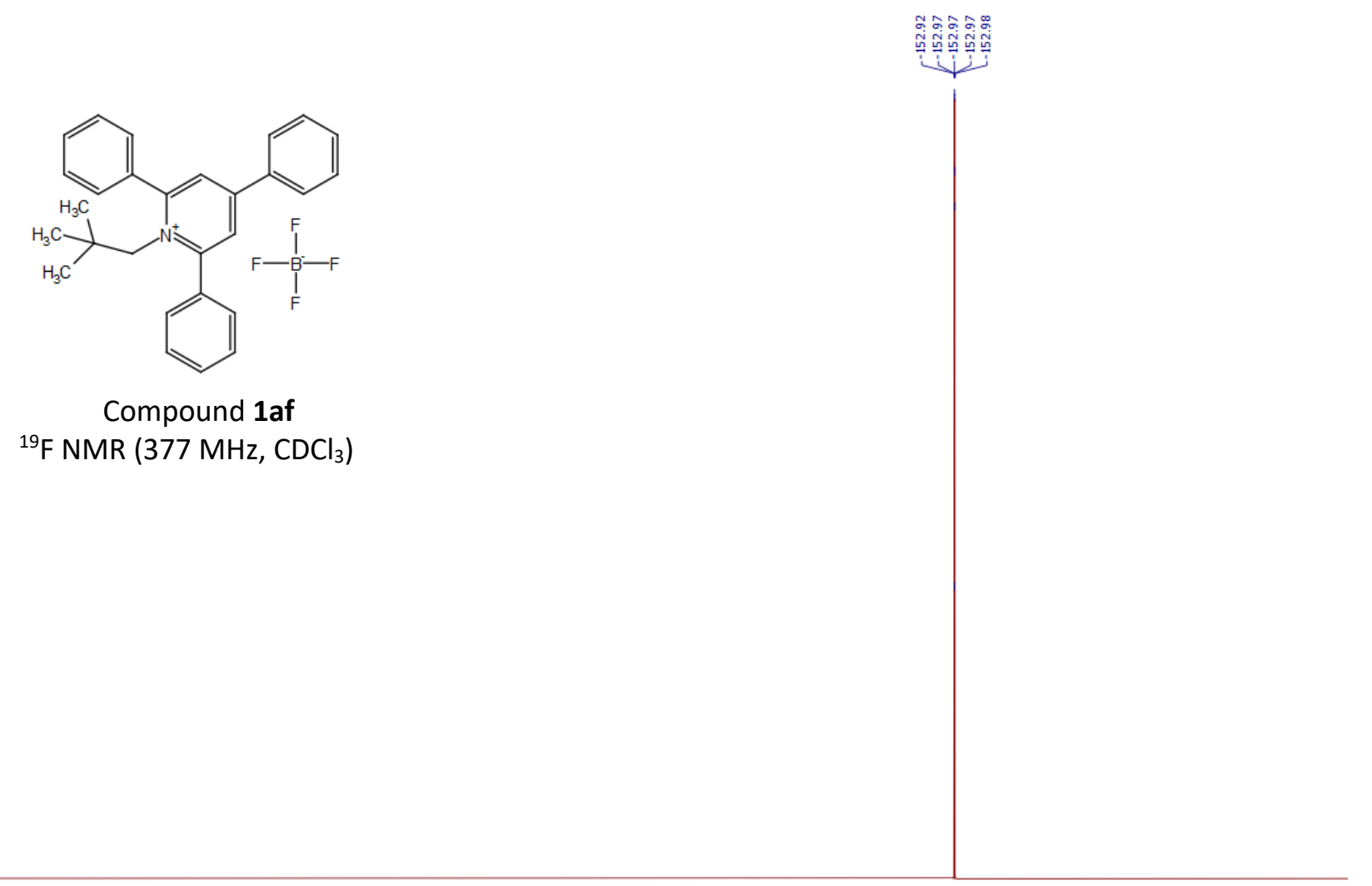

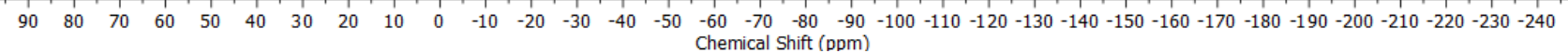


NMR spectra of 1-octyl-2,4,6-triphenylpyridin-1-ium tetrafluoroborate (1ag)

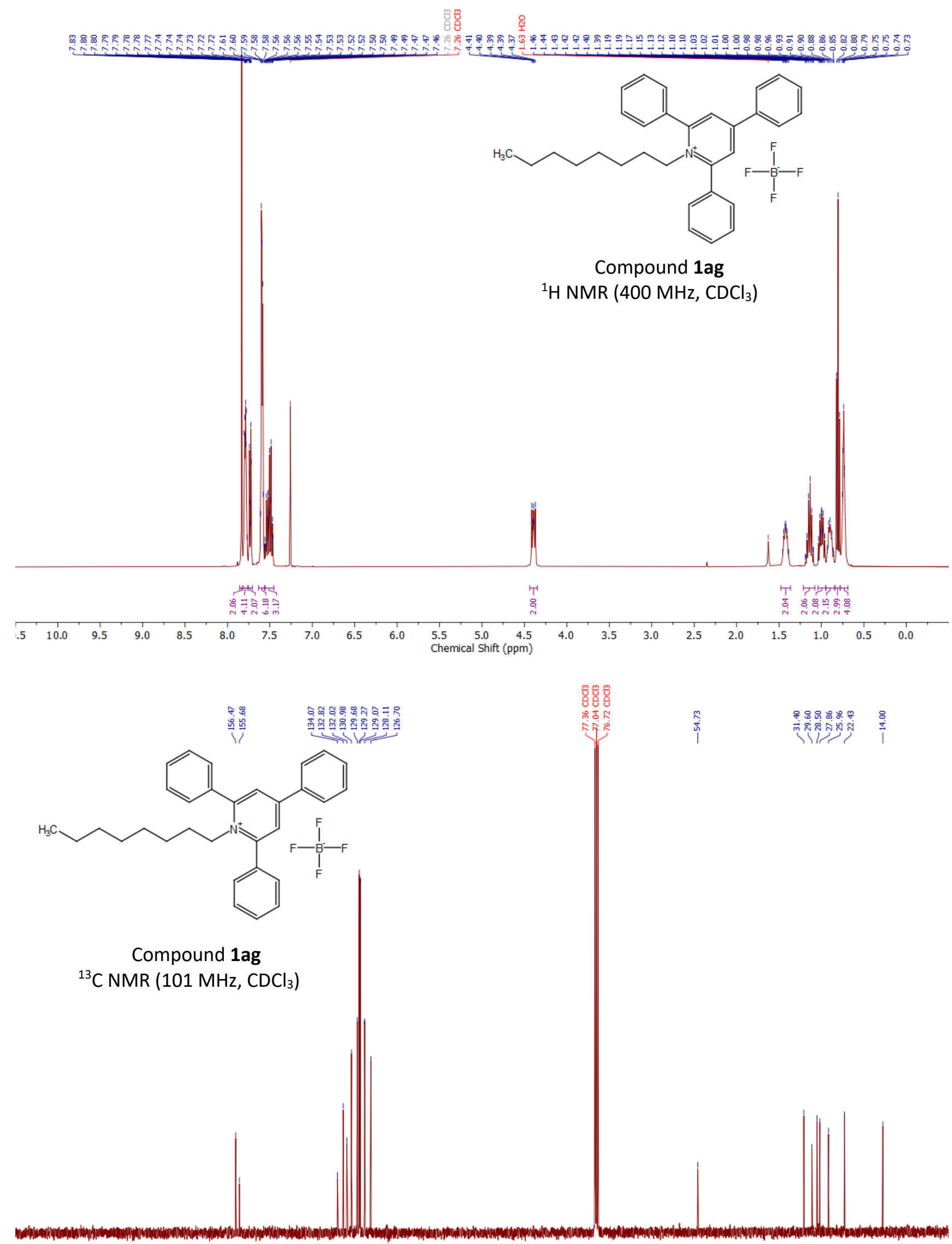

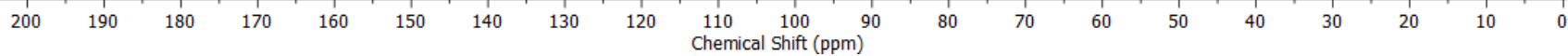




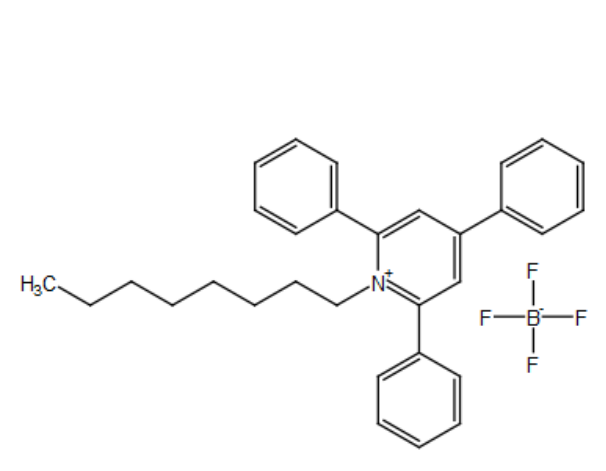

Compound 1ag

${ }^{19} \mathrm{~F} \mathrm{NMR}\left(377 \mathrm{MHz}, \mathrm{CDCl}_{3}\right)$

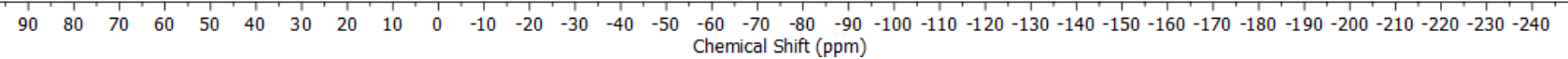


NMR spectra of 1-(2-(cyclohex-1-en-1-yl)ethyl)-2,4,6-triphenylpyridin-1-ium tetrafluoroborate (1ah)

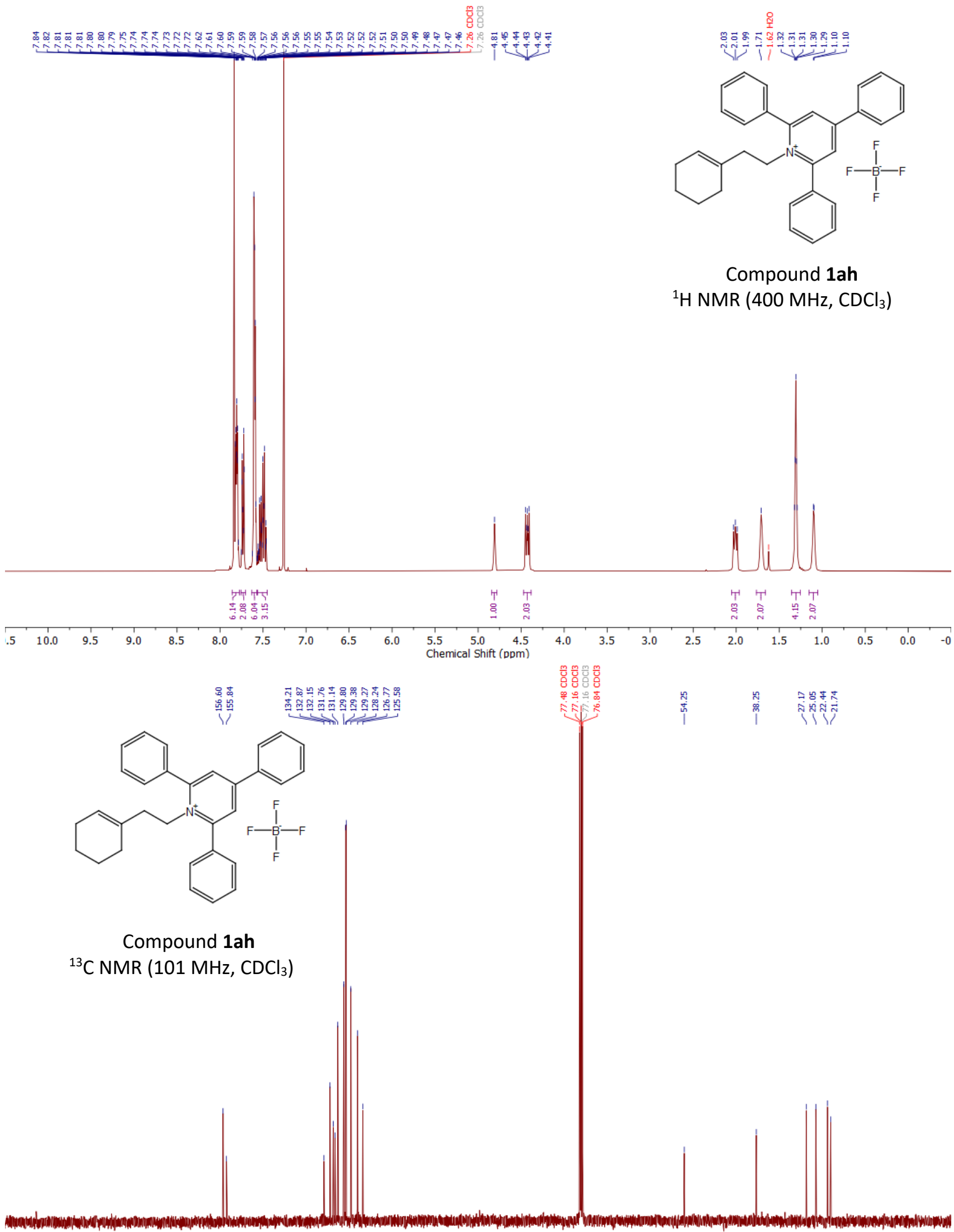

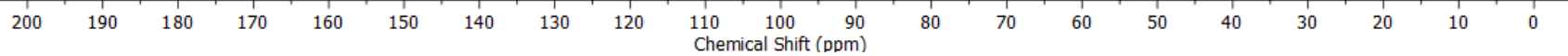




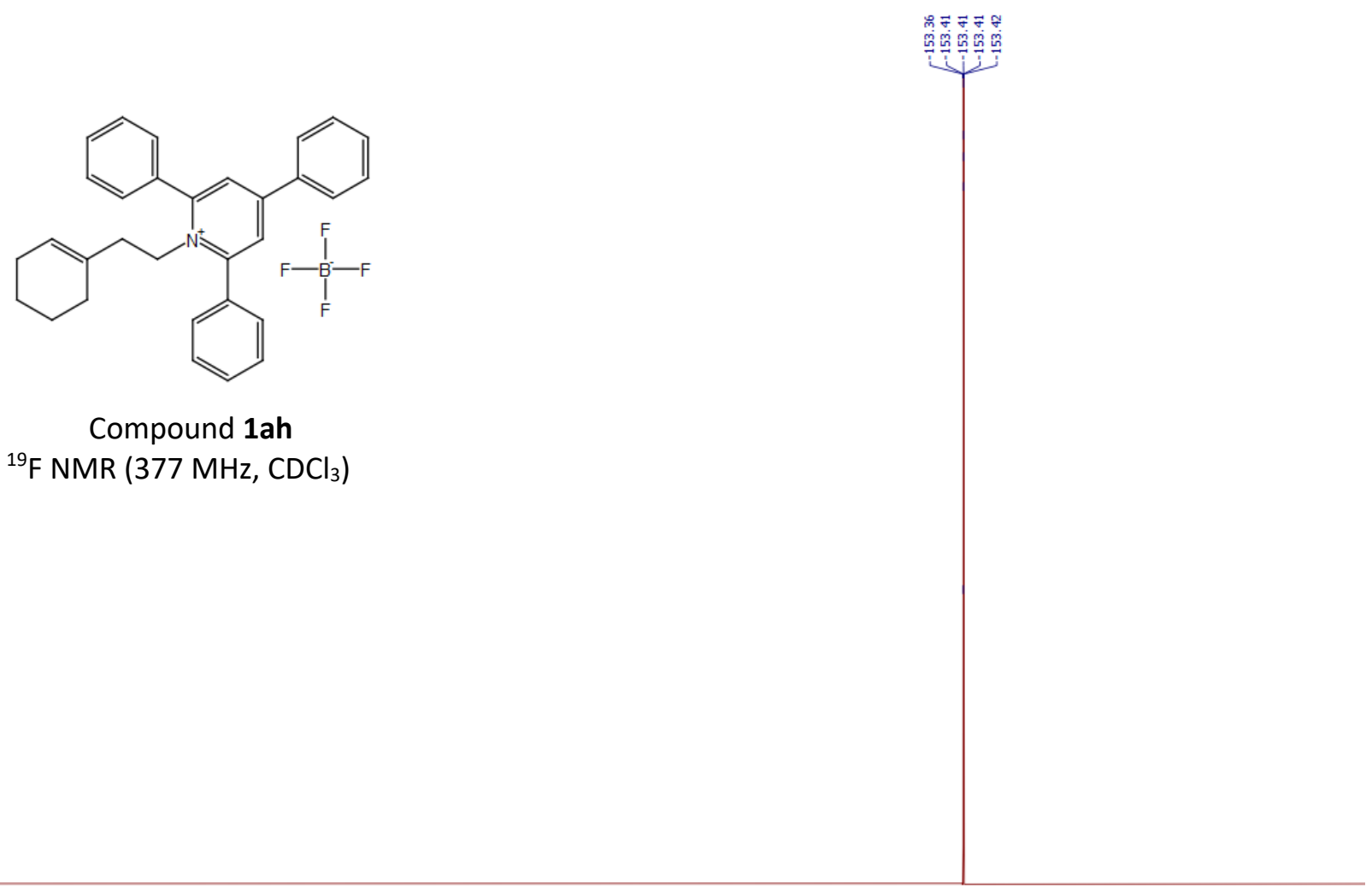

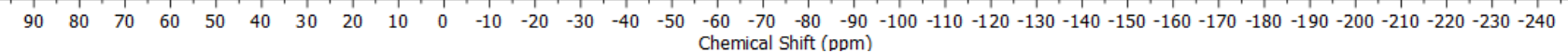


NMR spectra of 1-(2,2-dimethoxyethyl)-2,4,6-triphenylpyridin-1-ium tetrafluoroborate (1ai)

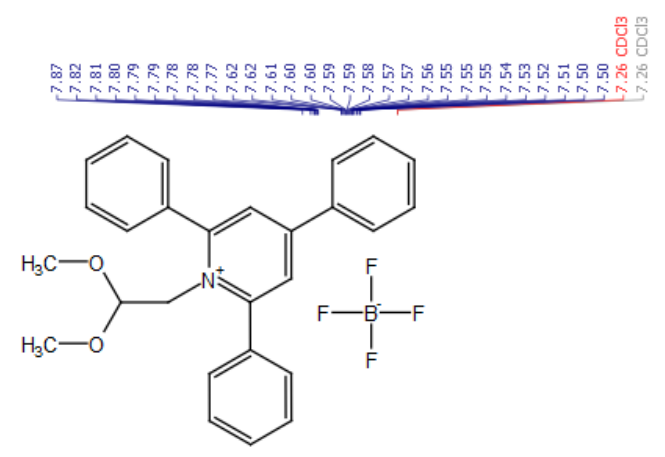

Compound 1ai

${ }^{1} \mathrm{H} \mathrm{NMR}\left(400 \mathrm{MHz}, \mathrm{CDCl}_{3}\right)$
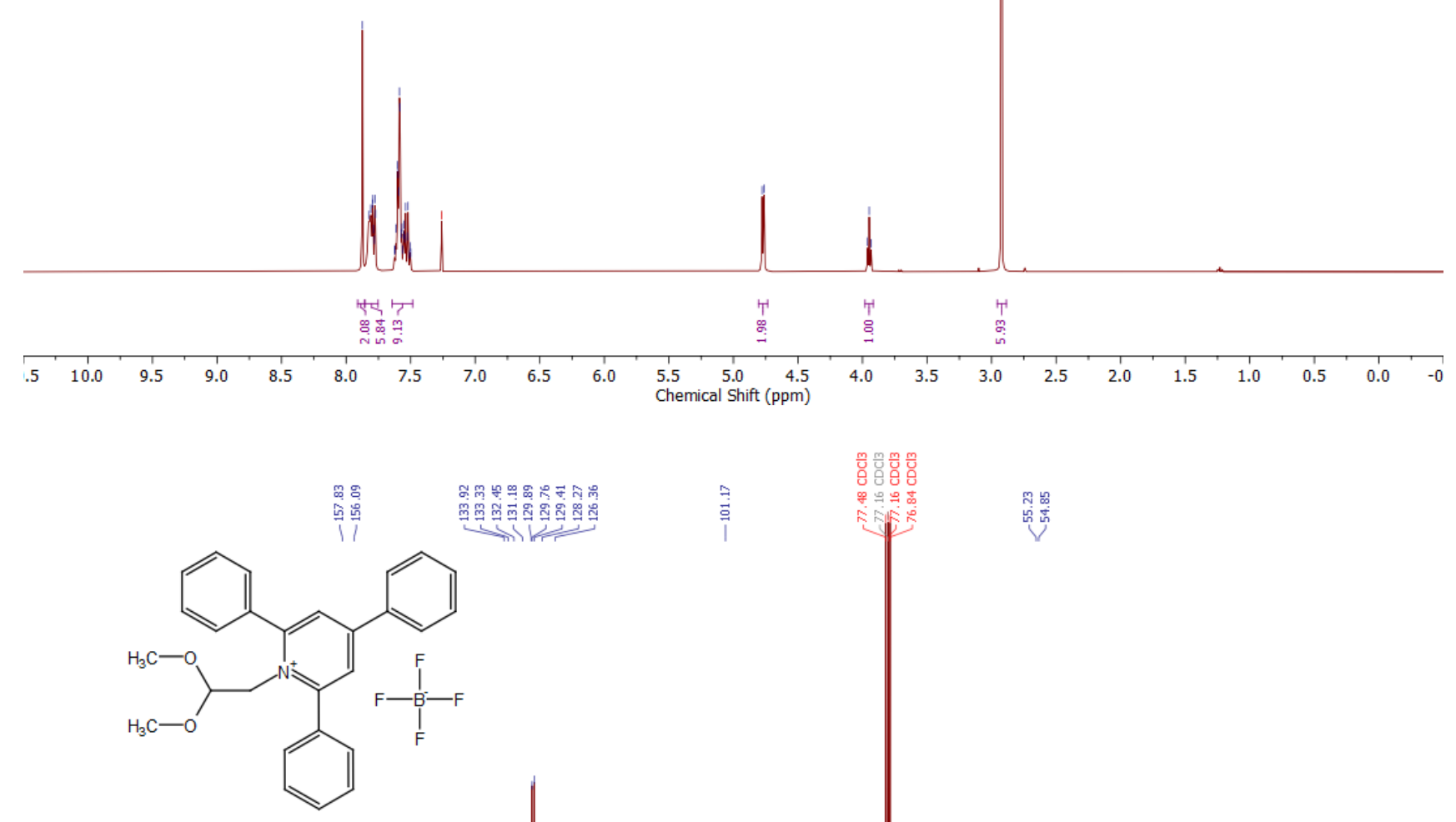

Compound 1ai

${ }^{13} \mathrm{C} \mathrm{NMR}\left(101 \mathrm{MHz}, \mathrm{CDCl}_{3}\right)$

$\begin{array}{lllllllllll}200 & 190 & 180 & 170 & 160 & 150 & 140 & 130 & 120 & 110 & 100 \\ \text { Chemical Shift (ppm) }\end{array}$




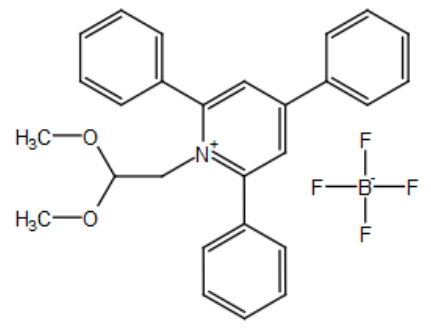

Compound 1ai

${ }^{19} \mathrm{~F}$ NMR (377 MHz, $\mathrm{CDCl}_{3}$ )

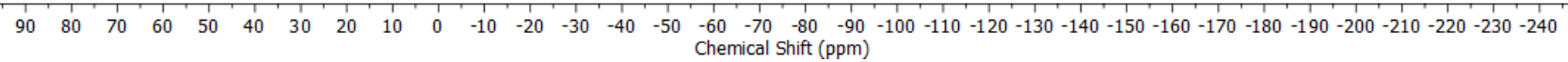


NMR spectra of 1-(4-cyanophenethyl)-2,4,6-triphenylpyridin-1-ium tetrafluoroborate (1aj)
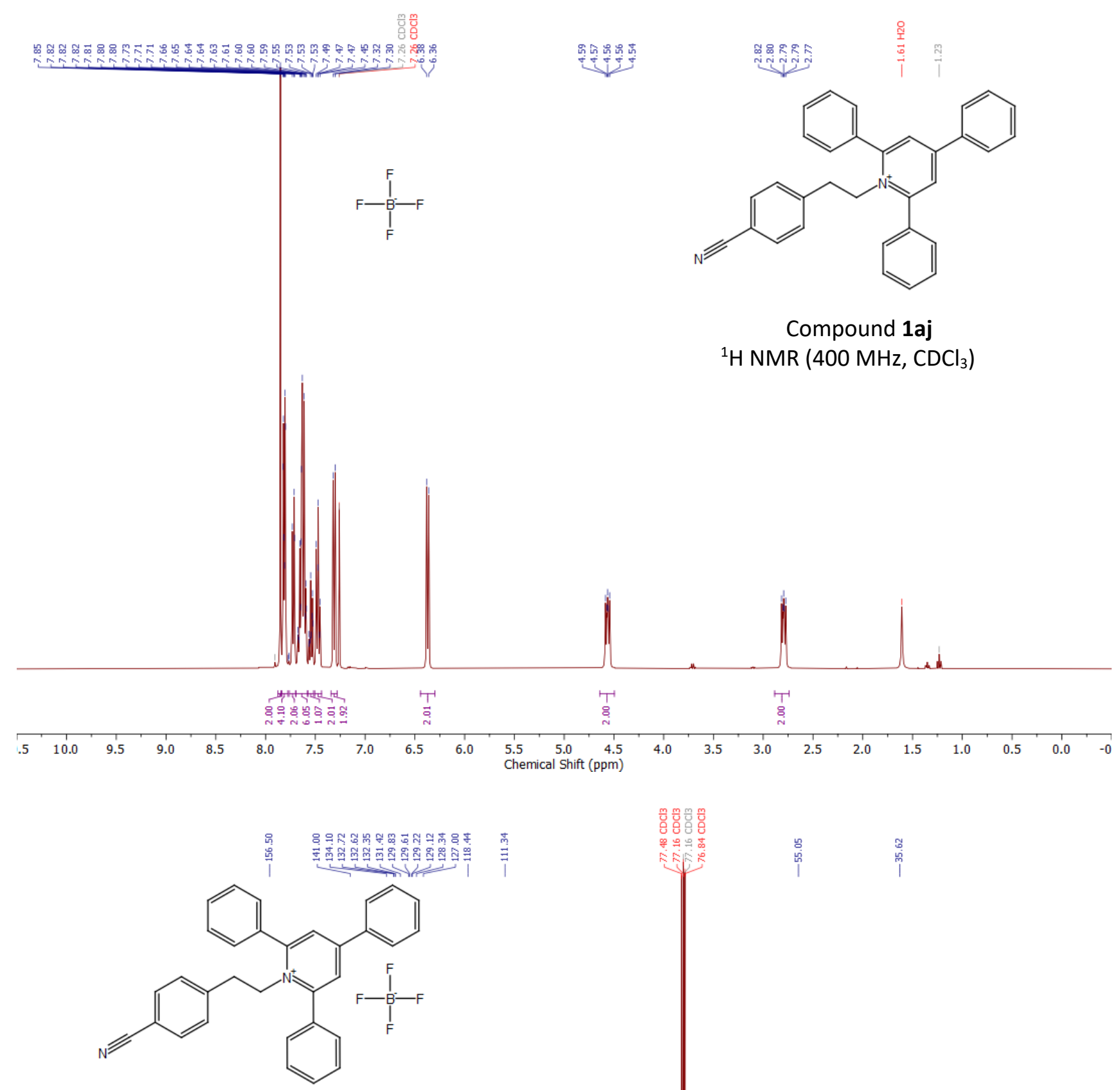

Compound 1aj

${ }^{13} \mathrm{C}$ NMR $\left(101 \mathrm{MHz}, \mathrm{CDCl}_{3}\right)$

$\begin{array}{rrrrrrrrrrr}200 & 190 & 180 & 170 & 160 & 150 & 140 & 130 & 120 & 110 & 100 \\ \text { Chemical Shift (ppm) }\end{array}$




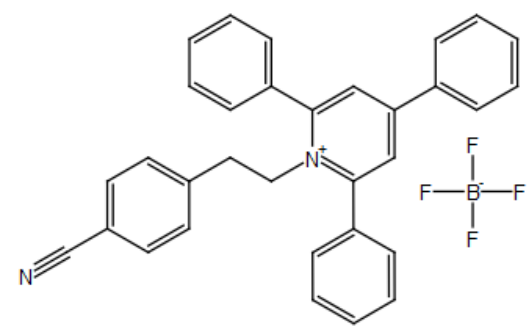

Compound 1aj

${ }^{19} \mathrm{~F} \mathrm{NMR} \mathrm{(377} \mathrm{MHz,} \mathrm{CDCl}{ }_{3}$ )

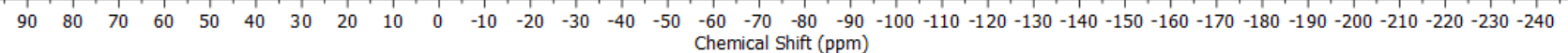


NMR spectra of 1-(4-(tert-butoxy)-4-oxobutyl)-2,4,6-triphenylpyridin-1-ium tetrafluoroborate (1ak)<smiles></smiles>

Compound 1ak

${ }^{1} \mathrm{H}$ NMR $\left(400 \mathrm{MHz}, \mathrm{CDCl}_{3}\right)$

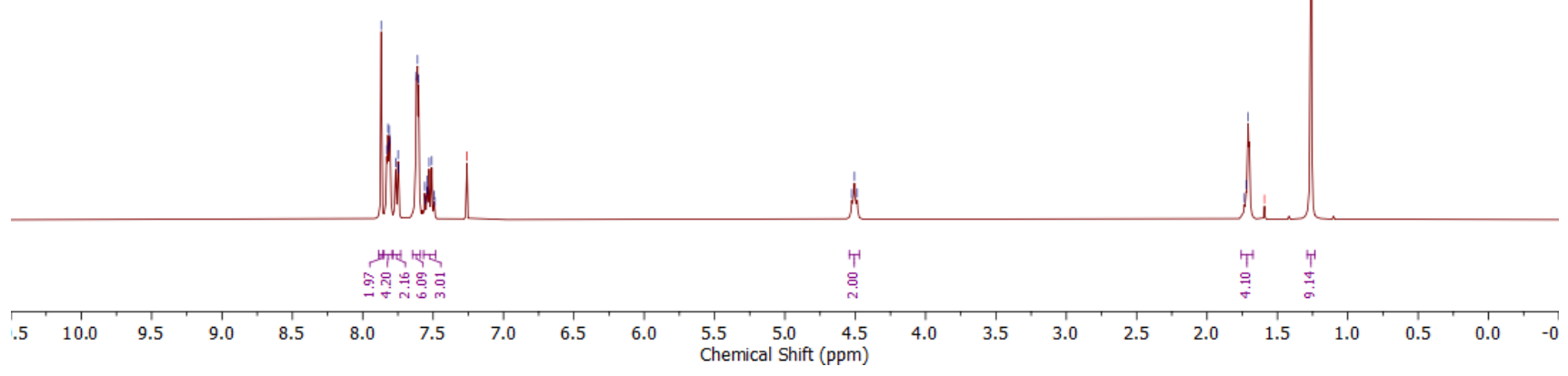<smiles></smiles>

Compound 1ak

${ }^{13} \mathrm{C}$ NMR $\left(101 \mathrm{MHz}, \mathrm{CDCl}_{3}\right)$

$\begin{array}{lllllllllll}200 & 190 & 180 & 170 & 160 & 150 & 140 & 130 & 120 & 110 & 100 \\ \text { Chemical Shift (ppm) }\end{array}$




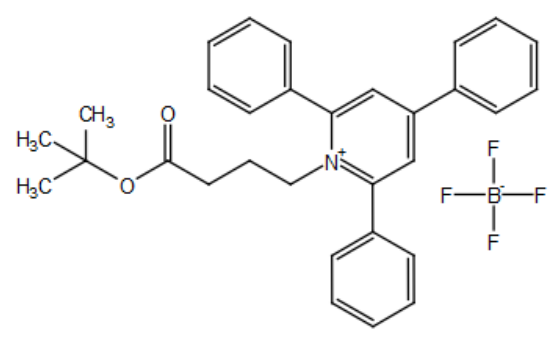

Compound 1ak

${ }^{19} \mathrm{~F}$ NMR (377 MHz, $\mathrm{CDCl}_{3}$ )

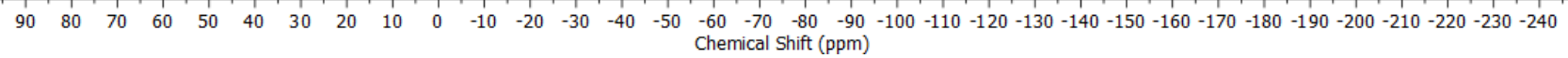


NMR spectra of 1-(2-(1H-indol-3-yl)ethyl)-2,4,6-triphenylpyridin-1-ium tetrafluoroborate (1al)<smiles></smiles>

Compound 1al

${ }^{1} \mathrm{H}$ NMR (400 MHz, $d_{6}$-DMSO)

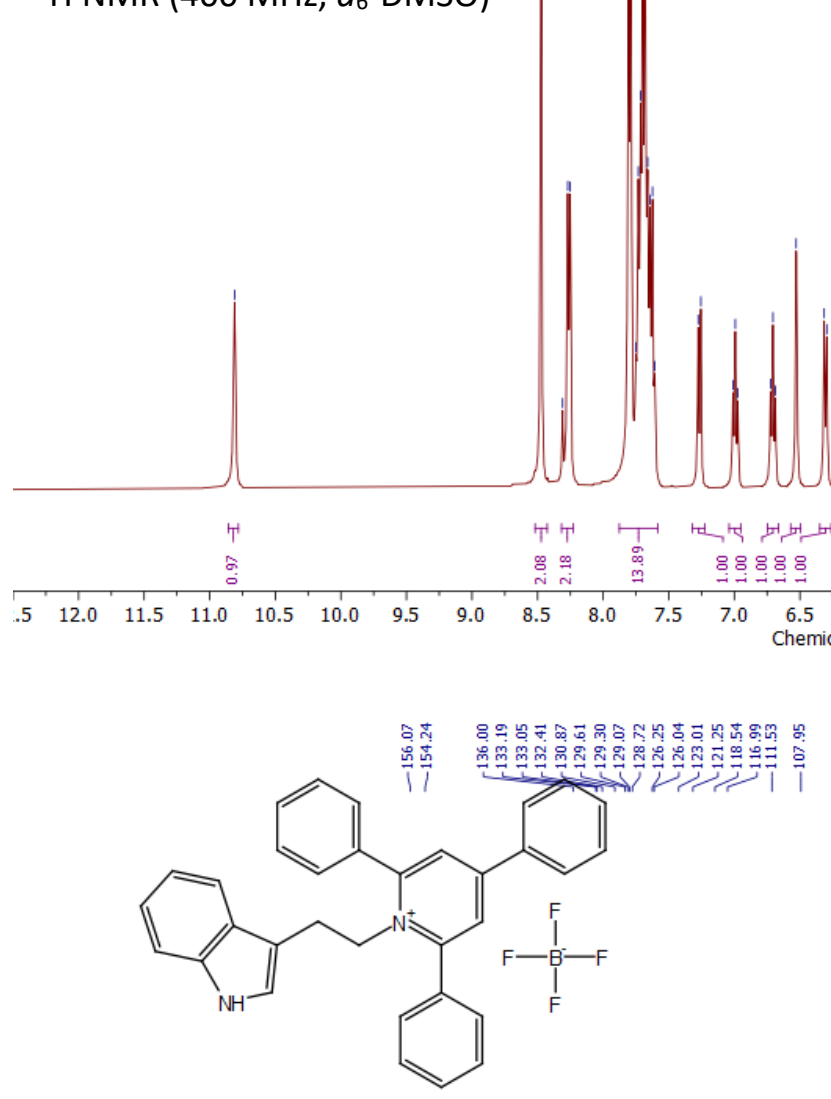

Compound 1al

${ }^{13} \mathrm{C}$ NMR (101 MHz, $d_{6}$-DMSO)

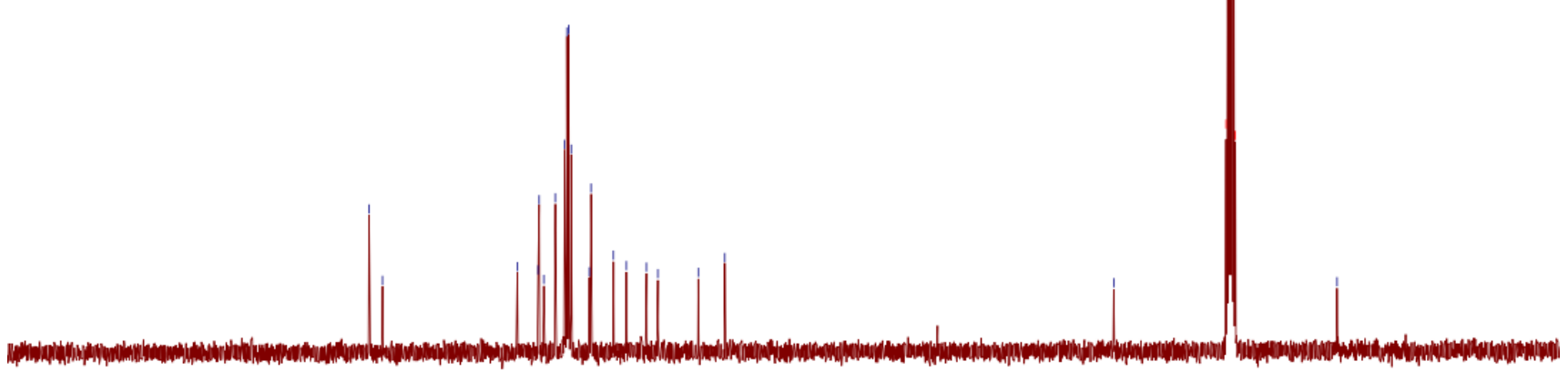

$\begin{array}{llllllllll}200 & 190 & 180 & 170 & 160 & 150 & 140 & 130 & 120 & \begin{array}{c}110 \\ \text { Chemical Shift (bom) }\end{array}\end{array}$ 


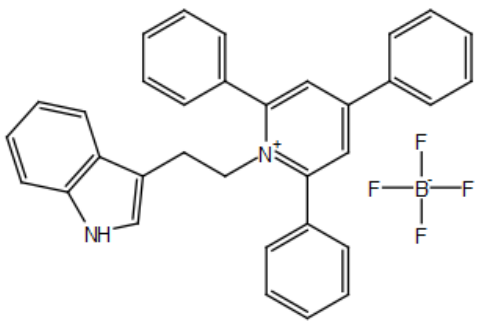

Compound 1al

${ }^{19} \mathrm{~F}$ NMR (377 MHz, $d_{6}$-DMSO)

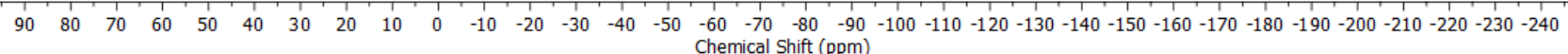


NMR spectra of 1-(2-(dimethylamino)ethyl)-2,4,6-triphenylpyridin-1-ium tetrafluoroborate (1am)<smiles></smiles>

Compound 1am

${ }^{1} \mathrm{H}$ NMR (400 MHz, $d_{6}$-DMSO)
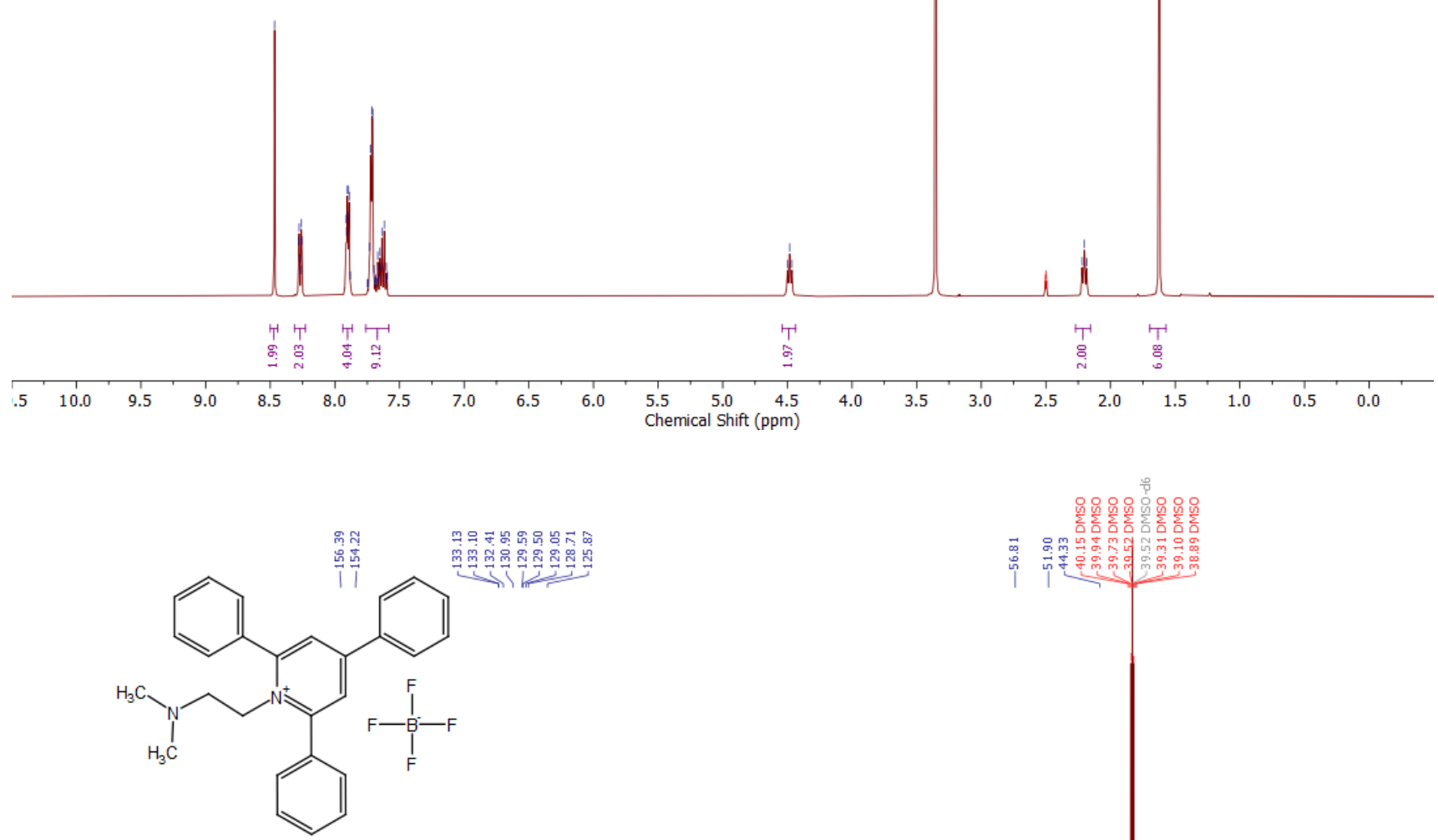

Compound 1am

${ }^{13} \mathrm{C}$ NMR (101 MHz, $d_{6}$-DMSO)

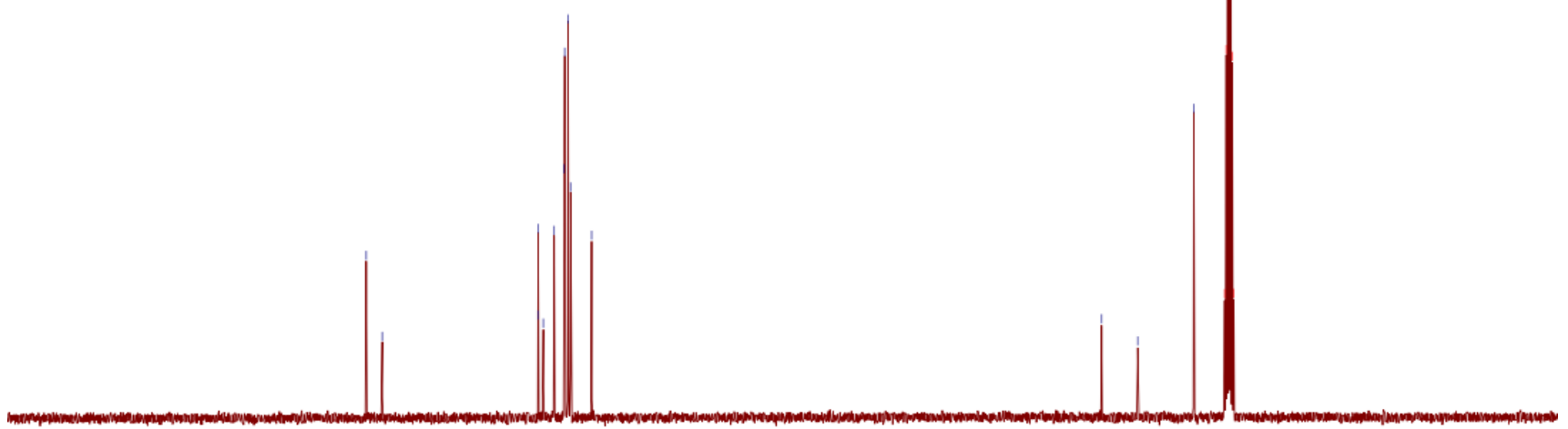


<smiles></smiles>

Compound 1am

${ }^{19} \mathrm{~F}$ NMR (377 MHz, $d_{6}$-DMSO)

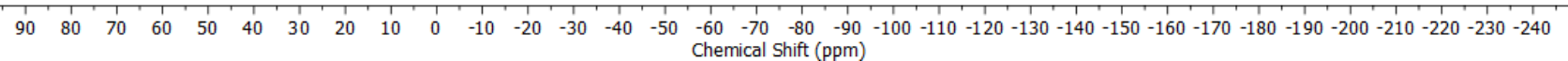


NMR spectra of (S)-1-(5-((tert-butoxycarbonyl)amino)-5-carboxypentyl)-2,4,6-triphenylpyridin-1-ium tetrafluoroborate (1an)

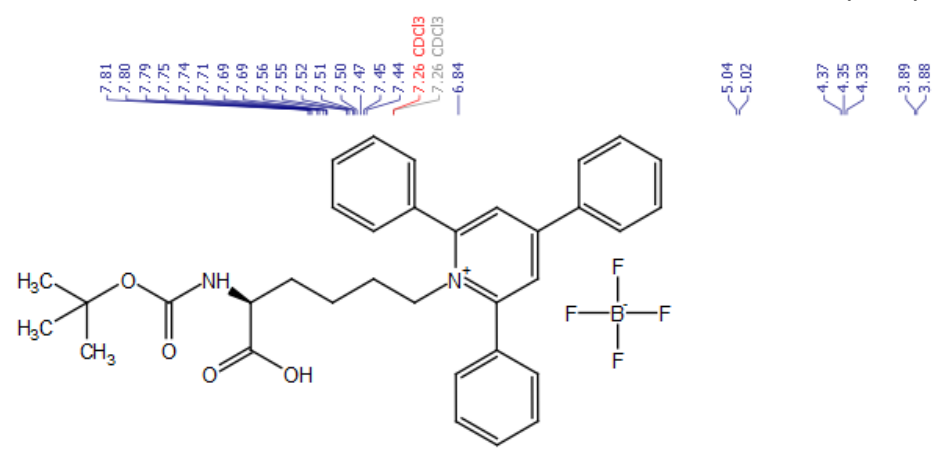

Compound 1an

${ }^{1} \mathrm{H}$ NMR $\left(400 \mathrm{MHz}, \mathrm{CDCl}_{3}\right)$

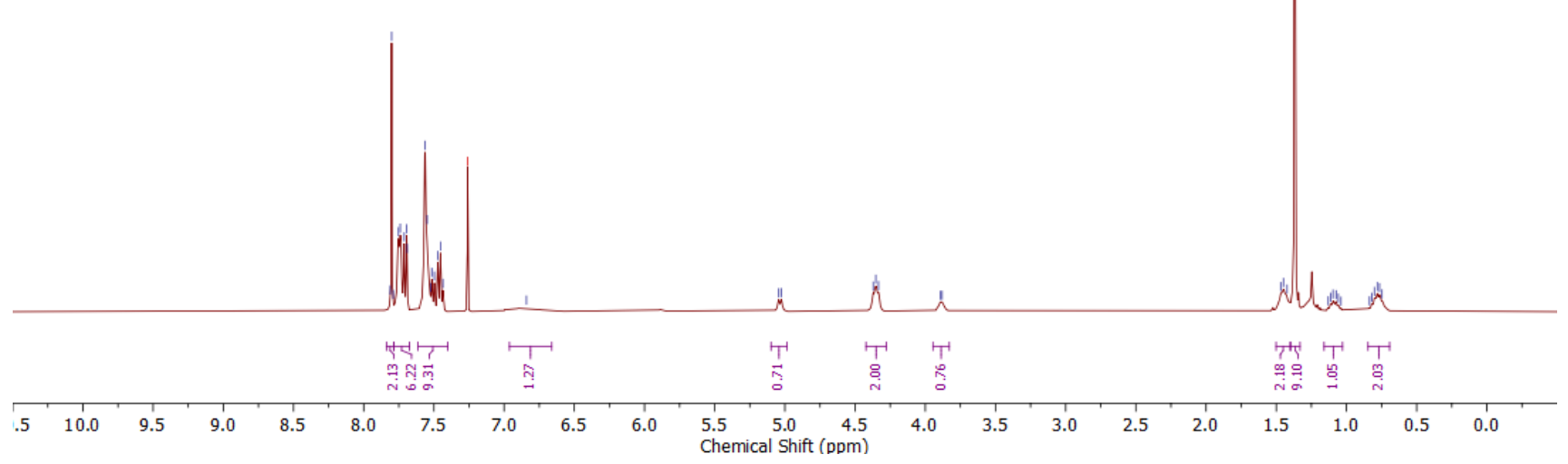<smiles></smiles>

Compound 1an

${ }^{13} \mathrm{C}$ NMR (101 MHz, $\left.\mathrm{CDCl}_{3}\right)$

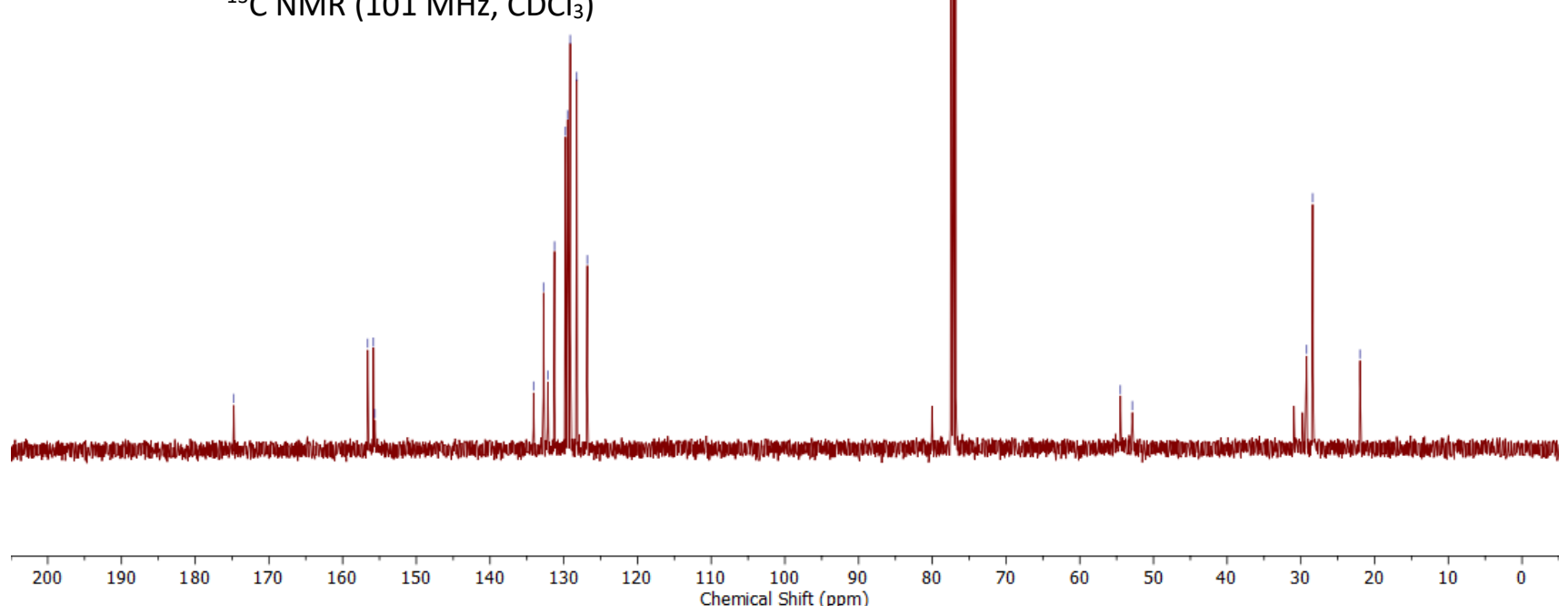




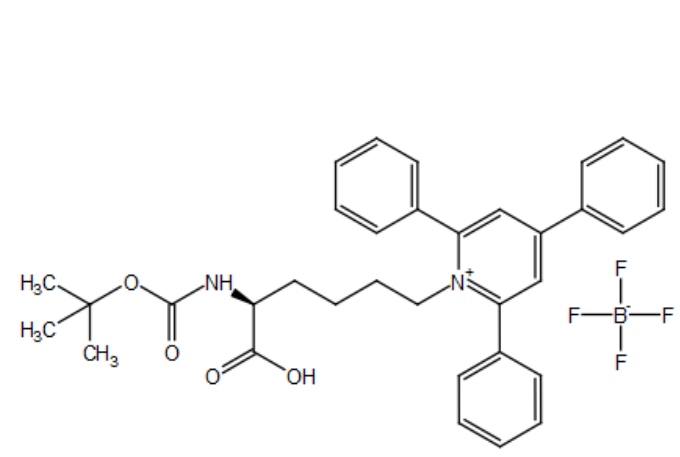

Compound 1an

${ }^{19} \mathrm{~F} \mathrm{NMR}\left(377 \mathrm{MHz}, \mathrm{CDCl}_{3}\right)$

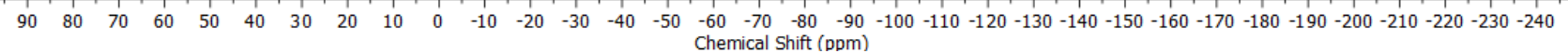


NMR spectra of 2,4,6-triphenyl-1-(2-sulfoethyl)pyridin-1-ium tetrafluoroborate (1ao)
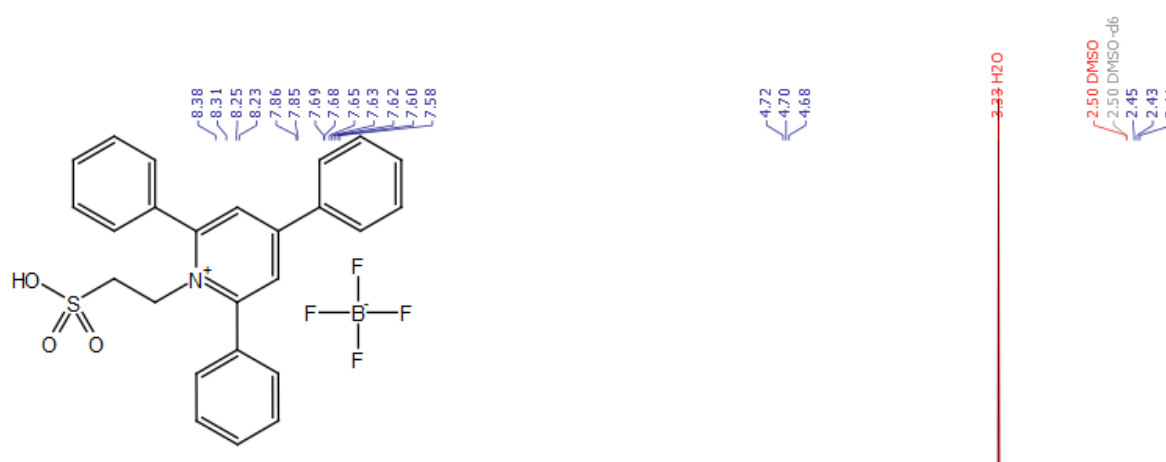

Compound 1ao

${ }^{1} \mathrm{H}$ NMR (400 MHz, $d_{6}$-DMSO)
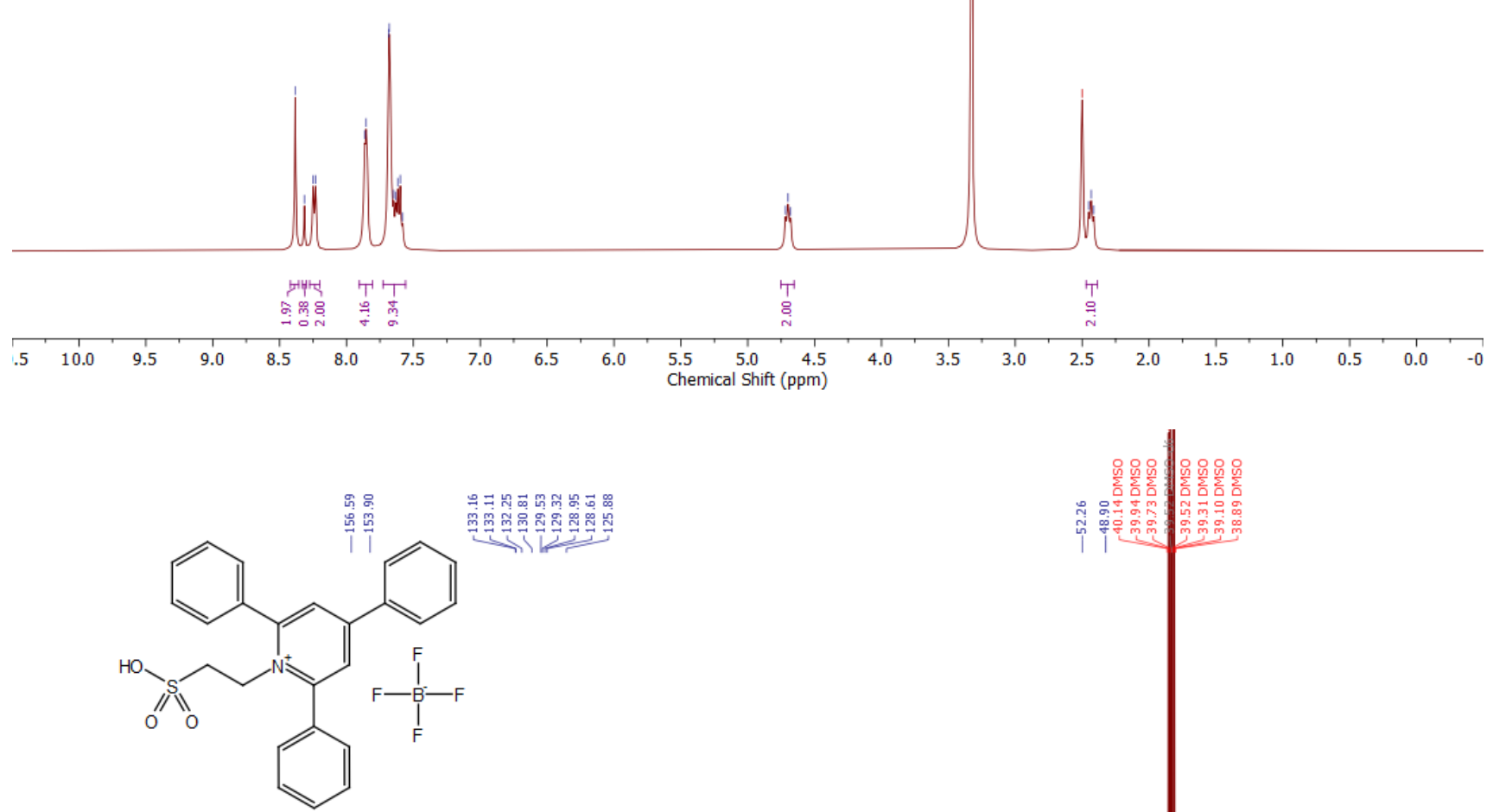

Compound 1ao

${ }^{13} \mathrm{C}$ NMR (101 MHz, $d_{6}$-DMSO)

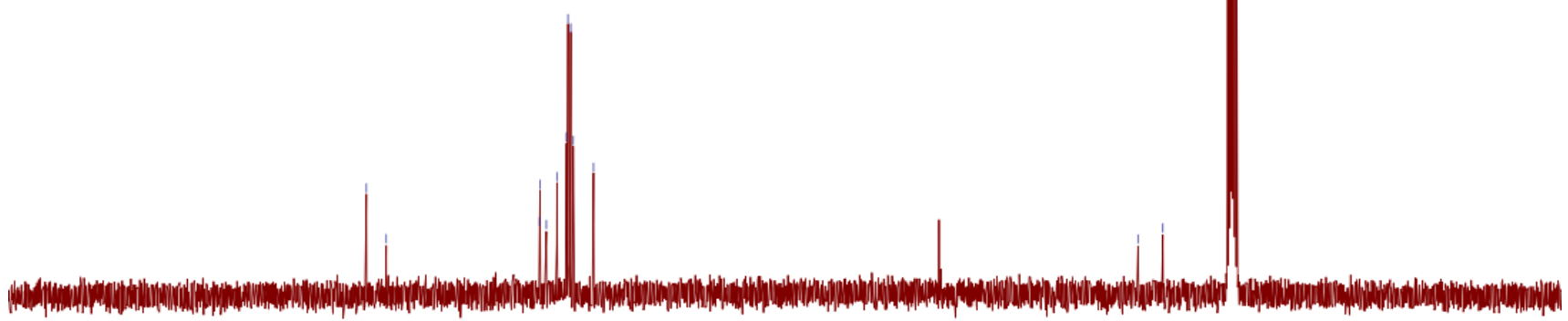

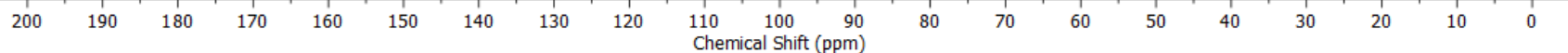




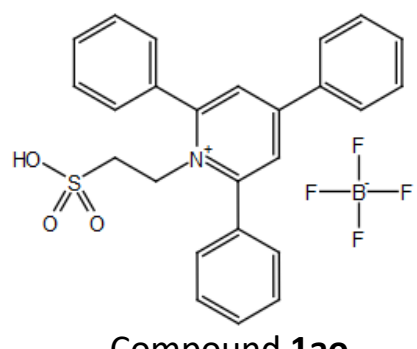

Compound 1ao

${ }^{19} \mathrm{~F}$ NMR (377 MHz, $d_{6}$-DMSO)

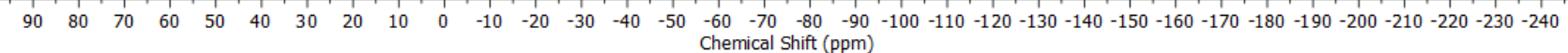


NMR spectra of 2,4,6-triphenyl-1-(4-sulfamoylphenethyl)pyridin-1-ium tetrafluoroborate (1ap)

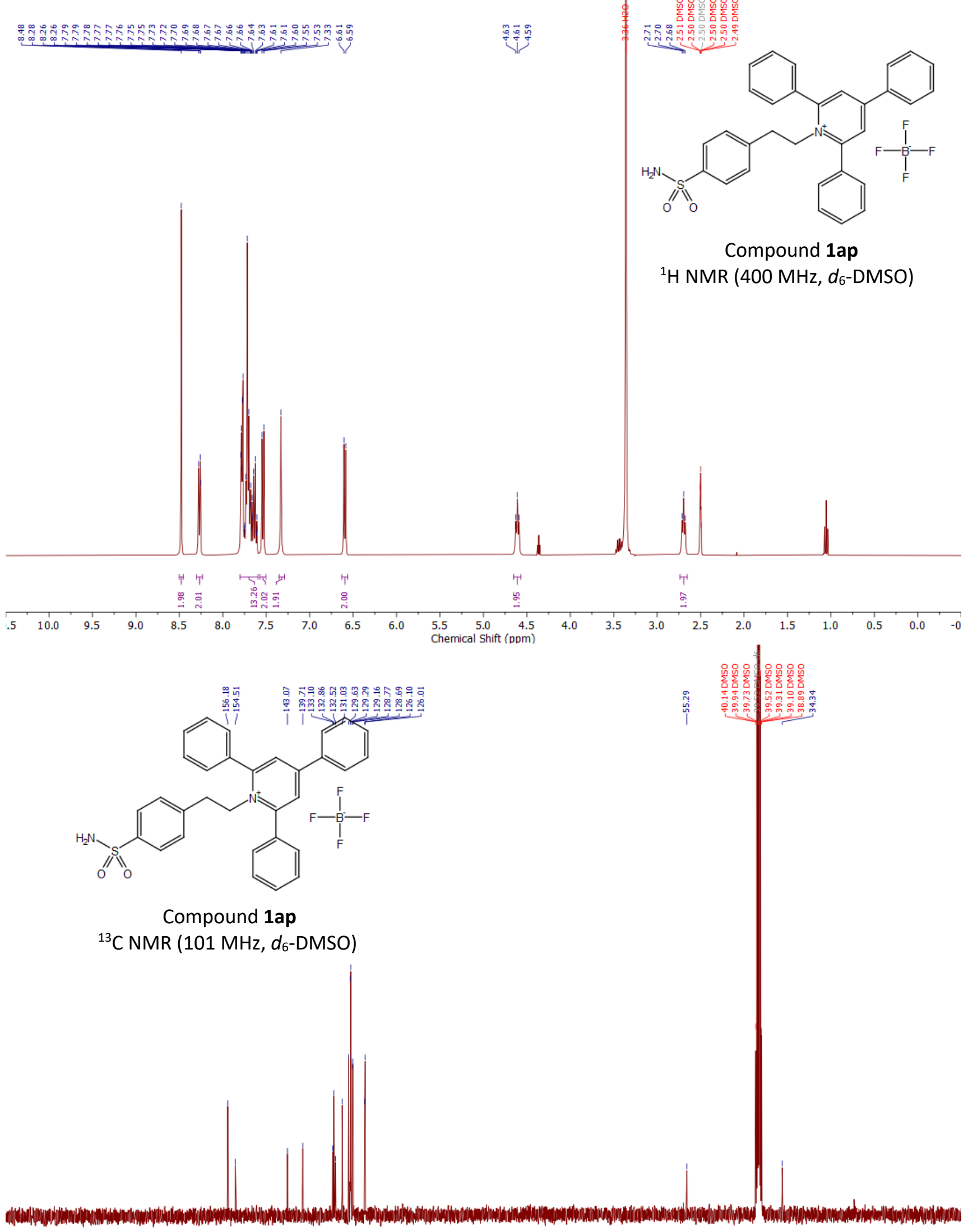

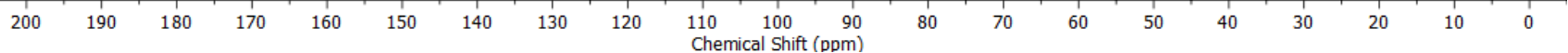




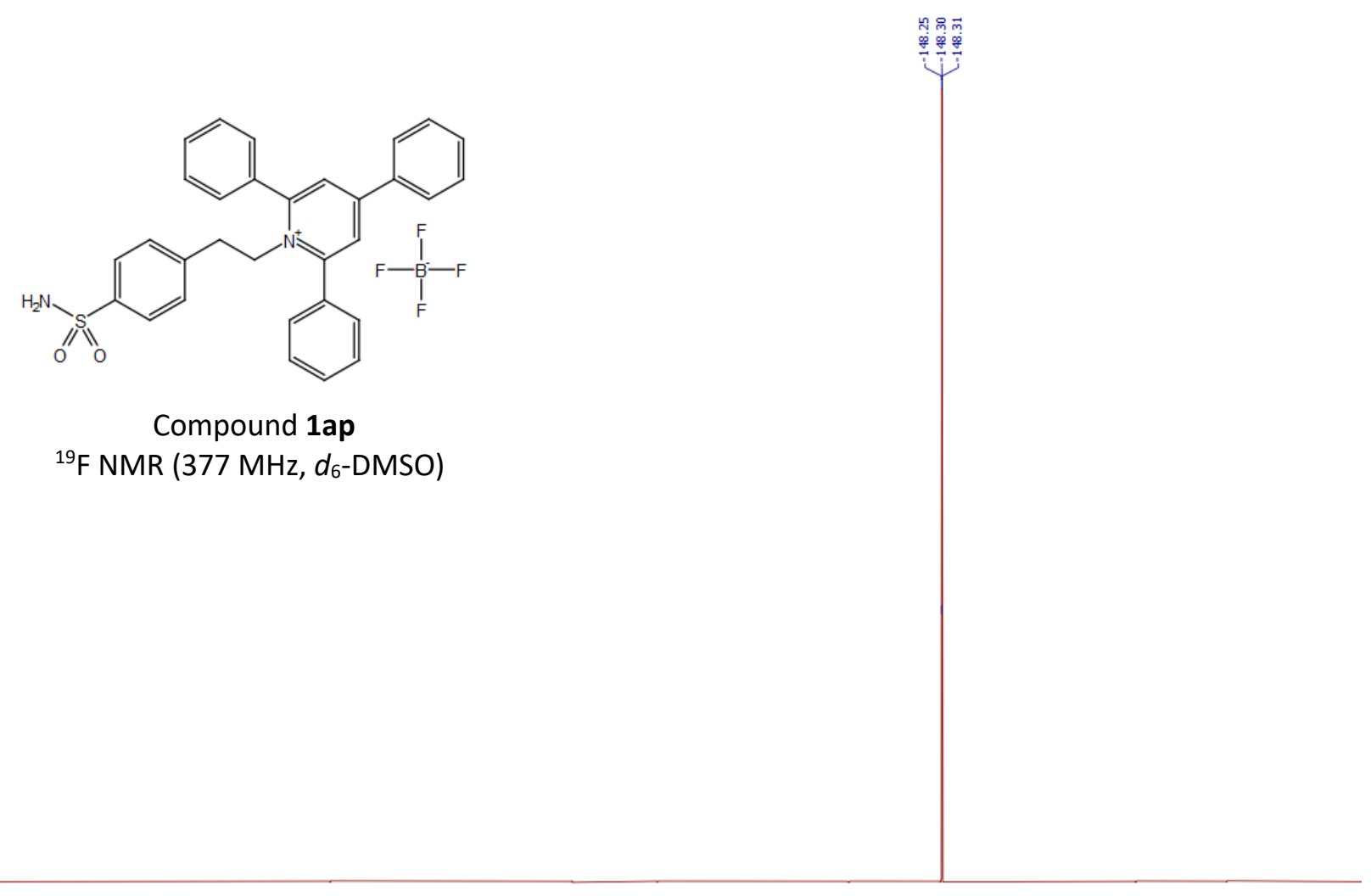

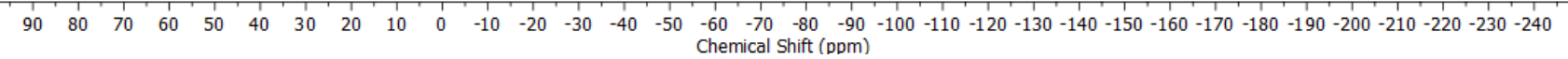


NMR spectra of benzyl 4-((2-(tert-butoxy)-2-oxoethyl)sulfonyl)piperidine-1-carboxylate (3a)
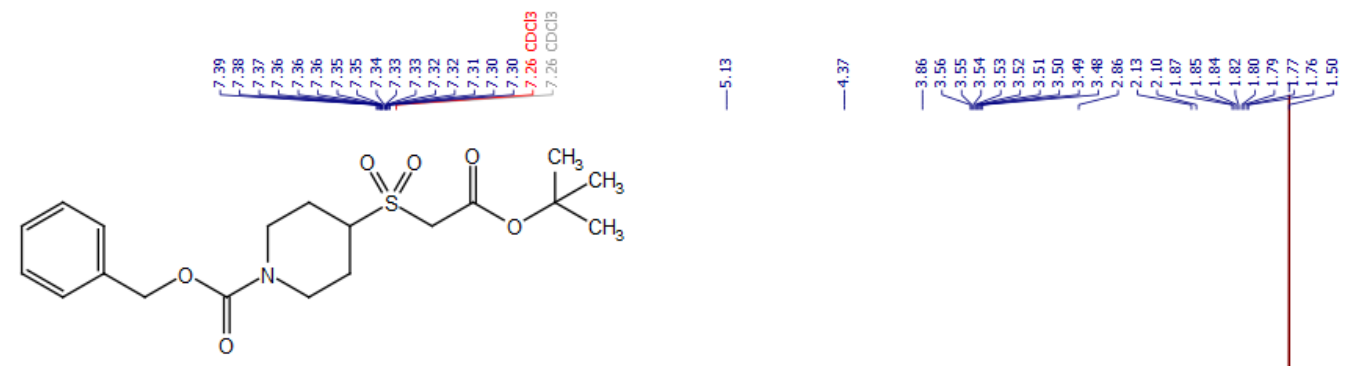

Compound 3a

${ }^{1} \mathrm{H}$ NMR $\left(400 \mathrm{MHz}, \mathrm{CDCl}_{3}\right)$
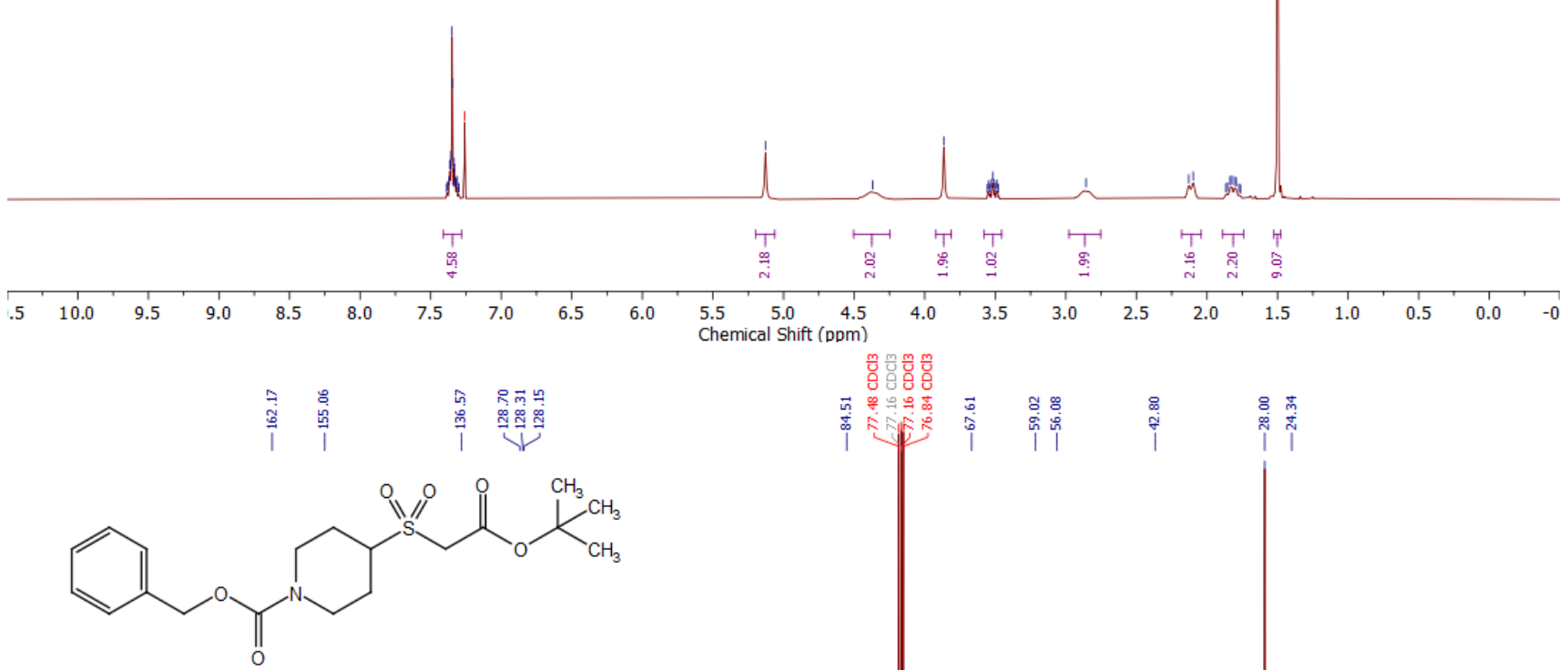

Compound 3a

${ }^{13} \mathrm{C}$ NMR $\left(101 \mathrm{MHz}, \mathrm{CDCl}_{3}\right)$

$\begin{array}{rrrrrrrrrr}200 & 190 & 180 & 170 & 160 & 150 & 140 & 130 & 120 & \begin{array}{c}110 \\ \text { Chemical Shift (bom) }\end{array}\end{array}$

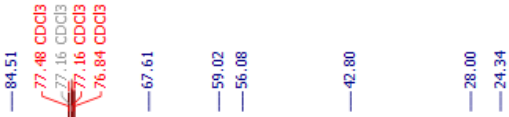


NMR spectra of tert-butyl 4-((2-(tert-butoxy)-2-oxoethyl)sulfonyl)piperidine-1-carboxylate (3d)<smiles>CC(=O)OCCCNNC(=O)OC(C)(C)C</smiles>

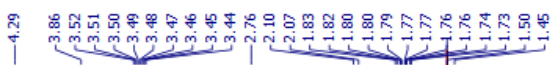
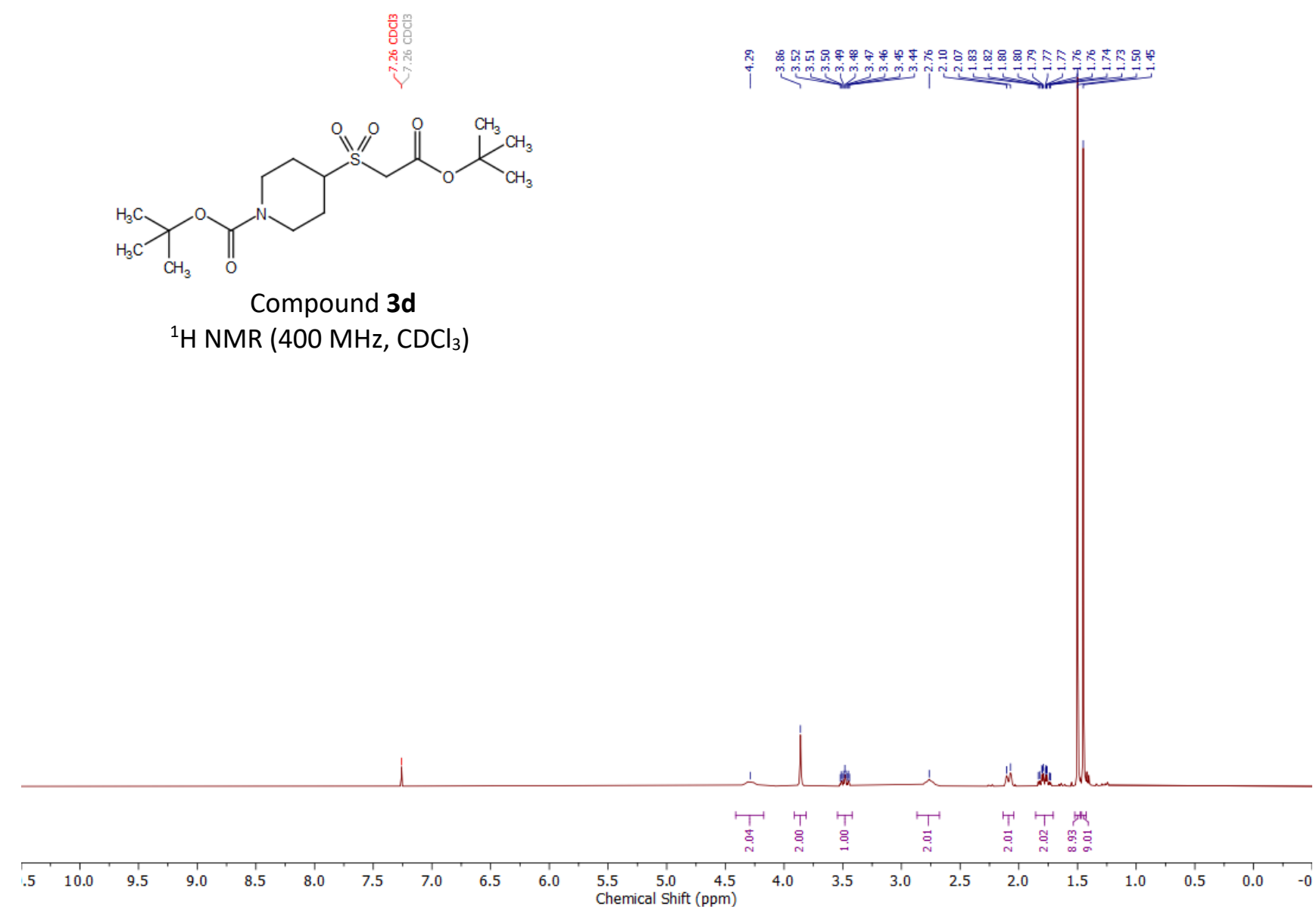<smiles>CC(C)(C)OC(=O)CS(=O)(=O)C1CCN(C(=O)OC(C)(C)C)CC1</smiles>

Compound 3d

${ }^{13} \mathrm{C}$ NMR (101 MHz, $\left.\mathrm{CDCl}_{3}\right)$

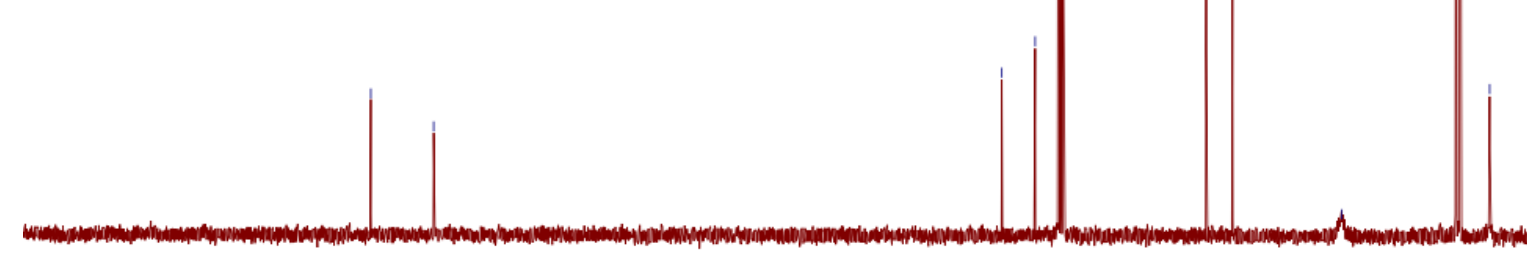


NMR spectra of tert-butyl 2-((1-tosylpiperidin-4-yl)sulfonyl)acetate (3e)

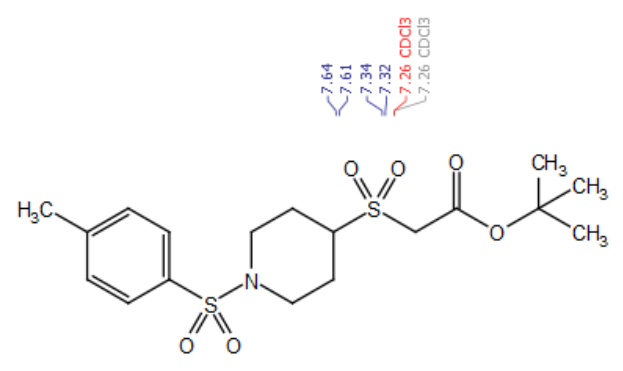

屯.

Compound $3 \mathbf{e}$

${ }^{1} \mathrm{H}$ NMR $\left(400 \mathrm{MHz}, \mathrm{CDCl}_{3}\right)$
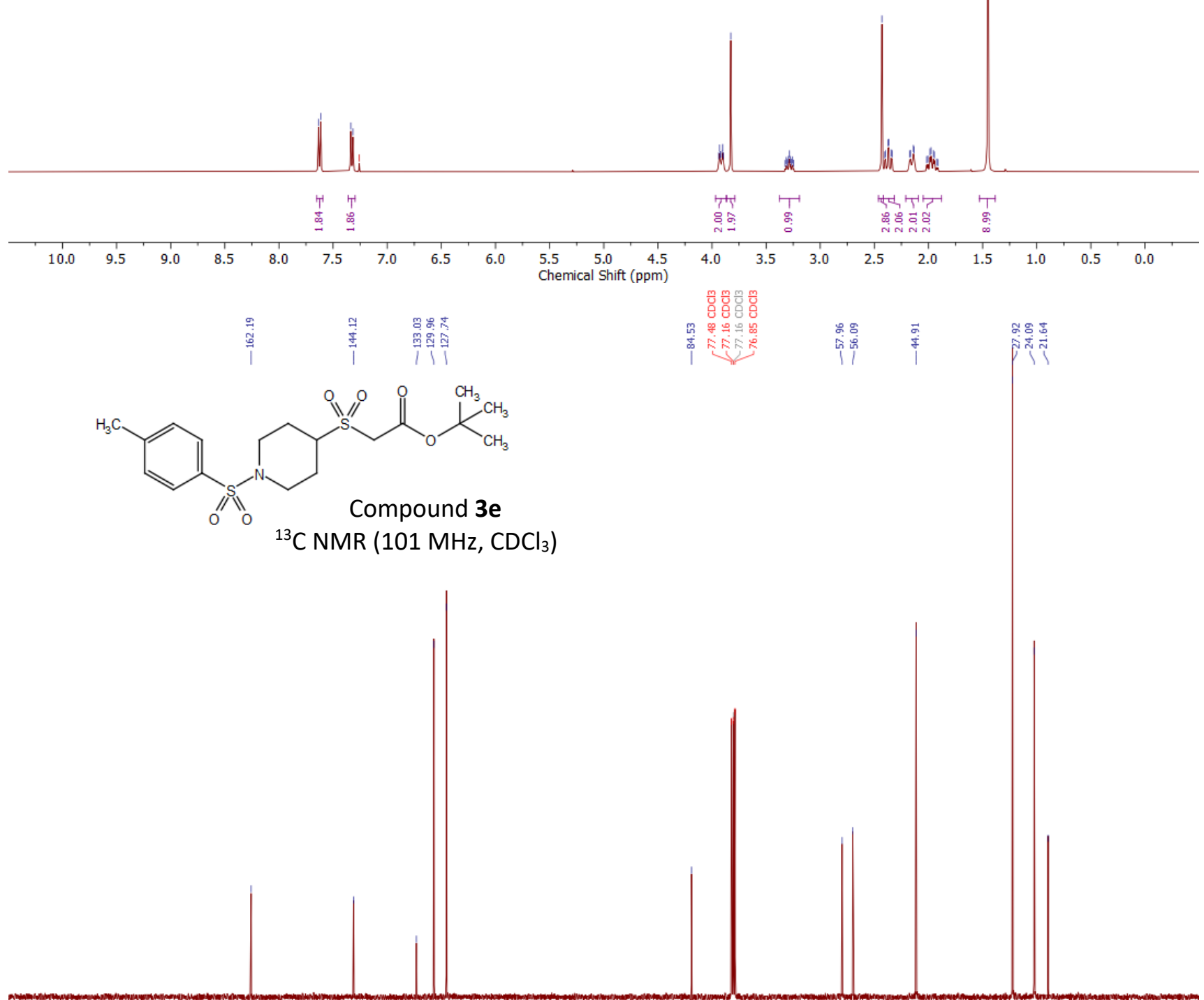
NMR spectra of tert-butyl 2-(cyclobutylsulfonyl)acetate (3f)

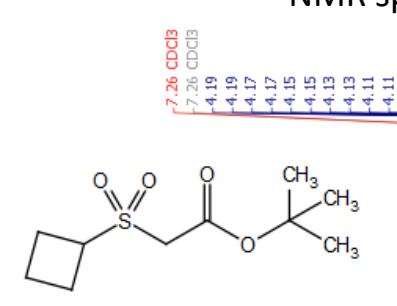

Compound $\mathbf{3 f}$

${ }^{1} \mathrm{H}$ NMR $\left(400 \mathrm{MHz}, \mathrm{CDCl}_{3}\right)$

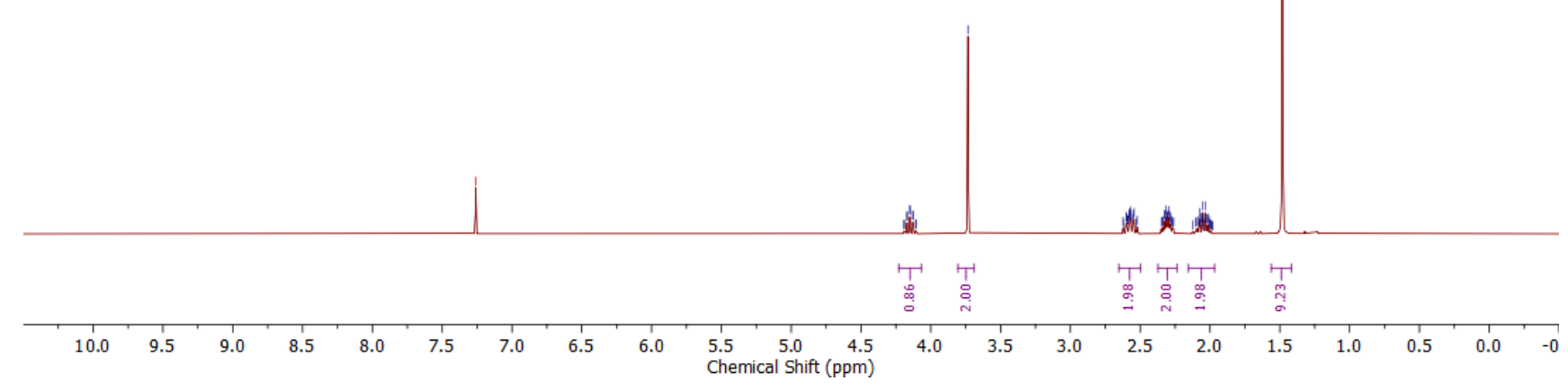

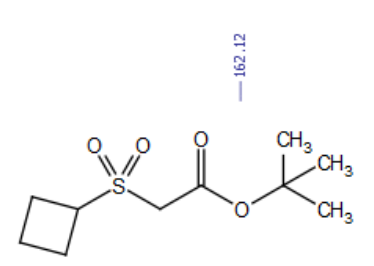

Compound $3 f$

${ }^{13} \mathrm{C}$ NMR $\left(101 \mathrm{MHz}, \mathrm{CDCl}_{3}\right)$

\section{齐}

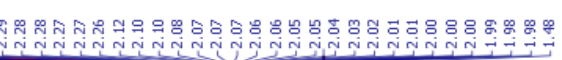

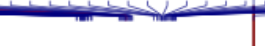


NMR spectra of tert-butyl 2-(cyclopentylsulfonyl)acetate (3g)
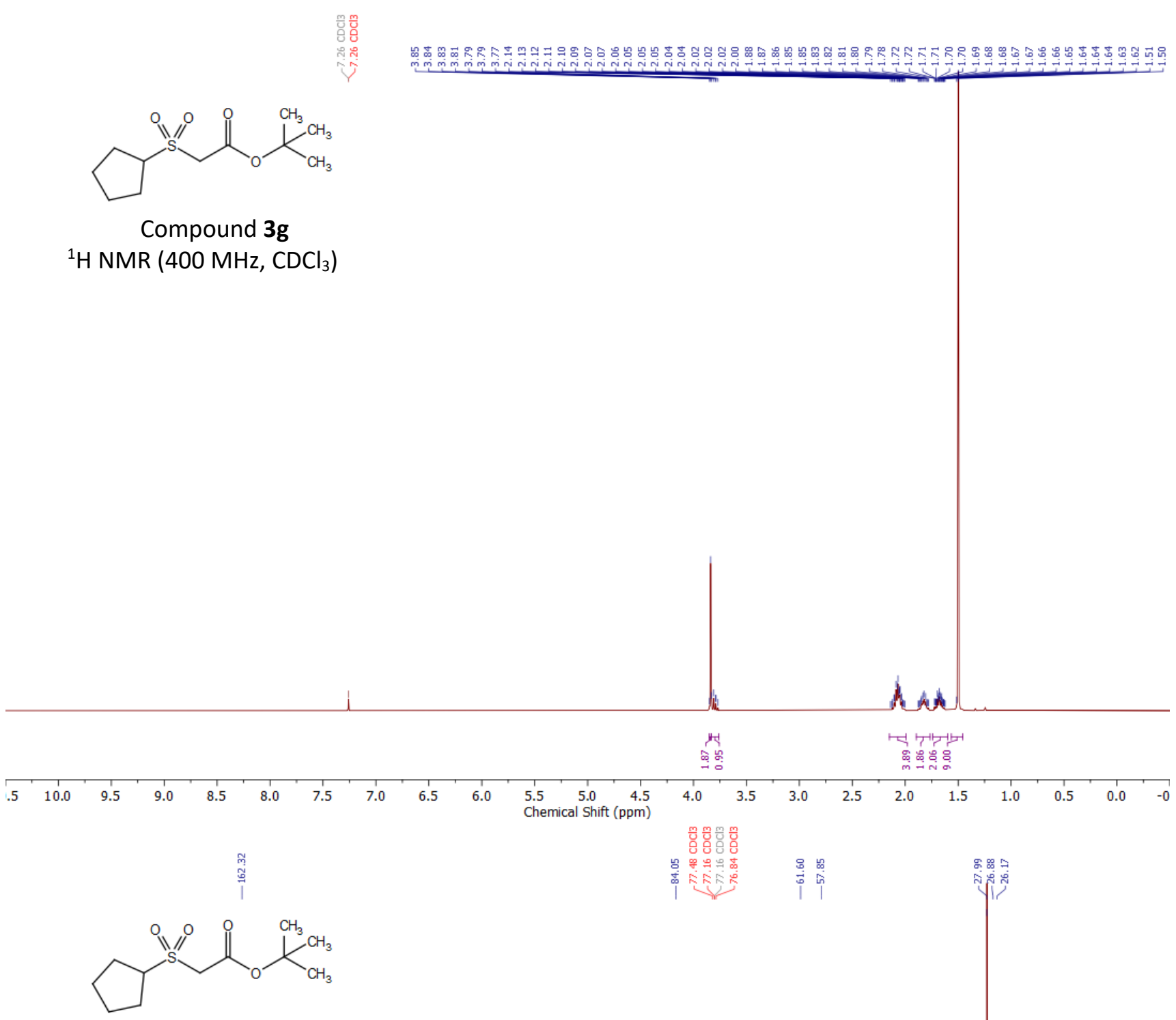

Compound 3g

${ }^{13} \mathrm{C}$ NMR $\left(101 \mathrm{MHz}, \mathrm{CDCl}_{3}\right)$

$\begin{array}{lllllllllll}200 & 190 & 180 & 170 & 160 & 150 & 140 & 130 & 120 & \begin{array}{l}110 \\ \text { Chemical Shift (bom) }\end{array}\end{array}$


NMR spectra of tert-butyl 2-(cyclohexylsulfonyl)acetate (3h)

$\frac{\mathrm{m}}{\mathrm{O}} \frac{m}{\mathrm{O}}$

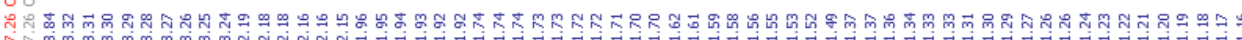<smiles>CC(C)(C)OC(=O)CS(=O)(=O)C1CCCCC1</smiles>

Compound $\mathbf{3 h}$

${ }^{1} \mathrm{H}$ NMR $\left(400 \mathrm{MHz}^{\mathrm{CDCl}}{ }_{3}\right)$

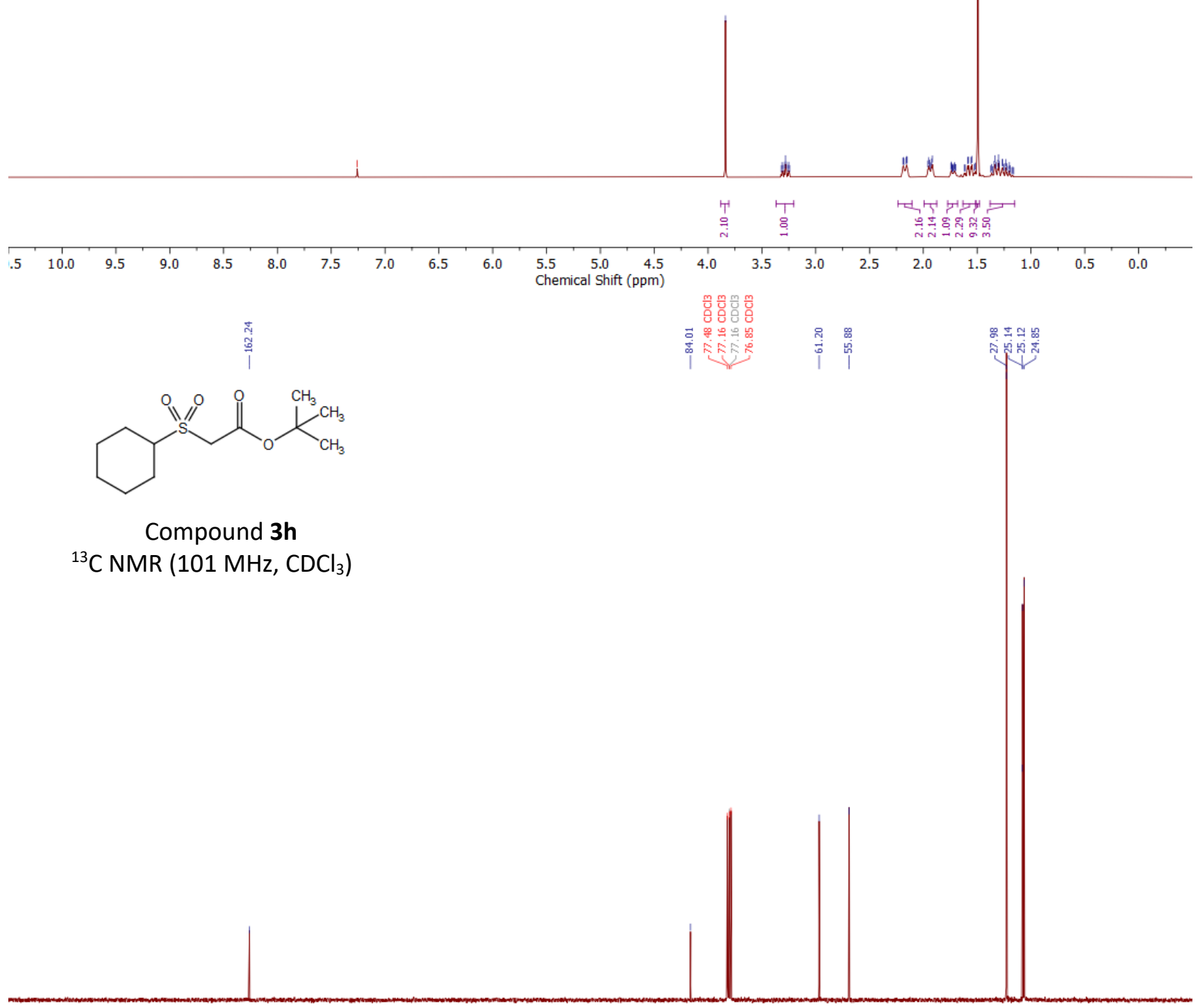

$\begin{array}{llllllllll}200 & 190 & 180 & 170 & 160 & 150 & 140 & 130 & 120 & \begin{array}{c}110 \\ \text { Chemical Shift (bom) }\end{array}\end{array}$ 
NMR spectra of tert-butyl 2-(isopropylsulfonyl)acetate (3i)

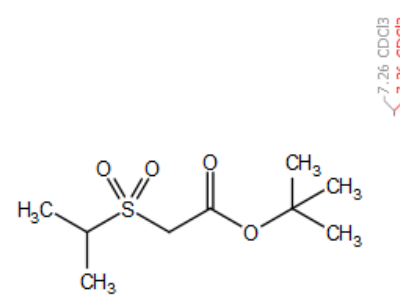

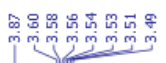

Compound $\mathbf{3 i}$

${ }^{1} \mathrm{H}$ NMR $\left(400 \mathrm{MHz}, \mathrm{CDCl}_{3}\right)$

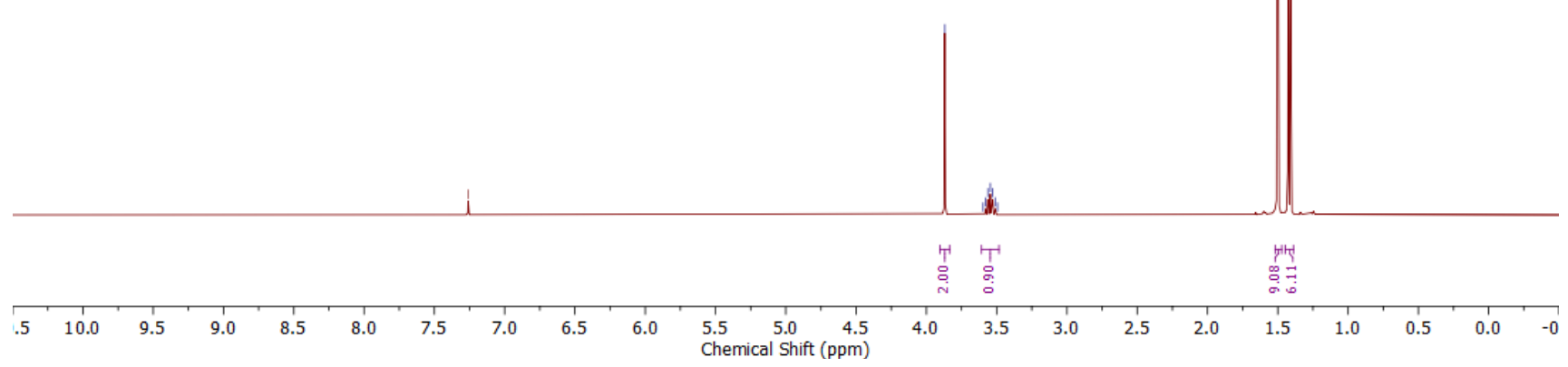

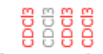<smiles>CC(C)S(=O)(=O)CC(=O)OC(C)(C)C</smiles>

Compound 3i

${ }^{13} \mathrm{C}$ NMR (101 MHz, $\mathrm{CDCl}_{3}$ )

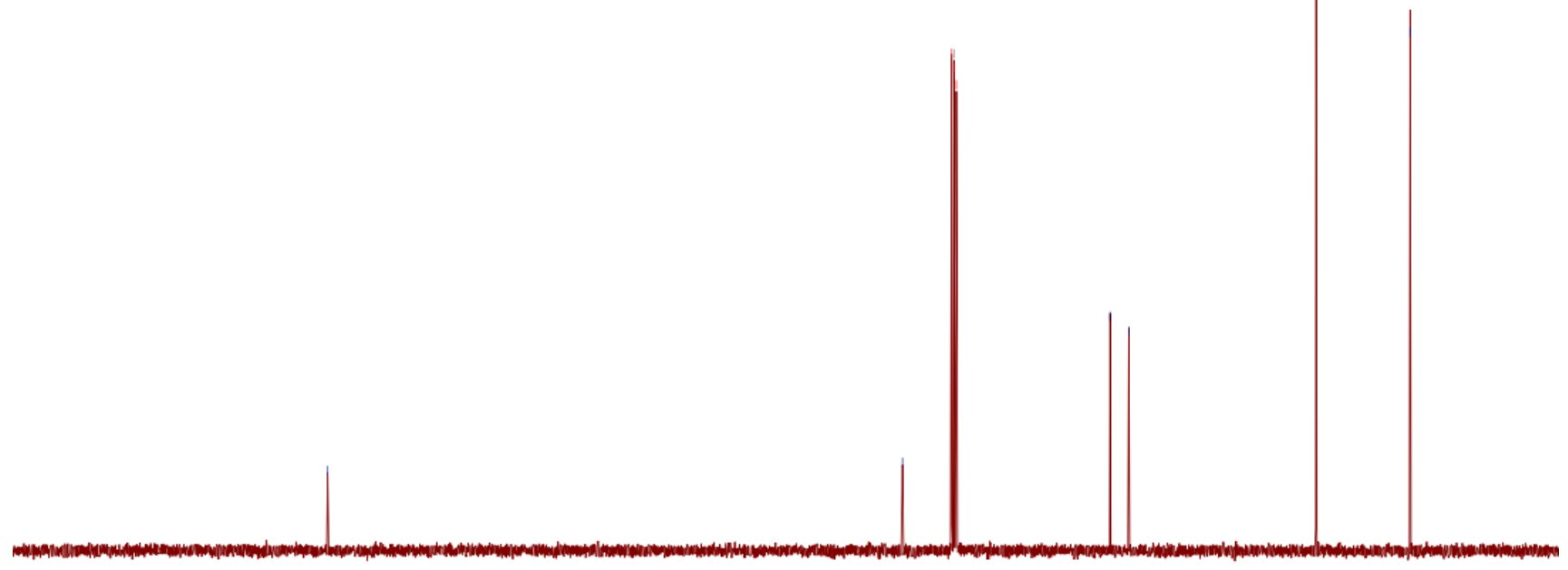

$\begin{array}{llllllllll}200 & 190 & 180 & 170 & 160 & 150 & 140 & 130 & 120 & \begin{array}{c}110 \\ \text { Chemical Shift (dom) }\end{array}\end{array}$ 
NMR spectra of tert-butyl 2-(cyclooctylsulfonyl)acetate (3j)
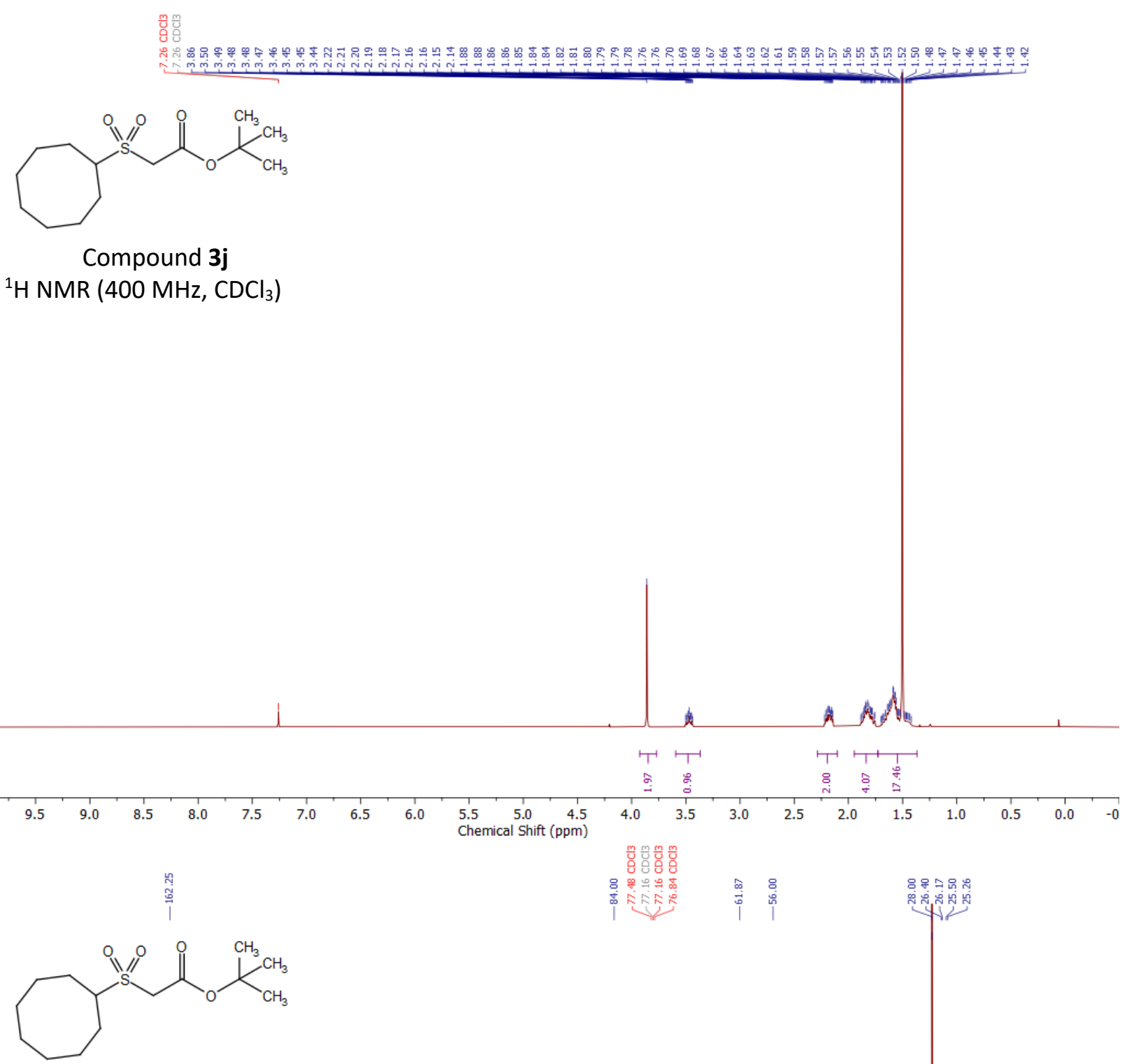

Compound 3j

${ }^{13} \mathrm{C}$ NMR (101 MHz, $\mathrm{CDCl}_{3}$ )

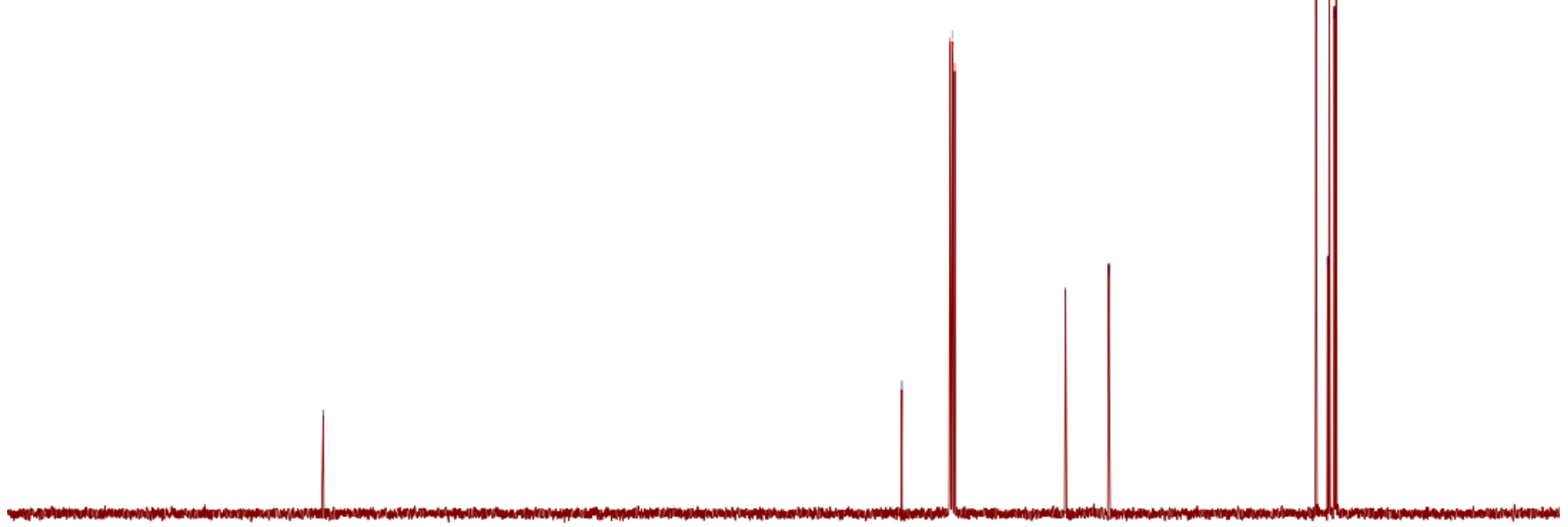

$\begin{array}{llllllllll}200 & 190 & 180 & 170 & 160 & 150 & 140 & 130 & 120 & \begin{array}{c}110 \\ \text { Chemical Shift (pdm) }\end{array}\end{array}$ 


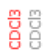

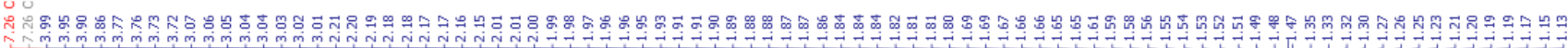<smiles>CC1CCCCC1S(=O)(=O)CC(=O)OC(C)(C)C</smiles>

Compound 3k

${ }^{1} \mathrm{H} \mathrm{NMR}\left(400 \mathrm{MHz}, \mathrm{CDCl}_{3}\right)$

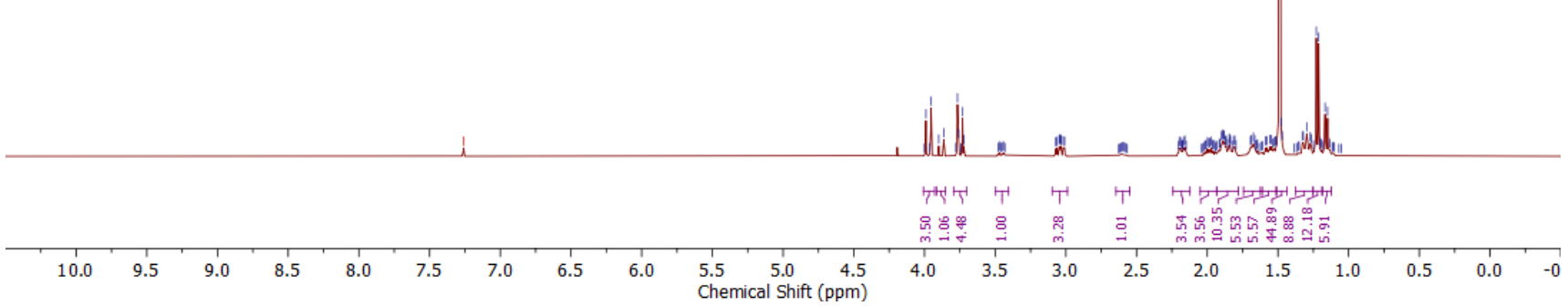<smiles>CC1CCCCC1S(=O)(=O)CC(=O)OC(C)(C)C</smiles>

Compound 3k

$\left.{ }^{13} \mathrm{C} \mathrm{NMR} \mathrm{(101} \mathrm{MHz,} \mathrm{CDCl}_{3}\right)$

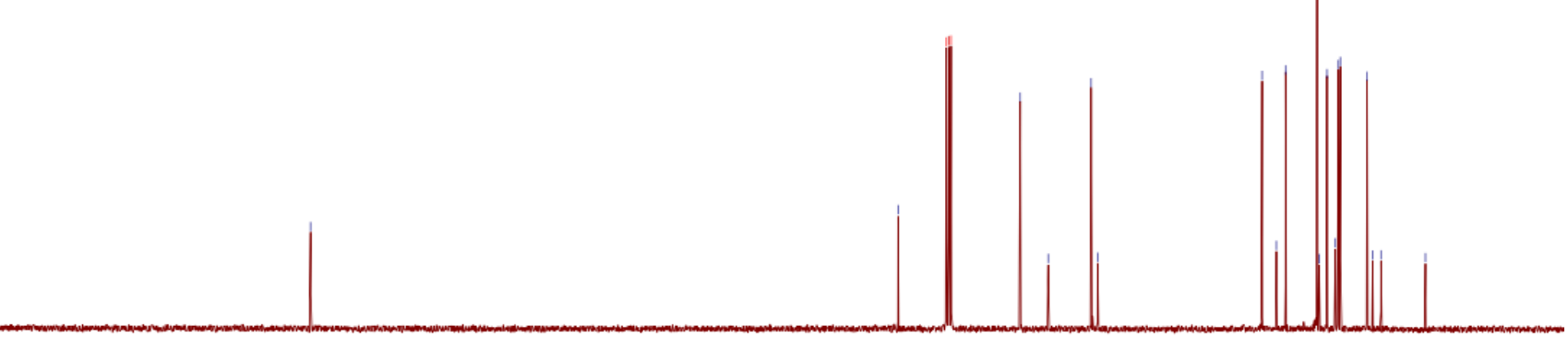


NMR spectra of tert-butyl 2-(heptan-2-ylsulfonyl)acetate (3l)

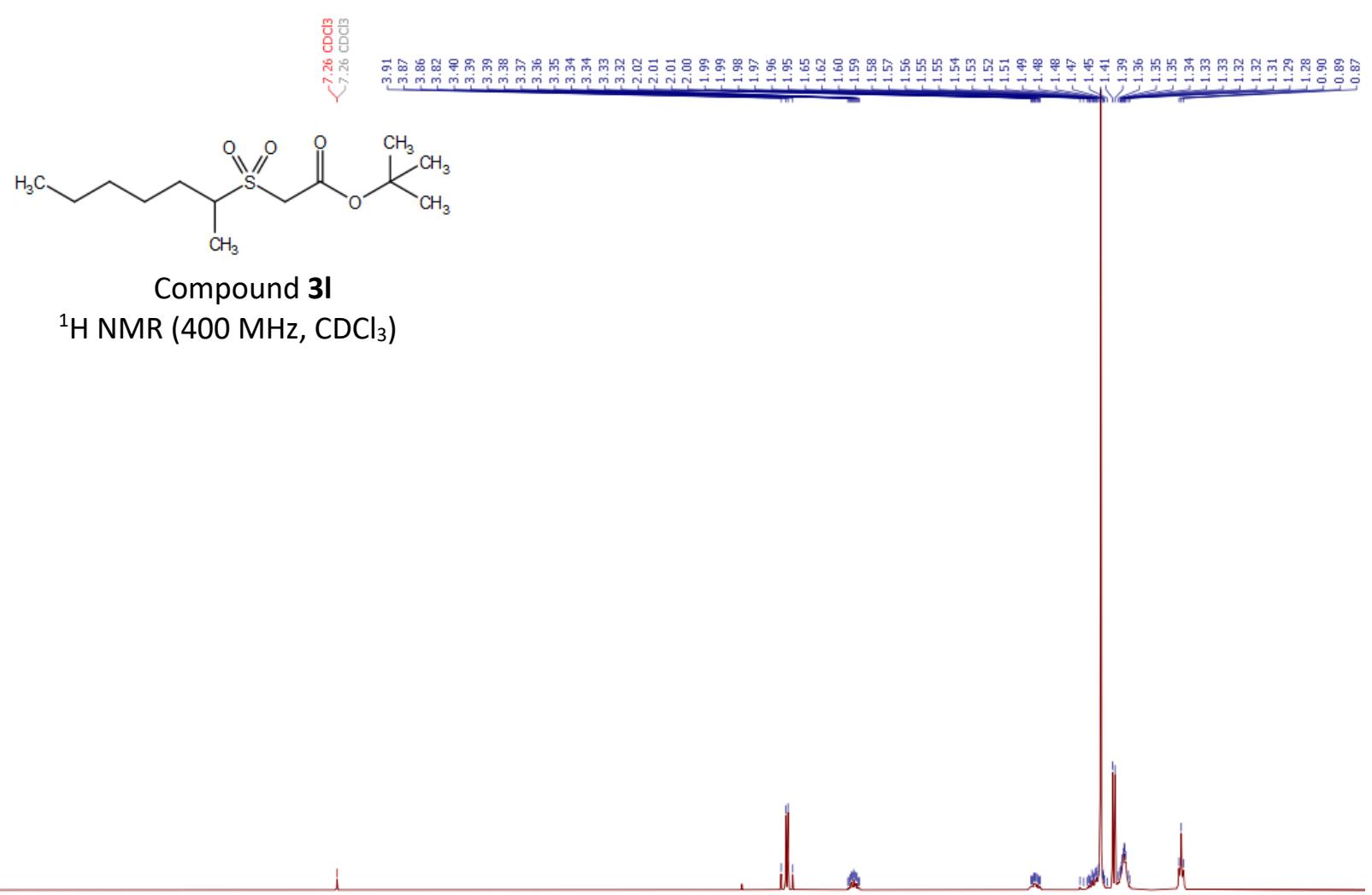

Tा मा मा ग ग ग

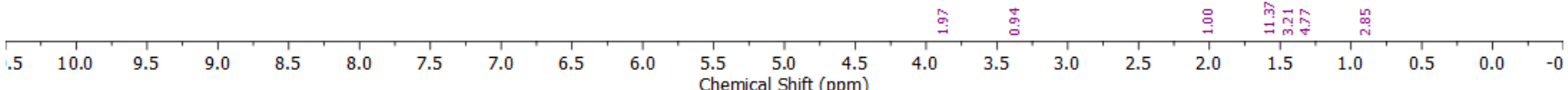<smiles>CCCCCC(C)S(=O)(=O)CC(=O)OC(C)(C)C</smiles>

Compound $\mathbf{3 l}$

${ }^{13} \mathrm{C}$ NMR $\left(101 \mathrm{MHz}, \mathrm{CDCl}_{3}\right)$

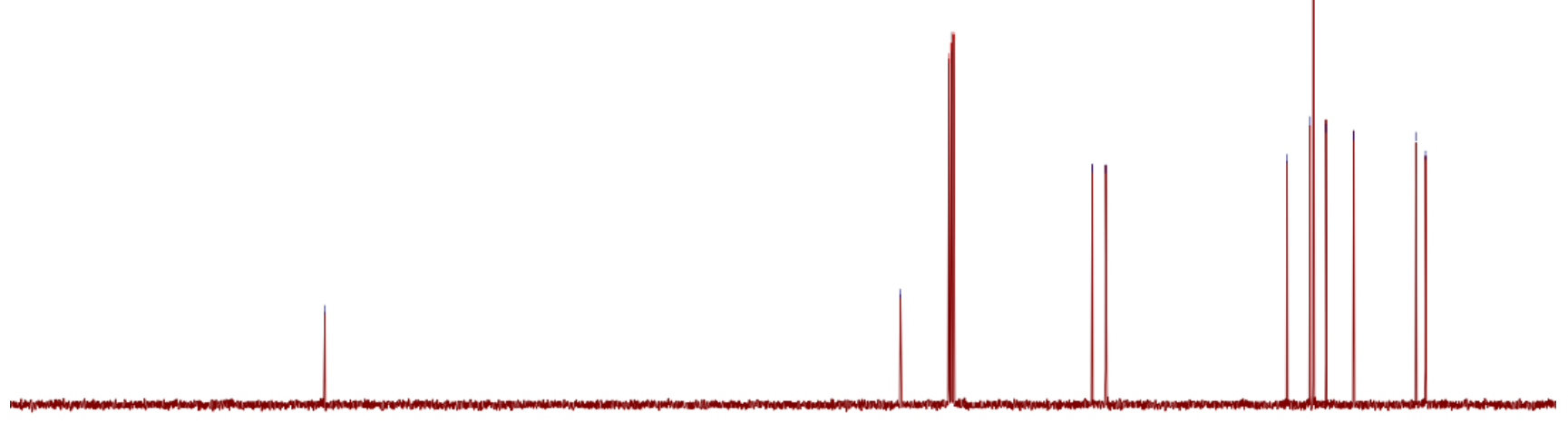

$\begin{array}{rrrrrrrrrr}200 & 190 & 180 & 170 & 160 & 150 & 140 & 130 & 120 & \begin{array}{c}110 \\ \text { Chemical Shift (bom) }\end{array}\end{array}$ 
NMR spectra of tert-butyl 2-((tetrahydro-2H-pyran-4-yl)sulfonyl)acetate (3m)

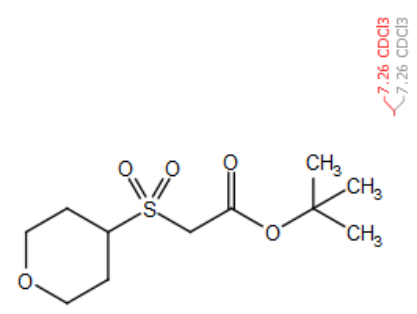

进甶

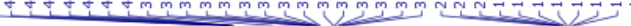

Compound 3m

${ }^{1} \mathrm{H} \mathrm{NMR}\left(400 \mathrm{MHz}, \mathrm{CDCl}_{3}\right)$

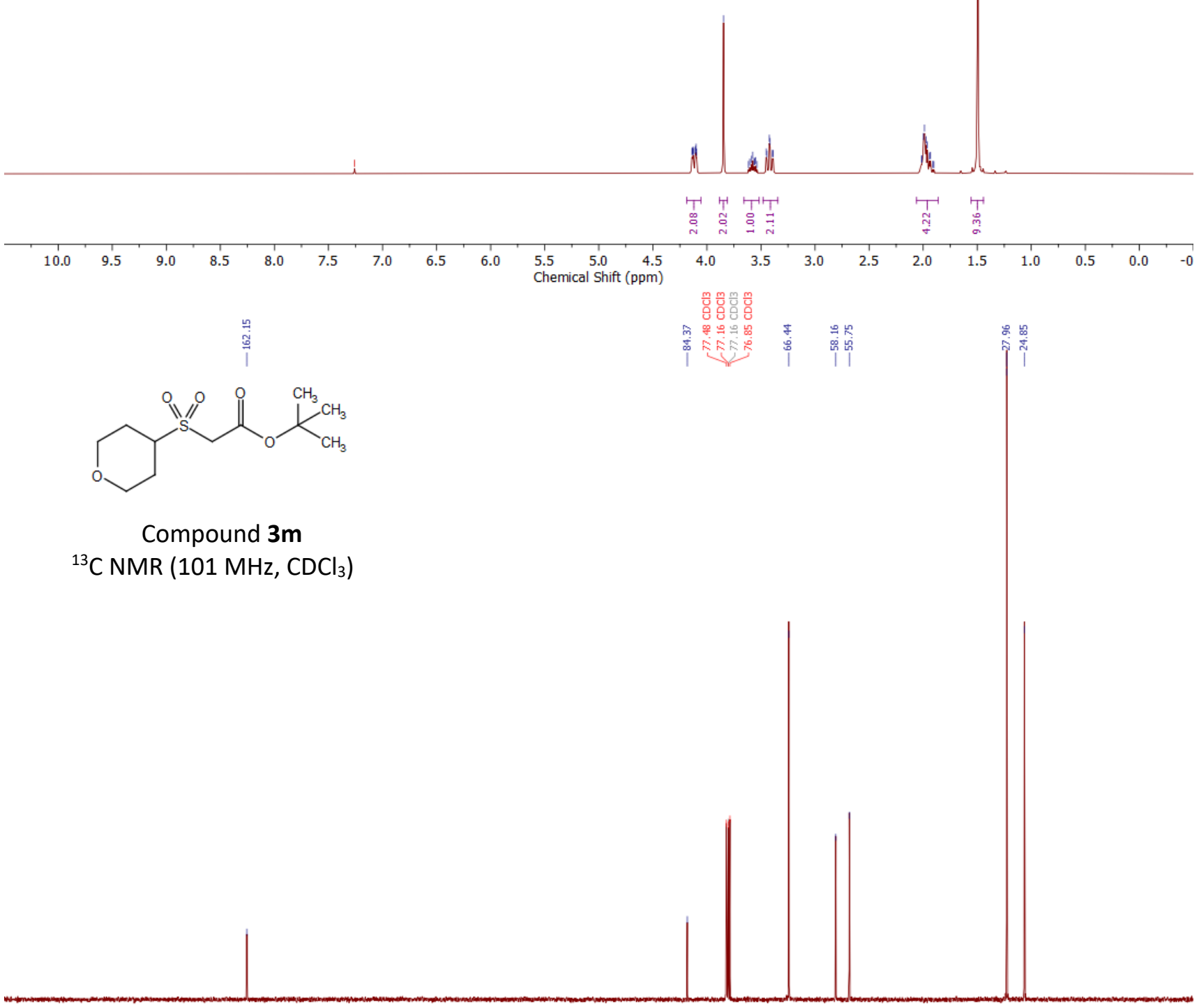

$\begin{array}{llllllllll}200 & 190 & 180 & 170 & 160 & 150 & 140 & 130 & 120 & \begin{array}{c}110 \\ \text { Chemical Shift (bom) }\end{array}\end{array}$ 
NMR spectra of tert-butyl 2-((tetrahydro-2H-thiopyran-4-yl)sulfonyl)acetate (3n)

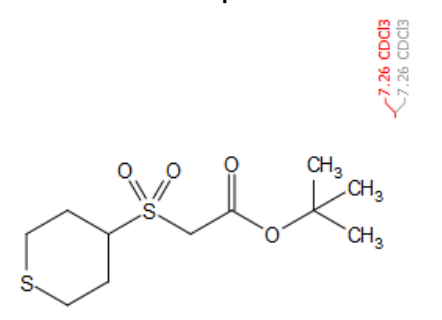

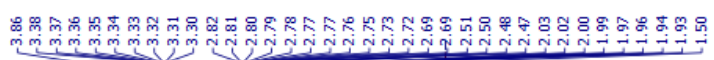

Compound 3n

${ }^{1} \mathrm{H}$ NMR $\left(400 \mathrm{MHz}, \mathrm{CDCl}_{3}\right)$
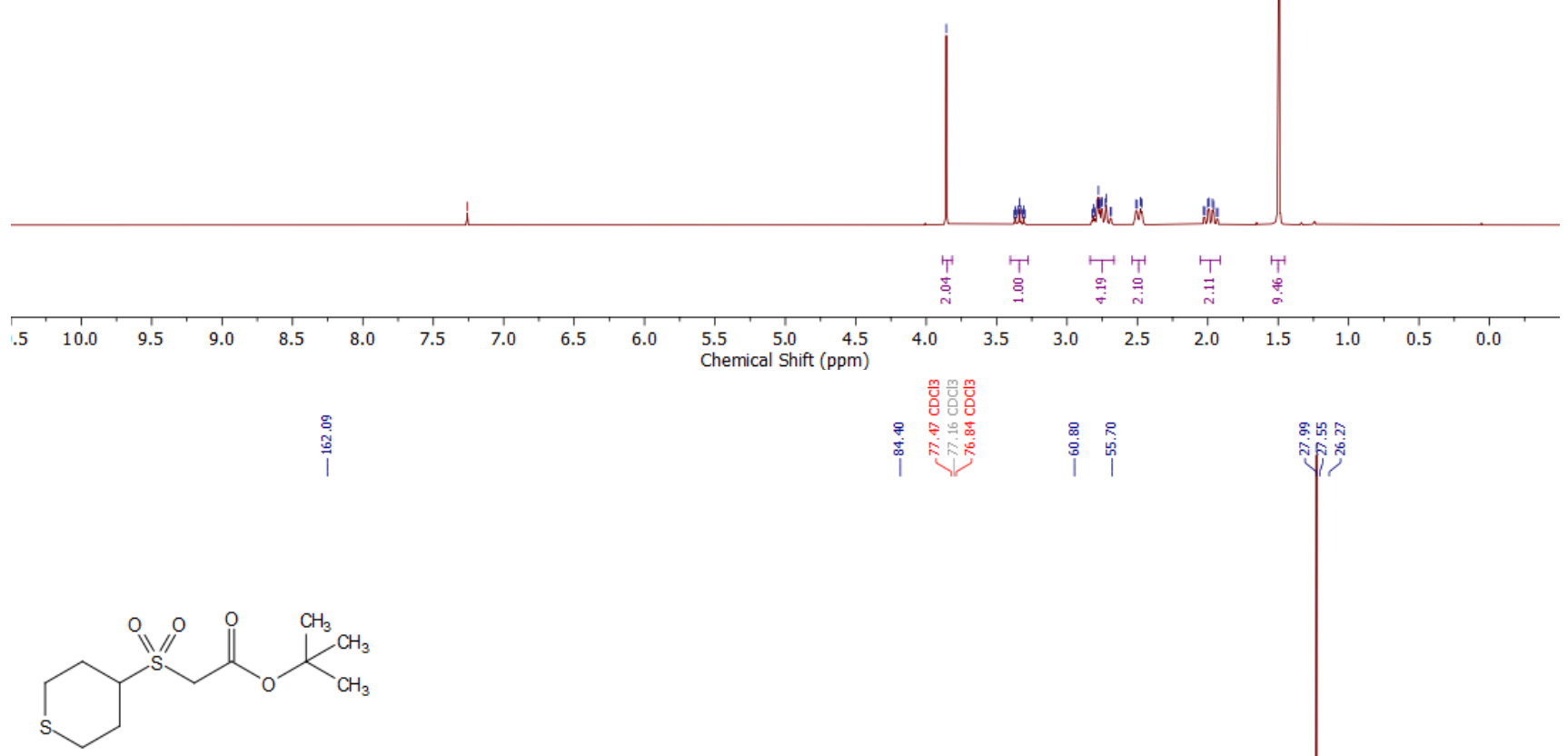

Compound 3n

$\left.{ }^{13} \mathrm{C} \mathrm{NMR} \mathrm{(101} \mathrm{MHz,} \mathrm{CDCl}_{3}\right)$

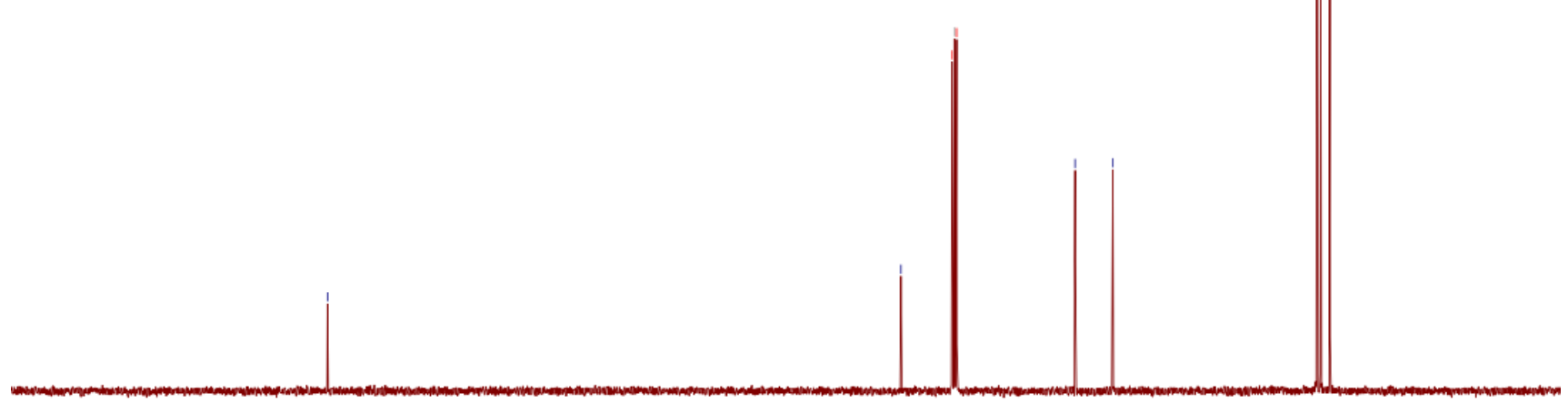

$\begin{array}{rrrrrrrrrr}200 & 190 & 180 & 170 & 160 & 150 & 140 & 130 & 120 & \begin{array}{c}110 \\ \text { Chemical Shift (ppm) }\end{array}\end{array}$ 
NMR spectra of tert-butyl 2-((1,1-dioxidotetrahydro-2H-thiopyran-4-yl)sulfonyl)acetate (30)

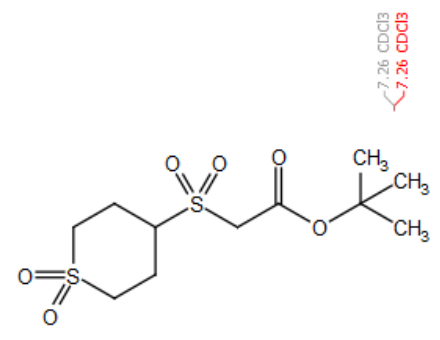

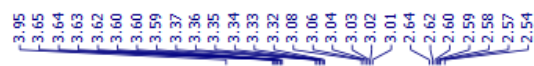

Compound 30

${ }^{1} \mathrm{H} \mathrm{NMR}\left(400 \mathrm{MHz}, \mathrm{CDCl}_{3}\right)$
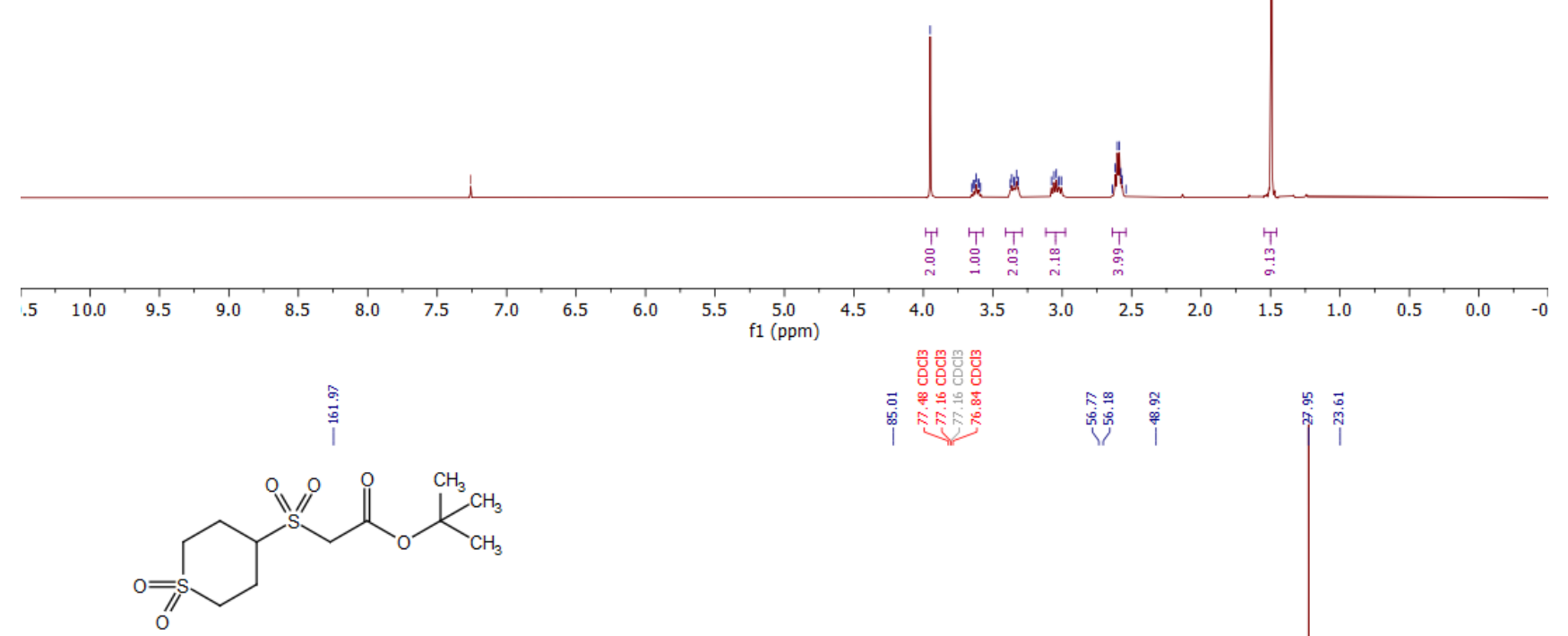

Compound 30

$\left.{ }^{13} \mathrm{C} \mathrm{NMR} \mathrm{(101} \mathrm{MHz,} \mathrm{CDCl}_{3}\right)$

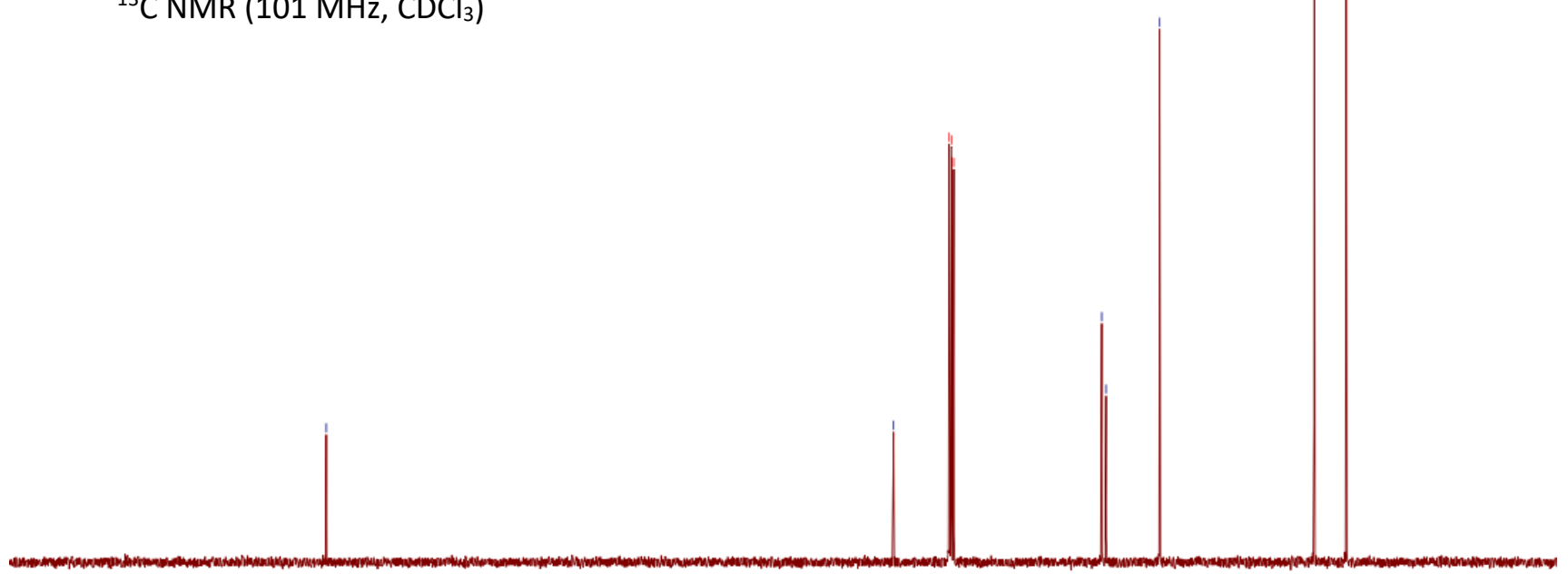

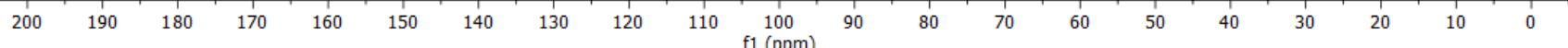


NMR spectra of tert-butyl 2-((4-hydroxycyclohexyl)sulfonyl)acetate (3p)

爱器

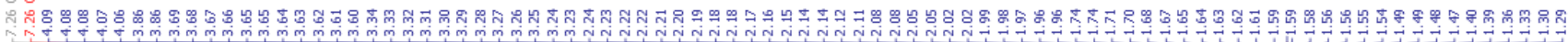<smiles>CC(C)(C)OC(=O)CS(=O)(=O)C1CCC(O)CC1</smiles>

Compound 3p

${ }^{1} \mathrm{H}$ NMR $\left(400 \mathrm{MHz}^{\mathrm{CDCl}}{ }_{3}\right)$

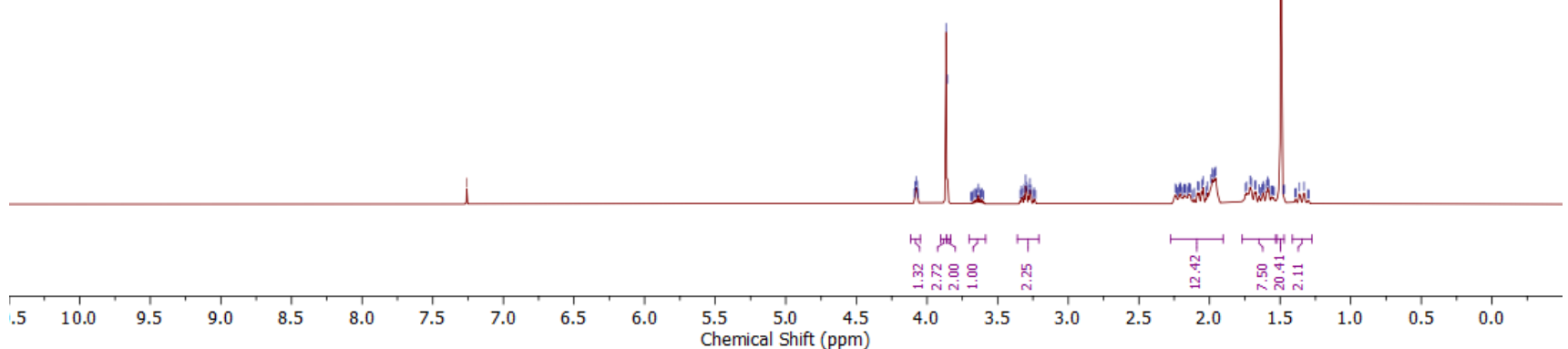<smiles>CC(C)(C)OC(=O)CS(=O)(=O)C1CCC(O)CC1</smiles>

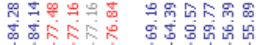

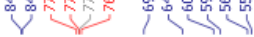

Compound 3p

${ }^{13} \mathrm{C}$ NMR $\left(101 \mathrm{MHz}, \mathrm{CDCl}_{3}\right)$

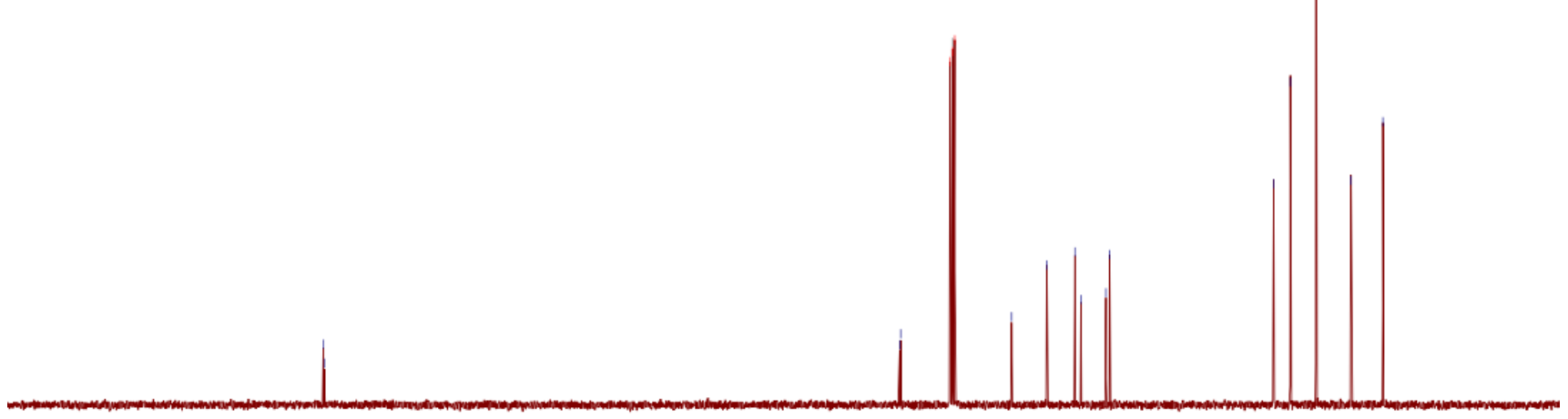

$\begin{array}{llllllllll}200 & 190 & 180 & 170 & 160 & 150 & 140 & 130 & 120 & \begin{array}{c}110 \\ \text { Chemical Shift (bom) }\end{array}\end{array}$ 
NMR spectra of tert-butyl 2-((4-carboxycyclohexyl)sulfonyl)acetate (3q)

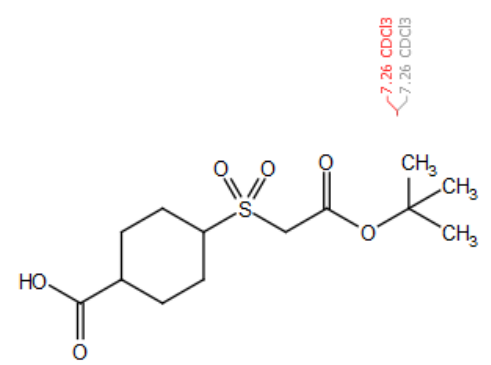

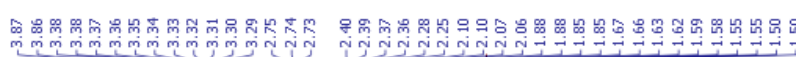

Compound 3q

${ }^{1} \mathrm{H}$ NMR $\left(400 \mathrm{MHz}, \mathrm{CDCl}_{3}\right)$
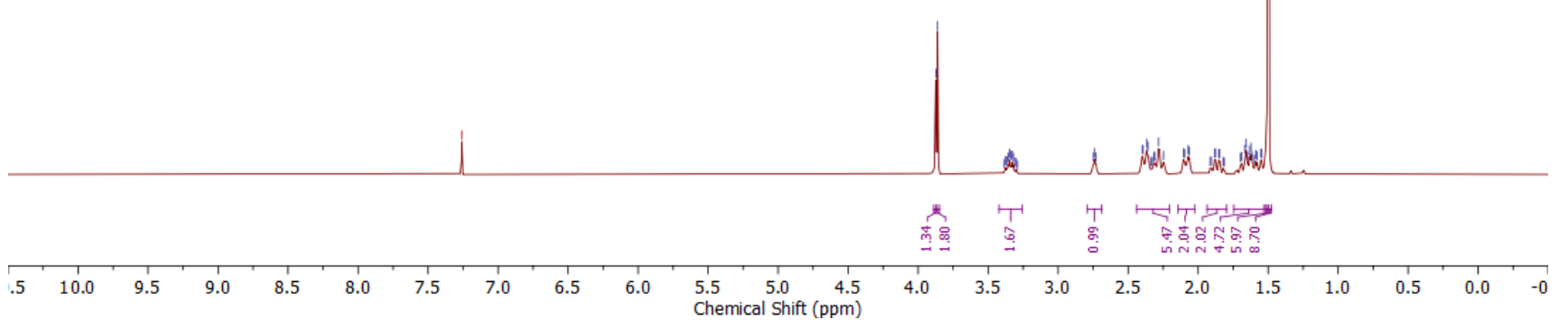<smiles>CC(C)(C)OC(=O)CS(=O)(=O)C1CCC(C(=O)O)CC1</smiles>

Compound 3q

${ }^{13} \mathrm{C}$ NMR $\left(101 \mathrm{MHz}, \mathrm{CDCl}_{3}\right)$ $\begin{array}{lllllllllll}200 & 190 & 180 & 170 & 160 & 150 & 140 & 130 & 120 & \begin{array}{l}110 \\ \text { Chemical Shift (ppm) }\end{array} & \begin{array}{l}90 \\ \text { (ppm }\end{array}\end{array}$ m.

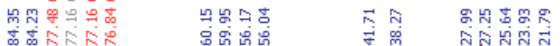


NMR spectra of methyl 4-((2-(tert-butoxy)-2-oxoethyl)sulfonyl)cyclohexane-1-carboxylate (3r)
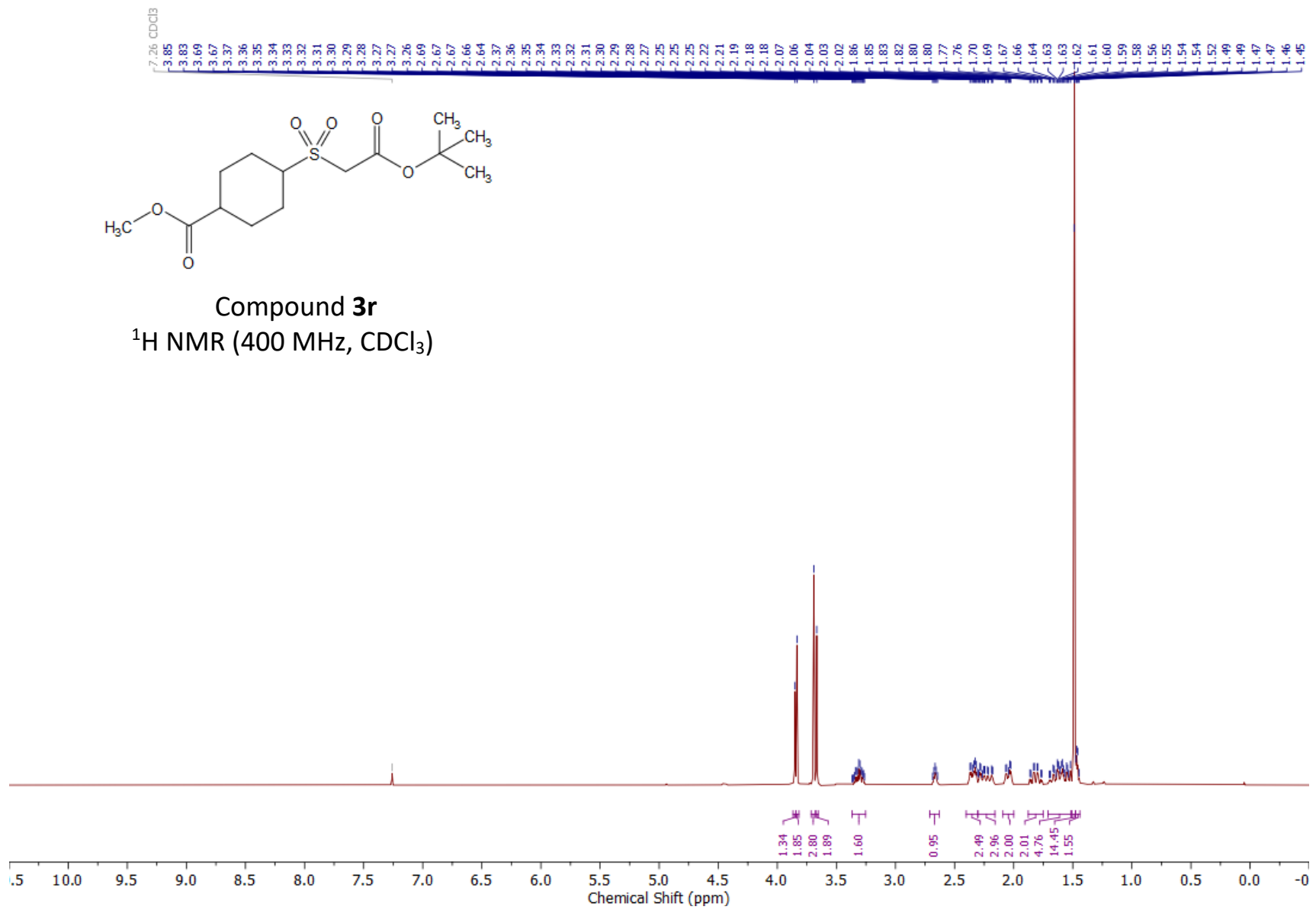

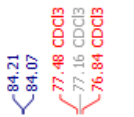

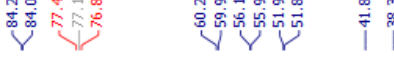

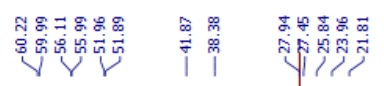

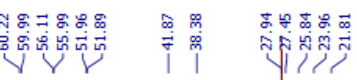

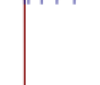

Compound $3 r$

${ }^{13} \mathrm{C} \mathrm{NMR}\left(101 \mathrm{MHz}, \mathrm{CDCl}_{3}\right)$

$\begin{array}{lllllllllll}200 & 190 & 180 & 170 & 160 & 150 & 140 & 130 & 120 & \begin{array}{l}110 \\ \text { Chemical Shift (ppm) }\end{array}\end{array}$


NMR spectra of tert-butyl 2-((1-(2,6-dimethylphenoxy)propan-2-yl)sulfonyl)acetate (3s)

웅응

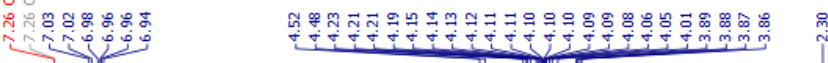<smiles>Cc1cccc(C)c1OCC(C)S(=O)(=O)CC(=O)OC(C)(C)C</smiles>

Compound 3s

${ }^{1} \mathrm{H} \mathrm{NMR}\left(400 \mathrm{MHz}, \mathrm{CDCl}_{3}\right)$
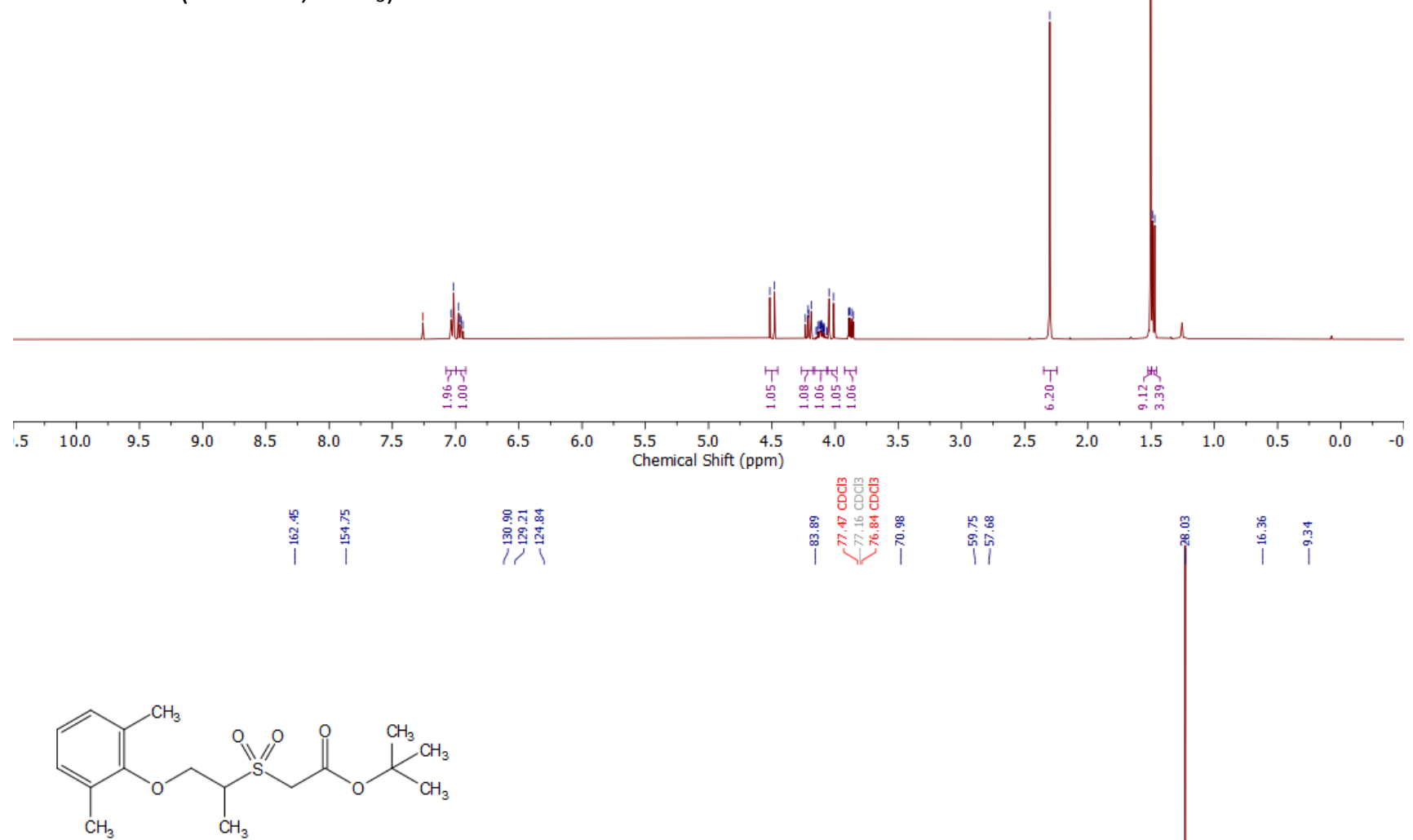

Compound 3s

${ }^{13} \mathrm{C}$ NMR (101 MHz, $\left.\mathrm{CDCl}_{3}\right)$ 
NMR spectra of tert-butyl 2-((1-hydroxy-3-phenylpropan-2-yl)sulfonyl)acetate (3t)
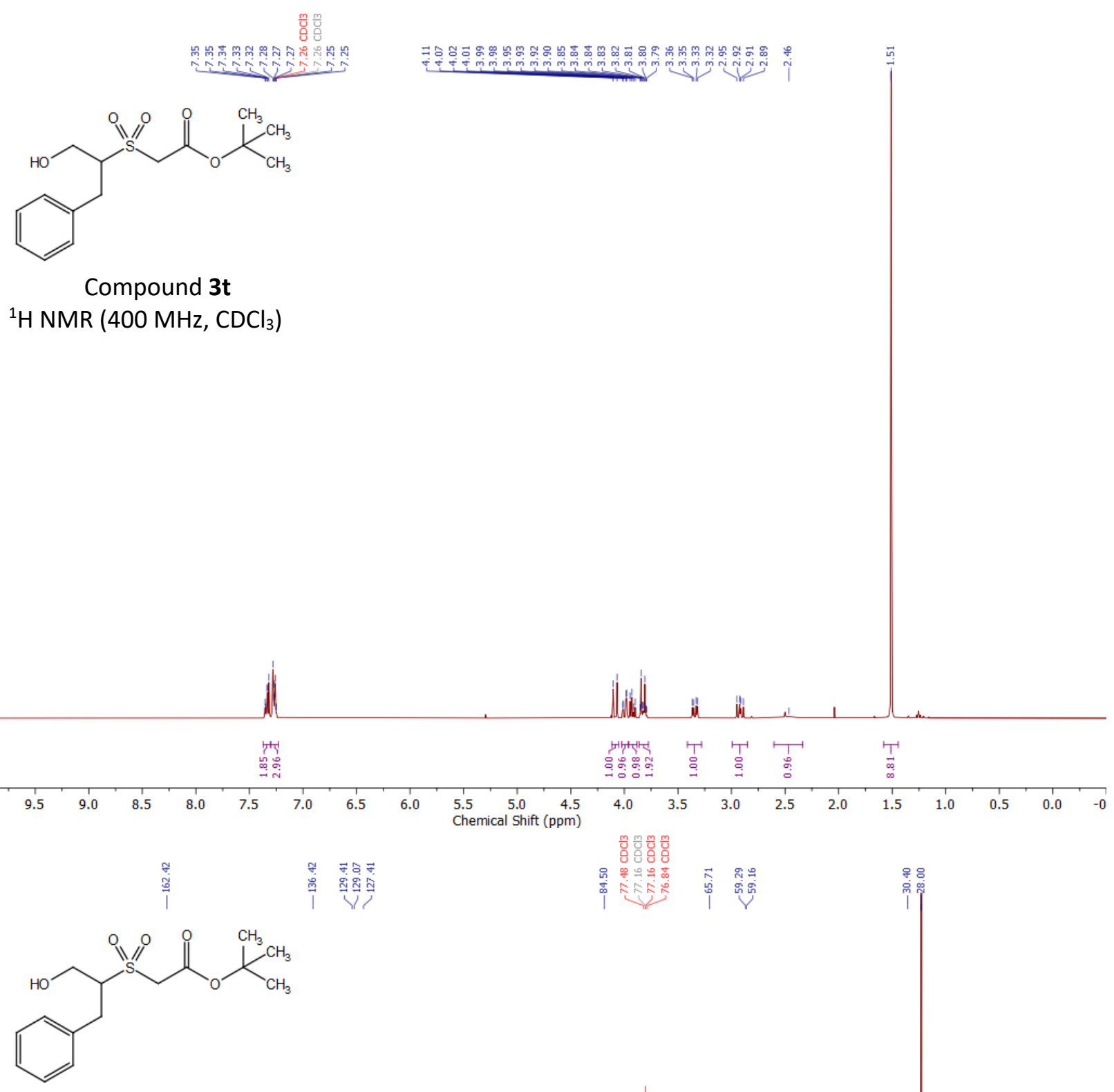

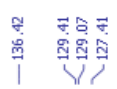

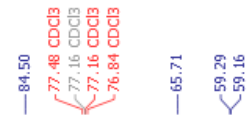

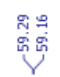

Compound 3t

$\left.{ }^{13} \mathrm{C} \mathrm{NMR} \mathrm{(101} \mathrm{MHz,} \mathrm{CDCl}_{3}\right)$

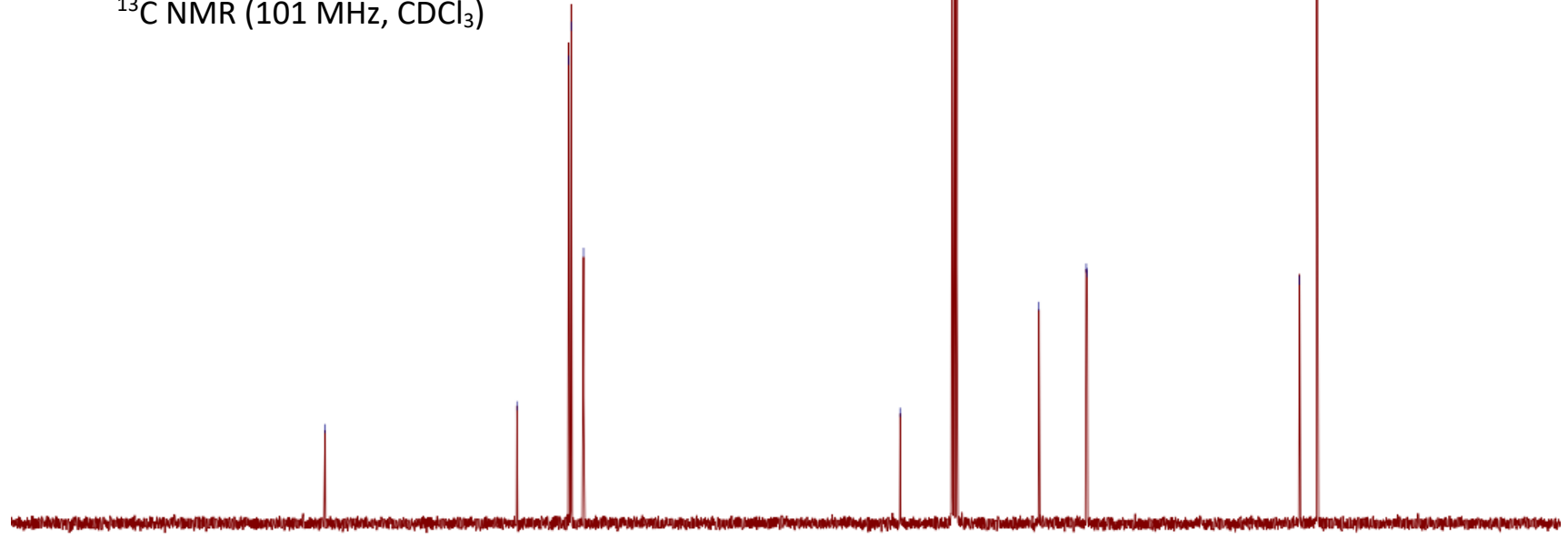

$\begin{array}{llllllllll}200 & 190 & 180 & 170 & 160 & 150 & 140 & 130 & 120 & \begin{array}{c}110 \\ \text { Chemical Shift (dom) }\end{array}\end{array}$ 
NMR spectra of tert-butyl 2-(benzylsulfonyl)acetate (3b)
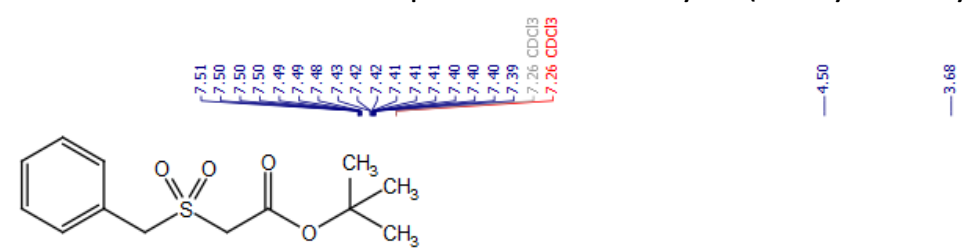

Compound $\mathbf{3 b}$

${ }^{1} \mathrm{H}$ NMR $\left(400 \mathrm{MHz}, \mathrm{CDCl}_{3}\right)$
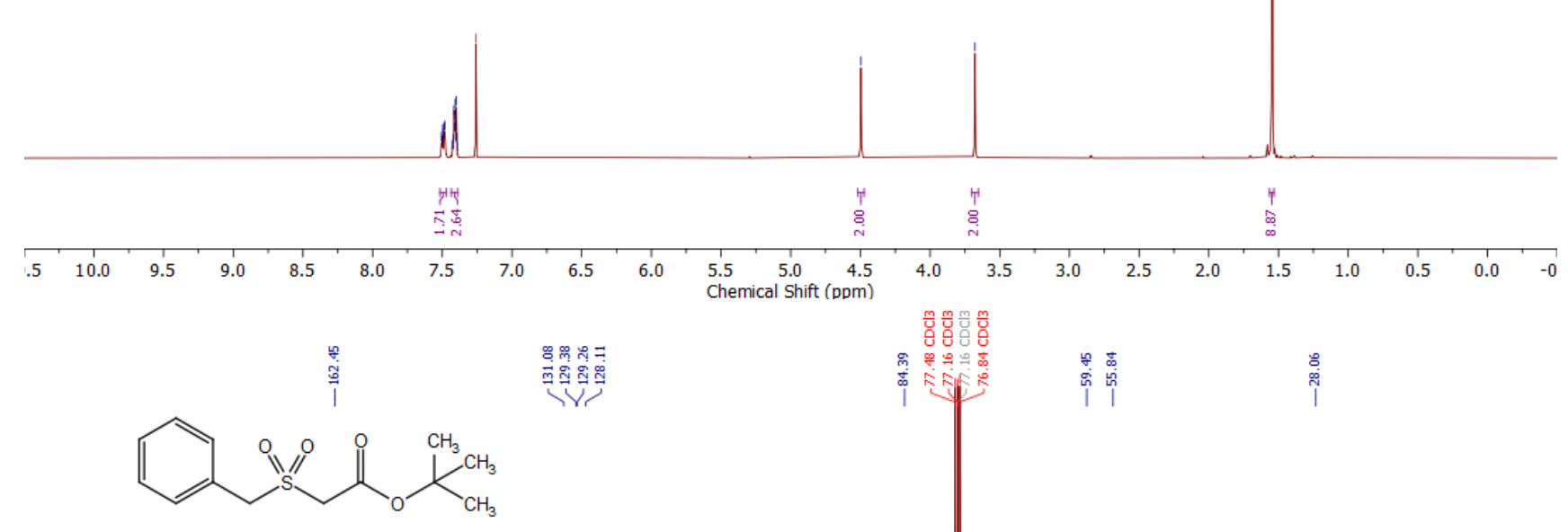

Compound $\mathbf{3 b}$

${ }^{13} \mathrm{C}$ NMR (101 MHz, $\mathrm{CDCl}_{3}$ ) 
NMR spectra of tert-butyl 2-((4-methoxybenzyl)sulfonyl)acetate (3v)
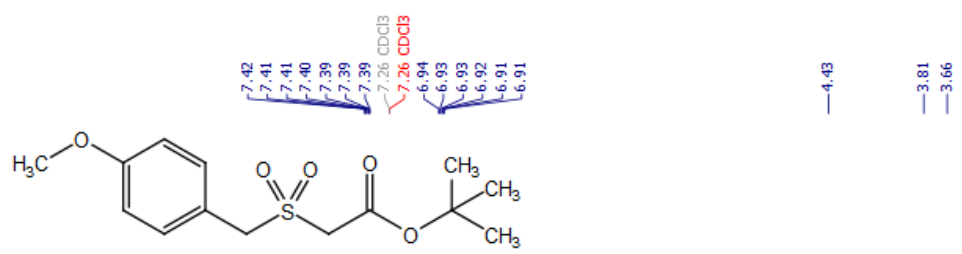

\section{Compound 3v}

${ }^{1} \mathrm{H}$ NMR $\left(400 \mathrm{MHz}, \mathrm{CDCl}_{3}\right)$
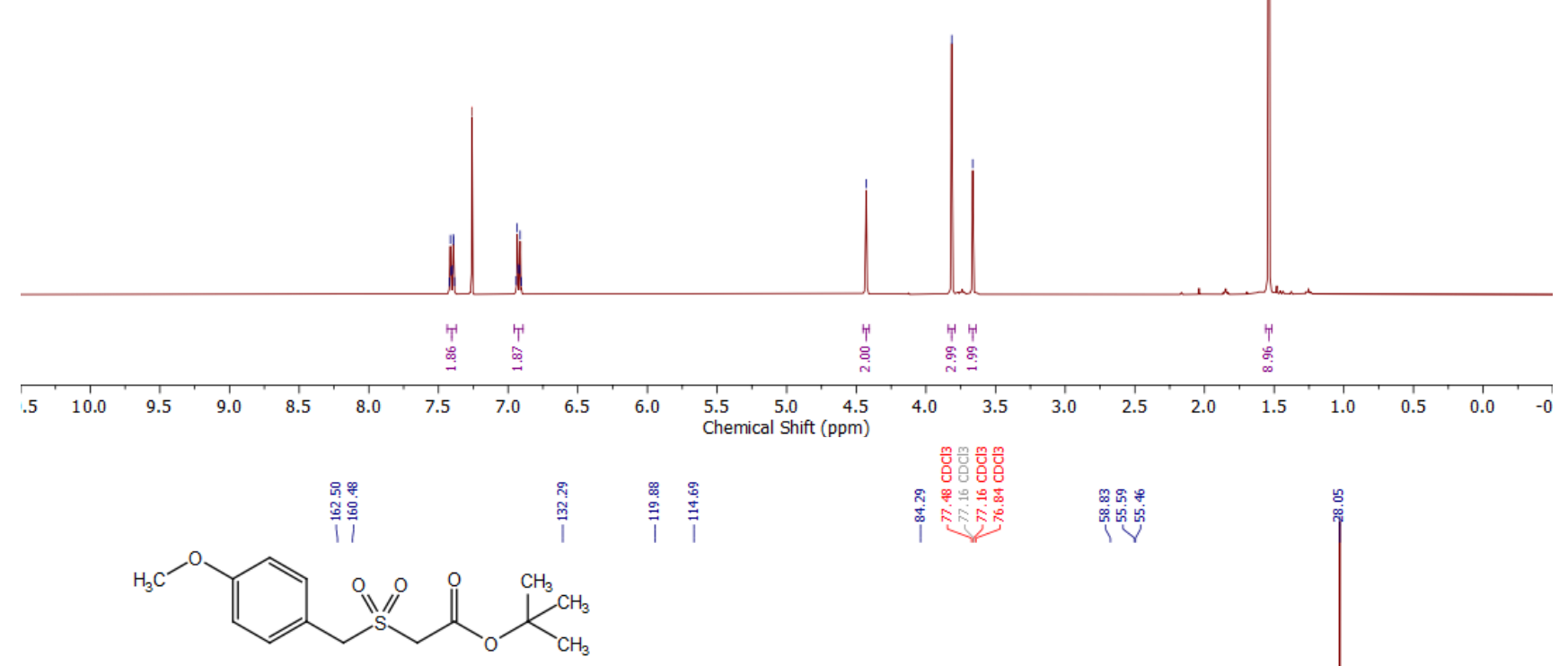

Compound 3v

${ }^{13} \mathrm{C}$ NMR (101 MHz, $\mathrm{CDCl}_{3}$ )

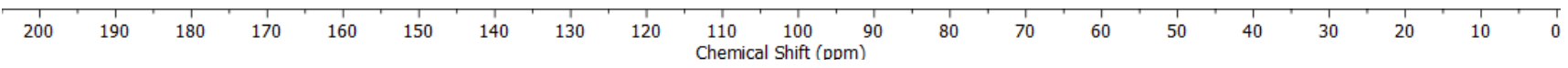


NMR spectra of tert-butyl 2-((3-methoxybenzyl)sulfonyl)acetate (3w)

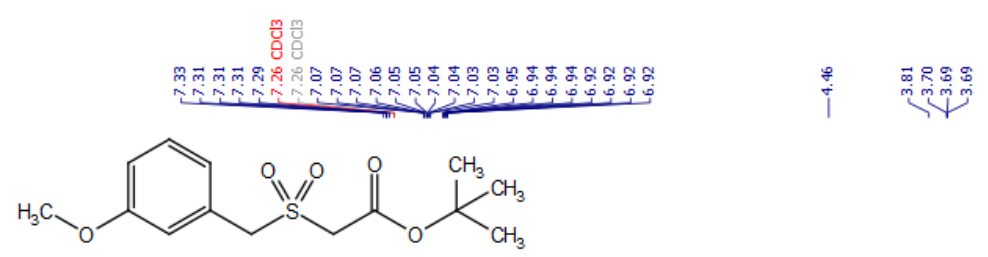

Compound 3w

${ }^{1} \mathrm{H}$ NMR $\left(400 \mathrm{MHz}, \mathrm{CDCl}_{3}\right)$
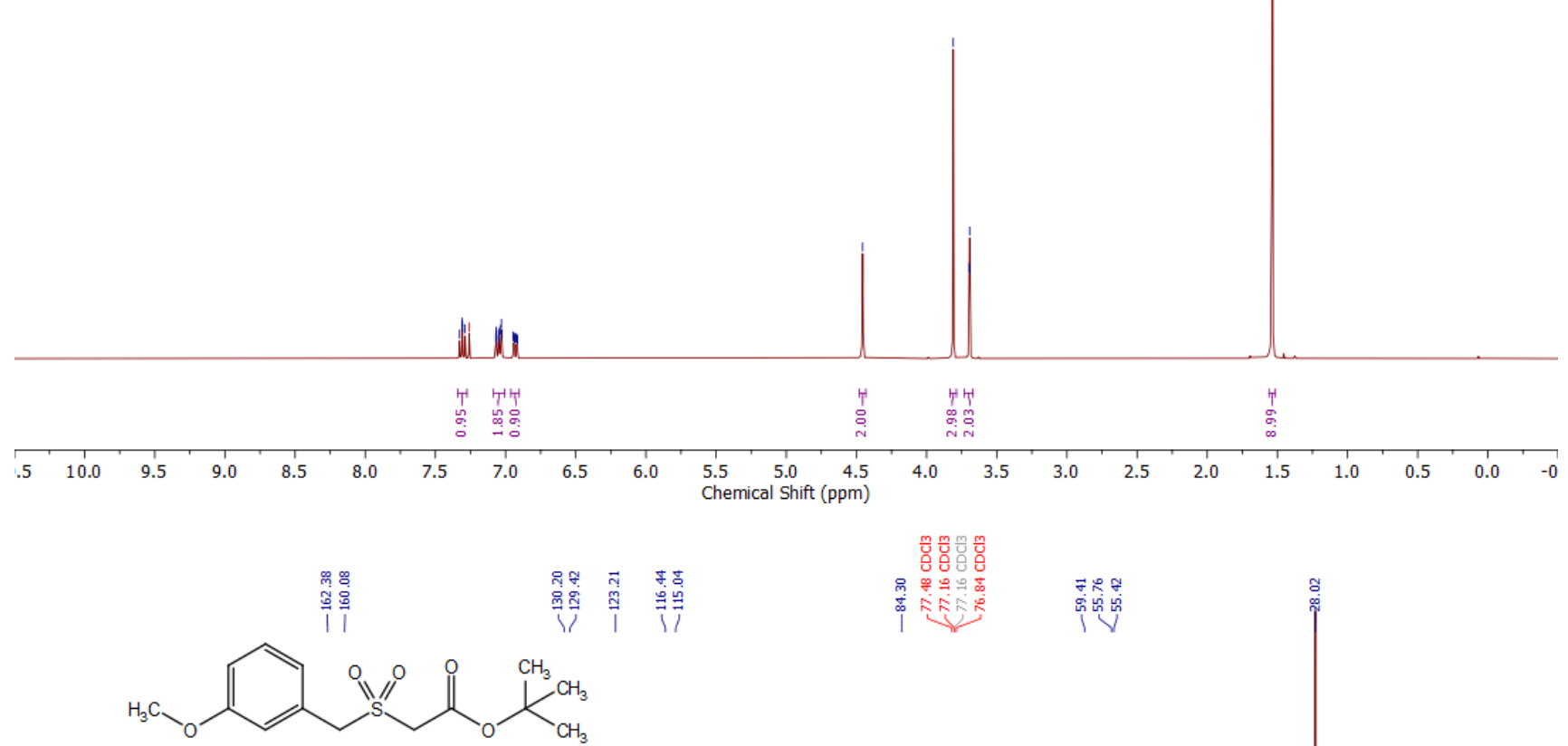

Compound 3w

${ }^{13} \mathrm{C}$ NMR $\left(101 \mathrm{MHz}, \mathrm{CDCl}_{3}\right)$

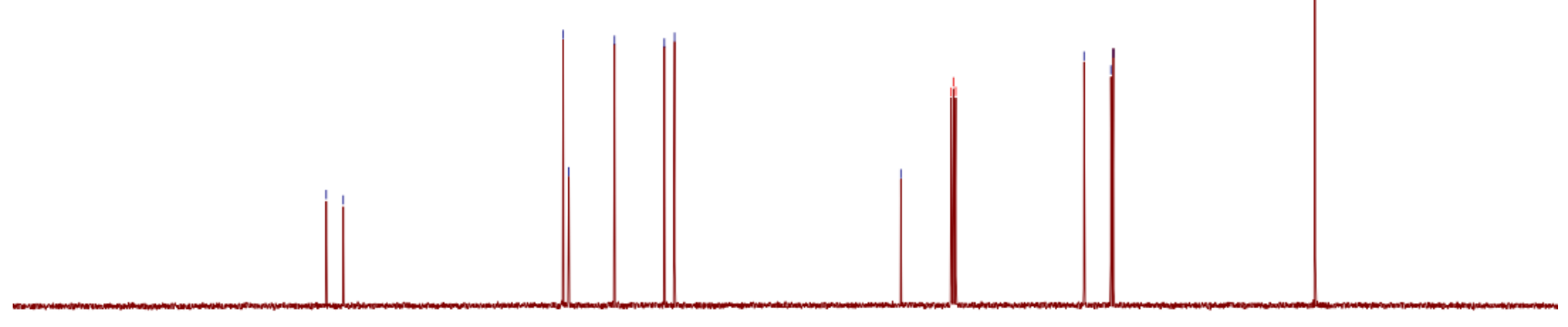

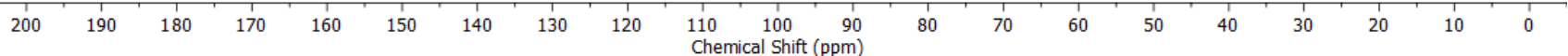


NMR spectra of tert-butyl 2-((2-methoxybenzyl)sulfonyl)acetate (3x)

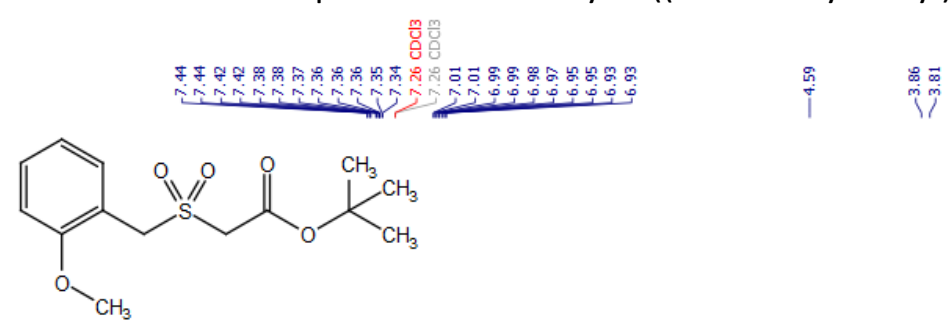

Compound $3 \mathbf{x}$

${ }^{1} \mathrm{H}$ NMR $\left(400 \mathrm{MHz}, \mathrm{CDCl}_{3}\right)$
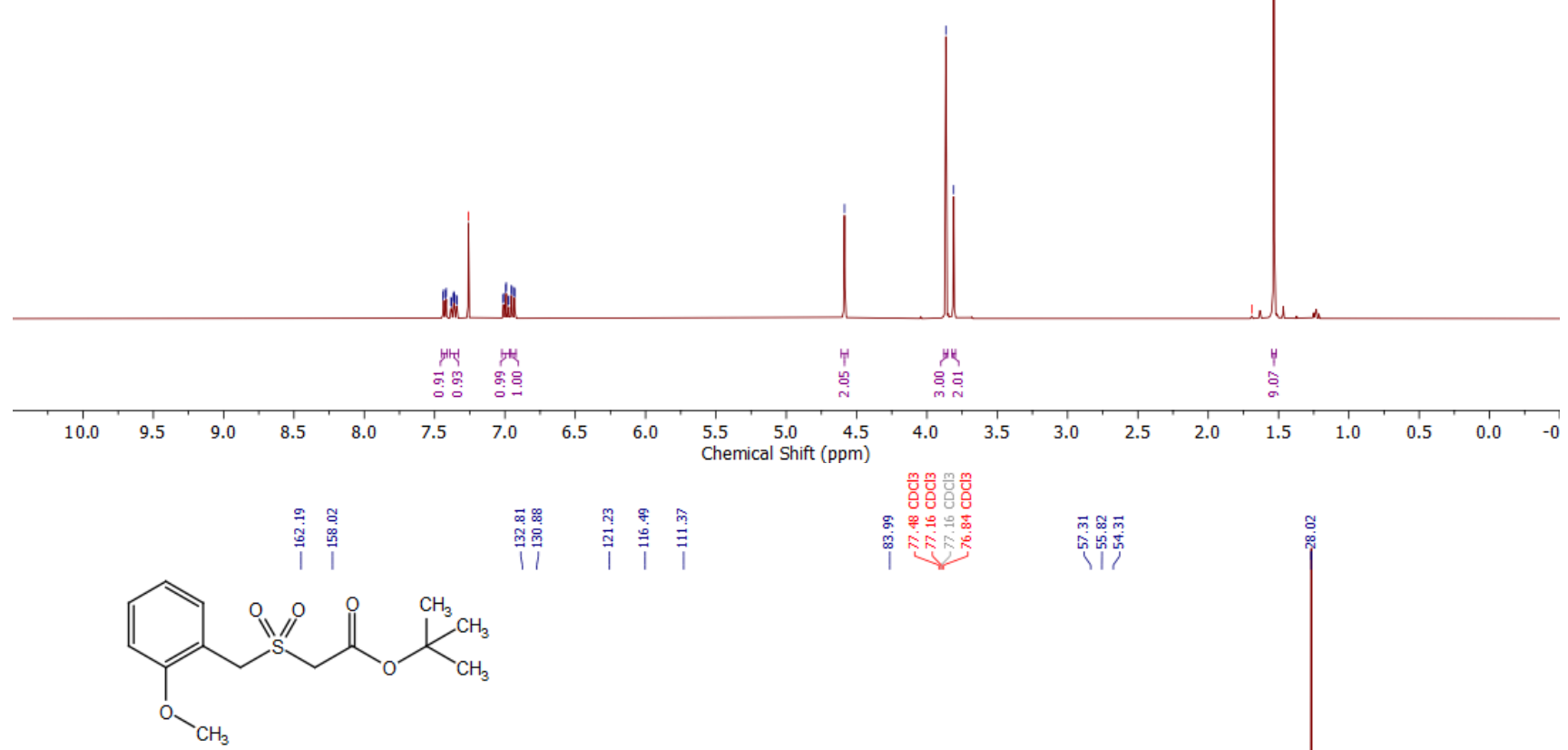

Compound 3x

${ }^{13} \mathrm{C}$ NMR $\left(101 \mathrm{MHz}, \mathrm{CDCl}_{3}\right)$

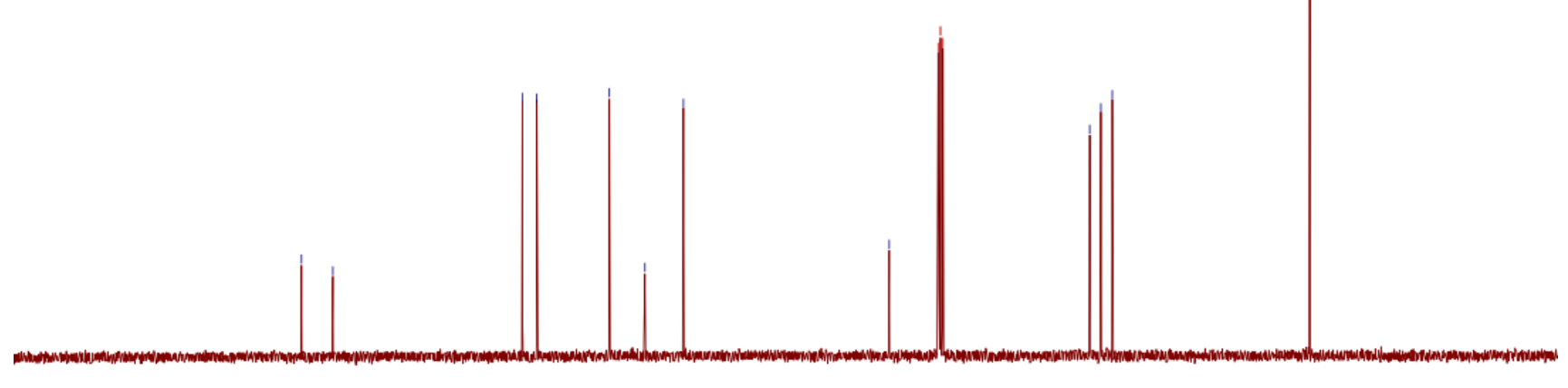

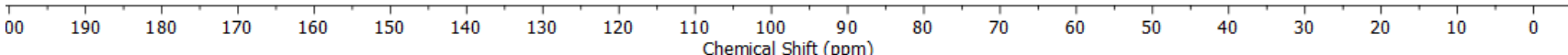


NMR spectra of tert-butyl 2-((4-chlorobenzyl)sulfonyl)acetate (3y)

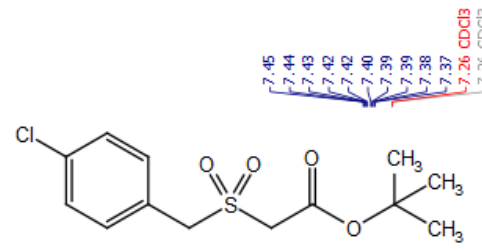

Compound 3y

${ }^{1} \mathrm{H}$ NMR $\left(400 \mathrm{MHz}, \mathrm{CDCl}_{3}\right)$
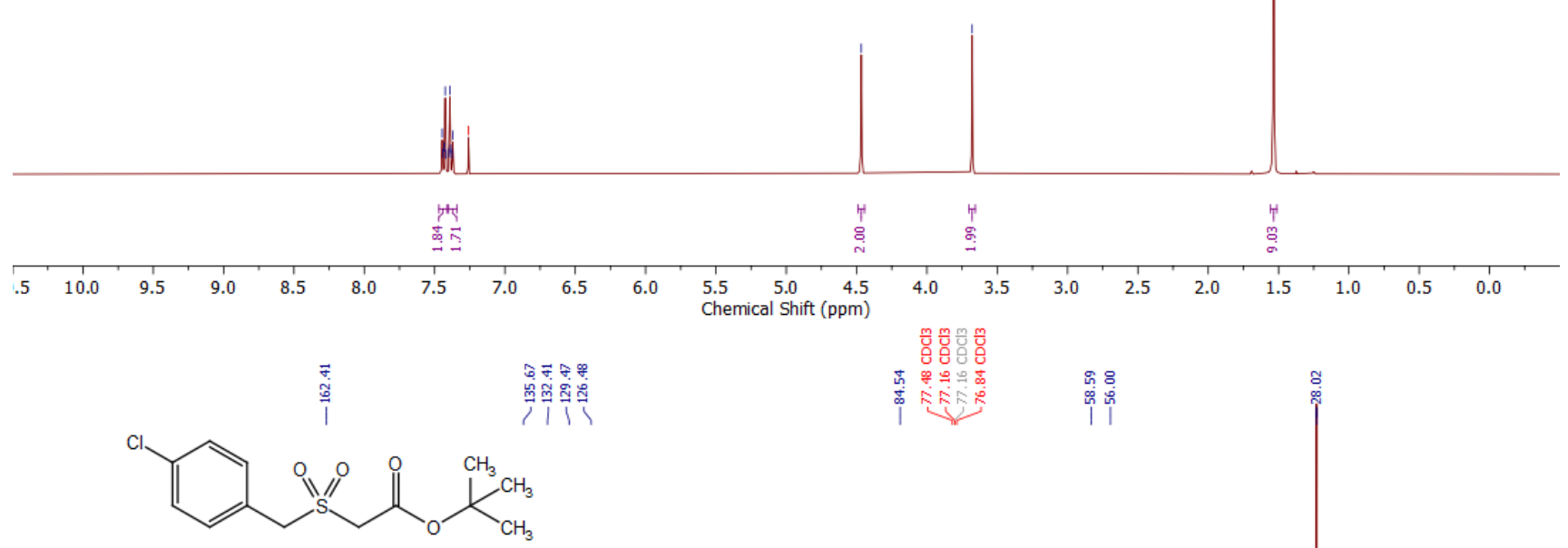
NMR spectra of tert-butyl 2-((4-(trifluoromethyl)benzyl)sulfonyl)acetate (3z)
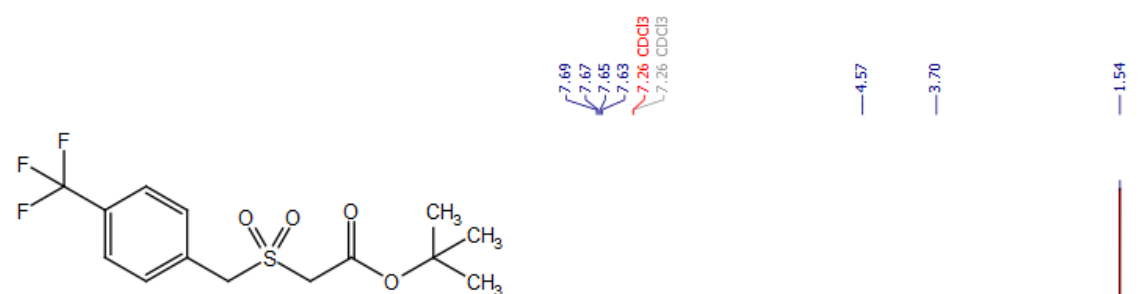

Compound $3 z$

${ }^{1} \mathrm{H}$ NMR (400 MHz, $\mathrm{CDCl}_{3}$ )

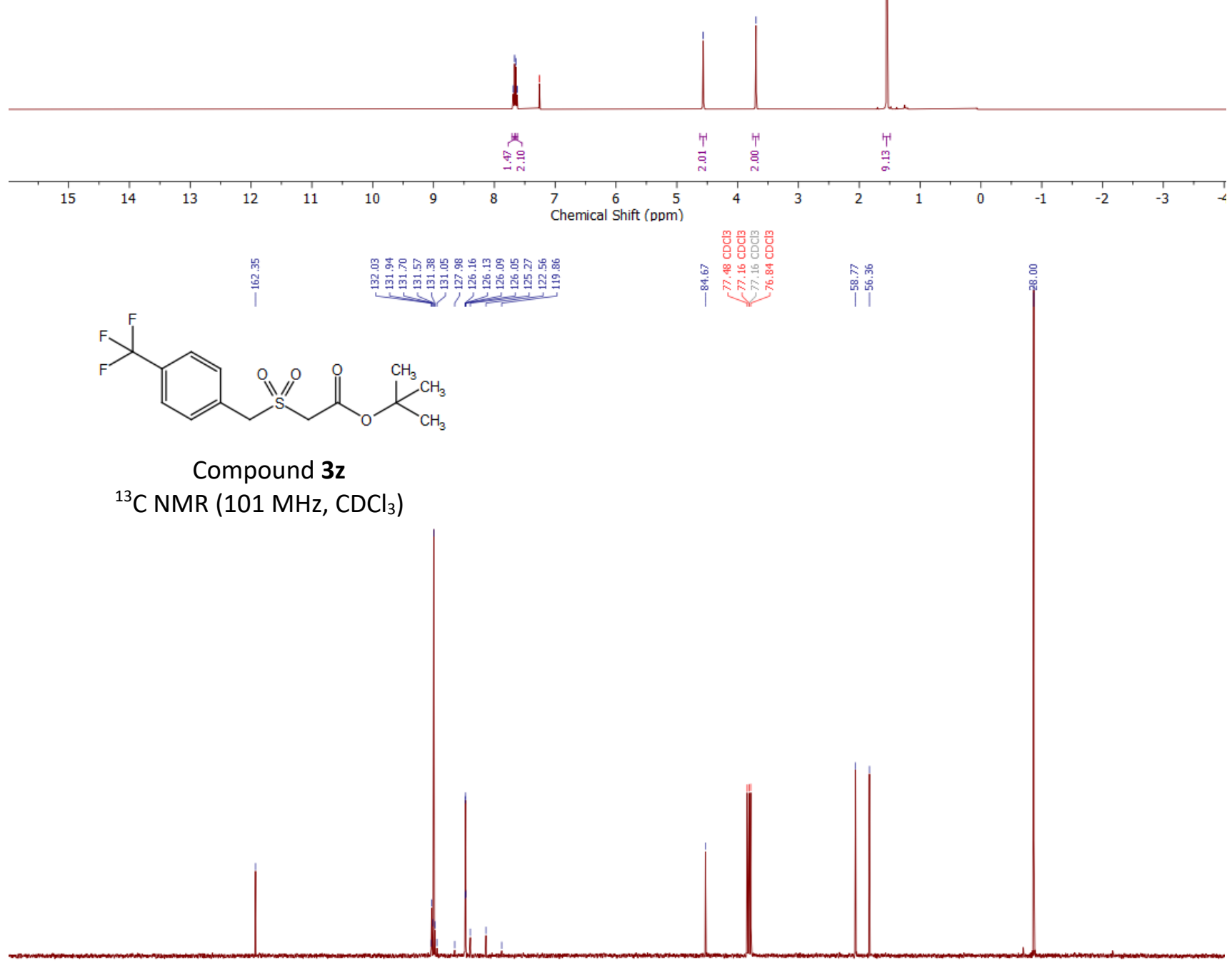

$\begin{array}{llllllllll}200 & 190 & 180 & 170 & 160 & 150 & 140 & 130 & 120 & \begin{array}{c}110 \\ \text { Chemical Shift (bom) }\end{array}\end{array}$ 


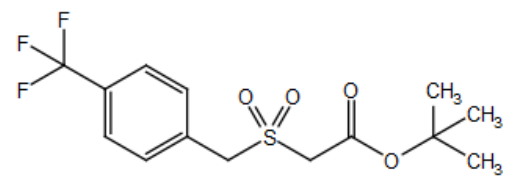

Compound $\mathbf{3 z}$

${ }^{19} \mathrm{~F} \mathrm{NMR}\left(377 \mathrm{MHz}, \mathrm{CDCl}_{3}\right)$

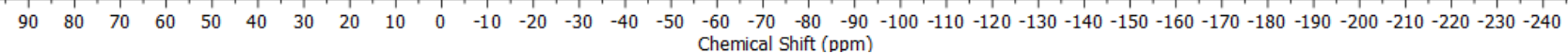


NMR spectra of tert-butyl 2-((thiophen-2-ylmethyl)sulfonyl)acetate (3aa)

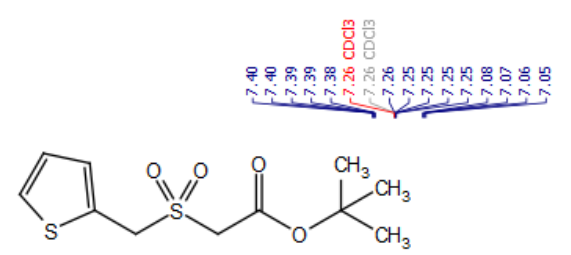

Compound 3aa

${ }^{1} \mathrm{H}$ NMR $\left(400 \mathrm{MHz}, \mathrm{CDCl}_{3}\right)$
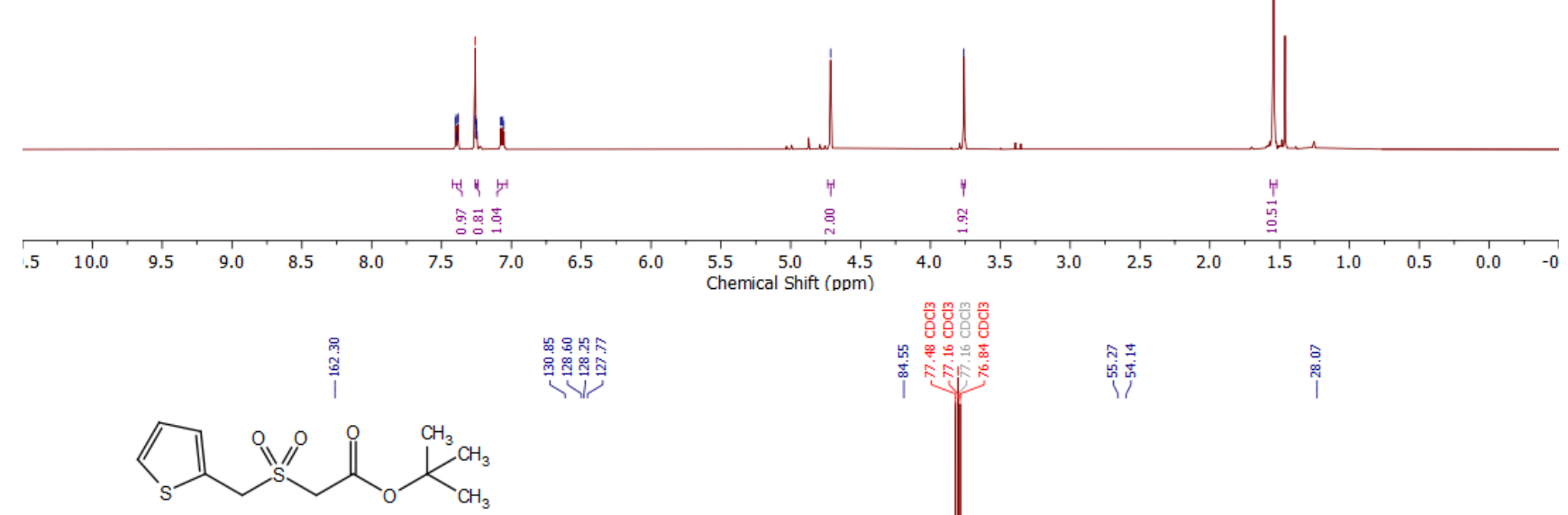

Compound 3aa

${ }^{13} \mathrm{C}$ NMR $\left(101 \mathrm{MHz}, \mathrm{CDCl}_{3}\right)$ 
NMR spectra of tert-butyl 2-((pyridin-3-ylmethyl)sulfonyl)acetate (3ab)

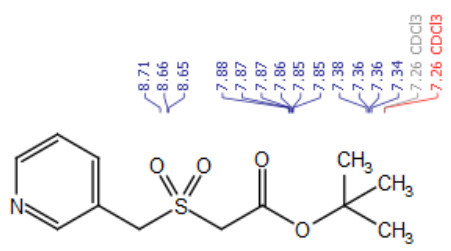

Compound $3 a b$

${ }^{1} \mathrm{H}$ NMR $\left(400 \mathrm{MHz}, \mathrm{CDCl}_{3}\right)$
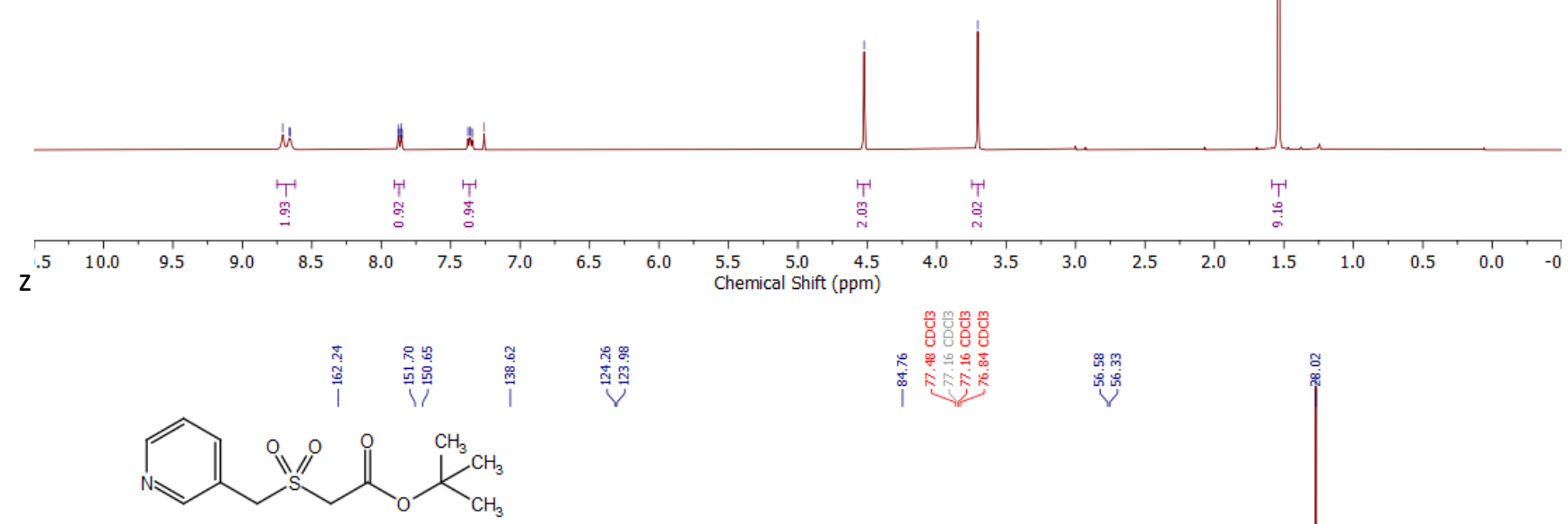

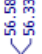

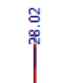

Compound 3ab

${ }^{13} \mathrm{C}$ NMR (101 MHz, $\mathrm{CDCl}_{3}$ )

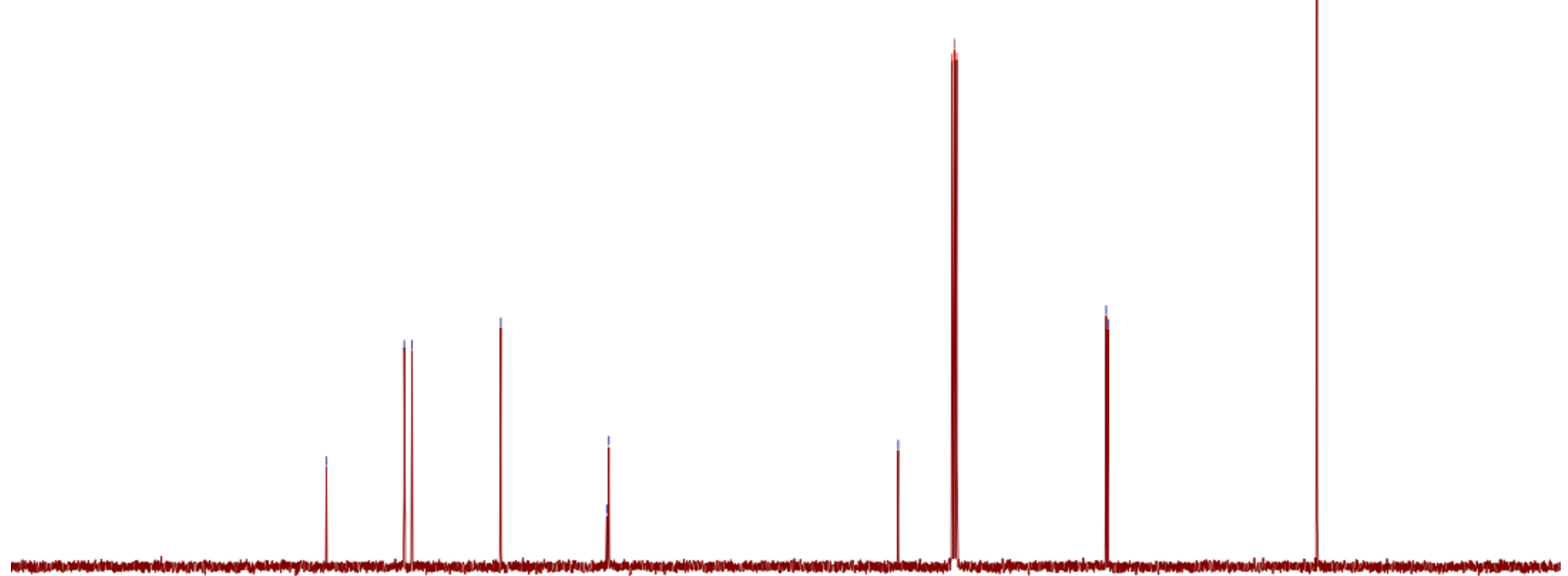

$\begin{array}{llllllllll}200 & 190 & 180 & 170 & 160 & 150 & 140 & 130 & 120 & \begin{array}{c}110 \\ \text { Chemical Shift (ppm) }\end{array}\end{array}$ 
NMR spectra of tert-butyl 2-(cinnamylsulfonyl)acetate (3ad)

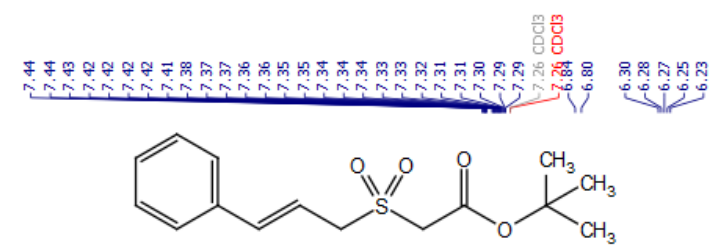

Compound 3ad

${ }^{1} \mathrm{H}$ NMR $\left(400 \mathrm{MHz}, \mathrm{CDCl}_{3}\right)$
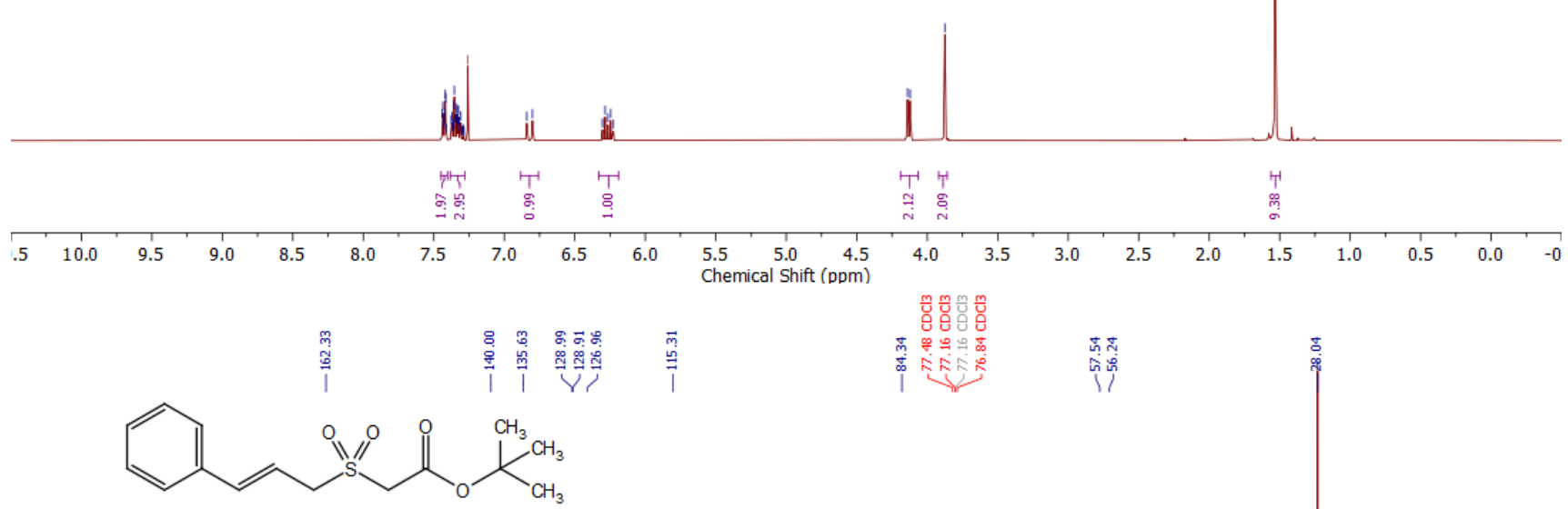

Compound 3ad

${ }^{13} \mathrm{C}$ NMR (101 MHz, $\mathrm{CDCl}_{3}$ )

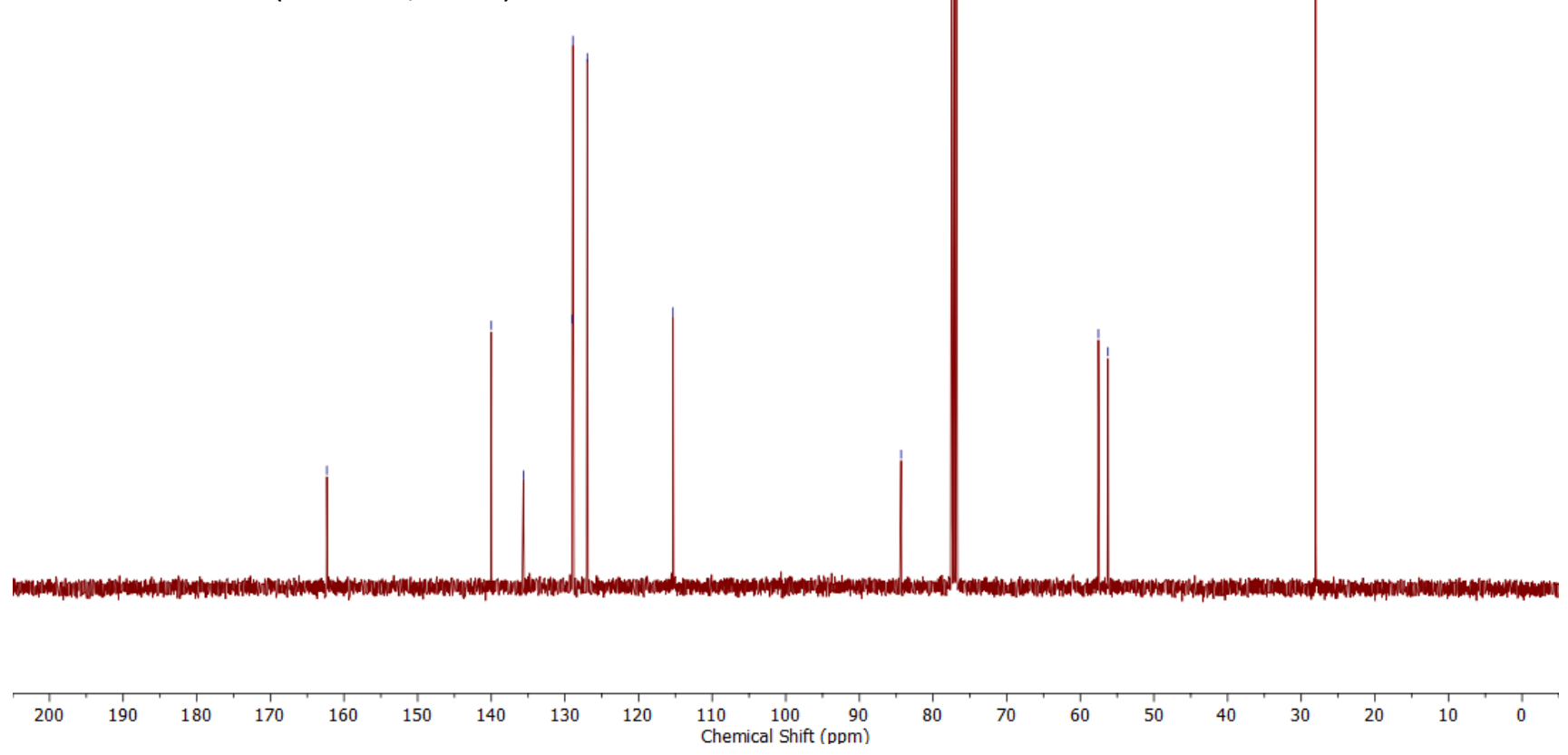


NMR spectra of tert-butyl 2-((1-phenylethyl)sulfonyl)acetate (3ae)
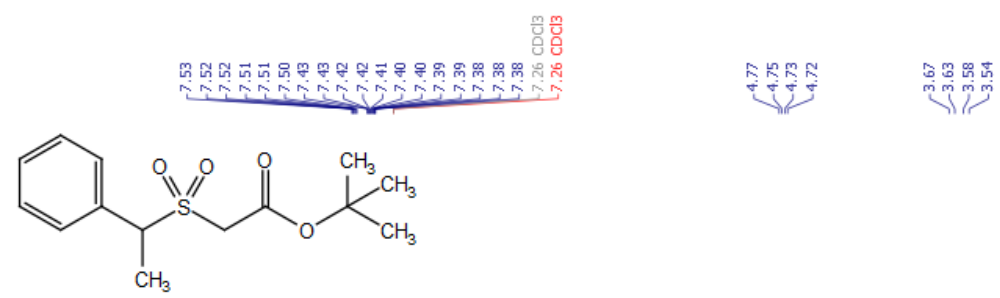

Compound 3ae

${ }^{1} \mathrm{H}$ NMR $\left(400 \mathrm{MHz}, \mathrm{CDCl}_{3}\right)$
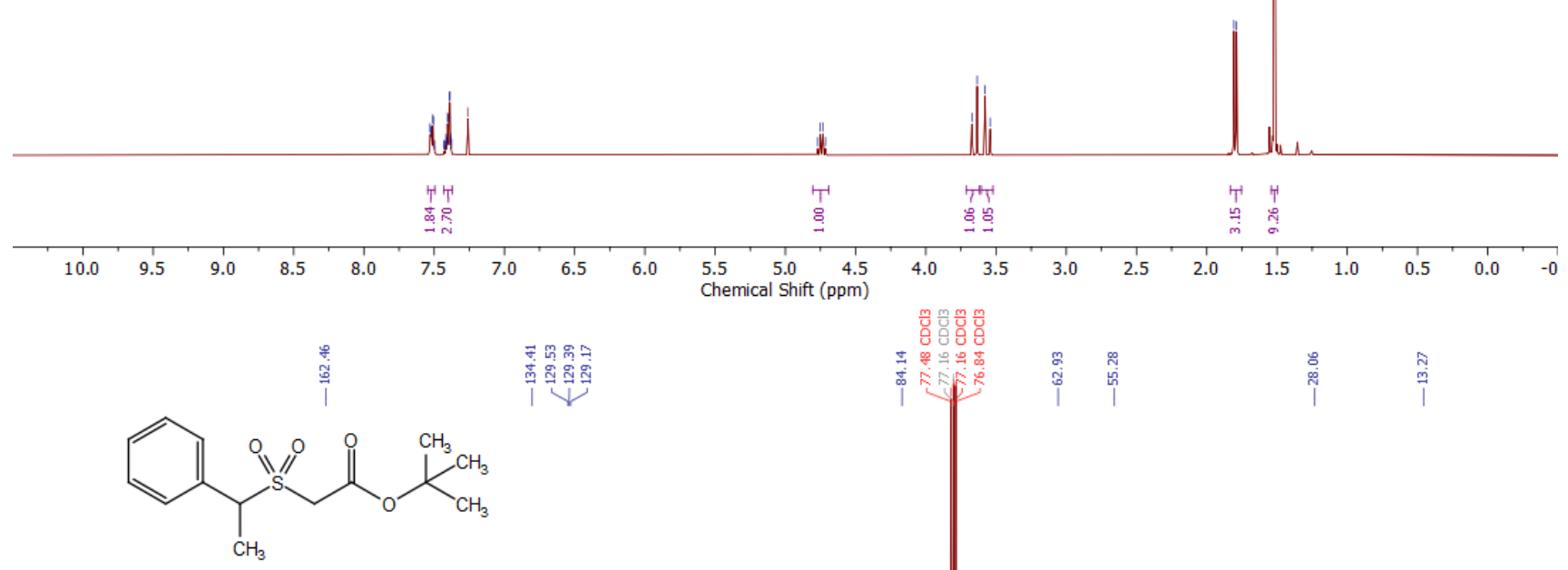

Compound 3ae

${ }^{13} \mathrm{C}$ NMR $\left(101 \mathrm{MHz}, \mathrm{CDCl}_{3}\right)$

$\begin{array}{lllllllllll}200 & 190 & 180 & 170 & 160 & 150 & 140 & 130 & 120 & \begin{array}{l}110 \\ \text { Chemical Shift (bom) }\end{array}\end{array}$


NMR spectra of benzyl 2-(phenethylsulfonyl)acetate (3c-1)

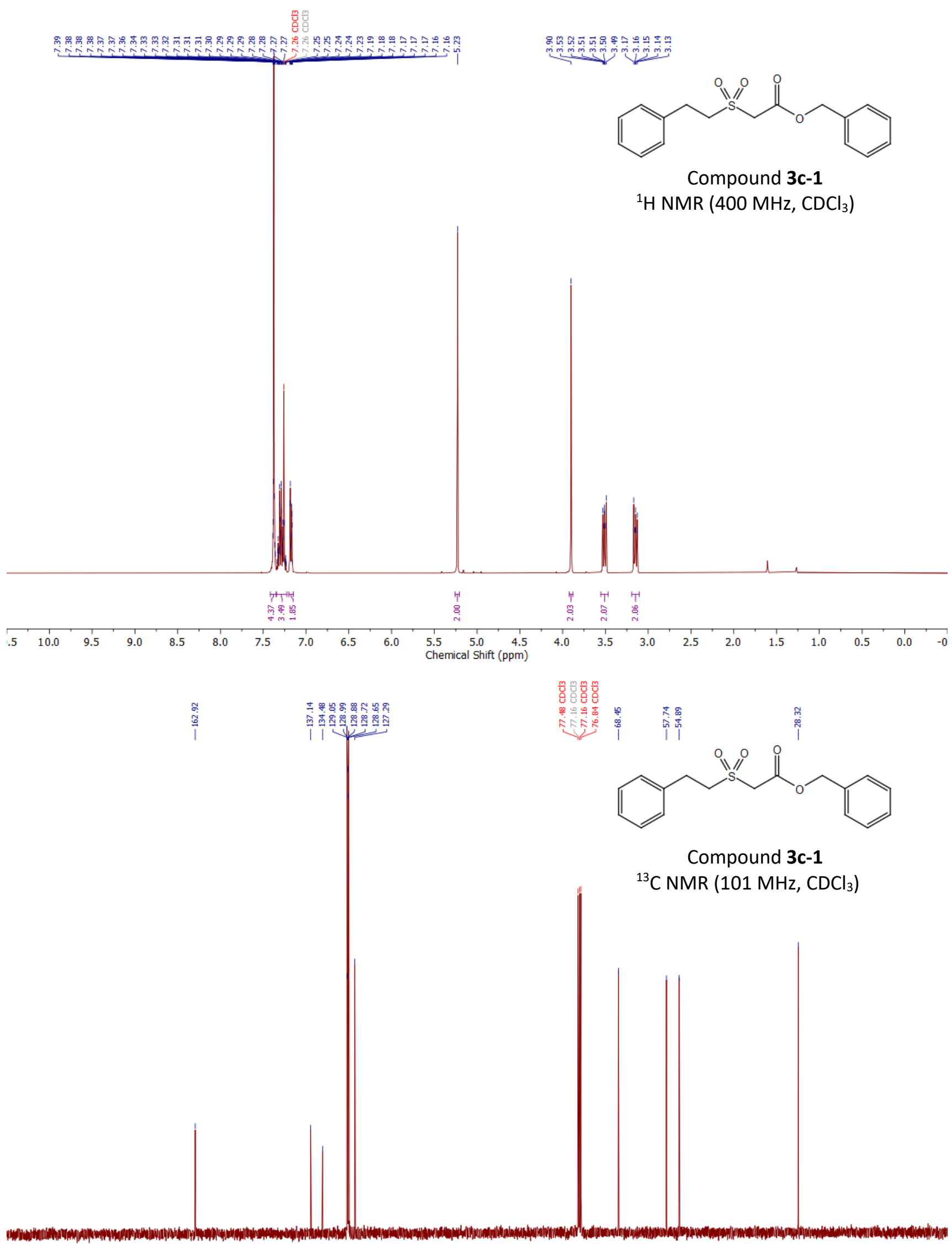

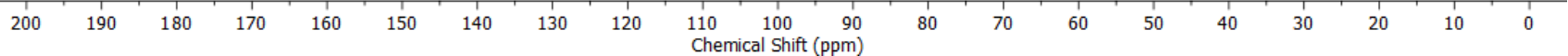


NMR spectra of tert-butyl 2-(phenethylsulfonyl)acetate (3c-2)
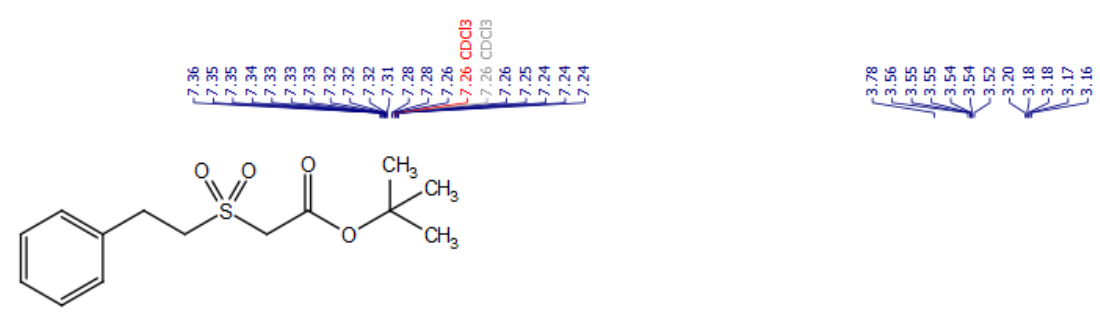

Compound 3c-2

${ }^{1} \mathrm{H}$ NMR $\left(400 \mathrm{MHz}, \mathrm{CDCl}_{3}\right)$
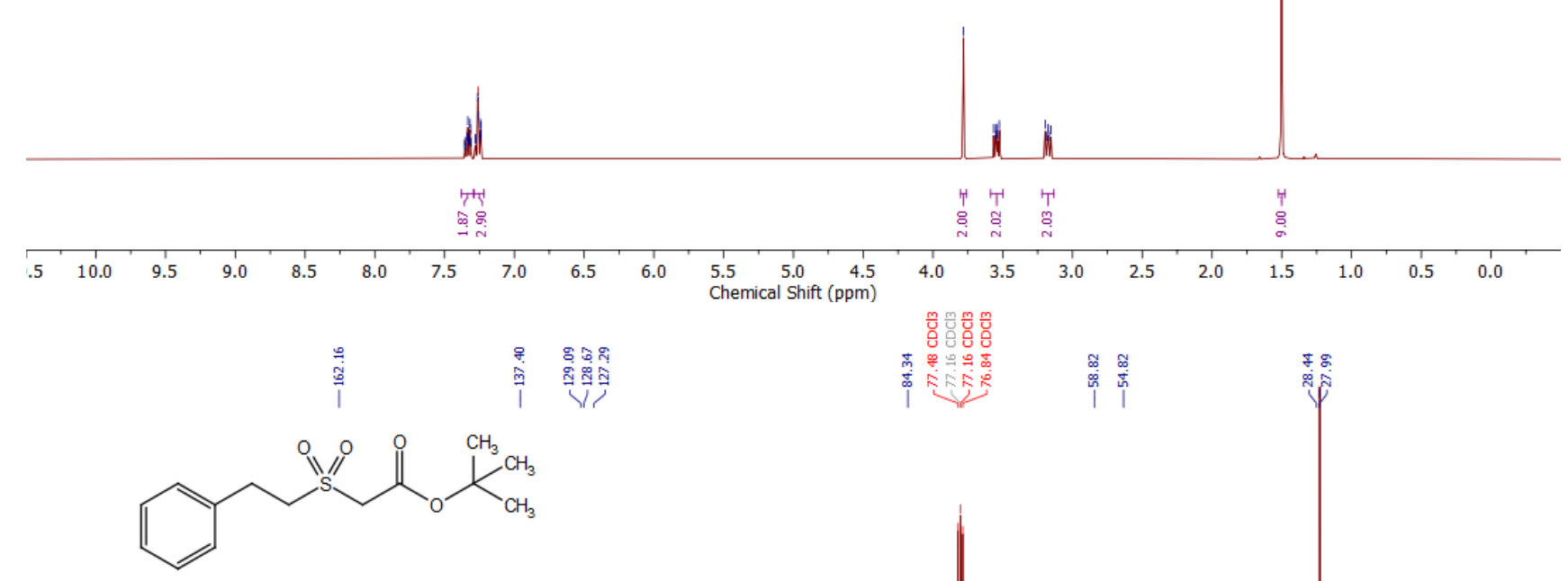

Compound 3c-2

${ }^{13} \mathrm{C}$ NMR $\left(101 \mathrm{MHz}, \mathrm{CDCl}_{3}\right)$

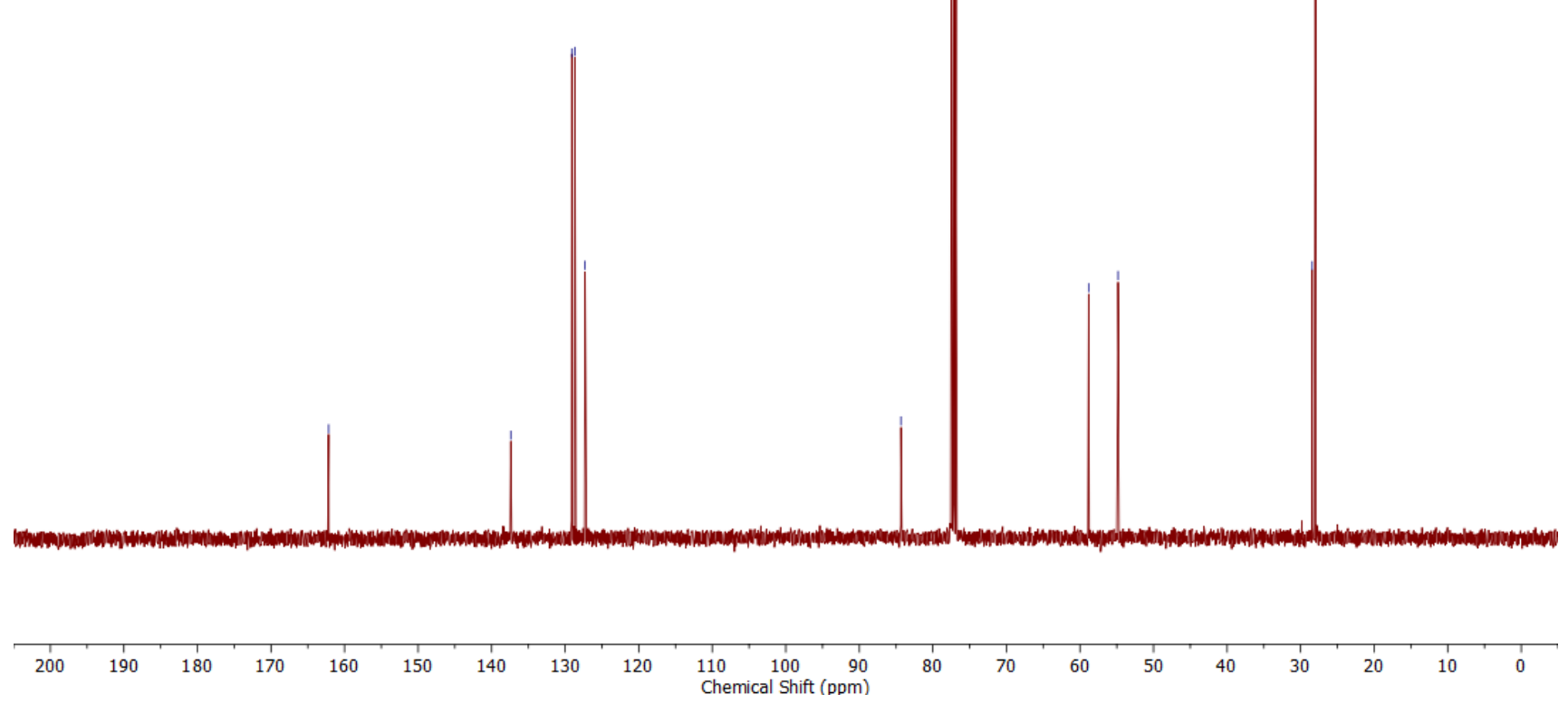


NMR spectra of tert-butyl 2-(neopentylsulfonyl)acetate (3af)

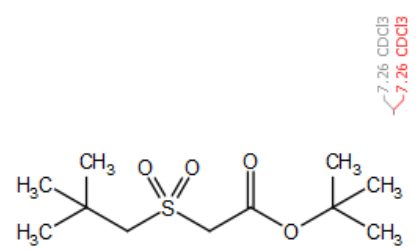

Compound 3af

${ }^{1} \mathrm{H}$ NMR (400 MHz, $\mathrm{CDCl}_{3}$ )
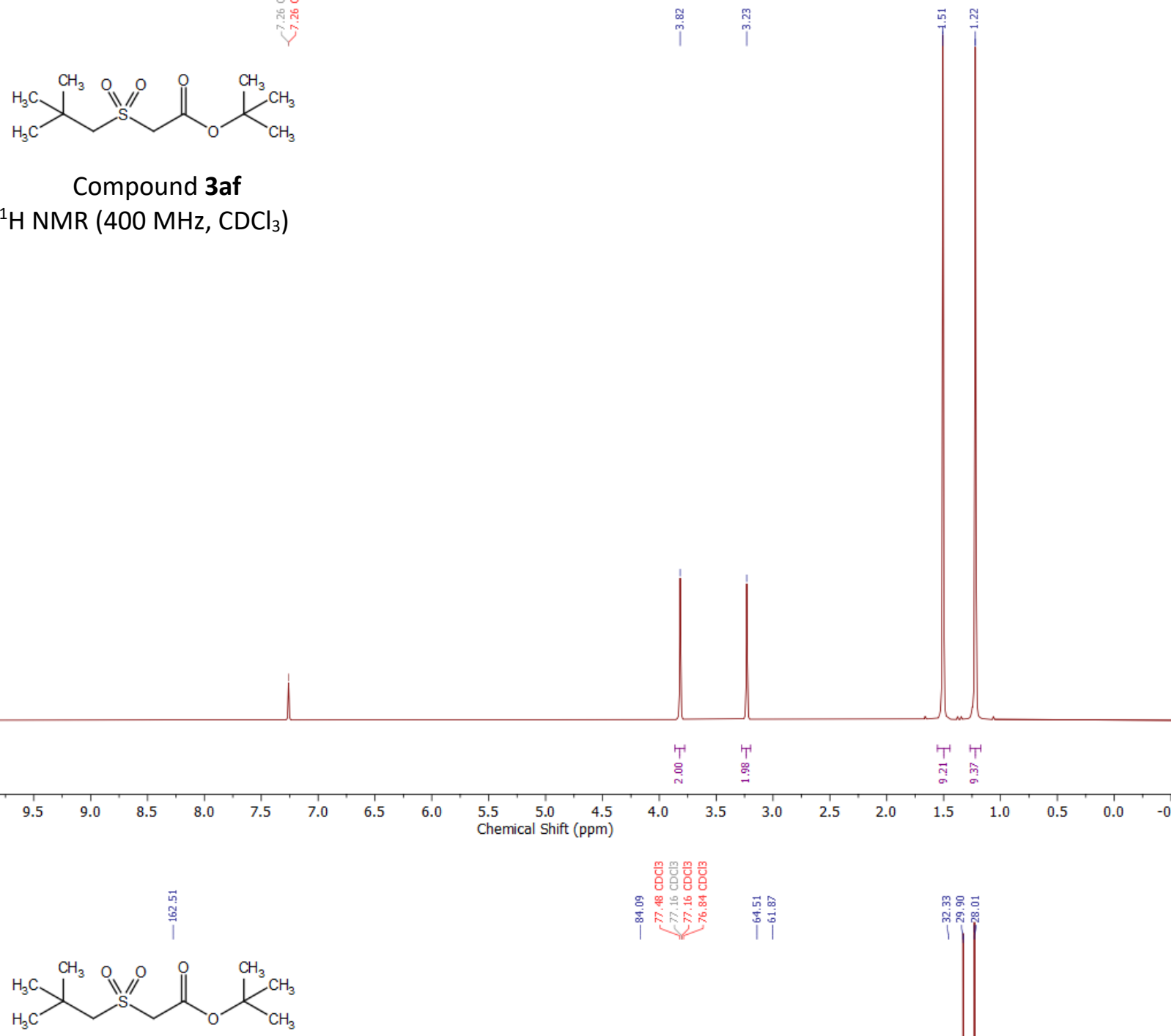

Compound 3af

$\left.{ }^{13} \mathrm{C} \mathrm{NMR} \mathrm{(101} \mathrm{MHz,} \mathrm{CDCl}_{3}\right)$ 
NMR spectra of tert-butyl 2-(octylsulfonyl)acetate (3ag)

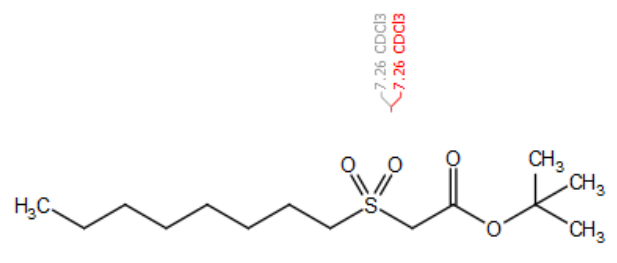

Compound 3ag

${ }^{1} \mathrm{H} \mathrm{NMR}\left(400 \mathrm{MHz}, \mathrm{CDCl}_{3}\right)$

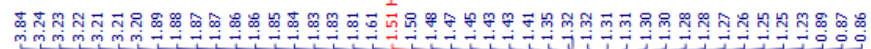
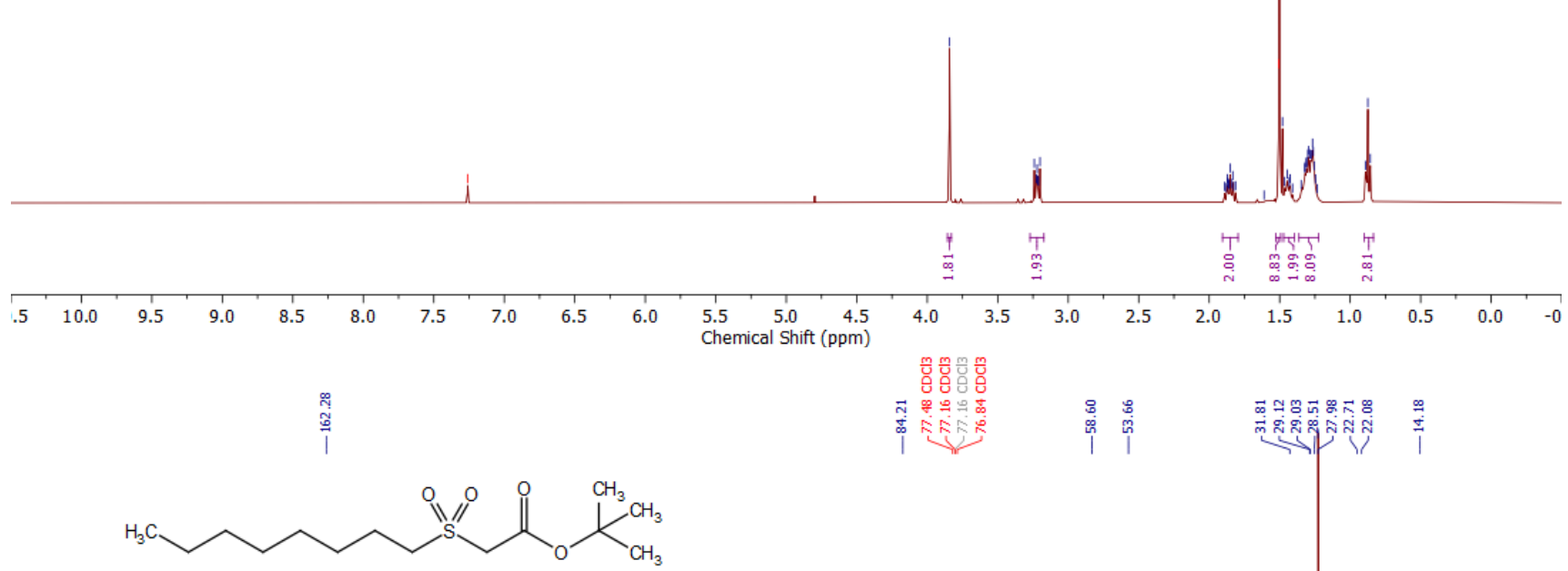

Compound 3ag

${ }^{13} \mathrm{C}$ NMR $\left(101 \mathrm{MHz}, \mathrm{CDCl}_{3}\right)$ 
NMR spectra of tert-butyl 2-((2-(cyclohex-1-en-1-yl)ethyl)sulfonyl)acetate (3ah)

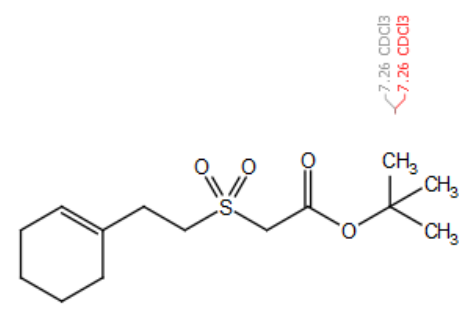

Compound 3ah

${ }^{1} \mathrm{H}$ NMR $\left(400 \mathrm{MHz}, \mathrm{CDCl}_{3}\right)$
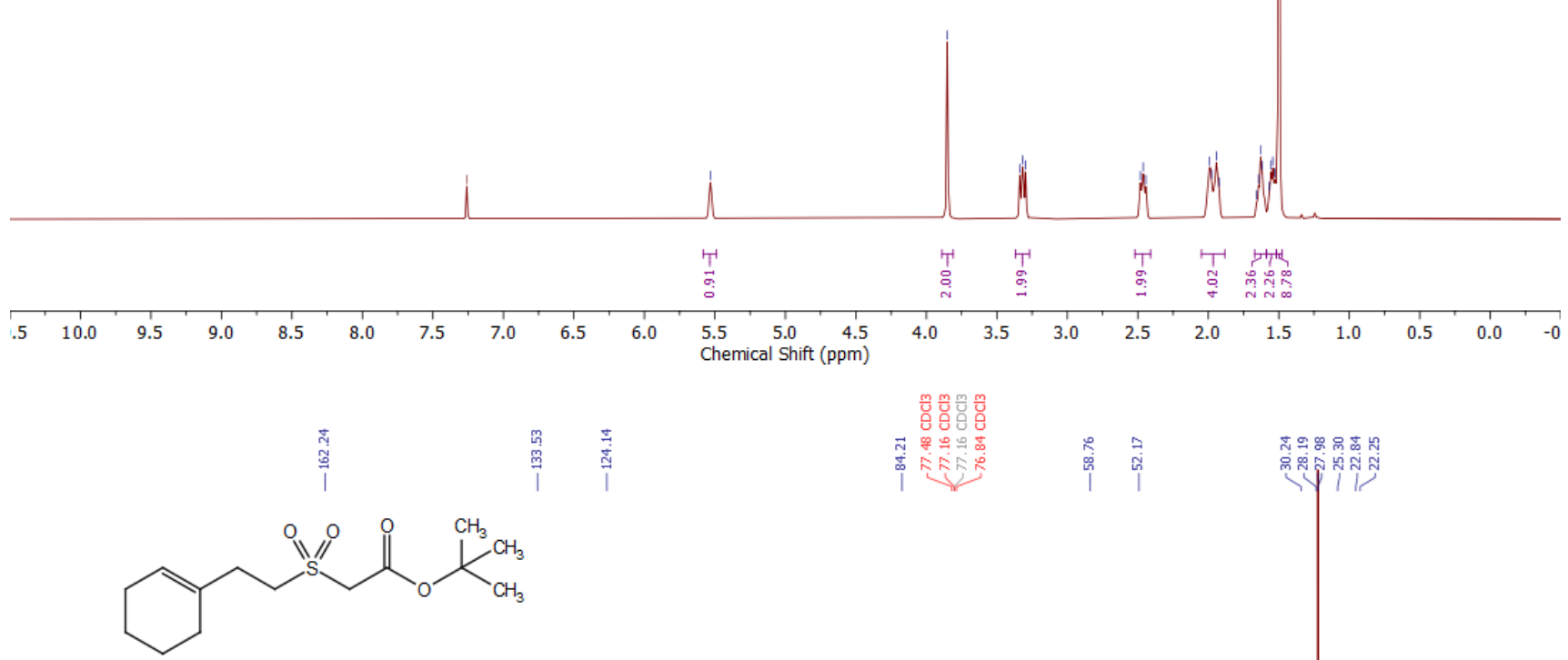

Compound 3ah

$\left.{ }^{13} \mathrm{C} \mathrm{NMR} \mathrm{(101} \mathrm{MHz,} \mathrm{CDCl}_{3}\right)$

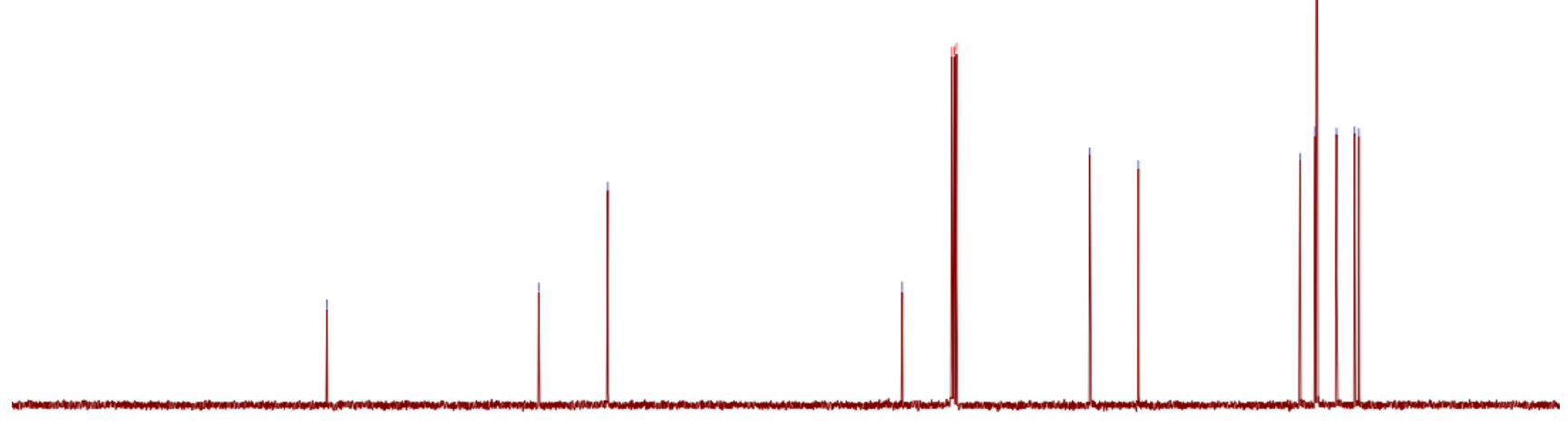

$\begin{array}{llllllllll}200 & 190 & 180 & 170 & 160 & 150 & 140 & 130 & 120 & \begin{array}{l}110 \\ \text { Chemical Shift }(\mathrm{ppm})\end{array}\end{array}$ 
NMR spectra of tert-butyl 2-((2,2-dimethoxyethyl)sulfonyl)acetate (3ai)
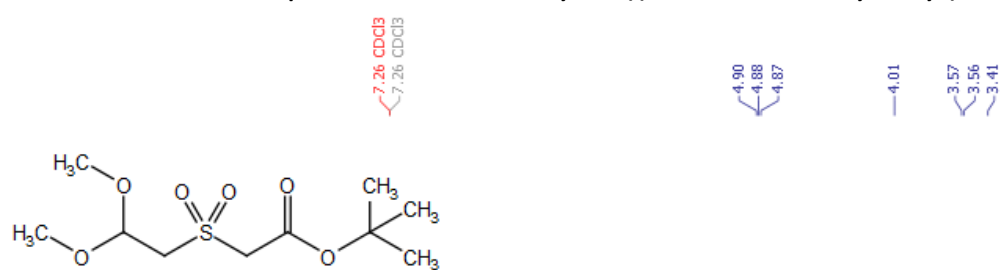

Compound 3ai

${ }^{1} \mathrm{H}$ NMR $\left(400 \mathrm{MHz}, \mathrm{CDCl}_{3}\right)$
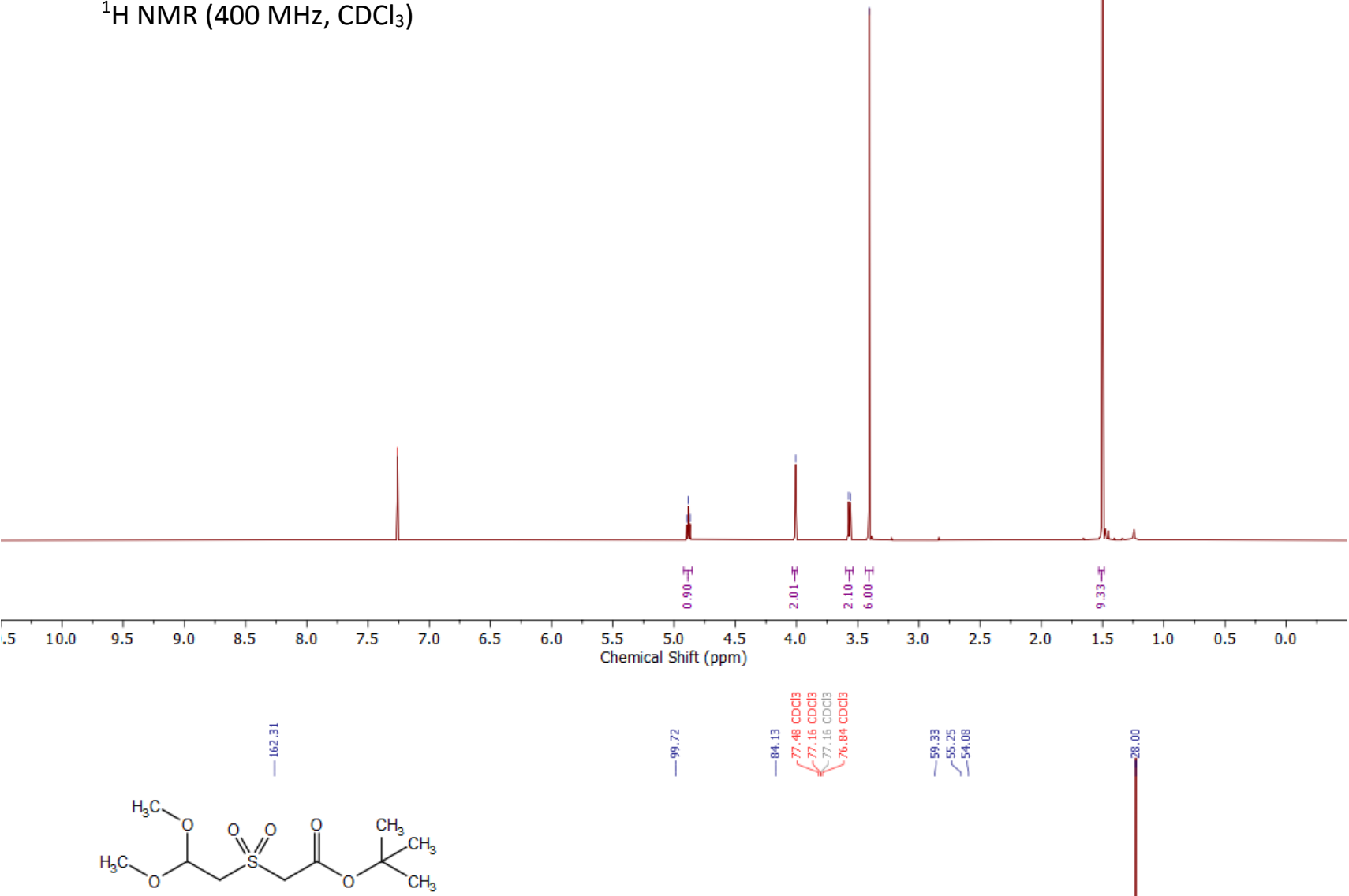

Compound 3ai

${ }^{13} \mathrm{C}$ NMR (101 MHz, $\left.\mathrm{CDCl}_{3}\right)$ 
NMR spectra of tert-butyl 2-((4-cyanophenethyl)sulfonyl)acetate (3aj)

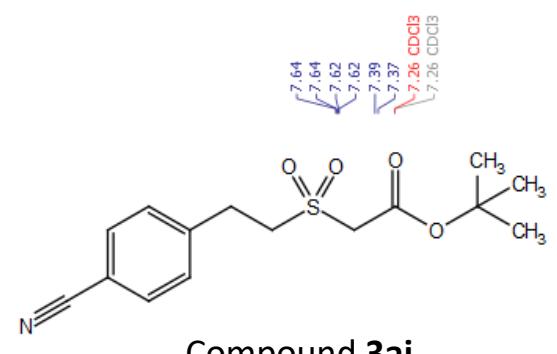

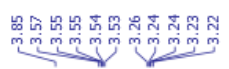

Compound 3aj

${ }^{1} \mathrm{H} \mathrm{NMR}\left(400 \mathrm{MHz}, \mathrm{CDCl}_{3}\right)$
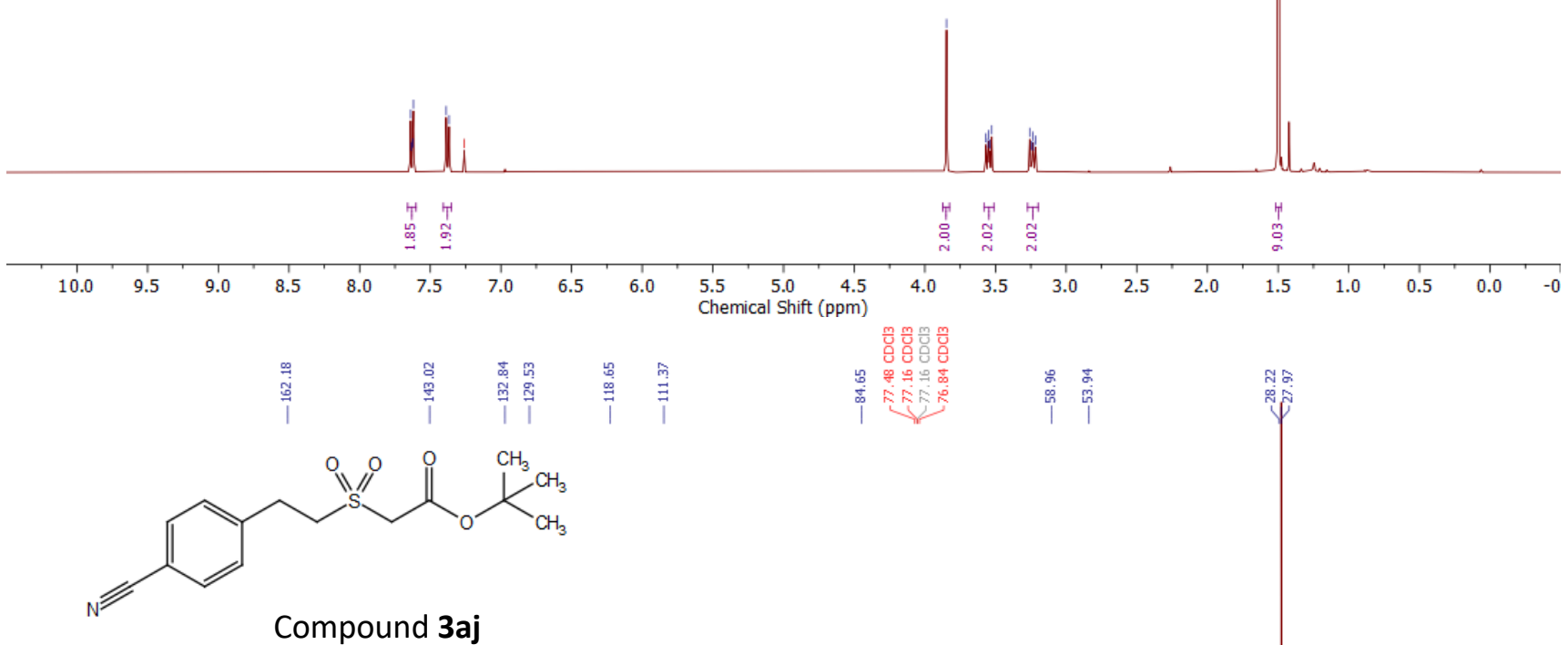

${ }^{13} \mathrm{C}$ NMR $\left(101 \mathrm{MHz}, \mathrm{CDCl}_{3}\right)$

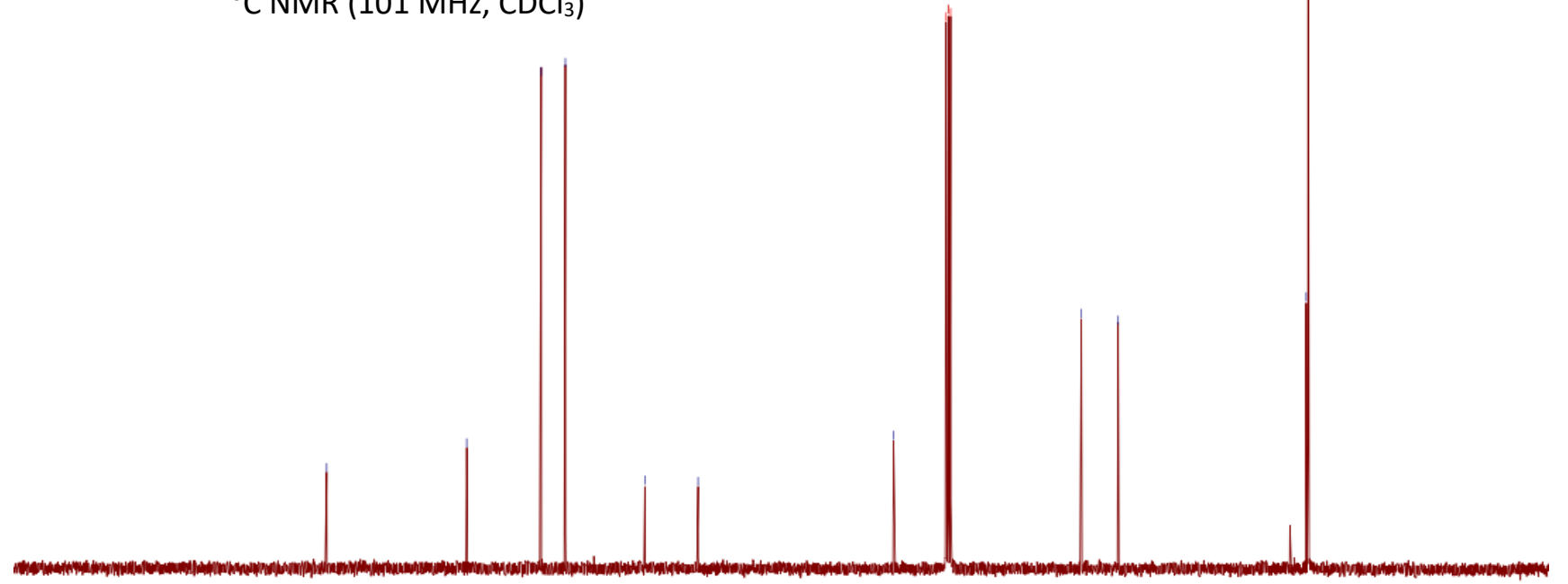

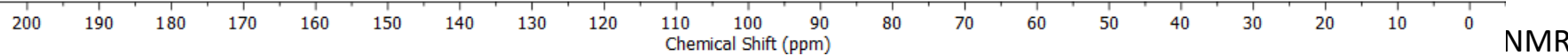


NMR spectra of tert-butyl 4-((2-(tert-butoxy)-2-oxoethyl)sulfonyl)butanoate (3ak)

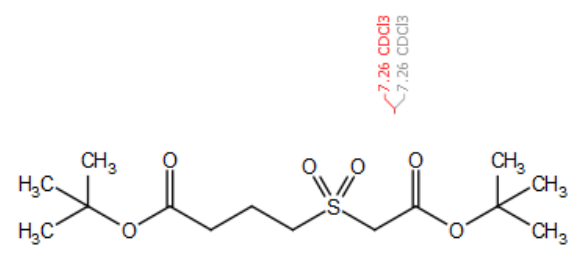

Compound 3ak

${ }^{1} \mathrm{H}$ NMR $\left(400 \mathrm{MHz}, \mathrm{CDCl}_{3}\right)$

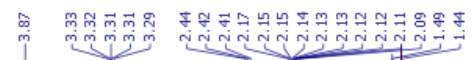

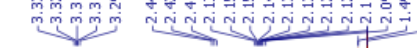

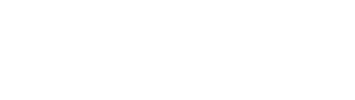


NMR spectra of tert-butyl 2-((2-(1H-indol-3-yl)ethyl)sulfonyl)acetate (3al)

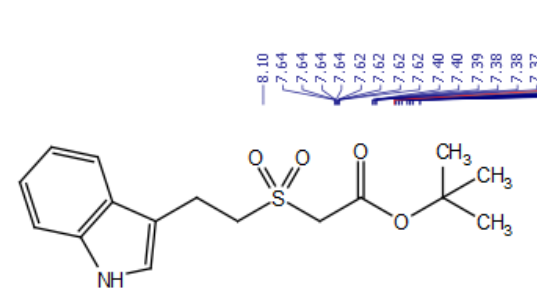

Compound 3al

${ }^{1} \mathrm{H}$ NMR (400 MHz, $\mathrm{CDCl}_{3}$ )
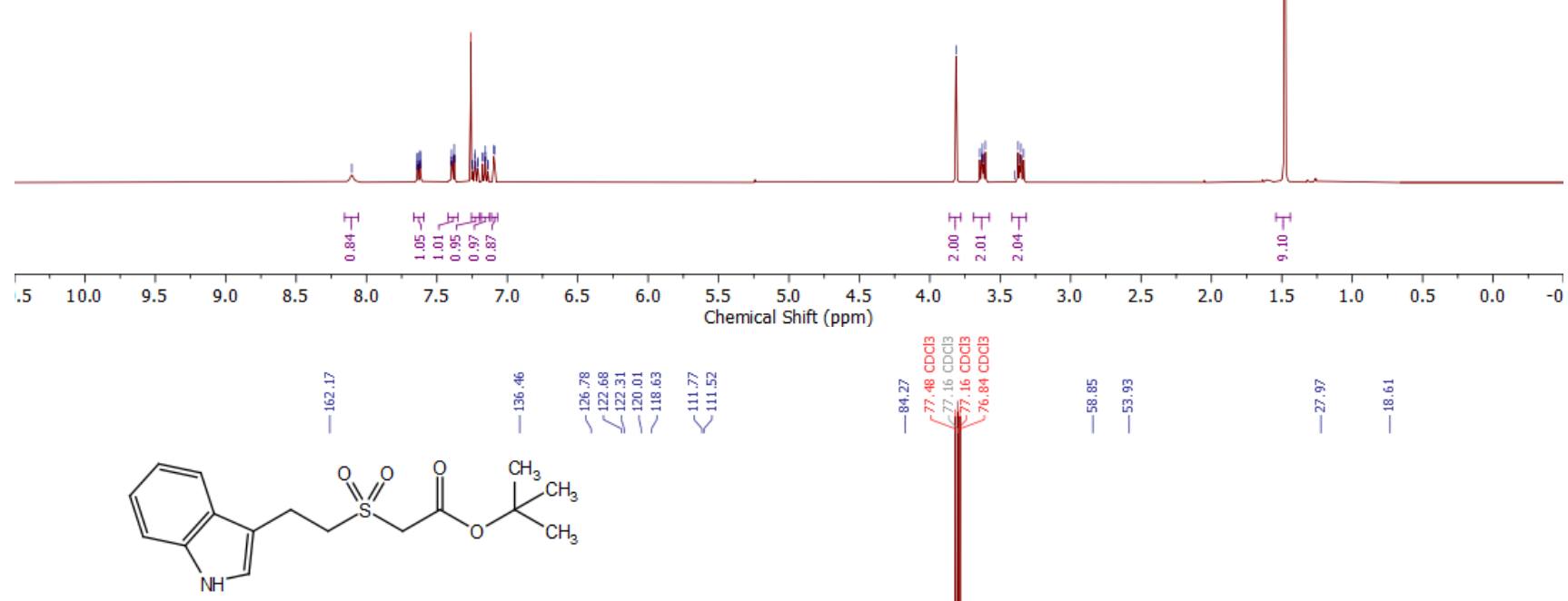

Compound 3al

${ }^{13} \mathrm{C}$ NMR (101 $\mathrm{MHz}, \mathrm{CDCl}_{3}$ ) 
NMR spectra of tert-butyl 2-((2-(dimethylamino)ethyl)sulfonyl)acetate (3am)<smiles>CN(C)CCS(=O)(=O)CC(=O)OC(C)(C)C</smiles>

Compound 3am

${ }^{1} \mathrm{H}$ NMR (400 MHz, $d_{6}$-DMSO)
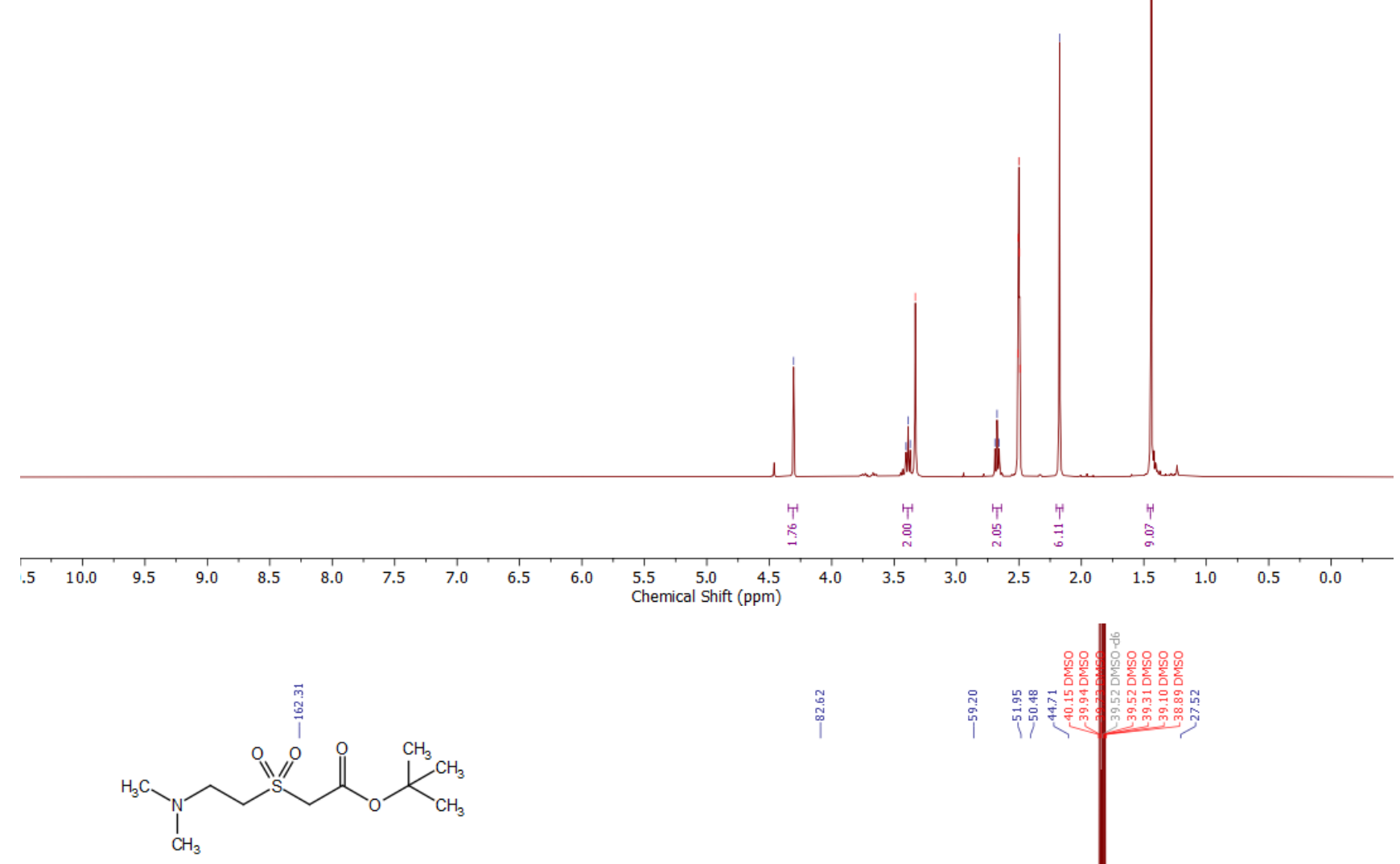

Compound 3am

${ }^{13} \mathrm{C}$ NMR (101 MHz, $d_{6}$-DMSO)

$\begin{array}{llllllllll}200 & 190 & 180 & 170 & 160 & 150 & 140 & 130 & 120 & \begin{array}{l}110 \\ \text { Chemical Shift (ppm) }\end{array}\end{array}$ 
NMR spectra of (S)-6-((2-(tert-butoxy)-2-oxoethyl)sulfonyl)-2-((tert-butoxycarbonyl)amino)hexanoic acid (3an)
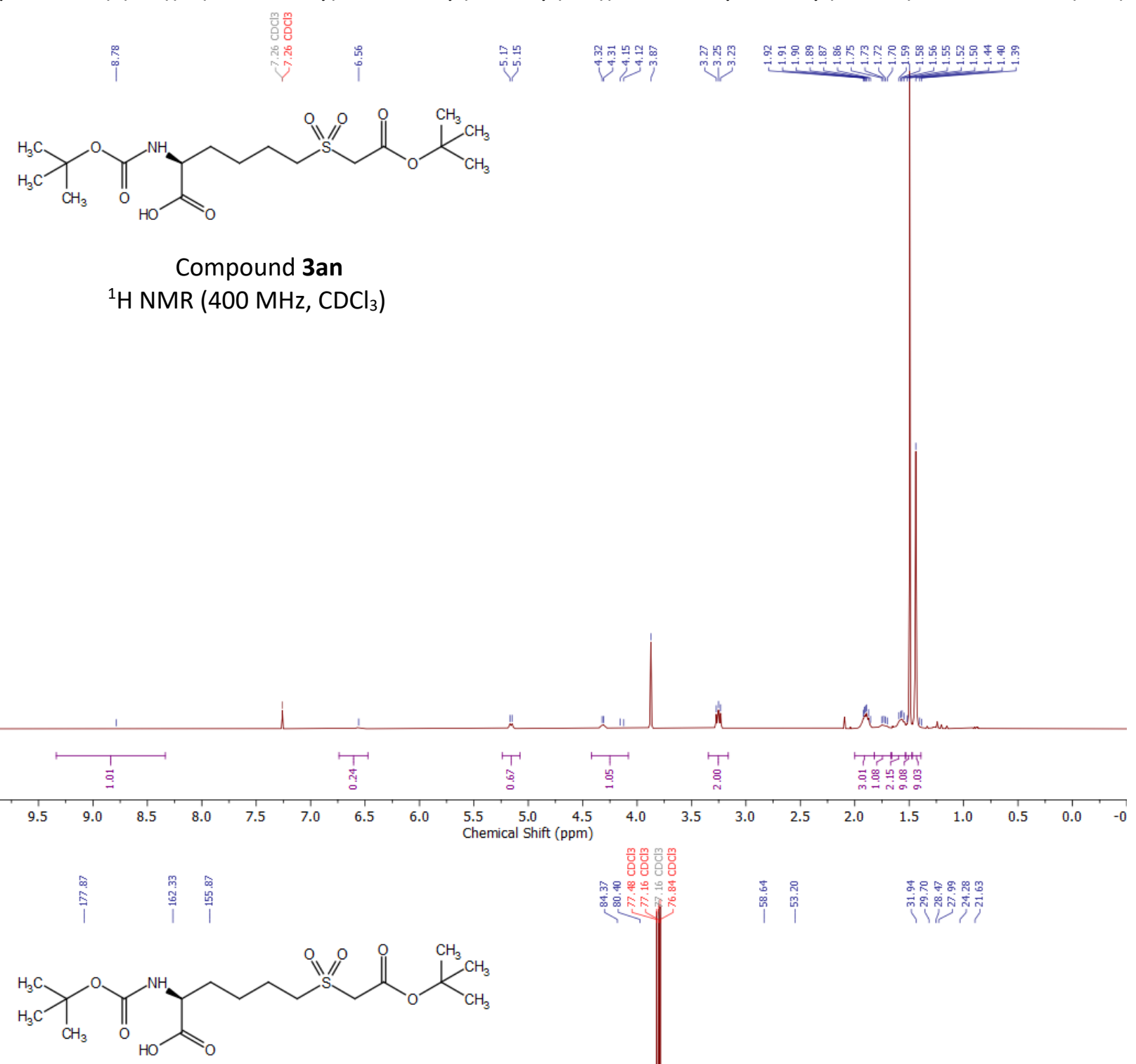

Compound 3an

${ }^{13} \mathrm{C}$ NMR $\left(101 \mathrm{MHz}, \mathrm{CDCl}_{3}\right)$

$\begin{array}{lllllllllll}200 & 190 & 180 & 170 & 160 & 150 & 140 & 130 & 120 & 110 & 100 \\ \text { Chemical Shift (Dom) }\end{array}$


NMR spectra of 2-((2-(tert-butoxy)-2-oxoethyl)sulfonyl)ethane-1-sulfonic acid (3ao)<smiles>CC(C)(C)OC(=O)CS(=O)(=O)CCS(=O)(=O)O</smiles>

Compound 3ao

${ }^{1} \mathrm{H}$ NMR (400 MHz, $d_{6}$-DSMO)
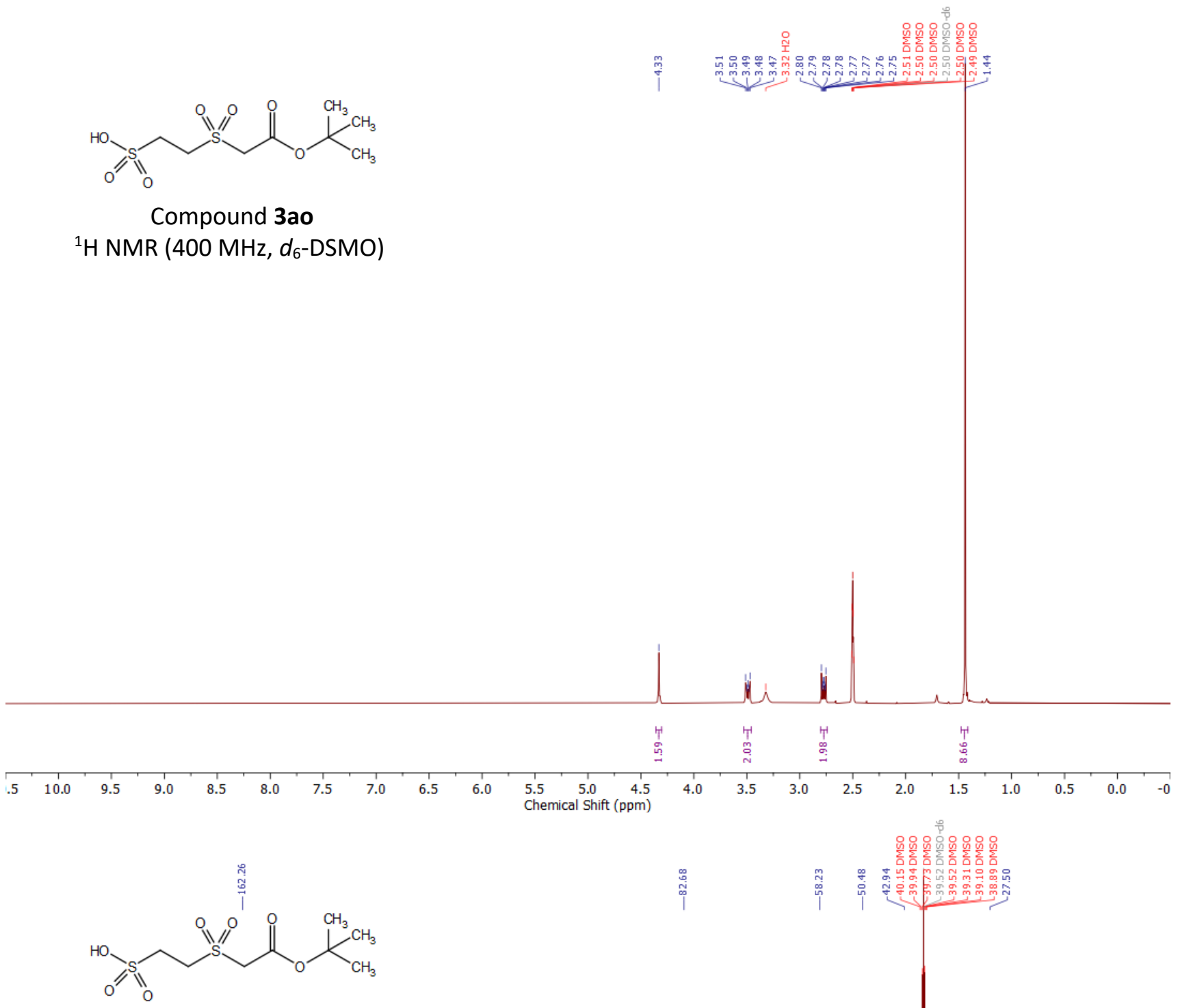

Compound 3ao

${ }^{13} \mathrm{C}$ NMR (101 MHz, $d_{6}$-DSMO) 
NMR spectra of tert-butyl 2-((4-sulfamoylphenethyl)sulfonyl)acetate (3ap)

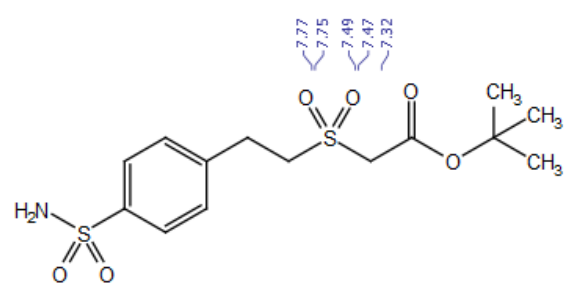

Compound 3ap

${ }^{1} \mathrm{H}$ NMR $\left(400 \mathrm{MHz}, \mathrm{CDCl}_{3}\right)$
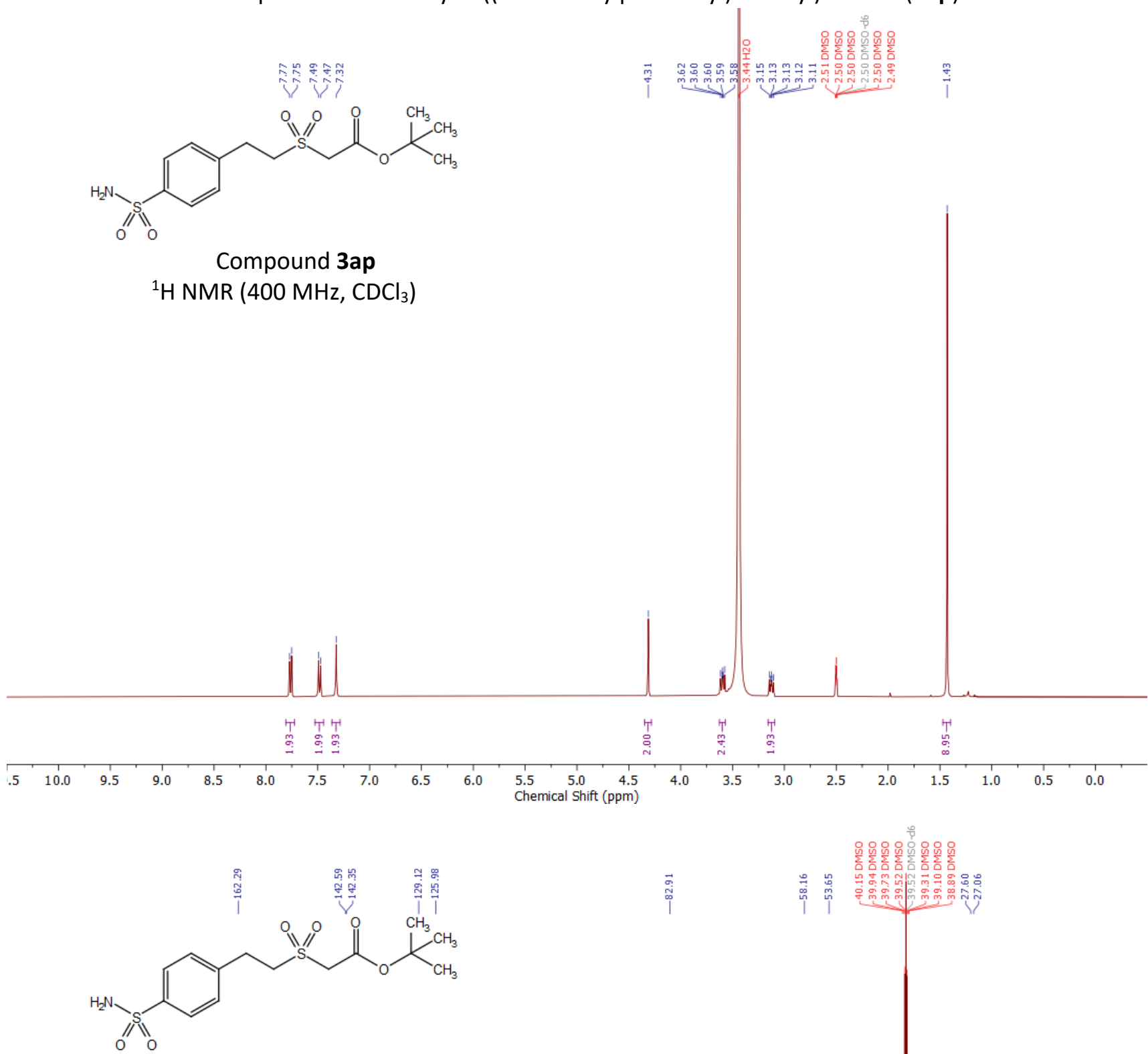

Compound 3ap

${ }^{13} \mathrm{C}$ NMR (101 MHz, $\mathrm{CDCl}_{3}$ )

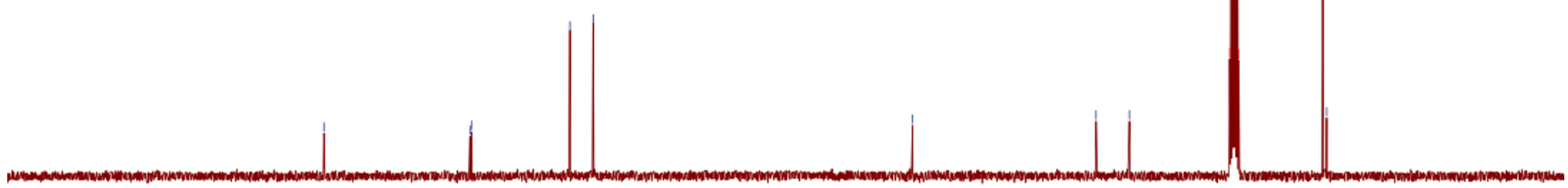

$\begin{array}{llllllllll}200 & 190 & 180 & 170 & 160 & 150 & 140 & 130 & 120 & \begin{array}{c}110 \\ \text { Chemical Shift (ppm) }\end{array}\end{array}$ 
NMR spectra of sodium 1-((benzyloxy)carbonyl)piperidine-4-sulfinate (2a)
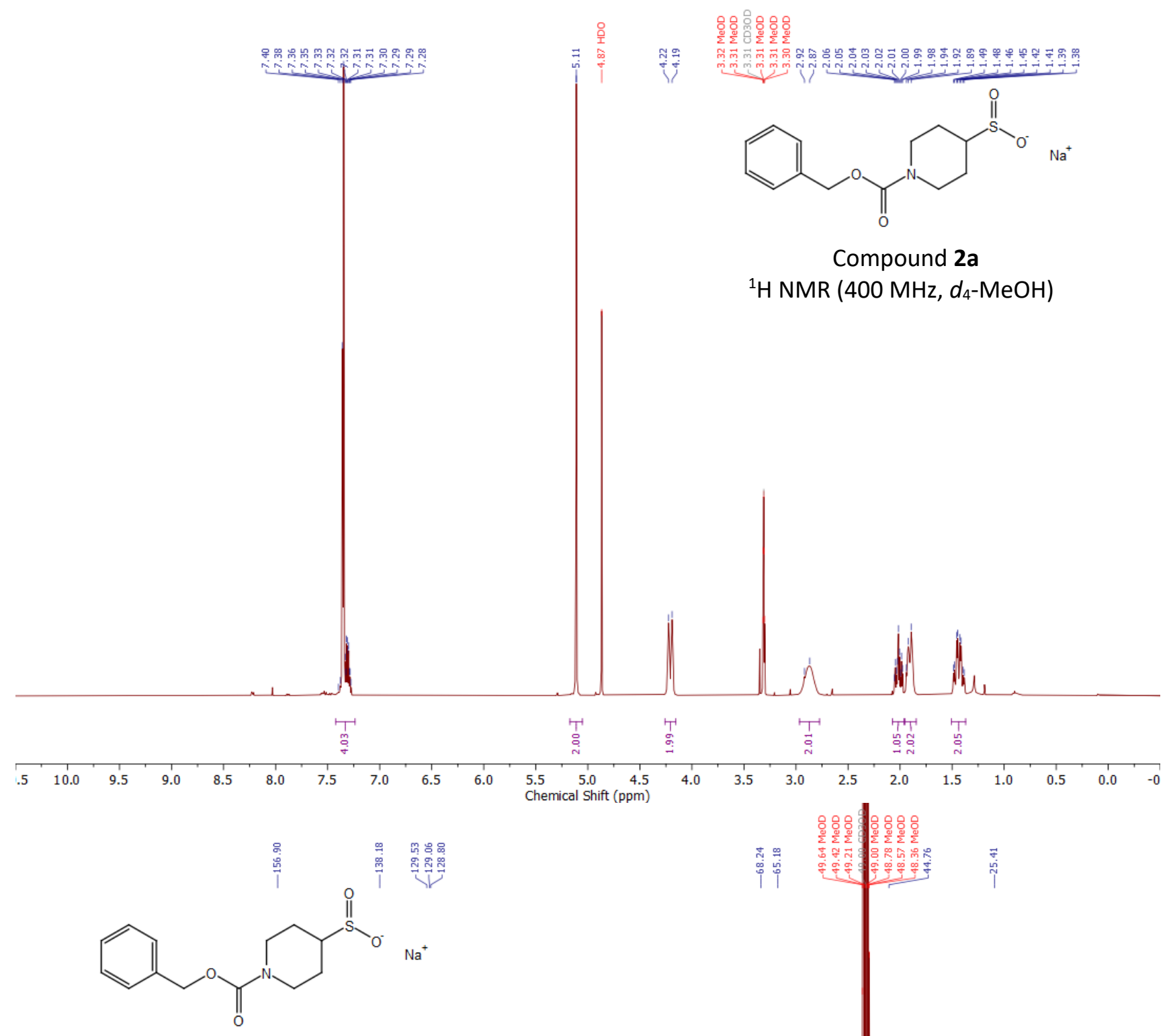

Compound 2a

${ }^{13} \mathrm{C}$ NMR $\left(101 \mathrm{MHz}, d_{4}-\mathrm{MeOH}\right)$

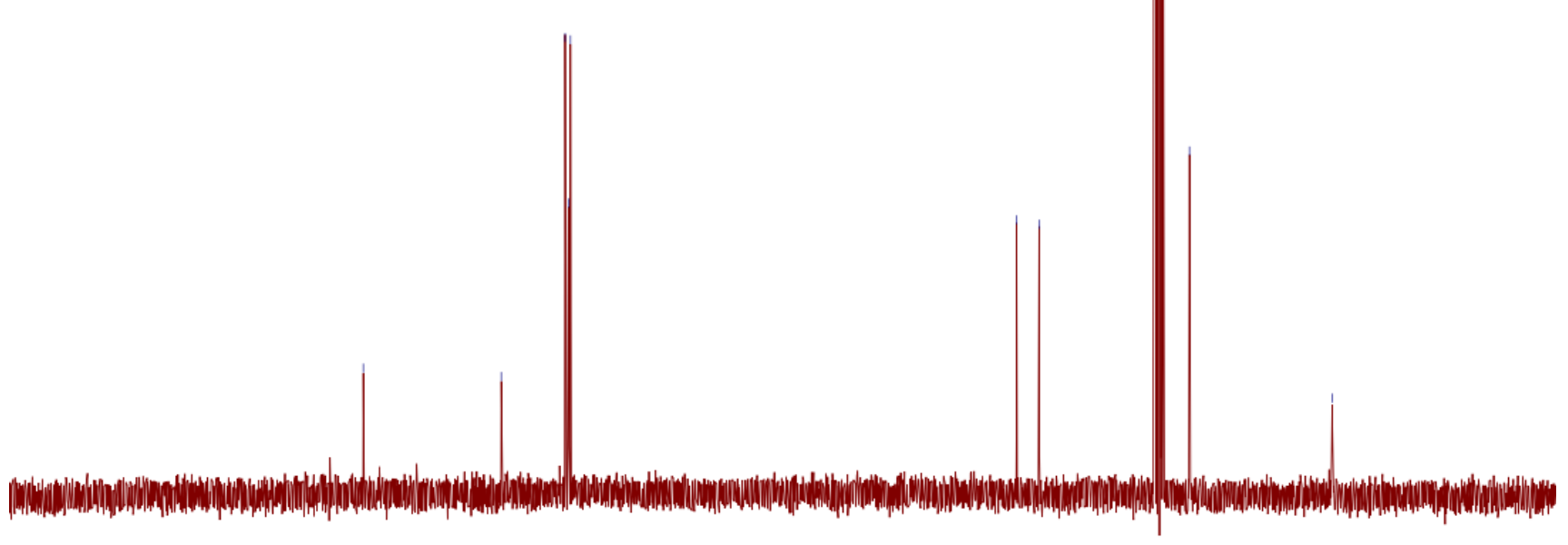

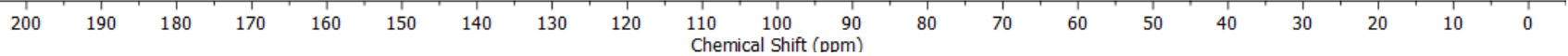


NMR spectra of sodium phenylmethanesulfinate (2b)

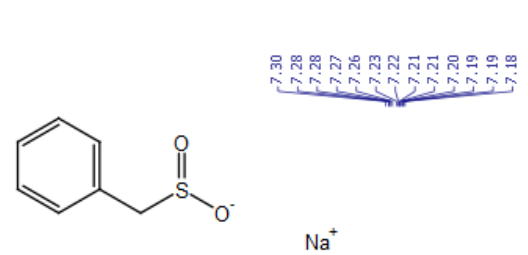

Compound $\mathbf{2 b}$

${ }^{1} \mathrm{H} \mathrm{NMR}\left(400 \mathrm{MHz}, d_{4}-\mathrm{MeOH}\right)$<smiles>O=S(O)Cc1ccccc1</smiles>

\section{Compound $\mathbf{2 b}$}

${ }^{13} \mathrm{C}$ NMR (101 MHz, $\left.d_{4}-\mathrm{MeOH}\right)$

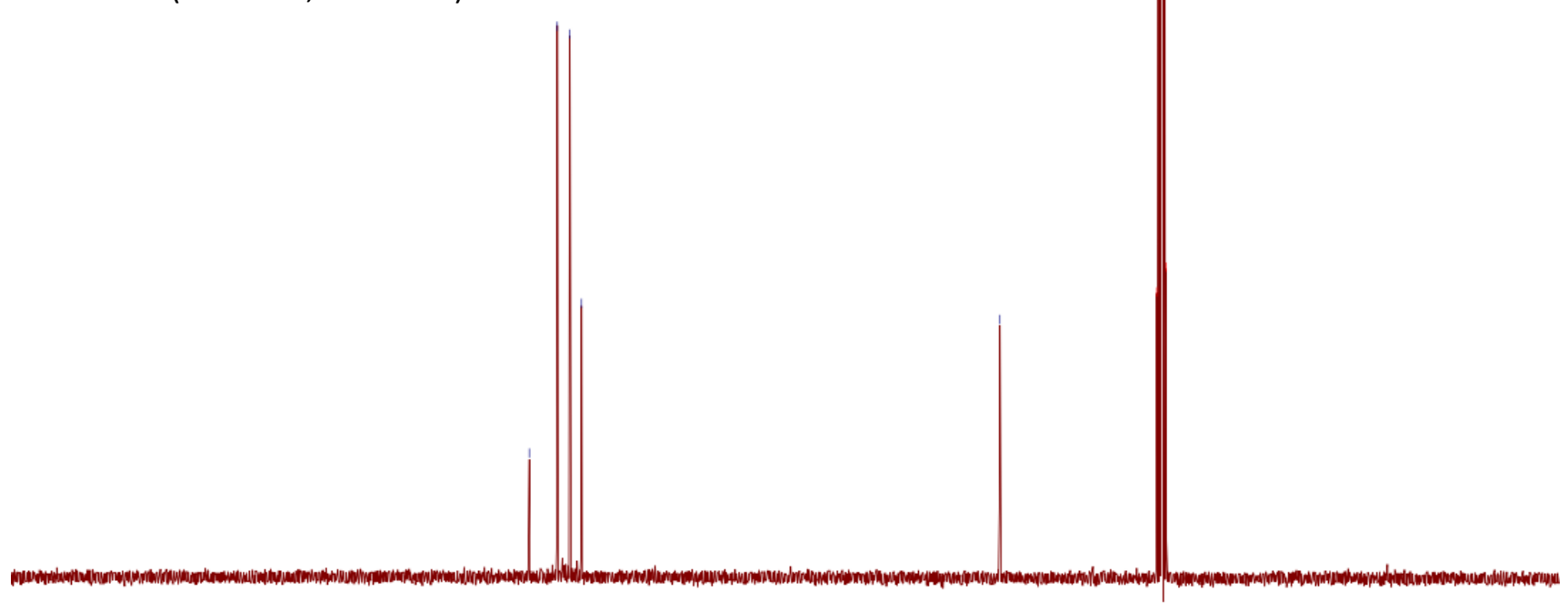


NMR spectra of sodium 2-phenylethane-1-sulfinate (2c)

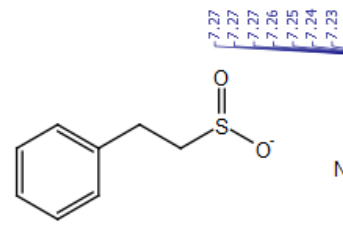

Compound 2c

${ }^{1} \mathrm{H}$ NMR $\left(400 \mathrm{MHz}, d_{4}-\mathrm{MeOH}\right)$

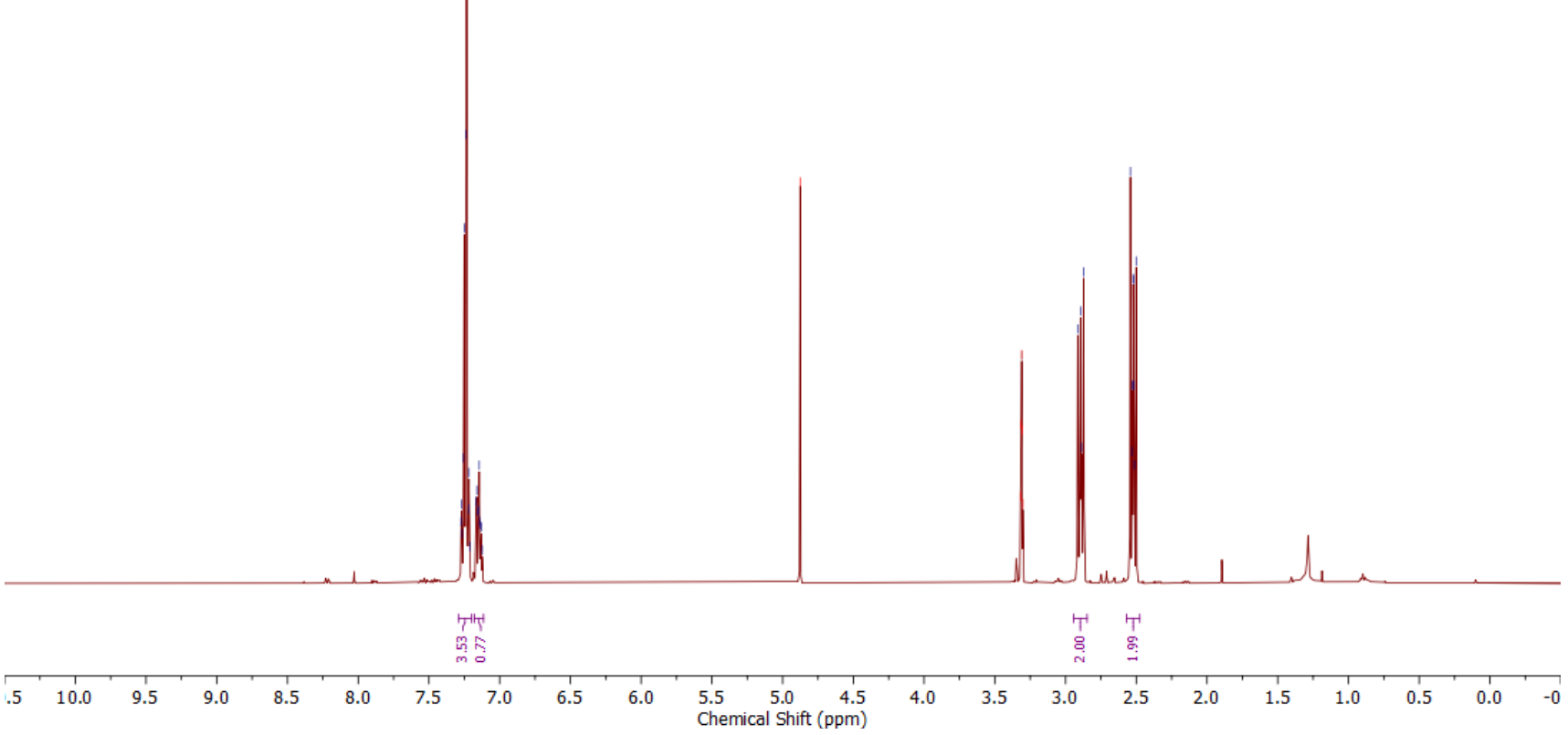<smiles>N#[W]c1ccccc1</smiles>

Compound 2c

${ }^{13} \mathrm{C}$ NMR $\left(101 \mathrm{MHz}, d_{4}-\mathrm{MeOH}\right)$

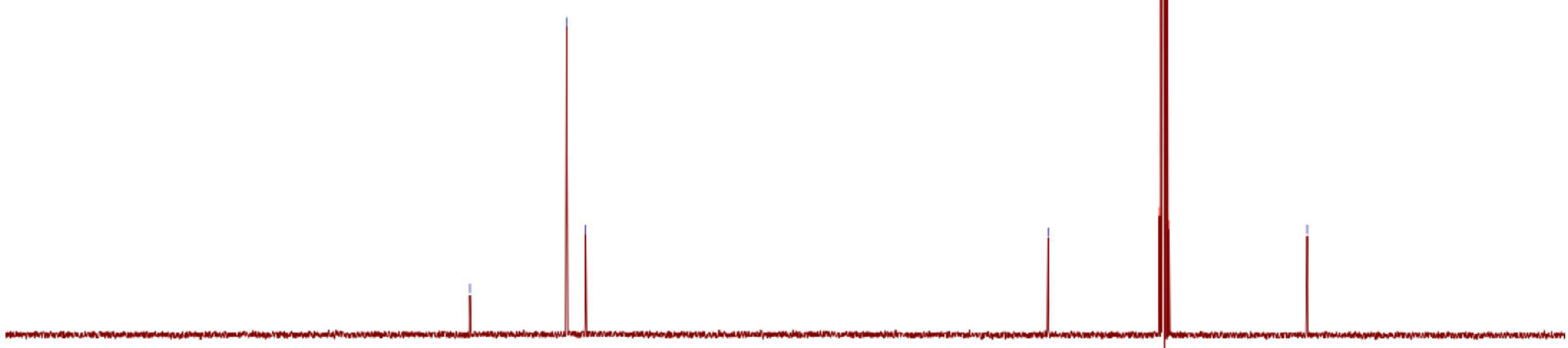

$\begin{array}{llllllllll}200 & 190 & 180 & 170 & 160 & 150 & 140 & 130 & 120 & \begin{array}{c}110 \\ \text { Chemical Shift (bom) }\end{array}\end{array}$ 
NMR spectra of benzyl 4-(propylsulfonyl)piperidine-1-carboxylate (6a)

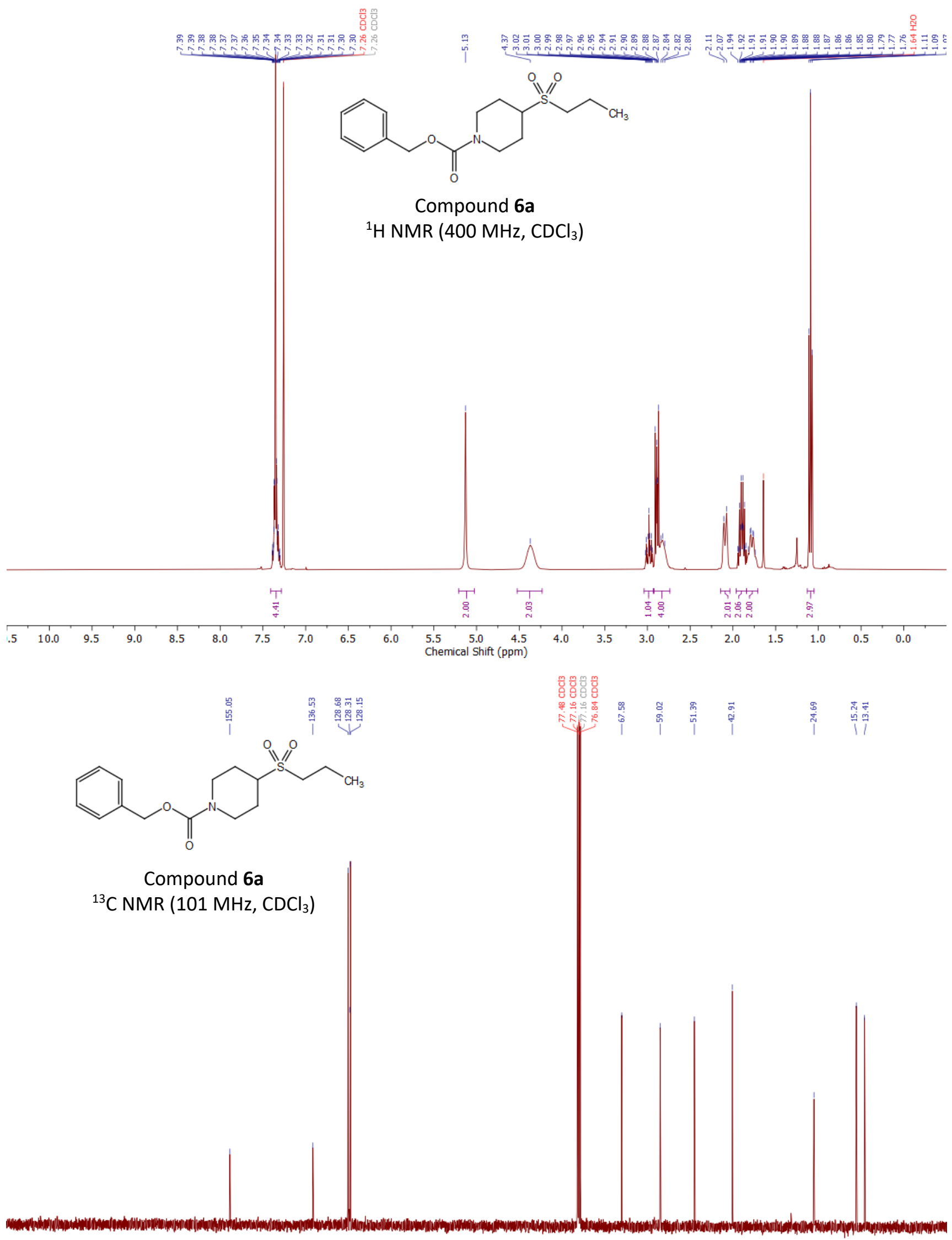

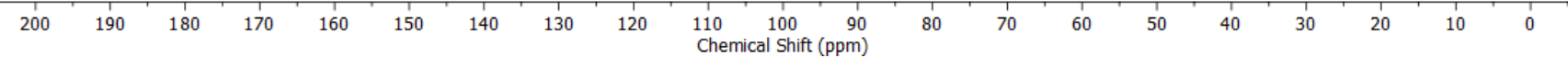


NMR spectra of benzyl 4-(benzylsulfonyl)piperidine-1-carboxylate (6b)

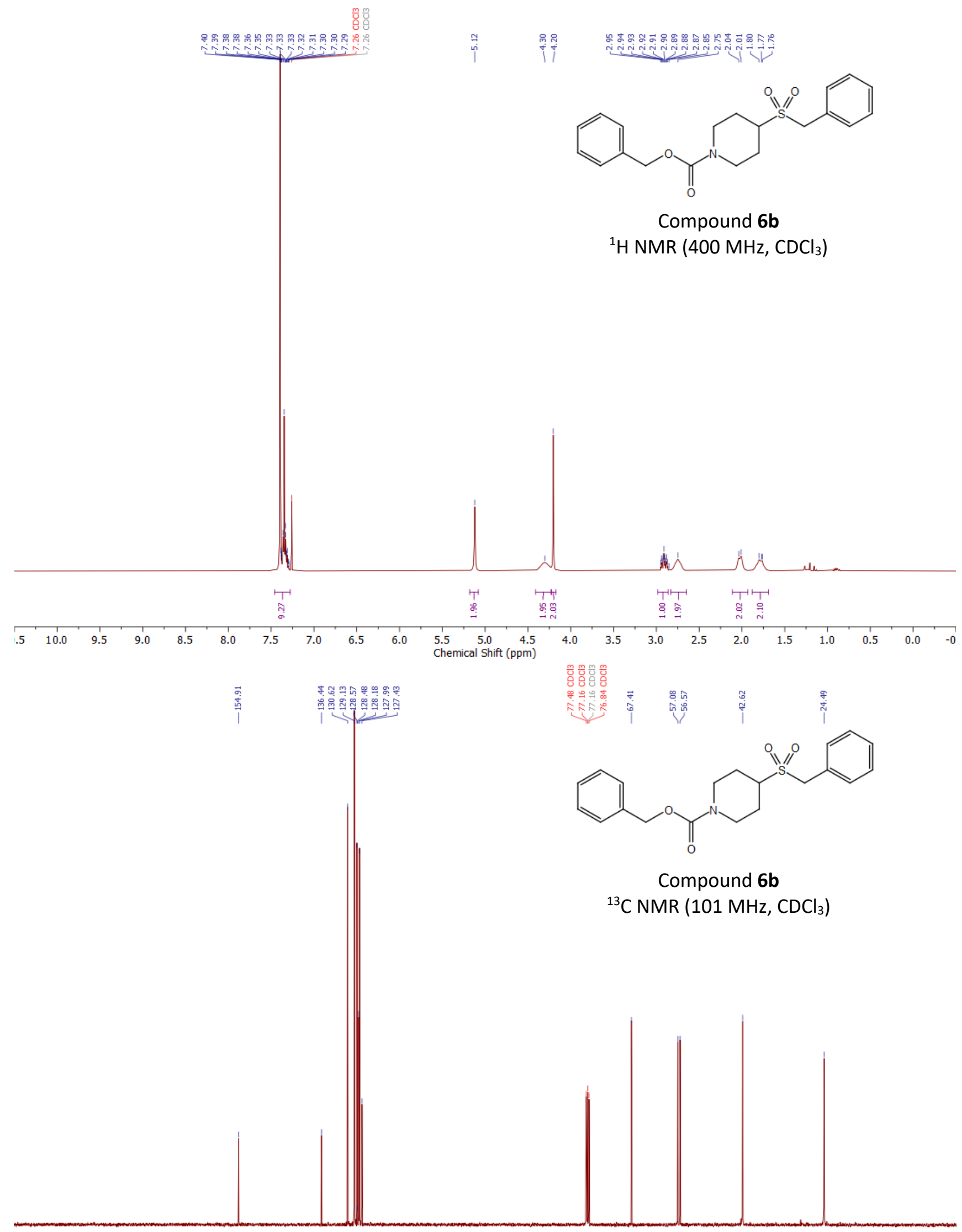

$\begin{array}{lllllllllll}200 & 190 & 180 & 170 & 160 & 150 & 140 & 130 & 120 & \begin{array}{c}110 \\ \text { Chemical Shift (ppm) }\end{array}\end{array}$ 
NMR spectra of benzyl 4-(allylsulfonyl)piperidine-1-carboxylate (6c)

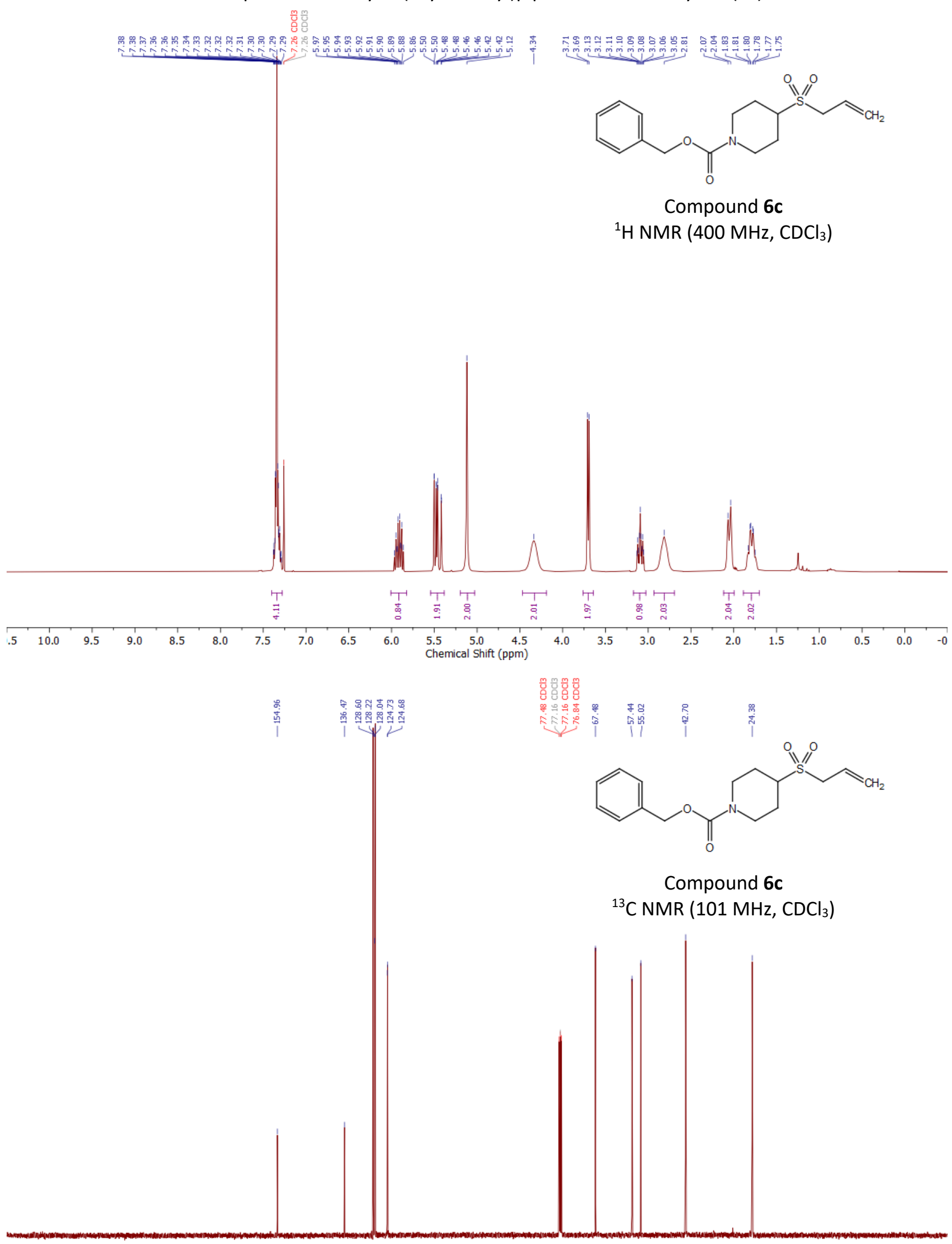

$\begin{array}{llllllllllllllllllllllllllll}220 & 210 & 200 & 190 & 180 & 170 & 160 & 150 & 140 & 130 & 120 & 110 & 100 & 90 & 80 & 70 & 60 & 50 & 40 & 30 & 20 & 10 & 0 & -10 & -20 & \end{array}$ 
NMR spectra of benzyl 4-(phenylsulfonyl)piperidine-1-carboxylate (6d)

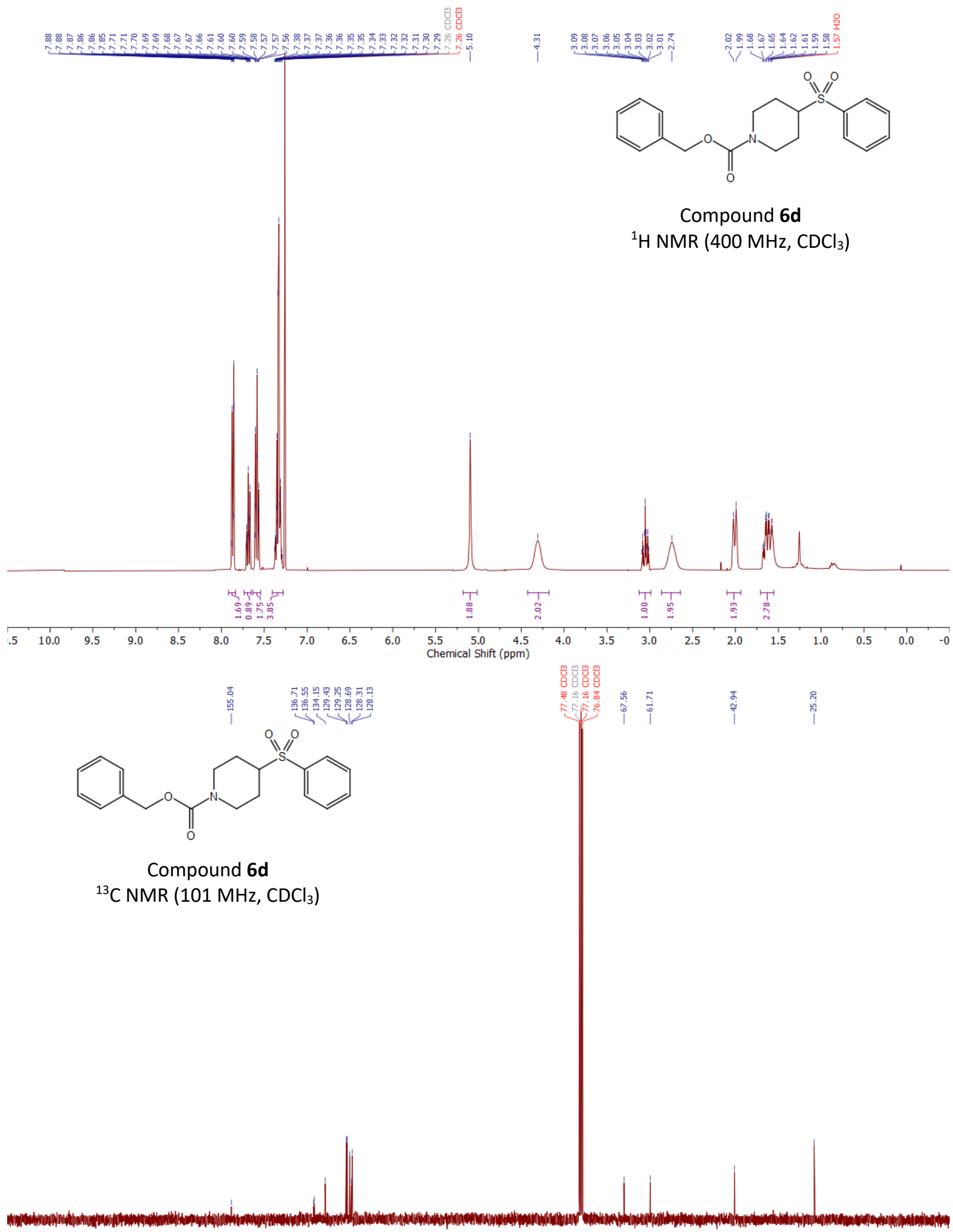

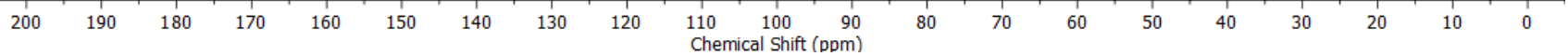


NMR spectra of benzyl 4-((2-hydroxycyclohexyl)sulfonyl)piperidine-1-carboxylate (6e-1)

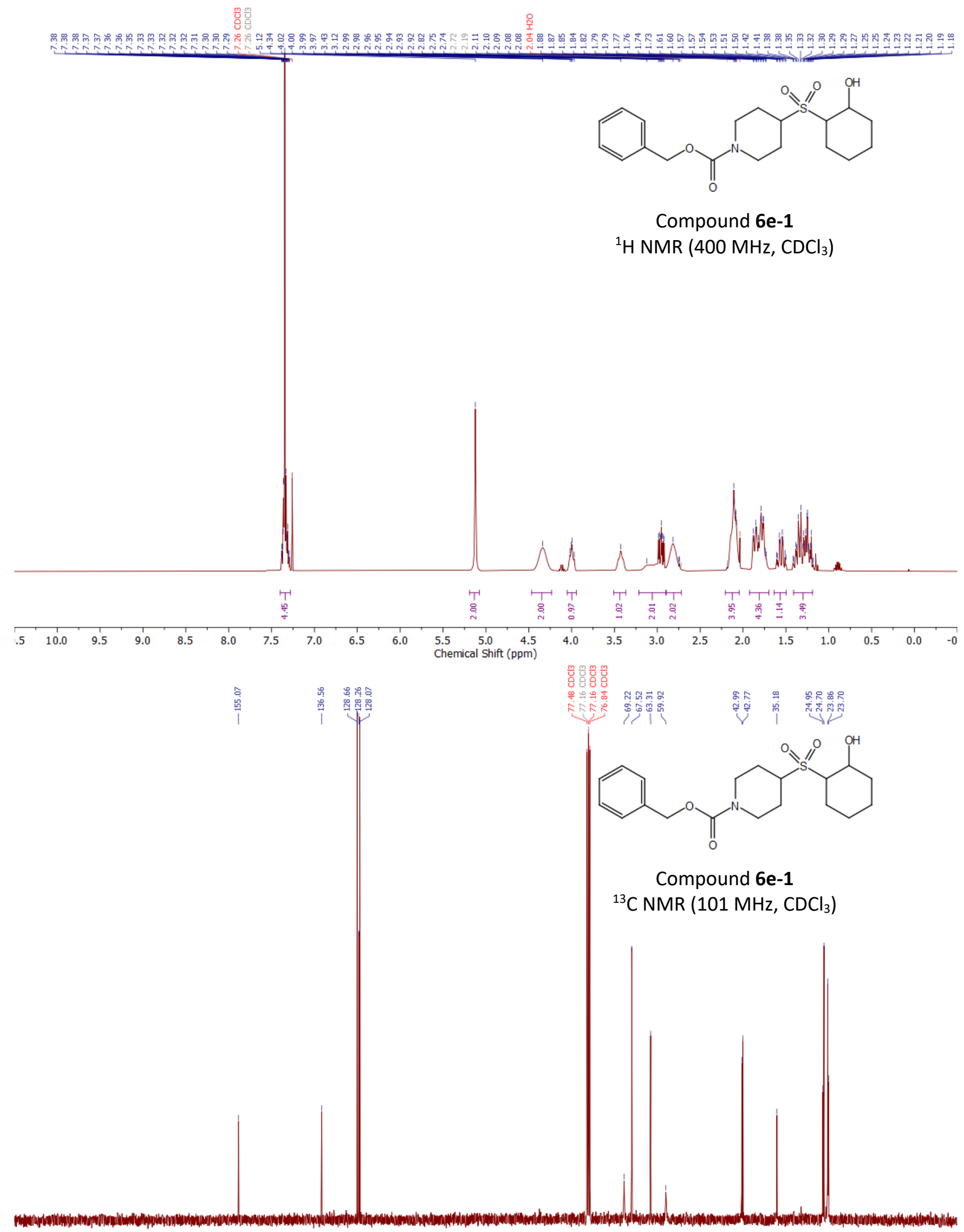

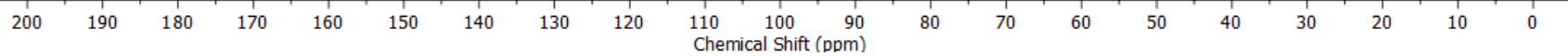


NMR spectra of benzyl 4-((2-hydroxycyclohexyl)sulfonyl)piperidine-1-carboxylate (6e-2)

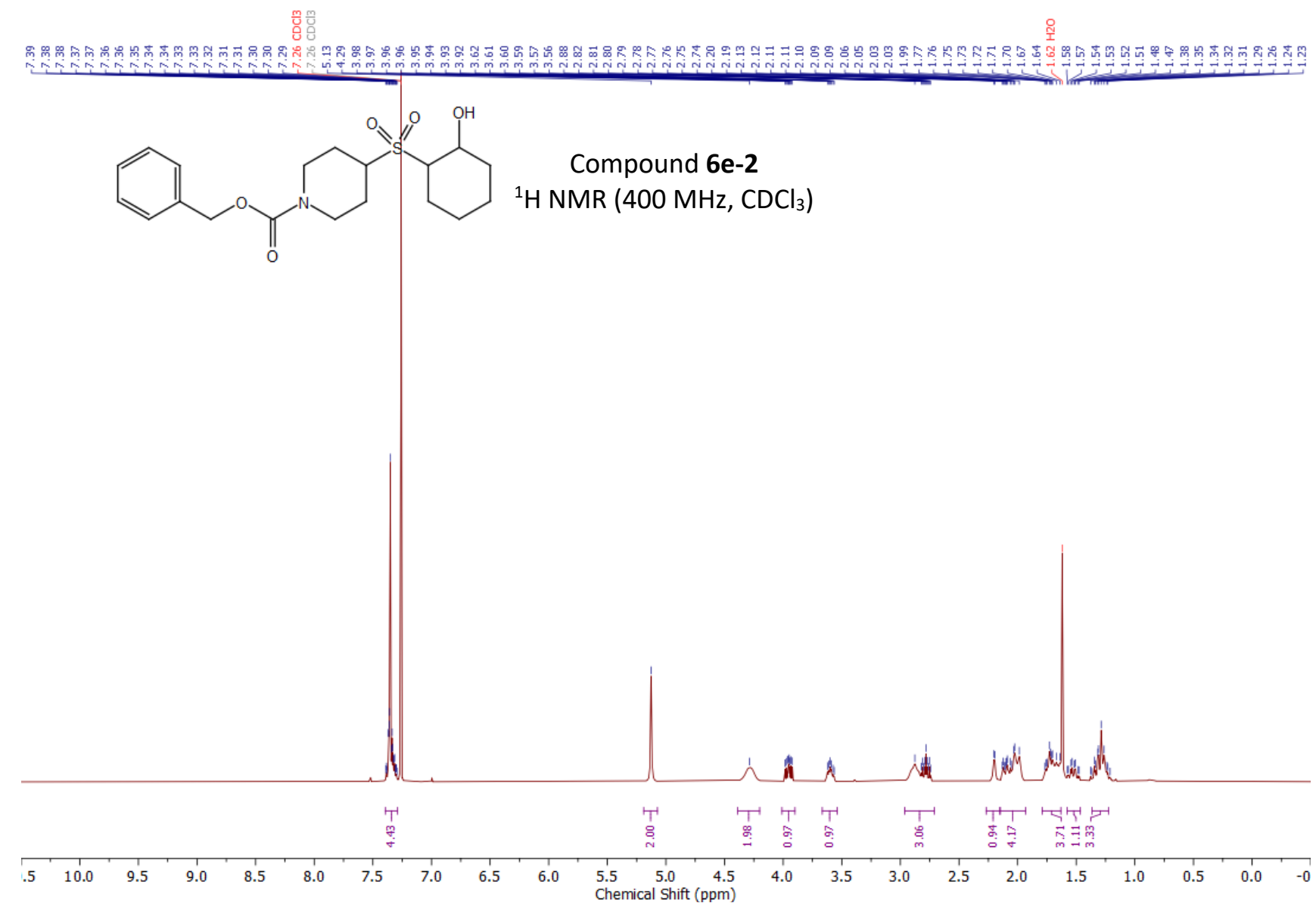<smiles>O=C(OCc1ccccc1)N1CCC(S(=O)(=O)C2CCCCC2O)CC1</smiles>

Compound 6e-2

$\left.{ }^{13} \mathrm{C} \mathrm{NMR} \mathrm{(101} \mathrm{MHz,} \mathrm{CDCl}_{3}\right)$ 
NMR spectra of benzyl 4-(benzo[d]thiazol-2-ylsulfonyl)piperidine-1-carboxylate (6f)

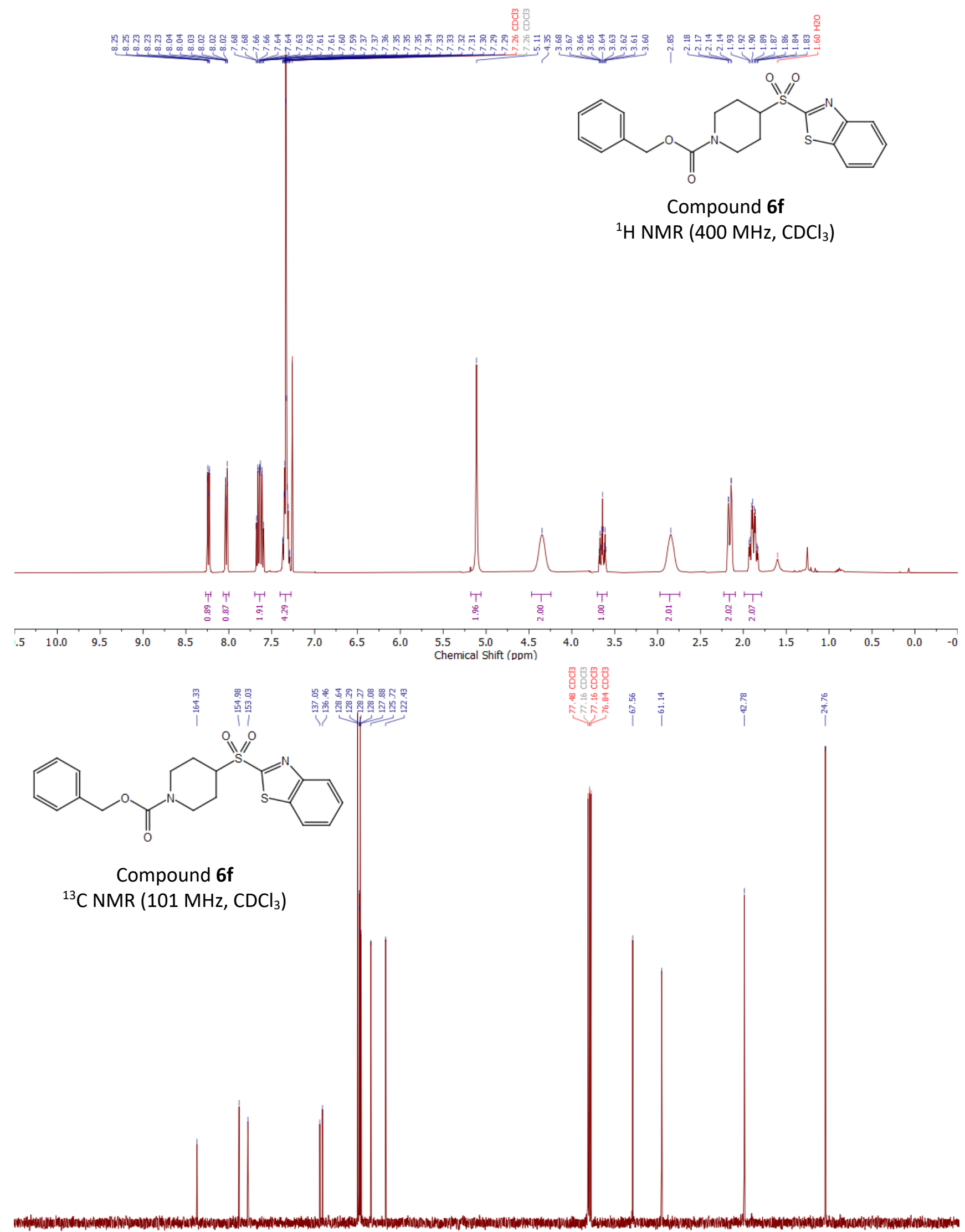

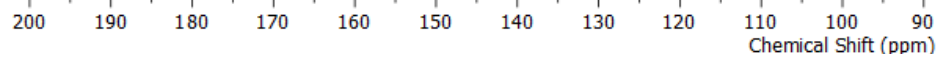


NMR spectra of benzyl 4-(methylsulfonyl)piperidine-1-carboxylate $(6 \mathrm{~g})$
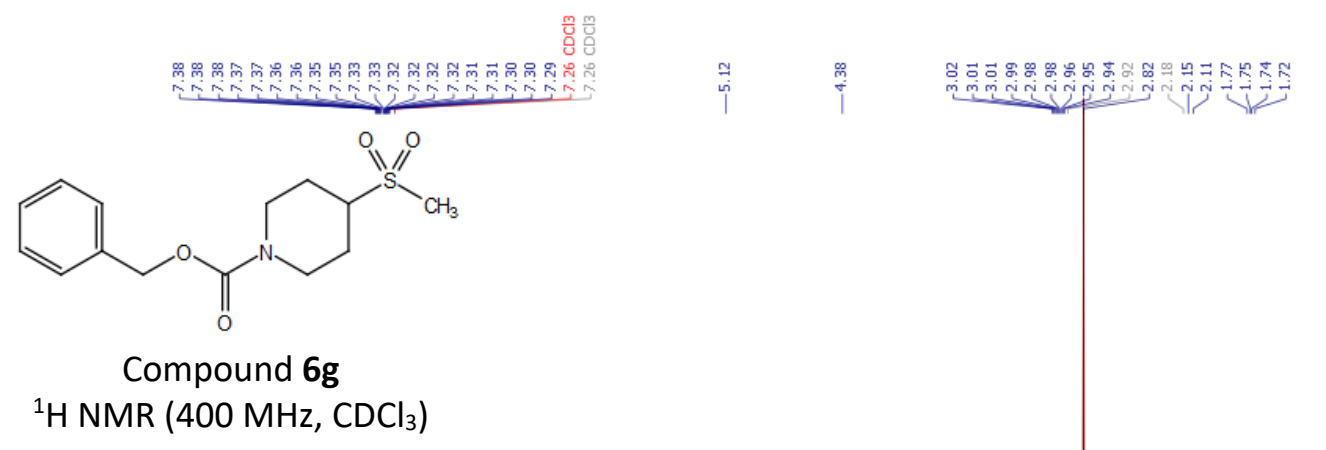

${ }^{1} \mathrm{H}$ NMR $\left(400 \mathrm{MHz}, \mathrm{CDCl}_{3}\right)$

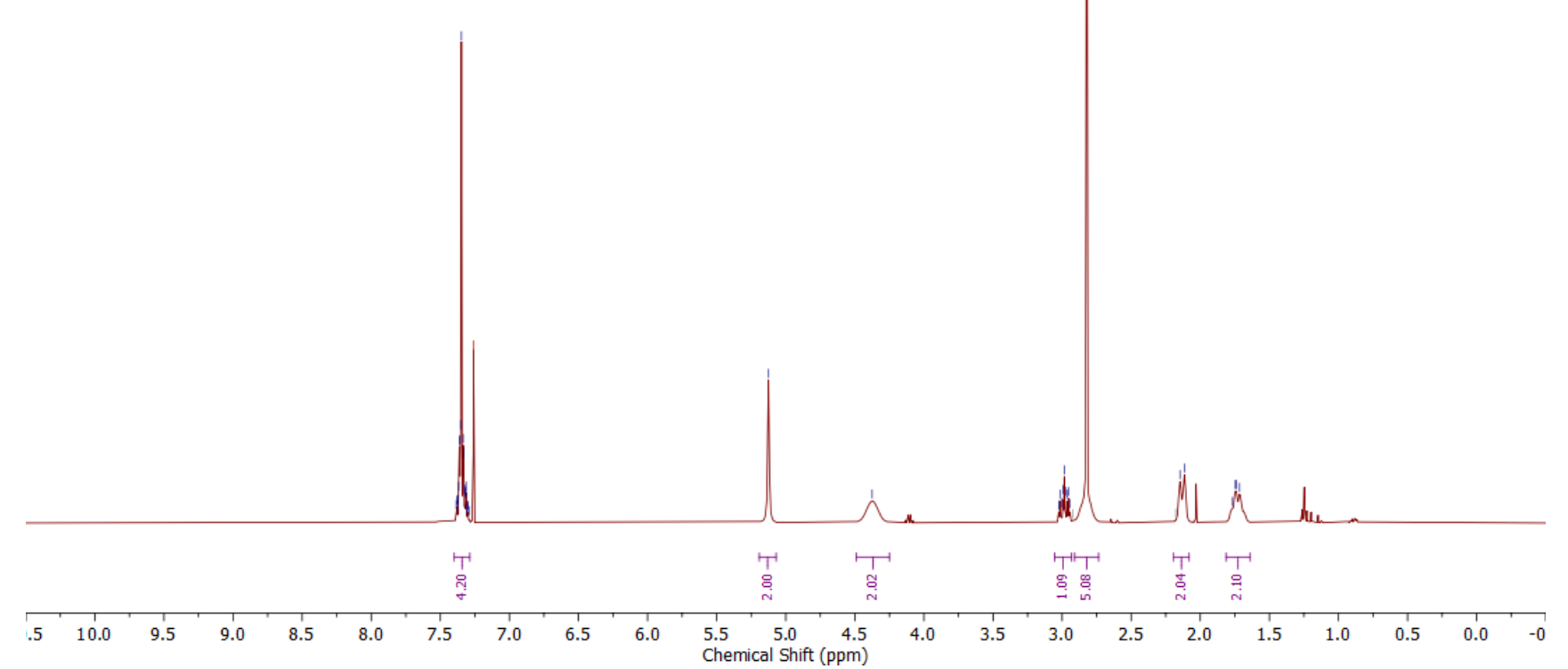<smiles>CS(=O)(=O)C1CCN(C(=O)OCc2ccccc2)CC1</smiles>

Compound $6 \mathrm{~g}$

$\left.{ }^{13} \mathrm{C} \mathrm{NMR} \mathrm{(101} \mathrm{MHz,} \mathrm{CDCl}_{3}\right)$ 
NMR spectra of ((methylsulfonyl)methyl)benzene (6h)
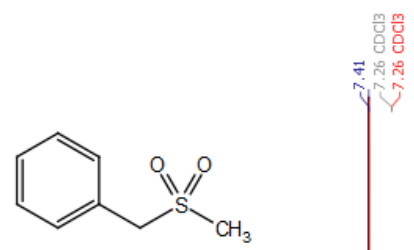

Compound $\mathbf{6 h}$

${ }^{1} \mathrm{H}$ NMR $\left(400 \mathrm{MHz}, \mathrm{CDCl}_{3}\right)$
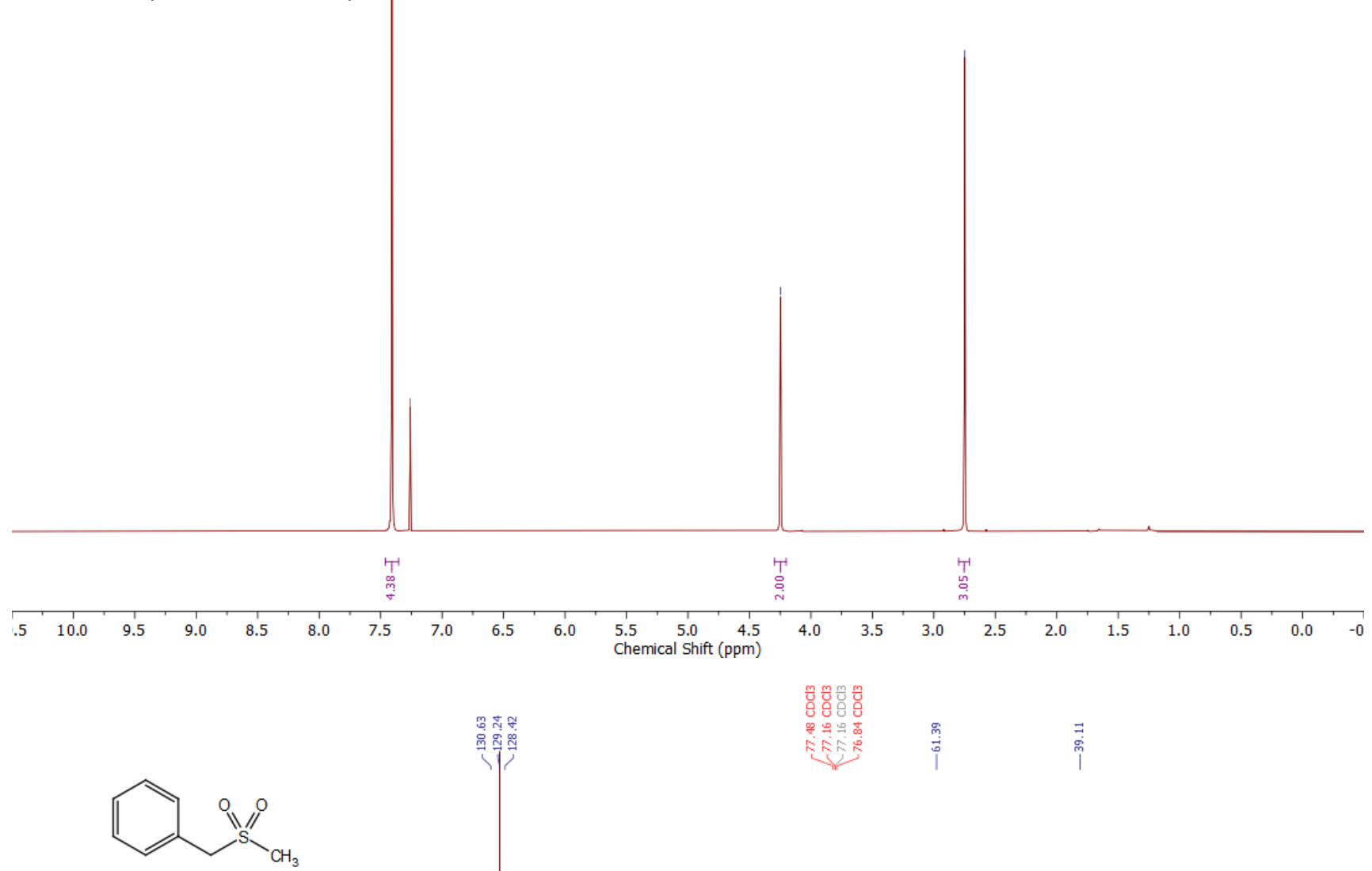

Compound $6 \mathbf{h}$

${ }^{13} \mathrm{C}$ NMR $\left(101 \mathrm{MHz}, \mathrm{CDCl}_{3}\right)$ 
NMR spectra of (2-(methylsulfonyl)ethyl)benzene (6i)

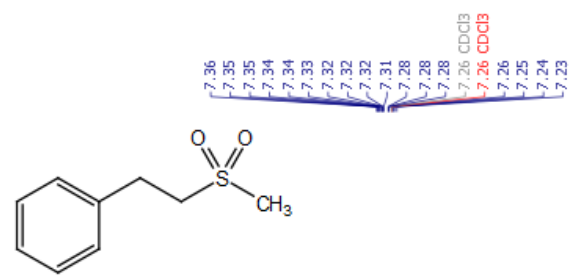

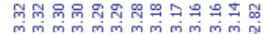

Compound $6 \mathbf{i}$

${ }^{1} \mathrm{H} \mathrm{NMR}\left(400 \mathrm{MHz}, \mathrm{CDCl}_{3}\right)$

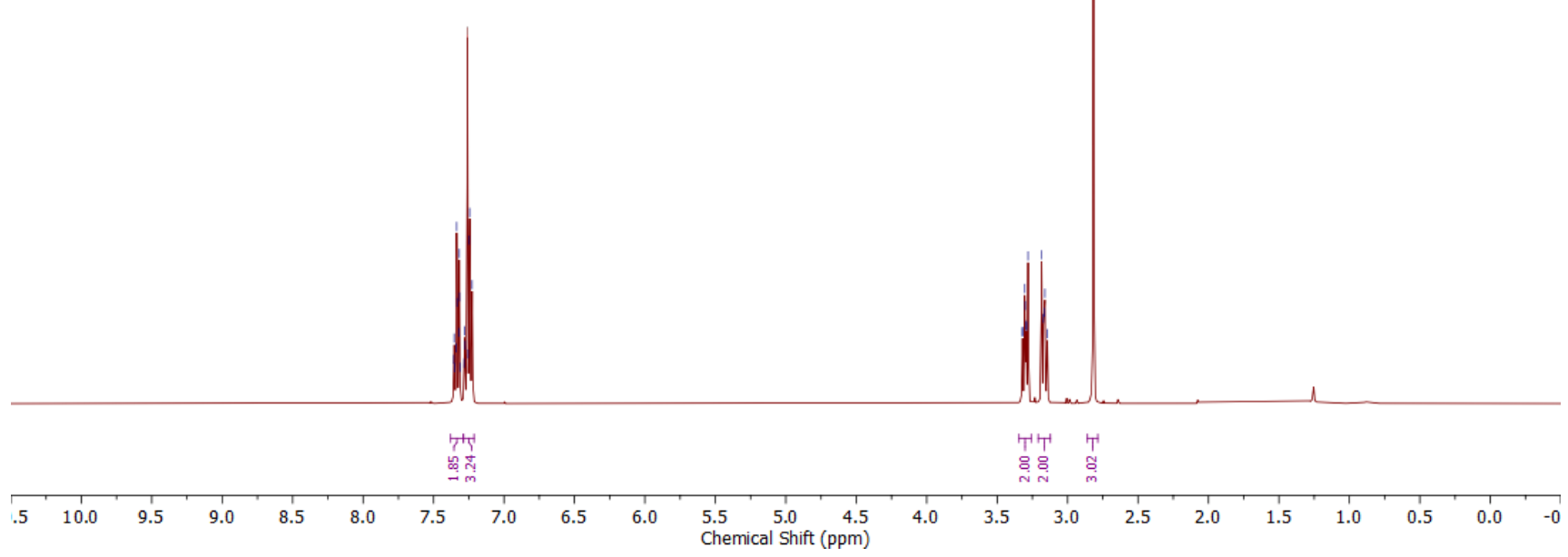

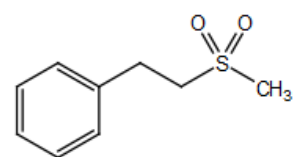

Compound $\mathbf{6 i}$

${ }^{13} \mathrm{C}$ NMR (101 MHz, $\left.\mathrm{CDCl}_{3}\right)$

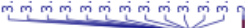


NMR spectra of benzyl 4-(fluorosulfonyl)piperidine-1-carboxylate (7a)
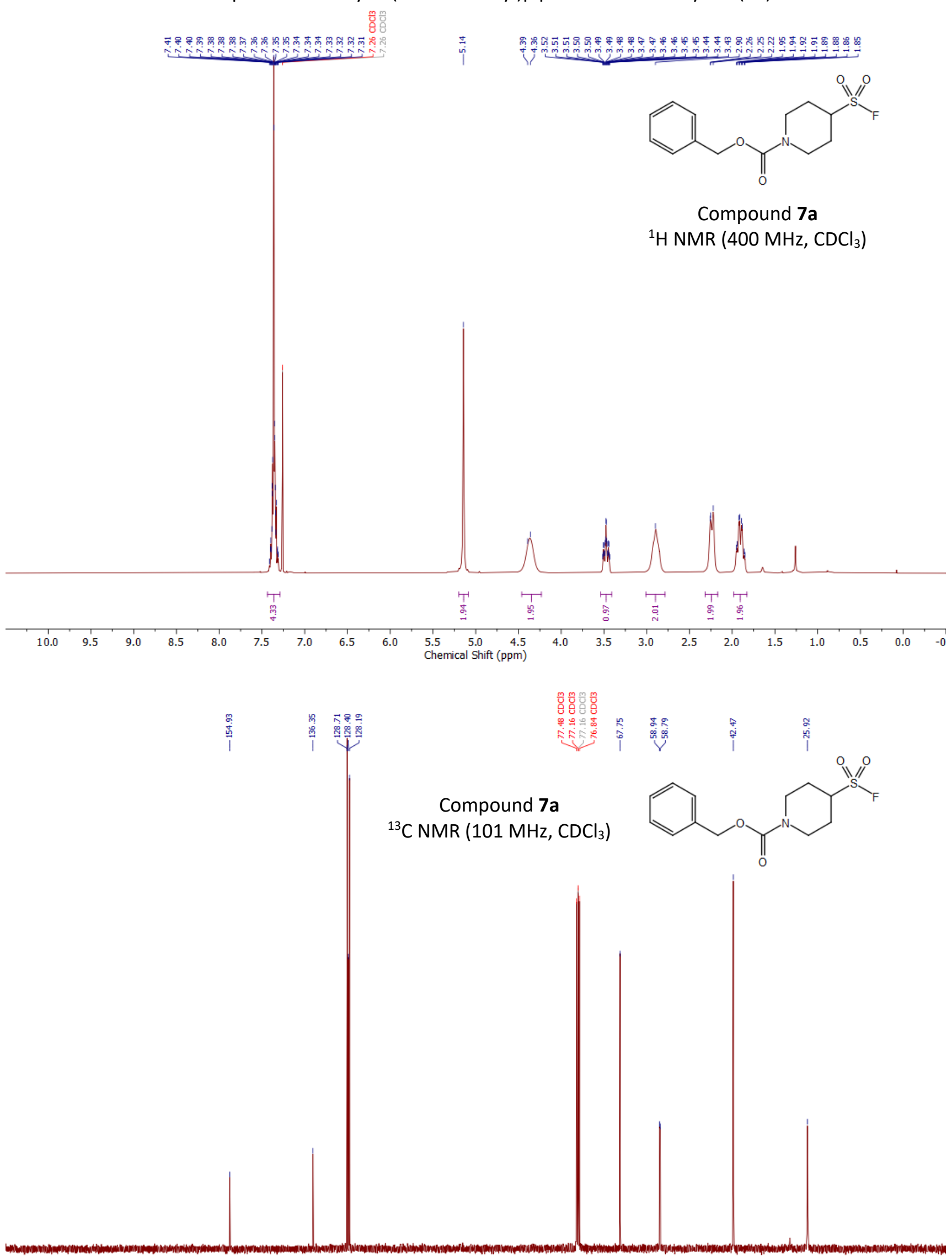

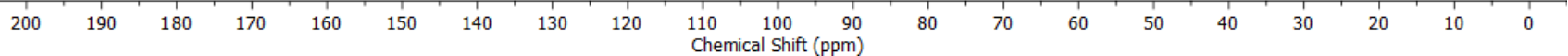




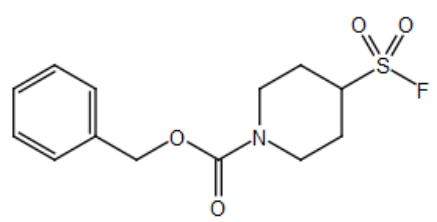

Compound 7a

${ }^{19} \mathrm{~F}$ NMR $\left(377 \mathrm{MHz}, \mathrm{CDCl}_{3}\right)$ 
NMR spectra of phenylmethanesulfonyl fluoride (7b)

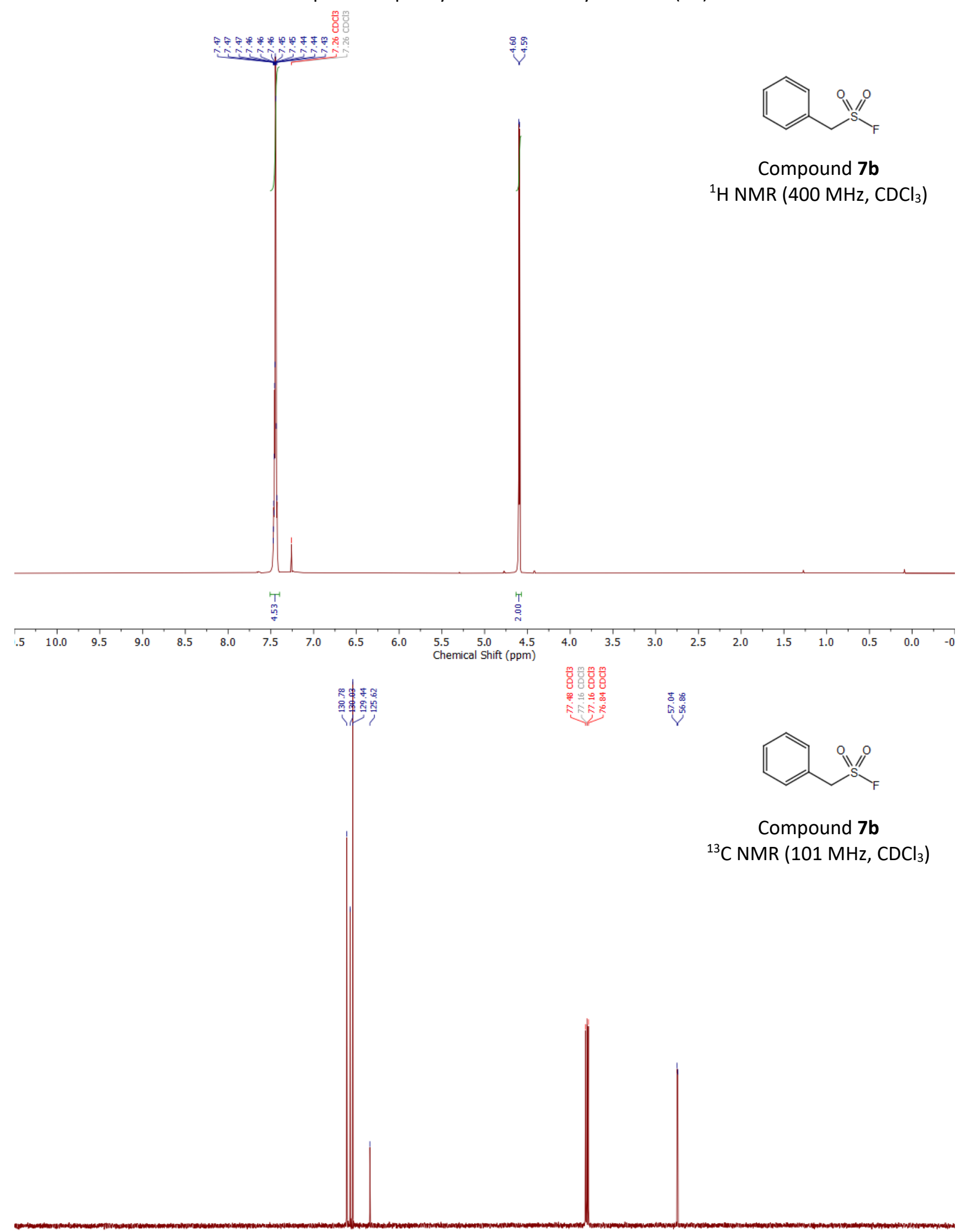

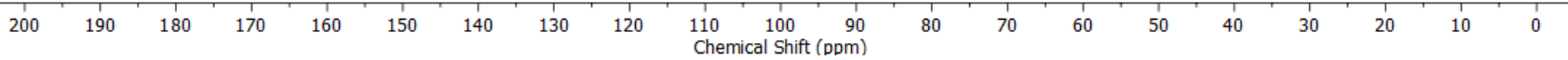




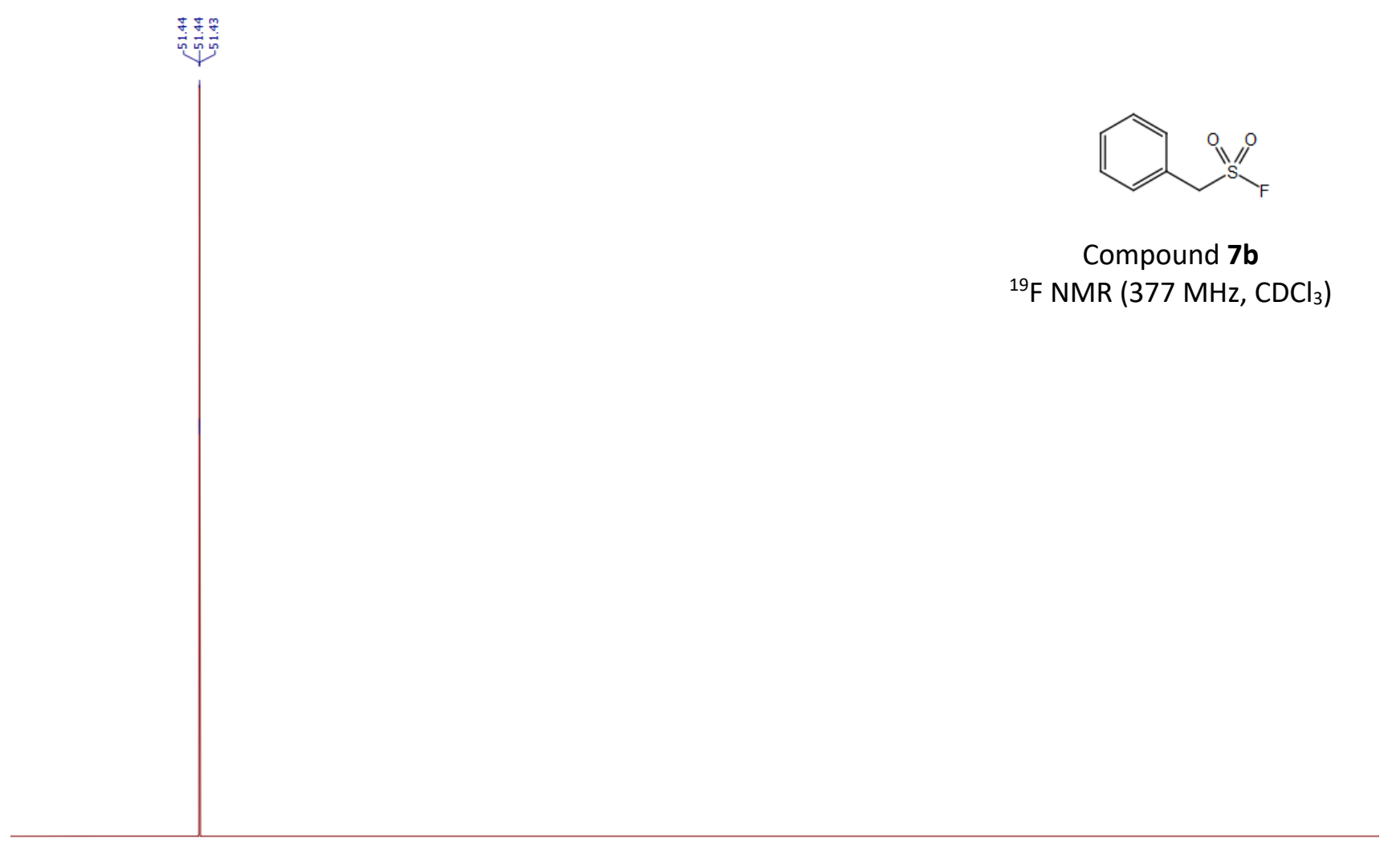

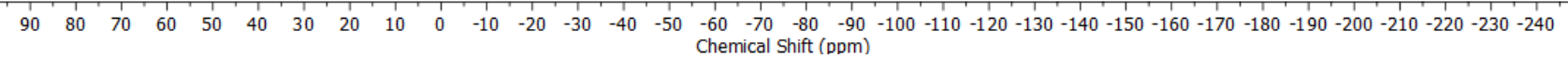


NMR spectra of 2-phenylethane-1-sulfonyl fluoride (7c)
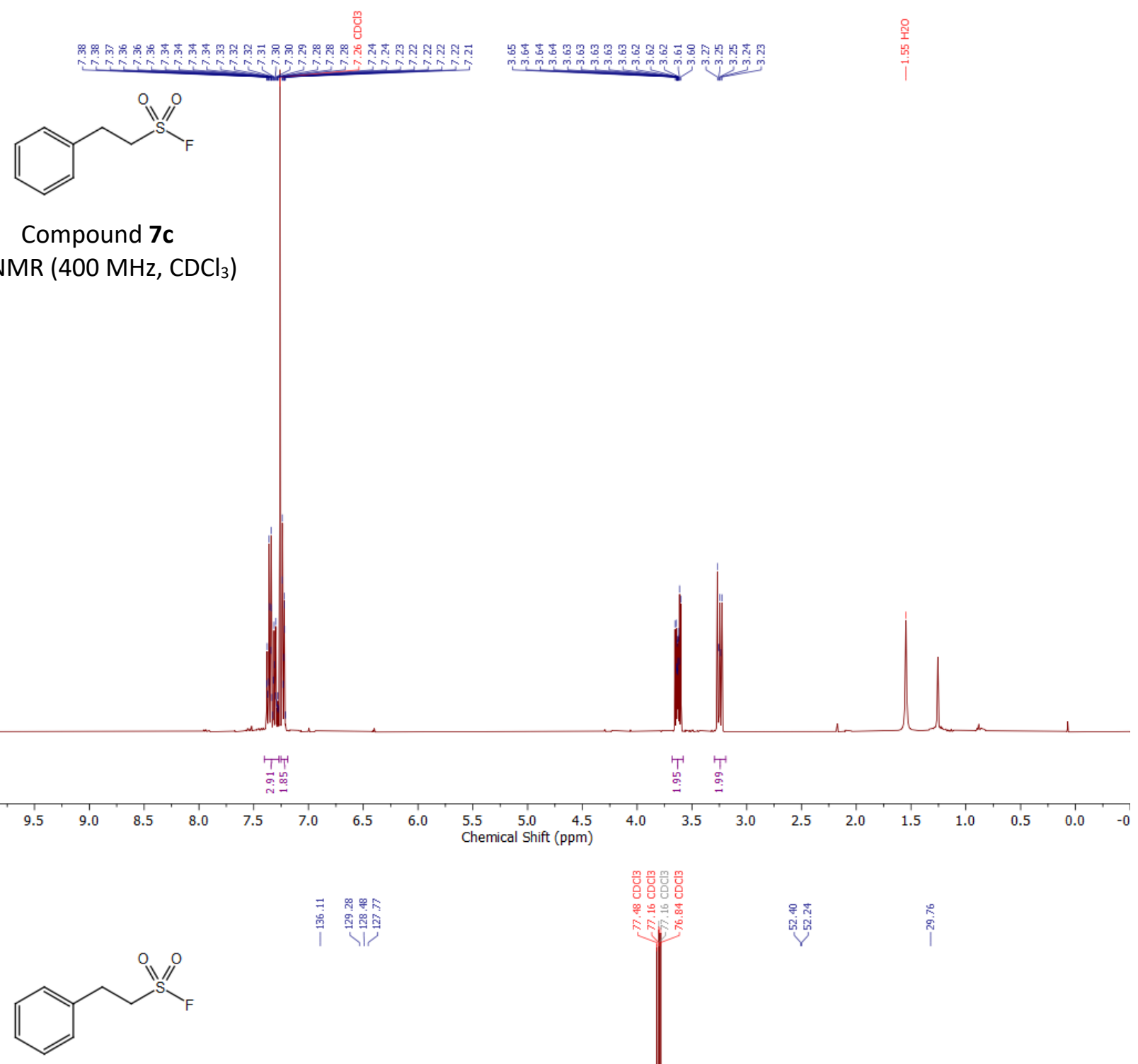

Compound 7c

${ }^{13} \mathrm{C}$ NMR (101 MHz, $\left.\mathrm{CDCl}_{3}\right)$

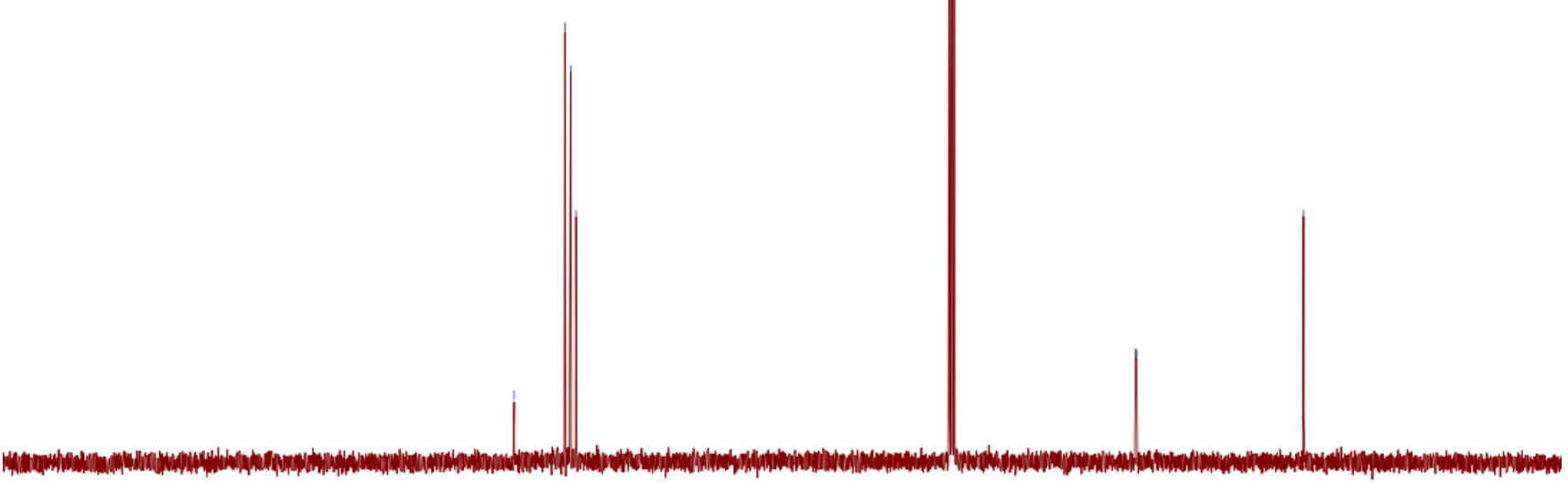

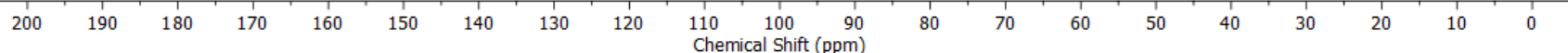




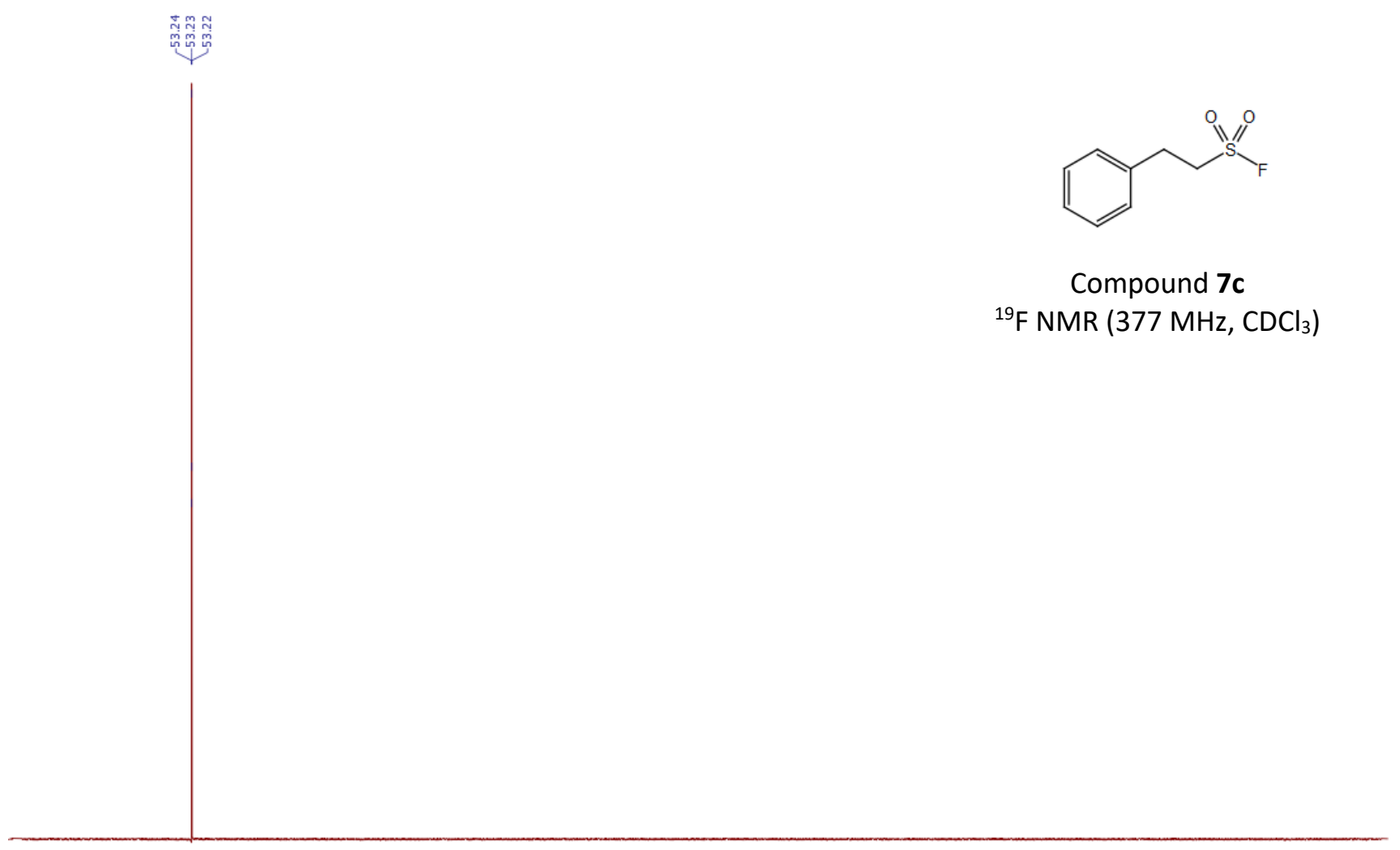

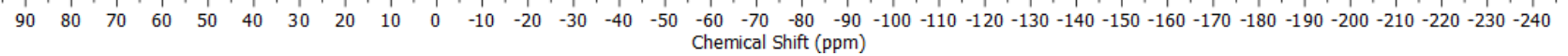


NMR spectra of benzyl 4-sulfamoylpiperidine-1-carboxylate (8a)

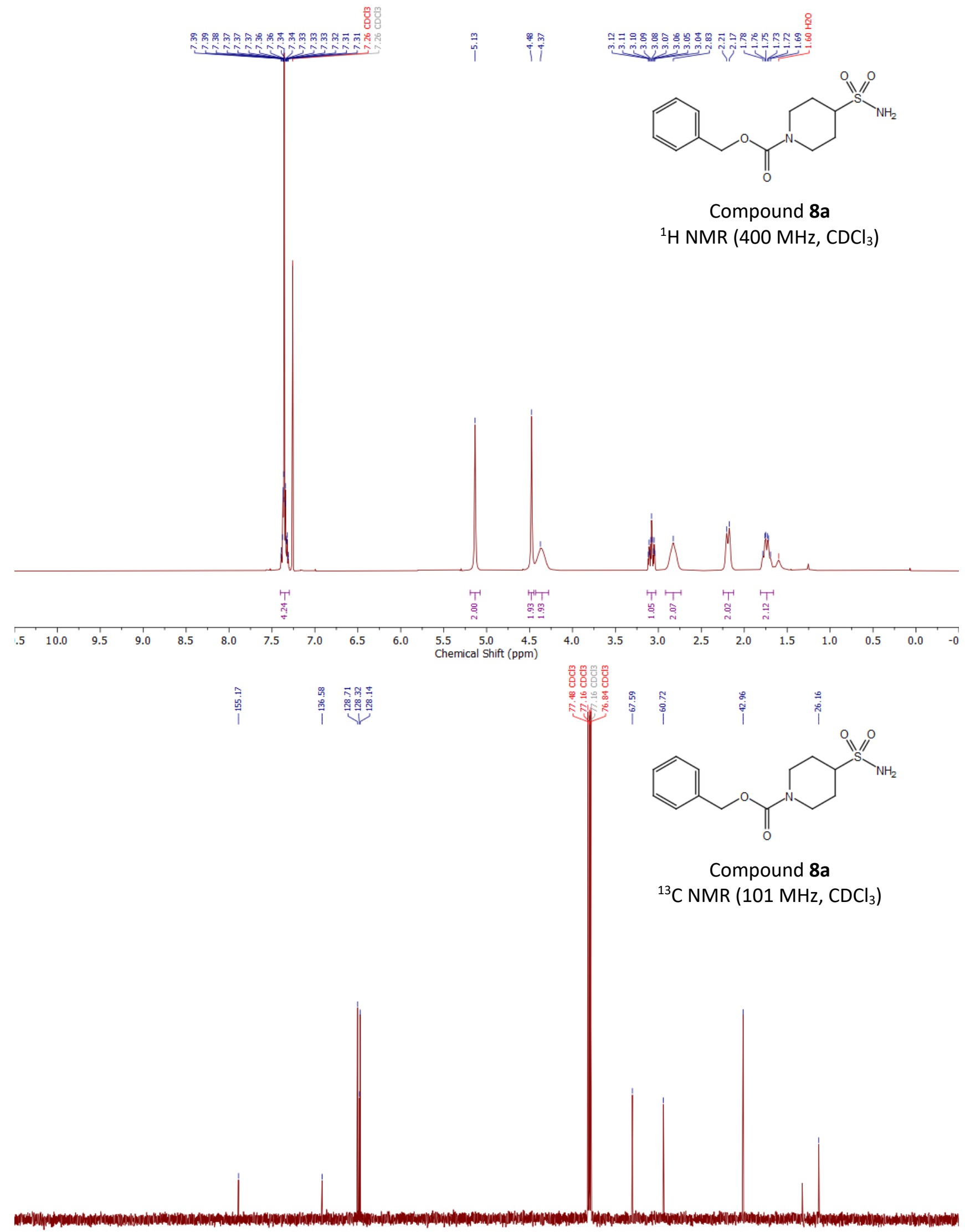

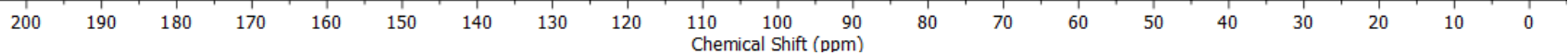


NMR spectra of phenylmethanesulfonamide (8b)
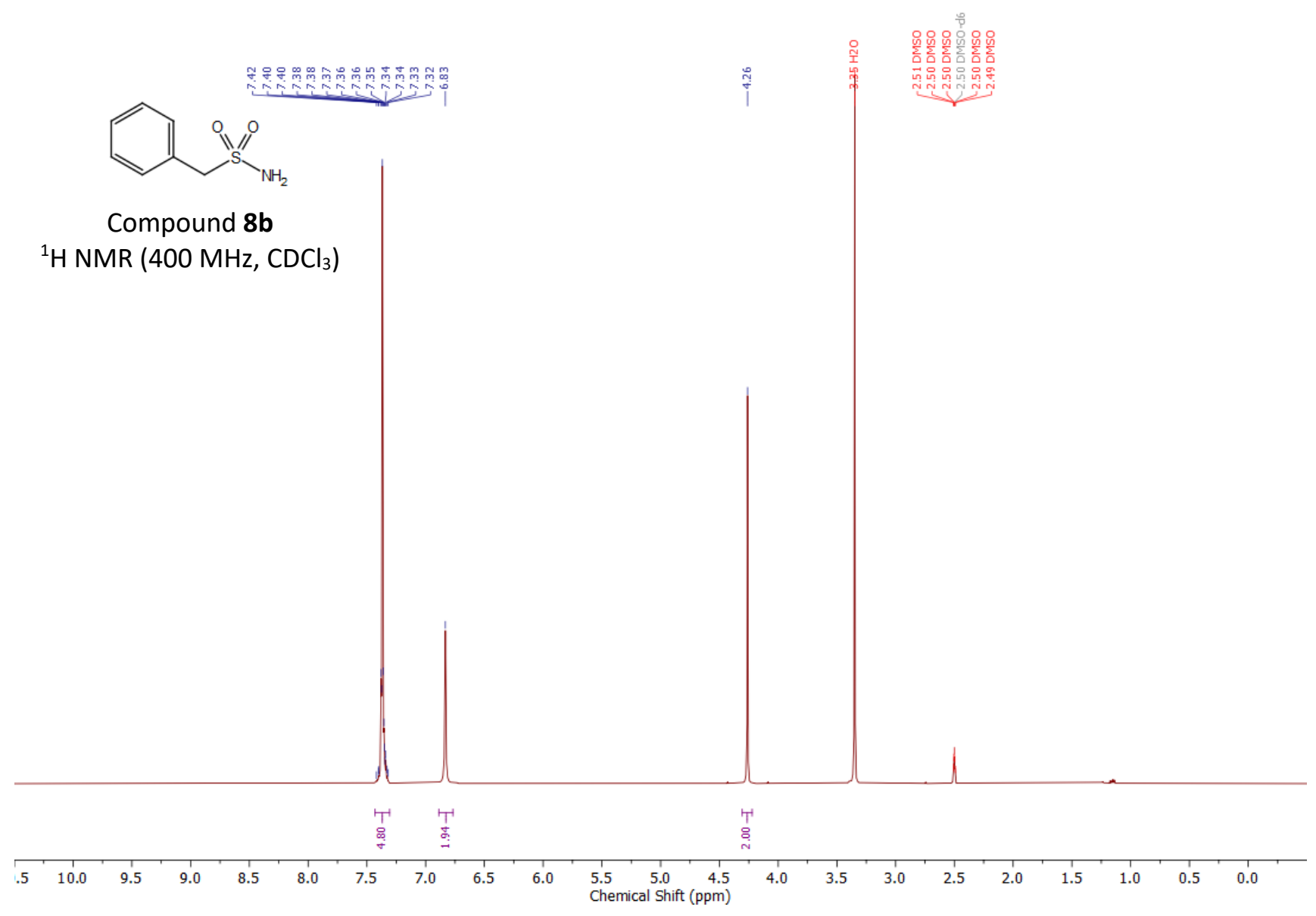

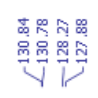
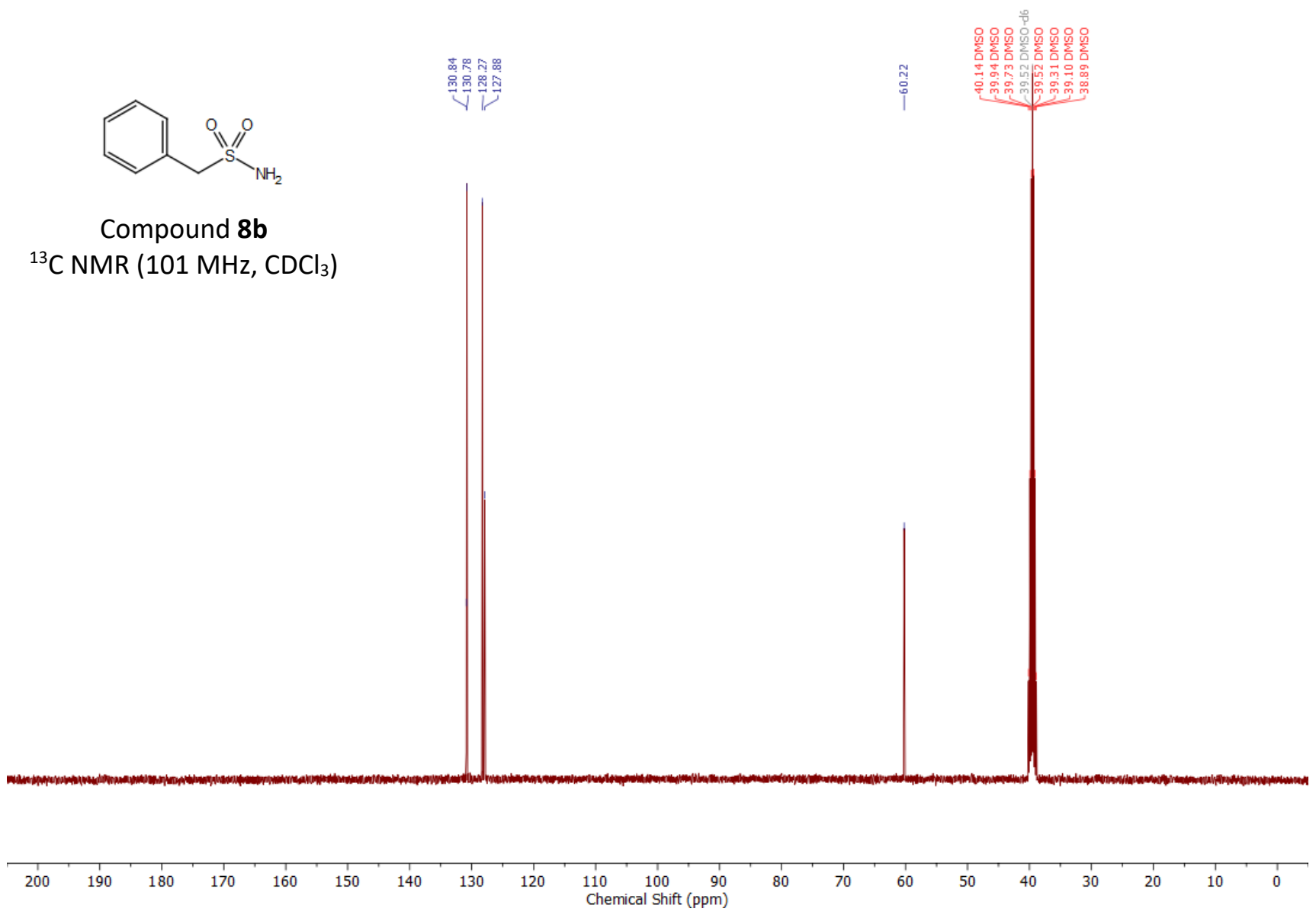

S-234 
NMR spectra of 2-phenylethane-1-sulfonamide (8c)
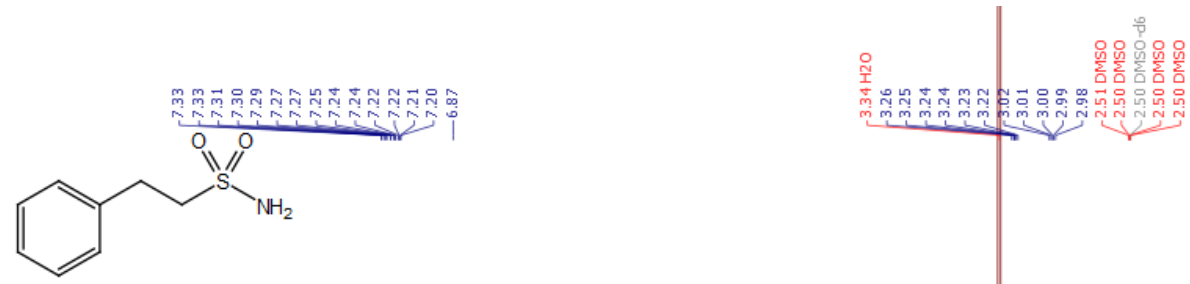

Compound 8c

${ }^{1} \mathrm{H}$ NMR $\left(400 \mathrm{MHz}, \mathrm{CDCl}_{3}\right)$
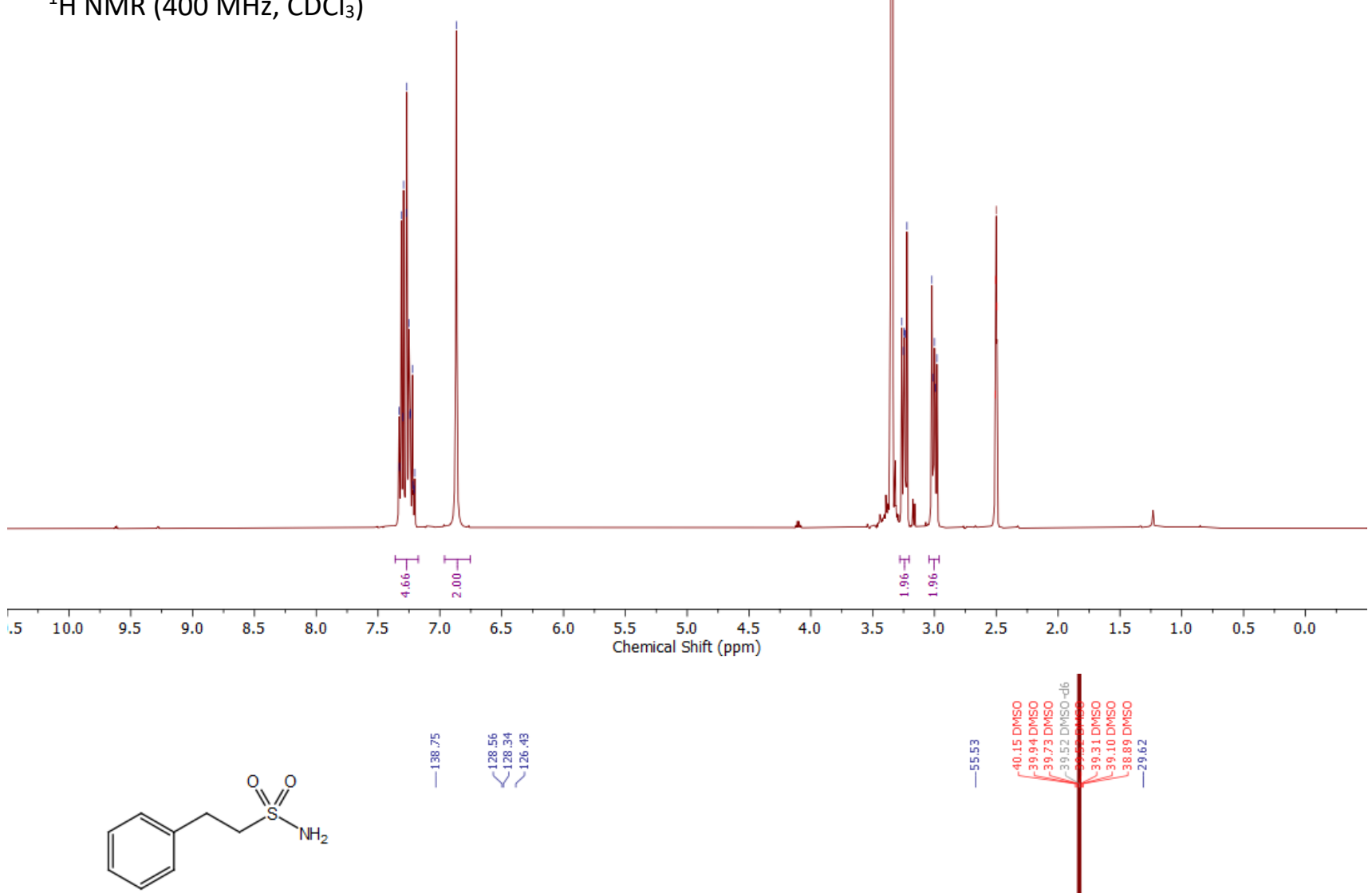

Compound 8c

$\left.{ }^{13} \mathrm{C} \mathrm{NMR} \mathrm{(101} \mathrm{MHz,} \mathrm{CDCl}_{3}\right)$

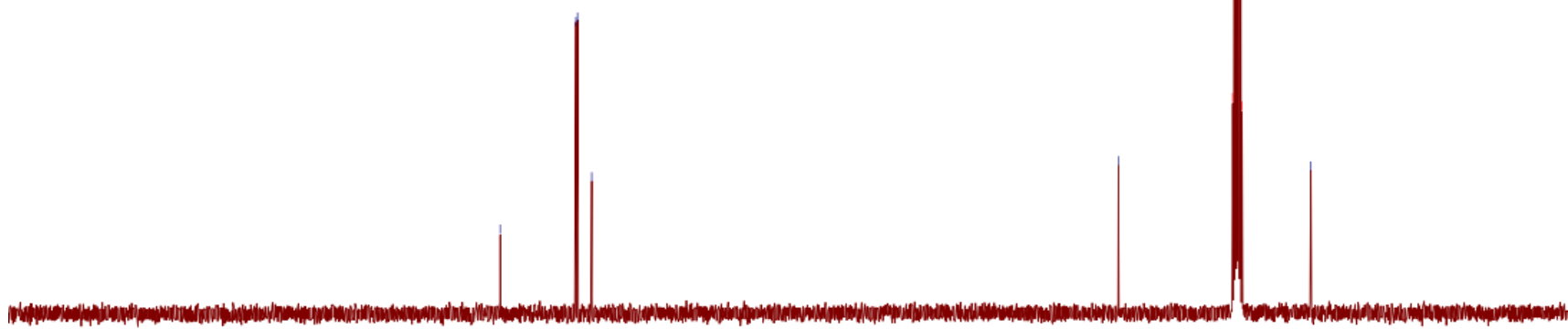

$\begin{array}{llllllllll}200 & 190 & 180 & 170 & 160 & 150 & 140 & 130 & 120 & \begin{array}{c}110 \\ \text { Chemical Shift (ppm) }\end{array}\end{array}$ 
NMR spectra of benzyl 4-(morpholinosulfonyl)piperidine-1-carboxylate (9a)

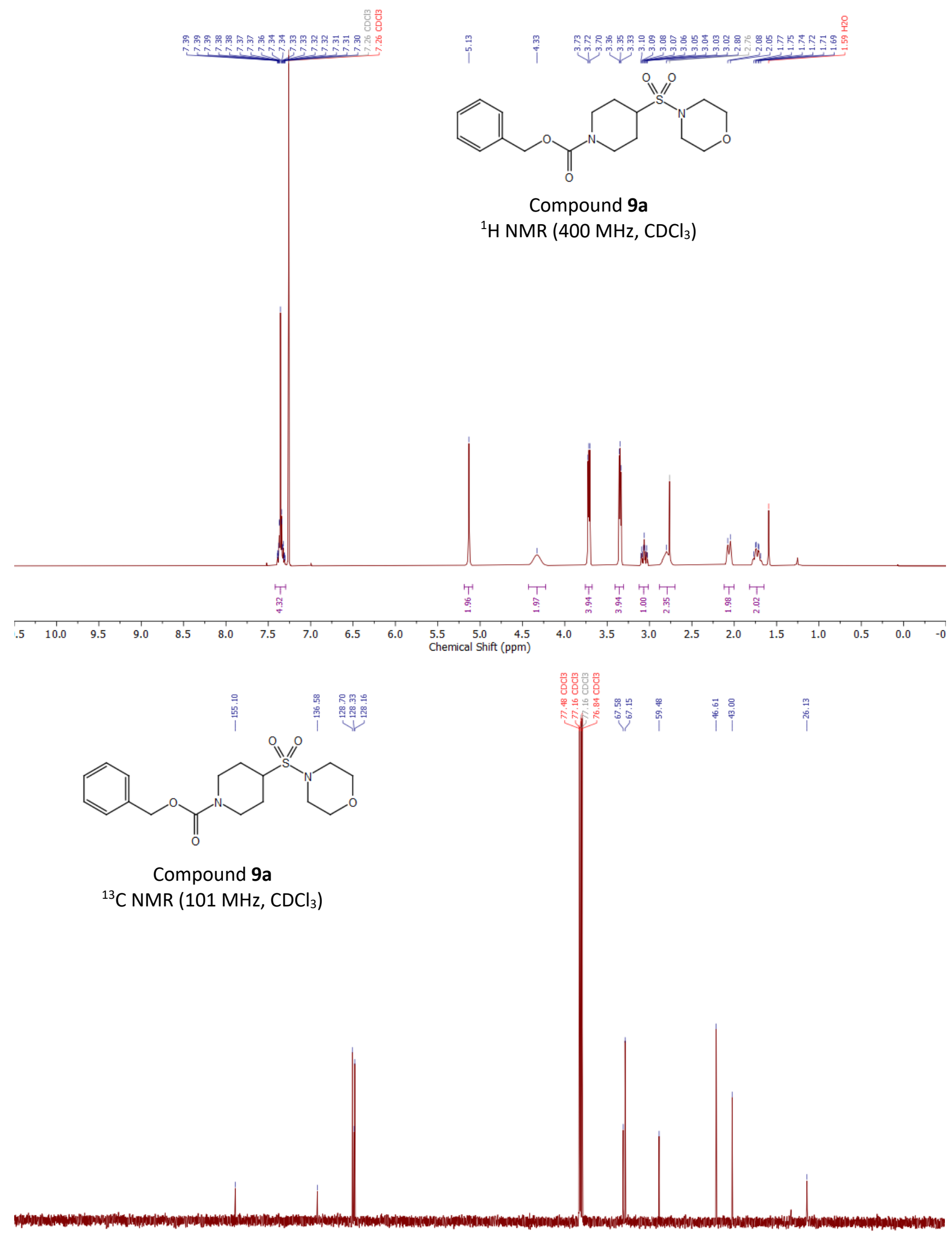

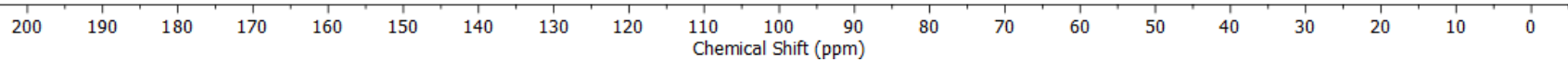


NMR spectra of benzyl 4-((4-(2-chlorodibenzo[b,f][1,4]oxazepin-11-yl)piperazin-1-yl)sulfonyl)piperidine-1carboxylate (9b)

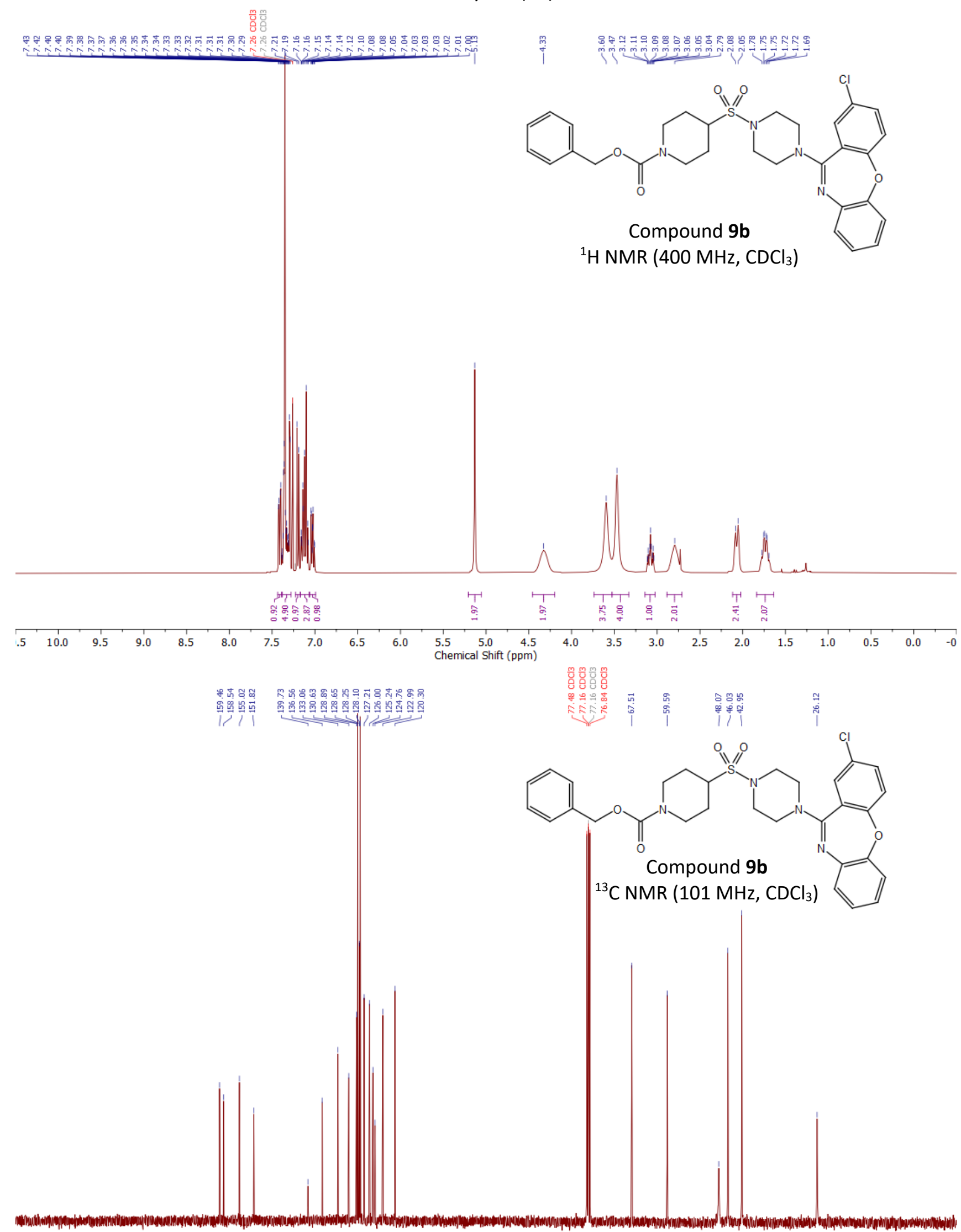

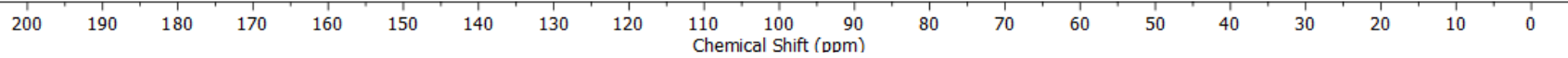


NMR spectra of benzyl 4-((6,7-dihydrothieno[3,2-c]pyridin-5(4H)-yl)sulfonyl)piperidine-1-carboxylate (9c)

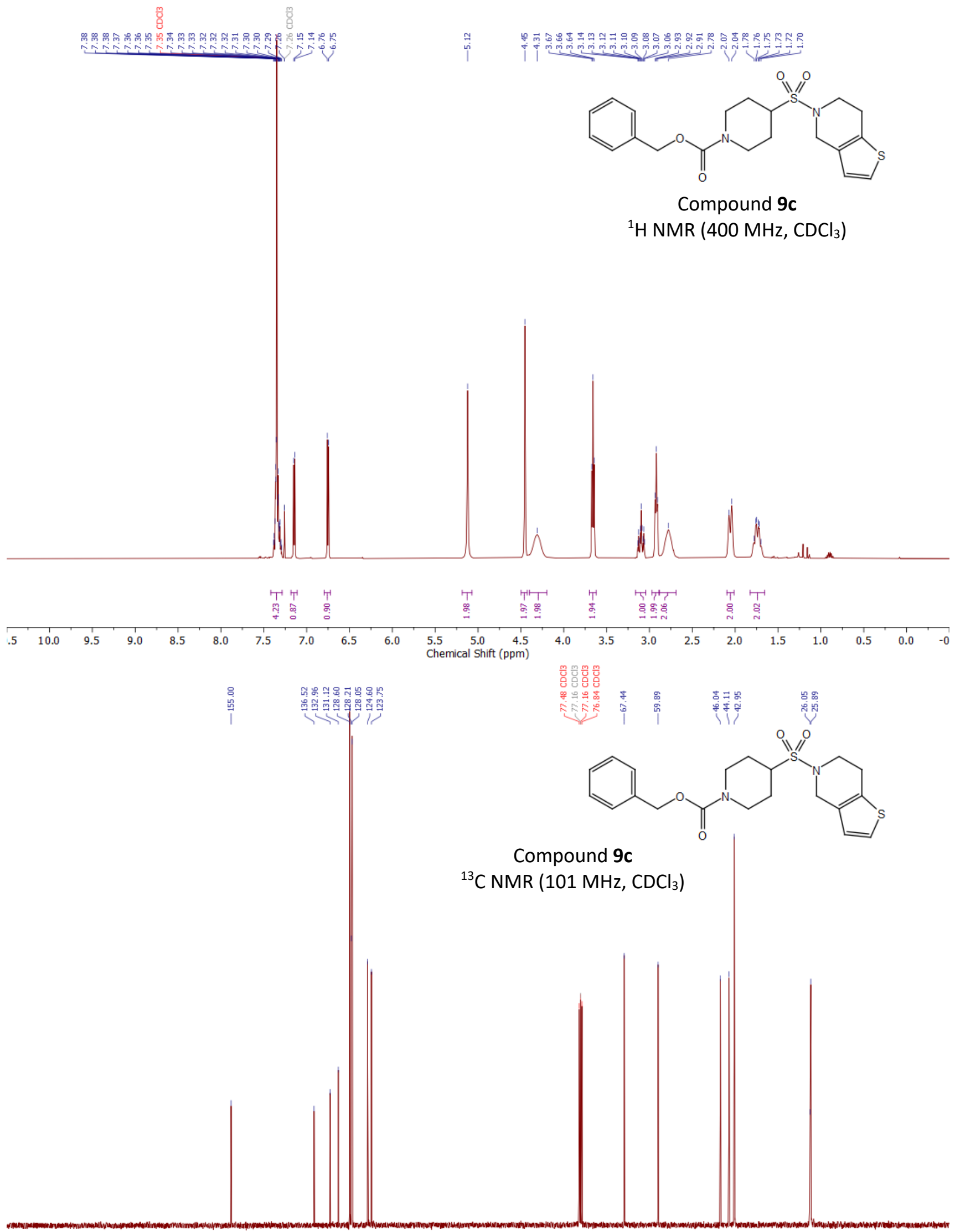

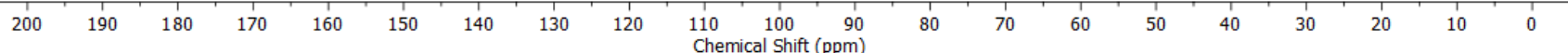


NMR spectra of benzyl 4-((1H-imidazol-1-yl)sulfonyl)piperidine-1-carboxylate (9d)

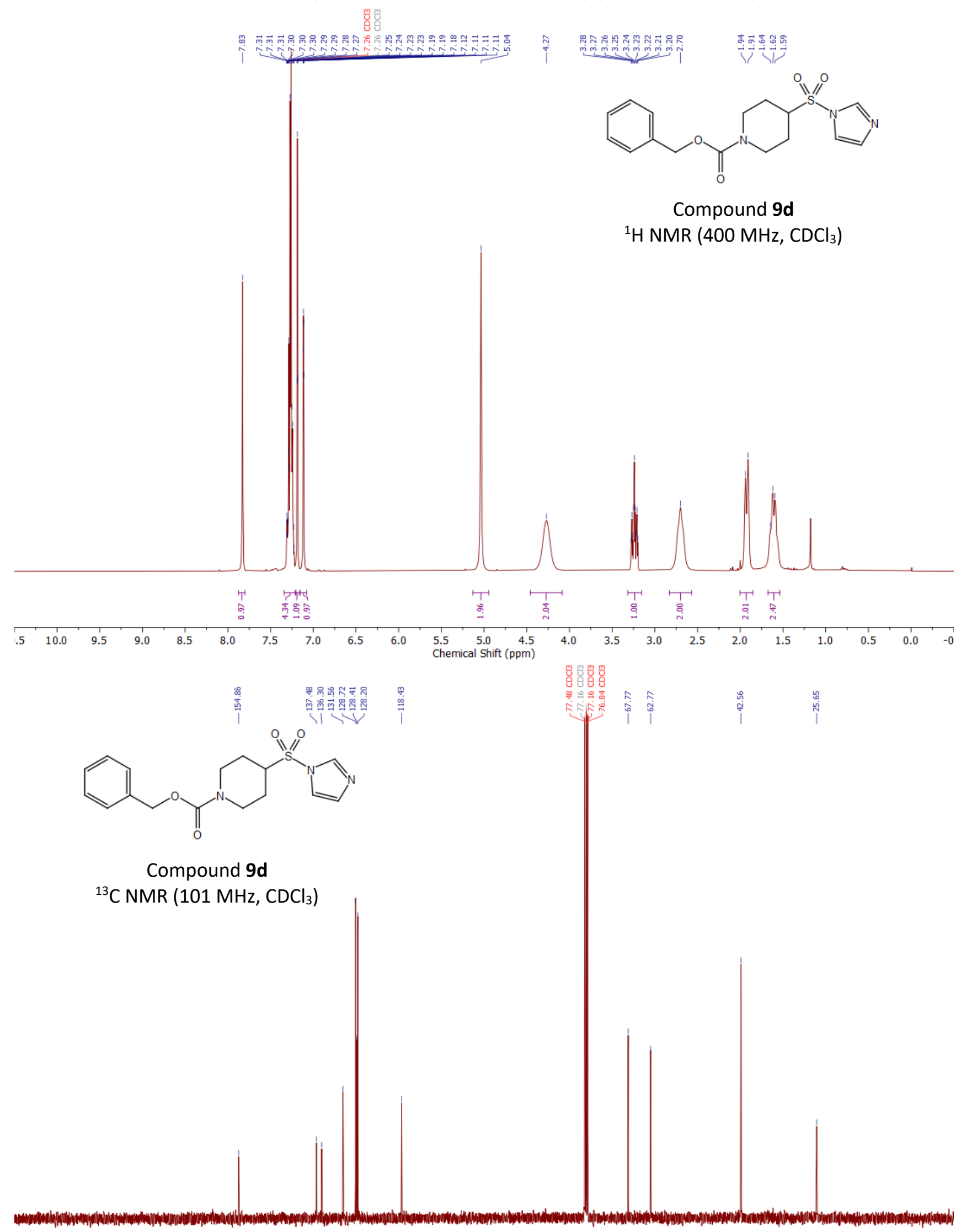

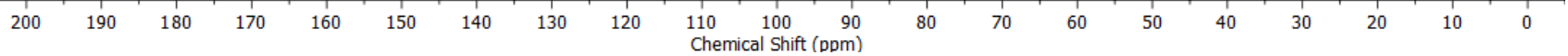


NMR spectra of 4-(benzylsulfonyl)morpholine (9e)

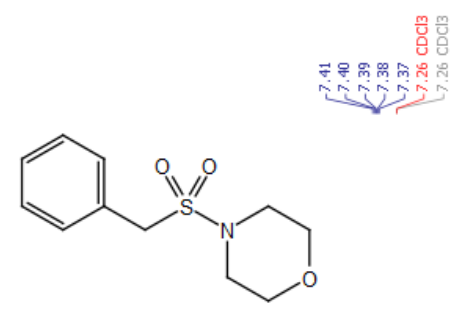

Compound 9e

${ }^{1} \mathrm{H} \mathrm{NMR}\left(400 \mathrm{MHz}, \mathrm{CDCl}_{3}\right)$
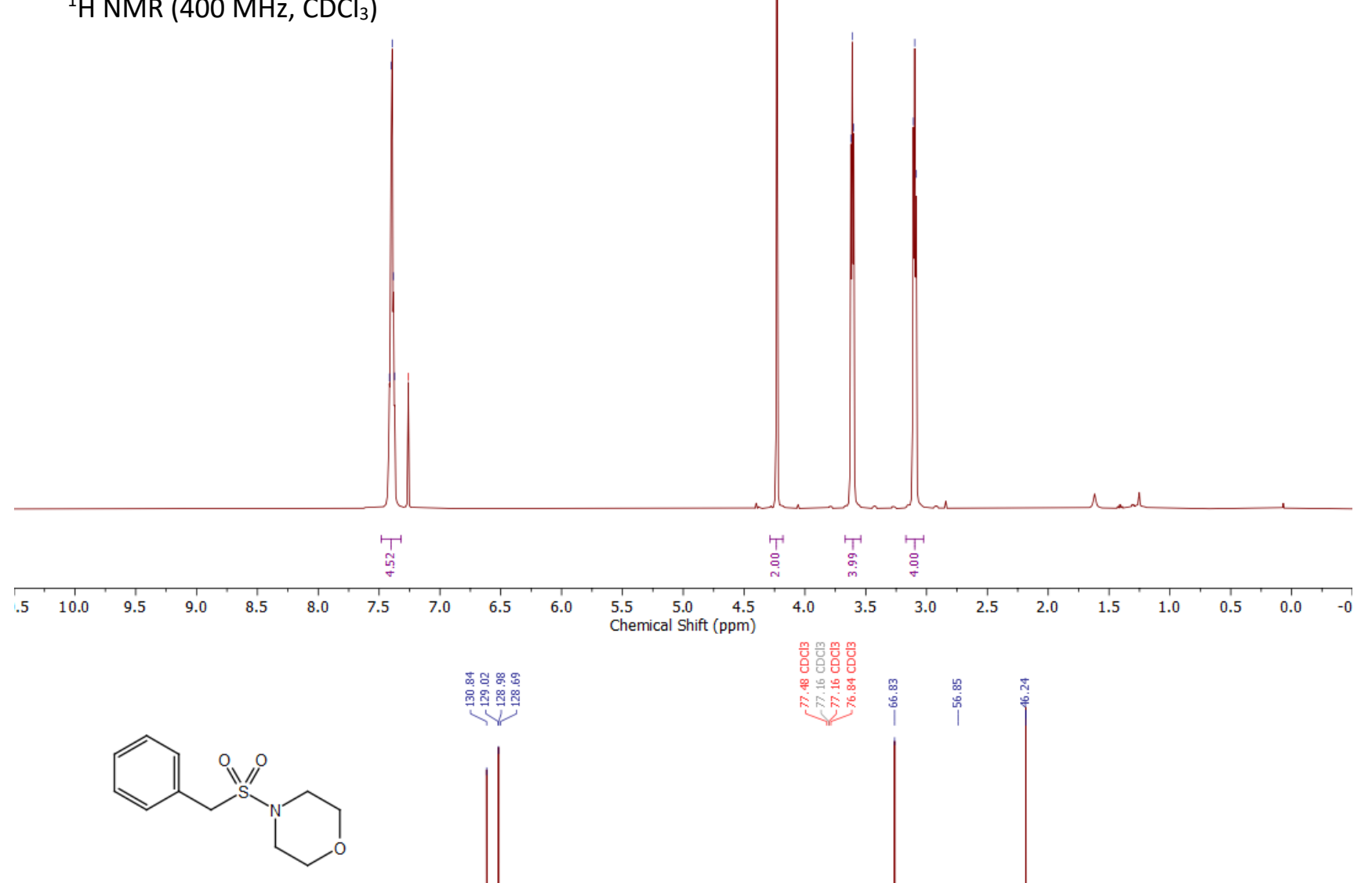

Compound 9e

$\left.{ }^{13} \mathrm{C} \mathrm{NMR} \mathrm{(101} \mathrm{MHz,} \mathrm{CDCl}_{3}\right)$ 
NMR spectra of 4-(phenethylsulfonyl)morpholine (9f)

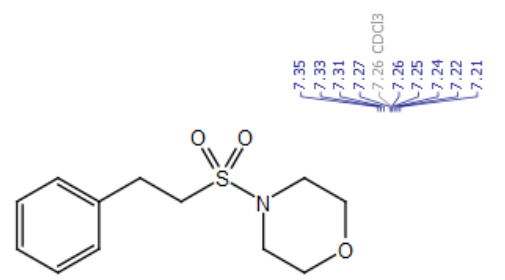

Compound $9 f$

${ }^{1} \mathrm{H}$ NMR $\left(400 \mathrm{MHz}, \mathrm{CDCl}_{3}\right)$
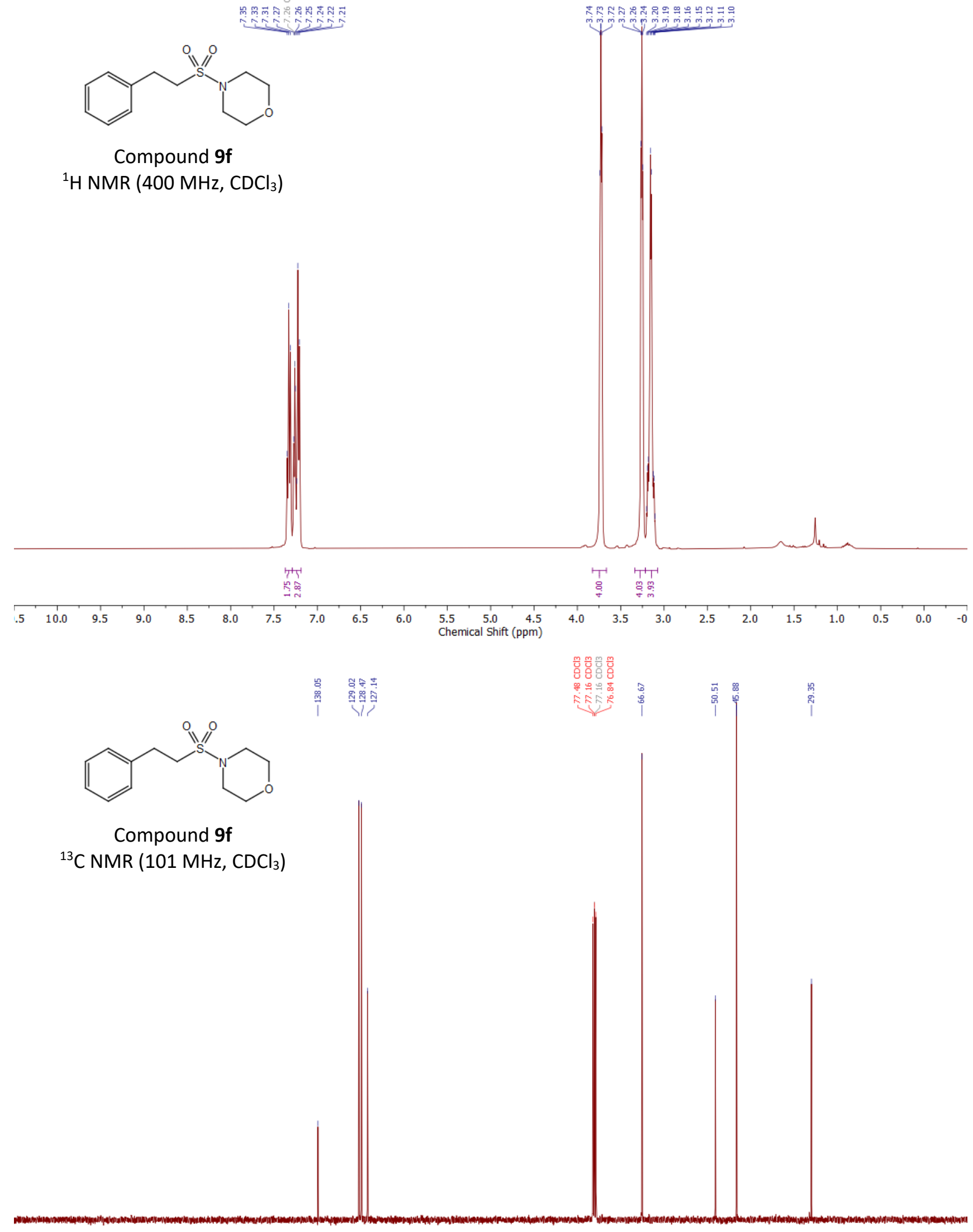

Compound 9f

${ }^{13} \mathrm{C}$ NMR $\left(101 \mathrm{MHz}, \mathrm{CDCl}_{3}\right)$

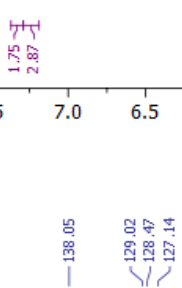

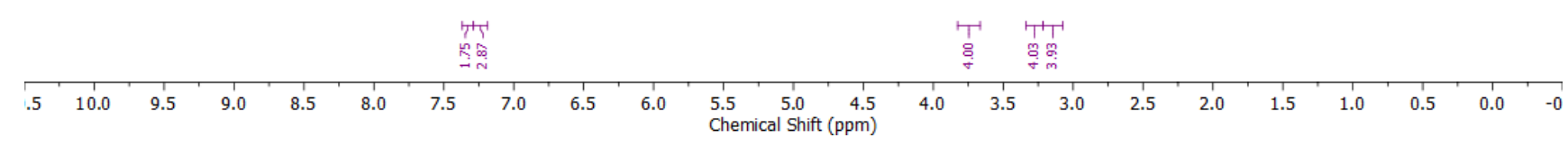

$\begin{array}{llllllllll}200 & 190 & 180 & 170 & 160 & 150 & 140 & 130 & 120 & \begin{array}{c}110 \\ \text { Chemical Shift (ppm) }\end{array}\end{array}$ 
NMR spectra of benzyl 4-(morpholinosulfonyl)piperidine-1-carboxylate (9g)
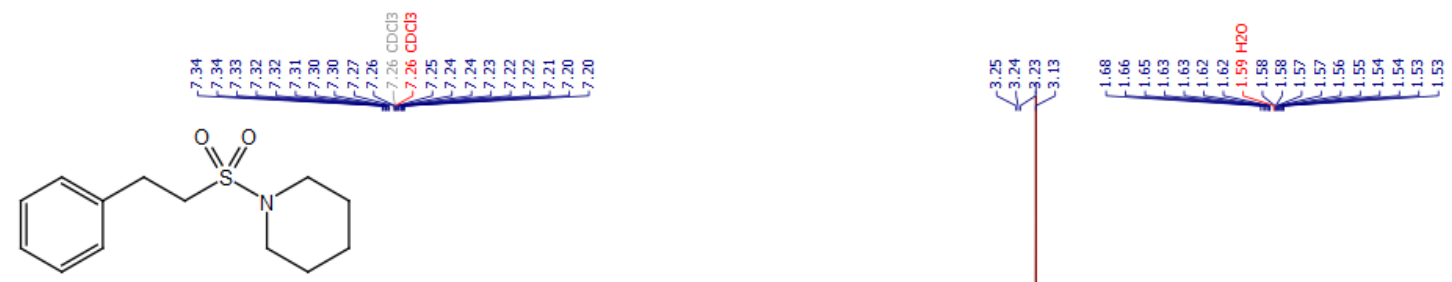

Compound 9g

${ }^{1} \mathrm{H} \mathrm{NMR}\left(400 \mathrm{MHz}, \mathrm{CDCl}_{3}\right)$
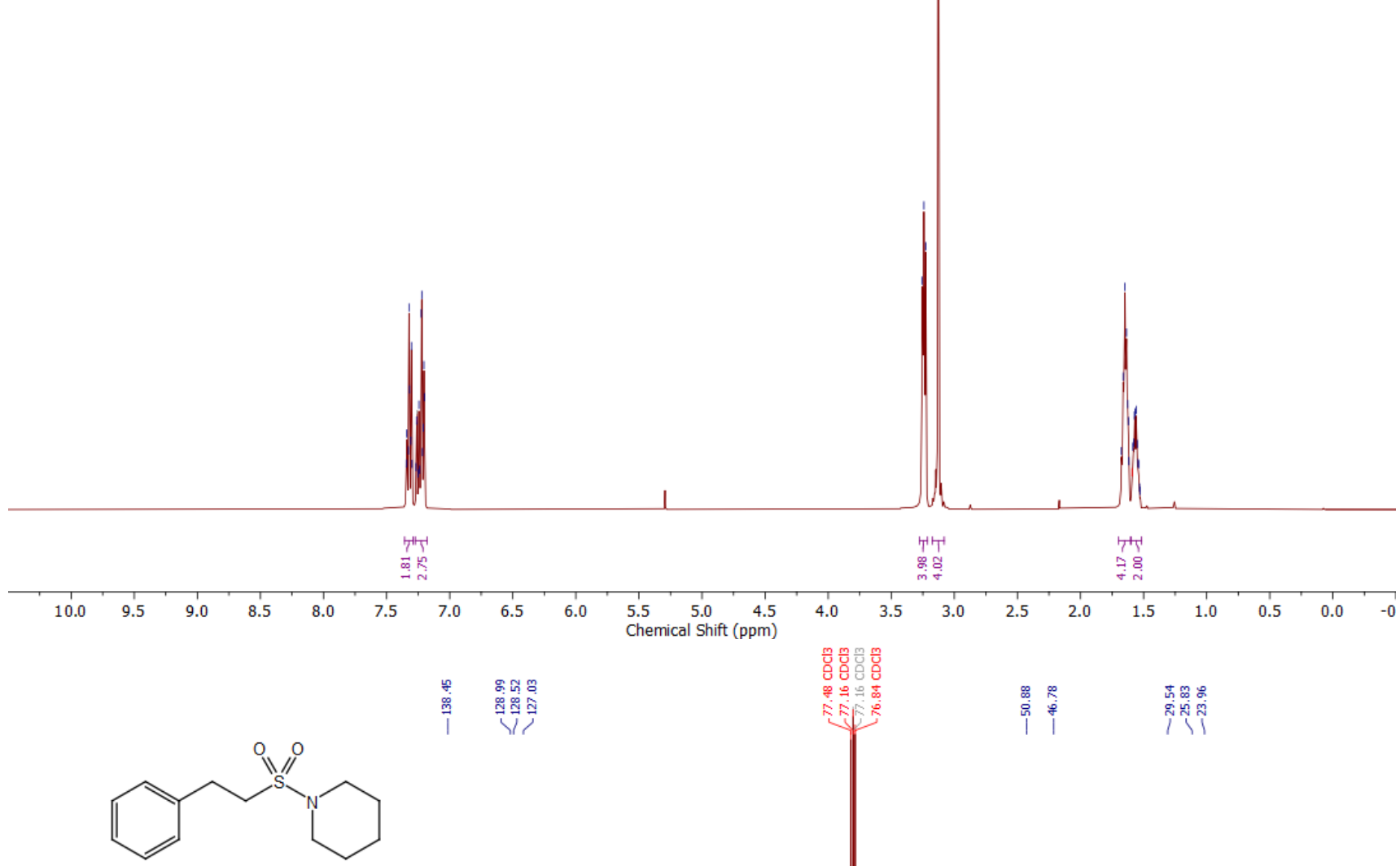

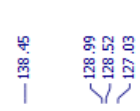

Compound 9g

${ }^{13} \mathrm{C}$ NMR (101 MHz, $\left.\mathrm{CDCl}_{3}\right)$ 
NMR spectra of benzyl 4-(N-benzylsulfamoyl)piperidine-1-carboxylate (10a)

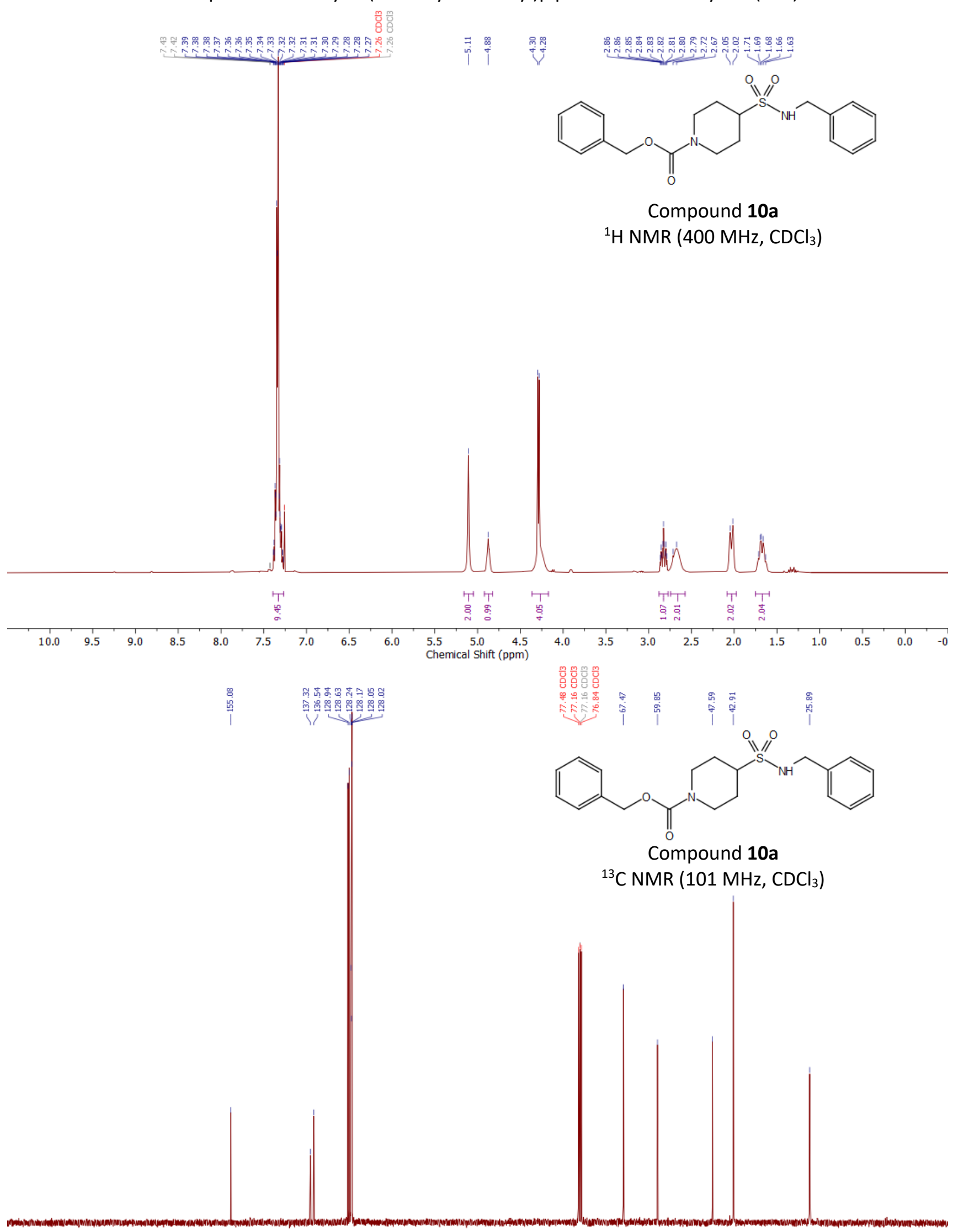

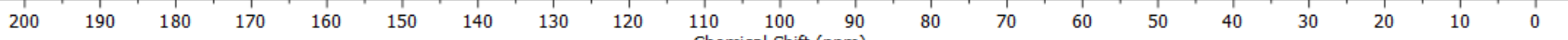


NMR spectra of benzyl 4-(N-(4-methoxyphenyl)sulfamoyl)piperidine-1-carboxylate (10b)

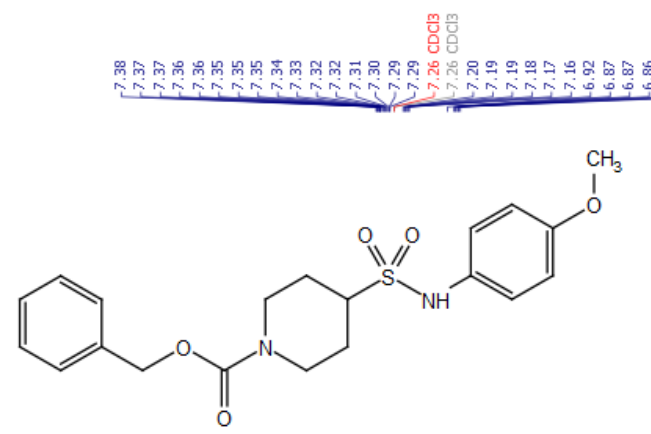

Compound 10b

${ }^{1} \mathrm{H} \mathrm{NMR}\left(400 \mathrm{MHz}, \mathrm{CDCl}_{3}\right)$
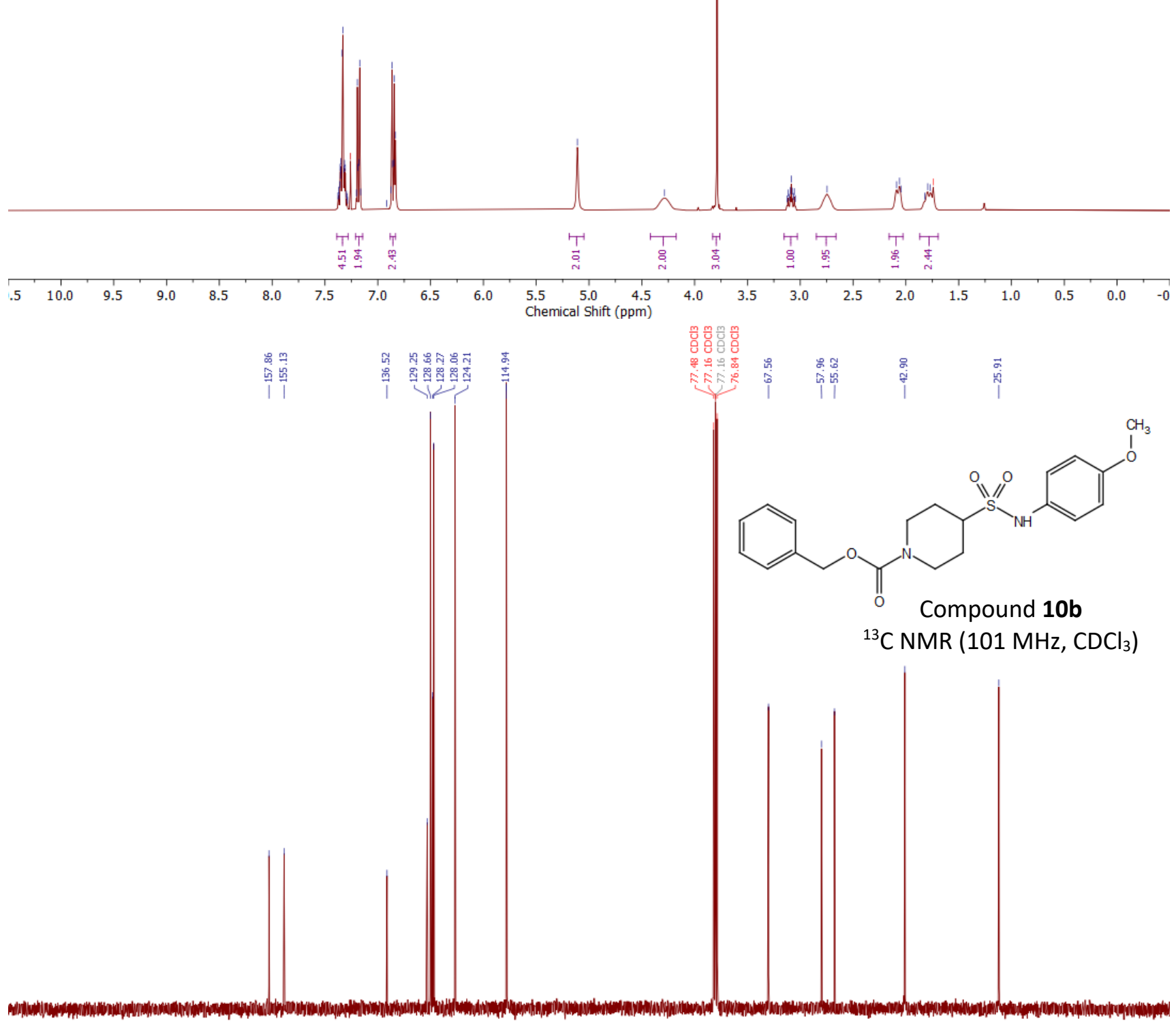

$\begin{array}{llllllllll}200 & 190 & 180 & 170 & 160 & 150 & 140 & 130 & 120 & \begin{array}{c}110 \\ \text { Chemical Shift (ppm) }\end{array}\end{array}$ 
NMR spectra of benzyl (S)-4-(N-(1-methoxy-1-oxo-3-phenylpropan-2-yl)sulfamoyl)piperidine-1-carboxylate (10c)
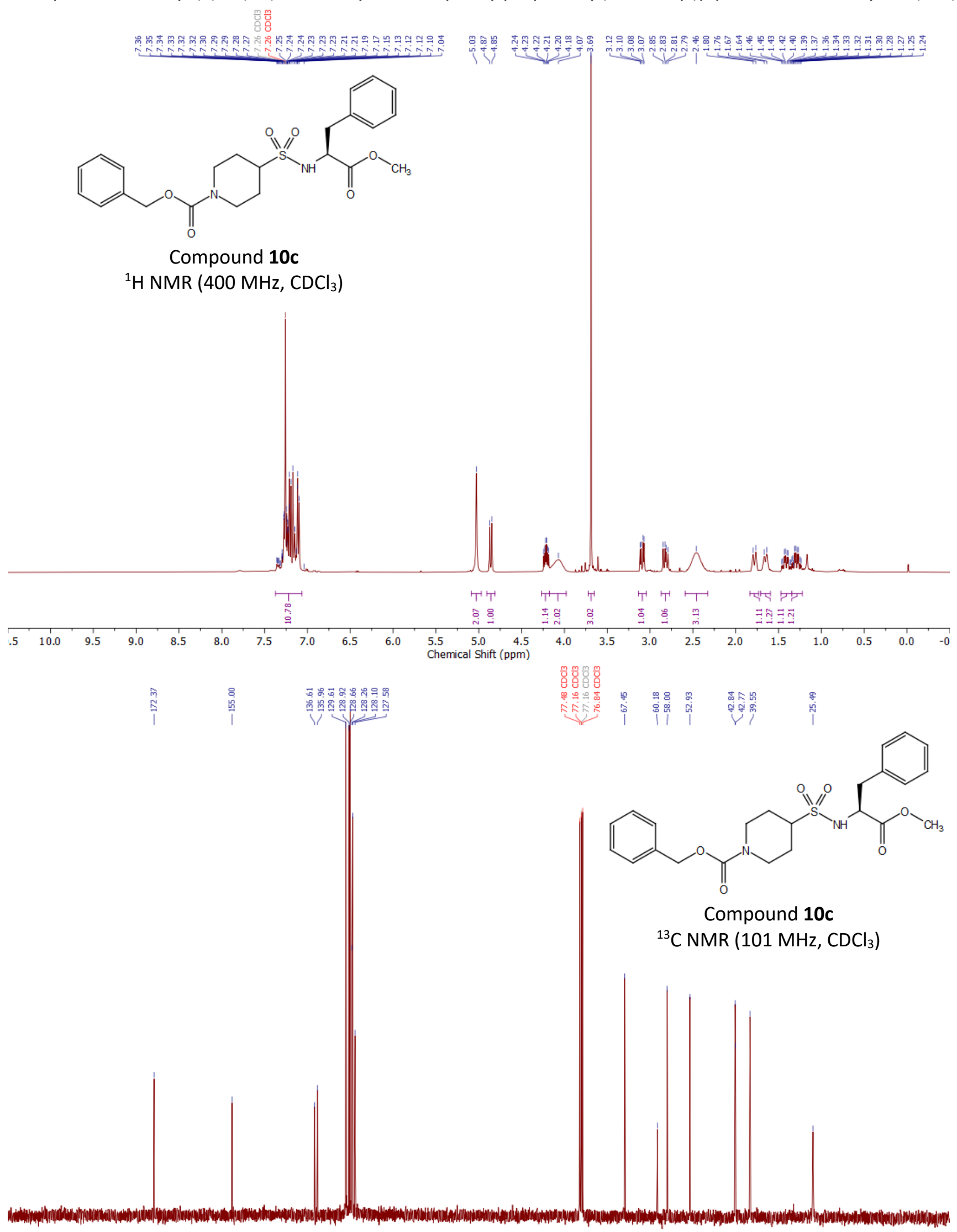

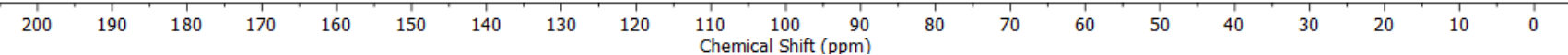


NMR spectra of 1,4-dibenzyl-2,4,6-triphenyl-1,4-dihydropyridine (5b)

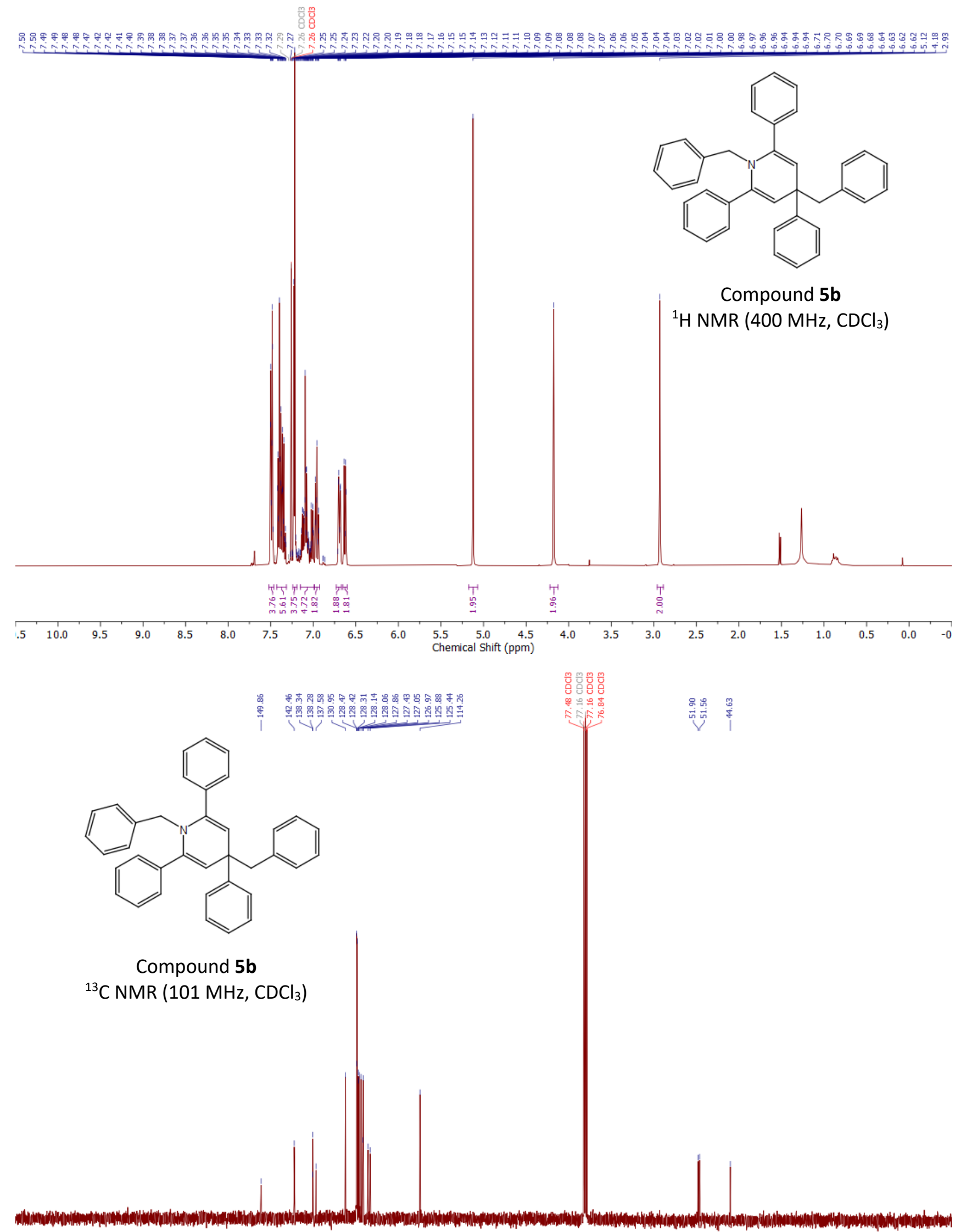

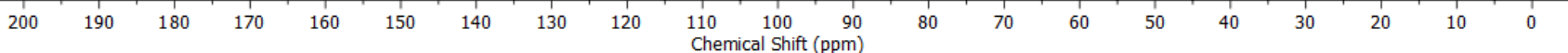


NMR spectra of 1,4-diphenethyl-2,4,6-triphenyl-1,4-dihydropyridine (5c)
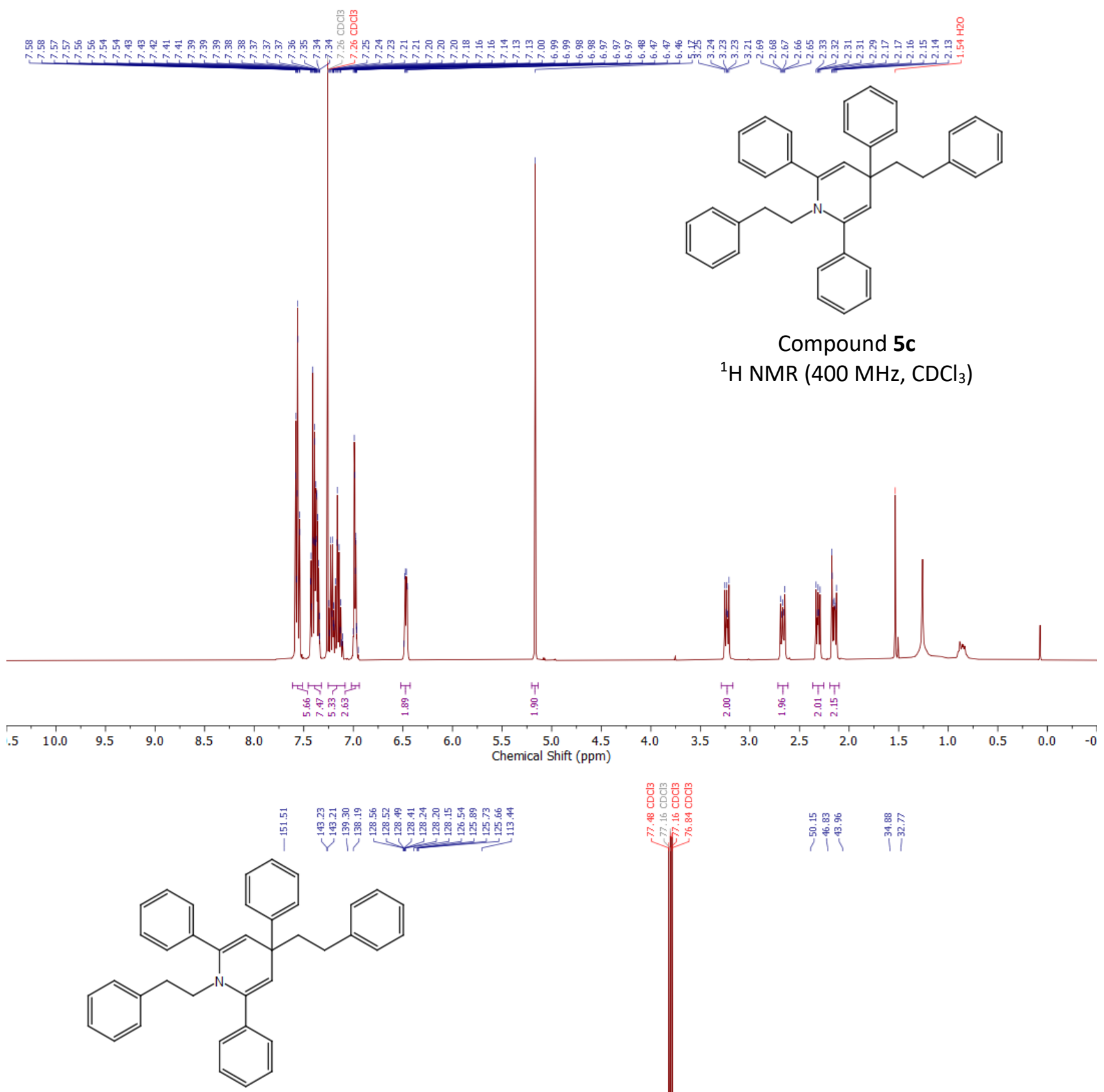

Compound $\mathbf{5 c}$

${ }^{13} \mathrm{C}$ NMR $\left(101 \mathrm{MHz}, \mathrm{CDCl}_{3}\right)$

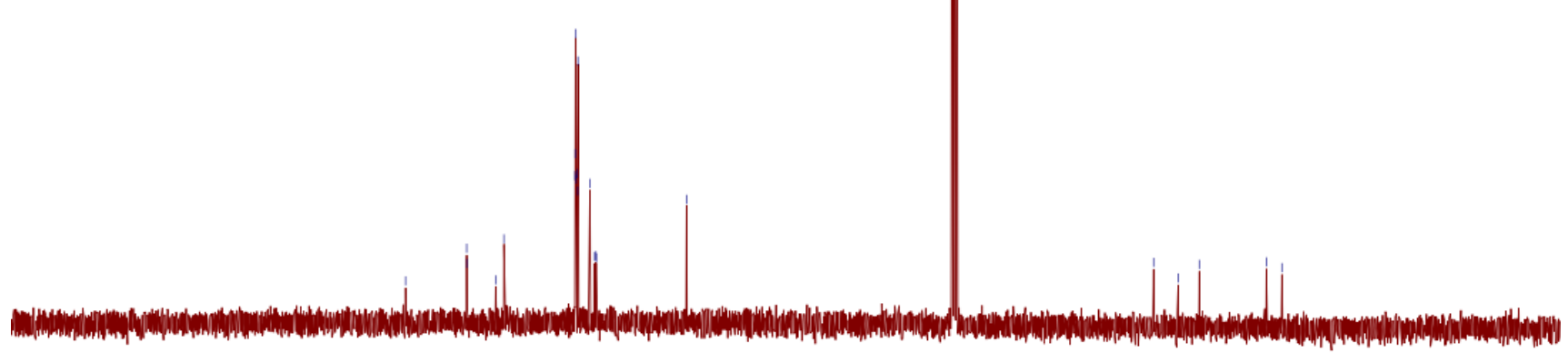

$\begin{array}{lllllllllll}200 & 190 & 180 & 170 & 160 & 150 & 140 & 130 & 120 & \begin{array}{c}110 \\ \text { Chemical Shift (ppm) }\end{array}\end{array}$ 
NMR spectra of 1-(benzyloxy)-2,2,6,6-tetramethylpiperidine (11)

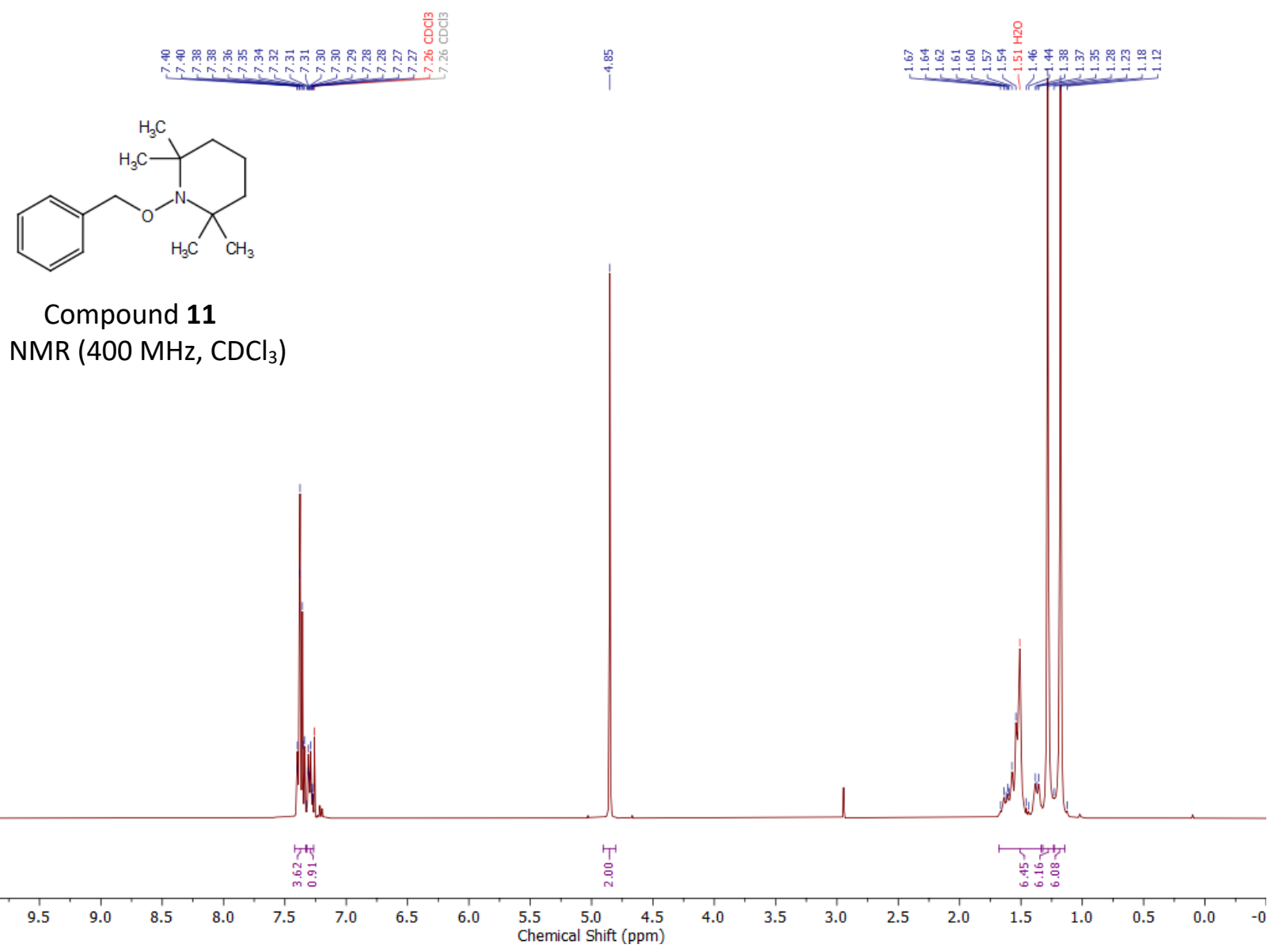

$\begin{array}{llllllllllll}.5 & 10.0 & 9.5 & 9.0 & 8.5 & 8.0 & 7.5 & 7.0 & 6.5 & 6.0 & 5.5 & 5.0 \begin{array}{r}4.5 \\ \text { Chemical Shift (ppm) }\end{array}\end{array}$

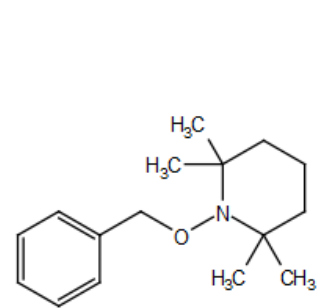

|

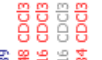

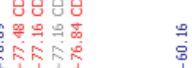

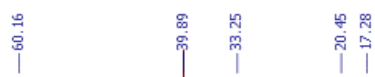

Compound 11

${ }^{13} \mathrm{C}$ NMR $\left(101 \mathrm{MHz}, \mathrm{CDCl}_{3}\right)$

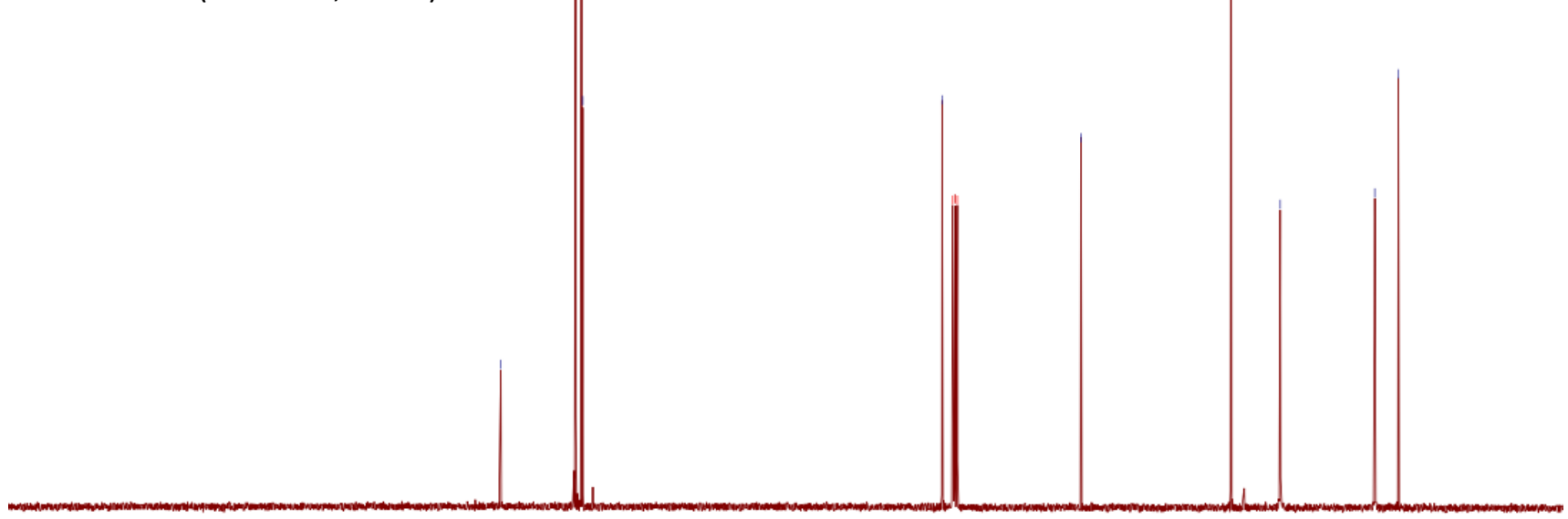

$\begin{array}{lllllllllll}200 & 190 & 180 & 170 & 160 & 150 & 140 & 130 & 120 & \begin{array}{c}110 \\ \text { Chemical Shift }(\mathrm{ppm})\end{array}\end{array}$ 
7. Chiral HPLC Traces

Benzyl (S)-4-(N-(1-methoxy-1-oxopropan-2-yl)sulfamoyl)piperidine-1-carboxylate (10c)<smiles>COC(=O)[C@H](Cc1ccccc1)NS(=O)(=O)C1CCN(C(=O)OCc2ccccc2)CC1</smiles>

ee determined by chiral SFC using a Chiralpak ${ }^{\circledR}$ IC column, 1500 psi, $30^{\circ} \mathrm{C}$; flow: $1.5 \mathrm{~mL} / \mathrm{min} ; 1-30 \% \mathrm{MeOH}$ in $5 \mathrm{~min}$, $98 \%$ ee (major enantiomer $\mathrm{tR}=4.305 \mathrm{~min}$, minor enantiomer $\mathrm{tR}=4.497 \mathrm{~min}$ ).
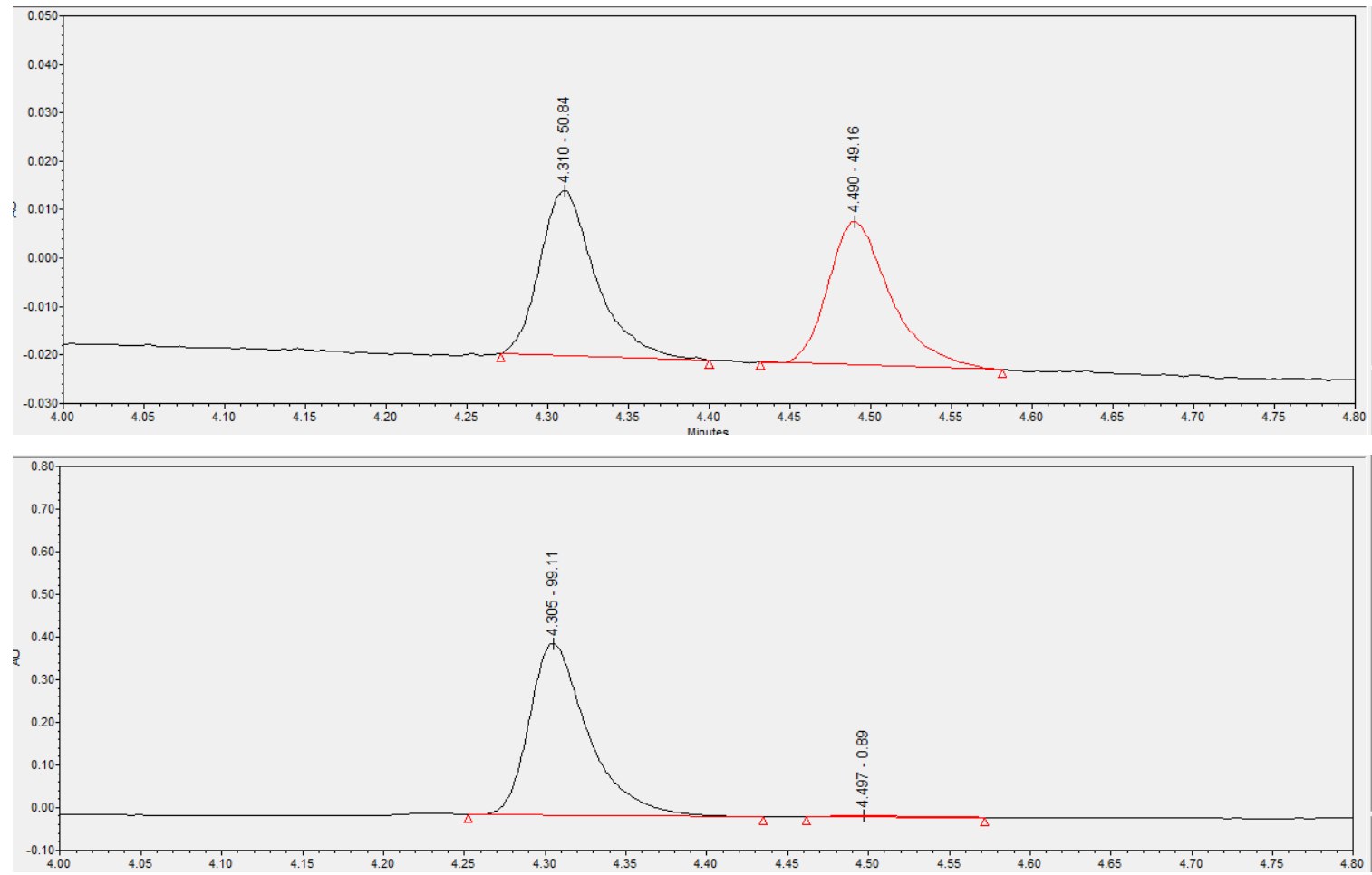

Figure S5: SFC traces of racemic $( \pm)-10 c$ (top) and enantioenriched 10c (bottom). 